Portland State University

PDXScholar

Winter 3-9-2015

\title{
Aberration Corrected Photoemission Electron Microscopy with Photonics Applications
}

Joseph P. S. Fitzgerald

Portland State University

Follow this and additional works at: https://pdxscholar.library.pdx.edu/open_access_etds

Part of the Physics Commons

Let us know how access to this document benefits you.

\section{Recommended Citation}

Fitzgerald, Joseph P. S., "Aberration Corrected Photoemission Electron Microscopy with Photonics Applications" (2015). Dissertations and Theses. Paper 2192.

https://doi.org/10.15760/etd.2190

This Dissertation is brought to you for free and open access. It has been accepted for inclusion in Dissertations and Theses by an authorized administrator of PDXScholar. Please contact us if we can make this document more accessible: pdxscholar@pdx.edu. 
Aberration Corrected Photoemission Electron

Microscopy with Photonics Applications

by

Joseph P. S. Fitzgerald

A dissertation submitted in partial fulfillment of the requirements for the degree of

Doctor of Philosophy

in

Applied Physics

Dissertation Committee:

Rolf Könenkamp, Chair

Dean Atkinson

Erik Bodegom

Andrew Fountain

Erik Sanchez

Robert C. Word

Portland State University

2015 


\begin{abstract}
Photoemission electron microscopy (PEEM) uses photoelectrons excited from material surfaces by incident photons to probe the interaction of light with surfaces with nanometer-scale resolution. The point resolution of PEEM images is strongly limited by spherical and chromatic aberration. Image aberrations primarily originate from the acceleration of photoelectrons and imaging with the objective lens and vary strongly in magnitude with specimen emission characteristics. Spherical and chromatic aberration can be corrected with an electrostatic mirror, and here I develop a triode mirror with hyperbolic geometry that has two adjacent, field-adjustable regions. I present analytic and numerical models of the mirror and show that the optical properties agree to within a few percent. When this mirror is coupled with an electron lens, it can provide a large dynamic range of correction and the coefficients of spherical and chromatic aberration can be varied independently. I report on efforts to realize a triode mirror corrector, including design, characterization, and alignment in our microscope at Portland State University (PSU). PEEM may be used to investigate optically active nanostructures, and we show that photoelectron emission yields can be identified with diffraction, surface plasmons, and dielectric waveguiding. Furthermore, we find that photoelectron micrographs of nanostructured metal and dielectric structures correlate with electromagnetic field calculations. We conclude that photoemission is highly spatially sensitive to the electromagnetic field intensity, allowing the direct visualization of the interaction of light with material surfaces at nanometer scales and over a wide range of incident light frequencies.
\end{abstract}




\section{Acknowledgments}

I would like to thank Rolf Könenkamp and Robert C. Word for significant help and guidance in all aspects of the work presented here. Specifically, Rolf supplied the original idea of investigating a three electrode mirror, and Rob provided all PEEM images presented here and technical advice. In addition, both Rolf and Rob lended valuable editorial advice and insightful scientific guidance. Furthermore, Athavan Nadarajah helped in sample preparation of gold nanoparticles, and Thomas Dornan prepared technical drawings of the triode mirror and microscope column modifications. The work reported here was supported by DOE contract number DE-FG02-13ER46406. 
Table of Contents

I Introduction $\quad 1$

II Aberration correction 4

1 Electron optics and image resolution 4

2 Resolution before aberration correction $\quad 13$

3 Aberration correction with a diode mirror $\quad 25$

3.1 Analytic model of a hyperbolic diode mirror . . . . . . . . . . . . 26

3.2 Aberration correction applied to our PEEM . . . . . . . . . . . 30

4 Dynamic aberration correction with a triode mirror 35

4.1 Analytic model of hyperbolic triode mirror . . . . . . . . . . . . . 37

4.2 Comparison to realistic model . . . . . . . . . . . . . . . . . 45

4.3 Triode mirror and lens with fixed object distance . . . . . . . . . . 48

4.4 Interacting triode mirror-lens corrector . . . . . . . . . . . . 53

5 Experimental adaptation and preparation $\quad 57$

5.1 Corrector design parameters . . . . . . . . . . . . . . . . . . 57

5.2 Numerical simulation via SIMION . . . . . . . . . . . . . . . . 59

5.3 Quality control and beam alignment . . . . . . . . . . 66

$\begin{array}{ll}\text { III Photonics } & 73\end{array}$

$\begin{array}{lll}6 & \text { Photoemission and photonics } & 73\end{array}$

7 Methods and materials for photonics $\quad 85$

7.1 Light sources . . . . . . . . . . . . . . . . . . . 85

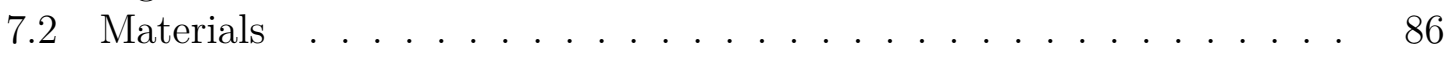

7.3 Nanostructures . . . . . . . . . . . . . . . . . . . . . . . . 90

8 Theory of electromagnetic fields as relevant to photoemission $\quad 96$

9 Photonics results \& discussion 108

9.1 Metal nanostructures . . . . . . . . . . . . . . . . . . . . . . . . . 108

9.2 Dielectric waveguide structures . . . . . . . . . . . . . . . . 114

9.3 Diffracted waves . . . . . . . . . . . . . . . . . . . 129 
IV Conclusions

V References \& Appendices

References

Appendix A Scale drawings: diode PEEM

Appendix B Code, Mathematica: hyperbolic mirror theory

Appendix C Code, Mathematica: mirror theory, updated

Appendix D Code, simion: swept-back lens

Appendix E Code, Mathematica: swept-back lens

Appendix F Code, Mathematica: objective branch

Appendix G Code, simion: diode hyperbolic mirror

Appendix H Code, simion: triode hyperbolic mirror

Appendix I Code, Mathematica: triode hyperbolic mirror

Appendix J PEEM control: triode mirror branch tables

Appendix K PEEM control: objective branch tables 261

Appendix K.1 Objective lens potential . . . . . . . . . . . . . 261

Appendix K.2 Auxiliary lens potential . . . . . . . . . . . . . . 262

Appendix K.3 Spherical aberration . . . . . . . . . . . . . . . . 262

Appendix K.4 Chromatic aberration . . . . . . . . . . . 263 


\section{List of Tables}

2.1 Resolution of realistic UV and multiphoton PEEM specimens. . . 17

2.2 Resolution with objective lens and $30 \mu \mathrm{m}$ aperture stop. . . . . . . 22

8.1 Interference wavenumbers resulting from two guided modes. . . . . 100

9.1 Waveguide mode numbers in ITO thin film. . . . . . . . . . . . . 119

9.2 Reflection distances ITO thin film. . . . . . . . . . . . . . . . . . 121 


\section{List of Figures}

1.1 Photoelectron cathode emission diagram. . . . . . . . . . . . 5

2.1 Photoelectron cathode detailed imaging diagram. . . . . . . . . . 14

2.2 Optical properties of the accelerating field. . . . . . . . . . . 15

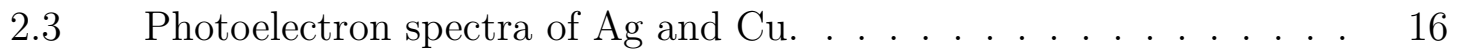

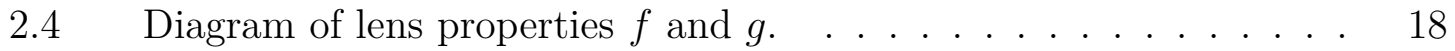

2.5 Objective lens diagram and optical properties. . . . . . . . . . . 19

2.6 Effect of the aperture stop on beam width and energy spectrum. . 21

2.7 Auxiliary lens diagram. . . . . . . . . . . . . . . 23

2.8 Total aberration coefficients of the objective branch. . . . . . . . 24

3.1 Diagram of an aberration-corrected PEEM with a mirror. . . . . . 26

3.2 Theoretical model of a diode hyperbolic electron mirror. . . . . . . 27

3.3 Plot of the optical properties of a diode hyperbolic mirror. . . . . 31

3.4 Plot of the aberration coefficient ratio of a diode hyperbolic mirror. 31

3.5 Aberration correction of diode compared to PEEM aberration. . . 34

4.1 Theoretical model of a triode hyperbolic electron mirror. . . . . . . 37

4.2 Plots of diode and triode mirror optical properties. . . . . . . . . . 41

4.3 Plots of diode and triode mirror aberration ratios. . . . . . . . . . 41

4.4 Triode mirror optical properties can be adjusted with geometry. $\quad 42$

4.5 Aberration coefficients of a triode mirror for fixed $\ell / \mathcal{L}$ and $z_{0} / \mathcal{L}$. . 44

4.6 Model of a triode mirror. . . . . . . . . . . . . . . . . 45

4.7 Comparison of the analytic and simulated triode models. . . . . . 49

4.8 Triode mirror optical properties and potentials, fixed image. . . . 50

4.9 Mirror-lens system diagrams. . . . . . . . . . . . . . . . . 52

4.10 Triode mirror optical properties and potentials, variable image. . . 54

5.1 Comparison of diode and triode correctors as installed. . . . . . 58

5.2 Electric potential within the triode corrector in SIMION. . . . . . . 61

5.3 Triode mirror real images have limited voltage ranges. . . . . . . . 62

5.4 Aberration correction range of the triode mirror corrector. . . . . 64

5.5 Aberration correction of triode compared to PEEM aberration. . . 65

5.6 Triode mirror parts and assembly. . . . . . . . . . . . 66

5.7 Interface lens and spacer atop the triode mirror. . . . . . . . . 67

5.8 Diode and triode assemblies. . . . . . . . . . . . . . . 67

5.9 Electron gun straight bench with triode mirror and lens. . . . . . . 68

5.10 Electron gun straight bench testing of the triode mirror. . . . . . . 69

5.11 Numerical studies of the triode mirror as a lens in the straight bench. 69

5.12 Interface lenses from diode and triode installations. . . . . . . . . 70 
5.13 Images of the triode mirror without the grounded electrode. . . . . 71

5.14 First images from the triode PEEM. . . . . . . . . . . . . 72

6.1 Energy level diagram representation of the photoelectric effect. . . 75

6.2 Graphical representation of the photoelectric effect interaction. . . 77

6.3 Two-photon excitation pathways in metals. . . . . . . . . . 81

6.4 Dielectric slab model. . . . . . . . . . . . . . . . . . . 83

7.1 Schematic representation of sample conditions in PEEM. . . . . 86

$\begin{array}{lll}7.2 & \text { Dispersion relationship for surface plasmons in ITO with vacuum. } 88\end{array}$

7.3 Polycrystalline gold thin film on ITO-glass substrate. . . . . . . . 89

7.4 Single-crystalline gold nanoflakes. . . . . . . . . . . . . 91

$\begin{array}{lll}\text { 7.5 Random FIB patterns milled into Au deposited on ITO thin film. } & 92\end{array}$

7.6 FIB fabrication of high-quality plasmonic structures. . . . . . . . 93

7.7 Straight and curved FIB-milled grooves in ITO-glass. . . . . . . 94

7.8 SEM image of Au platelets on ITO with a diffracting groove. . . . 95

8.1 Model for stationary interference pattern in PEEM. . . . . . . . 98

8.2 Solutions of the asymmetric slab model for vacuum/ITO/glass. . . 102

8.3 SPP modes of a vacuum/Au/ITO slab model. . . . . . . . . . 104

8.4 Optical excitations are coupled to incident light via diffraction. . . 105

9.1 Plasmonic hot spots on gold random nanostructures in PEEM. . . 109

9.2 Intensity and photon-energy dependence of photoemission yields. . 109

9.3 Hot spots emit from ITO in gaps of the Au nanostructure. . . . . 110

9.4 Au Y-structures with 410-nm and $\pm 45^{\circ}$-polarized light in PEEM. . 112

9.5 Au Y-structures with 780/244-nm and $\pm 45^{\circ}$ polar. light in PEEM. 113

9.6 PEEM images of diffracting ITO structure. . . . . . . . . . . 115

9.7 Interference pattern profiles near the diffracting ITO structure. . . 117

9.8 Fourier transforms of patterns near the diffracting ITO structure. 118

9.9 Waveguide mode numbers in ITO thin film. . . . . . . . . . . 120

9.10 PEEM micrographs of a waveguide bounded by a circular groove. 123

9.11 PEEM micrographs of a semicircle of holes. . . . . . . . . . . . 124

9.12 Experimental and simulated 2PPE patterns near a single hole. . . 125

9.13 2PPE line profiles across a single $0.5-\mu \mathrm{m}$ hole. . . . . . . . 125

9.14 Calculated coupling efficiency \& 2PPE pattern for the disc. . . . . 127

9.15 Calculated 2PPE pattern for the holes. . . . . . . . . . . . 128

9.16 UV-PEEM image of diffraction on Si. . . . . . . . . . . . . . . . 129

9.17 UV-PEEM images of diffraction on Si compared to calculations. . 131

9.18 2P-PEEM image of diffraction on Au triangle platelet. . . . . . . . 132

9.19 PEEM images of Au platelets on ITO with a diffracting groove. . 132 


\section{Introduction}

Photoemission electron microscopy (PEEM) has long been an invaluable tool in investigating physics at the surfaces of materials. PEEM has played an intrinsic and diverse role in high resolution microscopy, from pioneering work in early electron microscopy [1] to understanding biological proteins [2, 3], magnetic domains [4-7], and recent studies of plasmonic structures [8-48]. PEEM has had such wide-ranging impact due to a simple and versatile apparatus, relatively intuitive image interpretation, and an extensive breadth of accessible phenomena, with different photon energies highlighting different material processes and structures.

In photoemission, an electron can be excited with a single UV or higher energy photon or with multiple $(n)$ lower energy photons, where the number of photons satisfies the energy requirement $n \times(h c / \lambda)>W$, and $W$ is the photoemission threshold energy at the surface $[49,50]$. The quantum efficiency of photoemission with photons above the photoemission threshold is $\sim 10^{-4}[51]$. At longer wavelengths, the probability decreases with the power of $n$, necessitating high electromagnetic field intensities for visible and infrared excitation photons. Photoelectrons are excited with little excess energy, and primarily unscattered photoelectrons are emitted to the vacuum [52], limiting the emission depth to less than a nanometer in most cases [53]. Spatially varying photoemission rates are indicative of the underlying surface structures and processes, revealing surface topology, material and threshold differences, electron densities, electromagnetic interactions, as well as other contrasts, with the dominant process often varying strongly with incident photon energy [8, 54-56]. With such a wide variety of influences, the spatial and spectral distributions of photoelectrons can reveal significant information about the surface environment. 
Photoemission electron microscopy is concerned with the spatial distribution of the photoelectron emission yield. In PEEM, photoelectrons are accelerated away from the surface, collimating the electrons into a beam with a cross-sectional distribution preserving relative emission site locations. The beam is then focused with electron lenses, which magnifies the emission pattern, and photoelectrons register on a phosphor screen or other device to generate an image of the photoemission yield. Since electrons are used to create the image, the fundamental resolution is unaffected by the diffraction limit of the light used to illuminate the sample [54]. Instead, the electron de Broglie wavelength, the nature of the emission process, and the quality of the electron optical system set the resolution limit for PEEM. Electron lenses, which focus electrons with electric or magnetic fields, provide high magnification images but also introduce significant image aberrations that limit the maximum width of the aperture angle and the range of photoelectron energies that can used to produce a focused imaged. As a result, resolution is typically worse than than $20 \mathrm{~nm}$. Addressing aberration with multipole lenses or a mirror could significantly improve resolution to as little as 1-3 $\mathrm{nm}[57,58]$.

Using light to excite electrons, PEEM is at the intersection of light optics and electron microscopy. An intuitive application of aberration-corrected PEEM is nanoscale photonics. Here the advantages of PEEM can be used to great potential, naturally combining sensitivity to surface electromagnetic fields with the resolution of an electron microscope for visualization of light as it interacts with surfaces of photonic structures. To achieve this goal one must overcome the inherent challenges of singleand multi-photon photoemission electron microscopy: low electron emission rates and the imperfect focusing of electron lenses. This can be done by utilizing high intensity light sources and by introducing aberration correction to improve the quality of the electron images, gaining brightness, contrast, and resolution. In realizing these 
targets, we increase the scope of PEEM applications by developing a tool well suited to the investigation of light at material surfaces at the smallest scales, with broad applicability to weak and strong field phenomena alike.

In the next two parts, I describe two parallel efforts to advance the optical performance of PEEM and to apply PEEM to the the study of photonics. First, Part II Aberration correction investigates the resolution limit of PEEM with improved correction of spherical and chromatic aberrations. This begins with a discussion of how image aberrations arise in the acceleration and focusing of focusing of photoelectrons and the impact on micrograph point resolution. Then several models of aberration correction with a mirror are reviewed, followed by the full analytic derivation of the optical properties of a hyperbolic geometry with two variable potentials. When this triode mirror model is combined with an electron lens, aberration correction can be adjusted to match image aberrations, which vary with sample emission characteristics. Subsequently, I describe the optimization and characterization of a design of the triode mirror and lens corrector specifically tailored to our PEEM, replacing the previous diode-based corrector, as well as initial alignment efforts. Second, Part III Photonics explores the relationship between photoelectron emission and optical excitations in the surface near-field. This begins with an outline of the photoemission process and a basic theory relating the surface electromagnetic field intensity and the photoelectron yield. Then I relate the theory to experiments, starting with metals and high-optical-intensity, plasmon-enhanced photoemission and progressing to the optically weaker phenomena of diffraction and dielectric waveguides. Throughout, the incident light wavelength, intensity, and polarization play critical roles as the primary variables of investigation of the photoemission response. 


\section{Aberration correction}

\section{Electron optics and image resolution}

The smallest feature that can be resolved by a traditional optical microscope is determined by the wavelength and aperture angle of the imaging beam

$$
R_{d}=0.61 \lambda / \sin \alpha_{\max }
$$

where $R_{d}$ is the spatial resolution, $\lambda$ is the wavelength of the imaging beam, and $\alpha_{\max }$ is the aperture angle of the lens [59]. In electron microscopy, the wavelength is determined by the de Broglie relation for electrons $\lambda=h / p$, where $h$ is Planck's constant and $p$ is the electron momentum. In terms of the electron kinetic energy $E=e V_{a}$ the wavelength is

$$
\lambda=h c / \sqrt{E^{2}+2 E \cdot m_{e} c^{2}},
$$

where $e$ and $m_{e}$ are the charge and rest mass of an electron, and $c$ is the speed of light. The energy of electrons used in PEEM is primarily set by the potential difference between the sample held at the $V_{a}=-V_{C}$ and a grounded anode a distance $\ell_{a}$ away, as illustrated in Fig. 1.1. For the $20 \mathrm{kV}$ potential difference used in our instrument, the wavelength of electrons is $0.0086 \mathrm{~nm}$. The accelerating field and anode aperture collimates emitted electrons into a beam with angular width

$$
\alpha_{\max }=\sqrt{V_{e} / V_{a}},
$$




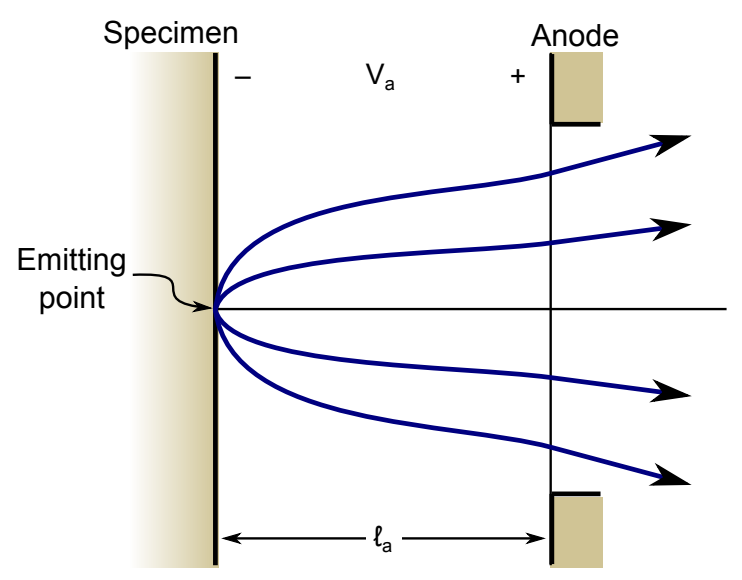

Figure 1.1: (a) Trajectories of electrons emitted from a point on the axis showing the curved paths in the accelerating region and the diverging action of the aperture lens. Radial distances have been exaggerated. Adapted from Rempfer and Griffith [57].

where $e V_{e}$ is the emission energy, $e V_{a}$ is the accelerating energy, and the small angle approximation is used [57]. In UV and multiphoton PEEM, the electrons are emitted with less energy than the photoemission threshold. For example, $1 \mathrm{eV}$ photoelectrons accelerated to $20 \mathrm{keV}$, as in our PEEM, have an aperture angle of $7 \mathrm{mrad}$ or $0.4^{\circ}$. Taking the electron wavelength and aperture angle into account, the resolution of a photoelectron micrograph composed of electrons emitted at $1 \mathrm{eV}$ and accelerated to $20 \mathrm{kV}$ is $0.75 \mathrm{~nm}$ when considering electron diffraction alone.

However, PEEM is far from realizing the resolution inherent to the electron beam. Electron lenses, which focus electrons with electric or magnetic fields, introduce image aberrations that reduce the quality of images. In an electron beam with a range of emission angles, the point resolution is determined not only by the diffraction limit, Eq. 1.1, but also by spherical aberration,

$$
R_{s}=C_{s} \alpha_{\max }^{3}
$$

where $C_{s}$ is the coefficient of spherical aberration. Using simple trigonometry and the 
small angle approximation, the coefficient can be defined by its effect on the image distance,

$$
z_{0}^{\prime}-z^{\prime}=C_{s} \alpha^{2},
$$

where $z^{\prime}$ is the image distance of an electron trajectory originating from an object with angle $\alpha$ to the optical axis, and $z_{0}^{\prime}=\lim _{\alpha \rightarrow 0} z^{\prime}$ is the paraxial image distance. A range of emission energies $e V_{e}$ similarly produces a point resolution governed by chromatic aberration,

$$
R_{c}=C_{c}(\Delta E /\langle E\rangle) \alpha_{\max },
$$

where $\langle E\rangle=e\left(\left\langle V_{e}\right\rangle+V_{a}\right)$ is the mean electron energy, $\Delta E=E-\langle E\rangle$ is the difference in energy from the mean, and $C_{c}$ is the coefficient of chromatic aberration. This coefficient too can be defined along the optical axis,

$$
z_{0}^{\prime}-\left\langle z_{0}^{\prime}\right\rangle=C_{c}(\Delta E /\langle E\rangle),
$$

where $\left\langle z_{0}^{\prime}\right\rangle$ is the paraxial image distance of the mean electron energy. Imaging errors due to lens aberrations combine with diffraction, limiting the point resolution to approximately

$$
R^{2}=R_{d}^{2}+\left(R_{s} / 4\right)^{2}+\left(R_{c} / 2\right)^{2} .
$$

The factors of $1 / 4$ and $1 / 2$ reflect that the aberration is evaluated not at the Gaussian plane, where Eqs. (1.4)-(1.7) are defined, but at the circles of least confusion optimized for spherical and chromatic aberration separately [60]. The axial locations of the spherical and chromatic circles of least confusion are generally not coincident, so the prefactors will typically be different than those given here. Nonetheless, Eq. 1.8 provides a simple and reasonably accurate estimate of resolution that compares favorably with more complicated methods [61]. 
Equations 1.5 and 1.7 represent the lowest order relationship of focus plane deviations with changes in ray image angle and energy. Higher order coefficients are possible with deviations proportional to $\alpha^{2 n-1}$ or $\alpha^{2 n-1}(\Delta E / E)^{m}$, where $m, n \in$ natural numbers. When including these coefficients it is common to denote $C_{s}$ as $C_{3}$; then higher order spherical aberration coefficients are $C_{5}$ (for the $\alpha^{5}$ term), $C_{7}\left(\alpha^{7}\right.$ term), etc., and higher order chromatic coefficients are $C_{3 c}$ (for the $\alpha^{3} \Delta E / E$ term), $C_{c c}$ (for the $\alpha(\Delta E / E)^{2}$ term), etc. These terms become increasingly important at wider aperture angles, with wider energy spectra, or at very high spatial resolution. Higher order terms are explicitly compensated in aberration corrected TEMs, which can resolve single atoms in a lattice spaced less than $0.1 \mathrm{~nm}$ apart [62,63], but are neglected in this discussion. With a normal distribution of emission energies spread out over $\Delta E=1 \mathrm{eV}$, typical aberration coefficients in the range $C_{s} \approx C_{c} \approx 0.4 \mathrm{~m}$ [64], and at an optimally limited aperture angle of $0.7 \mathrm{mrad}$, this formula predicts a best resolution of $10 \mathrm{~nm}$, an order of magnitude greater than the diffraction limit. Including higher order aberration terms, beam coherence, and microscope instability, which will be much greater with the reduced aperture, raises this limit to $15 \mathrm{~nm}$ [61], as experimentally reported by Griffith and Rempfer [65]. Clearly aberration has an effect on imaging in PEEM, so it is desirable to minimize or eliminate the magnitude of spherical and chromatic aberration in order to improve resolution. Correcting aberration also allows imaging with a larger aperture acceptance angle, which is of particular importance to multiphoton PEEM where the photoelectron yield can be quite limiting and the spectrum of emission energies can be relatively large.

With the exception of Eqs. (1.2) and (1.3), the formulae in this discussion apply equally well to electron and light microscopy. In light microscopy, methods of aberration correction were developed long ago by combining glasses with different shapes and refractive indices. Correcting the aberrations of glass lenses has developed such 
that diffraction is the main limitation, and Eq. 1.1 can be used to directly estimate image resolution. However, refraction in electron optics is qualitatively different; electron trajectories change direction continuously whereas light rays do so discretely at lens surfaces. In light optics, the aberration coefficient of multiple refractions is the sum of the aberration of each individual refraction,

$$
C_{\mathrm{tot}}=\sum_{j}^{n} a_{j} C_{j} \text {, }
$$

where the weighting coefficients $a_{j}$ depend on changes in magnification and effective refractive index $[60,66]$. For a continuous refraction, the aberration coefficients of a lens in electron optics are best expressed as integrals over the axial potential $V=$ $V(z)$. Munro [67] gives computationally useful expressions for electrostatic lenses,

$$
\begin{aligned}
C_{s}= & \frac{1}{16 r_{i}^{\prime 4} V_{C}^{1 / 2}} \int_{o}^{i}\left\{\left[\frac{5}{4}\left(\frac{V^{\prime \prime}}{V}\right)^{2}+\frac{5}{24}\left(\frac{V^{\prime}}{V}\right)^{2}\right] r^{2}+\frac{14}{3}\left(\frac{V^{\prime}}{V}\right)^{3} r^{\prime} r\right. \\
& \left.-\frac{3}{2}\left(\frac{V^{\prime}}{V}\right)^{2} r^{\prime 2} r^{2} V^{1 / 2}\right\} d z, \\
C_{c}= & \frac{V_{C}^{1 / 2}}{r_{i}^{\prime 2}} \int_{o}^{i}\left\{\left(\frac{V^{\prime}}{2 V}\right) r^{\prime}+\left(\frac{V^{\prime \prime}}{4 V}\right) r\right\} \frac{r}{V^{1 / 2}} d z,
\end{aligned}
$$

where $z$ is the axial position, taken as the sole independent variable, integrated from the location of the object $o$ to the image $i ; r=r(z)$ is the electron trajectory radial position; $e V_{C}$ and $r_{i}$ are the electron energy and radial position at the image; and the zero potential is chosen such that $V>0$ for all $z$. Through integration by parts, the 
integrands can be recast as [68]

$$
\begin{aligned}
C_{s}= & \frac{1}{32 V_{C}^{1 / 2}} \int_{o}^{i}\left\{\left[\frac{V^{\prime \prime}}{V}+\frac{V^{\prime}}{V} \frac{r^{\prime}}{r}-\frac{5}{4}\left(\frac{V^{\prime}}{V}\right)^{2}\right]^{2}+\frac{3}{2}\left[\frac{V^{\prime \prime}}{V}+\frac{V^{\prime}}{V} \frac{r^{\prime}}{r}-\left(\frac{V^{\prime}}{V}\right)^{2}\right]\right. \\
& \left.+2\left(\frac{V^{\prime}}{V}\right)^{2}\left(\frac{r^{\prime}}{r}+\frac{5}{6} \frac{V^{\prime}}{V}\right)^{2}+\frac{1}{36}\left(\frac{V^{\prime}}{V}\right)^{4}\right\} V^{1 / 2} r^{4} d z, \\
C_{c}= & \frac{3 V_{C}^{1 / 2}}{8} \int_{o}^{i}\left(\frac{V^{\prime}}{V}\right)^{2} \frac{r^{2}}{V^{1 / 2}} d z .
\end{aligned}
$$

For a derivation of these formulas, I refer the reader to any number of good textbooks [69-72], which also present formulae for magnetic lenses. The integrands in the previous formulas are a sum of squared terms, and subsequently the coefficients are positive definite. Thus, aberration in electron lenses is unavoidable: all standard electron lenses have positive spherical and chromatic aberrations. This result was originally derived for magnetic lenses by Scherzer [73]. The Scherzer theorem can be bypassed by breaking rotational symmetry (multipole lenses, cf. [74-78]), placing charge on the optical axis (mesh or foil lenses, cf. [74, 79-81]), reflecting electron trajectories so that $r(z)$ is no longer a one-to-one function (mirrors, cf. [64, 74, 82-84]), or using time-varying fields (cf. $[74,85,86]$ ). All of these approaches have seen recent development, though only multipole and mirror correctors have achieved notable success. Specifically, hexapole and quadrupole-octupole lenses have proven successful for correcting spherical aberration [76, 77], and more recently a system of quadrupole lenses has been employed to correct chromatic aberration [78] (though without improved resolution [87]). These systems use a sophisticated computer system to control the numerous electrode potentials and adapt aberration correction.

While spherical aberration affects all electron microscopies, PEEM, low energy electron microscopy (LEEM), and other emission or low energy electron microscopies must also deal with a relatively large range of electron energies and cannot neglect 
chromatic aberration. Electrostatic mirrors can be designed with both spherical and chromatic aberration coefficients of the opposite sign to electron lenses, opening the possibility for their use as aberration correctors [73]. To obtain correction, the electron beam is incident and reflected along the same axis, necessitating a magnet to separate the two beams. Despite early troubles, as presented by Ramberg [88] and others, Rempfer [64] constructed an analytic description of electron trajectories in a diode (two-electrode) mirror with a hyperbolic field distribution and subsequently showed that such a mirror can be employed to achieve spherical and chromatic aberration correction. Indeed, electron mirrors have proven experimentally successful at correcting spherical and chromatic aberration simultaneously [82, 89-92].

The theoretical foundations of these systems are mirrors with two or four electrodes [64, 93], and success at correcting aberration is determined by the number of independent parameters and accurate determination of instrument aberration. A diode mirror is designed to simultaneously correct spherical and chromatic aberration through appropriate choices of its length, spacing from other elements, and operating potential. Choices made during the design stage confine the parameter space to a narrow path traversed by a single potential. Furthermore, the lone degree of freedom link spherical and chromatic aberration correction - they cannot be varied independently. As a result, the correction offered by the diode mirror becomes locked into one optimal potential. This situation is undesirable since aberration may not be well known in the first place, and it is also may change with operating conditions, e.g., sample positioning, surface topology, and particularly photoelectron energy distribution. A three electrode (triode) mirror could potentially address this issue by introducing a second adjustable potential. Subsequently, the triode mirror can simultaneously and independently correct spherical and chromatic aberration, although not without also changing focal length of the mirror significantly. If focal length is held constant, dy- 
namic adjustment may be limited to a small range around an extremum in either spherical or chromatic aberration [94]. Thus, to achieve the full range of adaptive aberration correction a lens must be used in conjunction with a mirror to maintain constant focus on the beam separating magnet. This is no real problem since in practice a lens is nearly always paired with a mirror to control image distance to the beam-separating magnet and to set magnification. An interactive triode mirror and lens corrector involves three variable electrode potentials. Alternatively, these elements can be built into a single, four-electrode (tetrode) mirror. This device has been studied numerically $[90,93,95]$ and successfully incorporated into a LEEM for record resolution [91, 92], though PEEM resolution was not particularly improved. It was found that three adjustable potentials made possible simultaneous, independent, and dynamic manipulation of all three optical properties, $z_{0}, C_{s}$, and $C_{c}$.

However, without an analytic form of the electron trajectories and subsequently derived optical properties to inform design, it is difficult to develop physical intuition for the correcting behavior and the device cannot be readily optimized for other instruments. Without physical insight, the mirror will be difficult to operate; and without optimization capability, it may be difficult to adapt the aberration corrector to other instruments. Therefore I propose to a similar mirror-based corrector incorporating three adjustable electrode potentials, to give fully adaptable correction of spherical and chromatic aberration, but rooted in an analytic description that lends itself to optimization so that it can be used to improve upon an existing microscope. A firm analytic foundation can be found in the mirror described by Rempfer [64], which serves as a starting point here. In the following chapters, I begin with a description of electron optical aberration in PEEM, particularly that of the cathode and objective lens. I then discuss the correction scheme currently implemented in our PEEM. Subsequently, I introduce a compound, three-variable electrostatic mirror based on 
two hyperbolic fields and demonstrate its potential for providing adaptive aberration correction. Then I discuss the design, testing, and implementation of the mirror corrector, which requires high-precision control of the PEEM, with work currently in progress. 


\section{Resolution before aberration correction}

Several types of aberration potentially degrade image resolution [66], of which defocus, astigmatism, coma, and distortion can be corrected in electron microscopes. In UV and multiphoton PEEM, the next most severe aberrations are chromatic and spherical aberration of the accelerating field and the spherical aberration of the ob-

jective lens [57]. The aberrations of the accelerating field result from variations in image location with photoelectron emission angle, $\Delta_{s} z^{\prime}=\left(z^{\prime}-z_{0}^{\prime}\right)_{\text {const } E}$, and emission energy, $\Delta_{c} z_{0}^{\prime}=z_{0}^{\prime}-\left\langle z_{0}^{\prime}\right\rangle_{E}$. From Rempfer and Griffith [57], the virtual image is located a distance

$$
z_{a}^{\prime}=2 \ell_{a}\left(1-\sqrt{V_{e} / V_{a}}\right) \cos \alpha_{e}
$$

behind the anode plane (Fig. 1.1 and 2.1), where $\alpha_{e}$ is the photoelectron emission angle from a flat specimen surface relative to normal. Equation (1.3) gives the maximum angle when the beam is not limited by an aperture stop. At emission angles $\alpha_{e} \leq \pi / 2$, the trajectory angle after acceleration $\alpha_{a}$ varies with both emission energy and emission angle,

$$
\alpha_{a}=\sqrt{V_{e} / V_{a}} \sin \alpha_{e} .
$$

The paraxial image location is

$$
z_{a, 0}^{\prime}=2 \ell_{a}\left(1-\sqrt{V_{e} / V_{a}}\right) .
$$

The leading order difference between the image distance and its paraxial limit gives the spherical aberration coefficient

$$
C_{s, a}=\ell \sqrt{V_{a} / V_{e}}
$$




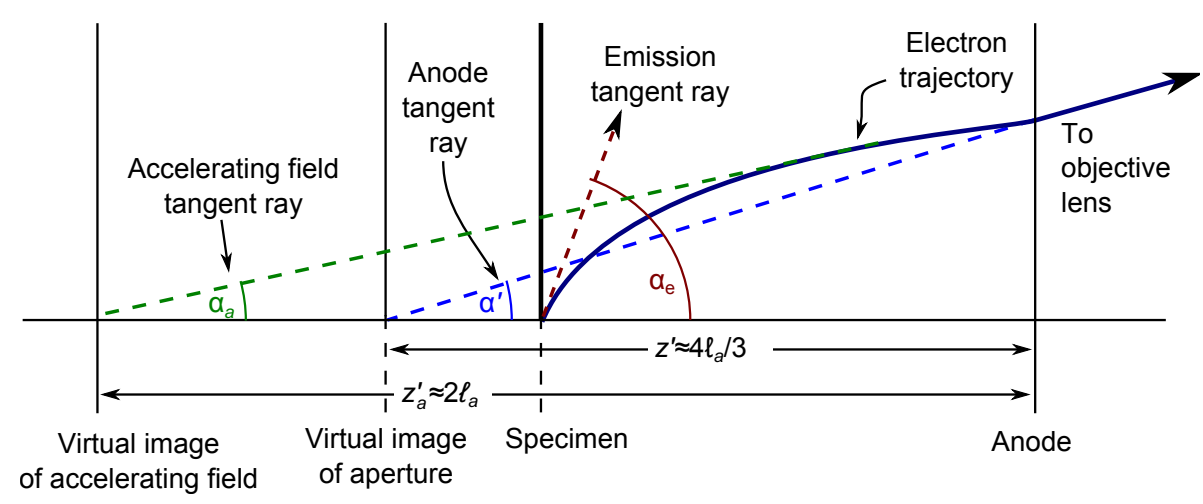

Figure 2.1: Detail showing a trajectory and a tangent ray defining the position of the virtual specimen before and after deflection by the anode aperture at the distances $z_{a}^{\prime} \approx 2 \ell_{a}$ and $z^{\prime} \approx 4 \ell_{a} / 3$ from the anode. The aperture deflection forms an image with magnification $2 / 3$ relative to the accelerating field image. The initial, intermediate, and final tangents make angles $\alpha_{e}, \alpha_{a}$, and $\alpha^{\prime}$ with the axis, respectively. Adapted from Rempfer and Griffith [57].

at a particular emission energy $e V_{e}$. The chromatic aberration coefficient is evaluated directly from Eq. 1.7. Plots of $z_{a, 0}^{\prime}, \alpha_{a}$, and $C_{s}$ are given in Fig. 2.2. The aperture angle, aberration coefficients, and point resolution can be calculated from these formulas and the axial aberration coefficient definitions. Mean values are obtained by averaging over the photoelectron energy distribution. For example, with UV emission from $\mathrm{Cu}$ at $257 \mathrm{~nm}$, the photoelectron beam mean aperture angle is $5 \mathrm{mrad}$, the mean spherical and chromatic aberration coefficients of $810 \mathrm{~mm}$ and $800 \mathrm{~mm}$, respectively, and the estimated point resolution of $28 \mathrm{~nm}$. Similar calculations for the photoelectron spectra in Fig. 2.3 are summarized in Table 2.1. The anode aperture deflects electron trajectories further from the optical axis, forming a demagnified image a distance $z_{0}^{\prime}=4 \ell_{a} / 3$ from the anode plane, as illustrated in Fig. 1.1. The lateral magnification of the image is $m_{a}=2 / 3$, such that a ray exiting the aperture makes an angle with the axis of $\alpha^{\prime}=3 \alpha_{a} / 2$. This deflection does not contribute significantly to the aberration [57].

The virtual image produced by the accelerating field and anode aperture serves 


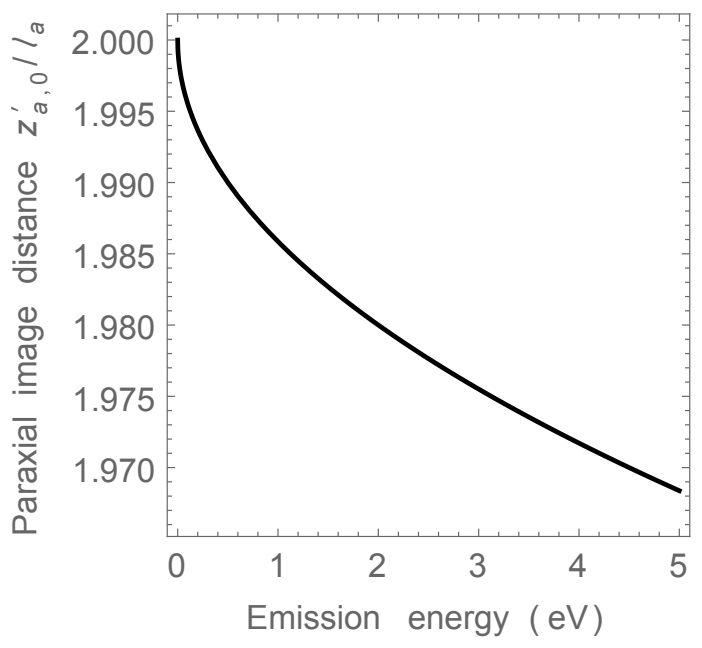

(a)

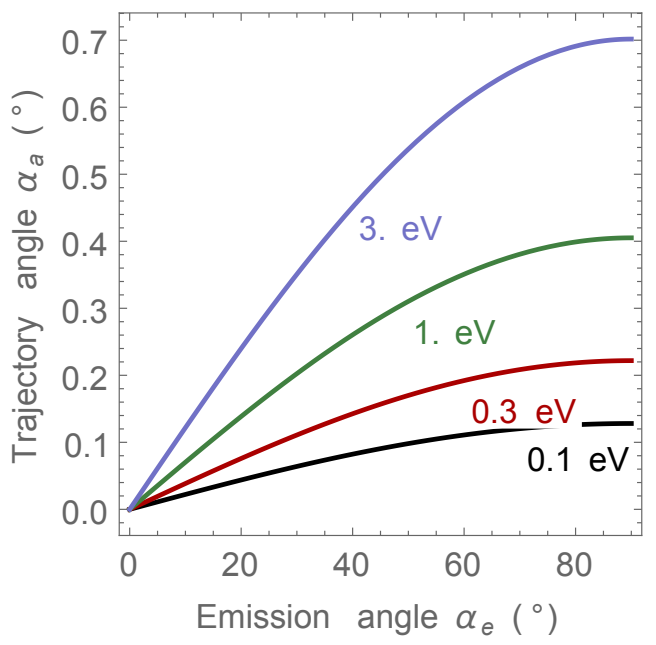

(b)

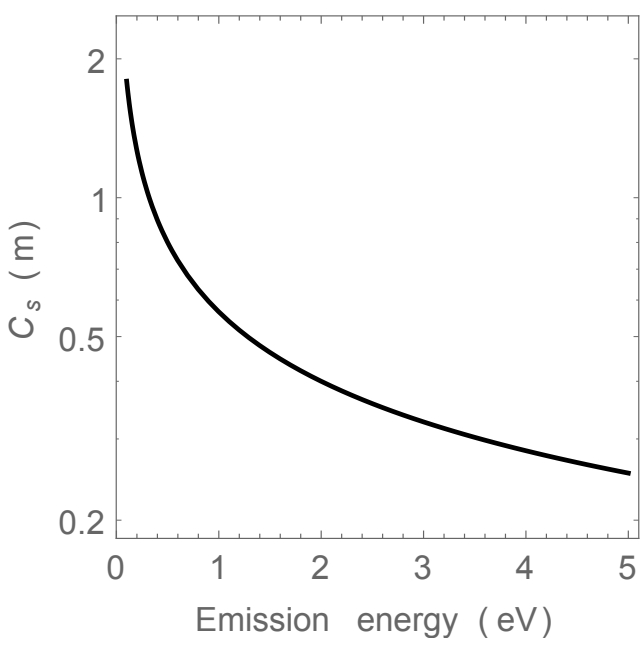

(c)

Figure 2.2: (a) The paraxial image distance of photoelectrons accelerated by a $V_{a}=20$ $\mathrm{kV}$ potential as a function of emission energy $V_{e}$ in units of cathode-anode spacing $\ell_{a}$. (b) The trajectory angle of photoelectrons emitted at the angle $\alpha_{e}$ with energy $V_{e}$, as indicated by the contour labels, and accelerated to $20 \mathrm{kV}$. (c) The spherical aberration coefficient of $20 \mathrm{kV}$ photoelectrons. 


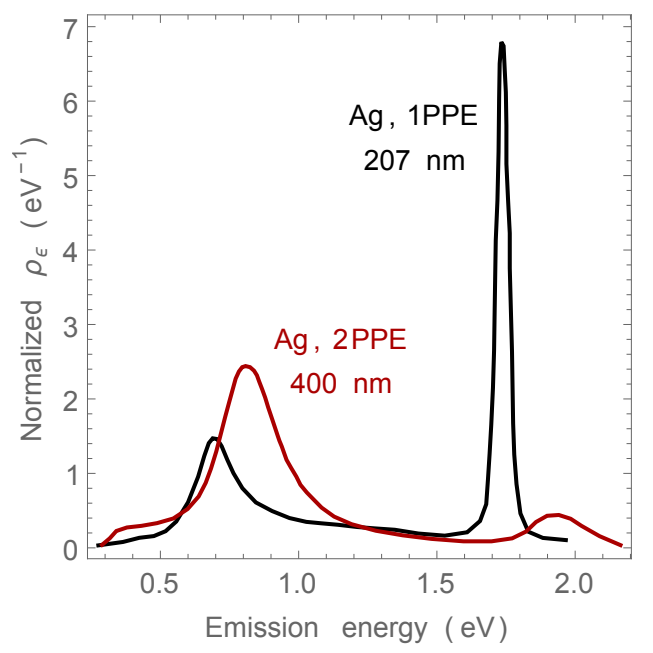

(a)

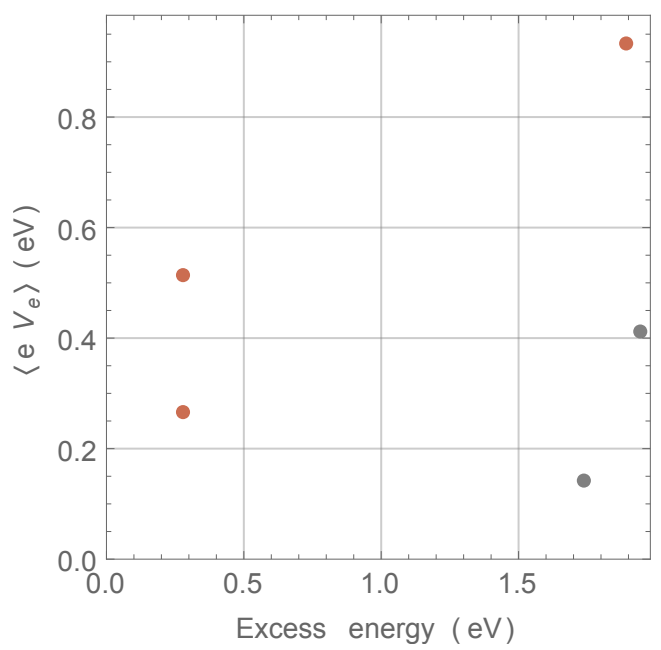

(c)

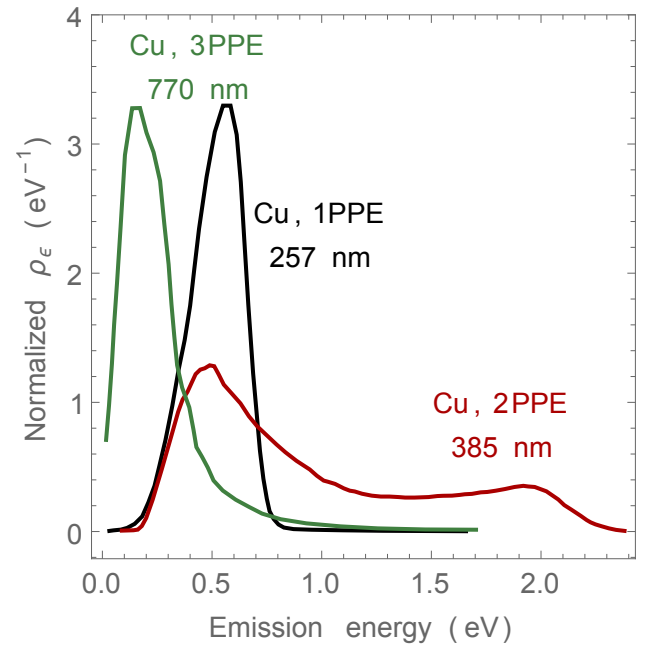

(b)

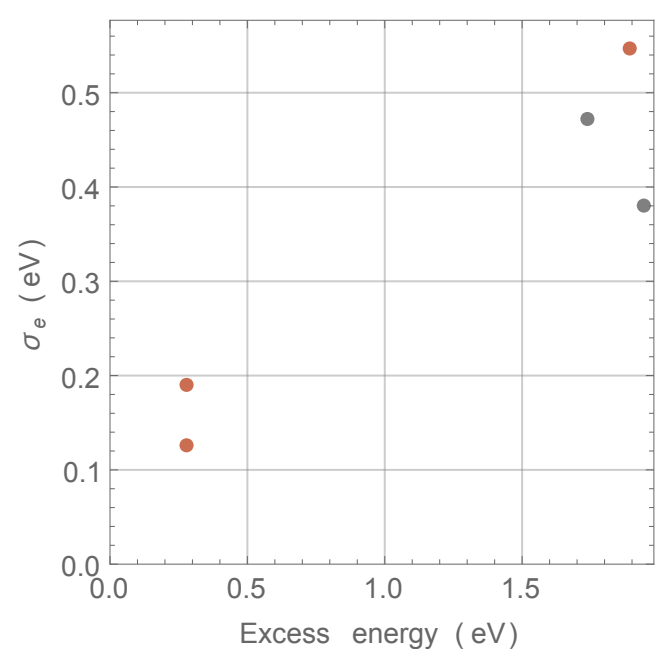

(d)

Figure 2.3: Photoelectron spectra of (a) silver and (b) copper. Photon energies are $0.3 \mathrm{eV}$, for $\mathrm{Cu} 1$ - and 3-PPE, and 1.7-2 eV for others in excess of the material photoemission threshold, which is $4.5 \mathrm{eV}$ for $\mathrm{Cu}$ and 4.3 for Ag. Statistical properties of the spectra, (c) the mean emission energy $\left\langle e V_{e}\right\rangle$ and (d) distribution width $\sigma_{e}$, are plotted against the excess energy. Adapted from Refs. [96-98]. 


\begin{tabular}{|c|c|c|c|c|c|}
\hline Material & $n \mathbf{P P E}$ & $\lambda(\mathrm{nm})$ & $\left\langle\alpha_{a}\right\rangle(\operatorname{mrad})$ & $\left\langle C_{s}\right\rangle(\mathbf{m})$ & $\left\langle C_{c}\right\rangle(\mathbf{m})$ \\
\hline $\mathrm{Ag}$ & 1 & 207 & 8.0 & 0.5 & 0.5 \\
\hline $\mathrm{Ag}$ & 2 & 400 & 7.0 & 0.6 & 0.6 \\
\hline $\mathrm{Cu}$ & 1 & 257 & 5.0 & 0.8 & 0.8 \\
\hline $\mathrm{Cu}$ & 2 & 385 & 6.5 & 0.7 & 0.6 \\
\hline $\mathrm{Cu}$ & 3 & 770 & 3.5 & 1.3 & 1.2 \\
\hline
\end{tabular}

(a)

\begin{tabular}{ccccc}
$\mathbf{M a t} / n \mathbf{P}$ & $\left\langle R_{d}\right\rangle(\mathbf{n m})$ & $\left\langle R_{s} / 4\right\rangle(\mathbf{n m})$ & $\left\langle R_{c} / 2\right\rangle(\mathbf{n m})$ & $\langle R\rangle(\mathbf{n m})$ \\
\hline $\mathrm{Ag} / 1$ & 0.7 & 63 & 44 & 79 \\
$\mathrm{Ag} / 2$ & 0.8 & 48 & 27 & 58 \\
$\mathrm{Cu} / 1$ & 1.1 & 26 & 10 & 28 \\
$\mathrm{Cu} / 2$ & 0.9 & 47 & 46 & 68 \\
$\mathrm{Cu} / 3$ & 1.7 & 13 & 13 & 20
\end{tabular}

Table 2.1: Aperture angles, aberration coefficients, mean point resolution contributions, and total point resolution of the photoelectron spectra given in Fig. 2.3. The brackets denote the mean emission energy value, $\langle x\rangle=\int x \rho(E) d E$, where $\rho$ is the normalized photoelectron energy distribution.

as the object of the objective lens. The aberration of the objective lens image is

$$
\Delta z_{o}^{\prime}=\left(1+m_{o}^{2}\right) \Delta g_{o}+2 m_{o} \Delta f_{o}+m_{o}^{2} \Delta z
$$

where $m_{o}=\alpha^{\prime} / \alpha_{o}^{\prime}=m_{a} \alpha_{a} / \alpha_{o}^{\prime}$ is the paraxial magnification of the objective lens, $\Delta g_{o}$ and $\Delta f_{o}$ are the variations in the focal point distance $g_{o}$ and focal length $f_{o}$ with angle and energy, and $\Delta z$ is the aberration of the accelerating field, i.e., $\Delta z=z_{a}^{\prime}-\left\langle z_{0, a}^{\prime}\right\rangle$. Definitions of the focal point distance $g$ and focal length $f$ are illustrated in Fig. 2.4. The focal variations are of the lens at a particular potential $V_{o} / V_{C}$ can be measured experimentally, as in Rempfer [60], or computed using suitable software. The properties of the objective lens in our PEEM are shown in Fig. 2.5, as computed using SIMION [100]. The aberration coefficients given in Fig. 2.5 are related to the 


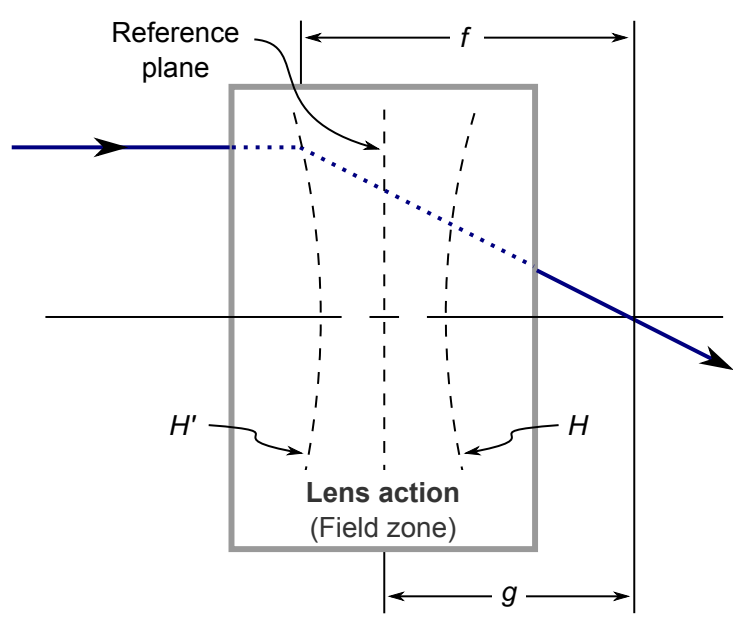

Figure 2.4: The focal properties of a lens are illustrated for a ray parallel incidence ray. The gray region denotes the refracting zone of the electron lens where values of the electric or magnetic field are non-zero. The focal properties of the lens are symmetric about the reference plane. The principle surfaces, marked $H$ and $H^{\prime}$, are positioned and curved in a way typical of electron lenses (though atypical in light microscopy). $f$ and $g$ are sufficient to describe this curvature, and change with the field strength of the lens. Adapted from Rempfer [60].

previously defined image aberration coefficients by

$$
C_{s}=-\left[\left(1+m^{-2}\right) S_{g}+2 m^{-1} S_{f}\right]\left(1+m^{-1}\right)^{2} f,
$$

and

$$
C_{c}=\left[\left(1+m^{-2}\right) C_{g}+2 m^{-1} C_{f}\right] f .
$$

The unitless aberration coefficients - $S_{f}$ and $S_{g}$ for spherical aberration, $C_{f}$ and $C_{g}$ for chromatic aberration - do not depend on magnification. The relationship between the unitless coefficients and the image coefficients is derived from the Newtonian relations

$$
\left(z^{\prime}-g\right) / f=m=f /(z-g)
$$

and the variations in focal point distance and length, given by Rempfer [60]. Re- 


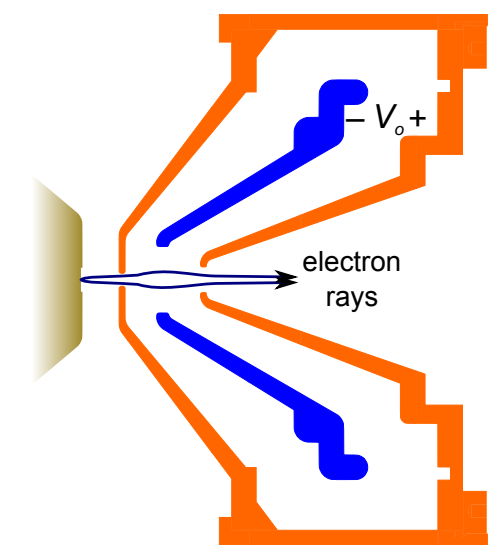

(a)

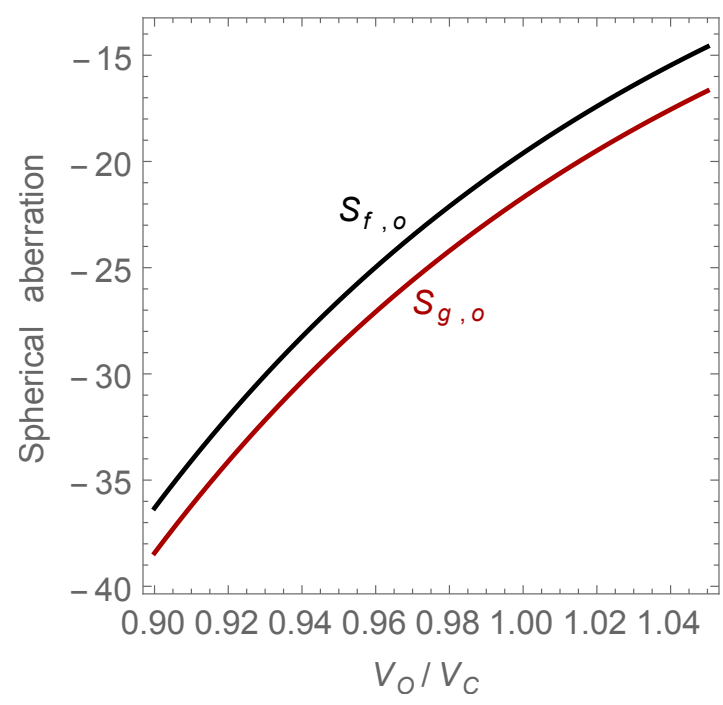

(c)

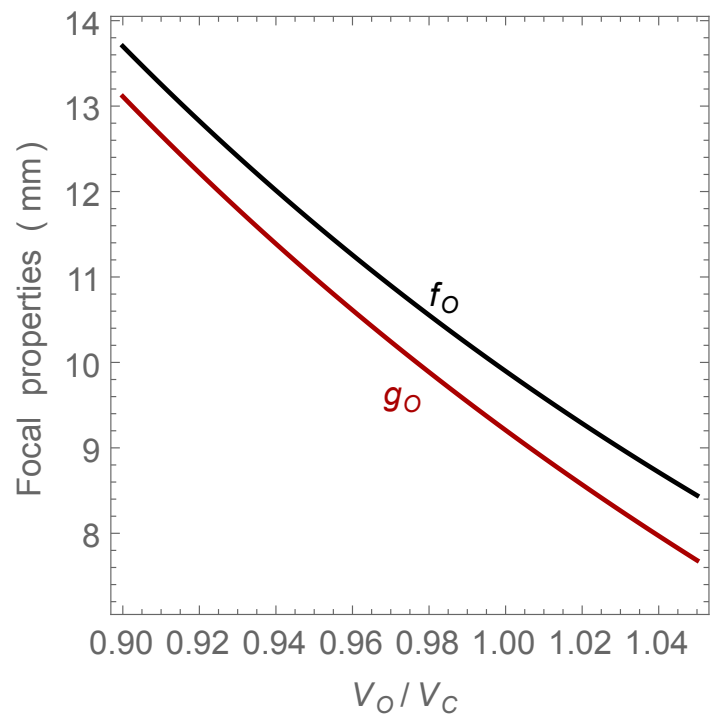

(b)

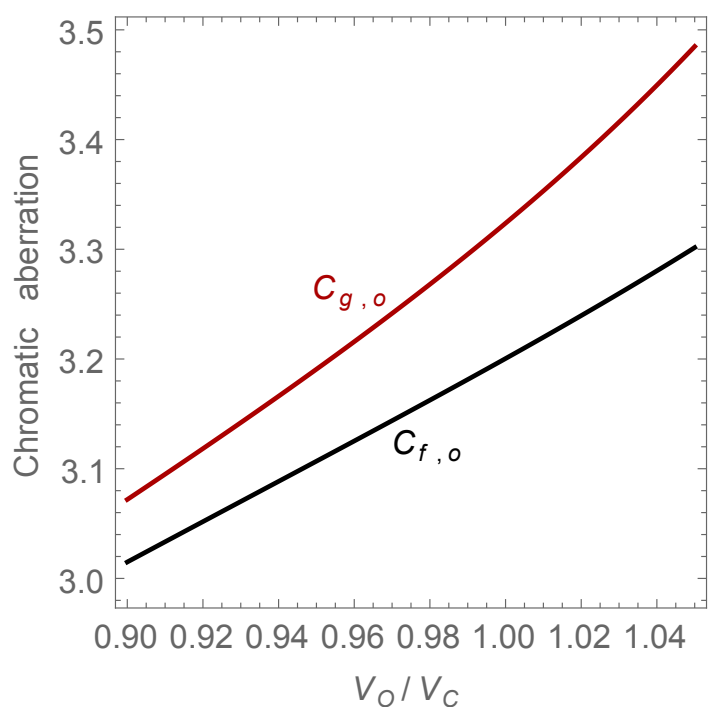

(d)

Figure 2.5: The objective lens used in our PEEM. (a) A diagram of the objective lens in cross-section, which is of the swept-back design of Rempfer with a central bore diameter of $6.71 \mathrm{~mm}$ [99]. The cathode, shown in gold on left, is a distance $3.95 \mathrm{~mm}$ from the front of the objective lens. A potential $V_{o}$ is applied between the grounded outer electrodes (orange) and center electrode (blue). (b) The objective lens focal length and point distance, $f$ and $g$, as a function of potential $V_{o}$. (c) The spherical aberration coefficients coefficients; and (d) the chromatic aberration coefficients. The properties scale with the cathode potential $V_{C}$, so they are plotted on a scale of $V_{o} / V_{C}$. These properties were computed using simion and formulae from Rempfer [60]. 
ferred back to the virtual specimen space at unit magnification, the resolution limit contribution due to spherical aberration is

$$
R_{s, o}=\left(C_{s, a}+C_{s, o} m_{a}^{-4}\right) \alpha_{a}^{3}
$$

and that due to chromatic aberration is

$$
R_{c, o}=\left(C_{c, a}+C_{c, o} m_{a}^{-2}\right)(\Delta E /\langle E\rangle) \alpha_{a} .
$$

An aperture stop is positioned between the exit aperture and image of the objective lens. The aperture stop is a $30 \mu \mathrm{m}$ diaphragm located at $z_{a p} \approx 18 \mathrm{~mm}$ from the center of the lens. At this position, the radius of the electron beam is

$$
r_{a p, \max }=\left(z_{o}^{\prime}-z_{a p}\right) \tan \left(\alpha_{a} m_{a}^{-1} m_{o}^{-1}\right),
$$

where

$$
\begin{aligned}
z_{o}^{\prime} & =g_{o}+f_{o}^{2}\left(z_{o}-g_{o}\right)^{-1}, \\
m_{o} & =f_{o}\left(z_{o}-g_{o}\right)^{-1},
\end{aligned}
$$

and $z_{o}=z^{\prime}+\overline{O A}$ is the distance between the image of the anode aperture image and the center of the objective lens with $\overline{O A}$ representing the distance between the front of the anode and the center of the lens. The aperture stop limits the beam waist, effectively reducing the range of photoelectron emission angles and energies, as shown in Fig. 2.6. Limiting the beam can significantly improve resolution by decreasing the magnitude of image aberrations, though with some penalty in increased electron diffraction and decreased luminosity. The image resolutions of a $30-\mu \mathrm{m}$ aperture stop 


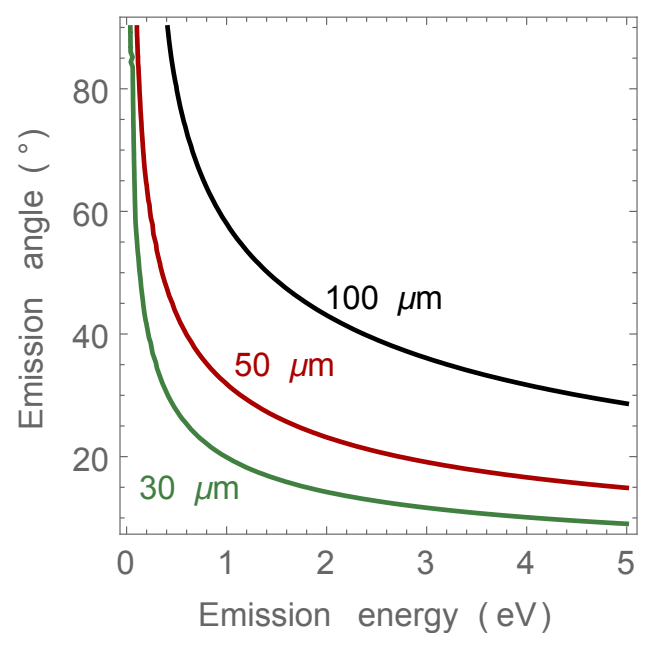

Figure 2.6: An aperture stop in the rear focal plane of the objective lens limits the photoelectron aperture angle and range of energies. Contours trace the maximum acceptance angle as a function of emission energy. The aperture stop used in our PEEM is $30 \mu \mathrm{m}$.

for some realistic photoelectron energy distributions are given in Table 2.2. Notice that using the aperture stop improves point resolution estimates by more than an order of magnitude when the objective lens aberrations are included. This is primarily due to a reduction in spherical aberration, which is particularly curbed for electron beams with large mean emission energies.

In our PEEM, the objective lens is zoom paired with an auxiliary lens, illustrated in Fig. 2.7 and described in Rempfer et al. [99]. The auxiliary lens decouples specimen focus from the position of the image plane. The potentials that maintain this image position for a given total image magnification $m_{a} m_{o} m_{x}$ are plotted in Fig. 2.8. The auxiliary lens impacts the point resolution in an analogous way to the objective, i.e.,

$$
\begin{aligned}
R_{s, x} & =\left[C_{s, a}+C_{s, o} m_{a}^{-4}+C_{s, x}\left(m_{a} m_{o}\right)^{-4}\right] \alpha_{a}^{3}, \\
R_{c, x} & =\left[C_{s, a}+C_{s, o} m_{a}^{-2}+C_{s, x}\left(m_{a} m_{o}\right)^{-2}\right](\Delta E /\langle E\rangle) \alpha_{a} .
\end{aligned}
$$




\begin{tabular}{cccccc}
$\mathbf{M a t} / n \mathbf{P}$ & $\left.\left\langle\alpha_{a}\right\rangle \mathbf{( m r a d}\right)$ & $\left\langle R_{d}\right\rangle(\mathbf{n m})$ & $\left\langle R_{s, a} / 4\right\rangle(\mathbf{n m})$ & $\left\langle R_{c, a} / 2\right\rangle(\mathbf{n m})$ & $\left\langle R_{a}\right\rangle(\mathbf{n m})$ \\
\hline $\mathrm{Ag} / 1$ & 2.4 & 2.2 & 1.9 & 14 & 14. \\
$\mathrm{Ag} / 2$ & 2.4 & 2.2 & 2.1 & 8.9 & 10. \\
$\mathrm{Cu} / 1$ & 2.3 & 2.3 & 2.6 & 4.6 & 6.1 \\
$\mathrm{Cu} / 2$ & 2.2 & 2.2 & 2.2 & 16. & 16. \\
$\mathrm{Cu} / 3$ & 2.1 & 2.5 & 3.2 & 7.3 & 8.8
\end{tabular}

(a) Cathode with aperture stop.

\begin{tabular}{cccc} 
Material $/ n \mathbf{P}$ & $\left\langle R_{s, a+o} / 4\right\rangle(\mathbf{n m})$ & $\left\langle R_{c, a+o} / 2\right\rangle(\mathbf{n m})$ & $\left\langle R_{a+o}\right\rangle(\mathbf{n m})$ \\
\hline $\mathrm{Ag} / 1$ & 13 & 17 & 22 \\
$\mathrm{Ag} / 2$ & 13 & 11 & 19 \\
$\mathrm{Cu} / 1$ & 12. & 5.3 & 14. \\
$\mathrm{Cu} / 2$ & 13. & 19. & 24. \\
$\mathrm{Cu} / 3$ & 11. & 8.1 & 15
\end{tabular}

(b) Cathode and objective with aperture stop.

\begin{tabular}{crrc} 
Material $/ n \mathbf{P}$ & $\left\langle R_{s, a+o} / 4\right\rangle(\mathbf{n m})$ & $\left\langle R_{c, a+o} / 2\right\rangle(\mathbf{n m})$ & $\left\langle R_{a+o}\right\rangle(\mathbf{n m})$ \\
\hline $\mathrm{Ag} / 1$ & 560 & 55 & 560 \\
$\mathrm{Ag} / 2$ & 380 & 34 & 380 \\
$\mathrm{Cu} / 1$ & 160 & 11 & 160 \\
$\mathrm{Cu} / 2$ & 380 & 56 & 390 \\
$\mathrm{Cu} / 3$ & 72 & 15 & 74
\end{tabular}

(c) Cathode and objective without aperture stop.

Table 2.2: Aperture angles, mean point resolution contributions, and total point resolution $\langle R\rangle$ of the photoelectron spectra given in Fig. 2.3. (a) Resolution of the accelerating field alone with aperture stop; compare to Table 2.1; (b) resolution of the accelerating field and objective lens operating at $0.985 V_{C}$ with aperture stop, and (c) without aperture stop. The aberration coefficients of the objective lens at $0.985 V_{C}$ are $C_{s, o}=630 \mathrm{~mm}$ and $C_{c, o}=55 \mathrm{~mm}$, and the magnification is $m_{o}=3.6 \times$. These values vary slightly with potential, as seen in Fig. 2.5, which may be adjusted to improve focus. 


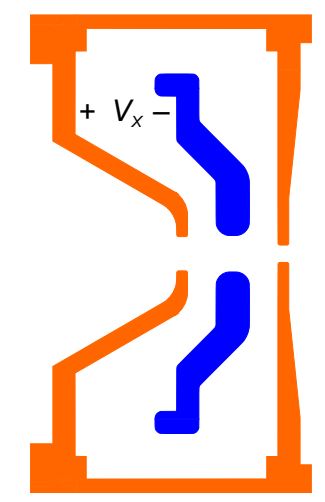

Figure 2.7: The Auxiliary Lens shown in cross-section, used as part of a zoom-pair with the objective lens. A potential $V_{X}$ is applied between the grounded outer electrodes (red) and center electrode (blue). The arrangement with the objective and aperture stop is shown in App. Appendix A.

The terms in brackets are the total aberration coefficients $C_{s, t o t}$ and $C_{c, t o t}$. Unlike before, however, the prefactor is much smaller since the objective lens provides a net image magnification in the range $m_{a} m_{o} \gtrsim 3$. With prefactors on the order of $\left(m_{a} m_{o}\right)^{-4} \lesssim 0.03$ and $\left(m_{a} m_{o}\right)^{-2} \lesssim 0.2$ the aberration imparted by the auxiliary lens is significantly less than that of the objective and accelerating field. The total aberration coefficients and point resolution are plotted in Fig. 2.8. The aberration coefficients and resolution are not significantly different than those of the cathode and objective lens alone, Table 2.2. Further magnifications downstream have negligibly small impact on the image resolution, with the exception of large demagnifications. Thus, the point resolution of our PEEM is effectively that of the accelerating field and the objective, e.g. Table 2.2 or Fig. 2.8 . 


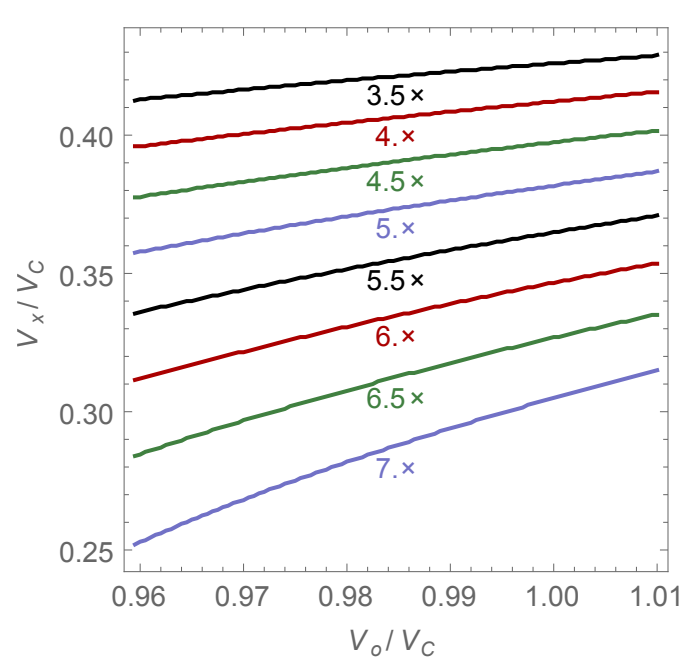

(a)

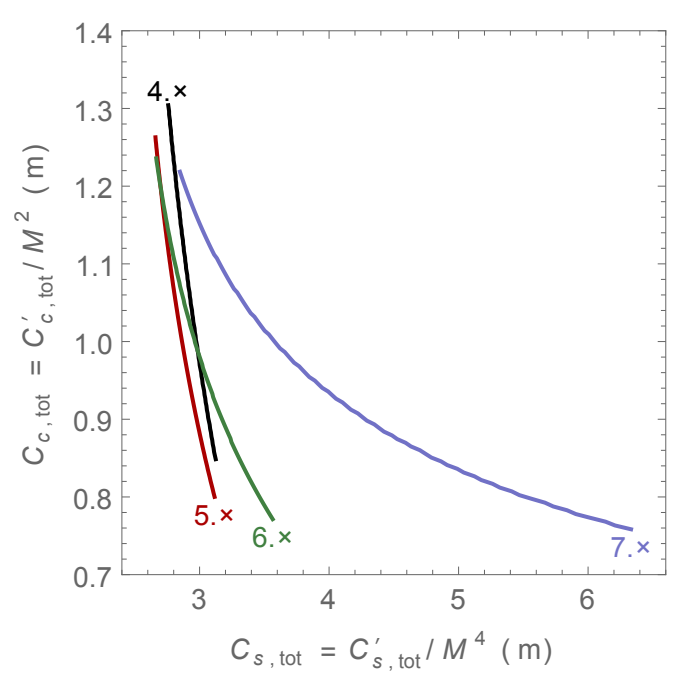

(b)

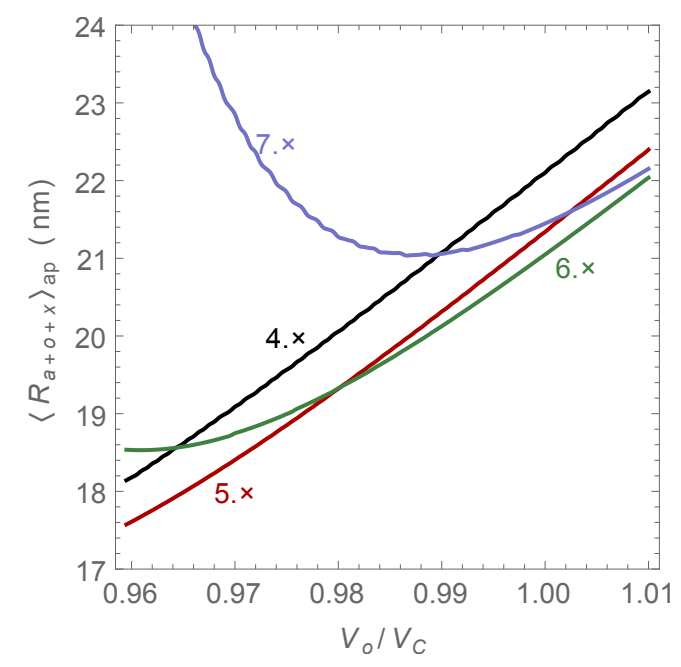

(c)

Figure 2.8: (a) Potentials for constant magnification in the objective and auxiliary lens zoom pair. Several magnifications are possible with the arrangement in our PEEM. Sample focus changes along the contour. (b) The total aberration coefficients and (c) point resolutions of the cathode, objective, and auxiliary lenses for the magnification contours from (a) with a copper specimen at $257 \mathrm{~nm}$. The total aberration coefficients and point resolutions refer to unit magnification in specimen space. 


\section{Aberration correction with a diode mirror}

Rempfer [64] gives the first definitive indications that an electrostatic mirror can correct spherical and chromatic aberration in an electron microscope. Aberration correction in our PEEM follows the scheme outlined there and subsequently refined in Refs. [82, 89, 99, 101, 102]. A schematic diagram of our PEEM incorporating a correcting mirror is shown in Fig. 3.1. The design possesses three branches separated by magnetic beam deflectors and transfer lenses. Following an electron from photoemission to detection, these elements are:

- Branch 1 (objective), cathode and objective lenses;

- Magnetic Deflector A and right transfer lenses;

- Magnetic Deflector B/beam separator;

- Branch 2 (mirror), mirror and interface lens; the beam then returns to Magnetic Deflector B/beam separator;

- Left transfer lenses and Magnetic Deflector C;

- Branch 3 (projection), housing three projection lenses.

An innovation perhaps unique to this design, the magnetic beam separator and deflectors have small deflections and left/right deflection pairs to minimize deflection distortions, instead of the alternative magnetic prism design with right angle deflections [90-92]. The deflections are provided by simple electromagnets, making alignment significantly simpler than the prism design. Though some image stretching occurs in the plane of the deflections, there are no measurable deflection aberrations. A scale drawing of the working instrument is given in App. Appendix A. 


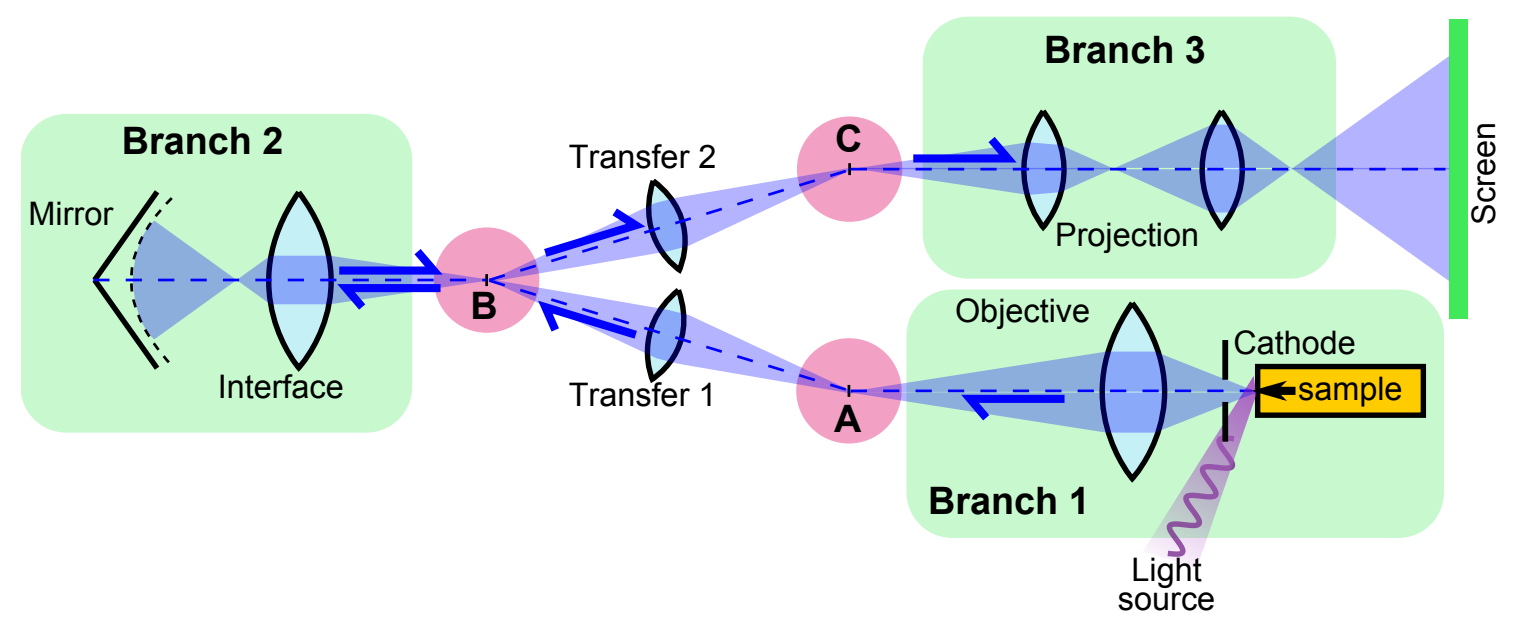

Figure 3.1: Diagram of an aberration-corrected PEEM with a Rempfer-type mirror. A detailed scale drawing of our PEEM is given in App. Appendix A. See text for description. Adapted from Rempfer et al. [82].

\subsection{Analytic model of a hyperbolic diode mirror}

The diode mirror design by Rempfer [64] is based upon a cylindrically symmetric hyperbolic field created between two infinite-radius, contour-conforming electrodes, as in Fig. 3.2. Between the electrodes is charge-free space, where the electric potential $V(\mathbf{x})$ is governed by Laplace's equation, $\nabla^{2} V=0$. Setting the slope of the asymptotic electrode to $2^{-1 / 2}$ gives the exact solution

$$
V(r, z)=V_{M}+k\left(z^{2}-r^{2} / 2\right),
$$

where $k=\left(V_{A}-V_{M}\right) / \ell^{2}$ and $\ell$ is the distance from vertex to terminating electrode, held at potentials $V_{M}$ and $V_{A}$, respectively. Due to this compact analytic description and solution to Laplace's equation, hyperbolic mirrors and lenses of this form have long been a favorite design in electron optics, particularly before the widespread availability of numerical methods $[64,103,104]$. 


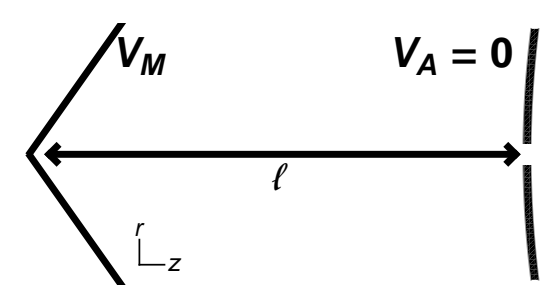

Figure 3.2: Theoretical model of the diode hyperbolic electron mirror. The mirror has cylindrical symmetry about the $z$-axis with $z=0$ at the cone vertex. The electric potentials $V_{M}$ and $V_{A}$ are applied to the mirror and terminating (here grounded) electrodes, respectively. The distance from the vertex to the opening of the aperture is $\ell$. A small aperture in the terminating electrode allows electrons to enter and exit the mirror field. Adapted from Rempfer [64].

The equations of motion for an electron in such a hyperbolic potential are

$$
\begin{aligned}
& \frac{d^{2} r}{d t^{2}}=\frac{e}{m} \frac{\partial V}{\partial r}=-\frac{e k}{m} r, \\
& \frac{d^{2} z}{d t^{2}}=\frac{e}{m} \frac{\partial V}{\partial z}=2 \frac{e k}{m} z,
\end{aligned}
$$

where $e$ and $m$ are the charge and mass of an electron, and $r$ and $z$ describe the particle trajectory at a given time $t[64,69]$. The general solutions for the position and velocity of the electron are

$$
\begin{aligned}
& r=r_{0} \cos (\theta-\phi) / \cos \phi, \\
& z=z_{0} \cosh \left(2^{1 / 2} \theta-\psi\right) / \cosh \psi, \\
& \dot{r}=-\omega r_{0} \sin (\theta-\phi) / \cos \phi, \\
& \dot{z}=2^{1 / 2} \omega z_{0} \sinh \left(2^{1 / 2} \theta-\psi\right) / \cosh \psi ;
\end{aligned}
$$

where $\theta=\omega t, \omega=(e k / m)^{1 / 2}$,

$$
\begin{aligned}
\tan \phi & =\dot{r}_{0} / \omega r_{0}, \\
\tanh \psi & =-\dot{z} / 2^{1 / 2} \omega z_{0} ;
\end{aligned}
$$


and the subscript "0" denotes initial values at time $t=0$. Expressions for the optical properties of a diode mirror can be fully derived from this description of the electron trajectories combined with the deflecting action of the aperture.

As previously discussed, an aberration-correcting electron mirror is operated in a symmetric mode, with ingoing and outgoing electron trajectories tracing approximately the same path along the optical axis and a magnet separating incident and reflected beams. In this case a returning electron retraces the path of the incident electron, with equal amounts of spherical and chromatic aberration accumulating along each arm of the trajectory. Thus, it is only necessary to follow one half of the trajectory, and here I follow the return path starting with the reflection point $\left(r_{C}, z_{C}\right)$ where the velocity components are zero. Choosing $t=0$ at this point, the boundary condition parameters are also $\varphi=\psi=0$, and the trajectory description simplifies to

$$
\begin{aligned}
& r=r_{C} \cos \theta, \\
& z=z_{C} \cosh 2^{1 / 2} \theta, \\
& \dot{r}=-\omega r_{C} \sin \theta, \\
& \dot{z}=2^{1 / 2} \omega z_{C} \sinh 2^{1 / 2} \theta .
\end{aligned}
$$

The maximum penetration of electrons into the mirror field coincides with the equipotential equal to the electron accelerating potential $V_{C}$. Points $\left(r_{C}, z_{C}\right)$ on this reflecting equipotential fall on the hyperbolic contour

$$
\ell^{2}\left(V_{C}-V_{M}\right) /\left(V_{A}-V_{M}\right)=z_{C}^{2}-r_{C}^{2} / 2 .
$$

The returning electron reaches the terminating electrode at time $t_{A}=\theta_{A} / \omega$ and at 
the coordinates $\left(r_{A}, z_{A}\right)$, which lie on the hyperbolic contour

$$
\ell^{2}=z_{A}^{2}-r_{A}^{2} / 2
$$

The slope of the electron trajectory at this point is

$$
\tan \alpha_{A}=-\dot{r}_{A} / \dot{z}_{A}=-\frac{r_{A} \tan \theta_{A}}{2^{1 / 2} z_{A} \tanh 2^{1 / 2} \theta_{A}} .
$$

As the electron exits the hyperbolic field, it is deflected by the exit aperture through angle $\delta_{A}$. The Davisson-Calbick approximation, gives the aperture focal length [105], $f_{A}=$ $-4\left(V_{A}-V_{C}\right) /\left(2 k z_{A}\right)$, which leads to a deflection of

$$
\tan \delta_{A}=r_{A} / f_{A}=-\frac{r_{A} z_{A} k}{2\left(V_{A}-V_{C}\right)} .
$$

Subsequently, the electron travels through field-free space, crossing the optical axis at the location

$$
z^{\prime}=z_{A}+r_{A} / \tan \left(\alpha_{A}+\delta_{A}\right) .
$$

In the absence of aberration, the distance from the axis crossing to the mirror vertex is the paraxial object/image distance

$$
z_{0}=\ell\left(1+1 / q_{0}\right)
$$

where

$$
q_{0}=\frac{\tan \theta_{A}^{(0)}}{2^{1 / 2} \tanh 2^{1 / 2} \theta_{A}^{(0)}}-\frac{V_{A}-V_{M}}{2\left(V_{A}-V_{C}\right)},
$$

and 


$$
2^{1 / 2} \tanh \theta_{A}^{(0)}=\left(\frac{V_{A}-V_{C}}{V_{A}-V_{M}}\right)^{1 / 2} .
$$

The time constant $\theta_{A}^{(0)}$ is the paraxial approximation of $\theta_{A}$, found by taking the limits $r_{C} \rightarrow 0$ and $r_{A} \rightarrow 0$ in the contour Eqs. (3.10-3.15). The spherical aberration coefficient is found from the variation of image distance with approach angle $\alpha^{\prime}=$ $\alpha_{A}+\delta_{A}$, using a modified version of Eq. (1.5),

$$
C_{s}=-2\left(z^{(1)}-z_{0}\right) / \alpha^{2},
$$

where $z^{(1)}$ is the first order correction to $z_{0}$, and the doubling is due to the path being traversed twice (in and out). Similarly, the coefficient of chromatic aberration is found from a modified version of Eq. (1.7),

$$
C_{c}=2 V_{C} \frac{\partial z_{0}}{\partial V_{C}},
$$

where $V_{C}$ is the accelerating potential of the electron, and thus related to its energy $-e V_{C}$. These three optical properties, $z_{0}, C_{s}$, and $C_{c}$, fully characterize the diode mirror, determining the aberration correction it provides and how to interface with it. A parametric plot of the three properties is shown in Fig. 3.3, where the mirror potential difference $V_{A}-V_{M}$ is the independent variable.

\subsection{Aberration correction applied to our PEEM}

The mirror properties scale with the physical length of the mirror, so to some extent the aberration correction provided by the mirror can be arbitrarily scaled. However, the aberration coefficient ratio $C_{c} / C_{s}$ is a unique function of mirror potential, as 


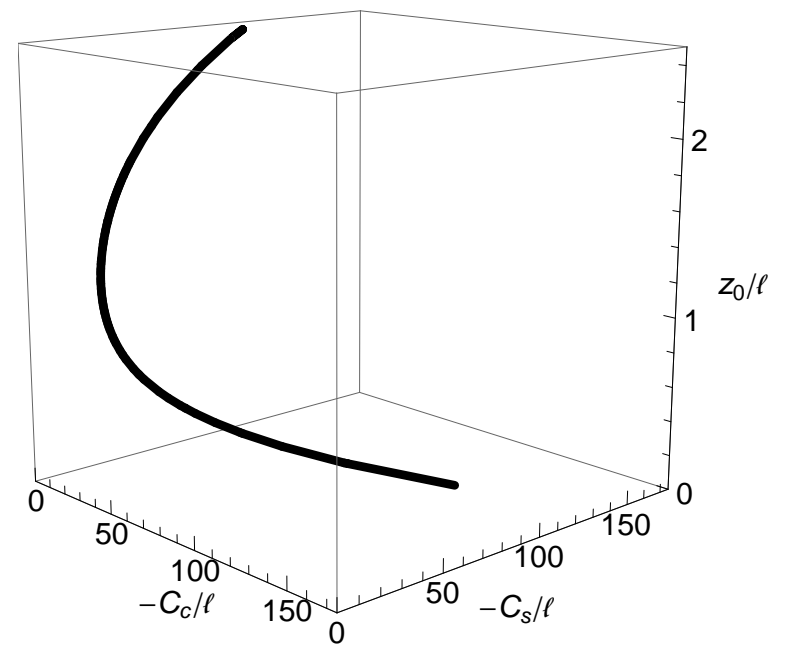

Figure 3.3: Parametric plot of the optical properties of the diode hyperbolic mirror as a function of the potential difference $V_{M}-V_{A}$. Since the properties scale with mirror length, they are plotted in units of mirror length. Adapted from Rempfer [64].

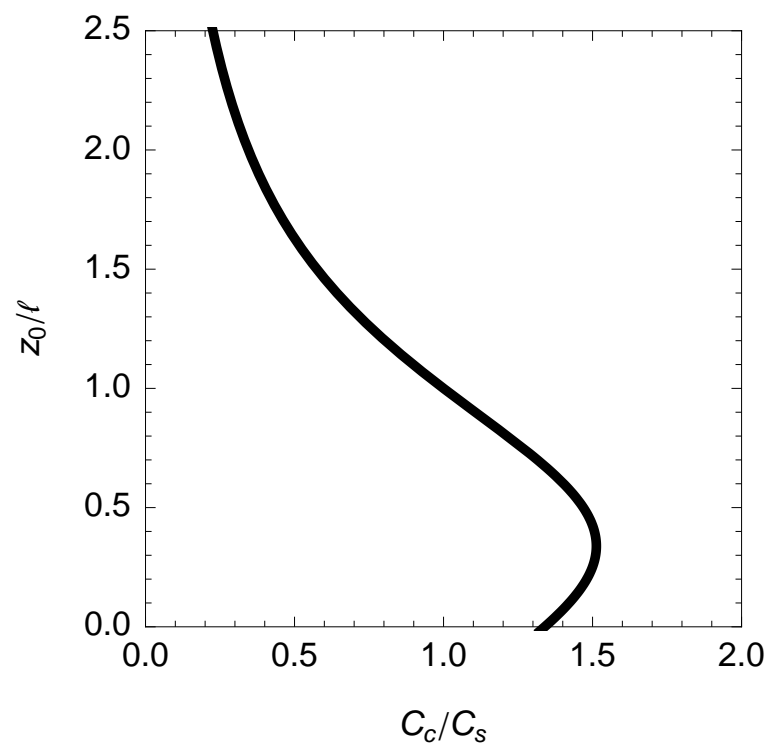

Figure 3.4: Parametric plot of the diode mirror aberration coefficient ratio versus the object/image location as a function of the potential difference $V_{A}-V_{M}$. Adapted from Rempfer [64]. 
shown in Fig. 3.4. This ratio must be matched to that of the instrument, deciding the operating potential of the diode mirror as well as the distance from mirror vertex to the beam axis-crossing point, $z_{0} / \ell$. This is accomplished in our PEEM with a mirror length of $\ell=25.4 \mathrm{~mm}$ and a lens-mirror spacing of $\sim 44 \mathrm{~mm}$, as seen in App. Appendix A. The transfer lenses between magnets and the projection lenses contribute negligibly to spherical and chromatic aberration. Thus, the total aberration of the objective branch can be compared to that of the mirror. The appropriate comparison is made not in the virtual specimen space of Eqs. (2.14) and (2.15), Table 2.2, or Fig. 2.8 , but rather in transfer space, where

$$
\begin{aligned}
& C_{s, a o x}^{\prime}=\left(m_{a} m_{o} m_{x}\right)^{4} C_{s, t o t} \approx 1300 \mathrm{~m}, \\
& C_{c, a o x}^{\prime}=\left(m_{a} m_{o} m_{x}\right)^{2} C_{c, t o t} \approx 18 \mathrm{~m} .
\end{aligned}
$$

The aberration coefficient ratio at this stage, $C_{c, a o x}^{\prime} / C_{s, a o x}^{\prime}=1 / 70$ is incompatible with the mirror ratio range shown in Fig. 3.4. In fact, the mirror is most compatible with the specimen space coefficients, $C_{c, a o x} / C_{s, a o x}=1 / 3$, or perhaps an even larger ratio close to unity. Reducing image magnification before correcting aberration with the mirror can accomplish this since the ratio scales with $\mathrm{m}^{-2}$. Consequently, instead of acting as a simple transfer lens, the lens interfacing the mirror with the beam separator (magnet B) should be run at magnification $m_{I}^{-1} \gtrsim 5$. As such, the aberration intrinsic to the interface lens becomes appreciable, and the condition for an aberration free image is

$$
\begin{aligned}
& C_{s M}+2 C_{s I}+m_{2}^{4} C_{s, a o x}^{\prime}=0 \\
& C_{c M}+2 C_{c I}+m_{2}^{2} C_{c, a o x}^{\prime},=0
\end{aligned}
$$


where $C_{s I}$ and $C_{C I}$ are the interface lens aberration coefficients with reference to the mirror side.

Given a particular objective branch lens aberration, the correction the mirror offers can be adjusted through the magnification of the interface lens $m_{I}$, the length of the mirror $\ell$, and the operating potential difference $V_{A}-V_{M}$. This is a sufficiently independent set of variables to address the three optical properties $z_{0}, C_{s M}$, and $C_{c M}$. Two of these variables are locked in once the microscope is assembled, the length of the mirror and the magnification of the lens, which is primarily determined by positioning. This leaves only one variable available for dynamic refinements, the potential difference $V_{A}-V_{M}$. To be clear, changes in the mirror voltage must also be accompanied by a change in lens magnification, but these variables are not independent. Subsequently, the system becomes overdetermined, i.e., it is impossible to improve aberration correction with a single variable. Unfortunately, this situation leads to less than optimal aberration correction for all specimens, as illustrated in Fig. (3.5). To expand the correctable range of aberration coefficient and furthermore introduce independent control $C_{s}$ and $C_{c} \mathrm{I}$ introduce a third electrode to the mirror design, as discussed in the next chapter. 


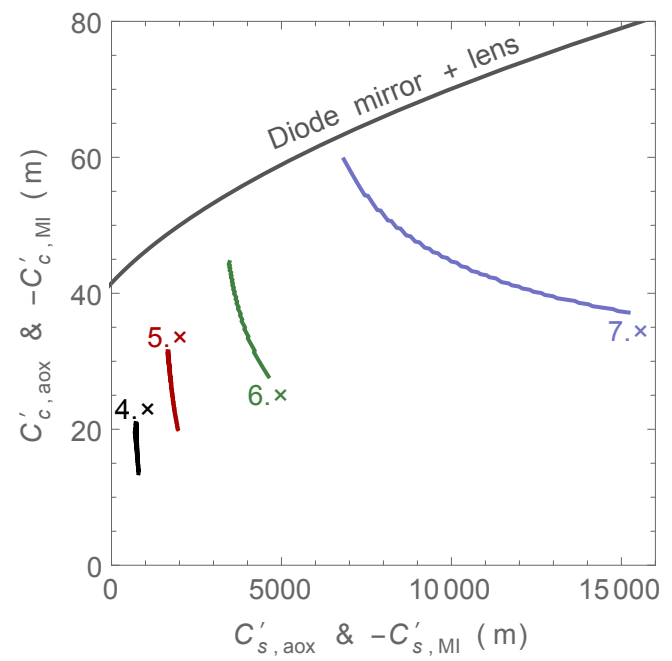

Figure 3.5: The aberration coefficients of the mirror branch (dark gray) and the objective branch with magnifications 4-7× compared in transfer space for $\mathrm{Cu}$ illuminated with $257 \mathrm{~nm}$ light. The mirror and lens overcorrect the objective branch chromatic aberration in this case, i.e., not only eliminate aberration but adding some of the opposite sign. Because the diode cannot be easily adjusted, not all specimens can have optimal aberration correction. 


\section{Dynamic aberration correction with a triode mirror}

As a result of the previous chapter's discussion, I propose that a mirror-based aberration corrector with independently and dynamically variable spherical and chromatic aberration correction may be possible with a hyperbolic mirror foundation. To provide fully independent control of the correcting spherical and chromatic aberration coefficients requires three variable potentials. In our design this is done with two in the mirror and a third in the the adjoining interface lens. In addition, the ideal corrector design incorporates closed form analytic expressions of the electron trajectories and their corresponding optical parameters, the image position and the aberration coefficients. Ultimately, an analytic expression will not be able to capture all of the nuances of real electron optical devices, and the final form of the corrector will be described by a numerical model. However, even approximate or idealized analytic expressions can lend significant physical intuition and guide design optimization. The new aberration corrector must also be able to work within our PEEM since it would be completely impractical to build a new microscope column to house the corrector. This puts space limitations of the arrangement of elements within the aberration correcting system. Three approaches to this problem immediately come to mind, which balance the number of lenses with the number of electrodes in the mirror.

A distinguishing characteristic of the diode mirror solution is the separation between the microscope and the mirror via an interfacing lens. The lens accommodates small changes in the spacing between the mirror and the beam separator and provides the high image magnification change necessary for the mirror correction to operate properly. An interface lens provides more intuitive operation by partitioning demagnification and alignment from aberration correction. A minimal accommodation for 
a third variable is a secondary lens coupled with the interface lens. Together, the two lenses act as a zoom pair, and with different lens aberration coefficients, they should be able to provide some range of independent spherical and chromatic aberration adjustment. Unfortunately, after surveying several available electron lenses [60], no lens satisfies the space requirements and leads to a sufficient dynamic range of aberration correction. Thus, a two-lens and diode mirror corrector is not a capable candidate, and the space between the mirror and the interface lens must accommodate additional electrodes.

The simplest approach is to extend the original diode mirror design to include a single additional electrode. It is otherwise paired with the same interface lens, and the lens potential $V_{I}$ is allowed to vary to accommodate changes in $z_{0}$ and magnification. The properties of such a triode mirror can be calculated analytically, applying the general solutions to the hyperbolic potential, Eqs. (3.4-3.7) if the additional electrode faces also follow hyperbolic contours. Once the properties of the triode mirror are analytically derived, the aberration correcting capabilities and dynamism of the combined triode mirror and lens system can be assessed. Alternatively, two adjustable potentials can be incorporated into a single tetrode mirror. Incorporation of all the electrodes into a single mirror reduces the possibility of optical axis misalignment. As found in the literature [93], a tetrode mirror is capable of correcting and dynamically adjusting spherical and chromatic aberration, although no analytic description of a tetrode mirror has been provided. In addition, it is still necessary to pair the tetrode mirror with a lens for alignment adjustments, as seen in one current implementation [91]. 


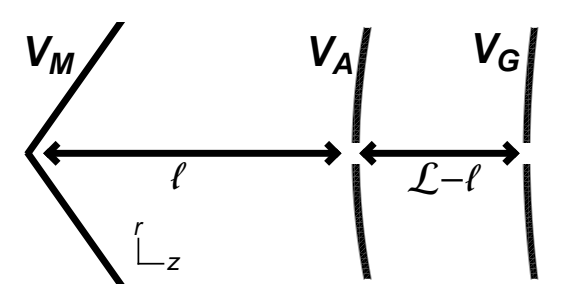

Figure 4.1: Theoretical model of the triode hyperbolic electron mirror. The mirror has cylindrical symmetry about the $z$-axis with $z=0$ at the cone vertex. The voltages $V_{M}, V_{A}$, and $V_{G}$ are on the mirror, center, and grounded/terminating electrodes. The distance from the vertex to the center electrode aperture is $\ell$, and from the vertex to the grounded electrode aperture is $\mathcal{L}$. small apertures in the center and terminating electrodes allow electrons to enter and exit both regions of the mirror field. Reprinted from Fitzgerald, Word, and Könenkamp [94].

\subsection{Analytic model of hyperbolic triode mirror}

In this section, I begin with a theoretical characterization of a triode hyperbolic mirror, as previously presented by Fitzgerald, Word, and Könenkamp [94, 106]. The analytic description builds on the work by Rempfer [64], matching an electron trajectory through two infinite hyperboloid regions joined by an aperture, as shown in Fig. 4.1. The new hyperbolic region exists between the diode mirror anode at $z=\ell$ and a new grounded outer electrode located at $z=\mathcal{L}$. The potential of the two regions is

$$
V(r, z)= \begin{cases}k_{1}\left(z^{2}-r^{2} / 2\right), & 0<z<\ell \\ -k_{2} \mathcal{L}^{2}+k_{2}\left(z^{2}-r^{2} / 2\right), & \ell<z<\mathcal{L}\end{cases}
$$

where the constants $k_{1}$ and $k_{2}$ are determined from the potential differences of the inner and outer region, i.e.,

$$
k_{1}=\left(V_{A}-V_{M}\right) / \ell^{2}, \text { and } \quad k_{2}=-V A /\left(\mathcal{L}^{2}-\ell^{2}\right),
$$

analogous to the diode constant $k$, Eq. 3.1. 
An electron trajectory in the region adjoining the asymptotic electrode can be treated as in the diode mirror description, Eqs. (3.10-3.17). The position and velocity at the boundary between the first and second regions are

$$
\begin{aligned}
& r_{1}=r_{C} \cos \theta_{1} ; \\
& z_{1}=z_{C} \cosh 2^{1 / 2} \theta_{1} ; \\
& \dot{r}_{1}=-\left[\sin \left(\alpha_{A}+\delta_{A}\right) / \sin \alpha_{A}\right] \omega_{1} r_{C} \sin \theta_{1} ; \\
& \dot{z}_{1}=\left[\cos \left(\alpha_{A}+\delta_{A}\right) / \cos \alpha_{A}\right] 2^{1 / 2} \omega_{1} z_{C} \sinh 2^{1 / 2} \theta_{1},
\end{aligned}
$$

where $\omega_{1}=\left(e k_{1} / m\right)^{1 / 2}$ and $\theta_{1}=\omega_{1} t_{1}$ is the time constant of the first region only. The angles $\alpha_{A}$ and $\delta_{A}$ are defined in Eqs. (3.16) and (3.17), respectively. The trajectory coordinates in the second region between potentials $V_{A}$ and $V_{G}=0$ (grounded) are given by the general solution, Eqs. (3.4-3.7), i.e.,

$$
\begin{aligned}
& r_{2}=r_{1} \cos \left(\theta_{2}-\phi_{2}\right) / \cos \phi_{2} ; \\
& z_{2}=z_{1} \cosh \left(2^{1 / 2} \theta_{2}-\psi_{2}\right) / \cosh \psi_{2} ; \\
& \dot{r}_{2}=-\omega_{2} r_{1} \sin \left(\theta_{2}-\phi_{2}\right) / \cos \phi_{2} ; \\
& \dot{z}_{2}=2^{1 / 2} \omega_{2} z_{1} \sinh \left(2^{1 / 2} \theta_{2}-\psi_{2}\right) / \cosh \psi_{2},
\end{aligned}
$$

where $\omega_{2}=\left(e k_{2} / m\right)^{1 / 2}, \theta_{2}=\omega_{2}\left(t_{2}-t_{1}\right)$ is the time constant of the second region, and $t_{2}-t_{1}$ is the time required for an electron to transit from the electron at potential $V_{A}$ to the grounded electrode. The coordinates $\left(r_{1}, z_{1}\right)$ and $\left(\dot{r}_{1}, \dot{z}_{1}\right)$ are the initial conditions for the second region trajectories, leading to nonzero values of parameters 
$\varphi_{2}$ and $\psi_{2}$,

$$
\begin{aligned}
\tan \phi_{2} & =-\left[\omega_{1} \sin \alpha_{A} / \omega_{2} \sin \left(\alpha_{A}+\delta_{A}\right)\right] \tan \theta_{1}, \\
\tanh \phi_{2} & =-\left[\omega_{1} \cos \alpha_{A} / \omega_{2} \cos \left(\alpha_{A}+\delta_{A}\right)\right] \tanh 2^{1 / 2} \theta_{1} .
\end{aligned}
$$

Reaching the aperture in the grounded electrode, an electron trajectory makes the angle $\tan \alpha_{2}=-\dot{r}_{2} / \dot{z}_{2}$ with the optical axis. Upon passing through the aperture, an electron is deflected by

$$
\tan \delta_{G}=r_{2} z_{2} k_{2} / 2 V_{C}
$$

Finally an electron enters field-free space, crossing the optical axis at

$$
z^{\prime}=z_{2}+r_{2} / \tan \left(\alpha_{2}+\delta_{G}\right)
$$

From Eq. (4.14) the optical properties of the triode mirror, the image/object distance $\left(z_{0}\right)$ and the coefficients of spherical and chromatic aberration $\left(C_{s}\right.$ and $\left.C_{c}\right)$, can be computed. This procedure is analogous to the diode description, using the formulas and method outlined in Eqs. (3.19-3.23), albeit with significantly more complicated expressions. For example, the paraxial image/object location

$$
z_{0}=\mathcal{L}\left(1+1 / q_{1}\right)
$$

where

$$
q_{1}=\left(k_{2} \mathcal{L} / 2 V_{C}\right)+\tan \left(\theta_{2}^{(0)}-\phi_{2}^{(0)}\right) / 2^{1 / 2} \tanh \left(2^{1 / 2} \theta_{2}^{(0)}-\psi_{2}^{(0)}\right),
$$

appears superficially similar to the diode expression Eq. (3.20). However, where the diode expression required the definition of one additional parameter, the triode 
expression requires five more:

$$
\begin{aligned}
\cosh \left(2^{1 / 2} \theta_{2}^{(0)}-\psi_{2}^{(0)}\right) & =(\mathcal{L} / \ell) \cosh \psi_{2}^{(0)} \\
\tan \phi_{2}^{(0)} & =-\left(k_{1} / k_{2}\right)^{1 / 2}\left[\left(\alpha_{A}+\delta_{A}\right) / \alpha_{A}\right]^{(0)} \tan \theta_{1}^{(0)}, \\
\tanh \psi_{2}^{(0)} & =-\left(k_{1} / k_{2}\right)^{1 / 2} \tanh 2^{1 / 2} \theta_{1}^{(0)}, \\
{\left[\left(\alpha_{A}+\delta_{A}\right) / \alpha_{A}\right]^{(0)} } & =1+\left(\ell^{2} / 2\right)\left[\left(k_{2}-k_{1}\right) /\left(V_{C}-V_{A}\right)\right] 2^{1 / 2} \tanh 2^{1 / 2} \theta_{1}^{(0)}, \\
\tanh 2^{1 / 2} \theta_{1}^{(0)} & =\left[\left(V_{A}-V_{C}\right) /\left(V_{A}-V_{M}\right)\right]^{1 / 2} .
\end{aligned}
$$

The coefficients of spherical and chromatic aberration can be calculated from Eqs. (3.22) and (3.23), i.e.,

$$
C_{s}=2 \frac{z^{(1)}-z_{0}}{\left(\alpha^{(1)}\right)^{2}}=2 \lim _{\varepsilon \rightarrow 0}\left(\frac{1}{2} \frac{\partial^{2} z^{(1)}}{\partial \varepsilon^{2}}\right)\left(\frac{\partial \alpha^{(1)}}{\partial \varepsilon}\right)^{-2}
$$

and

$$
C_{c}=\left(z_{0}-\mathcal{L}\right)^{2}\left(\sqrt{2} V_{C} \frac{\partial q_{1}}{\partial V_{C}}+\frac{\mathcal{L}^{2}\left(V_{A} / V_{C}\right)}{\left(\mathcal{L}^{2}-\ell^{2}\right)}\right) .
$$

$\varepsilon=r / \mathcal{L}$ is a small, unitless expansion parameter related to the maximum trajectory radial distance $r_{C}$. The (1) denotes the first-order approximation for $|\alpha|>0$, i.e., $\alpha$ is assumed to be very small but non-zero; $z^{(1)}$ and $\alpha^{(1)}$ are the first-order corrections to the paraxial position and angle of the electron trajectory where it crosses the optical axis. Complete closed-form expressions are sufficiently complicated that they will not be presented here, but App. Appendix B gives explicit calculation computer codes. The results are presented in Figs. (4.2)-(4.4).

At many points in the property space the surface described by the dynamic parameters is nearly flat in two out of the three properties. In these locally flat regions the third property can be adjusted almost independently. Each dynamic surface, e.g., Fig. 4.2 or one of the surfaces in Fig. 4.4, has one point surrounded by an optimally 


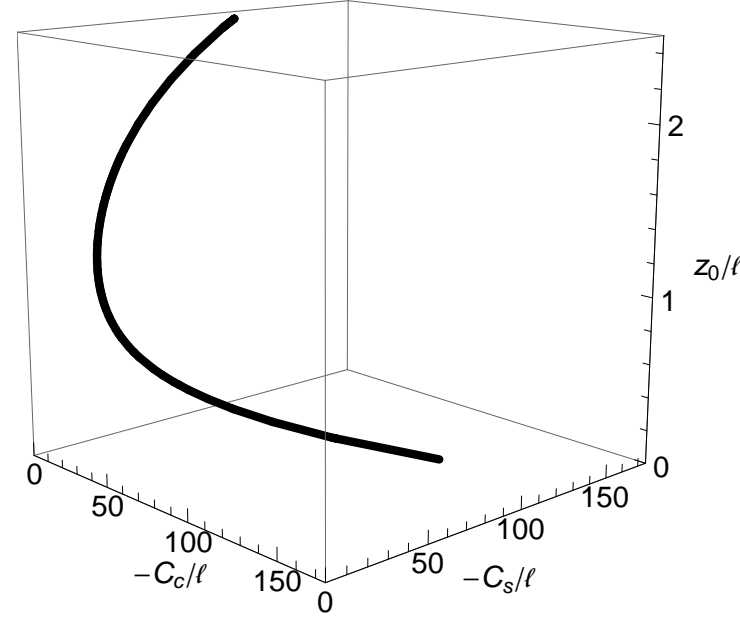

(a)

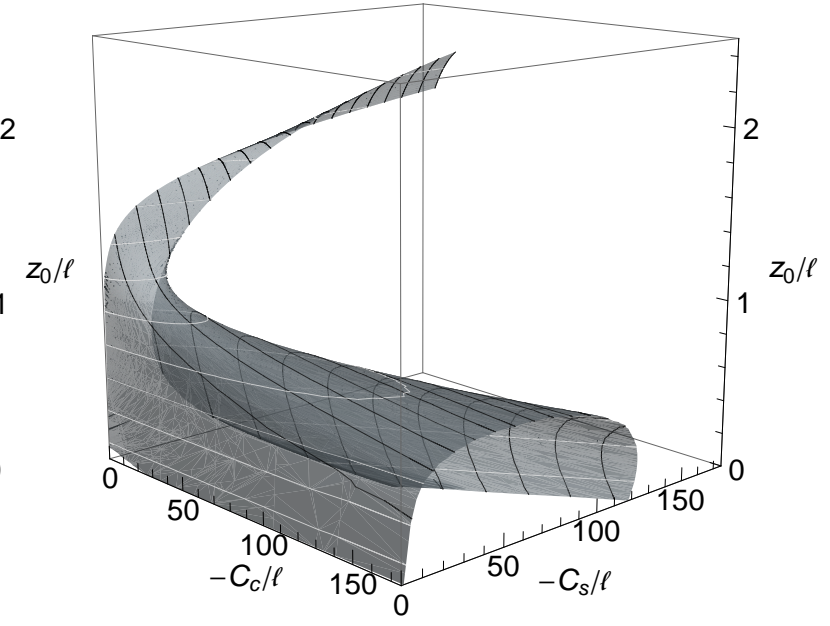

(b)

Figure 4.2: Parametric plots of the optical properties of the (a) diode and (b) triode hyperbolic mirrors as a function of the potential differences $V_{M}-V_{A}$ (diode and triode) and $V_{A}-V_{G}$ (triode only). The triode mirror geometric ratio is $\ell / \mathcal{L}=0.66$. Contour lines in the surface represent constant $z_{0}$ (light gray) and constant $C_{s}$ (black). Reprinted from Fitzgerald, Word, and Könenkamp [94].

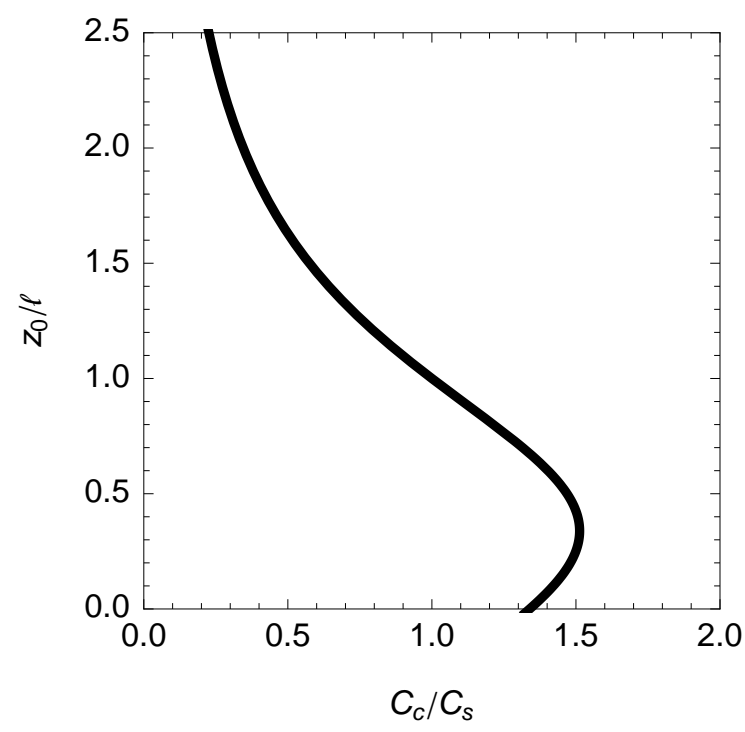

(a)

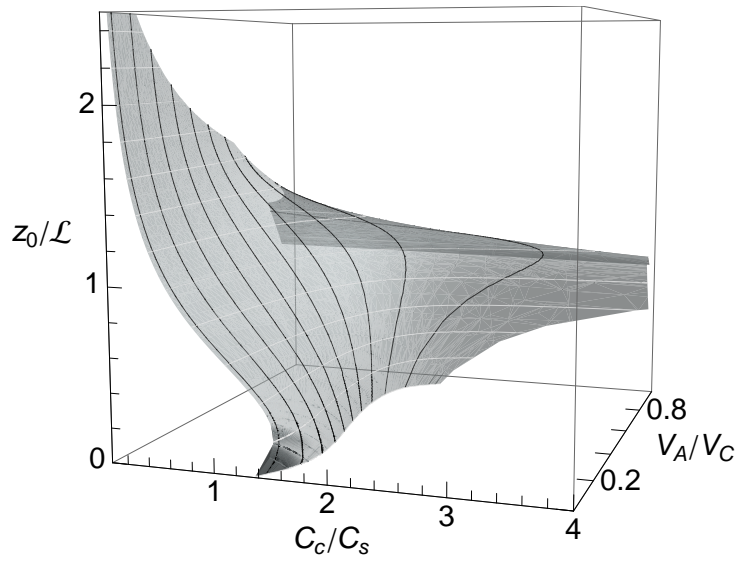

(b)

Figure 4.3: Parametric plot of the (a) diode and (b) triode aberration coefficient ratio versus the $z_{0}$ as a function of the potential difference differences $V_{M}-V_{A}$ (diode and triode) and $V_{A}-V_{G}$ (triode only), where $\ell / \mathcal{L}=0.66$. Contour lines in the surface represent constant $z_{0}$ (light gray) and constant $V_{A}$ (black). Reprinted from Fitzgerald, Word, and Könenkamp [94]. 


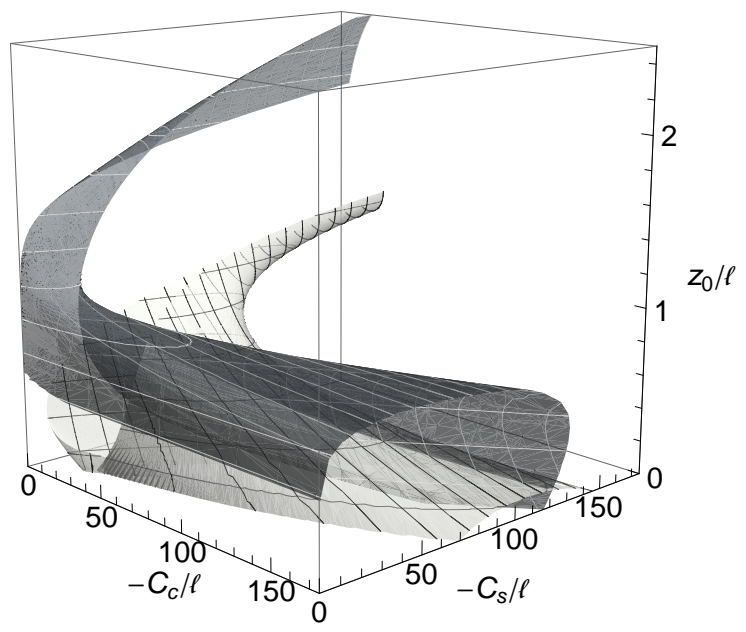

Figure 4.4: The parametric surface of the triode optical properties can be adjusted with the geometric ratio $\ell / \mathcal{L}$. The ratios $\ell / \mathcal{L}=0.3$ (dark gray) and 0.8 (light gray) are plotted here. Reprinted from Fitzgerald, Word, and Könenkamp [94].

flat region. Around that point one property can be dynamically adjusted while the other two remain fairly constant by changing $V_{A}$ and $V_{M}$. The ratio $\ell / \mathcal{L}$ can be tuned to match the flat region of the dynamic surface with the desired ratio of chromatic to spherical aberration. The ability to fine tune aberration correction is the most attractive feature the triode mirror offers over the diode mirror.

A recipe for the design of a triode mirror to compensate aberration follows:

1. Only one property can be dynamically adjusted independently. This recipe considers $C_{c}$ as the dynamic property, and consider $C_{s}$ and $z_{0}$ as (ideally) constant.

2. Minimize the change in $C_{s}$ and $z_{0}$ compared to the change in $C_{c}$ in a region around the desired ratio of aberration coefficients and on a surface of constant $\ell / \mathcal{L}$.

(a) The property functions in parameter space are nonlinear with many local extrema, so there may be many good optimization methods. One method is to minimize the angle $\theta$ between the voltage gradients of $C_{s}$ and $z_{0}$, where 
$\sin \theta \propto-\left(\partial z_{0} / \partial V_{M}\right)\left(\partial C_{s} / \partial V_{A}\right)+\left(\partial z_{0} / \partial V_{A}\right)\left(\partial C_{s} / \partial V_{M}\right)$. This identifies points where constant contours of $C_{s}$ and $z_{0}$ are the most parallel. These points can then be narrowed down by their aberration ratio and the curvature of the surface at that point.

(b) It may be necessary to consider the physical dimensions of the mirror during the minimization to limit the ranges of $C_{s} / \mathcal{L}$ and $C_{c} / \mathcal{L}$.

(c) This minimization gives a best parameter configuration $\left(V_{M} / V_{C}, V_{A} / V_{C}, \ell / \mathcal{L}\right)$ around which changes in voltage leave the object/image distance and the spherical aberration relatively constant. This fixes $\ell / \mathcal{L}$ and $z_{0} / \mathcal{L}$, and establishes a relationship between $V_{M}$ and $V_{A}$ for which the $z_{0}$ does not change.

(d) A survey shows that the function $V_{M}=f\left(V_{A}\right)$ that maintains constant $z_{0}$ is often linear throughout the tuning range and that the mirror is not highly sensitive to changes in $\ell / \mathcal{L}$, as shown in Fig. 4.4.

3. Next specify the length of the mirror $\mathcal{L}$ so that its value of $C_{s}$ matches that of the lens system. This will also set $z_{0}$.

4. Design the hyperbolic triode mirror with geometry $\ell / \mathcal{L}$ to length $\mathcal{L}$. The mirror should be positioned in the microscope such that paraxial object/image distance $z_{0}$ is tuned for the next element, similar to the location of the diode mirror.

5. Run the mirror electrodes at the potentials $V_{M}$ and $V_{A}$, as set by Step 2. This will provide aberration correction at the set aberration ratio. Change the potentials $V_{M}$ and $V_{A}$ according to a relationship that maintains $z_{0}$ to adjust the chromatic aberration compensation as needed. Assuming small changes in potential, this will have minimal effect on the spherical aberration compensation. 


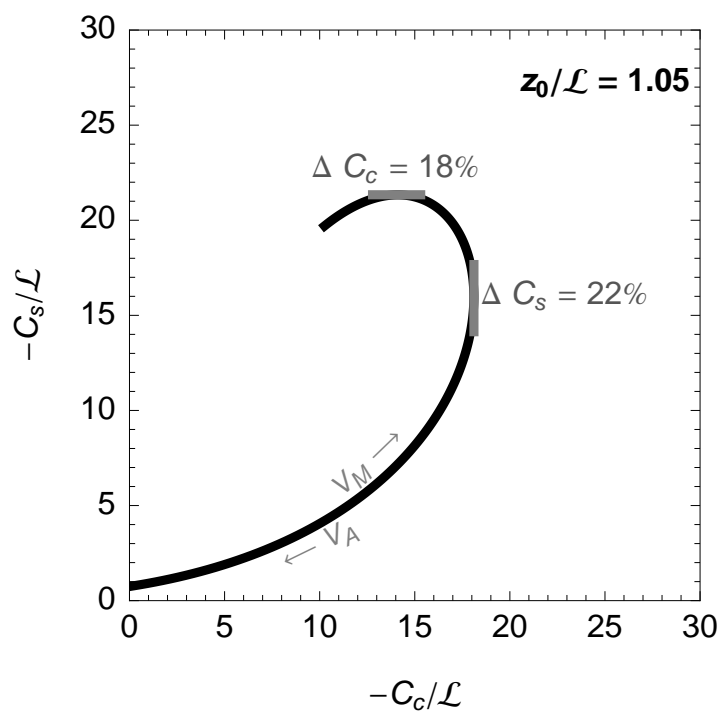

(a)

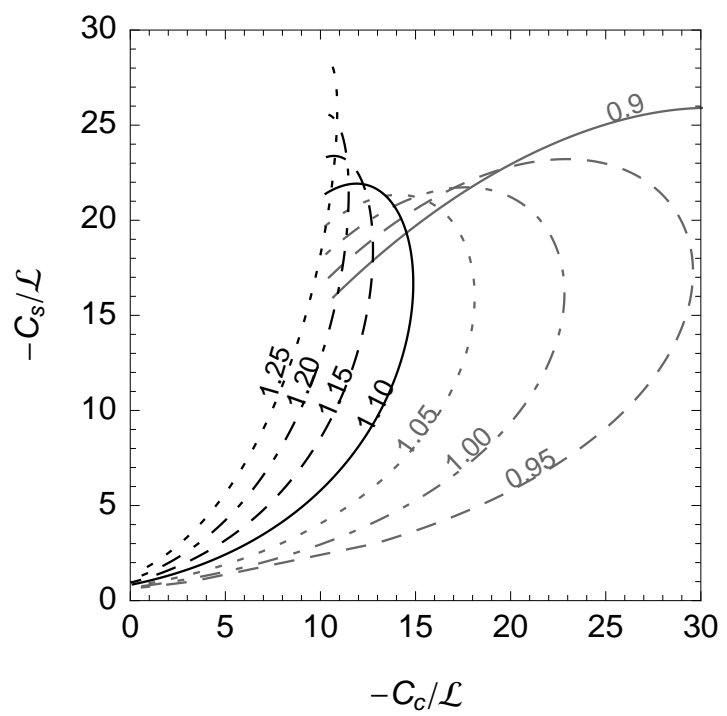

(b)

Figure 4.5: Aberration coefficients of a triode mirror for fixed $\ell / \mathcal{L}$ and object/image distance $z_{0} / \mathcal{L}$. (a) The relationship between spherical and chromatic aberration once the object/image distance is fixed. Each curve is labeled by its value of $z_{0} / \mathcal{L}$. (b) The parametric dependence on $\left(V_{A}, V_{M}\right)$ for $z_{0} / \mathcal{L}=1.05$, where the arrows point in the direction of increasing value. Also displayed are the dynamic adaptive aberration ranges for $1 \%$ variation in the other coefficient of aberration.

While this recipe describes dynamic chromatic aberration correction, slight procedural modifications would produce dynamic spherical aberration compensation or dynamic alignment control (touch up in $z_{0}$ ). Excluding extreme parameter values, Fig. 4.5 shows that a range of $0.2<C_{c} / C_{s}<4$ is very realistic, which is almost three times the range of the diode mirror. A survey of aberration ratios between 0.2 and 4 showed that a nominal $10 \%$ tuning range is possible for tuning in either $C_{c}$ or $C_{s}$ for less than $1 \%$ variation in the other property and constant $z_{0}$. By design, a triode mirror cannot dynamically adjust the correction values of both spherical and chromatic aberration without changing the focal length of the mirror. In essence, there are three properties $\left(z_{0}, C_{s}\right.$, or $\left.C_{c}\right)$ and only two variables $\left(V_{A}\right.$ and $\left.V_{M}\right)$; it is this underspecification which limits the adaptability of the triode mirror. With the introduction of a third dynamic 


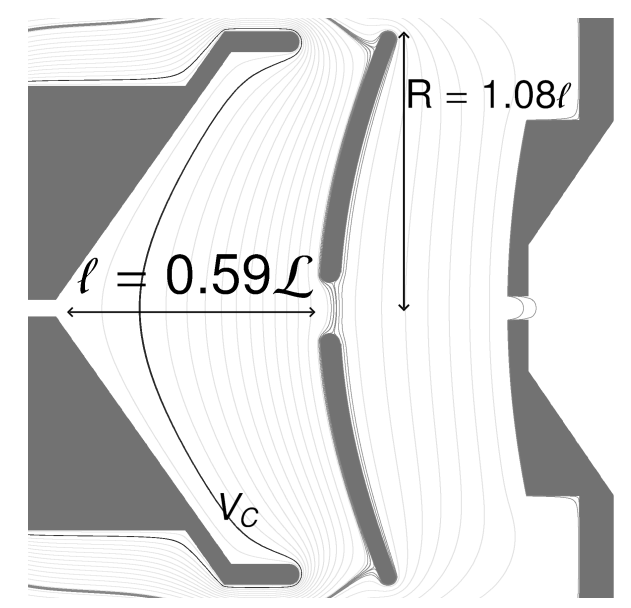

Figure 4.6: Model of the triode mirror used for numerical simulation. The electric potential distribution was determined by SIMION, with widely-spaced equipotentials between electrodes $\left(0.05 V_{C}\right.$ spacing), finer spacing $\left(0.005 V_{C}\right)$ near the apertures, and a dark equipotential highlighting the reflecting surface (at $V_{C}$ ). Reprinted from Fitzgerald, Word, and Könenkamp [94].

variable, i.e. the interface lens, an aberration-correcting system should be able to match any combination of the three properties over a wide range.

\subsection{Comparison to realistic model}

A realistic electron mirror can not have infinite radial extent nor infinitely thin apertures, as implied in the Davisson-Calbick formula, two major assumptions of the analytic model. The deviations from these approximations in a realistic mirror can be understood qualitatively and the scale of their impact can be calculated. The electron optical properties of a realistic geometry, shown in Fig. 4.6, are calculated using SIMION [100].

In a mirror without infinite radial extent, such as the one shown in Fig. 4.6, the potential distribution is only approximately hyperbolic near the optical axis. Define $D$ as the diameter of the mirror. The near-axis difference between the analytic and realistic potential distributions increases roughly as $(\ell / D)$ and $(d / D)$, where $\ell$ 
is the distance between the mirror and center electrodes, and $d$ is the maximum diameter of the electron beam. Additional elements, such as the cylindrical ring on the mirror electrode in Fig. 4.6, can mitigate the difference. Deviations from the hyperbolic distribution impact the spherical aberration strongly. As $(\ell / D)$ increases, the curvature of the potential distribution near the axis weakens. For $(\ell / D) \lesssim 0.5$ and $(d / D) \lesssim 0.03$ the maximum deviations in $C_{s}$ are about $15 \%$, though less than $5 \%$ is typical over the range of potentials of interest (the $10 \%$-variation-in- $C_{c}$ region). Geometries with $(\ell / D) \gtrsim 0.75$ are no longer accurately modeled by the analytic model, while geometries with $(\ell / D) \lesssim 0.25$ show deviations of less than $5 \%$ from the analytic model over a broad range of electrode potentials.

The analytic model uses the Davisson-Calbick formula for the aperture focal lengths. This model is a limiting case of an infinitely thin aperture which gives a sharp change in potential gradient across the aperture. For relatively weak lenses, the approximation works very well. For strong lenses or large trajectory angles, the focal length of the aperture is shorter than predicted by the formula [107], which results in a longer object/image distance $z_{0}$. In the triode mirror the strengths of the aperture lenses change with the electrode potentials, so chromatic aberration is also affected by the Davisson-Calbick approximation. Ideal apertures do not contribute to spherical aberration, and the effects of real apertures on spherical aberration can be safely ignored [107]. The object/image distances in consideration are typically $0.5<z_{0} / \mathcal{L}<1.5$, so the overall focal length of the mirror is $0.25<f / \mathcal{L}<0.75$. Apertures with focal length $\left|f_{a p} / \mathcal{L}\right|>2$ are relatively weak lenses that agree reasonably with the Davisson-Calbick formula, and any differences are minor perturbations to the overall mirror behavior. The grounded electrode aperture focuses weakly for 
all mirror electrode potentials if

$$
\left(V_{A} / V_{C}\right)<\left(1-\ell^{2} / \mathcal{L}^{2}\right) .
$$

The center electrode aperture focuses weakly when

$$
\begin{gathered}
\left(V_{M} / V_{C}\right)<1 /\left(1-\ell^{2} / \mathcal{L}^{2}\right), \text { and } \\
\frac{\left(\ell^{2} / \mathcal{L}^{2}\right)+\left(V_{M} / V_{C}\right)}{\left(\ell^{2} / \mathcal{L}^{2}\right)+\left(1-\ell^{2} / \mathcal{L}^{2}\right)^{-1}}<\frac{V_{A}}{V_{C}}<\frac{V_{M}}{V_{C}}\left(1-\ell^{2} / \mathcal{L}^{2}\right) .
\end{gathered}
$$

For example, if $\ell / \mathcal{L}=1 / 3\{2 / 3\}$ then $\left(V_{M} / V_{C}\right)<9 / 8\{9 / 5\}$ and $0.84\{0.29\}<$ $\left(V_{A} / V_{C}\right)<1$. Since a greater range of electrode potentials enhances the flexibility of the mirror, smaller $\ell / \mathcal{L}$ appears to be best. In practice, a greater range of $V_{A}$ can be accommodated by characterizing specific models with an extensive simulation.

A numerical model with realistic geometries provides more concrete validation of the analytic model, as in the analytic Fig. 4.6. The mirror lengths are $\mathcal{L}=42.3 \mathrm{~mm}$ and $\ell=0.48 \mathcal{L}$, the apertures have diameters $d=1.52 \mathrm{~mm}$, and the thicknesses of the center and outer electrodes are $1.5 \mathrm{~mm}$ and $2.54 \mathrm{~mm}$, respectively. Both faces of the center electrode different contours of the hyperbolic field. The mirror, center, and grounded electrodes extend radially $19 \mathrm{~mm}, 19 \mathrm{~mm}$, and $25.4 \mathrm{~mm}$, respectively, from the optical axis. The outer edge of the mirror electrode has been shaped to compensate for the finite radial extent with a ring $3.15 \mathrm{~mm}$ wide. A grounded cylinder of diameter $50.8 \mathrm{~mm}$ coaxial with the optical axis surrounds the entire model.

The potential distribution was determined by solving Laplace's equation with SIMION using an over-relaxation, finite difference technique to a convergence of one part in 20000 on a cylindrically symmetric mesh composed of $N=N_{z} \times N_{r}=12532 \times$ 4001 points spaced $6.35 \mu \mathrm{m}$ apart $\left(L_{z} \times L_{r}=79.6 \times 25.4 \mathrm{~mm}^{2}\right)$ in SIMION. A point 
source of twenty electrons with randomly distributed angles between 3 and 5 mrads was accelerated to $e V_{C}=20 \mathrm{keV}$. Electron trajectories were numerically integrated through the model to determine $z^{\prime}$ and $\alpha^{\prime}$ in symmetric mode, from which $z_{0}, C_{s}$, and $C_{c}$ were computed by fitting to Eqs. (4.15), (4.17), and (4.18). Electrode potentials were sampled over the range $-23.770 \mathrm{kV}<V_{M}<-22.08 \mathrm{kV}$ and $-14 \mathrm{kV}<V_{A}<$ $0 \mathrm{kV}$. Scan intervals were dynamically varied $1 \mathrm{~V}<\Delta V_{M}<10 \mathrm{~V}$ and $7 \mathrm{~V}<\Delta V_{A}<$ $400 \mathrm{~V}$, for a total of 26,375 configurations of $V_{M}$ and $V_{A}$ (non-focusing configurations were not recorded).

Fig. 4.7 shows the results of the simulation. The differences between the analytic and simulated optical properties, $z_{0}, C_{s}$, and $C_{c}$, are shown as contours plotted against the mirror potentials. Highlighted in black is the region centered around aberration corrections of $C_{s S y s 0} / \mathcal{L}=8.05 \times 10^{4}$ and $C_{c S y s 0} / \mathcal{L}=721$ and enclosing differences $\left(\Delta C_{s m} / C_{s S y s 0}\right)^{2}+\left(\Delta C_{c m} / C_{c S y s 0}\right)^{2}<0.3^{2}$ from that central point. Over the highlighted region, all three properties show agreement to within $5 \%$. The objec$\mathrm{t} /$ image distance has an offset from the analytic model of $1.26 \mathrm{~mm}(3 \%)$, but changes by less than $0.4 \mathrm{~mm}(1 \%)$. The spherical and chromatic aberration coefficients exhibit a wider range of variation, but also differ by less than $5 \%$ from the analytic model. Thus, while the analytic model may be too inaccurate to use for implementation, it is extremely useful for optimizing the configuration.

\subsection{Triode mirror and lens with fixed object distance}

The optimization of the mirror-lens system is complicated, so it is instructional to first consider a non-interacting model. This restricts the triode mirror to maintain a constant focus, as previously discussed, and the lens to maintain a constant magnification. The non-interactive corrector is a three-dimensional optimization problem, where the mirror-lens spacing $Z$, the geometry of the triode mirror $\ell / \mathcal{L}$, and the mir- 


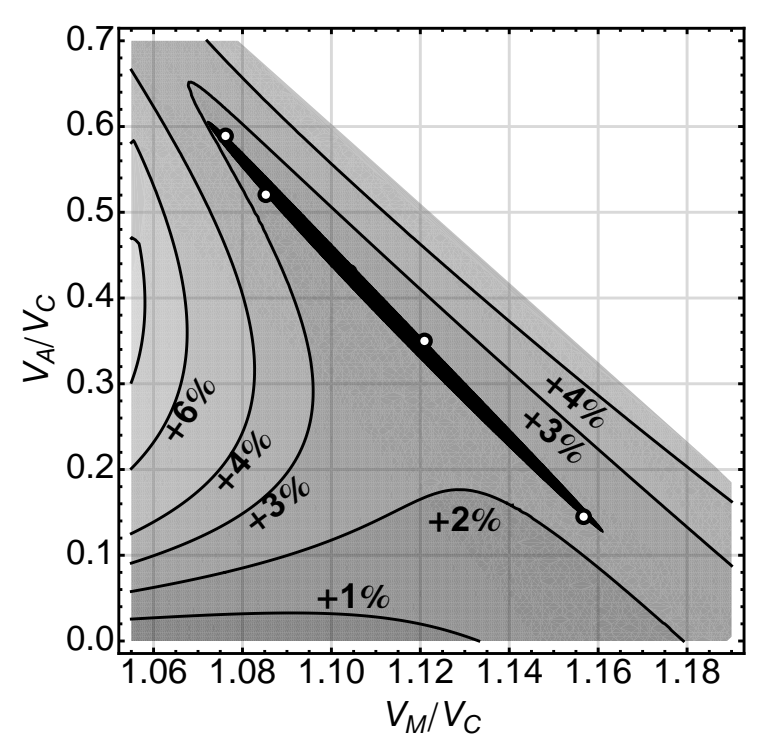

(a) $z_{0}$

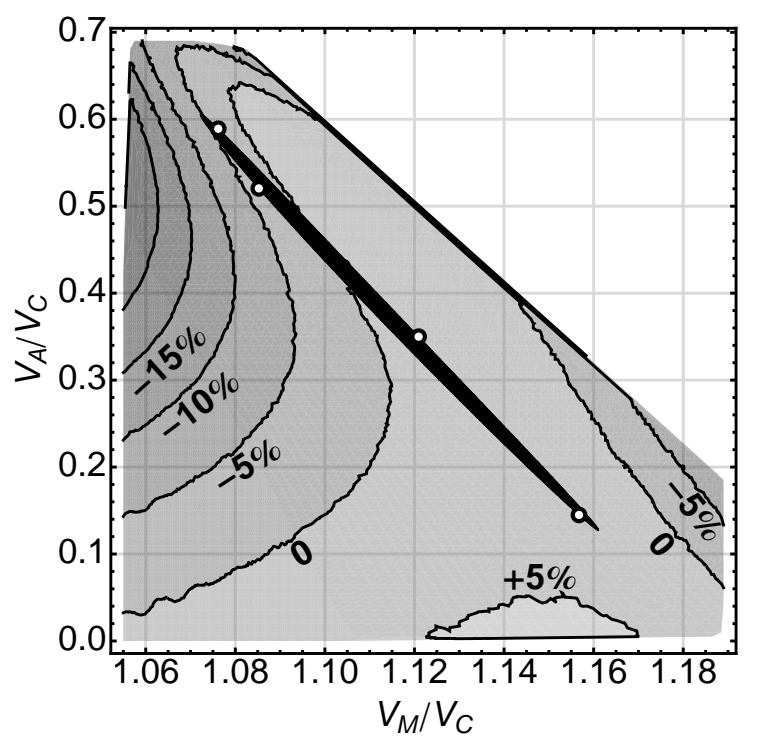

(b) $C_{s}$

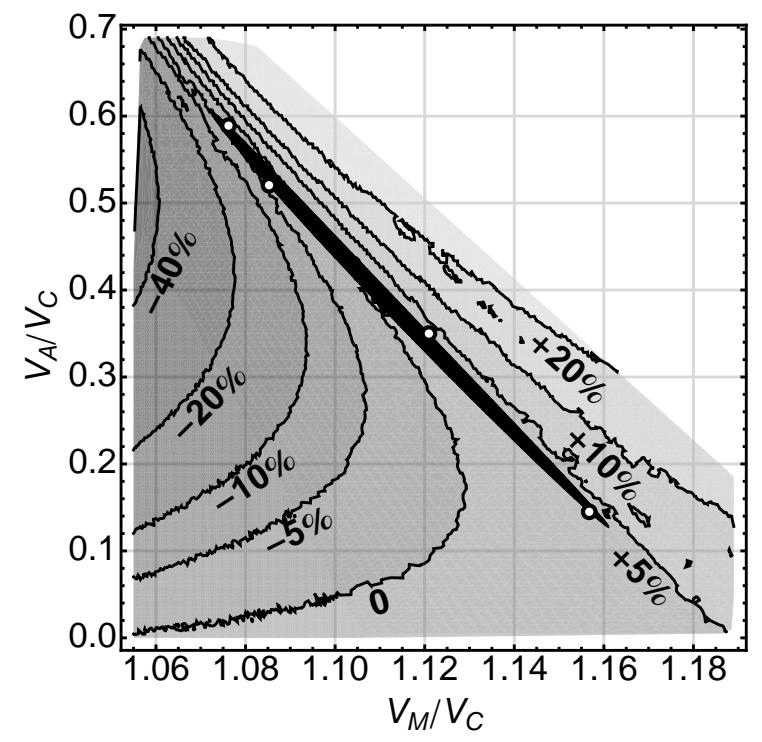

(c) $C_{c}$

Figure 4.7: Comparison of the optical properties of the triode mirror between analytic and simulated models. The 30\% aberration variation region is highlighted (black), over which region the difference changes by less than (a) 1\%, (b) 5\%, (c) 10\%. Contours are relative to: (a) the mirror length $\mathcal{L},(\mathrm{b})$ and $(\mathrm{c})$ spherical and chromatic aberration coefficients at the center of the $30 \%$ variation region $\left(C_{s} / \mathcal{L}=-30\right.$, $\left.C_{c} / \mathcal{L}=-14\right)$. Reprinted from Fitzgerald, Word, and Könenkamp [106]. 


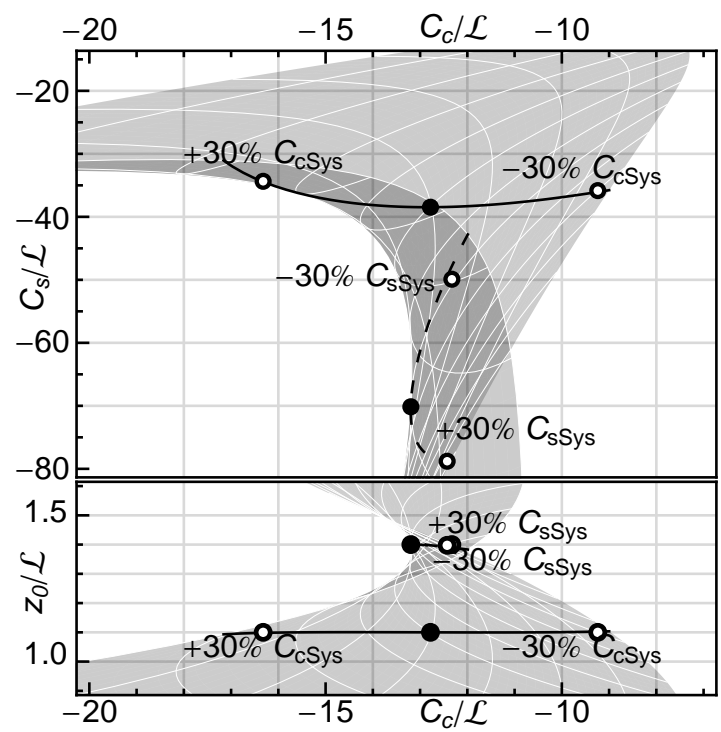

(a)

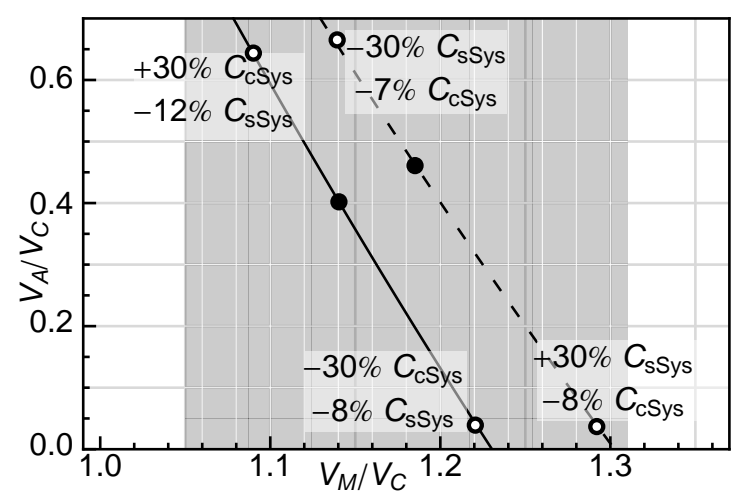

(b)

Figure 4.8: Properties of the triode mirror with $\ell=0.48 \mathcal{L}$ and $\mathcal{L}=42.3 \mathrm{~mm}$. Two constant focal length contours are plotted, with $z_{0}=1.1 \mathcal{L}$ (solid) and $z_{0}=1.4 \mathcal{L}$ (dashed). Three points along each constant- $z_{0}$ contour illustrate a range of aberration correction values. The solid, black point corrects the aberrations $-C_{s S y s}$ and $-C_{c S y s}$, where $C_{s \text { Sys }}$ and $C_{c S y s}$ are the spherical and chromatic aberrations of a cathode and objective lens. (a) The optical properties $C_{s}, C_{c}$, and $z_{0}$, plotted relative to the triode mirror length $\mathcal{L}$. The gray regions are projections in $C_{s} \times C_{c}$ and $z_{0} \times C_{c}$ of the $3 \mathrm{D}$ surface in mirror property space spanned by the potential range (shown in b) for any $z_{0}$. The white lines trace contours of constant potential. (b) The mirror and center electrode potentials, $V_{M}$ and $V_{A}$, plotted relative to the accelerating potential $V_{C}$. Reprinted from Fitzgerald, Word, and Könenkamp [106]. 
ror potential $V_{M}$ are the independent variables and the mirror length $\mathcal{L}$ and center electrode potential $V_{A}$ are the dependent variables.

An objective-type lens is used for its low spherical and chromatic aberration coefficients, $C_{s i}$ and $C_{c i}$, at relatively high focusing power. This is important since this lens images the electron beam twice. In this first treatment the lens-specific properties are determined from experimental data in Rempfer [60]. Subsequently the angular magnification, $m_{i}$, and the aberration coefficients (in $\mathrm{mm}$ ) are parameterized as functions of the incident image distance, $z_{i}^{\prime}$ (in $\mathrm{mm}$ ),

$$
\begin{aligned}
& m_{i}=-0.07474+0.0001108 z_{i}^{\prime}+66.19\left(z_{i}^{\prime}\right)^{-0.9637}, \\
& C_{s i}[\mathrm{~mm}]=-18.13+4.773 z_{i}^{\prime}+0.01598\left(z_{i}^{\prime}\right)^{3.388}, \\
& C_{c i}[\mathrm{~mm}]=4.347+1.442 z_{i}^{\prime}+0.01289\left(z_{i}^{\prime}\right)^{2.035} .
\end{aligned}
$$

The incident beam object distance is $72.9 \mathrm{~mm}$, as in Fig. 4.9b. Since the distance from the mirror electrode vertex to the lens center $Z$ also remains constant, the lens image and mirror object/image distances are related by $z_{i}^{\prime}=Z-z_{0}$. Hence, the lens properties become functions of the mirror potentials.

For each constant $z_{0}$ curve, the triode mirror has a fixed relationship between $V_{A}$ and $V_{M}$ (Fig. 4.8). Along a particular curve, e.g., $z_{0}=1.1 \mathcal{L}$, the amount of spherical aberration correction has a maximum value. Around this point, chromatic aberration correction can dynamically change without significantly affecting spherical aberration correction. Thus the triode mirror aberration correction system has an adaptive quality that allows for small corrections, at least in chromatic aberration. For a different curve, e.g., $z_{0}=1.4 \mathcal{L}$, the triode mirror has dynamically adjustable spherical aberration at nearly constant chromatic aberration. So for each $z_{0}$ there is one best combination of $V_{A}$ and $V_{M}$ around which aberration tuning is optimized. 


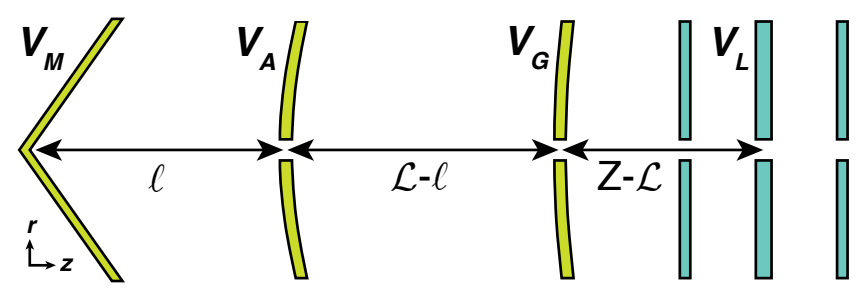

(a)

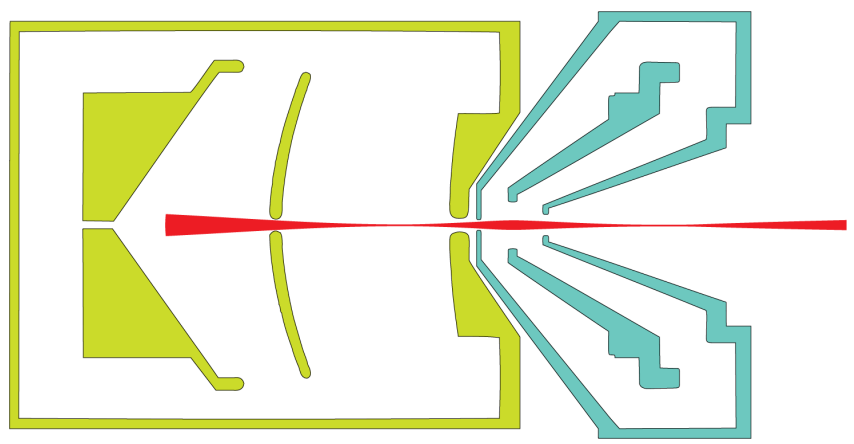

(b)

Figure 4.9: The mirror has cylindrical symmetry about the $z$-axis with $z=r=0$ at the cone vertex. The mirror electrode, at potential $V_{M}$, is a cone with opening angle $\Delta r / \Delta z=\sqrt{2}$. The center electrode, at potential $V_{A}$, follows the hyperbolic contour $z^{2}-\frac{1}{2} r^{2}=\ell^{2}$; and the outer electrode, at potential $V_{G}=0$, follows $z^{2}-\frac{1}{2} r^{2}=\mathcal{L}^{2}$. Small apertures in the center and outer electrodes allow electrons to enter and exit the mirror field. The distance from the mirror vertex to the opening of the first aperture is $\ell$, and the distance from the mirror vertex to the opening of the second aperture is $\mathcal{L}$. An einzel lens, at potential $V_{L}$, accompanies the mirror a distance $Z$ away. $Z$ is measured from $z=0$ to the center of the einzel lens. (a) Schematic diagram of the triode hyperbolic electron mirror with an einzel lens. (b) Simulation model of a mirror-lens combination showing the symmetric incident and exit beams. Reprinted from Fitzgerald, Word, and Könenkamp [106]. 
Matching the aberration of the mirror, $C_{s}$ and $C_{c}$ to that of the microscope, $C_{s T o t}$ and $C_{c T o t}$, at this value of $\ell / \mathcal{L}$ and $z_{0}$ fixes $Z$ and $\mathcal{L}$. For a given choice of these physical parameters- $Z, \ell / \mathcal{L}$, and $\mathcal{L}$ - the mirror potentials describe a surface in the threedimensional property space $C_{s} \times C_{c} \times z_{0}$, as shown in Fig. 4.8, which is accessible once the aberration correction system is installed. Examining the projections of this surface onto $C_{s} \times C_{c}$ and $z_{0} \times C_{c}$ it becomes clear that there is one best $z_{0}$ for spherical aberration tuning and another for chromatic aberration tuning.

Allowing $z_{0}$ to change would greatly enhance the dynamic range of correction, potentially yielding simultaneous and independent correction of spherical and chromatic aberration. However, for a fixed mirror to lens spacing, a dynamic $z_{0}$ also changes the magnification of the lens, which, in turn, changes the amount and ratio of the aberration to be corrected. The interacting mirror and lens system must be studied more carefully to determine whether it allows for independent variability of spherical and chromatic aberration correction and how the dynamic range or independent correction can be optimized.

\subsection{Interacting triode mirror-lens corrector}

The one-to-one relationship between the coefficients of spherical and chromatic aberration in the previous section is due to an overspecification of the system of equations: two variables $-V_{M}$ and $V_{A}$-are used to specify the two aberration coefficients and align the focus of the microscope, i.e., three properties. If the triode mirror were no longer constrained to constant focus, the resulting interacting mirror-lens system would be able to independently compensate spherical and chromatic aberration. In this way the focus and coefficients of aberration would be specified by three variable potentials, $V_{M}, V_{A}$, and $V_{I}$, where $V_{I}$ is the interface lens potential, and an exact specification of the system of equations is possible over the range in which the three 


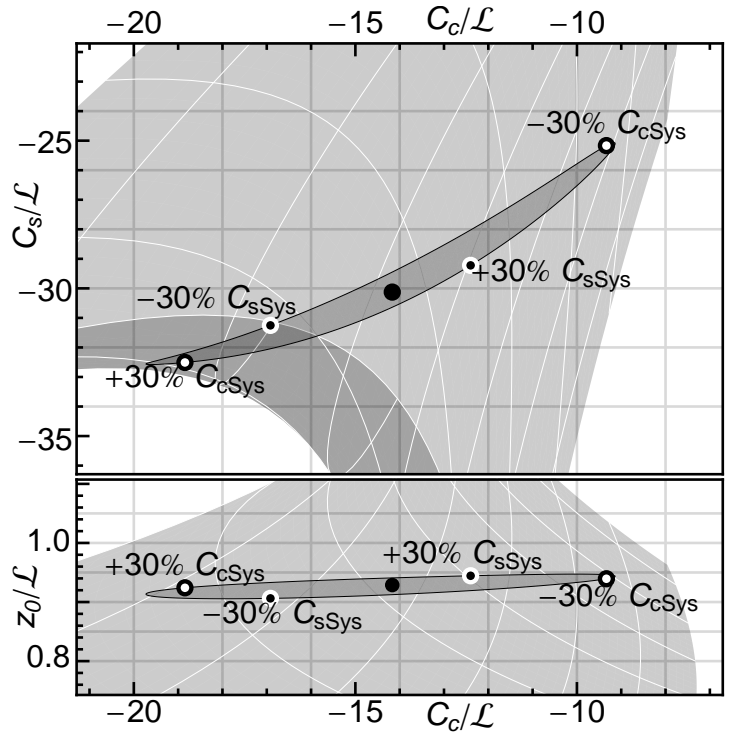

(a)

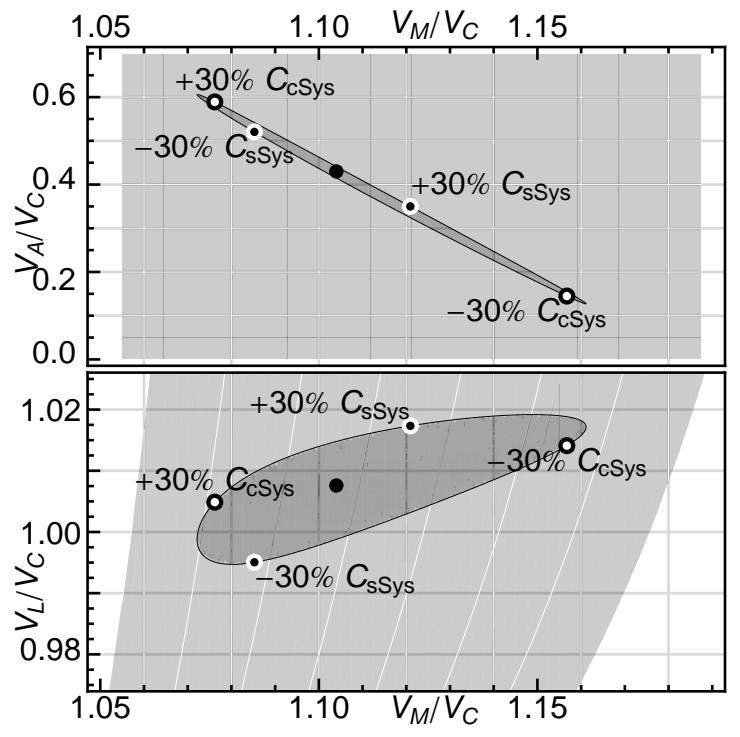

(b)

Figure 4.10: Properties of a triode mirror, with $\ell=0.48 \mathcal{L}$, used interactively with a lens in symmetric mode. The dark gray region highlights the property range that can be accessed by changing the potentials while maintaining a focus. This region encloses aberration correction changes of up $30 \%$ from the central point. The four perimeter points (black/white) indicate the maximum variation of each coefficient. (a) The triode optical properties, zoomed in from Fig. 4.8a. (b) The triode mirror and lens potentials. Compare to Fig. 4.8b. Reprinted from Fitzgerald, Word, and Könenkamp [106]. 
variables are linearly independent and physically meaningful. This system is quite similar to the non-interactive corrector described above. The focus equation,

$$
z_{0}+z_{i}^{\prime}=Z
$$

implies that $z_{i}^{\prime}$ is a function of the mirror object/image distance $z_{0}$ for fixed mirror to lens spacing $Z$. Thus, the lens properties in Eq. (4.22) are functions of the mirror potentials, and the two equations for the cancellation of spherical and chromatic aberration, Eqs. (3.26) and (3.27), depend only on the two variable potentials $V_{M}$ and $V_{A}$.

The optimization of the interactive system has only a few modifications from the previous section. Instead of a single best combination $V_{M}$ and $V_{A}$ for each $z_{0}$, the best interactive mirror-lens corrector maximizes the area enclosed by the range of spherical and chromatic aberration that can be corrected. This can be measured by the parametric area $\left(\Delta C_{s \text { Sys }} / C_{s \text { Sys } 0}\right) \times\left(\Delta C_{c \text { Sys }} / C_{c \text { Sys } 0}\right)$, where the range of aberration is $\Delta C_{S y s}=C_{S y s 0}-C_{S y s, c o r}$ and $C_{S y s, c o r r}$ is the value of aberration corrected as measured in transfer space, i.e., at the beam separator Magnet B. The current discussion uses $C_{s \text { Sys } 0}=3.40 \times 10^{6} \mathrm{~mm}$ and $C_{c S y s 0}=3.05 \times 10^{4} \mathrm{~mm}$. Optimization solutions are found by first setting $V_{A}=\frac{1}{2}\left(1-\ell^{2} / \mathcal{L}^{2}\right) V_{M}$ as the center of the correction area, and second the maximum correction area is limited by $\left(\Delta C_{s \text { Sys }} / C_{s \text { Sys } 0}\right)^{2}+\left(\Delta C_{s \text { Sys }} / C_{s S y s 0}\right)^{2} \leq 1$. This boundary traces out a unit circle in aberration correction coordinates, and the area of this circle can be used as a standard to judge the maximum correction region. The maximum aberration correction that this survey can find is $\pm 100 \%$.

The maximum coverage was found to be $69 \%$ for the parameters $\ell / \mathcal{L}=0.47$, $\mathcal{L}=42.3 \mathrm{~mm}$, and $Z=48.9 \mathrm{~mm}$. $C_{s S y s 0}$ and $C_{c S y s 0}$ are fully corrected with the potentials $V_{M} / V_{C}=1.104, V_{A} / V_{C}=0.43$, and $V_{I} / V_{C}=1.015$ and can independently 
correct any values of spherical and chromatic aberration within $38 \%$ of this central value since $z_{0}$ is only weakly constrained. The primary limitation of that region is that the range of $V_{A}$ is maximized. Spherical aberration correction, adjusted primarily by the magnification of the lens, can be freely adjusted to any value within $\pm 100 \%$, since the lens potential range is far from bounded. The potential relationships and the aberration and object/image distance of the triode mirror run in this configuration are shown in Fig. 4.10, with the 30\% aberration variation region highlighted. Note that a $\mp 5 \%$ change to triode mirror spherical aberration produces a $\pm 30 \%$ change in spherical aberration correction. The lens magnification change of $\sim 0.5 \mathrm{X}$ accounts for an effective correction change of $\sim \pm 33.5 \% C_{s S y s 0}$. The remaining $2.5 \%$ change is accounted for by the lens. For chromatic aberration, nearly all correction change is provided at constant $z_{0}$, and so it is the mirror potentials that adapt the correction.

In conclusion, I find that a triode mirror and lens can simultaneously correct spherical and chromatic aberration of a PEEM image when the two elements are used interactively. As implemented, the corrections can be independently varied over a range of greater than $100 \%$ and $30 \%$ for spherical and chromatic aberration, respectively. This allows for increased precision in aberration corrected microscopes, and opens the door to fine-tuning spherical and chromatic aberration correction for each imaging session. In addition, the analytic model developed here grants a degree of intuitive understanding that allows optimization of the mirror-lens interacting pair corrector. This model is accurate to within $5 \%$, so the optimized model relates well to a physical correction system. The triode mirror can correct aberration over a relatively large range because lens magnification can transform a wide range of microscope aberrations to the range of the mirror. 


\section{Experimental adaptation and preparation}

\subsection{Corrector design parameters}

The range of aberration correction of the triode mirror is matched to the calculated aberration range of our PEEM. The corrector is comprised of a two-region, hyperboloid-shaped mirror, as shown in Figs. 4.1 and 4.6, paired with a unipotential lens, as shown in Fig. 4.9. For comparison, the prior configuration of the PEEM used a diode mirror and lens, as shown in App. Appendix A.The new corrector is designed to work within the same physical space. To minimize changes to the established hardware, the interface lens is kept at the same distance from magnetic beam separator as in the diode design. The triode fits within the remaining $\sim 90 \mathrm{~mm}$ of the lens tube. Due to limited space, the triode hyperbolic potential regions are $\mathcal{L} \lesssim 55$ $\mathrm{mm}$. The central aberration range was estimated from the correction offered by the past diode mirror and interface lens, with the mirror operating at $-21.9 \mathrm{kV}$. The diode interface lens is a swept-back design (App. ??) with an estimated bore diameter of $6.71 \mathrm{~mm}(0.264 \mathrm{in})$. The diode mirror and interface lens were modeled in SIMION to accurately characterize their spherical and chromatic aberration coefficients. I wrote a Lua language program to automate SIMION simulations and a Mathematica program to analyze the simulation results (Apps. Appendix D, Appendix E, and Appendix G). From the diode mirror corrector simulation, I found aberration coefficients of $C_{s S y s 0}=3.40 \times 10^{6} \mathrm{~mm}$ and $C_{c S y s 0}=3.05 \times 10^{4} \mathrm{~mm}$, as measured in transfer space. Finally, the analytic triode mirror theory was geometrically optimized to provide the maximum aberration correction range, resulting in a geometric factor of $\ell / \mathcal{L}=0.47$ and a total distance $\mathcal{L}=42.3 \mathrm{~mm}$, as measured from the cone vertex to grounded electrode aperture. 


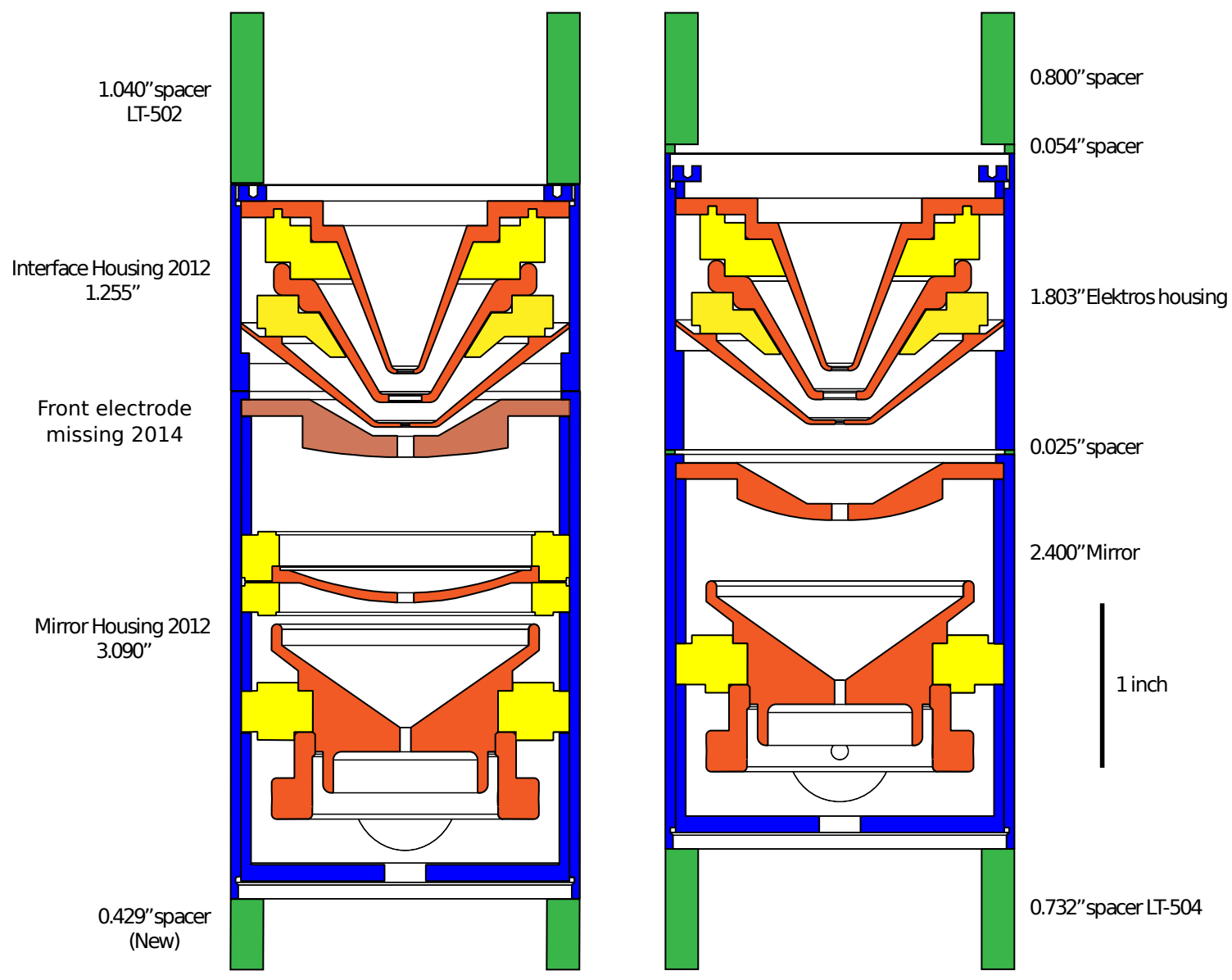

Triode Mirror

Diode Mirror

Figure 5.1: Direct, side-by-side comparison of the triode mirror and diode mirror branch designs. The final version of the triode mirror was installed without the grounded front electrode, as noted. Credit: R.C. Word.

The triode mirror with the optimized geometric parameters was based on the successful diode hyperbolic mirror designed by G. Rempfer. The part specifications and PEEM modifications were drawn by T. Dornan. A direct comparison of the diode and triode correctors as installed is given in Fig. 5.1. The design of the insulating spacers separating the additional mirror electrode at potential $V_{A}$ required a careful compromise between the demands of leakage currents, beam exposure, secure alignment, breakdown, and additional considerations, and may bear future investigation. The 
optimal aberration correction range is only provided when the interface lens is closely positioned to the triode mirror. It was determined that a spacing between of only $1.4 \mathrm{~mm}$ between the grounded mirror electrode and the grounded swept-back lens electrode could satisfy the design. However, the close proximity of the two apertures introduces some difficulties in characterization and alignment. In order to alleviate some of the potential problems with alignment, the triode mirror was ultimately installed without the grounded electrode. Instead, a spacer ring holds the remaining parts in place, and the outer electrode of the interface lens provides the grounding of the mirror field. It is expected that the modified geometry will compare favorably to a triode mirror lengthened by the distance between the grounded aperture of the mirror and the lens, $\sim 4.72 \mathrm{~mm}$. The lengthening of $\mathcal{L} \rightarrow \mathcal{L}^{\prime}$ changes the geometry ratio from $\ell / \mathcal{L}=0.47$ to $\ell / \mathcal{L}^{\prime}=0.42$. With the following simulation, I show that this change does not significantly alter the aberration correction properties.

\subsection{Numerical simulation via SIMION}

In the theoretical study of the mirror corrector, it was found that the analytic and numerical models differed by enough that only a numerical model could characterize the optical properties with sufficient precision. The precision of the power supplies in our PEEM is about 1-2 V, so the characterization has an electric potential resolution an order of magnitude larger or better, at $10^{-6}-10^{-7} \times V_{C}$. The useful mirror electrode potential ranges from $V_{M} / V_{C} \gtrsim 1.02$ to $V_{M} / V_{C} \lesssim 1.22$, and the useful additional electrode potential varies $0<V_{A} / V_{C} \lesssim 0.75$. At the desired resolution, the simulation space occupies up to $\left(4 \times 10^{5}\right) \times\left(1.5 \times 10^{6}\right)=6 \times 10^{11} \equiv N_{V, \text { max }}$ mirror potential configurations. Because of the close proximity of the mirror and lens, the two cannot be treated independently in a numerical characterization since the aperture fields of the two elements influence each other. When the grounded mirror electrode is 
removed, the interaction becomes stronger. Thus the lens voltage is a third dimension in the corrector configuration space $V_{M} \times V_{A} \times V_{I}$. The useful lens range is $0.9 \lesssim$ $V_{I} / V_{C} \lesssim 1.15$ for a $6.7 \mathrm{~mm}$ center bore diameter swept-back lens, leading to a upper limit to the corrector configuration space of $N_{V, \max }^{(\mathrm{tot})}=\left(4 \times 10^{5}\right) \times N_{V, \text { max }}=2 \times 10^{17}$ at a resolution of $5 \times 10^{-7} \times V_{C}$. If only $5 \times 10^{-6}$ is required-one volt in $20 \mathrm{kV}$ precision - then the configuration space is $2 \times 10^{14}$ potential combinations.

To determine each characterization in SIMION requires numerically tracing trajectories through the electric potential distribution of the corrector. The potential of a numerical model was refined in SIMION to a relative gradient precision of $5 \times 10^{-7}$ on an array with grid unit size of $6.25 \mu \mathrm{m}$. This was done for models both with and without the grounded front electrode, as shown in Fig. 5.2. The electric potential between $V_{A}$ and 0 differs by a few percent from a hyperbolic distribution in both models, and the difference between them is consistent with lengthening the triode mirror as expected. Examining the figure, it seems likely the deviation from a hyperbolic potential in this region is a large contributor to the difference between the analytic and numerical models of the triode mirror. Electron trajectories were numerically integrated through the potential, and information about those trajectories was collected with a Lua user program (App. Appendix H). The final implementation of this analysis includes several refinements over the initial code, similar to that used to analyze the diode mirror (App. Appendix G). First, three beam energies are used: the central energy of $20 \mathrm{keV}$, with the others differing by $+1 \mathrm{eV}$ and $-1 \mathrm{eV}$. The use of three beam energies increases the confidence in the chromatic aberration calculation, which is now a linear fit instead of a two-point slope calculation. Second, the computation only obtains a symmetric mode image distance for the central beam energy. It was found that tuning object position for symmetric mode in the other cases introduces chromatic aberration into the point source object since different beam energies 


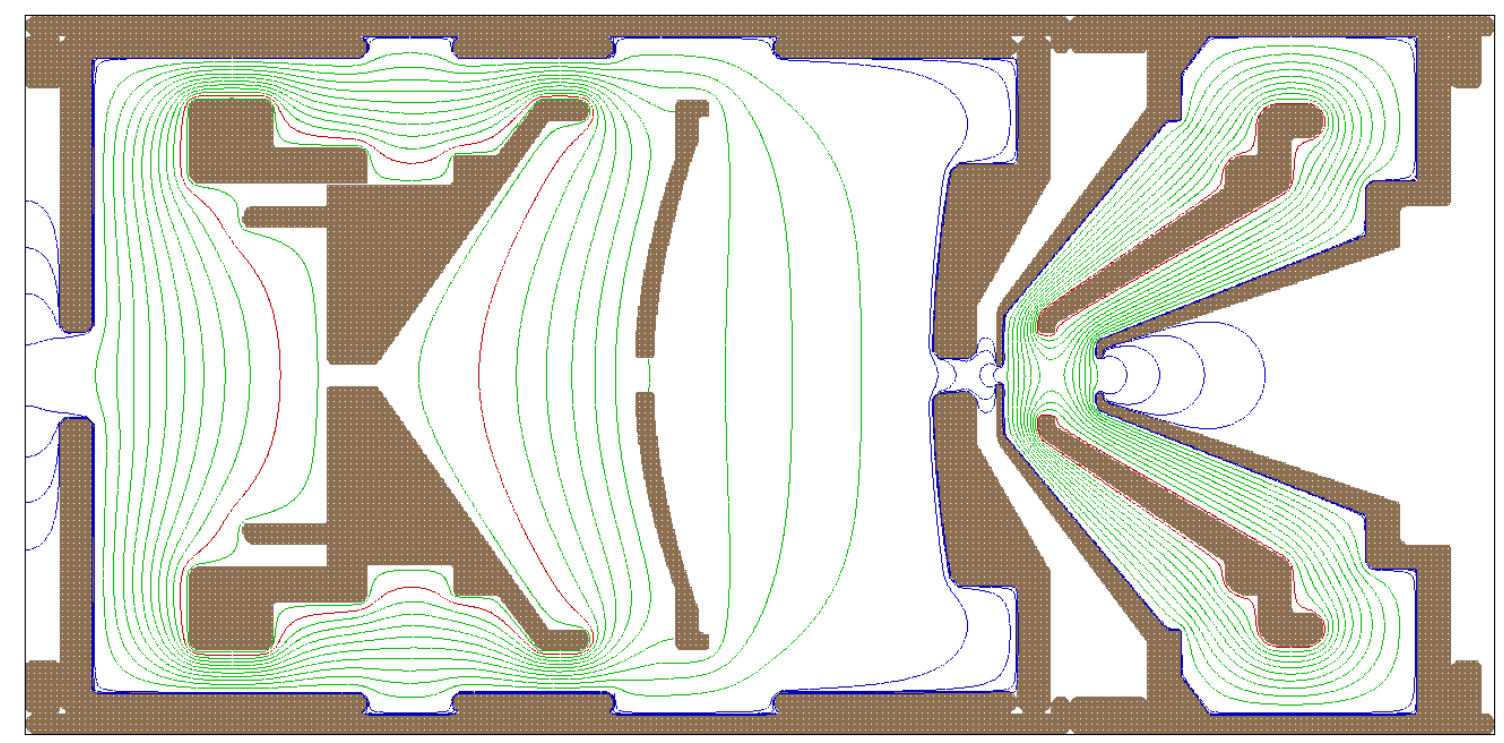

(a) Original model.

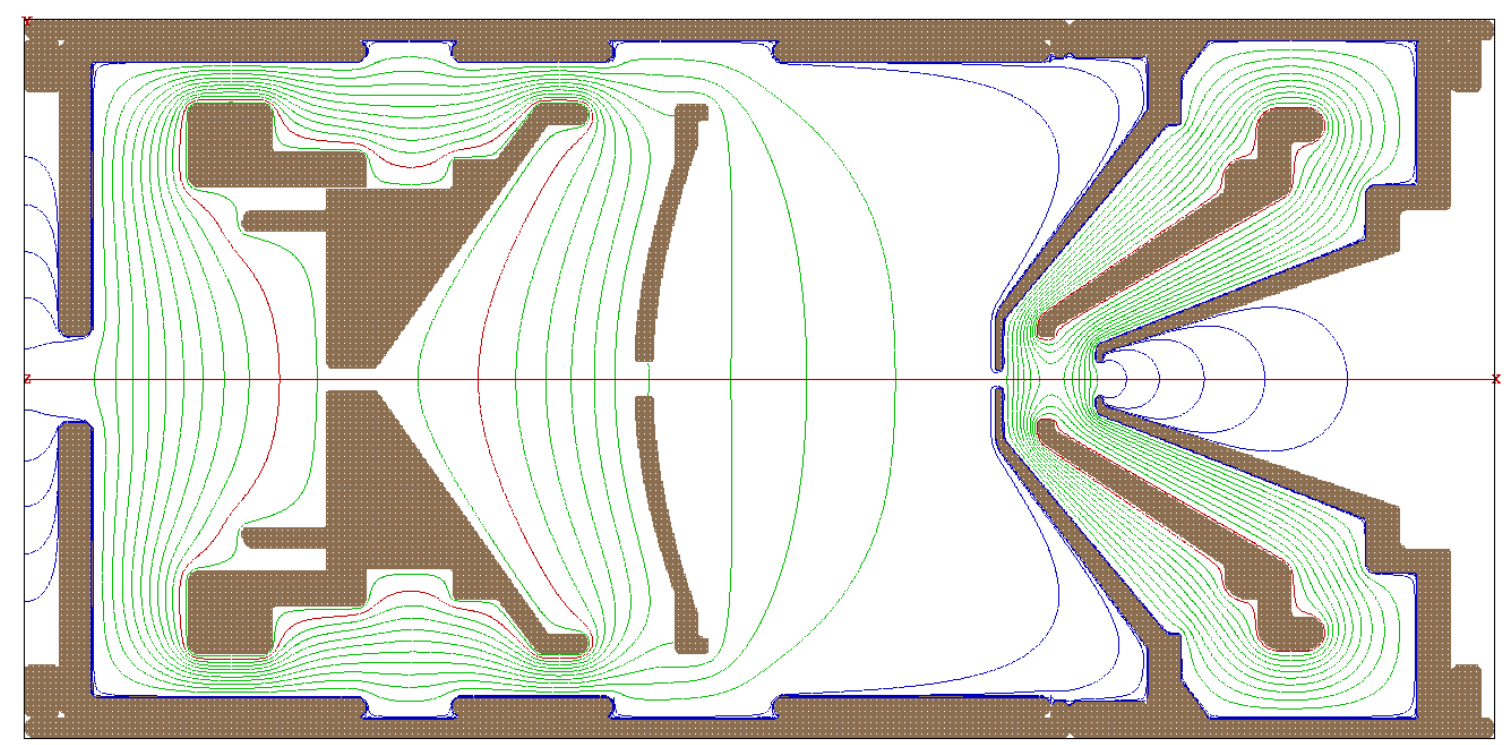

(b) Installed model without triode grounded electrode.

Figure 5.2: Electric potential distributions of triode correctors (a) with the original front electrode design and (b) without the electrode, as determined by SIMION. The electrodes are shown in brown, and potential contours are shown in blue, green, and red. The electrodes are at potentials $V_{M}=-22.5 \mathrm{kV}, V_{A}=-8 \mathrm{kV}$, and $V_{L}=-20.5$ $\mathrm{kV}$. The contours are color coded: blue contours range from $-0.1 \mathrm{~V}$ to $-100 \mathrm{~V}$ by powers of ten; green contours change by $2 \mathrm{kV}$ from $-2 \mathrm{kV}$ to $-22 \mathrm{kV}$; and the red contour shows the electron beam acceleration potential of $-20 \mathrm{kV}$. 


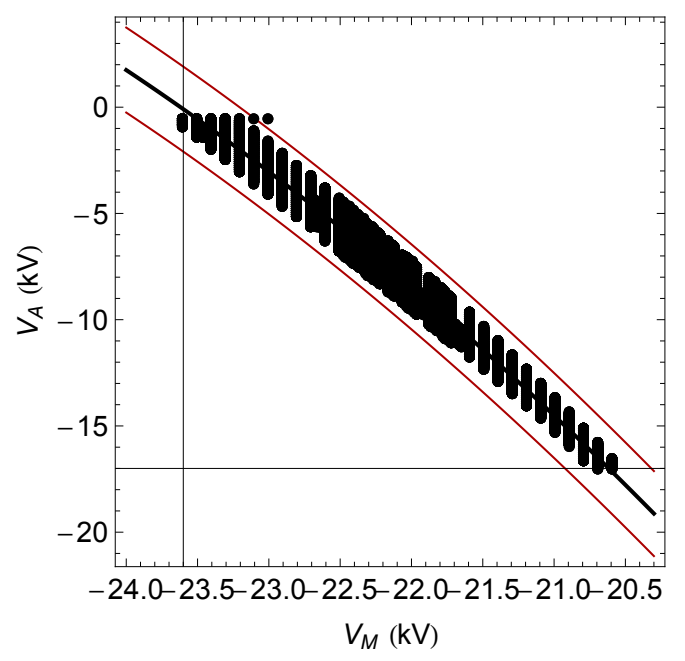

Figure 5.3: Relationship between triode mirror $V_{M}$ and $V_{A}$ that produces real images. All surveyed configurations that produced real images are plotted (black points) and fit to a quadratic polynomial $V_{A \text {,est }}\left(V_{M}\right)$ (black line). All points lie within $\mid V_{A}-$ $V_{A, \text { est }} \mid<2 \mathrm{kV}$, as highlighted the limits in red, and almost all points lie within 1.5 $\mathrm{kV}$, particularly at lower $V_{M}$.

have different object points. The desired model is one in which the object has no aberration and the image has all the aberration, instead of some in the image and some in the object, as before. Removing the additional object distance tuning also increased the speed of the computation and decreased the uncertainty of the final result, since there is explicitly no aberration in the object source.

It was also observed that only narrow ranges of potentials give real images with aberration correction. The interface lens is limited to between -20.0 and $-21.5 \mathrm{kV}$ for a $20 \mathrm{keV}$ electron beam. The mirror potentials vary together, such that configurations satisfying $\left|V_{A}-\left[-21.94-7.23\left(V_{M}+20\right)-0.319\left(V_{M}+20\right)^{2}\right]\right| \lesssim 1.5 \mathrm{kV}$ give real images, for $V_{M}$ and $V_{A}$ in $\mathrm{kV}$. This relationship was determined from an initial survey, and it is illustrated in Fig. 5.3. This reduces the maximum configuration space by two orders of magnitude to $N_{V_{M}} \times N_{V_{A}} \times N_{V_{I}}=\left(4 \times 10^{5}\right) \times\left(1.5 \times 10^{5}\right) \times\left(1.5 \times 10^{5}\right)=$ $9 \times 10^{15}$. The code is further optimized to scan through potential configurations 
more quickly when the previous configuration does not produce a real image, further decreasing the configuration space by a factor of $\sim 2$. Next, the object distance is fixed to the value assessed from the scaled drawings (Apps. Appendix A and ??), and symmetric mode is determined by tuning the lens potential instead of the object position. The tuning is limited to 5-20 attempts, depending on the convergence of the first few attempts. Over the range in interest, this potential is close to linear; however, over wider ranges this method breaks down somewhat. With most of the $V_{M} \times V_{A}$ configurations not producing a real image, on average there are only $\sim 10 V_{L}$ configurations attempted. This reduces the number of configurations significantly to

$$
N_{V, \text { tot }}=N_{V_{M}} \times N_{V_{A}} \times N_{V_{I}}=\left(4 \times 10^{5}\right) \times\left(1 \times 10^{5}\right) \times 10=2 \times 10^{12} .
$$

This is the estimated number of viable electric potential configurations of the triode mirror corrector to a voltage tolerance $5 \times 10^{-7} \times V_{C}$, or about $0.01 \mathrm{~V}$ in $20 \mathrm{kV}$. It is still too large to probe the complete configuration space in a reasonable amount of time, so instead the precision of $V_{M}$ and $V_{A}$ were limited to $10^{-3} V_{C}$ in the SIMION survey. Approximately $1.4 \times 10^{5}$ voltage configurations were simulated with over $5 \times 10^{6}$ electron trajectories, of which only $1.5 \times 10^{3}$ configurations give real images. The new Lua code dumps basic trajectory data into a file, and Mathematica is used to calculate the optical properties (App. Appendix I). For a given $V_{M}, V_{A}$ and $V_{L}$ could be extrapolated to an accuracy of $10^{-7} V_{C}$ with a 4-8 term polynomial. In the final result, only $V_{M}$ is limited to $\sim N_{V_{M}} / 400$ intervals in the final characterization, leading to an effective configuration space of $N_{V, \text { tot }} \sim 5 \times 10^{9}$.

This characterization of the mirror branch was found to give the desired spherical and chromatic aberration correction to within one percent, giving sufficient accuracy to test the effects of changes in aberration correction on the resolution of our PEEM. 


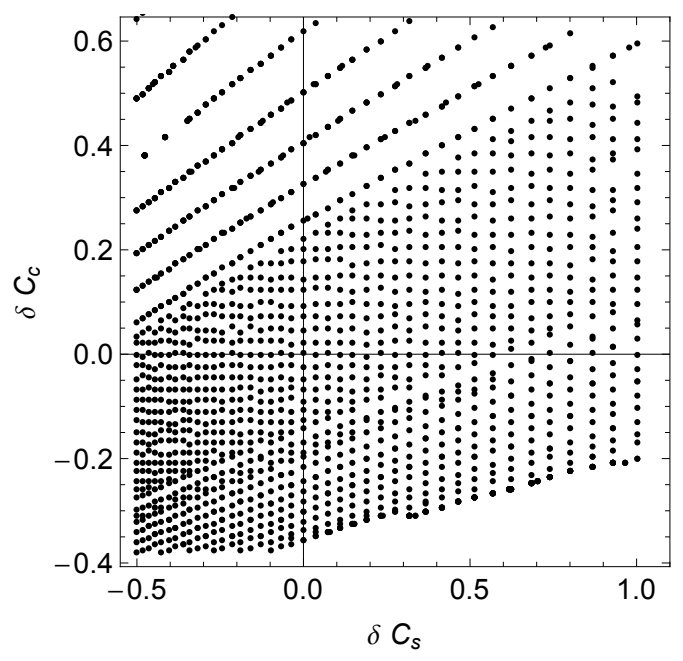

Figure 5.4: Discrete values of aberration correction provided by the triode mirror corrector. Changes in spherical and chromatic aberration coefficients of the mirror branch as measured in transfer space are presented relative to the aberration correction the diode mirror correction, $\delta C=\left(C_{\text {triode }}-C_{\text {diode }}\right) / C_{\text {diode }}$. Since coefficients vary with magnification according to a power law, values are varied logarithmically over a wide range. Correction is strongly limited in the direction of more spherical and less chromatic aberration (lower right corner), but is otherwise unbounded. The upper left corner was not predicted to be of significant interest, so has less dense coverage.

Tables of the mirror branch voltages and aberration coefficients in transfer space are given in App. Appendix J. These tables can be used to control the power supplies providing $V_{M}, V_{A}$, and $V_{I}$. The changes in aberration coefficient correction relative to the diode mirror of those tables are plotted in Fig. 5.4. Clearly, aberration can be corrected over a wide range. Other swept-back lenses can be used by adjusting the potential $V_{I}$ such that the thick-lens paraxial image distance between the lens and mirror is preserved. The effect on the aberration coefficients is approximately $10 \%$ in $C_{s}$ and $5 \%$ in $C_{c}$ for each $0.5 \mathrm{~mm}$ change in bore diameter.

The optical characterization presented in Ch. 2 also allows precise control of the objective branch, as well as an estimate of the aberration produced in configuration space. The main uncertainty in this estimate is from the cathode, which here has 


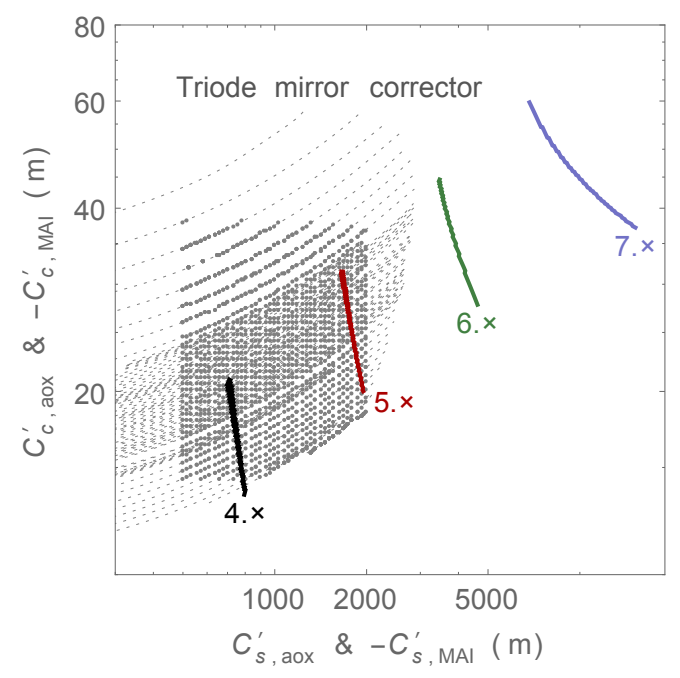

Figure 5.5: The aberration coefficients of the mirror branch (dark gray) and the objective branch with magnifications $4-7 \times$ compared in transfer space for $\mathrm{Cu}$ illuminated with $257 \mathrm{~nm}$ light. Compared to the diode, the triode corrector can accommodate a wider range of aberration correction values, and provides significant room for resolution optimization. Compare this to the diode corrector, Fig. 3.5.

only been characterized to no better than $\sim 10 \%$ precision. The overlap between the objective branch image aberration coefficients and the triode branch correction is presented in Fig. 5.5. From the results of the simulation, the triode mirror has has the potential to correct the image aberration of this specimen. For an another specimen, the image aberration can be approximately matched up with the ideal mirror branch correction by adjusting the magnification of the objective-auxiliary lens zoom pair. Subsequently, the mirror branch aberration can be varied by the microscope user to find the best image resolution, making UPS characterization perhaps less necessary. Examining Fig. 5.5, it appears that by varying objective branch magnification, the aberration correction space can accommodate almost any realistic combination of spherical and chromatic aberration. 


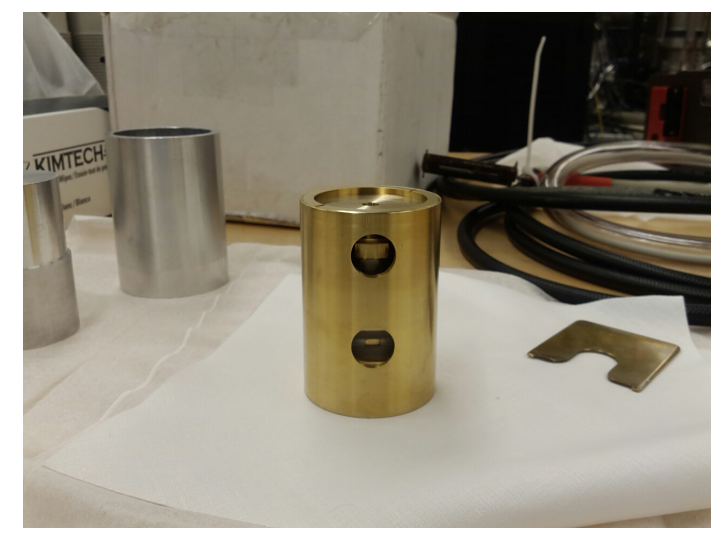

(a)

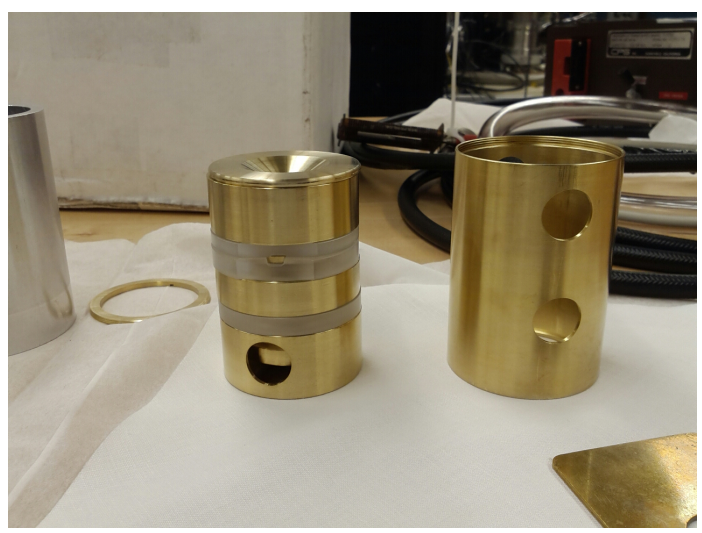

(b)

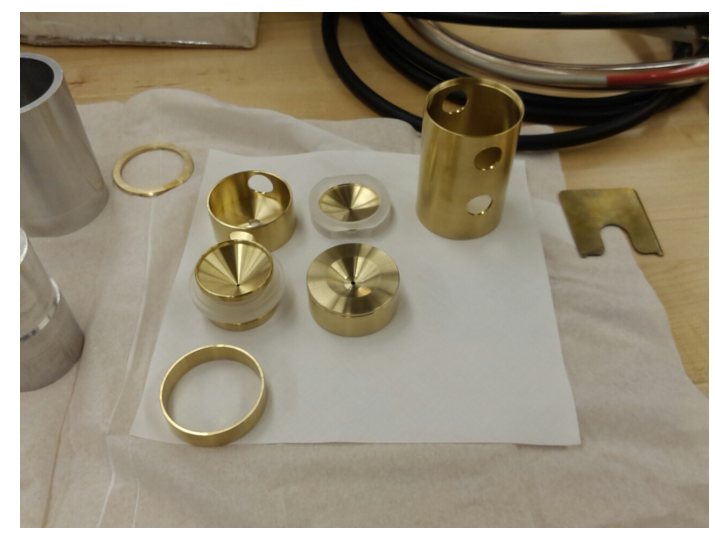

(c)

Figure 5.6: Triode mirror parts and assembly. The metal parts are constructed of naval brass and the insulators of rexolite.

\subsection{Quality control and beam alignment}

Work is underway to install and test the aberration correction of the triode mirror and lens. These two elements will replace the previous aberration correcting diode mirror and interface lens. Individual electrodes and spacers were built by Turk Manufacturing. They were assembled by hand, with various stages of assembly shown in Fig. 5.6. The triode interface lens is shown atop the mirror in Fig. 5.7, as it would be arranged in our PEEM. An as-built external comparison of the two lenses and the diode and triode mirrors is given in Fig. 5.8. 


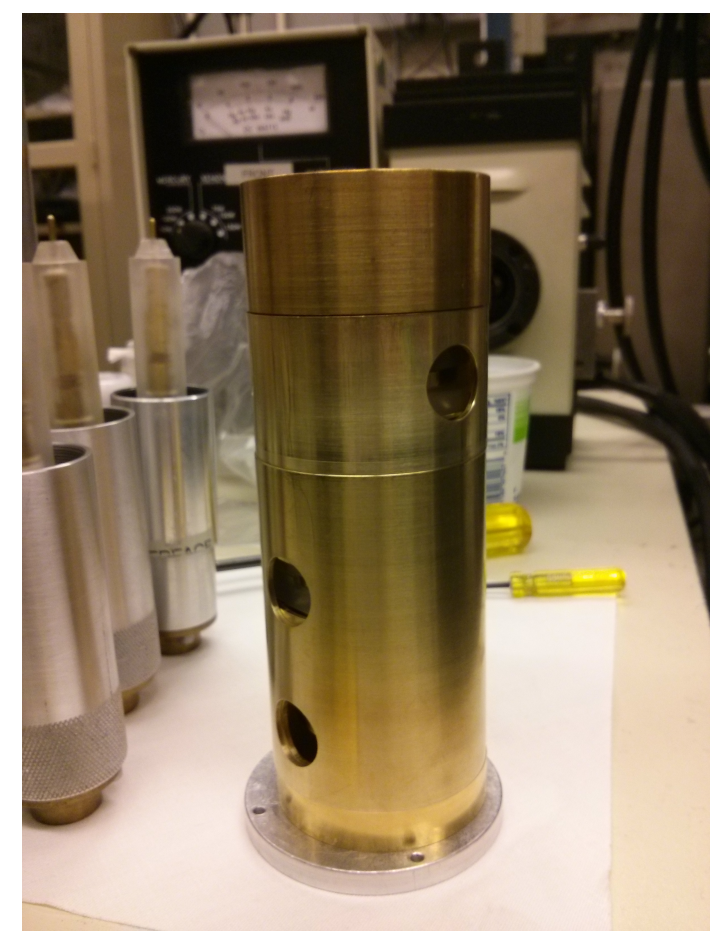

Figure 5.7: Interface lens and spacer atop the triode mirror, arranged as they would be installed in the PEEM. Three high-voltage feed-throughs are shown to the left, just behind.

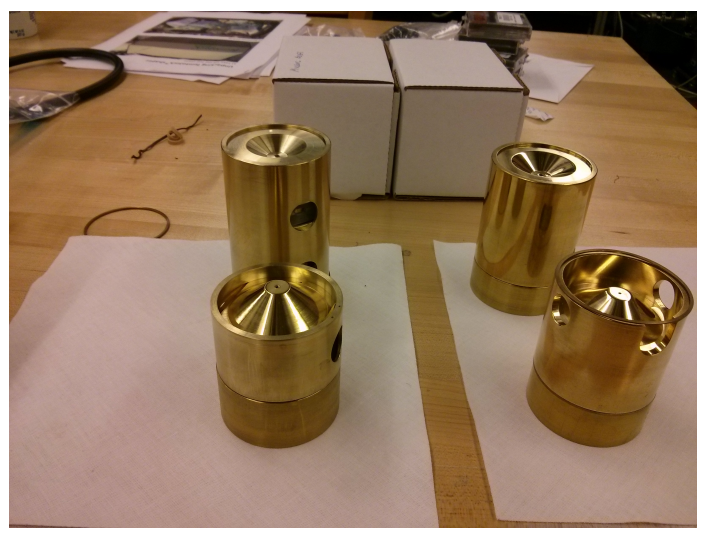

Figure 5.8: Diode (right) and triode (left) mirror branch assemblies, including the mirrors (back), interface lenses (forward), and appropriate spacers. 


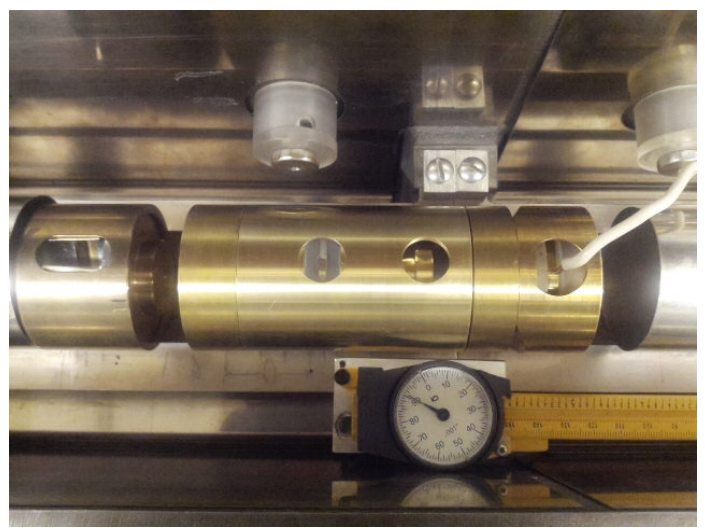

Figure 5.9: Electron gun straight bench with triode mirror (left) and interface lens (right). The interface lens is connected to a power supply by the white wire, while the triode mirror is not yet connected. The electron beam is incident from the left and the focused spot is imaged on a phosphor screen just off camera to the right.

The most sensitive part of the construction is the concentricity of the metal apertures, which is made increasingly difficult with the large number of apertures. Alignment of the apertures is tested in an electron gun straight bench, shown in Fig. 5.9. An electron beam is prepared so as to be incident on the test lens with nearly parallel rays. Then the test lens is run at the highest possible potential, up to the acceleration potential of the electron, such that a fine spot is formed by the electron beam on a phosphor screen at the end of the bench. Then, the lens is rotated $180^{\circ}$ degrees, and another spot is formed on the screen. The position of the two points is compared, as in Fig. 5.10, and internal alignment of the lens is assessed by the translation between the two spots. Typically, the test lens is used with a projection lens to increase this translation. This test is less indicative of misalignment with the triode mirror because the element is not designed to operate as a lens, as confirmed by simulations Fig. 5.11. As a result, the test reveals only the grossest of misalignments, and further testing must be done in the PEEM when the mirror is operated in reflection mode. The design of the mirror involves several axially thick pieces that ensure alignment with the optical axis, and alignment of the triode mirror was not a significant problem. 


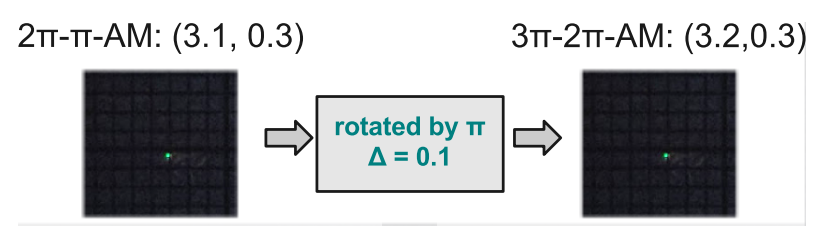

Figure 5.10: Visual comparison of electron beam focus points on a phosphor screen created the triode mirror operated as a transmission lens in the electron gun straight bench. These images compare a specific test where both electrodes in the mirror are at high potential. The second point is captured after the mirror was rotated by $180^{\circ}$. A total displacement of 0.1 grid units (about $0.1 \mathrm{~mm}$ ) is evident. This shift is at about resolution of the experiment, and any further mis-alignment must be checked or compensated for in our PEEM.

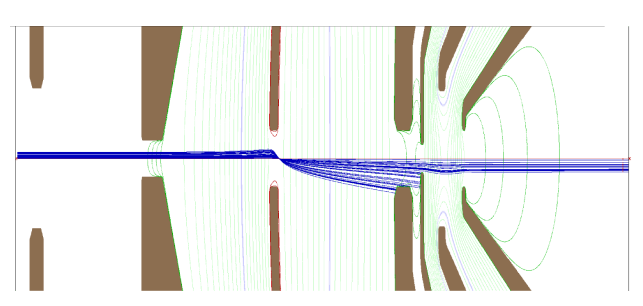

(a) A test.

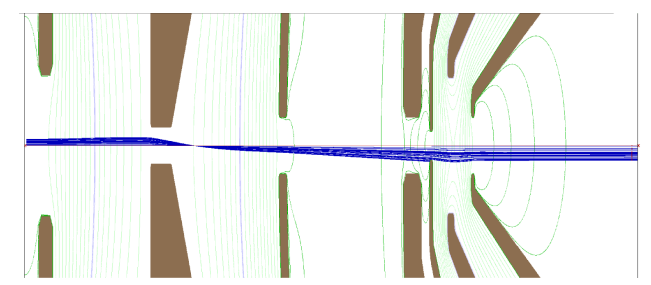

(b) M test.

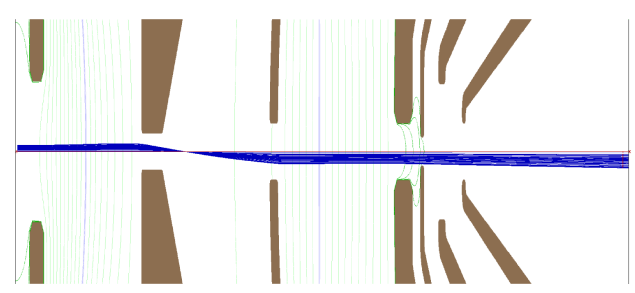

(c) AM test.

Figure 5.11: Numerical studies of the triode mirror as a lens in the straight bench. There are two electrodes, which allow three distinct tests with one electrode at high voltage and the other grounded (denoted A or M) or with both at potential (denoted $A M)$. The studies show that all three tests are sensitive to similar alignment problems, and that they are not particularly sensitive to alignment. 


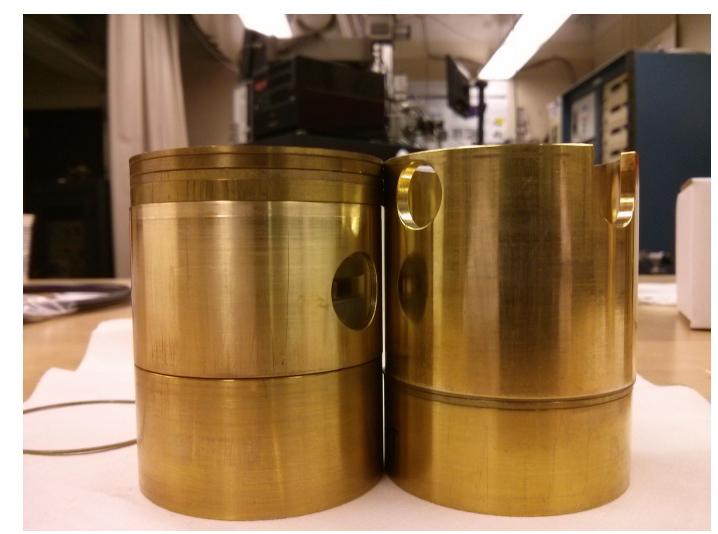

Figure 5.12: Interface lenses from diode (right) and triode (left) installations. Several spacers make up the difference in housing length. With these spacers, the lenses were interchangeable in the diode assembly. Testing the triode lens with the diode assembly allowed us to determine it alignment independent of the triode mirror.

In contrast, the swept-back design used for the interface lens does not give the most reliable part positioning. As a result, the interface lens required several assemblies and trials in the electron gun straight bench. The diode interface lens could not be used directly in the triode assembly because of it extra long housing. The difference is shown in spacers in Fig. 5.12.

Following initial tests for rotational symmetry of the lens and mirror in the straight bench, we continued tests in our PEEM. The electron image is sensitive to the total alignment of the mirror branch, including not only the internal alignment of the lens and mirror elements, but also the alignment of the lens and the mirror externally with each other and the magnetic beam deflector, Magnet B. This assumes that the alignment of the other branches is already acceptable. After several failed attempts, a triode mirror configuration without the front electrode successfully produced the most satisfactory image. The triode mirror with replacement spacers is shown in Fig. 5.13. Subsequently, beam alignment through the system was improved with electrostatic deflectors positioned throughout the PEEM. Finally, reasonable quality images could be produced with triode mirror corrector installed, as shown in Fig. 


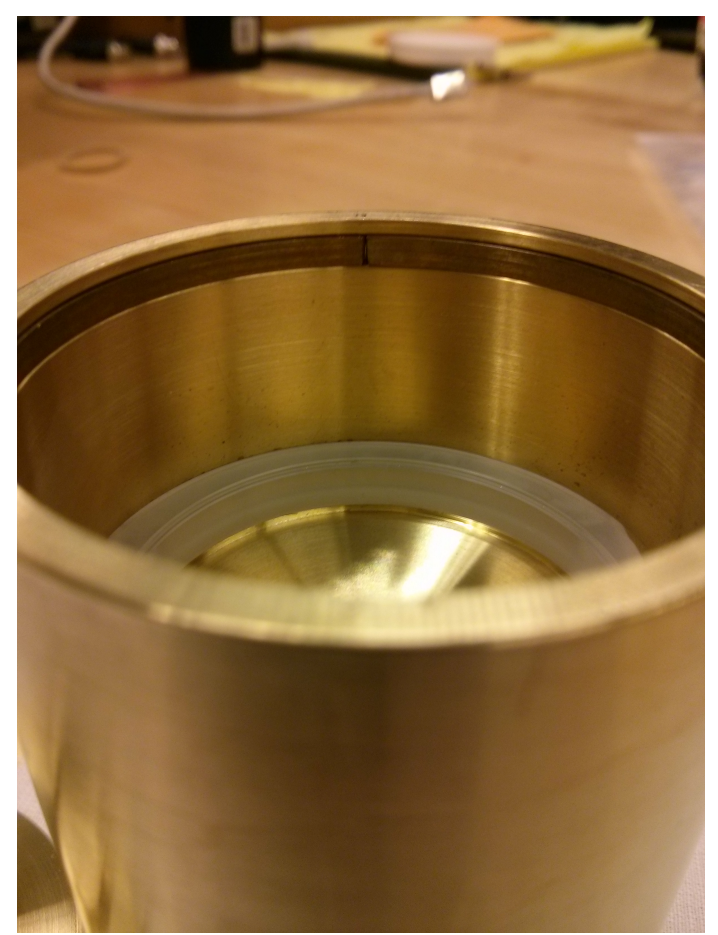

(a)

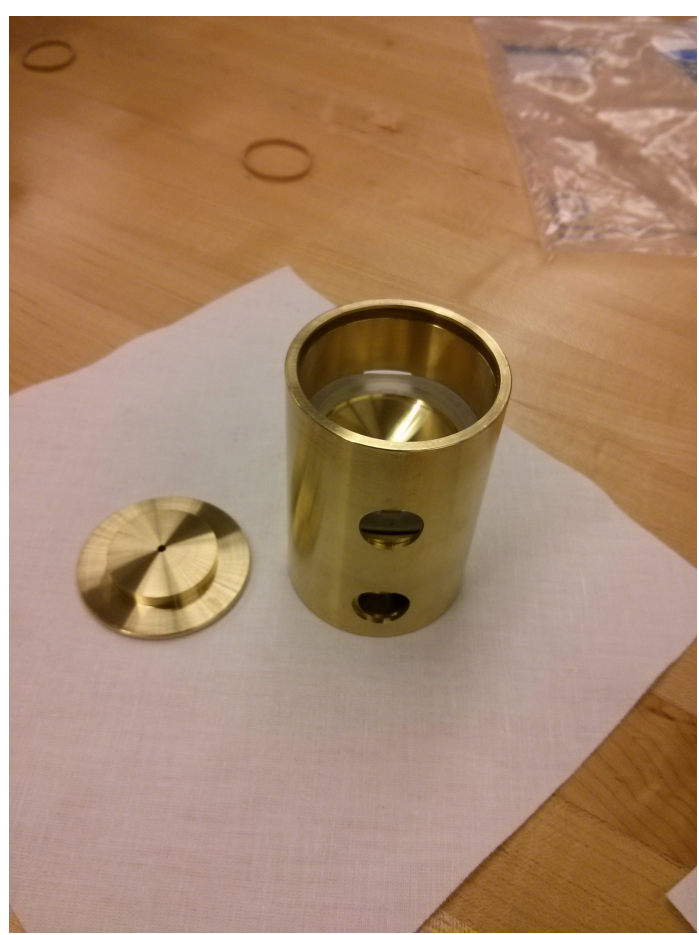

(b)

Figure 5.13: Triode mirror without the grounded electrode, showing the replacement spacer. The second image shows the grounded electrode to the side. 


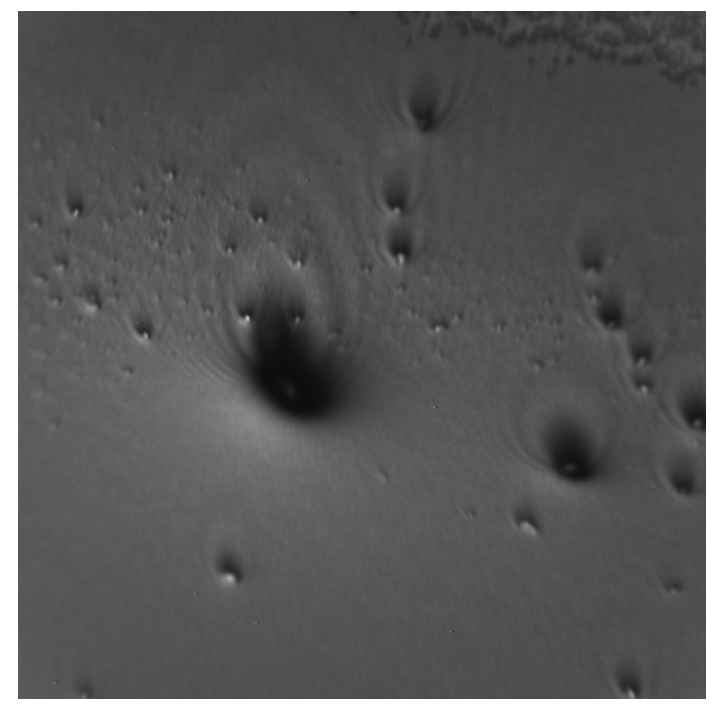

(a)

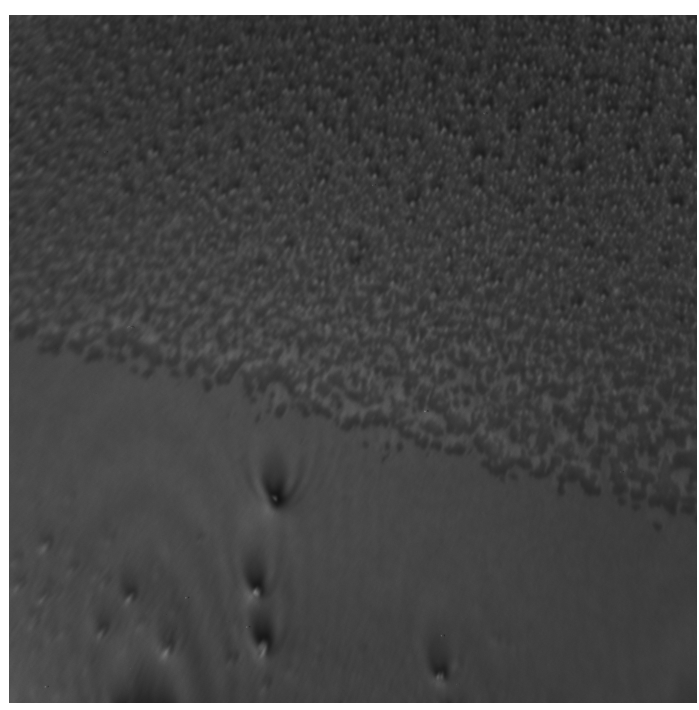

(b)

Figure 5.14: First images from the triode PEEM. The specimen is an evaporated gold film on ITO. Images are 5 second exposures with $244 \mathrm{~nm}$ light at $200 \mathrm{~mW}$ of two adjacent regions, (a) with and (b) without an aperture stop. Image resolution is low $(\sim 900 \times)$, with the field of view at several microns, so these images are not strongly sensitive to first-order image aberrations. However, they do confirm that the PEEM is basically aligned.

5.14. These images are at resolutions that are sensitive to the optical alignment of the electron beam but not sensitive to spherical and chromatic aberration.. Instead, image quality is significantly reduced by off-axis shifts, magnetic deflectors, and distortion. After correction of these defects, astigmatism and spherical and chromatic aberration become dominant contributions at higher magnifications where CCD pixel size limits are overcome. With astigmatism corrected by a multipole deflector, the bare effects of spherical and chromatic aberration can be seen, and with them, the image correcting properties of the triode mirror corrector can be confirmed. 


\section{Photonics}

\section{Photoemission and photonics}

The photoelectric effect, the emission of an electron from an atom upon absorption of an energetic photon, is among the most elementary quantum-mechanical phenomena. Hertz [108] originally discovered the effect in 1887 , when he noticed that the incidence of light on an electrode at high electric potential facilitated sparking across the gap. Furthermore, he determined that intercepting the light with glass removed this effect, while a quartz slide did not, leading him to conclude ultraviolet radiation was the relevant portion of the spectrum. Further investigation by Hallwachs [109], Stoletow [110], and others showed that the photocurrent is proportional to the incident light intensity above threshold some threshold. Finally, after almost two decades of experiments, Einstein [111] successfully explained the phenomenon in terms of the interaction of discrete packets of light with electrons, ushering in the modern era of quantum physics.

In the simplest picture, a light quantum gives all its energy to a single electron. Neglecting thermal effects, maximum energy of an electron emitted directly from the surface is given by

$$
E_{\max }=h c / \lambda-W,
$$

where $h$ is Planck's constant, $c$ is the vacuum speed of light, $\lambda$ is the wavelength of the incident light, and $W$ is the photoemission threshold energy, the minimum photon energy at which photoemission occurs. The photoemission threshold is closely related to the work function, a measure of the electron binding energy that is the difference between the Fermi energy and the vacuum energy. Above $W$, the photoelectron 
current $j_{e}$ is expected to increase linearly with the incident light intensity $I$,

$$
j_{e}=\eta I,
$$

where $c$ is the efficiency $[49,50]$. Photoemission is a low-probability process, typically yielding less than one electron per ten thousand incident photons [51]. At photon energies less than the threshold $W$ photoemission proceeds by an even lower probability, nonlinear processes, e.g., two-photon photoemission (2PPE), where multiple photons collude to excite a single electron. In general there is an $n$th order power law relationship for multiphoton photoemission $(n \mathrm{PPE})$,

$$
j_{e}=\eta_{n} I^{n} \text { for } h f>W_{n},
$$

where $\eta_{n}$ and $W_{n}$ are the $n$-photon efficiency and threshold $[8,112]$. The multiphoton values of the threshold and efficiency are typically comparable to ultraviolet (UV) photon values by $\eta_{n} \approx \eta^{1 / n}$ and $W_{n} \approx W / n$, and likewise show a general correlation with material surface composition. An energy level representation of one- and twophoton photoemission is shown in Fig. 6.1.

The energy of photoelectrons is the experimental quantity used to determine the paths of photoexcitation, either through spectroscopic measurement or by comparing incident photon energy to known binding energies. Some representative spectra from $\mathrm{Ag}$ and $\mathrm{Cu}$ are presented in Fig. 2.3. As one would guess from the simple picture presented above, photoelectron emission energy distributions depend primarily upon the energy in excess of a material's photoemission threshold energy. However, the nonGaussian spectral distributions and the differences between single- and multi-photon photoemission distributions (especially for $\mathrm{Cu} 1$ - vs. 3-PPE) serve to highlight the 


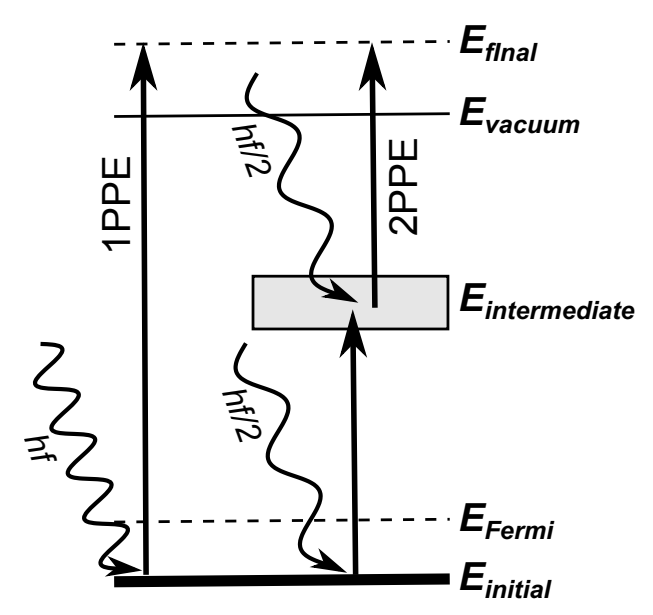

Figure 6.1: Energy level diagram representation of the photoelectric effect, adapted from [113]. An electron is excited from an energy level below the Fermi level by a photon of energy $h f$ or two photons of energy $h f / 2$ to above the vacuum level. Intermediate energy levels can be bulk, surface, virtual, or quasiparticle states.

complexity of the process, a point driven home by the independence of the mean emission energy $\left\langle e V_{e}\right\rangle$ and distribution width $\sigma_{e}$ on excess energy. Due to the complexity, photoemission in materials is usually discussed in terms of a three-step model: electrons are first optically excited, then travel to the solid surface (with or without scattering), and subsequently are emitted to the vacuum [49]. The spectral distribution of photoelectrons exhibits sensitivity to optical excitation processes and electron scattering processes. Because these processes vary within the material, spectra also change with sample position. In near-threshold studies, the mean electron energy and total yield shift in particular [96-98]. It is this contrast that PEEM utilizes, examining spatial variations in surface photoemission rates to reveal changes in photoexcitation pathways.

The photoemission threshold, efficiency, and photoelectron energy spectrum vary not only with the static electron environment and excess energy, determined primarily by the material and excitation energy, but also with dynamic processes and other experimental conditions, such as incident light intensity. These variations highlight a 
more intimate dependence on topological structure and energy redistribution channels and underscore that a single electron emitted from an atom that absorbs a photon is, strictly speaking, not a single-electron process. Hence, the properties of the emitted photoelectron [114], exhibit sensitivity to the effects of temperature and phonons [115], interband transitions, surface roughness [116], Tamm and Shockley surface states [117], collective oscillations and optical excitations [118].

The manifold paths of photoexcitation can be represented as various elements of a scattering matrix $T$ connecting the initial state $i\left(\psi_{i}\right)$ to the final electron state $f$ $\left(\psi_{f}\right)$. Starting with Fermi's Golden Rule, the transition probability per unit time (up to second order) is

$$
T_{i f}=\frac{2 \pi}{\hbar}\left|\left\langle f\left|H_{\mathrm{int}}\right| i\right\rangle+\sum_{n} \frac{\left\langle f\left|H_{\mathrm{int}}\right| n\right\rangle\left\langle n\left|H_{\mathrm{int}}\right| i\right\rangle}{\epsilon_{i}-\epsilon_{n}}\right|^{2} \delta\left(\epsilon_{f}-\epsilon_{i}-\hbar \omega\right)
$$

for an initial energy $\epsilon_{i}$, final energy $\epsilon_{f}$, and real or virtual intermediate states $n\left(\psi_{n}\right)$ in response to the Hamiltonian $H_{\text {int }}$ [119]. The interaction Hamiltonian $H_{\text {int }}$ and wave functions $\psi_{i}, \psi_{f}$, and $\psi_{n}$ are chosen to fit the system states and interactions with the electromagnetic field. Mahan [120] describes how to go about this with simple metals. The initial electron state is derived from the band structure, and it can be represented as a sum of Bloch function plane waves,

$$
\psi(\mathbf{k}, \mathbf{r})=e^{i \mathbf{k} \cdot \mathbf{r}}\left\{1+\sum_{G \neq 0} u_{k, G} e^{i \mathbf{G} \cdot \mathbf{r}}\right\}\left\{1+\sum_{G \neq 0} u_{k, G}^{2}\right\}^{-1 / 2},
$$

where an electron in the outgoing state $\mathbf{k}$ has plane-wave components in the directions $\mathbf{k}+\mathbf{G}$. Each of these components has an external distribution of electrons which may overlap with a free electron final state, represented by a Green's function evaluated 


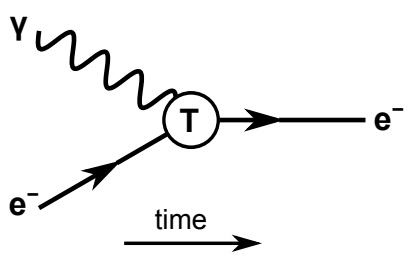

(a)

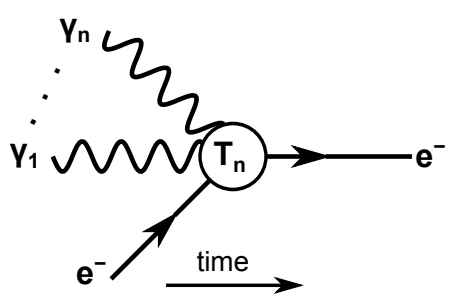

(b)

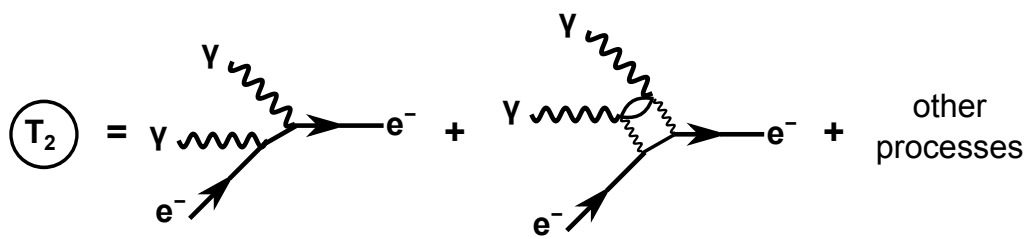

(c)

Figure 6.2: Graphical representation of the photoelectric effect using Feynman diagrams, where $\gamma$ represents a photon, $e^{-}$an electron, and $T$ is the transmission/scattering matrix standing in for the potentially mixed photoexcitation path; adapted from [121]. (a) Single photon photoemission (1PPE), (b) multiphoton photoemission $(n \mathrm{PPE})$, and (c) a diagrammatic representation of two-photon photoemission as the sum of several contributions, each with its own efficiency. The loop contribution is the interaction with surface plasmon polariton quasiparticles.

in the presence of the material surface,

$$
G_{E}\left(\mathbf{r}, \mathbf{r}^{\prime}\right)=\int d \mathbf{k}_{\|} \int_{0}^{\infty} d k_{z} \sum_{i} \frac{\psi_{i}(\mathbf{k}, \mathbf{r}) \psi_{i}\left(\mathbf{k}, \mathbf{r}^{\prime}\right)^{\star}}{E_{k}-E-i \delta}
$$

where $E_{k}=\left(k_{\|}^{2} / 2 m\right)+E_{k_{z}}$ and the wave functions $\psi_{i}$ are the initial electron wave functions. The amplitude of the overlap may be adequately described by a scattering $T$-matrix that connects the initial and final states through potentially several scattering events and intermediate states. Both virtual and real states are rigorously included, with the difference being largely semantic in this formalism. The interaction can be viewed as a perturbative sum of contributions, with individual terms often identified as quasiparticle interactions and represented by Feynman diagrams as in Fig. 6.2. Excitation paths can be direct, that is approximately a singular electron- 
photon interaction that conserves photon momentum, or indirect, involving one or several intermediate resonances or scattering events which may not conserve photon momentum [120]. In particular, multiphoton photoemission necessarily involves intermediate states, but these may be real or virtual states, with a range of lifetimes and potentially separately direct or indirect partial or complete pathways [98, 122]. The sum of excitation pathways, which depends on the density of states and the incident radiation, not only sets the total efficiency but also dictates the energy threshold. The sensitivity of photoemission efficiency to several processes invites careful scrutiny of the excited photoelectrons, allowing us understand the electron environment before emission and the processes in which photoelectrons participate [49, 123].

Low photoemission rates are the primary practical limitation in imaging with photoelectrons. Ultraviolet, single-photon quantum efficiencies for metals are on the order of $\eta=10^{-5}$ electrons per photon [51], so two- and three-photon quantum efficiencies are on the order of $\eta_{2} \approx \eta^{2}=10^{-10}$ and $\eta_{3} \approx \eta^{3}=10^{-15}$. Higher order processes are prohibitively rare for imaging. The availability of ultrafast, high-intensity lasers coupled with parallel developments in high sensitivity CCD cameras and aberration correction have increased the applicability of nonlinear $n \mathrm{P}$-PEEM, such that the near infrared and visible spectral region can be routinely used. Furthermore, contrast, brightness, and resolution are enhanced by corrected optics as discussed in Part II. These advances combine to make PEEM a potentially powerful tool for the study of all types of electromagnetic field excitations in materials, including guided optical modes, vacuum modes, and surface plasmons. With spatial resolution approaching 5 nm [89], PEEM may advance optical surface studies to scales well beyond standard optical microscopes and currently available super-resolution techniques.

Despite photoemission being an explicitly quantum process, imaging-i.e., the recording of emission yields of photoelectrons - requires such a high density of pho- 
tons that there is a nearly exact equivalence between quantum and classical descriptions, as given by the quantum correspondence principle [124]. This theorem states that in the limit of infinite quantum numbers, such as excitation state or particle density, that the difference between quantum and classical descriptions becomes merely semantic, i.e., that measurable quantities can be equally well explained either way. PEEM operates very close to this regime; therefore, no effort is made to distinguish between high photon densities and large field intensities in explaining the link between photoemission and optical phenomena. To better relate to optical literature, the electromagnetic field intensity is preferred in the following work. However, photoemission is a purely quantum effect, one that cannot be explained as a classical threshold process. Thus, PEEM images are more representative of the photon density, and only through the quantum equivalence principle can we conclude that this is also the electromagnetic field intensity.

With some notable exceptions $[52,125-129]$, the information reported by the lowenergy photoelectrons imaged in PEEM primarily originates from within a nanometer of the sample surface [53]. In this zone, optical interactions dominate the electron environment. PEEM allows us to visualize and scrutinize these processes on a scale significantly less than a wavelength of the incident light. The nanoscale observation of electromagnetic field intensity at material surfaces is receiving increased interest, as optical information processing now approaches frequencies in the visible spectral region and device sizes have moved into the sub-micron range [41]. Precise manipulation of the interaction between light and materials promises several applications, ranging from directing antenna radiation without moving parts [130] and micrometer-sized solid-state lasing cavities [131], to the more exotic pursuits of invisibility cloaking [132] and slow light [133].

Visual confirmation of electromagnetic processes in such nano-devices can only 
improve characterization and development. Near-field electromagnetic field distributions at surfaces are typically observed by scanning methods, such as photon scanning tunneling microscopy (PSTM) [134], near-field scanning optical microscopy (NSOM) [135], electron energy-loss spectroscopy (EELS) [136, 137], and cathodoluminescence [138]. In contrast, PEEM is a probe-free non-scanning technique in image resolution is independent of the light source $[139,140]$. The results are qualitatively similar to those obtained by PSTM [141] and NSOM [142].

Polarization is important to this investigation for two reasons. First, as a fundamental property of the incident light, photoemission should exhibit a strong sensitivity to the incident light polarization if it is at all sensitive to the optical fields. It is a variable along which the correlation between photoelectron and optical responses can be probed. Second, the optical control possible with polarization switching is of interest in a wide range of applications, particularly in ultrafast light-controlled optical switches, plasmonic routers, and path selectors. Even on slower time scales, the possibility of spatial control of optical fields and electron emission will be useful in sensors and actuators and in nanoscale electron sources.

Previous studies have demonstrated that photoemission from metal surfaces is highly sensitive to optical phenomena. In particular, high yielding multiphoton photoelectron sites, "hot spots," have been found from metallic surfaces and nanostructures $[7,10,15,143,144] 2 \mathrm{P}$ - and 3P-PEEM. As illustrated in Fig. 6.3, there are several potential photoexcitation paths in multiphoton photoemission, but observations indicate hot spots are the result of surface plasmons $[8,9,31,33,113,118]$. Plasmons are dynamic excitations that result from the collective oscillation of conduction-band electrons. At surfaces they couple to intense, highly-confined electromagnetic fields in a surface plasmon polariton (SPP) quasiparticle. While plasmon excitations are typically propagating waves, they can be localized in metal films with nanostructured 


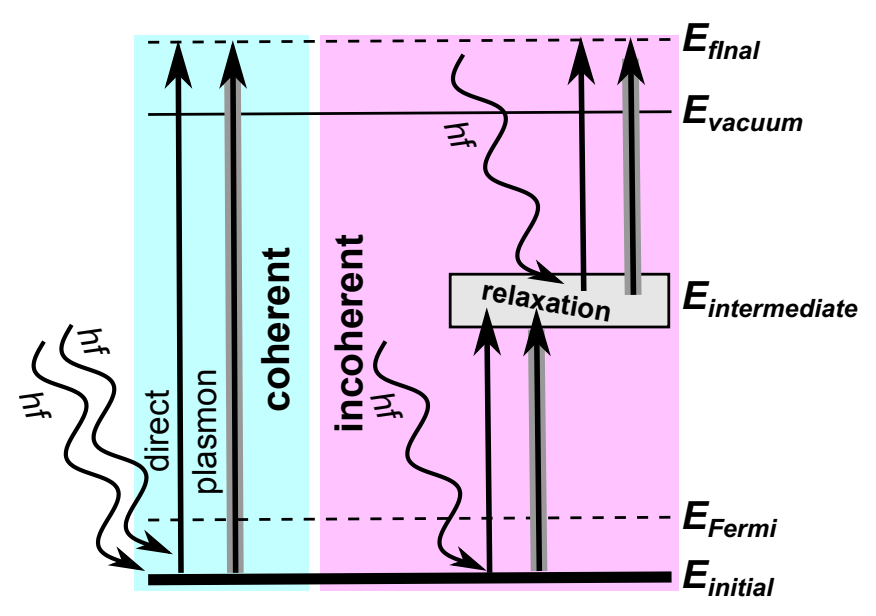

Figure 6.3: Two-photon excitation pathways in metals, including direct single-particle excitations (black, thin arrows) and surface plasmon excitations (gray, thick arrows). Coherent excitations proceed via a virtual intermediate state (direct) or through a multiply excited surface plasmon. Incoherent excitations involve relaxation time in an intermediate state, where electrons lose connection to the initial excitation, followed by excitation to the vacuum state.

surface, in random geometries, and in clustered metal films $[145,146]$. The localization occurs essentially by the confinement of local modes within the nanostructure. Intense localized surface plasmon resonances (LSPRs) increase optical field strengths by several orders of magnitude [147-150] and are thought to be the source for surfaceenhanced Raman scattering, enhanced luminescence, lasing, and other phenomena, including enhanced nanoscale photoemission in hot spots.

Surface plasmon polaritons have attracted wide interest for applications in ultrafast electronic and photonic devices, information devices, and for enhanced electron and light microscopy. Plasmon generation can be enhanced and manipulated in antenna structures [151-153]. Devices for controlling plasmon propagation have been demonstrated in the form of beam-splitters $[154,155]$ focusing lenses [156], and routers and multiplexers [157]. It is believed that the exploration of these basic device functions is needed for the realization of numerous applications ranging from ultra-sensing [158-161] and nano-lasing $[162,163]$ to cloaking $[164,165]$ and imaging 
$[166,167]$. Optical control is desirable for some of these applications and is essential for high-speed transfer of plasmonic signals, energy, or charge. As a next step in this development, it is desirable to motion the confined field region either to fine-tune the spatial overlap between probing field and sample, to carry out spatial scanning procedures or to selectively address separate receivers in routing applications.

While plasmonic phenomena have received significant attention lately, diffraction from particles or edges are also familiar image features in PEEM. These so-called 'fringe fields' can be used to obtain information on the diffracting object [13], but are usually ignored as image artifacts. Here I report that photoemission can also be used to image the near field of a photonic wave bound to a dielectric thin film, showing phase contrast in a manner similar to near field scanning optical microscopy [141]. Instead of responding strongly to incident light with collective oscillations as in metals, electrons in optical dielectrics are relatively tightly bound to lattice sites and allow light to pass through with little attenuation, albeit at a slightly reduced average speed. Layered dielectric structures, composed of electrically semiconducting or insulating materials, can guide and confine light in a specific layer through differences in refractive indices. Optical fibers are an exemplary application, where a graded index glass cylinder confines light to the center; signals in optical fiber can propagate several kilometers without significant signal deterioration. Planar, or "slab," waveguides (see schematic in Fig. 6.4) with spatially ordered holes in the central layer, so-called photonic crystals, can manipulate the properties of guided light [168]. Precision changes in the periodicity or defects can be used to create coupled micro-optical elements, such as micro-filters and micro-cavities, which scale communications processing and mirror arrangements down to micrometer scales $[131,169-171]$. The possibility of visualizing optical fields in dielectric media with photoelectrons has not been previously investigated. 


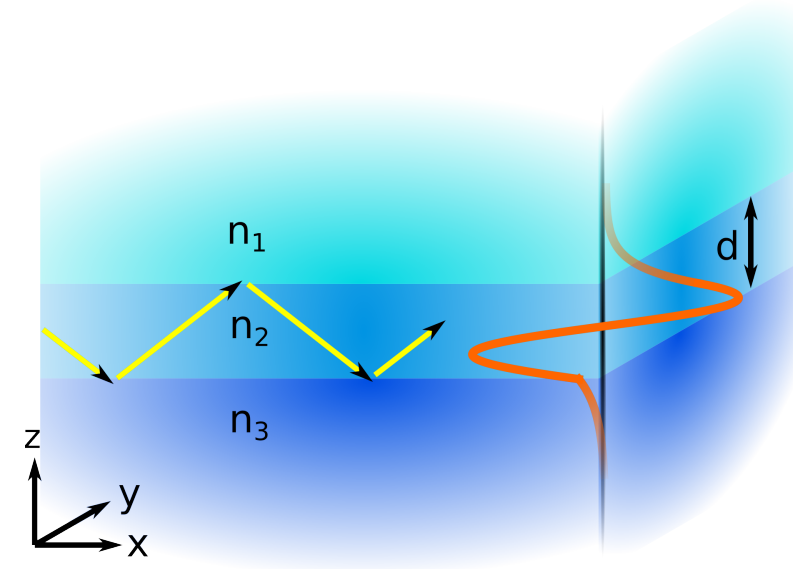

Figure 6.4: Dielectric slab model. Two semi-infinite dielectric layers, with refractive indices $n_{1}$ and $n_{3}$, bound a central layer with index $n_{2}$ and thickness $d$. The layers extend infinitely in $x$ and $y$ dimensions. Refracted electromagnetic waves can be guided and confined to the central layer if it has the appropriate wave vector. A geometric ray-tracing schematic and an intensity profile of one possible mode are overlaid in yellow and orange.

In the following, I explore the photoelectron response to these optical phenomena: surface plasmons in structured metals, photonic guided modes in optical dielectric structures, and diffraction at grooves, holes, and knife-edge steps. In the process I hope to demonstrate that PEEM can directly and quantitatively visualize optical phenomena in the near-field of solid surfaces. First, I examine localized and extended plasmon modes that arise in randomly $[28,30,42]$ and deliberately $[37,41,172]$ nanostructured metallic thin films, where diffraction and optical antenna resonances scatter the incident light wave vectors to match the plasmonic modes. Despite numerous experimental investigations of localized plasmon modes, a detailed understanding of the various conversion processes occurring in the photoemission process is still at the beginning. With high spatial resolution, PEEM can conclusively show that photoexcitation is enhanced from both participating metal surfaces and passive semiconducting surfaces in the vicinity, and that the location of emission is strongly responsive to the polarization of incident light. Similar wave vector matching conditions in more de- 
liberate diffracting structures $[41,173-175]$ lead to the emergence of photonic modes in the vicinity of transparent and absorbing semiconductors and of plasmonic modes at metal surfaces. A quantitative evaluation of the obtained images allows a distinction between modes that propagate inside the samples with propagation parameters given by the sample dielectric constant or refractive index, and modes propagating above the sample surface in vacuum and subject to the vacuum dielectric properties. In these extended modes, the surface electromagnetic near-field intensity can be calculated using a finite-element-method solution to Maxwell's equations or a more conceptually simple formula based on Huygens' principle and Fresnel diffraction. Using only a simple power-law relationship, I show that there is a close correspondence between photoemission patterns and calculated field intensities, demonstrating that PEEM is suitable for visualizing virtually any surface electromagnetic interaction. 


\section{Methods and materials for photonics}

Imaging with photoelectrons in an aberration corrected PEEM is described in detail in Part II. Here, I describe the illumination conditions and specimen preparations used to in photonics investigations.

\subsection{Light sources}

In all of our PEEM experiments, light is incident at $60^{\circ}$ to the sample surface normal for all investigations, as illustrated in Fig. 7.1. Furthermore, the laser beams were polarized to $>99 \%$ linearity by a tunable wave plate and focused to an incident spot of about $100-\mu \mathrm{m}$ diameter. Both linear and circular polarizations were investigated. Transverse magnetic (TM) polarized light induces a larger photoelectron response than transverse electric (TE) polarized light [176], typically by a factor of $2-5$, so images with different linear polarization angles have different mean intensities. This was often compensated by adjusting the exposure time, which varied widely with light source and material from a few seconds to several minutes.

Our PEEM is equipped with three light sources that provide limited ranges of IR, visible, and UV light. First, a mercury arc lamp provides low-intensity incoherent UV light, particularly at $253.7 \mathrm{~nm}$. Second, a continuous-mode, frequency-doubled $\mathrm{Ar}^{+}$-ion laser provides UV light at $244 \mathrm{~nm}$ and a power of $100-200 \mathrm{~mW}$. We found that a coherent and higher-power light source greatly increased the photoemission contrast in optical phenomena, so the $\mathrm{Ar}^{+}$-ion laser is the primary UV source. Third, a Spectra-Physics Mai Tai Ti:sapphire laser, produces $11-\mu \mathrm{J}, 80$-fs pulsed IR light at $780-900 \mathrm{~nm}$ at a frequency of $80 \mathrm{MHz}$. The output can be up-converted to $410 \mathrm{~nm}$ with pulse energies of 2-nJ and a 100-fs duration using a Del Mar Photonics second 


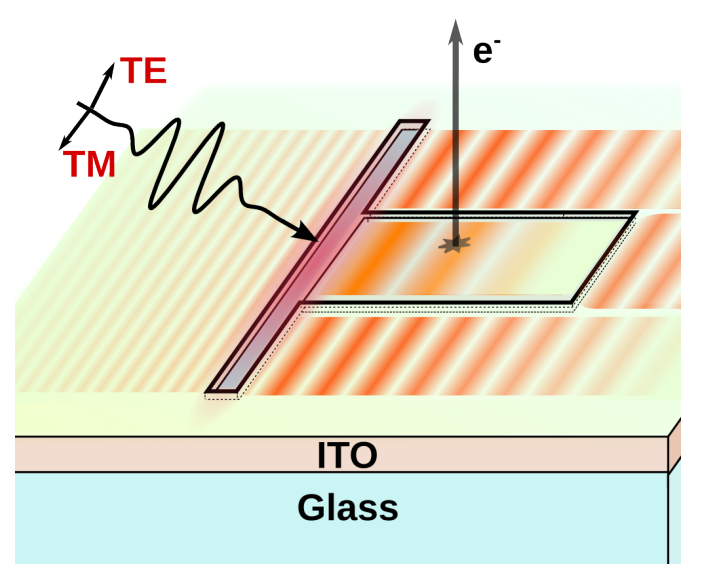

Figure 7.1: Schematic representation of sample conditions in PEEM, showing one of the diffracting nanostructures investigated here. The sample is illuminated by light at $60^{\circ}$ to surface normal, and emitted photoelectrons are imaged with electron lenses to produce a magnified image of the interaction. Reprinted from Fitzgerald, Word, Saliba, and Könenkamp [173].

harmonic generator. The up-converted pulses have a line-width of $4 \mathrm{~nm}$, full width at half-maximum. As a result of the limited spectrum of the light sources employed, I am only concerned with the optical properties of samples for wavelengths at 244 $\mathrm{nm}(5.1 \mathrm{eV}), 410 \mathrm{~nm}(3.0 \mathrm{eV})$, or between $780-900 \mathrm{~nm}(\sim 1.5 \mathrm{eV})$. In the case of each sample, I will also consider the photoemission threshold of the material and the excess photoemission energy, defined by $E_{\max }(n)=n \times(h c / \lambda)-W$, where $n$ is the order of the photoemission process. Actual photoelectron energy spectra (Fig. 2.3) and mean energies exhibit complex characteristics, but can be grossly distinguished by $E_{\max }$.

\subsection{Materials}

We used two types of PEEM specimens. The first was a single silicon magnification standard (10- $\mu \mathrm{m}$ pitch), commercially prepared by electron beam lithography (Planotec Si Test Specimen, Ted Pella, Inc.). At wavelengths less than $450 \mathrm{~nm}$, silicon is practically opaque with an absorption coefficient greater than $24,000 \mathrm{~cm}^{-1}[177]$. 
The photoemission thresholds of silicon and $\mathrm{SiO}_{2}$ range from $4.3 \mathrm{eV}$ [178] to $5.1 \mathrm{eV}$ [179], with a mean of $\sim 4.8 \mathrm{eV}$, so processes studied here are $1 \mathrm{PPE}(244 \mathrm{~nm})$ or $2 \mathrm{PPE}$ $(410 \mathrm{~nm})$, with an excess energies of $0.3 \mathrm{eV}$ and $1.2 \mathrm{eV}$, respectively. Silicon was not studied at longer wavelengths.

Other experiments employ conductive ITO slides, purchased from SPI Supplies, that consist of $2 \mathrm{~mm}$ borosilicate glass substrates sputter-coated with a 200-300 nm thick film of indium-tin-oxide (ITO). The borosilicate glass has a refractive index of 1.53 at $410 \mathrm{~nm}$. The ITO layer is a mixture of indium-oxide $\left(\mathrm{In}_{2} \mathrm{O}_{3}\right)$ and tin-oxide $\left(\mathrm{SnO}_{2}\right)$, approximately $90 \%$ indium oxide by weight with a sheet resistivity of 15 $20 \Omega / \square$ and a photoemission threshold of $4.1-4.25 \mathrm{eV}$ [180-187]. It is opaque to wavelengths less than $300 \mathrm{~nm}$, and 1PPE events with $244 \mathrm{~nm}$ light have an excess energy of $0.83 \mathrm{eV}$. At $410 \mathrm{~nm}$, the refractive index is $2.14+0.25 i$, corresponding to an absorption coefficient of $8000 \mathrm{~cm}^{-1}$; and photoelectrons are the result of $2 \mathrm{PPE}$ events with an excess energy of $1.8 \mathrm{eV}$. In the near-infrared, the index of refraction converges to 2.0 and the absorption coefficient drops below $500 \mathrm{~cm}^{-1}$. Photoemission using $780 \mathrm{~nm}$ light involve a $3 \mathrm{PPE}$ process with excess energy of $0.5 \mathrm{eV}$, but the photoelectron yield was generally not sufficient for independent study because of low absorption. At wavelengths significantly longer than $900 \mathrm{~nm}$, the optical response of ITO is plasmonic, as can be seen from the dispersion relation is shown in Fig. 7.2.

In experiments involving metallic thin films, conductive ITO-glass slides were used as a substrate. Gold was used for its low surface oxidation rates. In gold, the localized surface plasmon resonance typically lies between 400 and $800 \mathrm{~nm}$ depending on geometry. Photoelectrons emitted directly from gold at this wavelength are the result of a $4 \mathrm{PPE}$ process, with an excess energy of $\sim 1 \mathrm{eV}$ above the threshold of $4.5^{-}$ $5.3 \mathrm{eV}$ [188-190]. A non-plasmonic, optical antenna response is also possible across a wider spectrum of wavelengths, but is highly sensitive to the nanostructure geometry. 


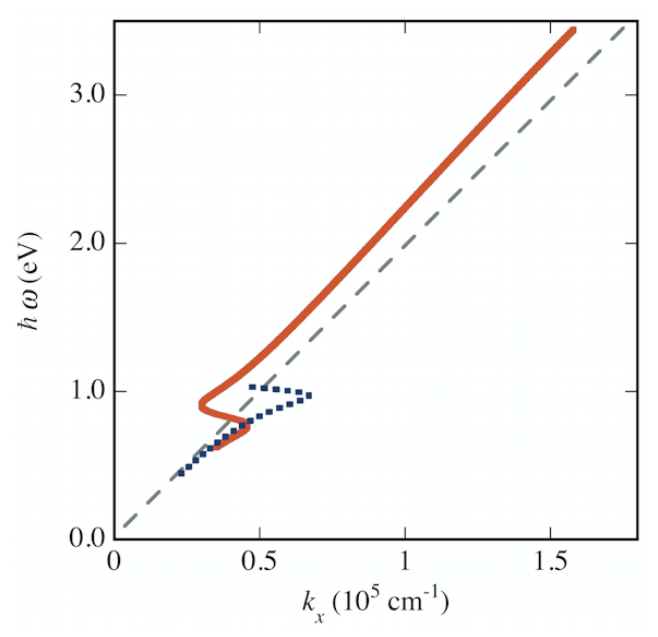

Figure 7.2: Dispersion relationship for SPPs propagating at the interface of indiumtin-oxide with vacuum. The visible light used here $(3.0 \mathrm{eV})$ is above the surface plasma frequency in the infrared $(0.8-1.0 \mathrm{eV})$. Calculated from optical constants of Refs. [185] (red line) and [186] (blue dotted line). Reprinted from Fitzgerald, Word, Saliba, and Könenkamp [173].

Photoelectrons are the result of 2PPE and 1PPE processes when excited with 410-nm and 244-nm light, with excess energies of $\sim 1 \mathrm{eV}$ and $\sim 0.3 \mathrm{eV}$, respectively.

Metallic thin films used here were composed of polycrystalline gold, single-crystalline gold, or polycrystalline gold and copper in separate layers, and were deposited using two distinct methods. Polycrystalline films were prepared by vacuum evaporation to thicknesses of 50-100 nm with a hexagonal mask for position determination, as shown in Fig. 7.3. In one case, a bi-layer arrangement of gold and copper, each $50 \mathrm{~nm}$ thick, was used because the layer of copper between the ITO and gold was found to increase the precision of sample structuring with a focused ion beam. In the other gold deposition procedure, single-crystalline gold platelets were obtained from an aqueous gold-chloride solution with aniline acting as a growth modifier, as described in Refs. [191, 192]. All compounds were analytical pure agents purchased from Sigma Aldrich. $50 \mathrm{~mL}$ of ethylene glycol solution containing $0.036 \mathrm{mM} \mathrm{HAuCl} 4 \cdot 4 \mathrm{H}_{2} \mathrm{O}$ was heated to $95^{\circ} \mathrm{C}$ for $20 \mathrm{~min}$. Then, $0.1 \mathrm{M}$ aniline solution in ethylene glycol was added 


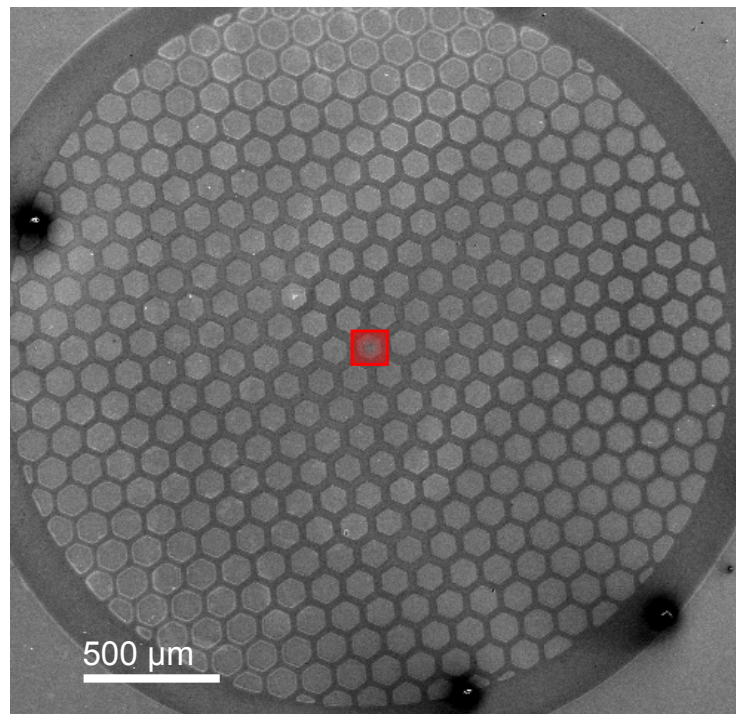

(a)

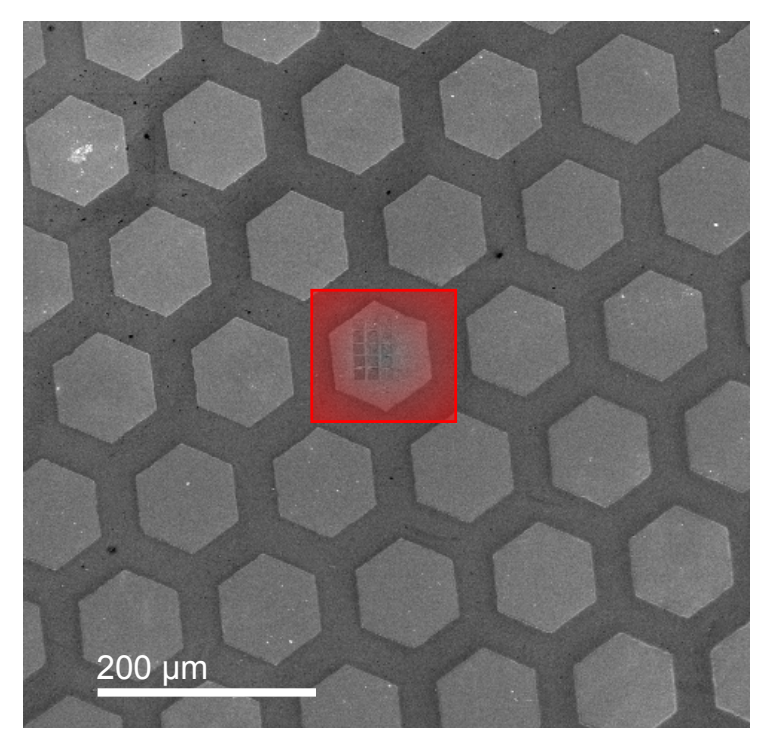

(b)

Figure 7.3: (a \& b) A thin layer of polycrystalline gold $(\sim 60 \mathrm{~nm})$ on ITO-glass substrate. The gold deposition was masked with a TEM grid, leaving a pattern of gold hexagons (lighter gray) surrounded by uncoated ITO (darker gray). The highlighted hexagon contains patterns milled by FIB. Both images taken at a beam energy of $10 \mathrm{keV}$ at a working distance of $4.9 \mathrm{~mm}$. 
under mild stirring to obtain a 1:1 molar ratio of aniline to gold. After 24 hours, a variety of gold flakes and platelets with diameters 3-15 $\mu \mathrm{m}$ and thicknesses 50-100 nm were obtained. These particles were washed of the solution with several cycles of ethanol and centrifuge separation, dispersed in water, and then cast on ITO-covered glass-substrates, as seen in Fig. 7.4.

\subsection{Nanostructures}

Diffracting nanostructures were milled using a focused ion beam (FIB) in a FEI DB237 dual-beam system, with the exception of the silicon magnification standard, which was used as purchased. Random and deliberate nanostructures were milled in gold and ITO, and the deliberate structures had varied complexity from simple rectangles and holes, the basic building blocks, to more elaborate patterns giving Y-shaped antennae.

Polycrystalline gold films on ITO were milled with a 100 pA Ga+ beam current over a $10 \mu \mathrm{m} \times 10 \mu \mathrm{m}$ area for varying times up to $300 \mathrm{~s}$. The approach exploits the varying response of gold crystal orientations to the gallium beam, creating random gold-ITO nanostructures with various degrees of connectivity and feature size, as seen in Fig. 7.5. The patterns shown here vary only in gallium beam exposure time, differing in increments of 10 seconds, allowing gold surface coverage to be optimized for photoemission during PEEM imaging without controlling for other experimental parameters, such as gold film thickness. The variations in gallium beam response of the polycrystalline film that worked in favor of random nanostructures inhibit sufficiently precise milling control for deliberate nanostructures. This can be somewhat remedied with an intermediate copper layer, as discussed by Word, Fitzgerald, and Könenkamp [172].

Alternatively, deliberate structures can be made from chemically grown single 


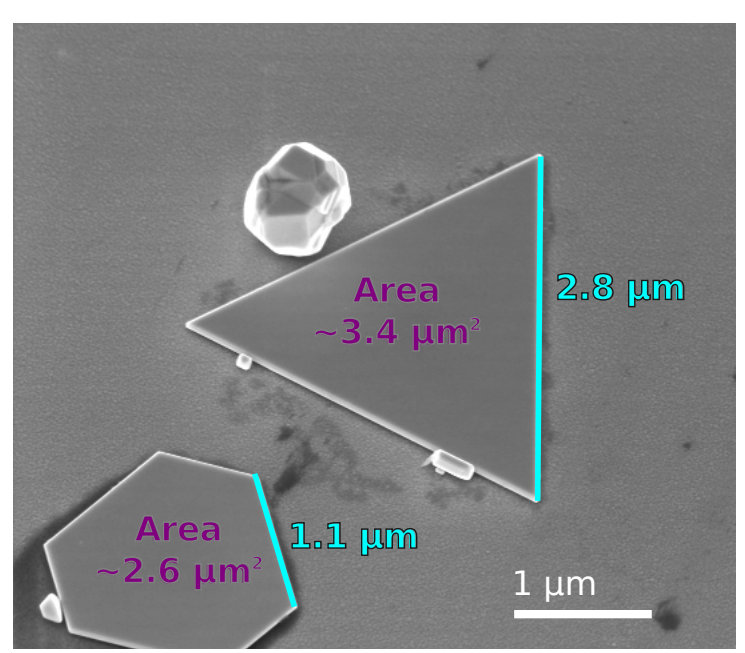

(a)

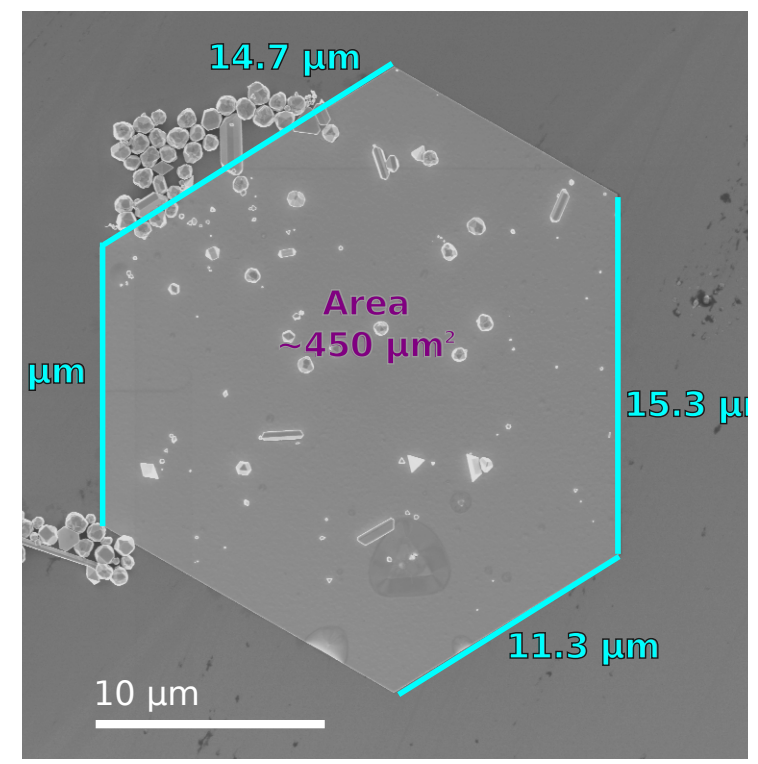

(b)

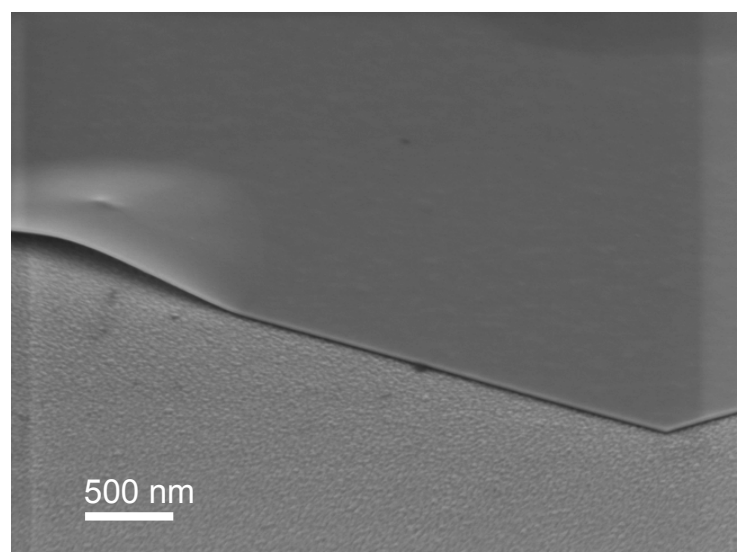

(c)

Figure 7.4: SEM images showing single-crystalline gold nanoflakes that were grown in solution, dispersed in water, and deposited on ITO-glass substrate. Gold crystals form equilateral triangles and hexagons with widths from $0.3-30 \mu \mathrm{m}$ and thicknesses ranging from 20-100 nm, though some spheroids also form. Thicknesses of very large flakes were as little as $20 \mathrm{~nm}$, as shown in (c), at a viewing angle of $60^{\circ}$ relative to normal. 


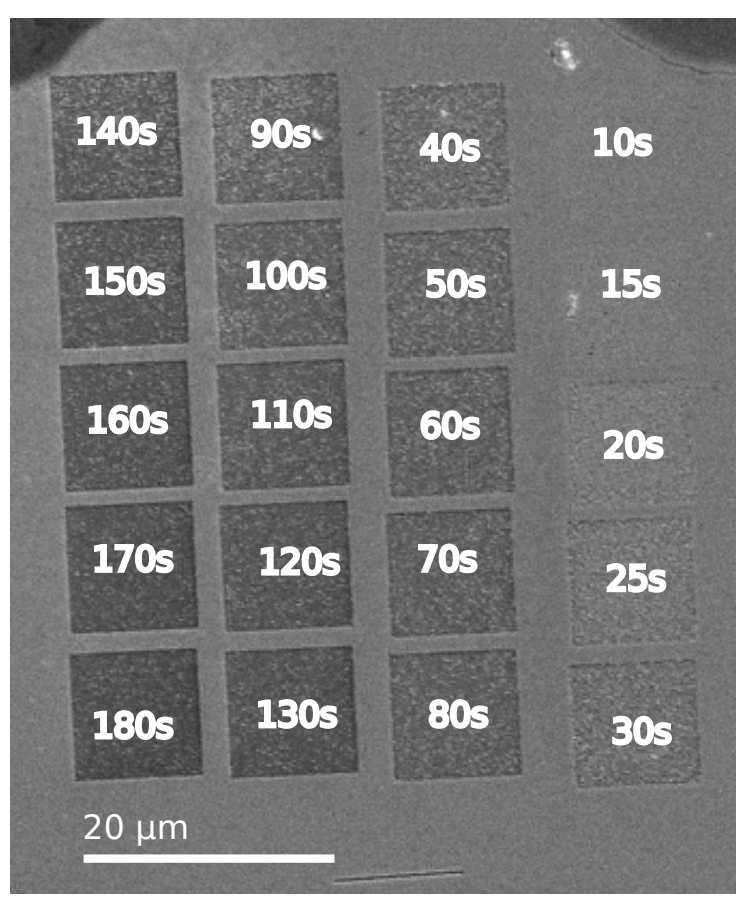

(a)

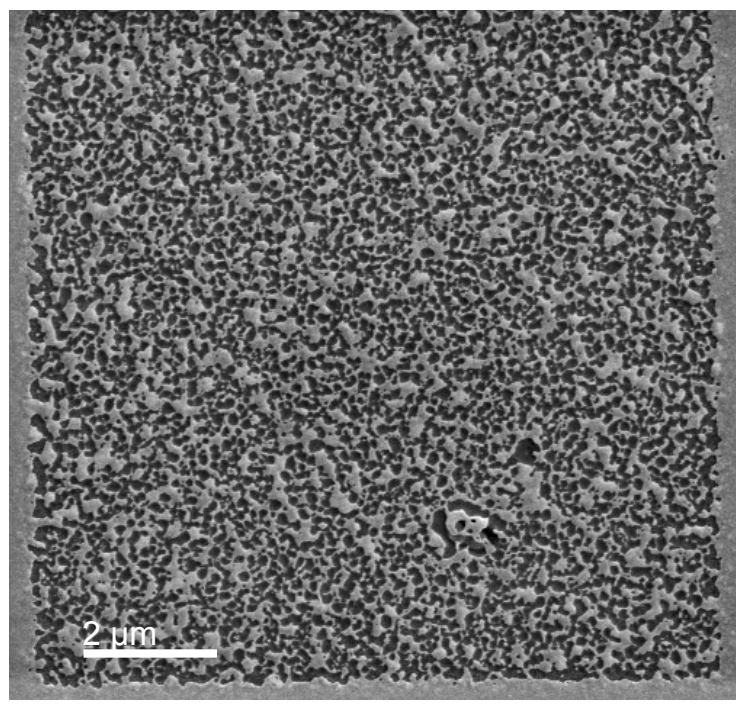

(b)

Figure 7.5: (a) Region of interest in specimen. Twenty boxes sized approximately 10 $\mu \mathrm{m} \times 10 \mu \mathrm{m}$ were created by imaging each box with a gallium ion beam with different exposure times. The boxes are labeled by their FIB imaging times in the figure. The resulting boxes have varying degrees of etching, ranging from very little material removal (top right corner, light gray) to nearly complete removal of the gold and ITO layers (bottom left corner, dark gray). (b) This area was FIB imaged for 30 seconds. The gallium beam imaging creates random metal and semiconductor structures which are quite complicated and narrow. Both images were taken at a beam energy of 10 $\mathrm{keV}$ at a working distance of $4.9 \mathrm{~mm}$. 


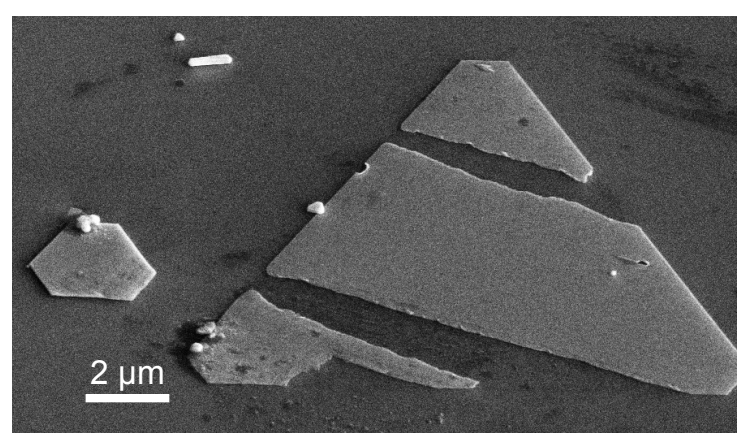

(a)

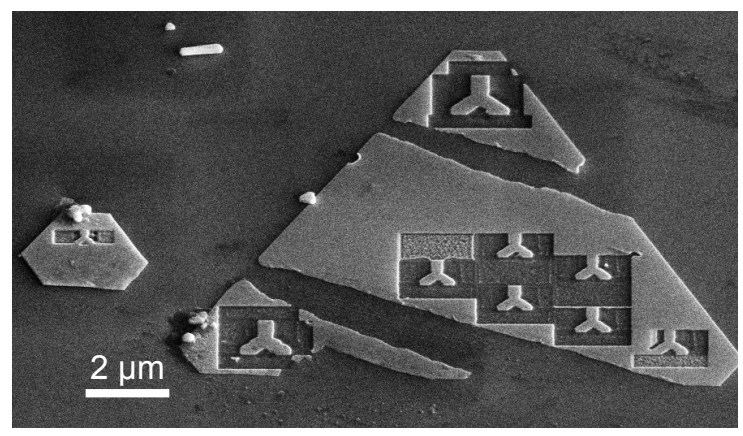

(c)

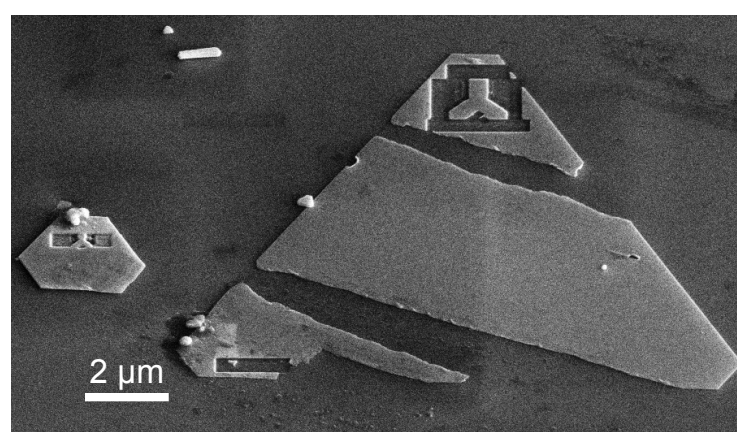

(b)

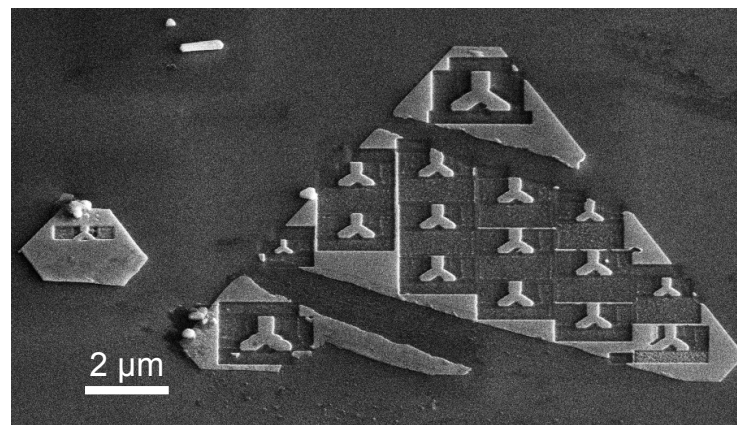

(d)

Figure 7.6: Y-shaped antenna nanostructures carved from a single-crystalline nanoflake using a FIB. The SEM images, taken at an angle of $52^{\circ}$, show a timelapse of the process as milling progresses over several minutes at low beam current (10 pA).

gold crystals. Following Huang et al. [192], nanostructures were milled from singlecrystalline gold nanoflakes using 10 pA beam currents, as shown in Fig. 7.6. A series of adjacent or overlapping rectangular milling regions define the edges of the structures by removing gold material. The complex patterns of rectangles forming Yshapes of various sizes were mapped out in advance by a Mathematica script, which subsequently output a file that the FIB could execute. The selected 20-nm-thick gold platelet platelet was large enough to carve eleven 500-nm Y-shaped antenna structures as well as a few larger and smaller size-structures.

For photonics experiments, several dielectric nanostructures were milled into ITO, with two representatives shown in Fig. 7.7. The nature of the investigation was much 


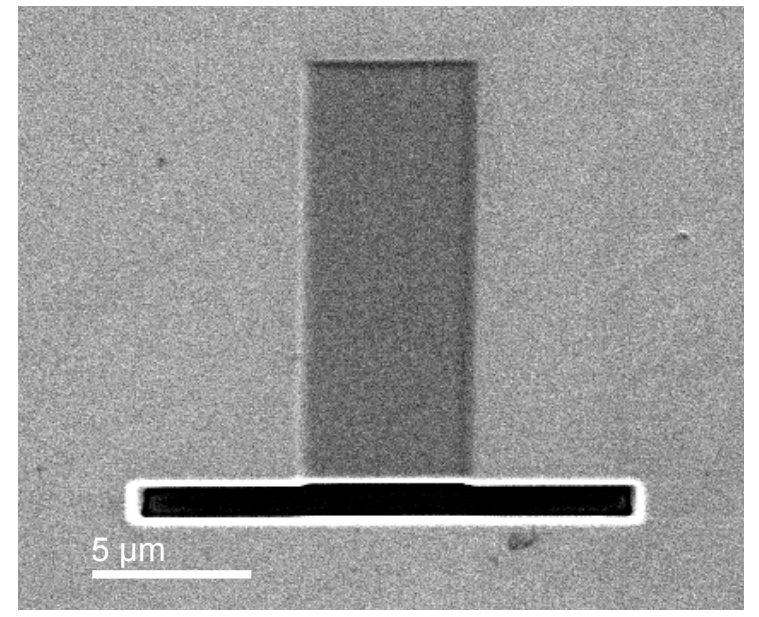

(a)

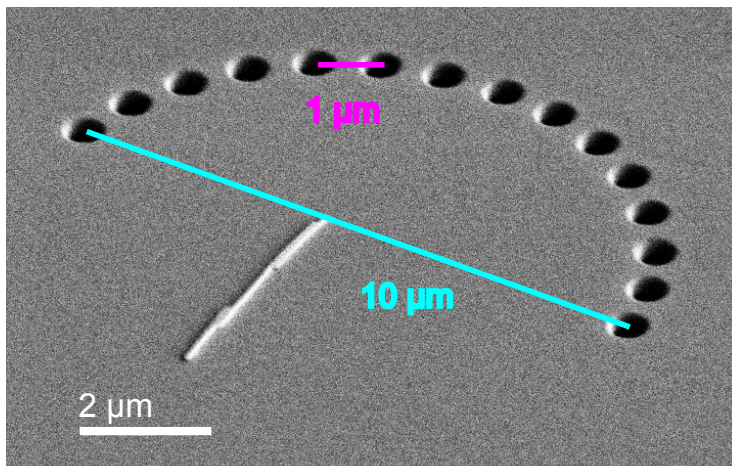

(b)

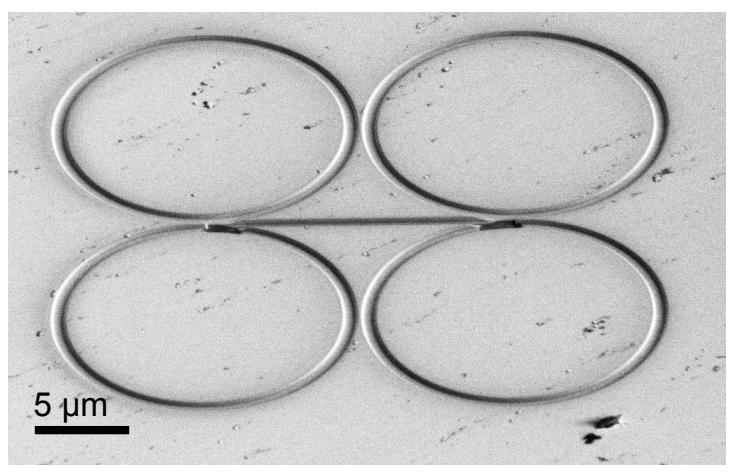

(c)

Figure 7.7: SEM images of a diffracting structures milled with a FIB into ITO thin film on glass, viewed at an angle of $52^{\circ}$. (a) A deep groove and an adjacent shallow groove. (b) A semicircular of holes. (c) Four adjacent circular grooves. 


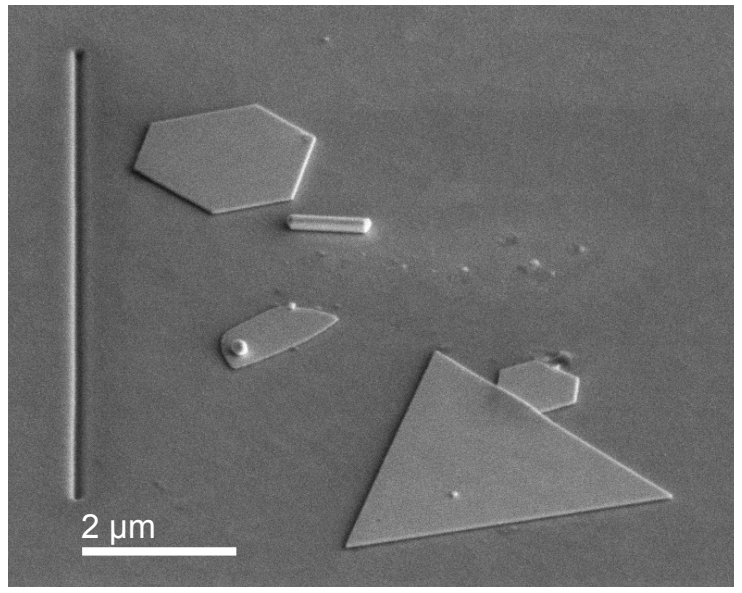

Figure 7.8: SEM micrograph of a cluster of crystalline Au platelets in the vicinity of a diffracting groove in the ITO substrate. The groove is $10 \mu \mathrm{m}$ long, and $650 \mathrm{~nm}$ from the closest flake. The SEM image is taken at a tilt of $52^{\circ}$.

more exploratory, and simple rectangles and holes were used. The simplest of these structures is a 320 -nm deep, $6.0 \times 0.4-\mu \mathrm{m}^{2}$ groove adjoined by a wide, shallow trench 50-nm deep and measuring $2.0 \times 5.0-\mu \mathrm{m}^{2}$ oriented perpendicular to the slit. The first groove is deeper than the 290-nm thick ITO film, exposing the glass layer at the bottom, while milling the shallower groove only served to thin the ITO layer to 240 $\mathrm{nm}$. The second structure is a semicircular array of holes $500 \mathrm{~nm}$ in diameter with centers spaced $1 \mu \mathrm{m}$ apart, following a circular arc with radius $5 \mu \mathrm{m}$. In the third structure the groove width is $500 \mathrm{~nm}$ with a diameter of $15 \mu \mathrm{m}$. The groove depth less than the ITO film thickness of $265 \mathrm{~nm}$. A straight groove was also milled into the ITO layer adjacent to single crystalline nanoflakes, as shown in Fig. 7.8. 


\section{Theory of electromagnetic fields as relevant to photoemission}

Spatial and temporal changes in photoemission rates are generally due to variations in the electron states (steady-state initial, intermediate, and final states), the electromagnetic field intensity (photon energy and density), and dynamic electron excitations (resonant or scattering events) [56]. The local density of states can be difficult to calculate or measure; however, in many cases, a simple model that incorporates the approximate photoemission threshold and any large band gaps is sufficient. Typically the incident light intensity is assumed to be spatially uniform since the field of view in PEEM images is usually less than the illumination area. Subsequently, local variations in light intensity or photon energy can be assumed to be dynamic responses of the sample as the result of surface features or electron excitations. These dynamic electron excitations also influence the local electromagnetic field. The local photoelectron yield is related to the local photon density, which corresponds to the intensity of the classical electromagnetic field $I \propto E_{\mathrm{tot}}^{2}[10,11,16,19,33] . \mathbf{E}_{\mathrm{tot}}$ is the total surface optical electric field, including the incident light and optical response of the material. At any surface location $\mathbf{x}$, there is a power law relationship between the photoelectron current $j_{e}$ and the intensity of the total electromagnetic field,

$$
j_{e} \propto \int E_{\mathrm{tot}}^{2 n} d t,
$$

where integration is over exposure time $t$, and $n$ is the order of the process, e.g., $n=2$ for two-photon photoemission. The order $n$ is typically assumed to be the minimum number of photons satisfying the energetic consideration $n(h c / \lambda)>W$, though this is only an approximation. Experimental determination from a plot of $j_{e}$ vs light intensity $I \propto E^{2}$ via Eq. (6.3) often gives fractional exponents, revealing 
several competing processes, some operating above threshold, i.e., proportional to $E^{2(n+1)}[96,193]$. When incident light is polarized, limited cross-polarized response is expected and a complex scalar field is sufficient.

The total scalar field is the superposition of incident and response fields, $E_{\mathrm{tot}}=$ $E_{\text {inc }}+E_{\text {exc }}$. The response field includes localized and propagating optical excitations, and may be separated into these constituents $E_{\mathrm{exc}}=E_{1}+E_{2}+\cdots$. Each component has a unique combination of spatial propagation wave vector (where appropriate), frequency (typically same as incident), excitation efficiency, phase delay relative to the incident wave, and absorption coefficient. For pulsed light sources, when the time between pulses is much greater than the duration of a single pulse, $j_{e}$ is directly proportional to the integration over one pulse. A steady state approximation $j_{e} \propto$ $\left\|E_{\text {tot }}\right\|^{2}$ is appropriate when the response field decay time is much less than the pulse duration. For propagating optical excitations, the steady state can be used when surface waves travel across the viewing window $\ell$ in significantly less time than the pulse duration $\tau$. With a maximum speed of $c$ the vacuum speed of light, this condition is met when $\ell / c \ll \tau$.. Optical responses in metals and semiconductors attenuate quickly in the visible and UV, so the viewing window is limited by the absorption length $\ell \sim(2 \alpha)^{-1}$. With $\alpha \gg 1000 \mathrm{~cm}^{-1},(2 \alpha c)^{-1} \ll 17 \mathrm{fs}$ and pulse durations of $\tau \gtrsim$ $100 \mathrm{fs}$ can be considered steady state. Differences from the steady state approximation for pulses even in the regime of $\sim 50$ fs are generally small enough to ignore since most attenuation constants are greater than $10,000 \mathrm{~cm}^{-1}$ in this spectral range. Working in the single-polarization and steady-state regimes, the time-independent photoelectron current at a position $\mathbf{x}$ is proportional to the $2 n$ power of the absolute value of the 


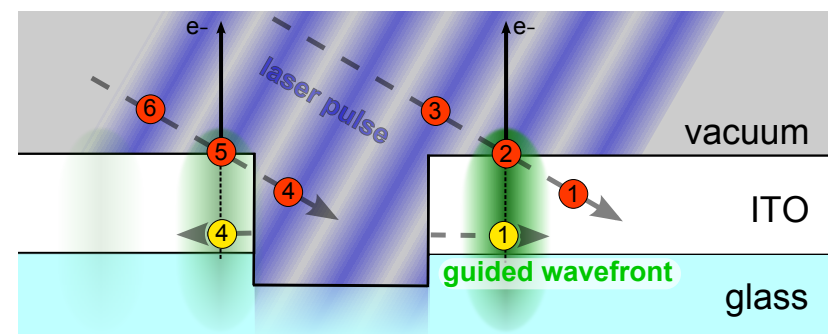

Figure 8.1: Cross section diagram of stationary interference pattern generation near a diffracting groove in a waveguide with propagating surface electromagnetic fields in PEEM. Incident wave fronts 1 and 4 (red) excite guided wave fronts 1 and 4 (yellow) through diffraction and scattering. The reverse-direction modes (4) are very weakly coupled. These propagate away from the excitation point and interfere constructively with the next incident wave fronts 2 and 5, enhancing photoemission there. Reprinted from Fitzgerald, Word, Saliba, and Könenkamp [173].

superposition of the incident and response fields at that location,

$$
j_{e}(\mathbf{x}) \propto\left\|E_{\text {inc }}+E_{1}+E_{2}+\cdots\right\|^{2 n}=\left\|E_{\text {inc }}+\sum_{j} E_{j}\right\|^{2 n} .
$$

For a localized oscillation, the photoelectron yield measures the correlation between the incident and response fields. Using a variable delay between two incident pulses, this correlation can be measured (cf. Refs. [8, 9]).

With a propagating optical excitation, the correlation takes on a spatial characteristic, producing a stationary interference pattern, as shown in Figs. 8.1 and 7.1. This pattern is readily imaged in PEEM, so it bears further elaboration. If the excitation amplitudes $B_{j}=\left|E_{j}\right|$ are much less than the incident amplitude $A=\left|E_{\text {inc }}\right| \gg B_{j}$, then spatial distribution of the electric field intensity is

$$
\left\|E_{\mathrm{tot}}\right\|^{2} \approx A^{2 n}\left[1+A^{-1}\left(B_{1} \cos k_{I}^{(0,1)} y+B_{2} \cos k_{I}^{(0,2)} y+\cdots\right)+\mathcal{O}\left(A^{-2} B_{j}^{2}\right)\right]
$$


with interference wave vectors

$$
k_{I}^{(0, j)}=k_{j}-k_{0} \sin \theta,
$$

where $\mathbf{k}_{0}=k_{0}(\hat{\mathbf{y}} \sin \theta-\hat{\mathbf{z}} \cos \theta), \theta$ is the angle of incidence relative to the surface normal $\hat{\mathbf{z}}, k_{0}=2 \pi / \lambda$ is the incident wave number, and $k_{j}=\mathbf{k}_{j} \cdot \hat{\mathbf{y}}$ is the surfaceparallel component of the excited wave vector. For a single mode, which is the typical case for surface plasmons, the interference maxima are separated by

$$
d_{I}=2 \pi / k_{I}
$$

and allows the ready determination of the excited wave vector $k_{1}$. This uniquely characterizes the response mechanism, especially if the analysis is repeated for more than one polarization $[16,33,38,44-46]$. For example, if the effective index $N_{1} \equiv$ $k_{1} / k_{0}=1$ is unitary, then the response wave must be traveling in the vacuum, a good indication of a diffracted surface wave [13] or a surface plasmon polariton with most of the field intensity in the vacuum (as occurs with gold and copper at frequencies above the plasmon frequency).

With more than one mode, the interference pattern is less readily interpreted. Instead, it becomes more convenient to examine the power spectrum of the spatial Fourier transform of the interference profile. Then spectrum has peaks centered on the interference wavenumbers with widths proportional to the absorption coefficient $\alpha$. The Fourier transform also typically shows higher order interference terms, such as the interference between modes with wavenumbers $k_{I}^{(j, l)}=\left|k_{j}-k_{l}\right|$. With particularly good signal to noise ratios, nonlinear interference terms are also identifiable in the Fourier spectrum, e.g., $k_{I}^{(0, j, l)}=k_{j}+k_{l}-2 k_{0} \sin \theta$ in $2 \mathrm{PPE}$. As an example, the largest 


\begin{tabular}{c|ccc}
\multicolumn{5}{c}{ Single-photon accessible } \\
\hline Label & 1 & 2 & 3 \\
Intensity & $A^{3} B_{1}$ & $A^{3} B_{2}$ & $A^{2} B_{1} B_{2}$ \\
Wavenumber $k_{I}$ & $k_{1}-k_{0} \sin \theta$ & $k_{2}-k_{0} \sin \theta$ & $k_{2}-k_{1}$ \\
\multicolumn{4}{|c}{ Multi-photon only } \\
\hline Label & 4 & 5 & 6 \\
Intensity & $A^{2} B_{1}^{2}$ & $A^{2} B_{2}^{2}$ & $A^{2} B_{1} B_{2}$ \\
Wavenumber $k_{I}$ & $2\left(k_{1}-k_{0} \sin \theta\right)$ & $2\left(k_{2}-k_{0} \sin \theta\right)$ & $k_{1}+k_{2}-2 k_{0} \sin \theta$
\end{tabular}

Table 8.1: Theoretical interference model signal wavenumbers, obtained from the Fourier transform of Eq. (8.3) with two modes $j=\{1,2\}$ at photoemission rank $n=2$. The signals are roughly sorted by intensity assuming $A>B_{1}>B_{2}$ and $k_{2}>$ $k_{1}$. Signals 4-6 are unique to multiphoton photoemission, but are often near the noise threshold. Six weaker signals of $\mathcal{O}\left(A B_{j}^{3}\right)$ are omitted. Additionally, the coefficients $B_{j}$ may contain attenuation and offset factors that reduce intensity, especially in signals 3 and 6. Adapted from Fitzgerald, Word, Saliba, and Könenkamp [173].

six interference signals of a two-mode, $2 \mathrm{PPE}$ interference pattern are presented in Table 8.1. Identifying all the peaks in the Fourier transforms allows an accurate computation of the excited wavenumbers and absorption coefficients, a calculation that becomes highly redundant as more interference signals can be identified.

Experimentally determined wavenumbers for propagating surface plasmon and photonic waveguide modes can be compared to the asymmetric slab waveguide model (see Fig. 6.4), as presented in [194-196]. The asymmetric slab model considers three semi-infinite layers and uses boundary matching conditions of Maxwell's equations to determine guided wave numbers. The discrete solutions depend on polarization, film thickness, and material optical parameters. For example, following the notation of Yariv [194], photonic modes are determined by solving

$$
\frac{p+q}{h\left(1-p q / h^{2}\right)}=\tan (h t),
$$

where

$$
h=k_{0} \sqrt{n_{2}^{2}-N^{2}},
$$




$$
\begin{gathered}
q=k_{0} \sqrt{N^{2}-n_{1}^{2}} \times \begin{cases}1 & (\mathrm{TE}) \\
n_{2}^{2} / n_{1}^{2} & (\mathrm{TM})\end{cases} \\
p=k_{0} \sqrt{N^{2}-n_{3}^{2}} \times \begin{cases}1 & (\mathrm{TE}) \\
n_{2}^{2} / n_{3}^{2} & (\mathrm{TM})\end{cases}
\end{gathered}
$$

$n_{j}$ are the layer refractive indices, and $N=k / k_{0}$ is the guided wave effective index. In photoemission experiments one outer layer is always vacuum, with $n=1$, and here the other layers are limited to gold, ITO, and glass, with optical properties and thicknesses given in the previous chapter. Effective indices solving this equation for vacuum/ITO/glass layers vary with film thickness, as shown in Fig. 8.2. Potentially several modes can be excited for each polarization, with the excited wave effective indices ranging between the refractive index of the central layer and the outer layers. In photonic waveguides, the lower limit is the larger refractive of the outer layers, while in PEEM plasmonics it is the vacuum index 1. These modes represent distinct standing wave patterns between the outer layer boundaries. With the thin films considered here, only symmetric and antisymmetric modes are relevant. Examples of the field intensities of symmetric modes are shown in Fig. 8.2.

The asymmetric slab model applies equally well to plasmonic propagating waves, though some adjustments on solution methods must be made to accommodate the unique role of the complex part of the refractive index (or dielectric constant) [197]. The three-layer model provides sufficient accuracy in the cases examined here, but many cases can be approximated by two layers. Specifically with gold films thicker than 60-100 $\mathrm{nm}$ at the wavelengths probed here, essentially all of the excitation energy is concentrated at the gold-vacuum interface or in the vacuum. In this case, 


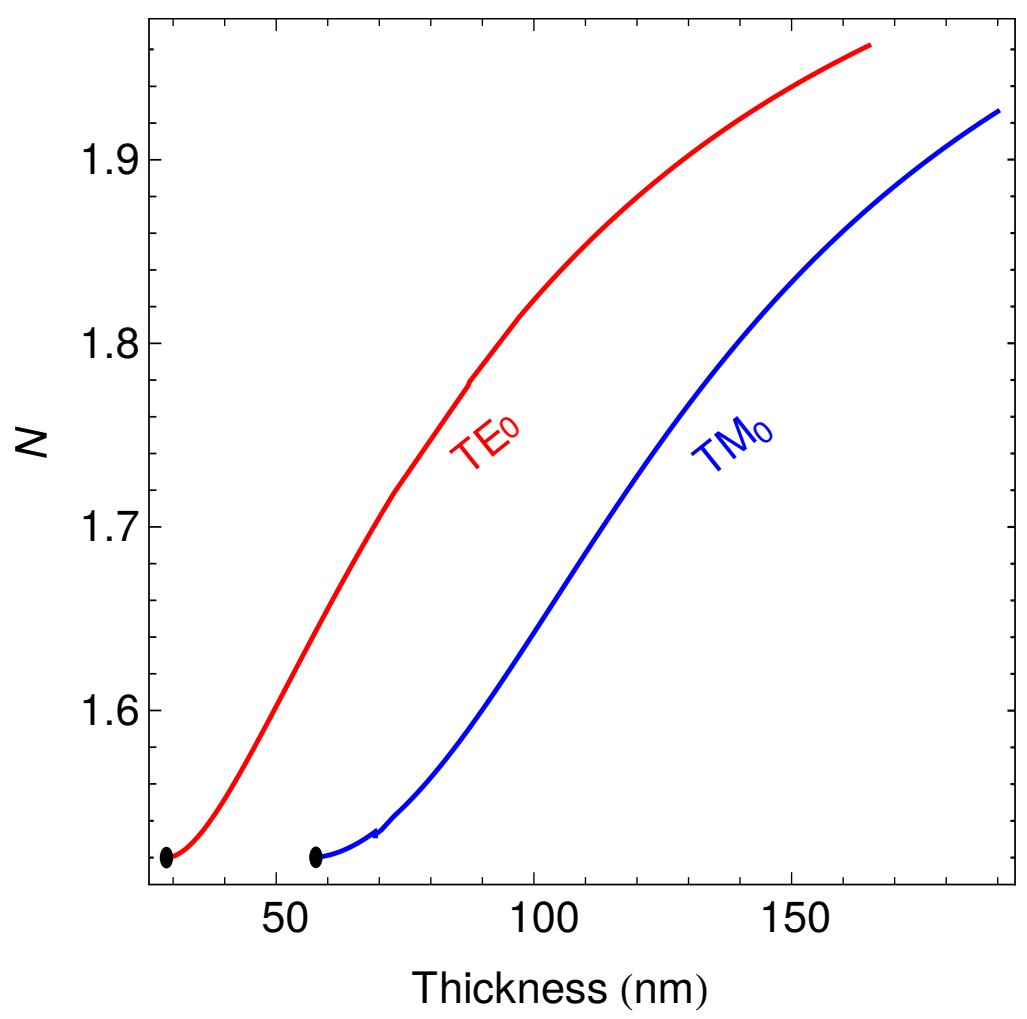

(a)

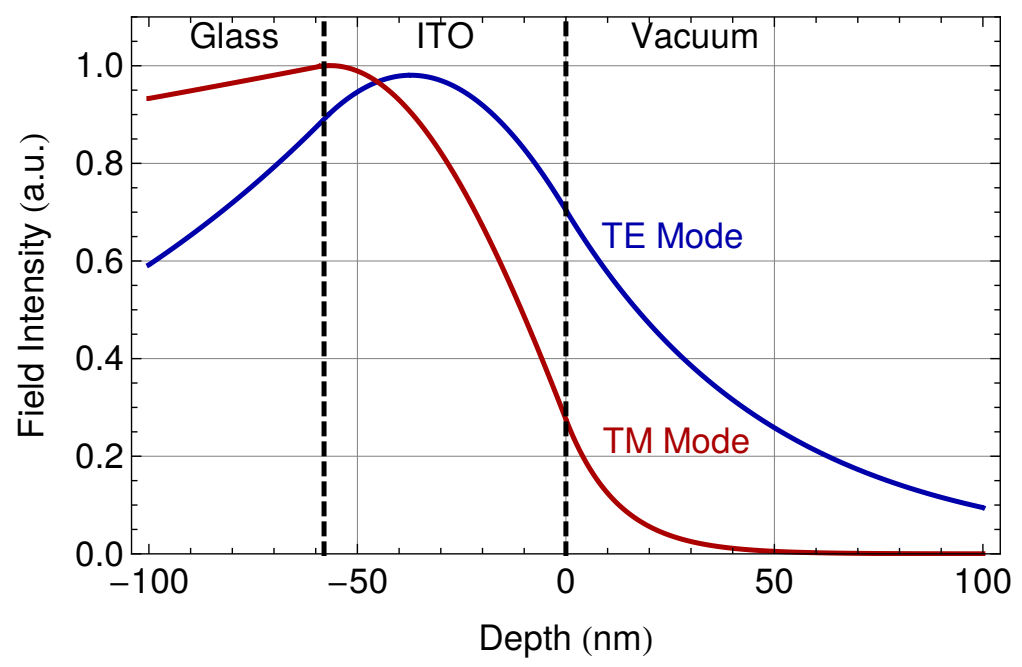

(b)

Figure 8.2: (a) Single-mode solutions to the dielectric asymmetric slab model consisting of vacuum/ITO/glass, as illustrated in Fig. 6.4. For thicker films, multiple modes exist. (b) Guided mode field intensity for an ITO film thickness of $60 \mathrm{~nm}$. The modes shown here are symmetric. 
the complex propagation constant $\beta$ is given by

$$
\beta=k_{0} \sqrt{\frac{\epsilon_{1} \epsilon_{2}}{\epsilon_{1}+\epsilon_{2}}},
$$

where the dielectric constant of the insulating layer (vacuum) is $\epsilon_{1}$ and complex dielectric constant of the metal (gold) is $\epsilon_{2}$ [198]. The SPP wavenumber $k_{j}=\Re(\beta)$ and its characteristic propagation length $L=[2 \Im(\beta)]^{-1}$ follow from $\beta$. For thinner metal films, the modes bound to the vacuum interface interact with those at the ITO (or glass) interface, and the full three-layer model is more appropriate. Solutions (Fig. 8.3) give symmetric and antisymmetric coupled modes that deviate significantly from the single-mode solutions of the simple vacuum/gold and gold/ITO interfaces for gold thicknesses less than $\sim 80 \mathrm{~nm}$. The symmetric mode generally has low spatial confinement with fields that extend far into the boundary dielectric layers (Fig. 8.3). As a result the symmetric mode has high velocity and hence low $N$. In contrast, the antisymmetric mode is more spatially confined, not limited by thickness, shorter ranged, and more importantly, has generally high $N$, which is more suitable to waveguide coupling.

Optical field excitations can be coupled to the surface or near-surface region only when the incident light wave vector is appropriately matched. Diffraction at sharp surface features bends rays to range of wave vectors, as illustrated for propagating modes in Fig. 8.4, some of which are resonant with the nanoparticle or surface geometry or material properties and can excite the optical response. For the metals and semiconductors, with absorption coefficients greater than $500 \mathrm{~cm}^{-1}$, diffraction is the most efficient and convenient coupling mechanism. The efficiency of this mechanism depends on the feature geometry and incident wavelength, but generally falls off quickly with increasing deflection angle. When more than one propagating optical 


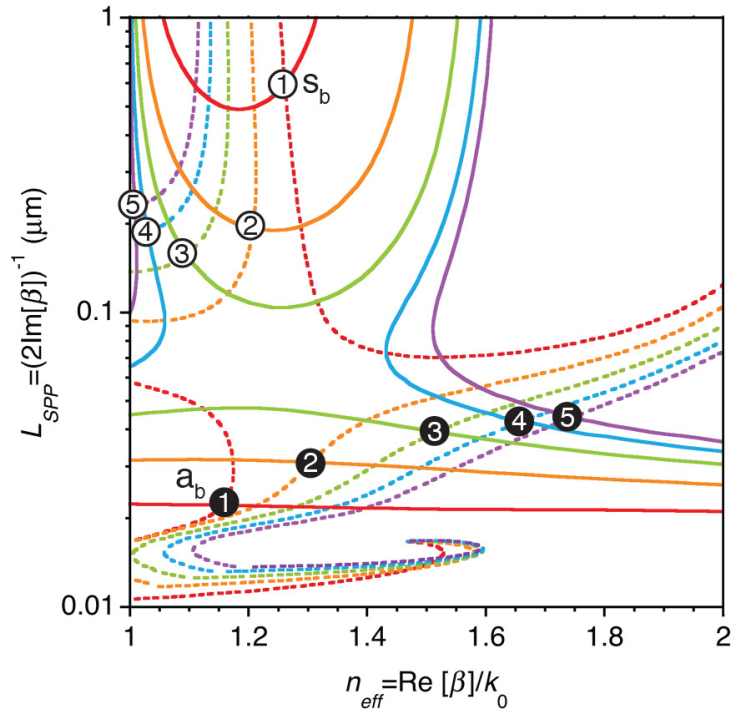

(a)

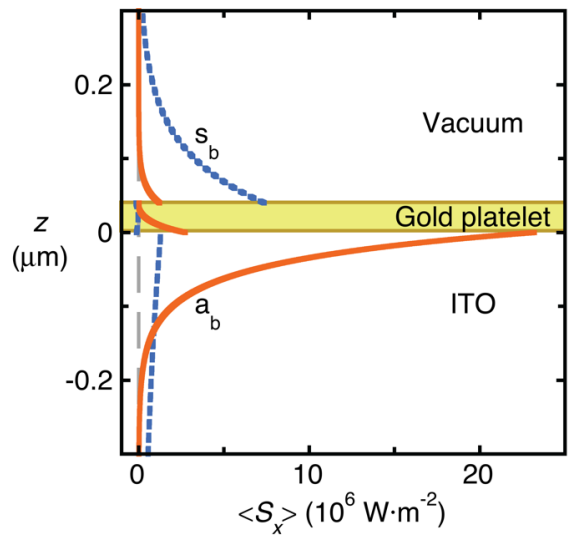

(b)

Figure 8.3: (a) Graphical method of solution for the SPP supermodes of a threelayer slab model of vacuum/gold/ITO. In this complex space graph, the propagation constants $\beta$ plotted here independently satisfy the real and imaginary parts of the equivalent of Eq. 8.6, which are shown as solid and dashed lines, respectively. Complete solutions exist where real and imaginary lines intersect. There are generally two solutions for each gold thickness $d$, which correspond to the symmetric $\left(s_{b}\right)$ and $\left(a_{b}\right)$ modes. Solutions for five gold film thicknesses shown: (1) $25 \mathrm{~nm},(2) 30 \mathrm{~nm}$, (3) $35 \mathrm{~nm},(4), 40 \mathrm{~nm}$, and (5) $45 \mathrm{~nm}$. (b) Time-averaged Poynting vector $\left\langle S_{x}\right\rangle$ of the asymmetric field $\left(a_{b}\right)$ and symmetric field $\left(s_{b}\right)$ SPP modes of a 3-layer slab model comprised of ITO, a 40-nm thick gold platelet, and vacuum. Scale normalized such that each SPP wave carries 1 watt per meter along the y-axis, with the x-axis being the propagation direction. Reprinted from Word, Fitzgerald, and Könenkamp [41]. 


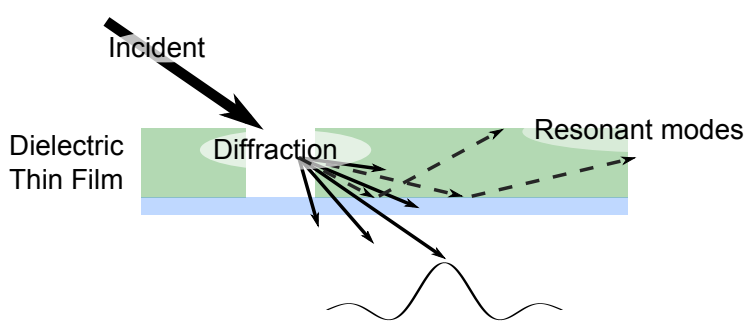

(a)

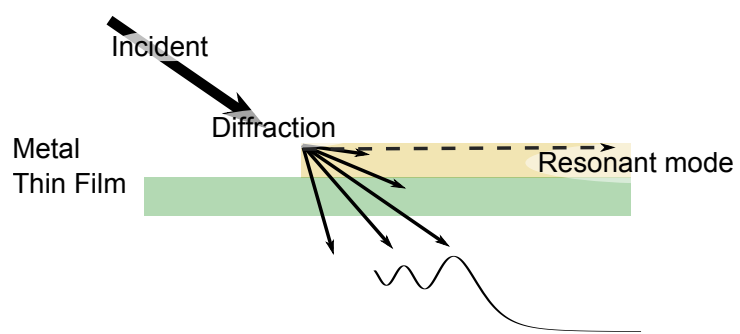

(b)

Figure 8.4: Incident light wave vectors can be matched to material and surface optical excitations near diffracting features, such as (a) a narrow slit or (b) and step or edge. The intensity of the diffracted waves is shown below plotted versus scattering angle, and contributes to the coupling efficiency.

mode is excited with coupling coefficients $B_{1}$ and $B_{2}$, then the ratio of excited mode amplitudes can be determined from the wavenumber and Fraunhofer diffraction as follows. The geometries considered here are groove, hole, and knife-edge diffraction. Adjacent to a groove, such as in Fig. 7.7, the relative mode coupling strength is

$$
B_{2} / B_{1}=\operatorname{sinc} \zeta_{2} / \operatorname{sinc} \zeta_{1},
$$

where $\zeta_{j}=k_{0} w \sin \left(\theta-\sin ^{-1} k_{j} / k_{0}^{\prime}\right), w$ is the slit width, and $k_{0}^{\prime}=n k_{0}$ is the wavenumber in the surface material with refractive index $n$. For a hole geometry, such as Fig. 7.7, diffraction intensity follows an Airy disc distribution, and the relative intensity is

$$
B_{2} / B_{1}=\left[J_{1}\left(\zeta_{2}\right) / \zeta_{2}\right] /\left[J_{1}\left(\zeta_{1}\right) / \zeta_{1}\right],
$$


where $J_{1}(x)$ is a Bessel function of the first kind, and $\zeta_{j}$ is the same as before with $w$ measuring the hole diameter. Knife-edge diffraction has a more complex relationship and here involved only single modes. Finally, the Fresnel transmission coefficients and phase changes must also be calculated in order to determine the coupling efficiency [199].

With both wavenumbers and coefficients readily calculable from a priori properties, it is possible to compute the electromagnetic field distribution of extended, two-dimensional diffracting structures. The structures can be considered apertures, and guided wave propagate away from the apertures across the surface according to Huygens' principle, which treats every point of a scalar wavefront as a new source wavelet propagating outward in all directions. Since guided waves are bound to the thin film (photonic) or the surface (plasmonic, vacuum-radiative), they can be represented by a two-dimensional wavelet [200],

$$
G_{j}\left(\mathbf{x}, \mathbf{x}^{\prime}\right)=\frac{i}{4} e^{-\alpha\left|\mathbf{x}-\mathbf{x}^{\prime}\right|} H_{0}^{(1)}\left(k_{j}\left|\mathbf{x}-\mathbf{x}^{\prime}\right|\right),
$$

where $\mathbf{x}$ is the sample location, $\mathbf{x}^{\prime}$ is the aperture source location, and $H_{0}^{(1)}(x)$ is a zero-order Hankel function of the first kind. The complex field $E_{j}$ at any point $\mathbf{x}$ is the superposition of all the wavelets that originated at the boundary of the waveguide $\mathbf{x}^{\prime}$. Fresnel, and later Kirchhoff, Rayleigh, and Sommerfeld, developed a rigorous method to give $E_{j}(\mathbf{x})$ by integrating over the boundary values $E_{j}\left(\mathbf{x}^{\prime}\right)$, which is the product of the incident wave phase $e^{i \mathbf{k}_{j} \cdot \mathbf{x}^{\prime}}$ and the complex coupling coefficient $B_{j}$. With both the wavelet function and the boundary value, it is now possible to calculate the complex scalar field $E_{j}(\mathbf{x})$ from the two-dimensional Fresnel-Kirchhoff integral [199],

$$
E_{j}(\mathbf{x})=\oint_{\mathcal{C}}\left\{E_{j}\left(\mathbf{x}^{\prime}\right) \mathbf{n}^{\prime} \cdot \nabla^{\prime} G_{j}\left(\mathbf{x}, \mathbf{x}^{\prime}\right)-G_{j}\left(\mathbf{x}, \mathbf{x}^{\prime}\right) \mathbf{n}^{\prime} \cdot \nabla^{\prime} E_{j}\left(\mathbf{x}^{\prime}\right)\right\} d \ell^{\prime},
$$


where $\mathcal{C}$ is a closed curve surrounding the sample points of interest $\mathbf{x}, \mathbf{n}^{\prime}$ is the inward-directed normal vector, and the integration is over the boundary (primed) terms. Suitable approximations exist for partially unbounded regions, such that many situations can be computed. The electromagnetic field distribution of each excited mode can be individually calculated at each sample point $\mathbf{x}$ with Eq. (8.11), and then the intensity distribution is the sum of terms, squared. A map can be created by repeating the computation for several thousand points $\mathbf{x}$. The $n$th power of the intensity distribution at each point $\mathbf{x}$, as given by Eq. 8.2, is directly comparable to the photoelectron micrograph, where $n$ is the rank of the photoexcitation process.

The Kirchhoff integration approach has the advantages of being relatively intuitive and computationally fast, but it works best for optical excitations with propagation distances longer than a half wavelength. For optical excitations with shorter attenuation lengths or geometries highly sensitive to the near-field effects, it is necessary to solve Maxwell's equations directly. In this case, the calculation can be carried out by the RF-Module of COMSOL 4.3, which solves Maxwell's equations for a triangular mesh to obtain the electric field distribution utilizing a finite-element approach with the full dielectric functions of the materials and the true, three-dimensional spatial dimensions of the device. Whether the field intensity is obtained from Eq. (8.11) or COMSOL, the field can be compared to the photoelectron yield by the power law relationship given in Eqs. (8.1) and (8.2). 


\section{Photonics results \& discussion}

In this chapter, I examine photoelectron images from several different materials and nanostructures. With Eq. 8.1 we can connect photoemission rates to surface fields. Going one step further with Eq. 8.11, we can compute the electromagnetic field distribution at the surface of a material in response to diffracting nanostructures. Looking at PEEM images in this light, we can them in terms of the electromagnetic fields and processes they represent.

\subsection{Metal nanostructures}

I begin by examining photoemission patterns from gold nanostructures on ITO, as originally presented in Refs. [28, 30, 37, 42, 172]. This work connects optical excitations at the material surface with the emission of electrons.

First, work done by Word, Dornan, and Könenkamp [28] as I joined the group establishes that specimens illuminated with IR light emit electrons via multiphoton processes. Figure 9.1 shows the UV and IR photoelectron response imaged in PEEM from a random gold nanostructure. The tunable pulsed laser provides photon energies between 1.37 and $1.6 \mathrm{eV}$. Since the photoemission thresholds for ITO and Au are significantly larger than the IR photon energies, the electrons are emitted via multiphoton processes. Figure 9.2 shows the emission intensity to be proportional to

the third power of the laser intensity, indicating a 3PPE process. In the $10 \times 10 \mu \mathrm{m}^{2}$ area shown in Fig. 9.1 there are $\sim 1000$ high-intensity photoemission sites, primarily located along the margins of the gold-ITO boundary. Figure 9.2 shows that the photoemission threshold is $4.1 \mathrm{eV}$, consistent with that of ITO and not that of gold, which is greater than $4.5 \mathrm{eV}$. Photoelectrons most likely originate from the ITO. A 


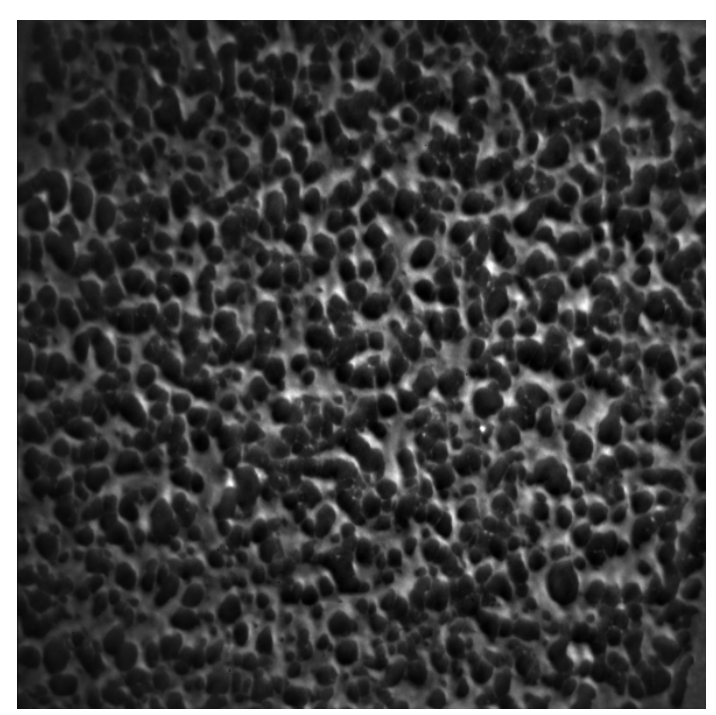

(a)

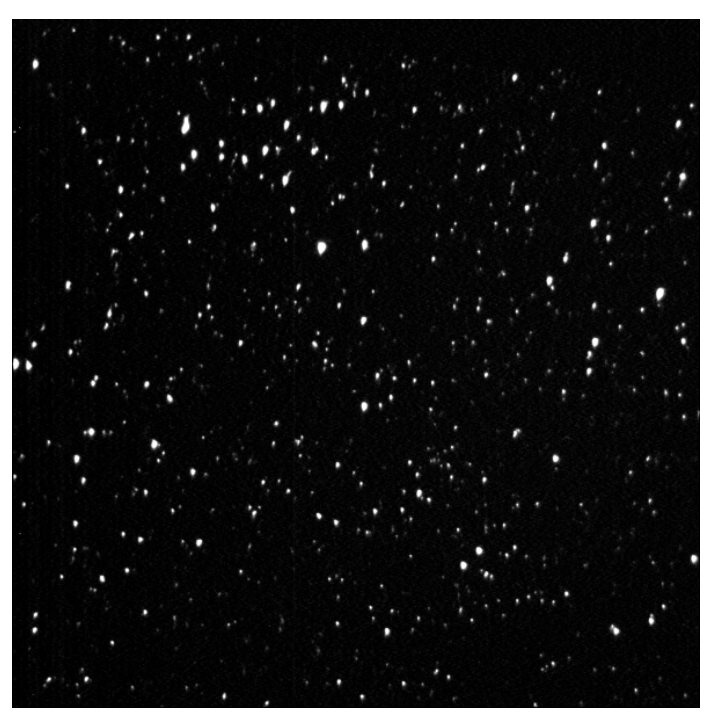

(b)

Figure 9.1: Plasmonic gold random nanostructures. (a) UV-PEEM image using 244 $\mathrm{nm}$ light of random gold-ITO nanostructures in one $10 \times 10$ micron square (field of view $9 \times 9$ microns). (b) 3 P-PEEM image using pulsed $790 \mathrm{~nm}$ light of the same region, clearly showing plasmonic hot spots.

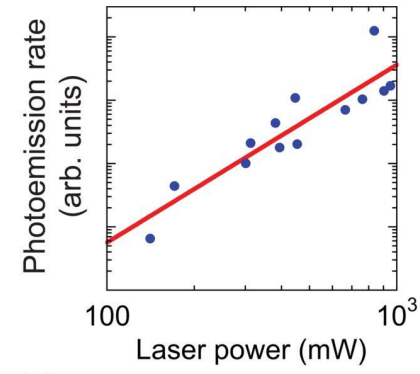

(a)

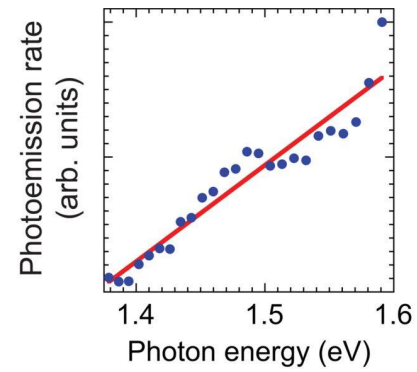

(b)

Figure 9.2: (a) Intensity dependence of the photoemission yield for $800 \mathrm{~nm}$ light. Inset: illumination scheme for nanostructured $\mathrm{Au}$ films on ITO. (b) Photon-energy dependence of the photoemission yield showing a threshold $3 \times 1.37 \mathrm{eV}$. Reprinted from Word, Dornan, and Könenkamp [28]. 


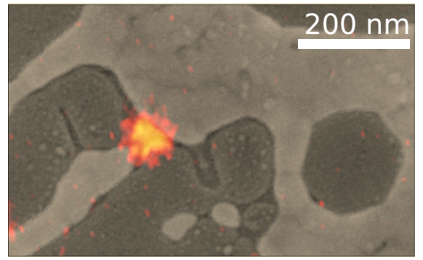

(a)

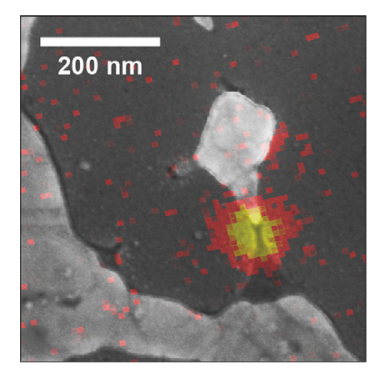

(b)

Figure 9.3: Composite micrographs of SEM and multiphoton PEEM images of localized photoemission from ITO in gaps of a plasmonic Au nanostructure at a wavelength of $792 \mathrm{~nm}$. Reprinted from Word, Dornan, and Könenkamp [28], Word, Fitzgerald, and Könenkamp [30].

high magnification image a photoemission hot spot, Fig. 9.3, reinforces this assertion. In this particular example a bridge-type geometry is depicted where the planar ITO film connects two separate $\mathrm{Au}$ areas with gaps of $\sim 100 \mathrm{~nm}$. The composite micrograph clearly shows that the photoemission originates from the ITO film. Since the ITO has a low absorption coefficient at $\lambda=780 \mathrm{~nm}$ [187], the photoemission is assumed to be due to energy transfer from the SPP fields of the adjacent, highly absorbing Au film.

The electron emission process is thought to involve the following steps: Infrared light is absorbed in the gold film exciting surface plasmon polaritons - predominantly with the same quantum energy as the exciting photons. The high peak intensities of the femtosecond pulses increase the rate of non-linear excitations to measurable amounts, and plasmons with doubled, tripled, and possibly higher quantum energies are also excited [113]. These coherently excited higher order collective excitations provide the energy needed for single-electron excitations beyond the photoemission threshold barrier, such that electron emission is observed. Alternatively, the high intensity pulses create a high population density of coherent singly-excited surface plasmons. There is enough overlap of the quantum wavefunctions of several quasi- 
particles to contribute to the emission of an electron. In either case, the electron emission is observed to originate from ITO in the optical near-field zone of the gold structures. Essentially, the gold films are found to act as optical receivers, inducing electron emission in adjacent ITO regions whose threshold is substantially lower than that of gold. For this near-field emission it is currently not known, if it involves a decay of surface plasmons into photons prior to the observed electron emission, or if the emission energy is also of plasmonic nature.

This photoemission process can be controlled with more selective nanostructures and polarization, as discussed by Könenkamp, Word, Fitzgerald, Nadarajah, and Saliba [37]. Figure 9.4 shows the photoemission from single-crystalline, Y-shaped antennae on ITO in response to an incident light pulse with wavelength $410 \mathrm{~nm}$. At this wavelength both gold and ITO require a two-quanta emission process for the 3.1 $\mathrm{eV}$ photons. However, the gold surface - with its higher electron density - dominates in the emission images and, on a linear scale, hardly any emission from the ITO is apparent. The micrographs in Fig. 9.4 show two superimposed electron emission images obtained under illumination with $-45^{\circ}$ and $+45^{\circ}$ polarizations. We see that emission sites are distributed quite differently for the two light polarizations. This is apparently due to polarization-dependent in-coupling of the optical power: The in-coupling of light is most efficient at the edge of the $\mathrm{Y}$ structure, where the light polarization vector can have a large component perpendicular to the metal surface. This is the case along the left edge of the $\mathrm{Y}$ for a polarization of $-45^{\circ}$ and along the right edge at a polarization of $+45^{\circ}$. The micrographs indicate a fairly high selectivity for the polarization direction based on the in-coupling process. Under infrared illumination, Fig. 9.5, electron emission originate from the near-field vicinity outside the plasmon excited metal structures instead of from the plasmonically active metal region itself, consistent with our previous interpretation. The eleven structures shown in Fig. 9.5 


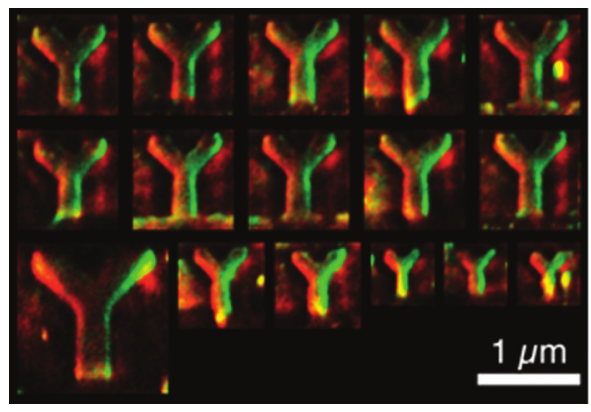

(a)

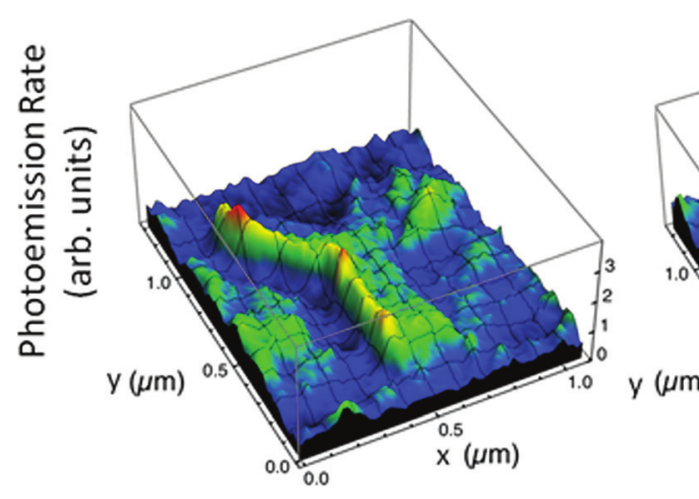

(c)

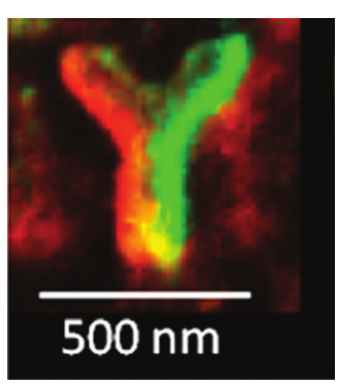

(b)

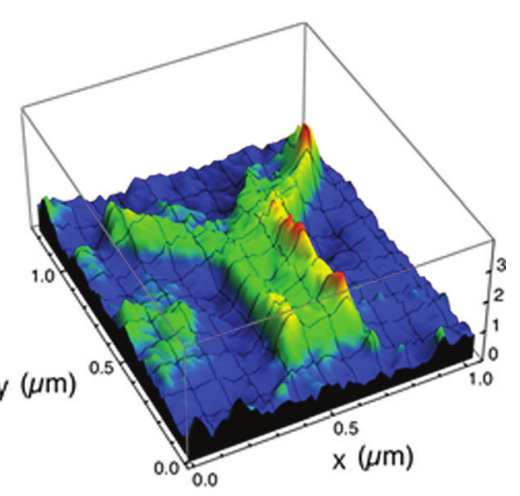

Figure 9.4: (a) Eleven routers carved from a triangular gold platelet. False color composite image of two PEEM micrographs taken with pulse illumination at $410 \mathrm{~nm}$ and $-45^{\circ}$ and $+45^{\circ}$ polarizations. Green brightness represents electron emission rate obtained for $+45^{\circ}$ polarization, red brightness represents electron emission obtained for $-45^{\circ}$ polarization. (b) Details of a structure from part (a). (c) Emission rate distribution for $-45^{\circ}$ polarization (left) and $+45^{\circ}$ polarization (right). Reprinted from Könenkamp, Word, Fitzgerald, Nadarajah, and Saliba [37]. 


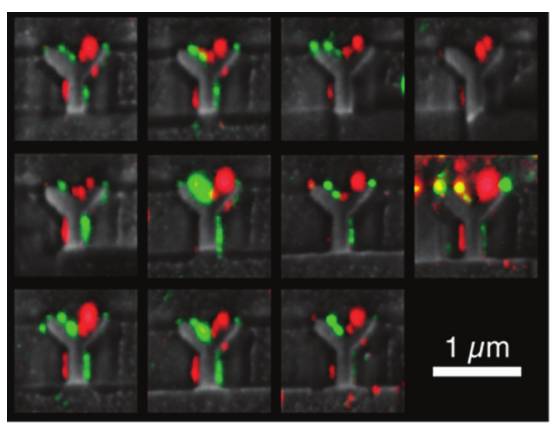

(a)
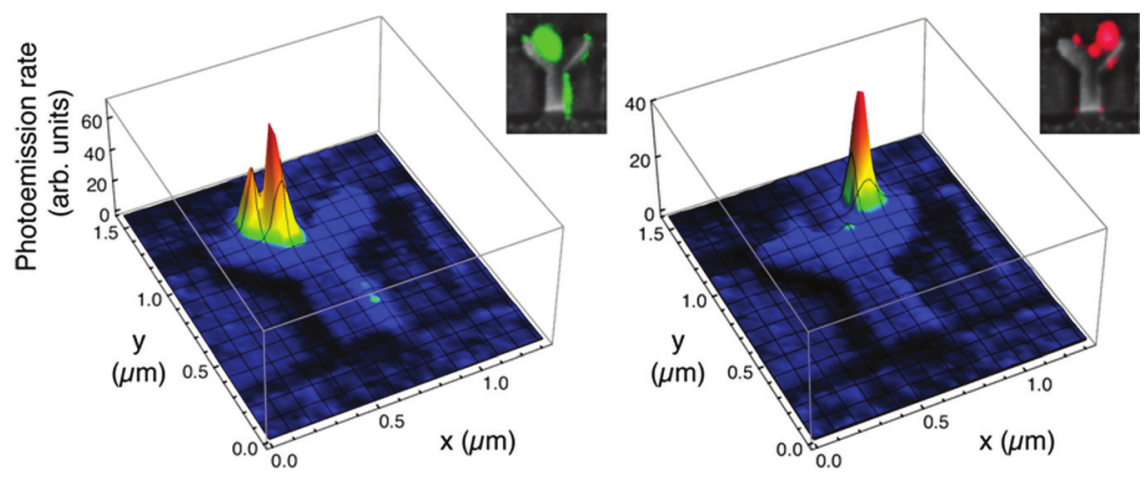

(b)

Figure 9.5: (a) Composite PEEM images obtained with DC-illumination at $244 \mathrm{~nm}$ (gray scale) and pulse illumination at $780 \mathrm{~nm}$ (color). Electron emission for $-45^{\circ}$ polarization is represented in red, for $+45^{\circ}$ electron emission is in green. Hot spots at the end points of the $\mathrm{Y}$ structure are clearly seen indicating polarization-selective emission from ITO. No emission from the front edge or the surface of the Y-structure is seen. (b) Digital plot of the emission rate for $-45^{\circ}$ degree polarization. This image is obtained with simultaneous illumination from a $\mathrm{Hg}$ lamp $(\sim 253.7 \mathrm{~nm})$ to provide the contour of the Y structure, and laser pulses at $780 \mathrm{~nm}$ for the plasmon excitation. When the emission from the $\mathrm{Hg}$ lamp is taken into account, the brightness ratio between the right and left-hand tips of the $\mathrm{Y}$ is found to be $>10$. Reprinted from Könenkamp, Word, Fitzgerald, Nadarajah, and Saliba [37]. 
show good reliability in the polarization response. Finally, Word et al. [172] reports similar results with another metallic antennae structure. This report goes one step further by calculating the optical field intensity of the circular antenna structure. The comparison shows good agreement for the lateral distribution and symmetry of the emission pattern across all four polarizations, making the connection between optical excitations and photoemission response even more concrete.

\subsection{Dielectric waveguide structures}

Photoemission electron microscopy allows the direct visualization of plasmonic near fields through nonlinear multiphoton photoemission. Each of these examples of emission from gold appears to adhere to Eq. (8.2), allowing predictable and precise position control of plasmon-enhanced optical fields and electron emission on a nanometer scale. I next investigate photoemission from dielectric structures, where electrons do not participate in plasmonic quasi-particles; this work was originally presented by Fitzgerald, Word, Saliba, and Könenkamp [173], Fitzgerald, Word, and Könenkamp [175].

Figure 9.6a shows a PEEM micrograph of a diffracting structure in ITO obtained at $244 \mathrm{~nm}$ from a frequency doubled $\mathrm{Ar}^{+}$-ion laser. The photon energy for this ultraviolet light is $5.1 \mathrm{eV}$, which is larger than the photoemission threshold energy of ITO, and the PEEM image is therefore obtained in a single-photon photoemission process. Mostly topological contrast is obtained in this imaging mode. The center region B is particularly bright due to surface roughness created by FIB milling.

When illuminated by pulsed light at a wavelength of $410 \mathrm{~nm}$, the character of the PEEM image changes dramatically, as in Fig. 9.6b-c. The vacuum wavelength of 410 $\mathrm{nm}$ corresponds to a photon energy of $3.0 \mathrm{eV}$. As this is less than the threshold, multiphoton photoemission must be assumed. In these images some of the topographical 


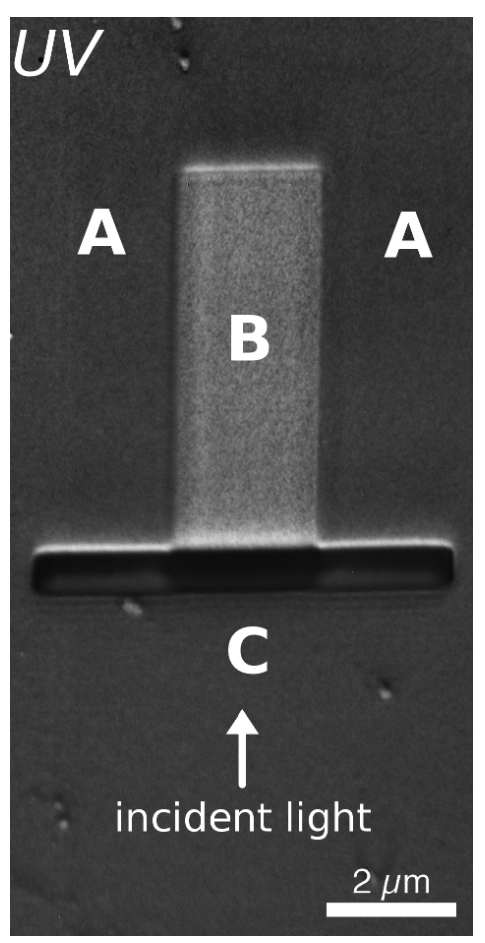

(a)

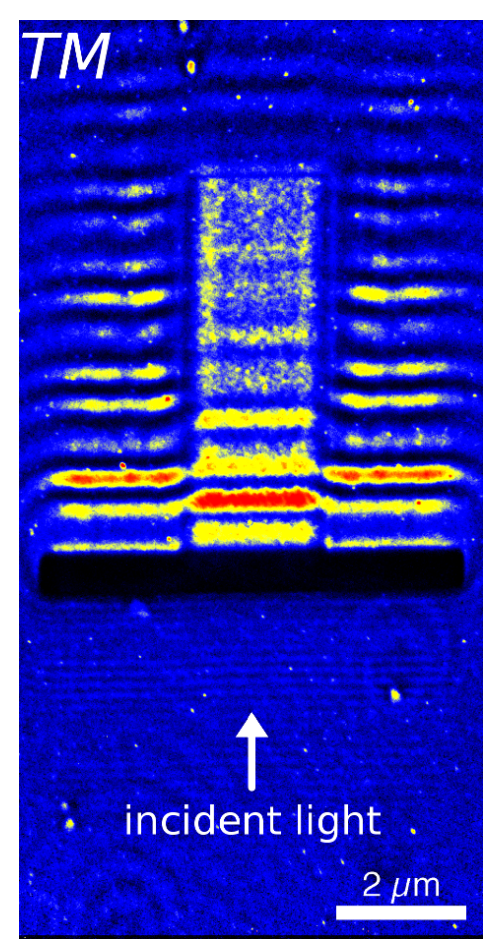

(b)

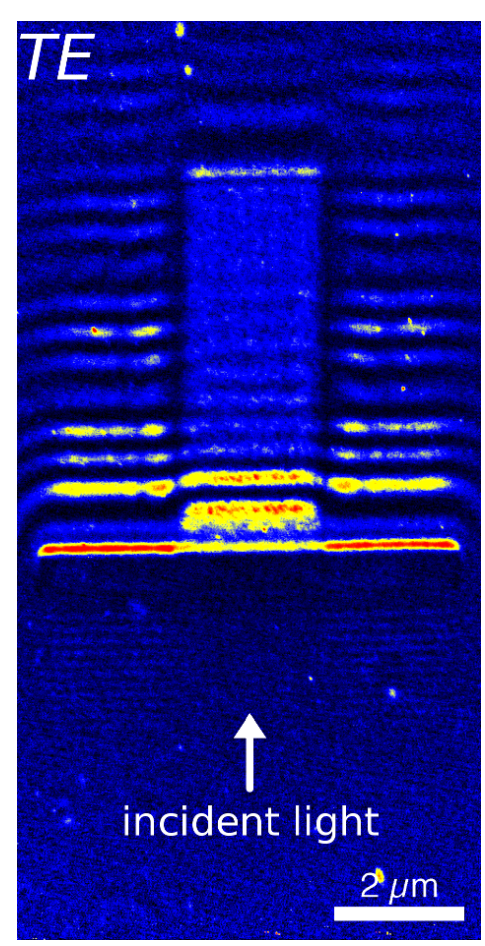

(c)

Figure 9.6: PEEM images of diffracting ITO structure. Laser is obliquely incident from the bottom edge at $60^{\circ}$ to surface normal. Regions of interest are indicated by A, B, and C. (a) Single-photon, 244-nm, continuous-wave (CW), TM polarized illumination. (b-c) Two-photon, 410-nm, 100-fs pulsed, (b) TM and (c) TE polarized illumination; shown in false color. Reprinted from Fitzgerald, Word, Saliba, and Könenkamp [173]. 
features are still recognizable, but many edges now appear highlighted and wave-like interference patterns with wavefronts perpendicular to the illumination direction are now visible. Edges facing the incident light have enhanced emission compared to edges at other orientations, especially in TE polarization. Strong localized emission due to surface inhomogeneities or deposited particles are evident across the sample surface, with varying intensities. These localized features are the signatures of diffraction and near-field radiative modes. Wave-like patterns surround the structure in Fig. 9.6b-c. The patterns exhibit different spacings, decay lengths, and intensity variations in different regions and for different polarizations. With closer inspection, all regions appear to have an offset maximum and beating phenomena. Three different wave fields can be identified; these have been labeled A, B, and C in Fig. 9.6. Region $\mathrm{A}$ is in the forward direction of the incident laser beam, i.e., above the slit and outside the trench area. Region B is the trench center region, and region $\mathrm{C}$ is in the reverse direction incident to the beam, i.e., below the slit. The average intensity profiles across each region and polarization are shown in Fig. 9.7.

These wave-like patterns are well explained as interference between the guided modes in the ITO layer and the incident light. The interference, illustrated in Fig. 8.1, is similar to those found in plasmonic metal structures, as reported, for example, in $[15,33,38]$. There are, however, significant differences between the plasmonic and the photonic cases: Due to the lower electron densities in the ITO material and the much lower optical absorptivities in ITO as compared to typical metals, the electron emission rates are lower in the photonic case. The observed interference fringes extend over distances of some 10 micrometers, which we can explain with the following formalism. The incident and guided waves have propagation vectors $\mathbf{k}$ and $\mathbf{k}_{j}$ with in-plane components $k \sin \theta$ and $\pm k N_{j}$, respectively, where $k=2 \pi / 410 \mathrm{~nm}, \omega=c k$, $\theta=60^{\circ}$, and $\pm N_{j}$ is the effective refractive index of guided wave $j=1,2$ traveling in 

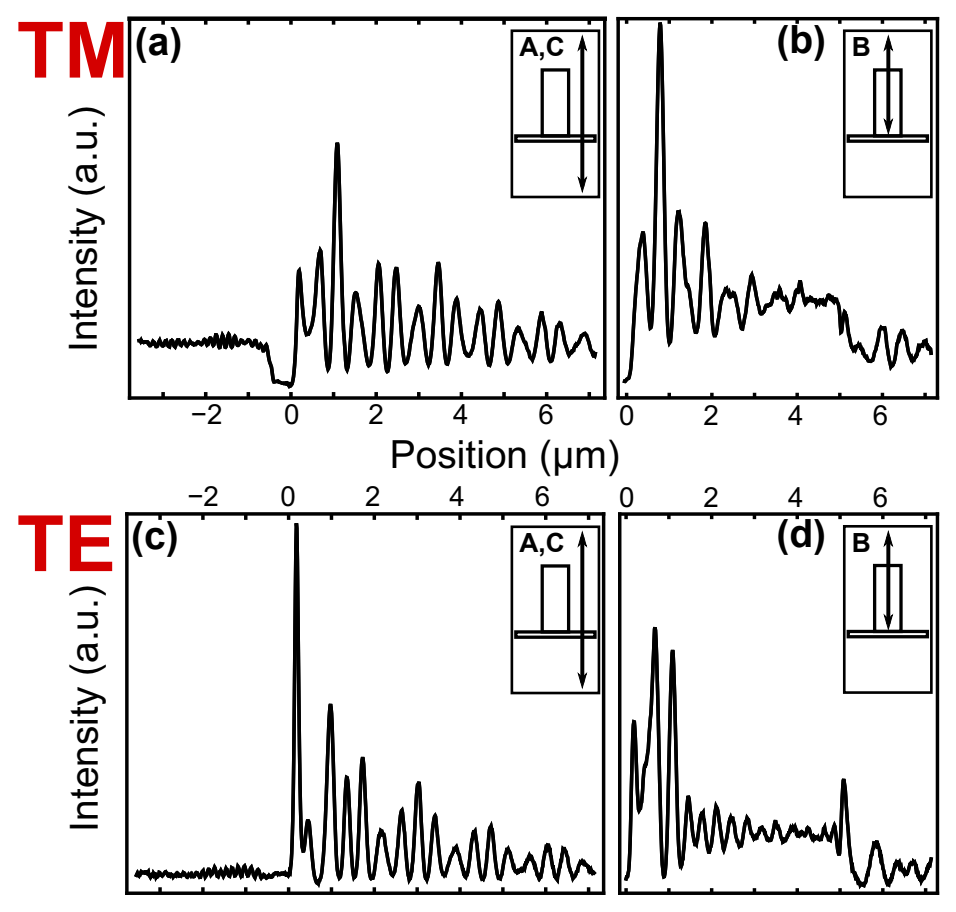

Figure 9.7: Two-photon PEEM interference pattern profiles, averaged from the regions marked in insets. (a-b) TM-polarized, (c-d) TE-polarized. Reprinted from Fitzgerald, Word, Saliba, and Könenkamp [173]. 

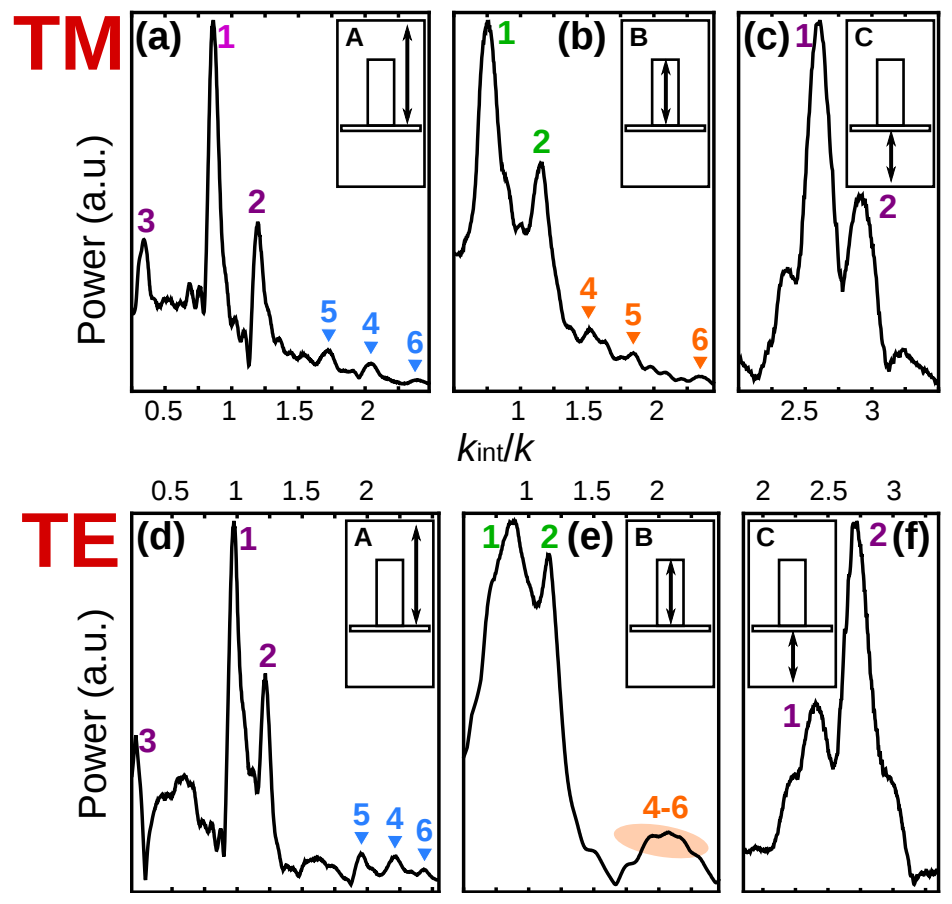

Figure 9.8: Fourier transform spectra of Fig. 9.7, where $k=2 \pi / 410 \mathrm{~nm}$. Six interference peaks are highlighted in each spectrum, with signals 4-6 unique to multiphoton interference. The six spectra are (a-c) TM-polarized, (d-f) TE-polarized; from the regions marked in insets. Reprinted from Fitzgerald, Word, Saliba, and Könenkamp [173].

the forward $(+)$ or reverse $(-)$ direction. Interference maxima are separated by distances $d_{j=1,2}=2 \pi / k_{I}^{(0, j)}=2 \pi / k\left(N_{j} \mp \sin \theta\right)$ and $d_{3}=2 \pi / k\left(N_{2}-N_{1}\right)$, where $N_{2}>N_{1}$, just as discussed the previous chapter. Furthermore, we may extract additional information by applying a Fourier transform (FT) to the interference pattern, which allows comparison of the image to the theory relating photoemission to the superposition of electric fields at the surface. The one-dimensional FT of the patterns in the three regions are shown in Fig. 9.8. There are a total of at most six different wave numbers $k_{I}$ in the interference patterns, which are apparent as peaks in the Fourier transform, and summarized in the previous chapter (Table 8.1). These six signals are labeled in the experimental fast Fourier transforms (FFTs), Fig. 9.8. With two $N_{j}$ for each surface region (A and B) and polarization (TM, TE), a total of eight modes in 


\begin{tabular}{c|c|cc|cc} 
Signal & $k / k_{0}$ & Theory, TM & Exp., TM & Theory, TE & Exp., TE \\
\hline 1 & $N_{1}-\sin \theta$ & 0.86 & 0.86 & 0.99 & 0.98 \\
2 & $N_{2}-\sin \theta$ & 1.17 & 1.19 & 1.20 & 1.22 \\
3 & $N_{2}-N_{1}$ & 0.30 & 0.34 & 0.22 & 0.22 \\
$4^{*}$ & $N_{1}+N_{2}$ & 2.03 & 2.05 & 2.19 & 2.22 \\
$5^{*}$ & $2\left(N_{1}-\sin \theta\right)$ & 1.73 & 1.72 & 1.97 & 1.95 \\
$6^{*}$ & $2\left(N_{2}-\sin \theta\right)$ & 2.34 & 2.39 & 2.40 & 2.44
\end{tabular}

Table 9.1: Effective indices calculated using asymmetric dielectric slab waveguide model: $N_{1}=1.73(\mathrm{TM}), 1.85(\mathrm{TE}), N_{2}=2.03(\mathrm{TM}), 2.07(\mathrm{TE})$. Signals $4-6$ are unique to multiphoton photoemission. These are compared to indices measured from the PE interference pattern.

the forward direction is obtained. There are also four modes in the reverse direction (region $\mathrm{C}$ ). These modes in the reverse direction have different interference spacing but the same effective indices as in region $\mathrm{A}$, as indicated in Table 8.1, providing an additional consistency check.

The Fourier spectra in Fig. 9.8 exhibit broadened resonances due to the short decay length of the surface waves. In the Fourier transforms, attenuation produces a Lorentzian peak shape whose half width at half maximum (HWHM) is the absorption coefficient, $\alpha$. In both TM and TE region A modes, HWHM $\approx 0.05 k=7700 \mathrm{~cm}^{-1}$. These values are in very good agreement with transmission measurement on ITO films $[180,185]$. This is a confirmation that the observed waves propagate through the ITO layer. The asymmetric slab waveguide model predicts two guided modes for both TM and TE polarizations, and also provides theoretical predictions for the effective indices, which can now be compared with the experimental results. Such a comparison is presented in Fig. 9.9 and Table 9.1. Table 9.1 demonstrates that the theoretical effective indices are consistent with those obtained experimentally. The predictions also nicely show the observed index difference for the two thicknesses available in the structure. The ratio of two mode coefficients, $B_{1} / B_{2}$, can be measured from the Fourier transform, Fig. 9.8, using the coefficients given in Table 8.1. The ratio can 


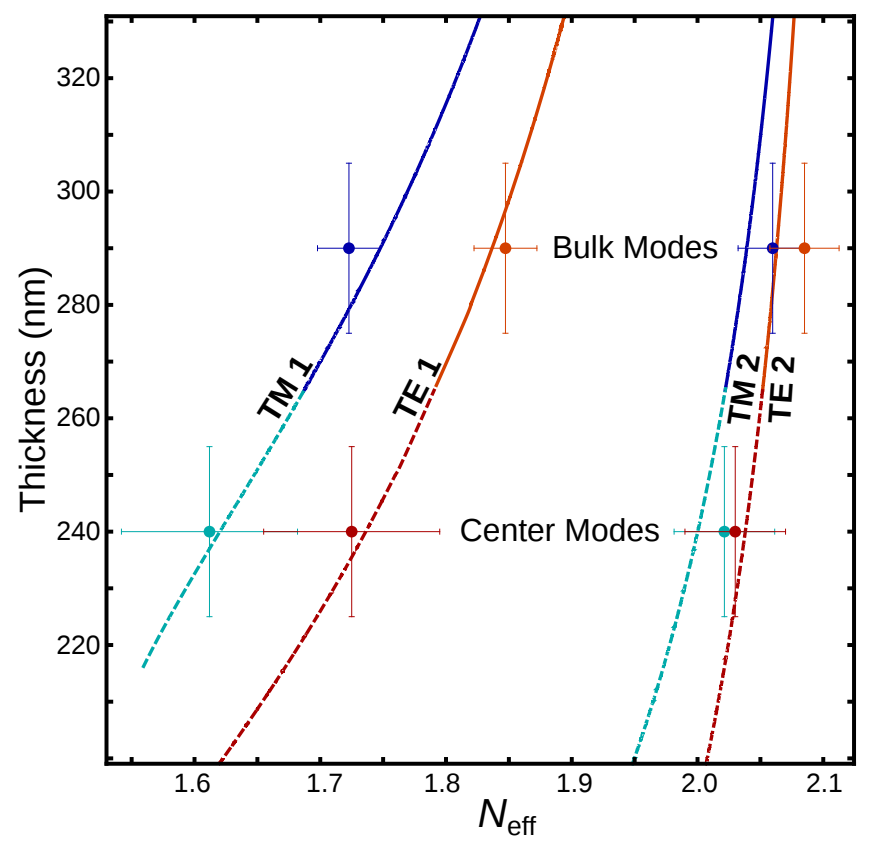

Figure 9.9: Allowed modes of the vacuum/ITO/glass waveguide model for ITO films of 200 to $320 \mathrm{~nm}$. Experimental results determined from FFT indicated with error bars. Reprinted from Fitzgerald, Word, Saliba, and Könenkamp [173].

also be calculated from the effective indices, independently measured from the Fourier transform. These two methods are found to be in good agreement. In addition, diffraction can explain why the primary propagating wave interference maxima are not coincident with the slit edge. Following an argument similar to [? ], light diffracted into the ITO layer at angle $\beta_{j}$ is reflected at the ITO-glass boundary. That ray returns to the ITO-vacuum surface a distance $\Delta y_{j}=2 t\left[1-\left(N_{j} / n_{\mathrm{ITO}}\right)^{2}\right]^{-1 / 2}$ from the slit, eliminating the angle $\beta_{j}=\sin ^{-1} N_{j} / n_{\text {ITO }}$ with Eq. (8.8). $\Delta y_{j}$ is the distance between the slit edge and the primary maximum. Using the effective indices measured from the Fourier spectrum, the computed distances are $0.6-1 \mu \mathrm{m}$ in the dominant waveguide modes, as given in Table (9.2), consistent with the observed phase offsets in regions $\mathrm{A}$ and $\mathrm{B}$ of Fig. 9.6b-c.

The center region modes have some obvious differences from the surrounding region modes. The most striking observation is that the spacing of the interference 


\begin{tabular}{c|c|cc|cc} 
& Expression & Thy., TM & Exp., TM & Thy., TE & Exp., TE \\
\hline$\Delta y_{1}$ & $2 t\left(n_{\mathrm{ITO}}^{2} / N_{1}^{2}-1\right)^{-1 / 2}$ & $0.77 \mu \mathrm{m}$ & $0.76 \mu \mathrm{m}$ & $1.03 \mu \mathrm{m}$ & $0.93 \mu \mathrm{m}$ \\
$\Delta y_{2}$ & $2 t\left(n_{\mathrm{ITO}}^{2} / N_{2}^{2}-1\right)^{-1 / 2}$ & $2.06 \mu \mathrm{m}$ & $2.06 \mu \mathrm{m}$ & $2.66 \mu \mathrm{m}$ & $2.69 \mu \mathrm{m}$
\end{tabular}

Table 9.2: Distances $\Delta y_{i}$ are the distance an equivalent ray would travel from trench edge to reflection at glass boundary and back to ITO surface.

pattern in Region B is smaller than in Region A, indicating a smaller effective refractive index. This is explained by the fact that the guided wave also probes the space outside the geometrical volume of the ITO layer in the trench. As Region B is thinner than Region A, the vacuum refractive index has a larger contribution to the effective index for the guided modes in Region B. As a consequence, a lower effective index, a longer wavelength, and a larger spacing in the interference pattern result. This is in complete agreement with the asymmetric slab waveguide model, as compared in Fig. 9.9. Another difference is the extremely rapid decay of the center modes, which have attenuation coefficients in the range $12-21 \times 10^{3} \mathrm{~cm}^{-1}, 2-3$ times larger than in Region A. This is most likely due to lateral confinement of the trench region. Guided modes are best confined if the refractive index of the confining layer is greater than the surrounding materials. Since the effective indices of Region B are less than in Regions A and C, the confinement in the center trench is "leaky" which results in a stronger damping of the propagating modes [194]. Region B has two additional interesting features which contribute to increased background brightness. At the far edge, reverse modes (counter propagating modes) are coupled in and interfere with the forward propagating waves. The result is that the region is generally brighter than the surrounding bulk surface but without a strong contrast in the interference pattern, as seen in the profiles Fig. 9.7. Secondly, the surface of the trench was FIB milled, unlike Regions A and C. FIB milling modifies surfaces, changing surface roughness and disrupting the surface homogeneity due to gallium impact [201]. The difference 
in surface roughness between the center and bulk surface regions is also visible in the 1PPE image, Fig. 9.6a. These changes are generally limited to a shallow surface depth, so FIB milling should have negligible effect on wave-guiding within the ITO layer.

It is worth briefly discussing other features of the two-photon photoemission images. The top edges of the slit and center region are significantly narrower than the interference pattern maxima, indicating tightly confined electromagnetic fields. Diffraction fringe fields extend around hot spot sites in the multiphoton PEEM micrographs and are significantly weaker under TE polarization. The occurrence of these fringes has been discussed in some detail in Chelaru et al. [13]. The width of the fringe maxima varies with particle size and is projected further in the forward direction than in the reverse. These features clearly distinguish the waveguide modes from diffraction phenomena. The fringe fields can be explained using Fraunhofer (far field) diffraction around an opaque aperture-like object, which predicts a minima immediately surrounding the particles and gives a first maximum spacing $\Delta y \approx 3.83 \lambda A / \pi \sin 30^{\circ}$ in the forward direction, where $A$ is the aspect ratio of height to diameter [13]. For $A \leq 1$, this formula predicts a variable spacing $\Delta y \lesssim 1 \mu \mathrm{m}$, consistent with the forward fringes observed in Fig. 9.6.

It is of interest to determine the potential accuracy of PEEM in this optical application, particularly for the experimentally observed optical parameters such as the refractive index, the absorption coefficient and relative phase shifts. The Fourier transform evaluation provides interference pattern spacings with an accuracy of $3 \%$, the effective indices of the guided modes with $5 \%$, and the absorption coefficient with $9 \%$. If the laser wavelength, the angle of incidence, and the film thickness could be determined with significantly better accuracy, then the optical parameters of the waveguide might be determined with high precision in areas as small as a few 


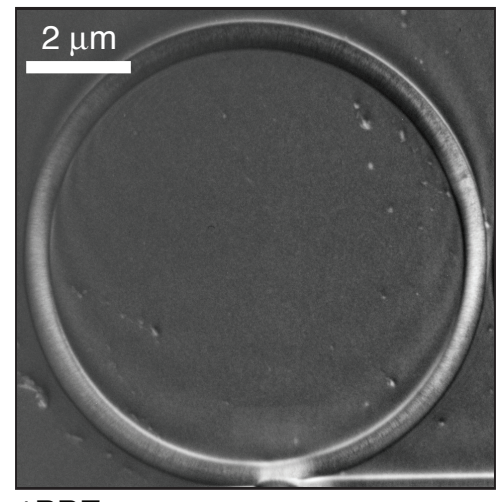

1PPE

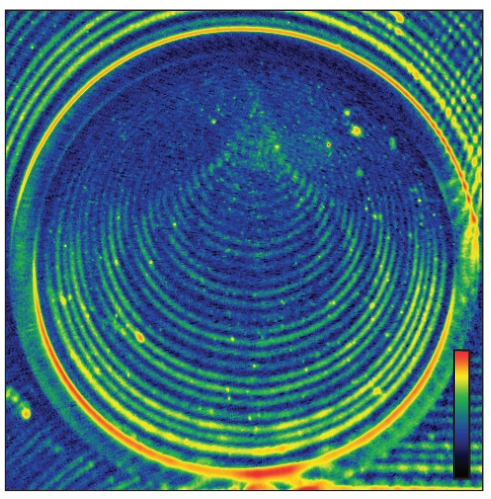

2PPE (TE)

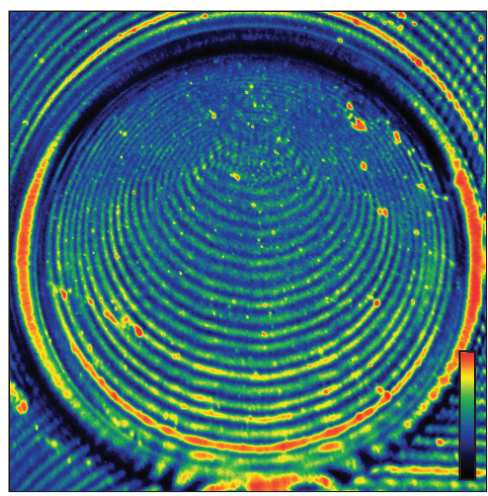

2PPE (TM)

Figure 9.10: PEEM micrographs of a waveguide bounded by a circular groove. Light is incident from the bottom edge of the images. The 1PPE image shows the milled groove down to the glass substrate as a lighter shade. The 2PPE images (in false color) show modulations in the surface electromagnetic field due to interference between incident and guided light. Reprinted from Fitzgerald, Word, and Könenkamp [175].

microns. The determination of relative phase shifts can be obtained from a direct image analysis of Fig. 9.7 once the periods are determined from the Fourier analysis. The slit edge can be taken as the reference position for this comparison. A basic analysis using fitted waveforms indicates that the two images provide an accuracy of $24 \mathrm{~nm}$ for the relative phase shifts of the two polarization modes depicted. This corresponds to corresponds to $\lambda / 17$. These results for local averages of the optical constants and the dynamic phase shifts in diffractive in-coupling nicely demonstrate the potential for PEEM imaging in planar optics applications.

Bolstered by the success of Eq. (8.2) in one-dimensional photonic waves, I next consider two-dimensional patterns, calculating the electromagnetic field from Eq. (8.11). Figures 9.10 and 9.11 show PEEM micrographs of the semicircular hole assembly and groove encircling a disc-shaped waveguide region, respectively. As before, the 1PPE images show topographic features, similar to an SEM image. Modulations in electron emission due to surface light interference are less pronounced. On the other hand, the 2PPE images show much stronger contrast resulting from the superposition 


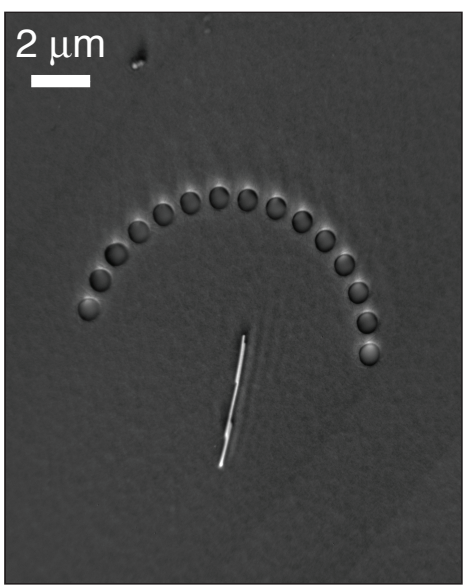

1PPE

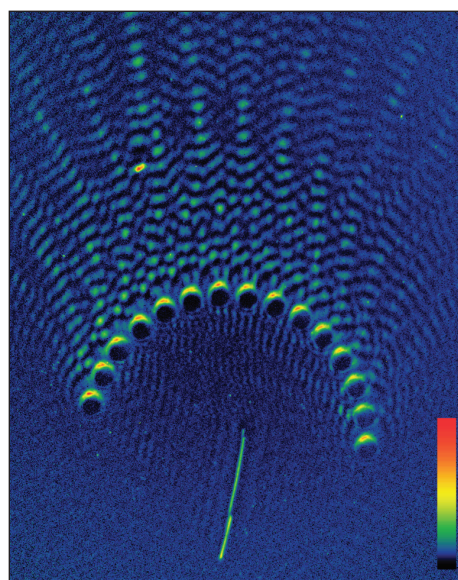

2PPE (TE)

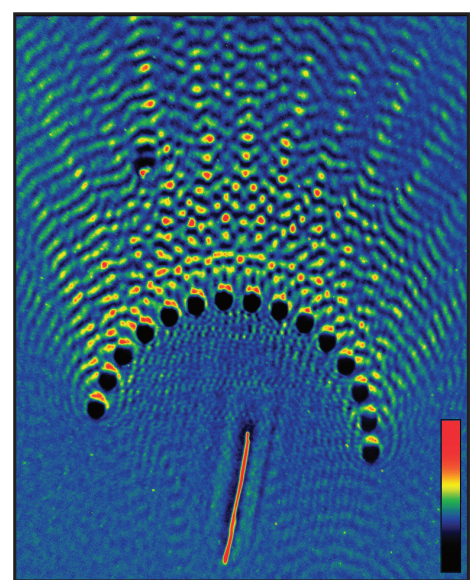

2PPE (TM)

Figure 9.11: PEEM micrographs of a semicircle of holes. Light is incident from the bottom edge of the images. The 1PPE image shows topographic features, similar to an SEM image, as well as some diffraction. The 2PPE images (in false color) show modulations in the surface electromagnetic field due to interference between incident and guided light. Reprinted from Fitzgerald, Word, and Könenkamp [175].

of guided fields from curved sources and the incident light. Guided waves refracted into the disc structure in Fig. 9.10 converge at multiple foci, with the dominant focus attributable to the forward direction and a weaker, secondary focus from the reverse direction found just below the primary focus. Bright line patterns diverge from the structure in Fig. 9.11 as a result of constructive interference between two or more holes. The presence of multiple waveguide modes leads to the fractured appearance of beating in Fig. 9.10 and the line patterns in Fig. 9.11.

Analysis of Figs. 9.10 and 9.11 yields the guided mode effective indices and relative mode intensities, which can be quantitatively compared to theoretical calculations to complement qualitative comparison of the experimental and theoretical images. The fields of a single, representative hole are shown in Fig. 9.12, which is reconstructed from an average of the fields surrounding a selection of holes in the assembly. Figure 9.13 shows line profiles bisecting the hole in the direction of incident light. The effective indices and relative strengths of the two guided modes can be more readily 


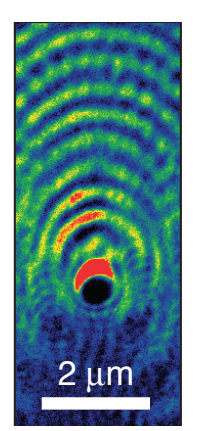

TE (exp.)

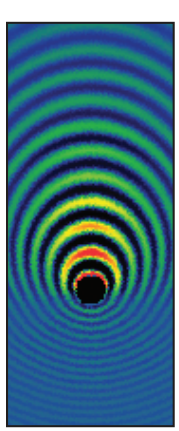

TE (sim.)

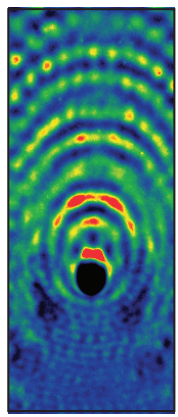

TM (exp.) TM (sim.)

Figure 9.12: Experimental and simulated 2PPE interference patterns generated by a single hole. The experimentally derived patterns are composite sums of the $2 \mathrm{PPE}$ near each hole in Figure (9.11). Reprinted from Fitzgerald, Word, and Könenkamp [175].
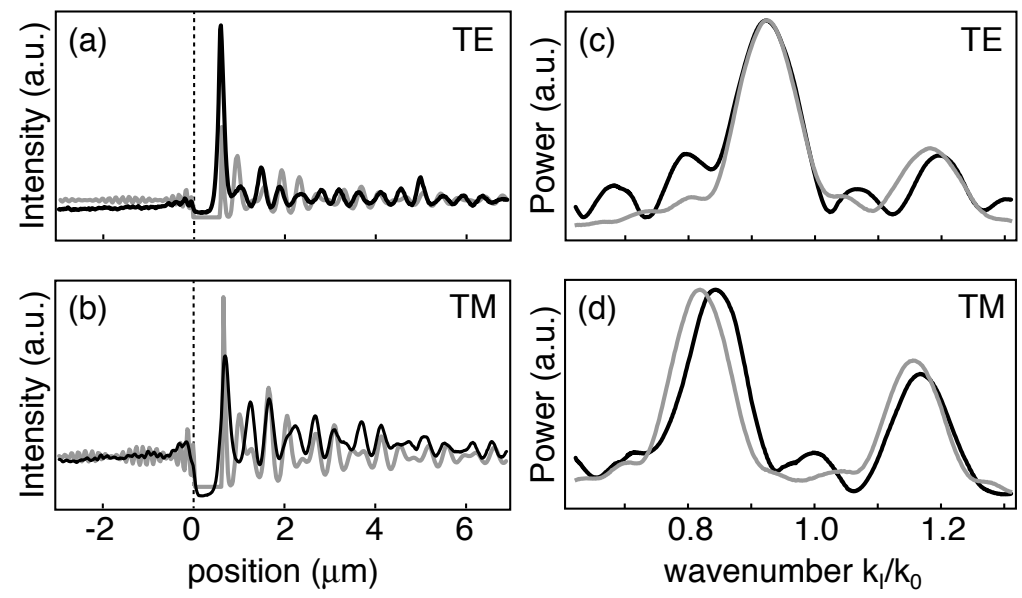

Figure 9.13: 2PPE pattern from a single 0.5- $\mu \mathrm{m}$ hole. (a,b) Line profiles taken in the direction of incident light, with simulated line profile shown in light gray. $(\mathrm{c}, \mathrm{d})$ Fourier transform power spectra of the line profiles plotted vs. interference wavenumbers normalized by vacuum wavenumber. Simulated spectra are shown in light gray, experimental data in heavy black. Reprinted from Fitzgerald, Word, and Könenkamp [175]. 
determined from the Fourier transform power spectra of the line profiles, also shown in Fig. 9.13. Adding the in-plane component of the incident wavenumber to the interference wavenumber gives the guided mode wavenumber, or, in terms of the effective index, $N=k_{I} /(k+\sin \theta)$. The measured values are $N_{1 \mathrm{TE}}=1.78, N_{2 \mathrm{TE}}=$ 2.05, $N_{1 \mathrm{TM}}=1.68$, and $N_{2 \mathrm{TM}}=2.02$. Using these effective indices, I calculate an ITO film thickness of $265 \mathrm{~nm}$, which is consistent with the $250 \pm 40 \mathrm{~nm}$ measured in SEM. Fig. 9.10 gives similar effective indices as the holes since the film is roughly the same thickness. The relative intensities can also be determined from the peak intensities of the Fourier transform power spectrum, giving 0.28 (TE) and 0.38 (TM) for the holes. These values conform to within $6 \%$ with a Fraunhofer diffraction model based on the Airy disc, Eq. (8.9). In the disc, $\left(B_{2} / B_{1}\right)_{\mathrm{TM}}=0.84$ and $\left(B_{2} / B_{1}\right)_{\mathrm{TE}}=0.41$, which agree with a slit diffraction model, Eq. (8.8), also to within $6 \%$.

Next, I compute the expected photoelectron yield intensity of our theory, beginning with the coupling coefficient, $B_{j}$. Fields diffracted through a hole could be approximately modeled with a real transmission coefficient that is unity for the top edge and 0.66 for TE and 1.0 for TM modes for the bottom edge of a hole. Because of the extended nature of the circular groove, it was necessary to use boundary values that vary with position around the circular groove edge [199]. The results for this calculation are shown in Fig. 9.14. Next, individual field distributions $E_{j}$ are calculated from a numerical integration of the Kirchhoff formula Eq. (8.11), with the calculated wave mode numbers in the Green's function wavelet. Subsequently the total surface fields are linear superpositions of the incident wave field and the two guided mode fields, as in Eq. (8.3), weighted according to their diffractive coefficients, given by Eqs. 8.8 and 8.9. Constructing the field distribution of multiple holes requires a further superposition of individual hole fields. The relative 2PPE intensities are then computed from the total surface fields using Eq. (8.2). Figure 9.12 shows the theo- 

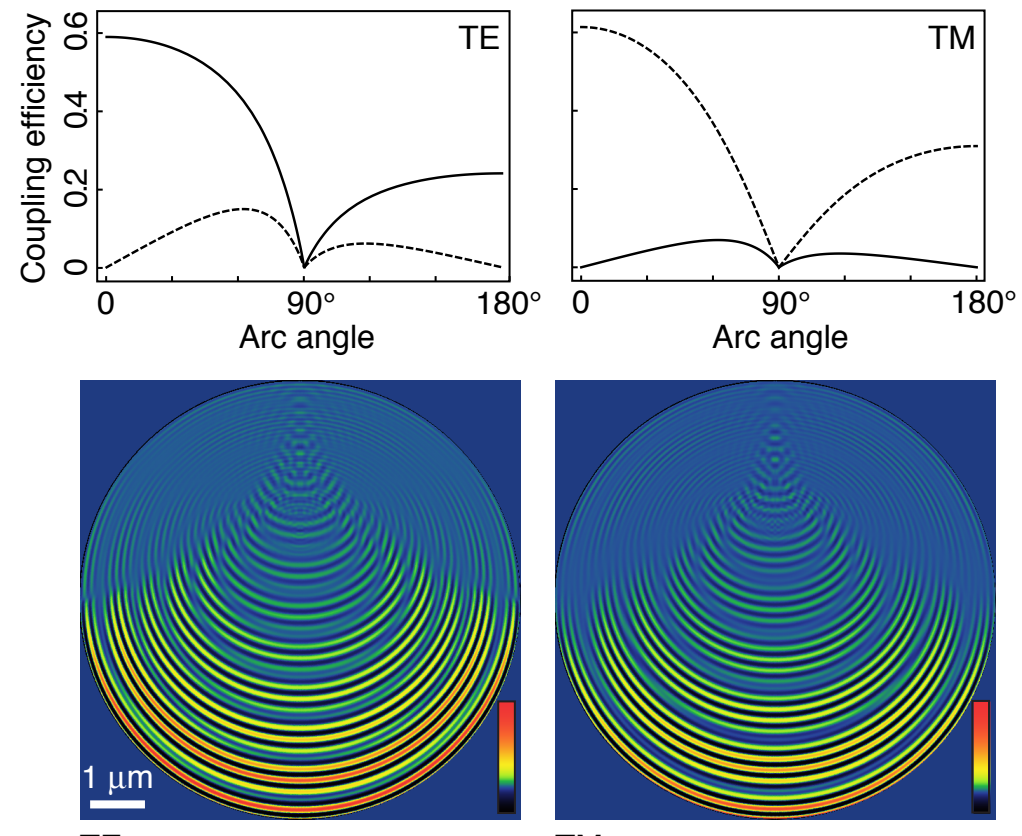

TE

TM

Figure 9.14: (Top) Calculated efficiency of TE and TM incident light for generating TE-like (solid line) and TM-like (dotted line) guided modes in the ITO, with $0^{\circ}$ position at the bottom and $180^{\circ}$ at the top of the disc. (Bottom) Simulated $2 \mathrm{PPE}$ pattern for the disc structure. Reprinted from Fitzgerald, Word, and Könenkamp [175]. 


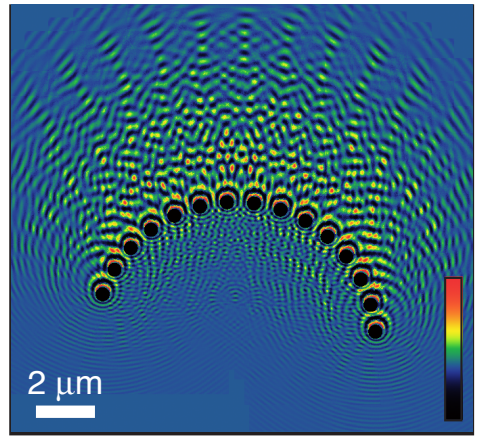

TE

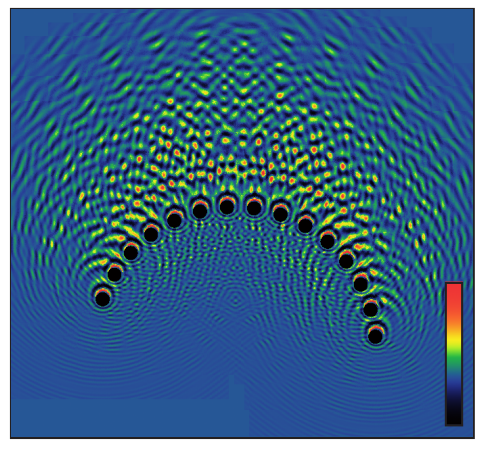

TM

Figure 9.15: Simulated 2PPE patterns constructed from a superposition of single hole simulations. Individual hole line profiles and spectra are compared to experiment in Fig. 9.13. Reprinted from Fitzgerald, Word, and Könenkamp [175].

retical photoelectron yield intensities of a single hole, with quantitative comparisons to experimental data given in Fig. 9.13. Theoretical PE yields of the more advanced structures of a disc and semicircle of holes are shown in Figs. 9.14 and 9.15.

Overall the calculations in Figs. 9.13, 9.14, and 9.15 show excellent agreement with the experimental 2PPE electron micrographs in Figs. 9.10 and 9.11. Single hole simulations reproduce the observed asymmetric interference patterns, with bright, widely-spaced interference maxima in the direction of the incident light wave vector, weak, finely-spaced maxima in the opposite direction, and a smooth gradient of maxima intensity and spacing along the sides. Both modeled and experimental images of the hole assembly have similar patterns of speckles, diverging lines of constructive interference maxima in the forward direction, and finer lines of interference maxima parallel to the incident wave vector in the reverse direction. In the disc structure, the model maxima follow the contour of the groove edge as observed. Additionally, the locations and intensities of foci as well as the beating between the two guided modes are accurately reproduced. Differences between model and observed 2PPE images highlight the depth of information in present 2P-PEEM electron micrographs. These are especially apparent in near-field zones, around groove and hole edges, and are 


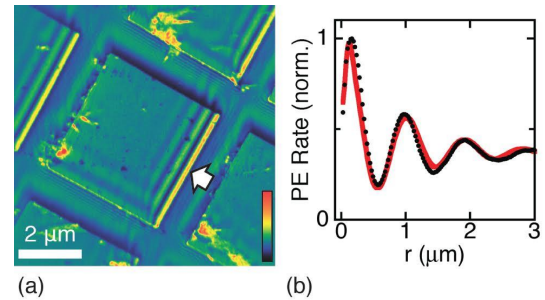

Figure 9.16: (a) Ultraviolet PEEM micrograph of diffraction at the edges of a nanostructured Si wafer. The arrow indicates the direction for the line scan. (b) Photoemission line profile in the direction of the arrow in (a). The experimental data are plotted as black dots, the calculated profile is plotted as a continuous line in red and is based on Eq. (8.5) with a propagation velocity $v=c$ and a decay length of 470 nm. Reprinted from Word, Fitzgerald, and Könenkamp [174].

likely the result of more complicated optical and geometric properties than in the idealized simulation.

\subsection{Diffracted waves}

Diffraction without guided modes and the interaction between plasmonic and photonic modes provide final examples of the close relationship between photoemission and optical near-fields. The results presented here were originally presented in Word, Fitzgerald, and Könenkamp [41, 174]. Replacing the transparent ITO film with a highly absorbing silicon wafer strongly suppresses wave propagation within the sample material, as the high optical absorption coefficient corresponds to an absorption length of only $3 \mathrm{~nm}$. Yet a diffraction-based interference pattern is still observed in this case as shown in Fig. 9.16. The analysis of the diffraction pattern shows that the diffracted wave propagates in an effective index $N=1$, indicating that the photoemission image is now due to interference between a diffracted wave propagating through vacuum and the incident light. This wave is apparently part of a wave scattered by a linear feature at the Si surface and propagating in the vacuum above the $\mathrm{Si}$ surface. The physical mechanism to generate the photoemission image in this case is 
the spatially varying electric field immediately above the samples surface. Apparently the spatially modulated field strength changes the photoemission rate. Figure 9.16 shows that similar to the waveguide case in ITO, the experimental data are good enough to establish phase shifts occurring within the near-field of the diffracting edge feature as well as losses along the propagation. Rotating the illumination vector and comparing two polarizations, Fig. 9.17a shows that the strength of the forward edge scattered wave varies significantly with polarization. By applying Eq. (8.11) to each electromagnetic field component, the Fresnel-Kirchhoff method of calculating the twodimensional field can more readily accommodate the differences in diffraction strength with polarization. The intensity and photoemission pattern can be computed from the norm of the vectorial field. Fitting the PE micrograph for the coupling coefficients and polarization yields Fig. 9.17b. The fitting indicates that the maximum diffracted wave coupling strength is $\sim 20 \%$, and the scattered wave couples more strongly with the forward perpendicular edge than parallel edges, by a factor of $\sim 2.5 \times$, or the reverse edge by $\sim 3 \times$. These factors are multiplicative to the coupling already built into the Kirchhoff diffraction formula.

A second example of optical diffraction is shown in Fig. 9.18. In this case, incident light diffracts at the edge of an unmodified, single-crystalline 60-nm-thick, gold platelet on an ITO substrate. Analysis of the interference pattern in the forward direction yields a wave traveling at the speed of light. Fig. 9.18c shows the photoemission distribution calculated by the same method as the silicon square. Near the triangle, a groove has been milled into the ITO layer, as seen in Fig. 7.8, and several nanoflakes are located in the vicinity. This cluster is of further interest because PEEM images in Fig. 9.19 capture the interaction between photonic modes, which are diffraction coupled into the ITO layer at the $10-\mu \mathrm{m}$ groove, and plasmonic excitations in the Au flakes. The presence of many types of optical phenomena makes description and 


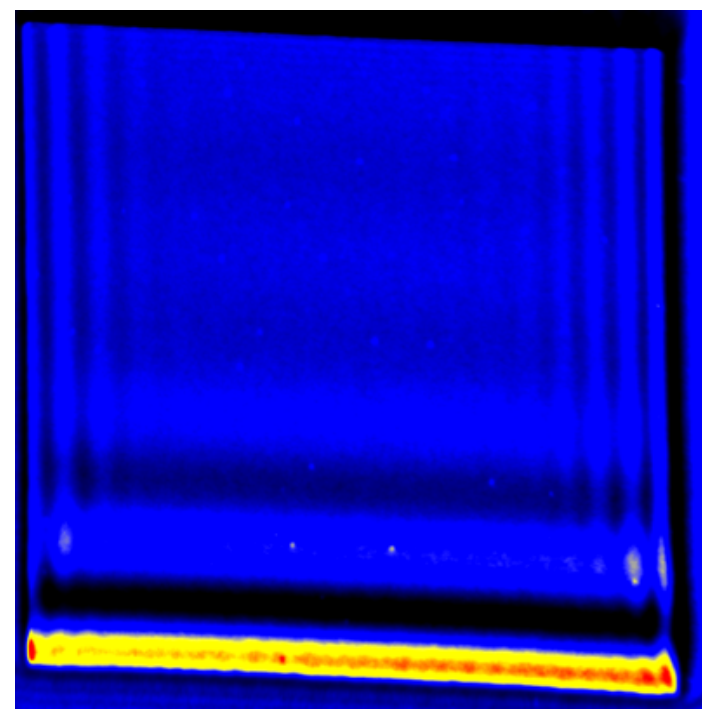

(a)

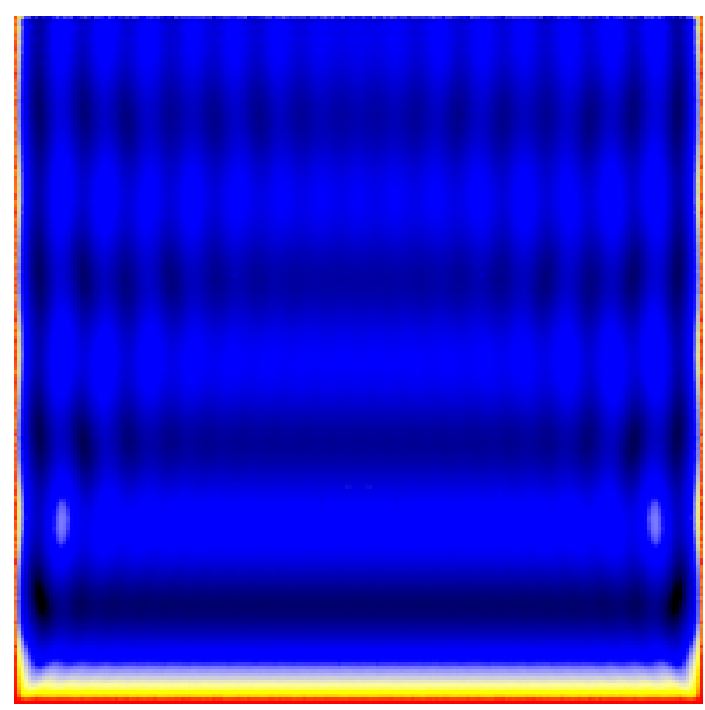

(c)

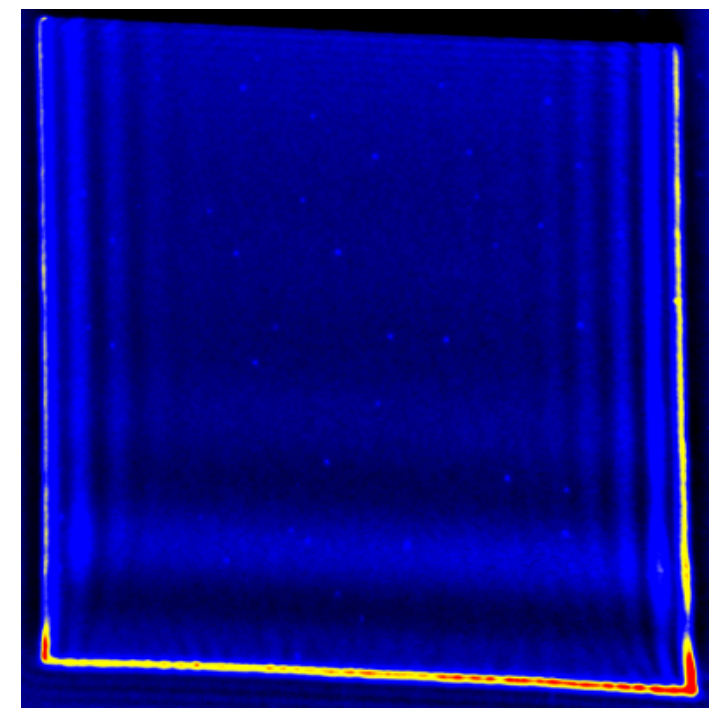

(b)

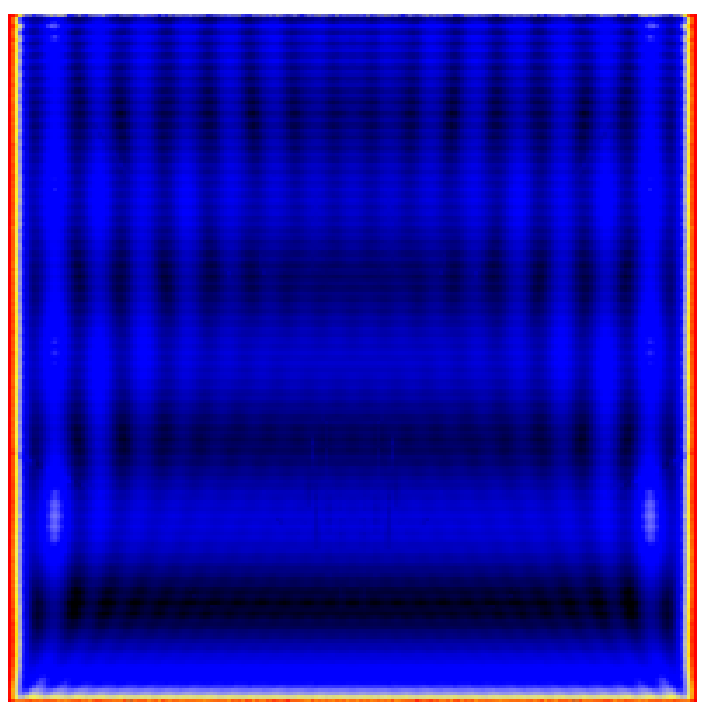

(d)

Figure 9.17: (a-b) PEEM micrographs of diffraction at the edges of a nanostructured Si wafer, rotated relative to Fig. 9.16. Ultraviolet light incident from the bottom edge is TM- (a) or TE- (b) polarized. (c-d) Photoemission patterns calculated using Eq. (8.11) and the optical properties of Si for a diffracted (radiative) wave for (a) TM and (b) TE incident light. 


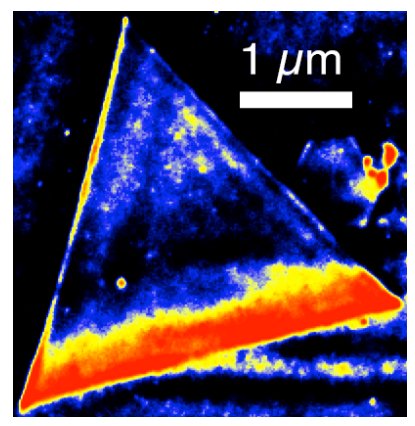

(a)

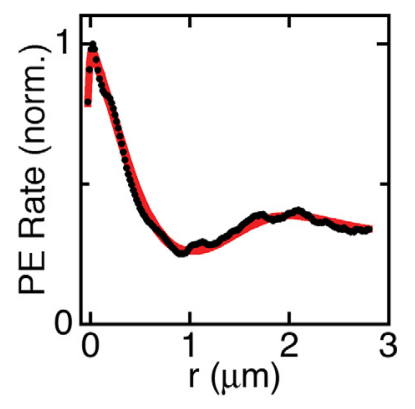

(b)

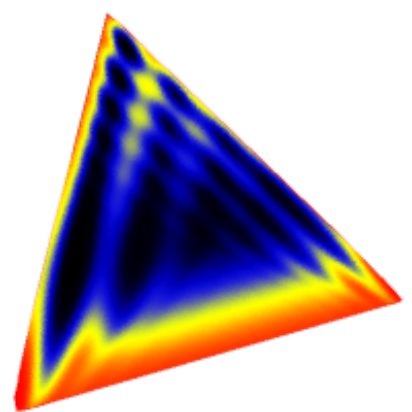

(c)

Figure 9.18: (a) 2P-PEEM micrograph of diffraction at the edges of a triangular Au platelet. Visible 410-nm, TM-polarized light is incident from the bottom. (b) Line profile of the photoemission rate taken perpendicular to the bottom edge. Black dots: experimental data, red line: fit based on optical data of Palik [202] with a decay length of $380 \mathrm{~nm}$. Reprinted from Word, Fitzgerald, and Könenkamp [174]. (c) Photoemission patterns calculated using Eq. (8.11) and the optical data of Au.

(a)

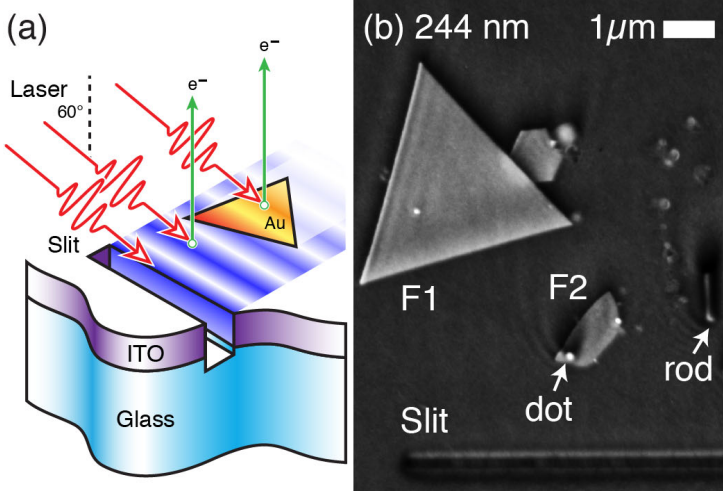

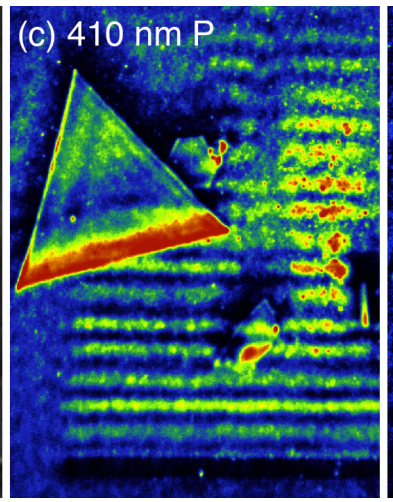

Figure 9.19: (a) Imaging photoelectrons are emitted as the incident laser interferes with guided photonic waves coupled into a slit or plasmonic waves bound to gold. (b) UV-PEEM of gold flakes on ITO. (c) False color 2P-PEEM taken with 410-nm TM-polarized light and (d) TE-polarized light. Reprinted from Word, Fitzgerald, and Könenkamp [41]. 
analysis complicated. In the UV, ITO is opaque and the micrograph shows the ITO substrate, FIB-milled groove in the ITO, and gold platelets and particles with mostly topographical detail plus some yield modulation due to diffraction. In 2P-PEEM the images are very different. The most obvious difference is a series of horizontal bands due to interference between the incident laser and the guided photonic modes.

Direct imaging of photonic and plasmonic waves is an important demonstration of the use of PEEM in the study of integrated photonic circuits. The experimental identification of a photonic vacuum mode above a solid surface indicates that PEEM can also be used to probe electric fields outside the electron emitting material. The examples discussed here indicate that a complete visualization of diffraction phenomena including plasmonic and photonic modes and their inter-conversion may be achievable. 


\section{Conclusions}

The work presented here demonstrates that photoemission microscopy is a sensitive, powerful, and versatile tool for exploring near-field optical phenomena. While aberration limits current resolution, in Part II demonstrate that spherical and chromatic aberration can be corrected with an electrostatic mirror. Part III shows evidence of vacuum modes, guided photonic modes, and plasmonic surface modes that can be directly visualized in conceptually simple interference experiments.

The hyperbolic mirror geometry presented here has the virtue of allowing analytic solutions in the calculation of electron trajectories and optical properties. The expressions derived for a single hyperbolic region can be coupled together to give an analytic description of a multi-electrode mirror. Such an analytic solution is a very powerful tool in understanding the behavior of a corrector as a tool. Analytic treatment also allows for efficient design optimization. I gave a description of a triode mirror, with two hyperbolic regions, and a simple einzel lens. This mirror-lens combination is the simplest arrangement that can provide a wide range of dynamic correction in which the spherical and chromatic coefficients of correction can be independently varied. All variations can be controlled with three electrode potentials. The analytic description of this corrector agrees well with results from a numerical model based on a simple version of a realistic device.

Implementation of a triode mirror corrector into our PEEM is underway. This process has required high precision characterization of the new corrector as well as of previously installed elements of the microscope. To better estimate the aberration of the microscope, I have presented an optical characterization of several photoemission specimens after imaging by the accelerating field, objective lens, and auxiliary lens. 
This characterization has already been implemented in the PEEM software, leading to a decoupling between specimen focusing and magnification of the objective-auxiliary lens zoom pair. Second, the new hyperbolic triode mirror was designed based on the proven diode hyperbolic mirror already installed in the PEEM, placing several physical constraints on the device. The spacing of the electrodes and the lens was optimized within this framework to nonetheless provide a large range of dynamic and independent aberration correction. A complete characterization of the full triode mirror configuration space demonstrates that this range should be more than enough to correct photoelectron image aberrations. With the characterization presented here, the objective and mirror branches of our PEEM can be controlled with high precision, promising aberration free photoelectron micrographs.

Part III shows evidence of photonic vacuum modes, guided photonic modes in transparent media, and plasmonic surface modes that can be directly visualized in conceptually simple interference experiments. The high spatial resolution of PEEM provided by aberration-corrected electron optics with a diode mirror allows for quantitative analysis of wave speed, propagation length, and relative intensities, differentiating between the various forms of surface electromagnetic phenomena and leading to a better understanding of the physics of nanophotonic structures. By extending the interferometric analysis, I have also analyzed the interactions between surface plasmons and guided photons. In addition, I also offered a method for calculating surface fields and relative photoemission rates of more complicated optical structures based on the wave diffraction theory of Kirchhoff. The calculated field intensity, either from this method or a finite-element simulation, can be simply and convincingly related to the observed photoemission patterns with a simple power-law relationship. These findings complement and significantly augment earlier work that has demonstrated the visualization of plasmon propagation in thin metallic films using a very similar 
approach [9, 24, 32, 33, 38, 44-46].

Thus, PEEM combines the ability to observe a wide range of electromagnetic phenomena with sub-wavelength sensitivity and excellent resolution. The high contrast inherent to multiphoton photoemission is particularly well suited to planar nanoscale optical devices where these parameters are of importance [203], as, for example, in high-confinement waveguides [204], high sensitivity biochemical sensing devices [205], quantum coherent devices [206], and ultrafast optical switches [207]. Such intimate knowledge opens near-field optics to quantitative experimental analysis and allows new approaches for the development of photonic and plasmonic metamaterials. 


\section{References \& Appendices}

\section{References}

[1] E. Brüche, Elektronenmikroskopische Abbildung mit lichtelektrischen Elektronen, Z. Physik 86 (7-8) (1933) 448-450.

[2] K. K. Nadakavukaren, G. F. Rempfer, O. H. Griffith, Photoelectron microscopy of cell surface topography, J. Microsc. 122 (Pt 3) (1981) 301-307.

[3] O. H. Griffith, G. F. Rempfer, Photoelectron imaging and photoelectron labeling, Ultramicroscopy 24 (2-3) (1988) 299-312.

[4] Nolting, Scholl, Stohr, Seo, Fompeyrine, Siegwart, Locquet, Anders, Luning, Fullerton, Toney, Scheinfein, Padmore, Direct observation of the alignment of ferromagnetic spins by antiferromagnetic spins, Nature 405 (6788) (2000) 767769 .

[5] J. Vogel, W. Kuch, M. Bonfim, J. Camarero, Y. Pennec, F. Offi, K. Fukumoto, J. Kirschner, A. Fontaine, S. Pizzini, Time-resolved magnetic domain imaging by x-ray photoemission electron microscopy, Appl. Phys. Lett. 82 (14) (2003) 2299-2301.

[6] W. Kuch, L. I. Chelaru, F. Offi, J. Wang, M. Kotsugi, J. Kirschner, Tuning the magnetic coupling across ultrathin antiferromagnetic films by controlling atomic-scale roughness, Nat. Mater. 5 (2) (2006) 128-133.

[7] G. Schönhense, H. Elmers, S. Nepijko, C. Schneider, Time-Resolved Photoemission Electron Microscopy, in: P. Hawkes (Ed.), Advances in Imaging and Electron Physics, vol. Volume 142, Elsevier, 159-323, 2006.

[8] O. Schmidt, M. Bauer, C. Wiemann, R. Porath, M. Scharte, O. Andreyev, G. Schönhense, M. Aeschlimann, Time-resolved two photon photoemission electron microscopy, Appl. Phys. B 74 (3) (2002) 223-227.

[9] A. Kubo, K. Onda, H. Petek, Z. Sun, Y. S. Jung, H. K. Kim, Femtosecond imaging of surface plasmon dynamics in a nanostructured silver film, Nano Lett. 5 (6) (2005) 1123-1127.

[10] M. Cinchetti, G. Schönhense, Two-photon photoemission spectromicroscopy of noble metal clusters on surfaces studied using time-of-flight photoemission electron microscopy, J. Phys. Condens. Matter 17 (16) (2005) S1319. 
[11] M. Cinchetti, A. Gloskovskii, S. A. Nepjiko, G. Schönhense, H. Rochholz, M. Kreiter, Photoemission electron microscopy as a tool for the investigation of optical near fields, Phys. Rev. Lett. 95 (4) (2005) 047601.

[12] M. Munzinger, C. Wiemann, M. Rohmer, L. Guo, M. Aeschlimann, M. Bauer, The lateral photoemission distribution from a defined cluster/substrate system as probed by photoemission electron microscopy, New J. Phys. 7 (1) (2005) 68.

[13] L. I. Chelaru, M. Horn-von Hoegen, D. Thien, F.-J. Meyer zu Heringdorf, Fringe fields in nonlinear photoemission microscopy, Phys. Rev. B Condens. Matter 73 (11) (2006) 115416.

[14] L. I. Chelaru, F.-J. Meyer zu Heringdorf, In situ monitoring of surface plasmons in single-crystalline Ag-nanowires, Surf. Sci. 601 (18) (2007) 4541-4545.

[15] A. Kubo, N. Pontius, H. Petek, Femtosecond microscopy of surface plasmon polariton wave packet evolution at the silver/vacuum interface, Nano Lett. 7 (2) (2007) 470-475.

[16] A. Kubo, Y. S. Jung, H. K. Kim, H. Petek, Femtosecond microscopy of localized and propagating surface plasmons in silver gratings, J. Phys. B At. Mol. Opt. Phys. 40 (11) (2007) S259.

[17] M. I. Stockman, M. F. Kling, U. Kleineberg, F. Krausz, Attosecond nanoplasmonic-field microscope, Nat. Photonics 1 (9) (2007) 539-544.

[18] L. Douillard, F. Charra, C. Fiorini, P. M. Adam, R. Bachelot, S. Kostcheev, G. Lerondel, M. L. de la Chapelle, P. Royer, Optical properties of metal nanoparticles as probed by photoemission electron microscopy, J. Appl. Phys. 101 (8) (2007) 083518.

[19] M. Aeschlimann, M. Bauer, D. Bayer, T. Brixner, F. J. García de Abajo, W. Pfeiffer, M. Rohmer, C. Spindler, F. Steeb, Adaptive subwavelength control of nano-optical fields, Nature 446 (7133) (2007) 301-304.

[20] M. Bauer, C. Wiemann, J. Lange, D. Bayer, M. Rohmer, M. Aeschlimann, Phase propagation of localized surface plasmons probed by time-resolved photoemission electron microscopy, Appl. Phys. A: Mater. Sci. Process. 88 (3) (2007) 473-480.

[21] A. Gloskovskii, D. Valdaitsev, S. Nepijko, G. Schönhense, B. Rethfeld, Coexisting electron emission mechanisms in small metal particles observed in fs-laser excited PEEM, Surf. Sci. 601 (20) (2007) 4706-4713.

[22] F.-J. Meyer zu Heringdorf, L. Chelaru, S. Möllenbeck, D. Thien, M. Hornvon Hoegen, Femtosecond photoemission microscopy, Surf. Sci. 601 (20) (2007) 4700-4705. 
[23] C. Wiemann, D. Bayer, M. Rohmer, M. Aeschlimann, M. Bauer, Local 2PPEyield enhancement in a defined periodic silver nanodisk array, Surf. Sci. 601 (20) (2007) 4714-4721.

[24] L. Douillard, F. Charra, Z. Korczak, R. Bachelot, S. Kostcheev, G. Lerondel, P.M. Adam, P. Royer, Short range plasmon resonators probed by photoemission electron microscopy, Nano Lett. 8 (3) (2008) 935-940.

[25] A. Gloskovskii, D. A. Valdaitsev, M. Cinchetti, S. A. Nepijko, J. Lange, M. Aeschlimann, M. Bauer, M. Klimenkov, L. V. Viduta, P. M. Tomchuk, G. Schönhense, Electron emission from films of Ag and $\mathrm{Au}$ nanoparticles excited by a femtosecond pump-probe laser, Phys. Rev. B Condens. Matter 77 (19) (2008) 195427.

[26] B. Barwick, D. J. Flannigan, A. H. Zewail, Photon-induced near-field electron microscopy, Nature 462 (7275) (2009) 902-906.

[27] M. Berndt, M. Rohmer, B. Ashall, C. Schneider, M. Aeschlimann, D. Zerulla, Polarization selective near-field focusing on mesoscopic surface patterns with threefold symmetry measured with PEEM, Opt. Lett. 34 (7) (2009) 959-961.

[28] R. C. Word, T. Dornan, R. Könenkamp, Photoemission from localized surface plasmons in fractal metal nanostructures, Appl. Phys. Lett. 96 (25) (2010) 251110 .

[29] M. Aeschlimann, M. Bauer, D. Bayer, T. Brixner, S. Cunovic, F. Dimler, A. Fischer, W. Pfeiffer, M. Rohmer, C. Schneider, F. Steeb, C. Strüber, D. V. Voronine, Spatiotemporal Near-Field Control in Nanostructures, in: Imaging and Applied Optics Congress, Optical Society of America, MTuC1, 2010.

[30] R. C. Word, J. Fitzgerald, R. Könenkamp, Photoelectron emission control with polarized light in plasmonic metal random structures, Appl. Phys. Lett. 99 (4) (2011) 041106.

[31] M. Aeschlimann, T. Brixner, A. Fischer, C. Kramer, P. Melchior, W. Pfeiffer, C. Schneider, C. Strüber, P. Tuchscherer, D. V. Voronine, Coherent twodimensional nanoscopy, Science 333 (6050) (2011) 1723-1726.

[32] P. Melchior, D. Bayer, C. Schneider, A. Fischer, M. Rohmer, W. Pfeiffer, M. Aeschlimann, Optical near-field interference in the excitation of a bowtie nanoantenna, Phys. Rev. B Condens. Matter 83 (23) (2011) 235407.

[33] L. Zhang, A. Kubo, L. Wang, H. Petek, T. Seideman, Imaging of surface plasmon polariton fields excited at a nanometer-scale slit, Phys. Rev. B Condens. Matter 84 (24) (2011) 245442. 
[34] L. Douillard, F. Charra, High-resolution mapping of plasmonic modes: photoemission and scanning tunnelling luminescence microscopies, J. Phys. D Appl. Phys. 44 (46) (2011) 464002.

[35] C. Hrelescu, T. K. Sau, A. L. Rogach, F. Jäckel, G. Laurent, L. Douillard, F. Charra, Selective excitation of individual plasmonic hotspots at the tips of single gold nanostars, Nano Lett. 11 (2) (2011) 402-407.

[36] S. J. Peppernick, A. G. Joly, K. M. Beck, W. P. Hess, Plasmonic field enhancement of individual nanoparticles by correlated scanning and photoemission electron microscopy, J. Chem. Phys. 134 (3) (2011) 034507.

[37] R. Könenkamp, R. C. Word, J. P. S. Fitzgerald, A. Nadarajah, S. D. Saliba, Controlled spatial switching and routing of surface plasmons in designed singlecrystalline gold nanostructures, Appl. Phys. Lett. 101 (14) (2012) 141114.

[38] C. Lemke, T. Leißner, S. Jauernik, A. Klick, J. Fiutowski, J. Kjelstrup-Hansen, H.-G. Rubahn, M. Bauer, Mapping surface plasmon polariton propagation via counter-propagating light pulses, Opt. Express 20 (12) (2012) 12877-12884.

[39] C. Awada, T. Popescu, L. Douillard, F. Charra, A. Perron, H. Yockell-Lelièvre, A.-L. Baudrion, P.-M. Adam, R. Bachelot, Selective Excitation of Plasmon Resonances of Single Au Triangles by Polarization-Dependent Light Excitation, J. Phys. Chem. C 116 (27) (2012) 14591-14598.

[40] C. Awada, G. Barbillon, F. Charra, L. Douillard, J.-J. Greffet, Experimental study of hot spots in gold/glass nanocomposite films by photoemission electron microscopy, Phys. Rev. B Condens. Matter 85 (4) (2012) 045438.

[41] R. C. Word, J. P. S. Fitzgerald, R. Könenkamp, Direct coupling of photonic modes and surface plasmon polaritons observed in 2-photon PEEM, Opt. Express 21 (25) (2013) 30507-30520.

[42] R. C. Word, J. Fitzgerald, R. Könenkamp, Electron emission in the near-field of surface plasmons, Surf. Sci. 607 (0) (2013) 148-152.

[43] Q. Sun, K. Ueno, H. Yu, A. Kubo, Y. Matsuo, H. Misawa, Direct imaging of the near field and dynamics of surface plasmon resonance on gold nanostructures using photoemission electron microscopy, Light: Science \& Applications 2 (12) (2013) e118.

[44] C. Lemke, T. Leißner, A. Klick, J. W. Radke, J. Fiutowski, J. Kjelstrup-Hansen, H.-G. Rubahn, M. Bauer, Measurement of surface plasmon autocorrelation functions, Opt. Express 21 (22) (2013) 27392-27401. 
[45] C. Lemke, T. Leißner, A. Klick, J. Fiutowski, J. W. Radke, M. Thomaschewski, J. Kjelstrup-Hansen, H.-G. Rubahn, M. Bauer, The complex dispersion relation of surface plasmon polaritons at gold/para-hexaphenylene interfaces, Appl. Phys. B (2013) 1-7.

[46] C. Lemke, C. Schneider, T. Leißner, D. Bayer, J. W. Radke, A. Fischer, P. Melchior, A. B. Evlyukhin, B. N. Chichkov, C. Reinhardt, M. Bauer, M. Aeschlimann, Spatiotemporal characterization of SPP pulse propagation in two-dimensional plasmonic focusing devices, Nano Lett. 13 (3) (2013) 10531058 .

[47] N. M. Buckanie, P. Kirschbaum, S. Sindermann, F.-J. Meyer zu Heringdorf, Interaction of light and surface plasmon polaritons in $\mathrm{Ag}$ islands studied by nonlinear photoemission microscopy, Ultramicroscopy 130 (2013) 49-53.

[48] L. Zhang, A. Kubo, L. Wang, H. Petek, T. Seideman, Universal Aspects of Ultrafast Optical Pulse Scattering by a Nanoscale Asperity, J. Phys. Chem. C 117 (36) (2013) 18648-18652.

[49] C. N. Berglund, W. E. Spicer, Photoemission Studies of Copper and Silver: Theory, Phys. Rev. 136 (4A) (1964) A1030-A1044.

[50] J. H. Bechtel, W. Lee Smith, N. Bloembergen, Two-photon photoemission from metals induced by picosecond laser pulses, Phys. Rev. B Condens. Matter 15 (10) (1977) 4557-4563.

[51] T. Srinivasan-Rao, J. Fischer, T. Tsang, Photoemission studies on metals using picosecond ultraviolet laser pulses, J. Appl. Phys. 69 (5) (1991) 3291-3296.

[52] G. K. L. Marx, P.-O. Jubert, A. Bischof, R. Allenspach, Probing depth of threshold photoemission electron microscopy, Appl. Phys. Lett. 83 (14) (2003) 2925-2927.

[53] H. C. Siegmann, Surface and 2D magnetism, J. Phys. Condens. Matter 4 (44) (1992) 8395.

[54] E. Bauer, Low energy electron microscopy, Rep. Prog. Phys. 57 (9) (1994) 895.

[55] J. Stohr, S. Anders, X-ray spectro-microscopy of complex materials and surfaces, IBM J. Res. Dev. 44 (4) (2000) 535-551.

[56] M. Bauer, M. Aeschlimann, Dynamics of excited electrons in metals, thin films and nanostructures, J. Electron Spectrosc. Relat. Phenom. 124 (2-3) (2002) 225-243. 
[57] G. F. Rempfer, O. H. Griffith, The resolution of photoelectron microscopes with UV, X-ray, and synchrotron excitation sources, Ultramicroscopy 27 (3) (1989) 273-300.

[58] S. M. Schramm, S. J. van der Molen, R. M. Tromp, Intrinsic instability of aberration-corrected electron microscopes, Phys. Rev. Lett. 109 (16) (2012) 163901.

[59] M. Born, E. Wolf, A. Bhatia, P. Clemmow, D. Gabor, A. Stokes, A. Taylor, P. Wayman, W. Wilcock, Principles of Optics: Electromagnetic Theory of Propagation, Interference and Diffraction of Light, Cambridge University Press, 1999.

[60] G. F. Rempfer, Unipotential electrostatic lenses: Paraxial properties and aberrations of focal length and focal point, J. Appl. Phys. 57 (7) (1985) 2385-2401.

[61] S. M. Schramm, A. B. Pang, M. S. Altman, R. M. Tromp, A Contrast Transfer Function approach for image calculations in standard and aberration-corrected LEEM and PEEM, Ultramicroscopy 115 (2011) 88-108.

[62] P. E. Batson, N. Dellby, O. L. Krivanek, Sub-ångstrom resolution using aberration corrected electron optics, Nature 418 (6898) (2002) 617-620.

[63] M. Lentzen, B. Jahnen, C. L. Jia, A. Thust, K. Tillmann, K. Urban, Highresolution imaging with an aberration-corrected transmission electron microscope, Ultramicroscopy 92 (3-4) (2002) 233-242.

[64] G. F. Rempfer, A theoretical study of the hyperbolic electron mirror as a correcting element for spherical and chromatic aberration in electron optics, J. Appl. Phys. 67 (10) (1990) 6027-6040.

[65] O. H. Griffith, G. F. Rempfer, Photoelectron imaging in cell biology, Annu. Rev. Biophys. Biophys. Chem. 14 (1985) 113-130.

[66] F. Jenkins, H. White, Fundamentals of Optics, McGraw-Hill New York, 2001.

[67] E. Munro, Computational Techniques for Design of Charged Particle, in: J. Orloff (Ed.), Handbook of Charged Particle Optics, Taylor \& Francis, 1$76,1997$.

[68] P. W. Hawkes, Aberrations, in: J. Orloff (Ed.), Handbook of Charged Particle Optics, Taylor \& Francis, 223-274, 1997.

[69] P. Grivet, M. Bernard, P. Hawkes, A. Septier, Electron optics, ulb.tudarmstadt.de, 1972 .

[70] J. Orloff, Handbook of Charged Particle Optics, Taylor \& Francis, 1997. 
[71] P. W. Hawkes, E. Kasper, Principles of Electron optics, vol. 1, Academic Press, 1989.

[72] M. Sedlacek, Electron Physics of Vacuum and Gaseous Devices, WileyInterscience publication, Wiley, 1996.

[73] O. Scherzer, Über einige Fehler von Elektronenlinsen, Z. Physik 101 (9-10) (1936) 593-603.

[74] O. Scherzer, The Theoretical Resolution Limit of the Electron Microscope, J. Appl. Phys. 20 (1) (1949) 20-29.

[75] P. Hawkes, Quadrupole optics, Springer, 1966.

[76] O. Krivanek, N. Dellby, A. Lupini, Towards sub- $\AA$ electron beams, Ultramicroscopy 78 (1-4) (1999) 1-11.

[77] J. Zach, M. Haider, Aberration correction in a low voltage SEM by a multipole corrector, Nucl. Instrum. Methods Phys. Res. A 363 (1-2) (1995) 316-325.

[78] B. Kabius, P. Hartel, M. Haider, H. Müller, S. Uhlemann, U. Loebau, J. Zach, H. Rose, First application of Cc-corrected imaging for high-resolution and energy-filtered TEM, J. Electron Microsc. 58 (3) (2009) 147-155.

[79] R. H. van Aken, C. W. Hagen, J. E. Barth, P. Kruit, Low-energy foil aberration corrector, Ultramicroscopy 93 (3-4) (2002) 321-330.

[80] R. van Aken, M. Lenc, J. Barth, Aberration integrals for the low-voltage foil corrector, Nucl. Instrum. Methods Phys. Res. A 519 (1-2) (2004) 205-215.

[81] R. H. van Aken, D. J. Maas, C. W. Hagen, J. E. Barth, P. Kruit, Design of an aberration corrected low-voltage SEM, Ultramicroscopy 110 (11) (2010) 1411-1419.

[82] G. F. Rempfer, D. M. Desloge, W. P. Skoczylas, O. H. Griffith, Simultaneous Correction of Spherical and Chromatic Aberrations with an Electron Mirror: An Electron Optical Achromat, Microsc. Microanal. 3 (01) (1997) 14-27.

[83] A. Recknagel, On the theory of the electron mirror, Zeitschrift fur Physik 104 (3) (1936) 381-394.

[84] D. Gabor, A zonally corrected electron lens, Nature 158 (1946) 198.

[85] G. Schönhense, H. Spiecker, Correction of chromatic and spherical aberration in electron microscopy utilizing the time structure of pulsed excitation sources, J. Vac. Sci. Technol. B 20 (6) (2002) 2526. 
[86] T. Koshikawa, H. Shimizu, R. Amakawa, T. Ikuta, T. Yasue, E. Bauer, A new aberration correction method for photoemission electron microscopy by means of moving focus, J. Phys. Condens. Matter 17 (16) (2005) S1371.

[87] S. Uhlemann, H. Müller, P. Hartel, J. Zach, M. Haider, Thermal magnetic field noise limits resolution in transmission electron microscopy, Phys. Rev. Lett. 111 (4) (2013) 046101.

[88] E. G. Ramberg, Electron Optics, Phys. Today 2 (3) (1949) 32.

[89] R. Könenkamp, R. C. Word, G. F. Rempfer, T. Dixon, L. Almaraz, T. Jones, $5.4 \mathrm{~nm}$ spatial resolution in biological photoemission electron microscopy, Ultramicroscopy 110 (7) (2010) 899-902.

[90] R. M. Tromp, J. B. Hannon, A. W. Ellis, W. Wan, A. Berghaus, O. Schaff, A new aberration-corrected, energy-filtered LEEM/PEEM instrument. I. Principles and design, Ultramicroscopy 110 (7) (2010) 852-861.

[91] R. M. Tromp, J. B. Hannon, W. Wan, A. Berghaus, O. Schaff, A new aberrationcorrected, energy-filtered LEEM/PEEM instrument II. Operation and results, Ultramicroscopy 127 (2012) 25-39.

[92] F. Nickel, D. M. Gottlob, I. P. Krug, H. Doganay, S. Cramm, A. M. Kaiser, G. Lin, D. Makarov, O. G. Schmidt, C. M. Schneider, Time-resolved magnetic imaging in an aberration-corrected, energy-filtered photoemission electron microscope, Ultramicroscopy 130 (2013) 54-62.

[93] Z. Shao, X. D. Wu, Adjustable four-electrode electron mirror as an aberration corrector, Appl. Phys. Lett. 55 (26) (1989) 2696-2697.

[94] J. P. S. Fitzgerald, R. C. Word, R. Könenkamp, Adaptive aberration correction using a triode hyperbolic electron mirror, Ultramicroscopy 111 (9-10) (2011) $1495-1503$.

[95] Z. Shao, X. D. Wu, Properties of a four-electrode adjustable electron mirror as an aberration corrector, Rev. Sci. Instrum. 61 (4) (1990) 1230-1235.

[96] M. Aeschlimann, C. A. Schmuttenmaer, H. E. Elsayed-Ali, R. J. D. Miller, J. Cao, Y. Gao, D. A. Mantell, Observation of surface enhanced multiphoton photoemission from metal surfaces in the short pulse limit, J. Chem. Phys. 102 (21) (1995) 8606-8613.

[97] S. Ogawa, H. Petek, Femtosecond dynamics of hot-electron relaxation in $\mathrm{Cu}(110)$ and $\mathrm{Cu}(100)$, Surf. Sci. 357-358 (0) (1996) 585-594. 
[98] N. Pontius, V. Sametoglu, H. Petek, Simulation of two-photon photoemission from the bulk s p-bands of Ag (111), Phys. Rev. B: Condens. Matter Mater. Phys. .

[99] G. F. Rempfer, W. P. Skoczylas, O. H. Griffith, Design and performance of a high-resolution photoelectron microscope, Ultramicroscopy 36 (1-3) (1991) $196-221$.

[100] D. A. Dahl, simion for the personal computer in reflection, Int. J. Mass Spectrom. 200 (1-3) (2000) 3-25.

[101] G. Rempfer, M. Mauck, Correction of chromatic aberration with an electron mirror, Optik .

[102] R. Könenkamp, T. Jones, J. Elstner, R. Word, G. Rempfer, T. Dixon, L. Almaraz, W. Skoczylas, Image properties in an aberration-corrected photoemission electron microscope - Proceedings of the Seventh International Conference on Charged Particle Optics (CPO-7), Phys. Procedia 1 (1) (2008) 505-511.

[103] R. Rudenberg, Electron lenses of hyperbolic field structure, J. Franklin Inst. 246 (5) (1948) 377-408.

[104] A. Septier, M. Ruytoor, Aberration spherique de lentilles electrostatiques de revolution pour des faisceaux de grande ouverture methodes de mesure, Comptes Rendus de laposAcadémie des Sciences. 249 (1959) 2175 and 2746.

[105] C. J. Davisson, C. J. Calbick, Electron Lenses, Phys. Rev. 42 (4) (1932) 580580 .

[106] J. P. S. Fitzgerald, R. C. Word, R. Könenkamp, Simultaneous and independent adaptive correction of spherical and chromatic aberration using an electron mirror and lens combination, Ultramicroscopy 115 (2012) 35-40.

[107] M. M. MacNaughton, The Focal Properties and Spherical Aberration Constants of Aperture Electron Lenses, Proceedings of the Physical Society. Section B 65 (8) (1952) 590.

[108] H. Hertz, Ueber einen Einfluss des ultravioletten Lichtes auf die electrische Entladung, Ann. Phys. 267 (8) (1887) 983-1000.

[109] W. Hallwachs, Ueber den Einfluss des Lichtes auf electrostatisch geladene Körper, Ann. Phys. 269 (2) (1888) 301-312.

[110] M. A. Stoletow, On a kind of electrical current produced by ultra-violet rays, Philosophical Magazine Series 526 (160) (1888) 317-319. 
[111] A. Einstein, Über einen die Erzeugung und Verwandlung des Lichtes betreffenden heuristischen Gesichtspunkt, Ann. Phys. 322 (6) (1905) 132-148.

[112] M. Göppert-Mayer, Über Elementarakte mit zwei Quantensprüngen, Ann. Phys. 401 (3) (1931) 273-294.

[113] M. Merschdorf, W. Pfeiffer, A. Thon, S. Voll, G. Gerber, Photoemission from multiply excited surface plasmons in Ag nanoparticles, Appl. Phys. A: Mater. Sci. Process. 71 (5) (2000) 547-552.

[114] M. Schultze, M. Fiess, N. Karpowicz, J. Gagnon, M. Korbman, M. Hofstetter, S. Neppl, A. L. Cavalieri, Y. Komninos, T. Mercouris, C. A. Nicolaides, R. Pazourek, S. Nagele, J. Feist, J. Burgdörfer, A. M. Azzeer, R. Ernstorfer, R. Kienberger, U. Kleineberg, E. Goulielmakis, F. Krausz, V. S. Yakovlev, Delay in photoemission, Science 328 (5986) (2010) 1658-1662.

[115] R. H. Fowler, The Analysis of Photoelectric Sensitivity Curves for Clean Metals at Various Temperatures, Phys. Rev. 38 (1) (1931) 45-56.

[116] Stuckless, Moskovits, Enhanced two-photon photoemission from coldly deposited silver films, Phys. Rev. B Condens. Matter 40 (14) (1989) 9997-9998.

[117] L. Apker, E. Taft, J. Dickey, Photoelectric Emission and Contact Potentials of Semiconductors, Phys. Rev. 74 (10) (1948) 1462-1474.

[118] Tsang, Srinivasan-Rao, Fischer, Surface-plasmon field-enhanced multiphoton photoelectric emission from metal films, Phys. Rev. B Condens. Matter 43 (11) (1991) 8870-8878.

[119] E. Bauer, Surface Microscopy with Low Energy Electrons, Springer, 2014 edition edn., 2014.

[120] G. D. Mahan, Theory of Photoemission in Simple Metals, Phys. Rev. B Condens. Matter 2 (11) (1970) 4334-4350.

[121] R. D. Mattuck, A guide to Feynman diagrams in the many-body problem, McGraw-Hill, New York, 1976.

[122] P. Monchicourt, M. Raynaud, H. Saringar, J. Kupersztych, Resonant electron emission of silver spheroids induced by laser surface plasmon excitation, J. Phys. Condens. Matter 9 (27) (1997) 5765.

[123] M. Cardona, L. Ley, Photoemission in Solids I: General Principles, vol. -1, Springer Berlin Heidelberg, 1978.

[124] N. Bohr, 'Uber die Serienspektra der Elemente, Z. Physik 2 (5) (1920) 423-469. 
[125] D. T. Pierce, H. C. Siegmann, Hot-electron scattering length by measurement of spin polarization, Phys. Rev. B Condens. Matter 9 (10) (1974) 4035-4037.

[126] O. Paul, S. Toscano, K. Totland, M. Landolt, The spatial origin of the spinpolarization of secondary-electron emission from Fe, Surf. Sci. 251-252 (0) (1991) 27-30.

[127] Pappas, Kämper, Miller, Hopster, Fowler, Brundle, Luntz, Shen, Spindependent electron attenuation by transmission through thin ferromagnetic films, Phys. Rev. Lett. 66 (4) (1991) 504-507.

[128] M. Donath, D. Scholl, H. C. Siegmann, E. Kay, Probing depth of the low energy cascade electrons from a transition metal, Appl. Phys. A: Mater. Sci. Process. 52 (3) (1991) 206-209.

[129] R. Knorren, G. Bouzerar, K. H. Bennemann, Theory for the dynamics of excited electrons in noble and transition metals, J. Phys. Condens. Matter 14 (27) (2002) R739.

[130] S. Enoch, G. Tayeb, P. Sabouroux, N. Guérin, P. Vincent, A metamaterial for directive emission, Phys. Rev. Lett. 89 (21) (2002) 213902.

[131] Y. Takahashi, Y. Inui, M. Chihara, T. Asano, R. Terawaki, S. Noda, A micrometre-scale Raman silicon laser with a microwatt threshold, Nature 498 (7455) (2013) 470-474.

[132] T. Ergin, N. Stenger, P. Brenner, J. B. Pendry, M. Wegener, Three-dimensional invisibility cloak at optical wavelengths, Science 328 (5976) (2010) 337-339.

[133] R. W. Boyd, D. J. Gauthier, Controlling the velocity of light pulses, Science 326 (5956) (2009) 1074-1077.

[134] J. R. Krenn, J.-C. Weeber, Surface plasmon polaritons in metal stripes and wires, Philos. Trans. A Math. Phys. Eng. Sci. 362 (1817) (2004) 739-756.

[135] P. Dvořák, T. Neuman, L. Bř́nek, T. Šamořil, R. Kalousek, P. Dub, P. Varga, T. Šikola, Control and near-field detection of surface plasmon interference patterns, Nano Lett. 13 (6) (2013) 2558-2563.

[136] M. Bosman, E. Ye, S. F. Tan, C. A. Nijhuis, J. K. W. Yang, R. Marty, A. Mlayah, A. Arbouet, C. Girard, M.-Y. Han, Surface plasmon damping quantified with an electron nanoprobe, Sci. Rep. 3 (2013) 1312.

[137] H. Ditlbacher, U. Hohenester, A. Hohenau, others, Universal dispersion of surface plasmons in flat nanostructures, Nature . 
[138] T. Coenen, E. J. R. Vesseur, A. Polman, Deep subwavelength spatial characterization of angular emission from single-crystal Au plasmonic ridge nanoantennas, ACS Nano 6 (2) (2012) 1742-1750.

[139] R. Vogelgesang, A. Dmitriev, Real-space imaging of nanoplasmonic resonances, Analyst 135 (6) (2010) 1175-1181.

[140] L. Douillard, F. Charra, Photoemission electron microscopy, a tool for plasmonics, J. Electron Spectrosc. Relat. Phenom. 189, Supplement (0) (2013) 24-29.

[141] E. Fluck, M. Hammer, A. M. Otter, J. Korterik, L. Kuipers, N. van Hulst, Amplitude and phase evolution of optical fields inside periodic photonic structures, J. Lightwave Technol. 21 (5) (2003) 1384-1393.

[142] L. Stern, B. Desiatov, I. Goykhman, G. M. Lerman, U. Levy, Near field phase mapping exploiting intrinsic oscillations of aperture NSOM probe, Opt. Express 19 (13) (2011) 12014-12020.

[143] G. H. Fecher, O. Schmidt, Y. Hwu, G. Schönhense, Multiphoton photoemission electron microscopy using femtosecond laser radiation, J. Electron Spectrosc. Relat. Phenom. 126 (1-3) (2002) 77-87.

[144] M. Cinchetti, A. Oelsner, G. H. Fecher, H. J. Elmers, G. Schönhense, Observation of $\mathrm{Cu}$ surface inhomogeneities by multiphoton photoemission spectromicroscopy, Appl. Phys. Lett. 83 (8) (2003) 1503-1505.

[145] H. Raether, Surface plasmons on smooth surfaces, Springer, 1988.

[146] A. V. Zayats, I. I. Smolyaninov, Near-field photonics: surface plasmon polaritons and localized surface plasmons, J. Opt. A: Pure Appl. Opt. 5 (4) (2003) S16.

[147] M. Moskovits, Surface-enhanced spectroscopy, Rev. Mod. Phys. 57 (3) (1985) 783-826.

[148] C. Douketis, T. L. Haslett, J. T. Stuckless, M. Moskovits, V. M. Shalaev, Direct and roughness-induced indirect transitions in photoemission from silver films, Surf. Sci. 297 (2) (1993) L84-L90.

[149] M. I. Stockman, Femtosecond Optical Responses of Disordered Clusters, Composites, and Rough Surfaces: "The Ninth Wave" Effect, Phys. Rev. Lett. 84 (5) (2000) 1011-1014.

[150] E. Ozbay, Plasmonics: merging photonics and electronics at nanoscale dimensions, Science 311 (5758) (2006) 189-193. 
[151] A. V. Akimov, A. Mukherjee, C. L. Yu, D. E. Chang, A. S. Zibrov, P. R. Hemmer, H. Park, M. D. Lukin, Generation of single optical plasmons in metallic nanowires coupled to quantum dots, Nature 450 (7168) (2007) 402-406.

[152] M. W. Knight, N. K. Grady, R. Bardhan, F. Hao, P. Nordlander, N. J. Halas, Nanoparticle-mediated coupling of light into a nanowire, Nano Lett. 7 (8) (2007) 2346-2350.

[153] H. Wei, D. Ratchford, X. E. Li, H. Xu, C.-K. Shih, Propagating surface plasmon induced photon emission from quantum dots, Nano Lett. 9 (12) (2009) 41684171.

[154] A. B. Evlyukhin, S. I. Bozhevolnyi, A. L. Stepanov, J. R. Krenn, Splitting of a surface plasmon polariton beam by chains of nanoparticles, Appl. Phys. B $84(1-2)(2006)$ 29-34.

[155] C.-Y. Tai, S. H. Chang, T. Chiu, Numerical optimization of wide-angle, broadband operational polarization beam splitter based on aniostropically coupled surface-plasmon-polariton waves, J. Opt. Soc. Am. B, JOSAB 25 (8) (2008) $1387-1392$.

[156] Z. Liu, J. M. Steele, W. Srituravanich, Y. Pikus, C. Sun, X. Zhang, Focusing surface plasmons with a plasmonic lens, Nano Lett. 5 (9) (2005) 1726-1729.

[157] Y. Fang, Z. Li, Y. Huang, S. Zhang, P. Nordlander, N. J. Halas, H. Xu, Branched silver nanowires as controllable plasmon routers, Nano Lett. 10 (5) (2010) 19501954 .

[158] Z.-Y. Li, Y. Xia, Metal nanoparticles with gain toward single-molecule detection by surface-enhanced Raman scattering, Nano Lett. 10 (1) (2010) 243-249.

[159] Z. Zhang, A. Weber-Bargioni, S. W. Wu, S. Dhuey, S. Cabrini, P. J. Schuck, Manipulating nanoscale light fields with the asymmetric bowtie nano-colorsorter, Nano Lett. 9 (12) (2009) 4505-4509.

[160] Y. Fang, H. Wei, F. Hao, P. Nordlander, H. Xu, Remote-excitation surfaceenhanced Raman scattering using propagating Ag nanowire plasmons, Nano Lett. 9 (5) (2009) 2049-2053.

[161] H. Xu, E. J. Bjerneld, M. K'all, L. B'orjesson, Spectroscopy of Single Hemoglobin Molecules by Surface Enhanced Raman Scattering, Phys. Rev. Lett. 83 (21) (1999) 4357-4360.

[162] S. Lal, S. E. Clare, N. J. Halas, Nanoshell-enabled photothermal cancer therapy: impending clinical impact, Acc. Chem. Res. 41 (12) (2008) 1842-1851. 
[163] M. A. Noginov, G. Zhu, A. M. Belgrave, R. Bakker, V. M. Shalaev, E. E. Narimanov, S. Stout, E. Herz, T. Suteewong, U. Wiesner, Demonstration of a spaser-based nanolaser, Nature 460 (7259) (2009) 1110-1112.

[164] N. Fang, H. Lee, C. Sun, X. Zhang, Sub-diffraction-limited optical imaging with a silver superlens, Science 308 (5721) (2005) 534-537.

[165] A. Alù, N. Engheta, Multifrequency optical invisibility cloak with layered plasmonic shells, Phys. Rev. Lett. 100 (11) (2008) 113901.

[166] I. I. Smolyaninov, Y.-J. Hung, C. C. Davis, Magnifying superlens in the visible frequency range, Science 315 (5819) (2007) 1699-1701.

[167] Z. Liu, H. Lee, Y. Xiong, C. Sun, X. Zhang, Far-field optical hyperlens magnifying sub-diffraction-limited objects, Science 315 (5819) (2007) 1686.

[168] J. C. Knight, Photonic crystal fibres, Nature 424 (6950) (2003) 847-851.

[169] Y. Akahane, T. Asano, B.-S. Song, S. Noda, High-Q photonic nanocavity in a two-dimensional photonic crystal, Nature 425 (6961) (2003) 944-947.

[170] T. Yoshie, A. Scherer, J. Hendrickson, G. Khitrova, H. M. Gibbs, G. Rupper, C. Ell, O. B. Shchekin, D. G. Deppe, Vacuum Rabi splitting with a single quantum dot in a photonic crystal nanocavity, Nature 432 (7014) (2004) 200203.

[171] Y. A. Vlasov, M. O'Boyle, H. F. Hamann, S. J. McNab, Active control of slow light on a chip with photonic crystal waveguides, Nature 438 (7064) (2005) 65-69.

[172] R. C. Word, J. P. S. Fitzgerald, R. Könenkamp, Positional control of plasmonic fields and electron emission, Appl. Phys. Lett. 105 (11) (2014) 111114.

[173] J. P. S. Fitzgerald, R. C. Word, S. D. Saliba, R. Könenkamp, Photonic nearfield imaging in multiphoton photoemission electron microscopy, Phys. Rev. B Condens. Matter 87 (20) (2013) 205419.

[174] R. C. Word, J. P. S. Fitzgerald, R. Könenkamp, Direct imaging of optical diffraction in photoemission electron microscopy, Appl. Phys. Lett. 103 (2) (2013) 021118 .

[175] J. P. S. Fitzgerald, R. C. Word, R. Könenkamp, Subwavelength visualization of light in thin film waveguides with photoelectrons, Phys. Rev. B Condens. Matter 89 (19) (2014) 195129.

[176] R. M. Broudy, Vectorial Photoelectric Effect, Phys. Rev. B Condens. Matter 3 (11) (1971) 3641-3651. 
[177] G. Vuye, S. Fisson, V. Nguyen Van, Y. Wang, J. Rivory, F. Abelès, Temperature dependence of the dielectric function of silicon using in situ spectroscopic ellipsometry, Thin Solid Films 233 (1-2) (1993) 166-170.

[178] R. Williams, Photoemission of Electrons from Silicon into Silicon Dioxide, Phys. Rev. 140 (2A) (1965) A569-A575.

[179] F. Allen, G. Gobeli, Work Function, Photoelectric Threshold, and Surface States of Atomically Clean Silicon, Phys. Rev. 127 (1) (1962) 150-158.

[180] D. Davazoglou, Optical properties of SnO_2 thin films grown by atmospheric pressure chemical vapour deposition oxiding SnCl_4, Thin Solid Films 302 (1) (1997) 204-213.

[181] K. Sugiyama, H. Ishii, Y. Ouchi, K. Seki, Dependence of indium-tin-oxide work function on surface cleaning method as studied by ultraviolet and x-ray photoemission spectroscopies, J. Appl. Phys. 87 (1) (2000) 295-298.

[182] J. S. Kim, B. L'agel, E. Moons, N. Johansson, I. D. Baikie, W. R. Salaneck, R. H. Friend, F. Cacialli, Kelvin probe and ultraviolet photoemission measurements of indium tin oxide work function: a comparison, Synth. Met. 111-112 (0) (2000) 311-314.

[183] H. Kim, J. S. Horwitz, G. Kushto, A. Piqué, Z. H. Kafafi, C. M. Gilmore, D. B. Chrisey, Effect of film thickness on the properties of indium tin oxide thin films, J. Appl. Phys. 88 (10) (2000) 6021-6025.

[184] R. Schlaf, H. Murata, Z. Kafafi, Work function measurements on indium tin oxide films, J. Electron Spectrosc. Relat. Phenom. 120 (1-3) (2001) 149-154.

[185] V. Craciun, C. Chiritescua, F. Kelly, others, Low temperature growth of smooth indium tin oxide films by ultraviolet assisted pulsed laser deposition, J. Optoelectron. Adv. Mater. 4 (1) (2002) 21-25.

[186] S. Franzen, Surface Plasmon Polaritons and Screened Plasma Absorption in Indium Tin Oxide Compared to Silver and Gold, J. Phys. Chem. C 112 (15) (2008) 6027-6032.

[187] G. V. Naik, J. Kim, A. Boltasseva, Oxides and nitrides as alternative plasmonic materials in the optical range [Invited], Opt. Mater. Express, OME 1 (6) (2011) 1090-1099.

[188] J. C. Rivière, THE WORK FUNCTION OF GOLD, Appl. Phys. Lett. 8 (7) (1966) 172-172.

[189] W. M. H. Sachtler, G. J. H. Dorgelo, A. A. Holscher, The work function of gold, Surf. Sci. 5 (2) (1966) 221-229. 
[190] D. Pescia, F. Meier, Spin polarized photoemission from gold using circularly polarized light, Surf. Sci. 117 (1-3) (1982) 302-309.

[191] Z. Guo, Y. Zhang, Y. DuanMu, L. Xu, S. Xie, N. Gu, Facile synthesis of micrometer-sized gold nanoplates through an aniline-assisted route in ethylene glycol solution, Colloids Surf. A Physicochem. Eng. Asp. 278 (1-3) (2006) 3338.

[192] J.-S. Huang, V. Callegari, P. Geisler, C. Brüning, J. Kern, J. C. Prangsma, X. Wu, T. Feichtner, J. Ziegler, P. Weinmann, M. Kamp, A. Forchel, P. Biagioni, U. Sennhauser, B. Hecht, Atomically flat single-crystalline gold nanostructures for plasmonic nanocircuitry, Nat. Commun. 1 (2010) 150.

[193] F. Bisio, M. Nývlt, J. Franta, H. Petek, J. Kirschner, Mechanisms of highorder perturbative photoemission from $\mathrm{Cu}(001)$, Phys. Rev. Lett. 96 (8) (2006) 087601.

[194] A. Yariv, Optical Electronics, Saunders College Publishing, Philadelphia, 1991.

[195] A. Buckman, Guided-Wave Photonics, Saunders College Pub., 1992.

[196] T. Tamir, Integrated optics, Topics in Applied Physics, Berlin: Springer, 1979, 2nd rev. ed., edited by Tamir, T. -1 .

[197] P. Berini, Plasmon-polariton waves guided by thin lossy metal films of finite width: Bound modes of asymmetric structures, Phys. Rev. B Condens. Matter 63 (12) (2001) 125417.

[198] S. A. Maier, Plasmonics: Fundamentals and Applications, Springer, softcover reprint of hardcover 1st ed. 2007 edition edn., 2010.

[199] J. D. Jackson, Classical Electrodynamics, Wiley, New York, 3rd edn., 1999.

[200] W. Singer, M. Totzeck, H. Gross, Handbook of Optical Systems, Physical Image Formation, Handbook of Optical Systems, Wiley, 2006.

[201] J. P. McCaffrey, M. W. Phaneuf, L. D. Madsen, Surface damage formation during ion-beam thinning of samples for transmission electron microscopy, Ultramicroscopy 87 (3) (2001) 97-104.

[202] E. D. Palik, Handbook of Optical Constants of Solids, Academic Press handbook series, Academic Press, 1998.

[203] O. Benson, Assembly of hybrid photonic architectures from nanophotonic constituents, Nature 480 (7376) (2011) 193-199. 
[204] R. F. Oulton, V. J. Sorger, D. A. Genov, D. F. P. Pile, X. Zhang, A hybrid plasmonic waveguide for subwavelength confinement and long-range propagation, Nat. Photonics 2 (8) (2008) 496-500.

[205] F. De Angelis, M. Patrini, G. Das, I. Maksymov, M. Galli, L. Businaro, L. C. Andreani, E. Di Fabrizio, A hybrid plasmonic-photonic nanodevice for label-free detection of a few molecules, Nano Lett. 8 (8) (2008) 2321-2327.

[206] A. Faraon, I. Fushman, D. Englund, N. Stoltz, P. Petroff, J. Vucković, Dipole induced transparency in waveguide coupled photonic crystal cavities, Opt. Express 16 (16) (2008) 12154-12162.

[207] T. Utikal, M. I. Stockman, A. P. Heberle, M. Lippitz, H. Giessen, All-optical control of the ultrafast dynamics of a hybrid plasmonic system, Phys. Rev. Lett. 104 (11) (2010) 113903. 


\section{Appendix A Scale drawings: diode PEEM}

Scale drawing of our PEEM before installation of the triode mirror corrector. This

drawing is significantly the same as the working instrument, with the exception of the objective lens and interface lens bore diameters, of which a variety of lenses were used. Credit: R.C. Word. 


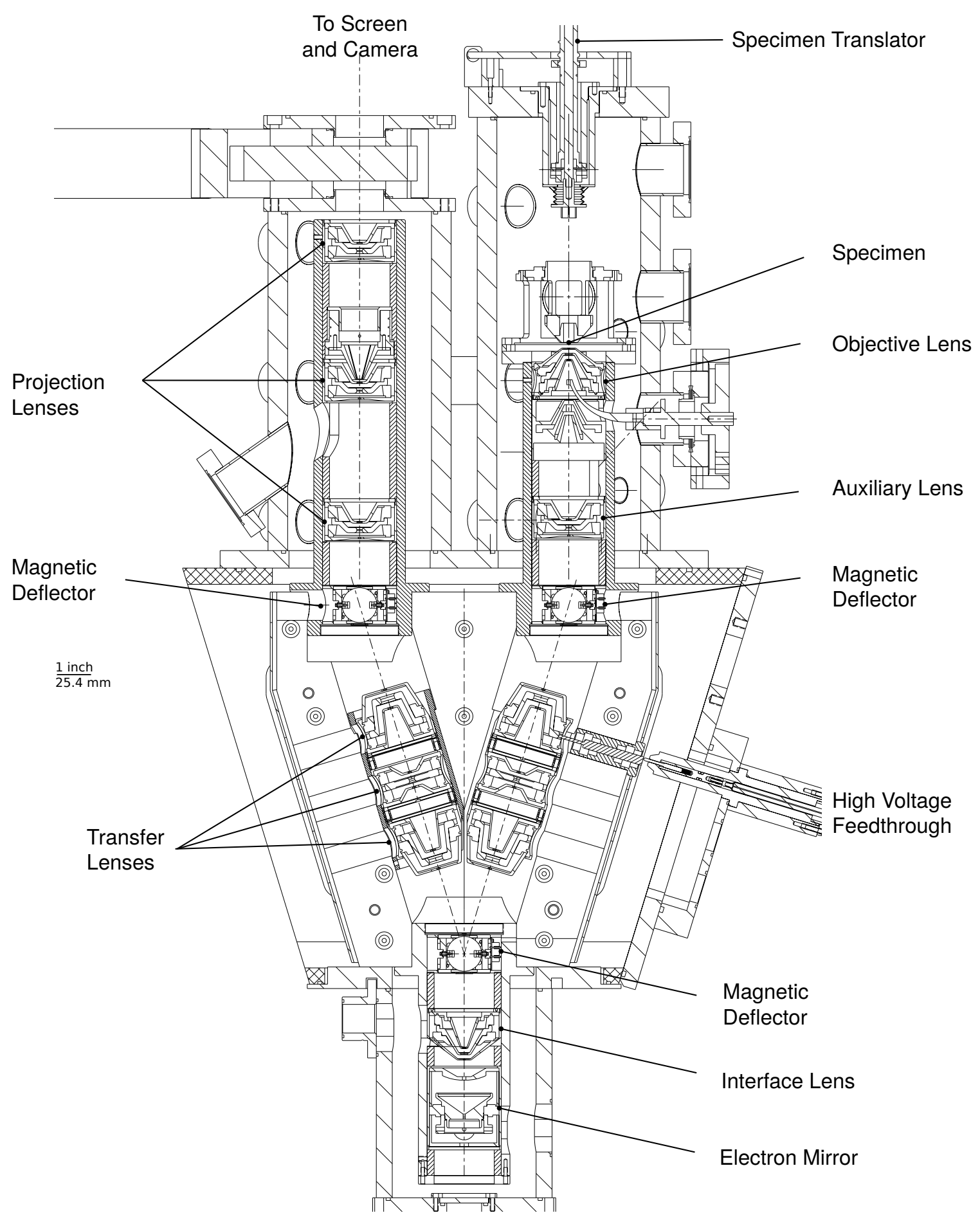




\section{Appendix B Code, Mathematica: hyperbolic mirror theory}

The optical properties in Figs. 3.3, 3.4, 4.2, 4.3, 4.4, 4.8a, 4.8b, and 4.8 were evaluated using Mathematica 8 running the following code. 


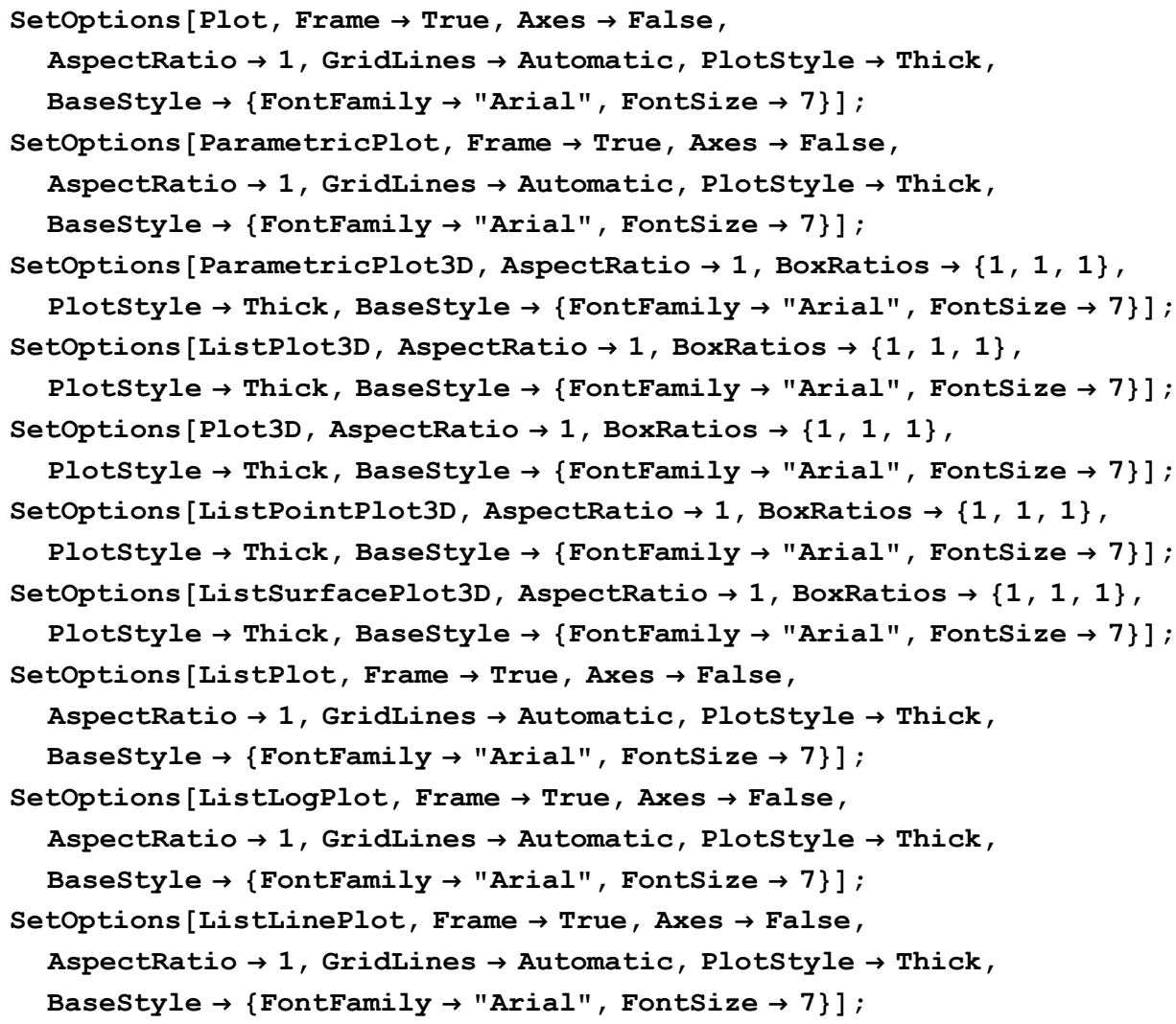

\section{Basic Theory}

This is a reproduction of Gert's paper (1990), specifically the graphs (fig 4).

\section{- Theory}

The position of an electron at the turn-around (potential there is $V_{C}$ ). The turn-around is a hyperboloid surface. This treats $\rho_{C}>0$ as the independent variable.

$$
\zeta_{C}=\sqrt{(v-1) l^{2}-\left(\frac{\rho C}{2}\right)^{2}} ;
$$

The position, velocity, and angle of an electron at electrode $V_{A} . t_{A}$ is the time of flight from the turn-around.

$$
\begin{aligned}
& v=\frac{V a-V c}{V a-V m} ; \\
& k=\frac{V a-V m}{\rho^{2}} ; \\
& \omega=\sqrt{e k / m} ; \\
& \theta=\omega t a ;
\end{aligned}
$$




$$
\begin{aligned}
& \zeta a=\zeta \cosh [\sqrt{2} \theta] ; \\
& \rho a=\rho \operatorname{Cos}[2 \theta] ; \\
& d \rho a=-\omega \rho \operatorname{Tan}[\theta] ; \\
& d \zeta a=\sqrt{2} \omega \zeta a \operatorname{Tanh}[\sqrt{2} \theta] ; \\
& \alpha a=\operatorname{ArcTan}\left[-\frac{d \rho a}{d \zeta a}\right] ;
\end{aligned}
$$

The $V_{A}$ position coordinates are also related by

$$
\zeta a=\sqrt{\rho^{2}-\left(\frac{\rho a}{2}\right)^{2}}
$$

The position, velocity, and angle of an electron when it crosses the axis. This includes the lensing effects of the opening in $V_{A}$.

$\rho \mathrm{x}=0$;

$$
\alpha x=\alpha a-\frac{\rho a \sqrt{1+\left(\frac{\rho a}{2 \ell}\right)^{2}}}{2 v \ell} ;
$$

\section{- Solution, Functions \& Plots}

\section{- Solving for $\theta^{(1)}$}

Simplifying in terms of some unitless parameters:

$\alpha=\mathrm{Va} / \mathrm{Vm} ; v=(\mathrm{Va}-\mathrm{VC}) /(\mathrm{Va}-\mathrm{Vm}) ; \mathrm{rc}=\rho \mathrm{c} / \rho ; \tau=\sqrt{-\frac{\mathrm{m} \rho^{2}}{\mathrm{eVm}}} ; \mathrm{zc}=\zeta_{\mathrm{C}} / \rho ;$

To first order, $\zeta_{A}=\ell$, which allows solution for $t_{A}$. Simplifying this equation into unitless parameters,

$$
\begin{aligned}
& \text { Solve }\left[\sqrt{1-v-\operatorname{rc}^{2}} \operatorname{Cosh}[\sqrt{2} \theta]=1, \theta\right] \\
& \theta 1=\sqrt{\frac{1}{2}} \operatorname{ArcCosh}\left[\sqrt{\frac{1}{1-v-r c^{2}}}\right] ;
\end{aligned}
$$

\section{- Solving for $\theta^{(2)}$}

Take the first order solution and use it to calculate $r_{A}=\rho_{A} / \ell, z_{A}=\zeta_{A} / \ell$ :

$\operatorname{ra1}=\operatorname{rc} \operatorname{Cos}[\theta 1] ;$

$\mathrm{za} 1=\sqrt{1+\frac{\mathrm{ra}^{2}}{2}} ;$

Solve $z_{A}{ }^{(1)}=\zeta_{C} \operatorname{Cosh} \sqrt{2} \theta^{(2)}$ : 


$$
\begin{aligned}
& \text { Solve }\left[z a 1==\sqrt{1-v v-\operatorname{rc}^{2}} \operatorname{Cosh}[\sqrt{2} \theta], \theta\right] \\
& \theta 2=\sqrt{\frac{1}{2}} \operatorname{ArcCosh}\left[\frac{\sqrt{1+\frac{r c^{2}}{2}\left(\operatorname{Cos}\left[\sqrt{\frac{1}{2}} \operatorname{ArcCosh}\left[\sqrt{\frac{1}{1-r c^{2}-v \nu}}\right]\right]\right)^{2}}}{\sqrt{1-\nu v-r c^{2}}} ;\right.
\end{aligned}
$$

- $z_{0} / \ell$

Only solve for paraxial rays $\left(\rho_{c}=0\right)$ :

$\theta 0=\sqrt{\frac{1}{2}} \operatorname{ArcCosh}\left[\frac{1}{\sqrt{1-v \nu}}\right] ;$

Using equation (15) from the paper, the paraxial object/image distance:

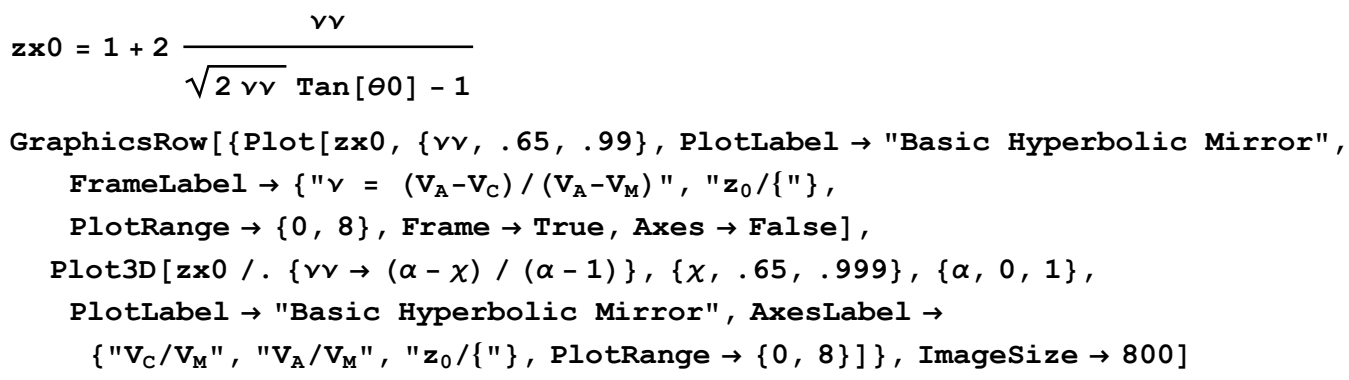

- $C_{c} / \boldsymbol{P}$ $\theta 0=\sqrt{\frac{1}{2}} \operatorname{ArcCosh}\left[\sqrt{\frac{1}{1-\nu \nu}}\right] ;$

From equation (17), $C_{c} / \ell$

$$
\begin{aligned}
& \text { Cc0 }=-\frac{v v}{1-v v}\left(1+2 \frac{v v}{\sqrt{2 v v} \operatorname{Tan}[\theta 0]-1}+\frac{3}{4} \frac{1}{v v^{2}}\left(\frac{2 v v}{\sqrt{2 v v} \operatorname{Tan}[\theta 0]-1}\right)^{2}\right) \\
& \text { GraphicsRow }[ \\
& \{\text { Plot }[-\mathrm{Cc} 0,\{v v, .65, .99\}, \text { PlotLabel } \rightarrow \text { "Basic Hyperbolic Mirror", } \\
& \quad \text { FrameLabel } \rightarrow\left\{" v=\left(\mathrm{V}_{\mathrm{A}}-\mathrm{V}_{\mathrm{C}}\right) /\left(\mathrm{V}_{\mathrm{A}}-\mathrm{V}_{\mathrm{M}}\right) ", "-\mathrm{C}_{\mathrm{C}} /\{"\},\right. \\
& \text { Frame } \rightarrow \text { True, Axes } \rightarrow \text { False, PlotRange } \rightarrow\{0,160\}], \\
& \text { Plot3D }[-\mathrm{Cc} 0 / .\{v v \rightarrow(\alpha-\chi) /(\alpha-1)\},\{\chi, .65, .99\},\{\alpha, 0.001,1\}, \\
& \text { PlotLabel } \rightarrow \text { "Basic Hyperbolic Mirror", AxesLabel } \rightarrow \\
& \left.\left.\quad\left\{" \mathrm{~V}_{\mathrm{C}} / \mathrm{V}_{\mathrm{M}} ", " \mathrm{~V}_{\mathrm{A}} / \mathrm{V}_{\mathrm{M}} ", "-\mathrm{C}_{\mathrm{c}} /\{"\}, \text { PlotRange } \rightarrow\{0,160\}\right]\right\}, \text { ImageSize } \rightarrow 800\right]
\end{aligned}
$$

- Solving for $C_{s} / \ell$

Need to calculate $z_{A}\left(\rho_{c}\right)$

ra2 $=r c \operatorname{Cos}[\theta 2] ;$

$\mathrm{za2}=\sqrt{1+\frac{\mathrm{ra2}^{2}}{2}} ;$ 
Use this to calculate $\alpha, z$ in the small angle limit $(\tan \alpha \approx \alpha)$

$$
\begin{aligned}
& \alpha \times 2=\frac{\mathrm{ra} 2 \operatorname{Tan}[\theta 2]}{\sqrt{2} \mathrm{za} 2 \operatorname{Tanh}[\sqrt{2} \theta 2]}-\frac{\mathrm{ra} 2 \mathrm{za} 2}{2 v v} ; \\
& \mathrm{zx} 2=\mathrm{za} 2+\frac{\mathrm{ra} 2}{\alpha \times 2}
\end{aligned}
$$

Now calculate $\Delta z / \alpha^{2}$

$$
\begin{aligned}
& \Delta \mathrm{zx} 2=\mathrm{zx} 2-\mathrm{zx} 0 ; \\
& \mathrm{Cs} 2=\operatorname{Series}\left[\frac{\Delta \mathrm{zx} 2}{\alpha \times 2^{2}},\{\mathrm{rc}, 0,0\}\right] / / \text { Normal // Chop }
\end{aligned}
$$

At high enough order $v$, this has poles in $r_{C}$, probably due to rounding errors.

\section{- $C_{s}$ / $\ell$ graphs}

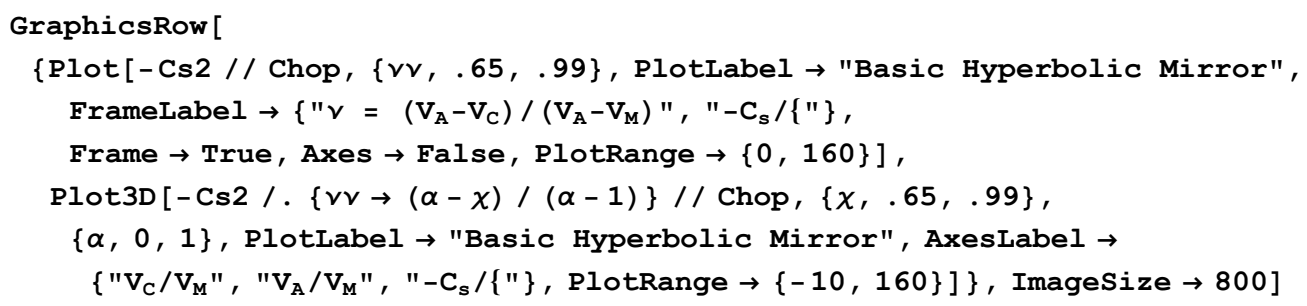

- Summary \& reproduction of graphs

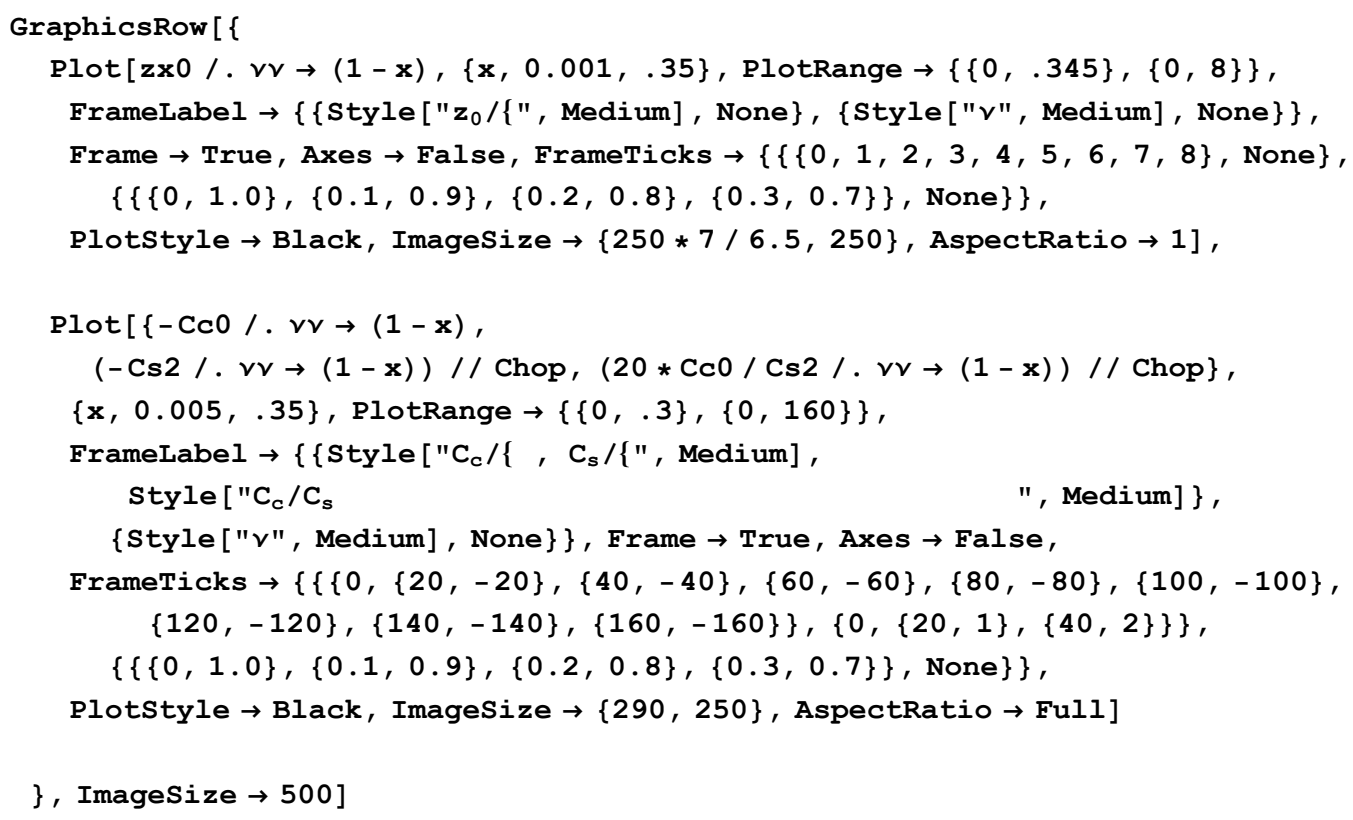

Aberration graph to compare to triode model 


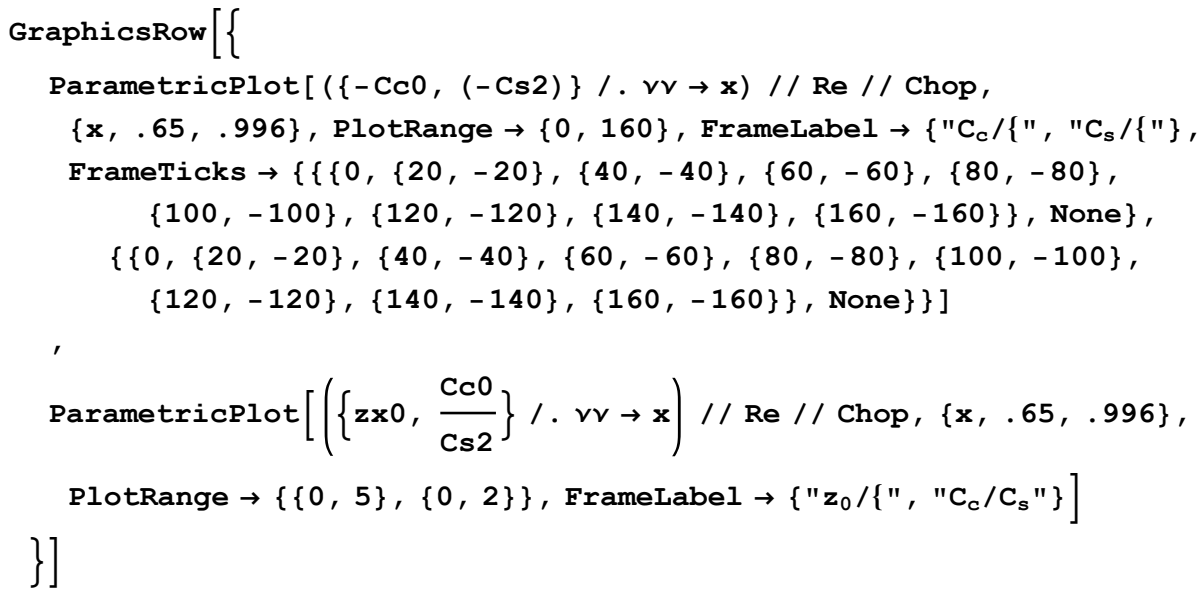

\section{Extended Mirror Theory}

This incorporates a mirror extension and hopes to find graphs similar to those in the paper

\section{- Theory}

\section{- Initial region electron trajectory}

Only symmetric electron trajectories are considered. At reflection, an electron has coordinate $(r, z, t)=\left(r_{C}, z_{C}, 0\right)$ and the speed is zero, $\dot{r}_{C}=\dot{z}_{C}=0$.

After traversing the initial region between the mirror electrode at $V_{M}$ and the adjustable electrode at $V_{A}$, an electron has coordinates $\left(r_{A}, z_{A}, t_{A}\right)$. In terms of the turn-around coordinates,

$$
\begin{aligned}
& r a=r c \cos [\theta i] \text {; } \\
& z a=z \cosh [\sqrt{2} \theta i] ; \\
& \alpha a=\frac{\operatorname{ra} \operatorname{Tan}[\theta i]}{\sqrt{2} z a \operatorname{Tanh}[\sqrt{2} \theta i]} ;
\end{aligned}
$$

where $\theta_{i}=\omega_{i} t, \omega_{i}=\sqrt{2 k_{i} / m}$, and $k_{i}=\left(V_{A}-V_{M}\right) / \ell^{2} . \theta_{i}$ can be approximated as follows:

zero-th order, $z_{A}=\ell$ and $z_{C}=\ell \sqrt{1-v}$, where $v=\left(V_{A}-V_{C}\right) /\left(V_{A}-V_{M}\right)$. 


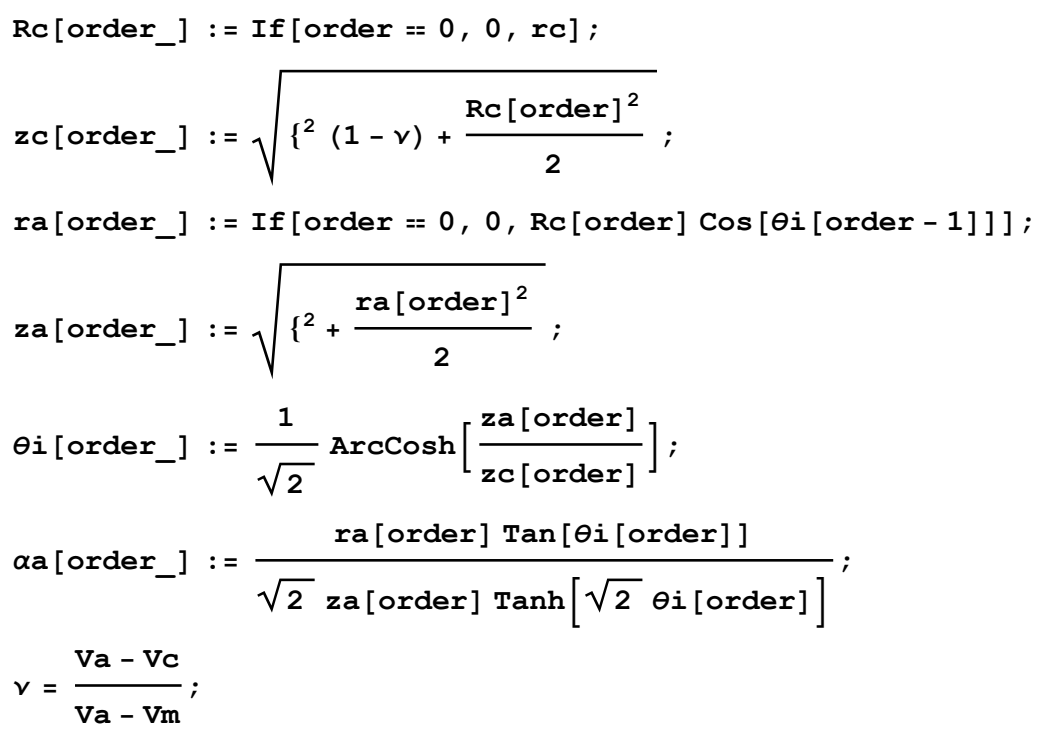

\section{- Aperture A deflection}

The aperture at the adjustable electrode acts to deflect the electron trajectory such that $\alpha_{A} \rightarrow \alpha_{A}+\delta_{A}=\alpha_{i}$, where the deflection is

$$
\begin{aligned}
& \delta a\left[o r d e r \_\right]:=r a[o r d e r] ~ z a[o r d e r] \frac{(k f-k i)}{2(\mathrm{Va}-\mathrm{VC})} \\
& \mathrm{ki}=\frac{\mathrm{Va}-\mathrm{Vm}}{\left\{^{2}\right.} ; \mathrm{kf}=\frac{-\mathrm{Va}}{\mathrm{L}^{2}-\left\{^{2}\right.} ;
\end{aligned}
$$

This deflection will have negligible effect on $\dot{z}$, and $\dot{r} \rightarrow \dot{r}\left(1+\delta_{A} / \alpha_{A}\right)$.

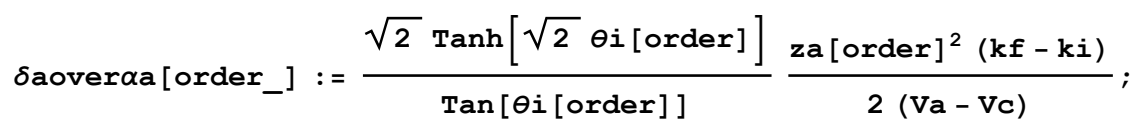

\section{- Check to see if good single-region results}

If the outer region is inactive, the distance $z_{i}$ at which the trajectory intersects the axis is $z_{i}=z_{A}+\frac{r_{A}}{\alpha_{i}}$. Let $q_{i} / \ell=\alpha_{i} / r_{A}$ is

$$
\begin{aligned}
& z i\left[\text { order_] }:=\left(z a \text { [order] }+\frac{\{}{q i[\text { order }]}\right) / . \mathrm{Va} \rightarrow 0\right. \text {; } \\
& q i\left[\text { order_] }:=\left\{\left(\frac{\operatorname{Tan}[\theta i \text { [order] ] }}{\sqrt{2} \text { za [order] } \operatorname{Tanh}[\sqrt{2} \theta i[\text { order] }]}+z a\left[\text { order] } \frac{(\mathbf{k f}-\mathbf{k i})}{2(\mathrm{Va}-\mathrm{Vc})}\right)\right.\right. \text {; }\right. \\
& \text { Plot }[z i[0] / .\{\mathrm{Vm} \rightarrow-1,\{\rightarrow 1\},\{\mathrm{Vc},-.999,-0.65\} \text {, } \\
& \text { Frame } \rightarrow \text { True, PlotRange } \rightarrow\{0,8\} \text {, Axes } \rightarrow \text { False }]
\end{aligned}
$$

- Final region electron trajectory

After traversing the second (final) region potential field, the coordinates are 


$$
\begin{aligned}
& \text { ro [order_] : = If }\left[\text { order }=0,0, \frac{\operatorname{ra}[\text { order }]}{\operatorname{Cos}[\phi[\text { order }]]} \operatorname{Cos}[\theta f[\text { order }-1]-\phi[\text { order }]]\right] ; \\
& \text { zo[order_] }:=\sqrt{\mathbf{L}^{2}+\frac{\text { ro[order }]^{2}}{2}} ; \\
& \theta f\left[\text { order_] }:=\frac{1}{\sqrt{2}}\left(\operatorname{ArcCosh}\left[\frac{\mathrm{zo}[\text { order }] \operatorname{Cosh}[\psi[\text { order }]]}{\mathrm{za}[\text { order }]}\right]+\psi[\text { order }]\right) ;\right. \\
& \phi\left[\text { order_] }:=\operatorname{ArcTan}\left[-\sqrt{\frac{k i}{k f}} \operatorname{Tan}[\theta i[\text { order] }](1+\text { oaoveraa[order] })] ;\right.\right. \\
& \psi\left[\text { order_] }:=\operatorname{ArcTanh}\left[-\sqrt{\frac{k i}{k f}} \operatorname{Tanh}[\sqrt{2} \text { ei [order }]\right]\right] ; \\
& \alpha \circ\left[\text { order_] }:=\frac{\operatorname{ro[order}] \operatorname{Tan}[\theta f[\text { order] }-\phi[\text { order] }]}{\sqrt{2} \text { zo[order] } \operatorname{Tanh}[\sqrt{2} \theta f \text { [order] }-\psi[\text { order] }]} ;\right.
\end{aligned}
$$

\section{- Aperture $\mathrm{O}$ deflection}

The aperture at the adjustable electrode acts to deflect the electron trajectory such that $\alpha_{O} \rightarrow \alpha_{O}+\delta_{O}=\alpha_{f}$, where the deflection is

$$
\begin{aligned}
& \delta \circ\left[\text { order_] }:=\frac{\mathrm{kf} \mathrm{zo[order]} \mathrm{ro[order]}}{2 \mathrm{Vc}} ;\right. \\
& \alpha f[\text { order_] }:=\alpha \circ[\text { order] }+\delta \circ[\text { order] } ;
\end{aligned}
$$

The distance $z_{f}$ at which the trajectory intersects the axis is $z_{f}=z_{O}+\frac{r_{O}}{\alpha_{f}}$, where $q / \mathcal{L}=\alpha_{f} / r_{O}$ is

$$
\begin{aligned}
& \text { zf [order_] : = zo[order] }+\frac{\mathbf{L}}{\text { q[order] }} ; \\
& \text { q[order_] : = } \\
& L\left(\frac{\operatorname{Tan}[\theta f[\text { order }]-\phi \text { [order] }]}{\sqrt{2} \text { zo[order] } \operatorname{Tanh}[\sqrt{2} \text { ef [order] }-\psi[\text { order] }]}-\frac{\mathrm{kf} \mathrm{zo[order]}}{2 \mathrm{Vc}}\right) ;
\end{aligned}
$$

\section{- Aberration}

The chromatic and spherical aberration

$$
\begin{aligned}
& \mathrm{Cc}=2 \mathrm{Vc} \partial_{\mathrm{Vc}}(\mathrm{zf}[0]) ; \\
& \mathrm{Cs}=-2\left(\frac{\frac{1}{2}(\mathrm{D}[\mathrm{zf}[1],\{\mathrm{rc}, 2\}] / . \mathrm{rc} \rightarrow 0)}{(\mathrm{D}[\alpha \mathrm{f}[1],\{\mathrm{rc}, 1\}] / . \mathrm{rc} \rightarrow 0)^{2}}\right) / . \mathrm{rc} \rightarrow 0 ;
\end{aligned}
$$




\section{Plots}

\section{- 3D Plots to compare to SHM}

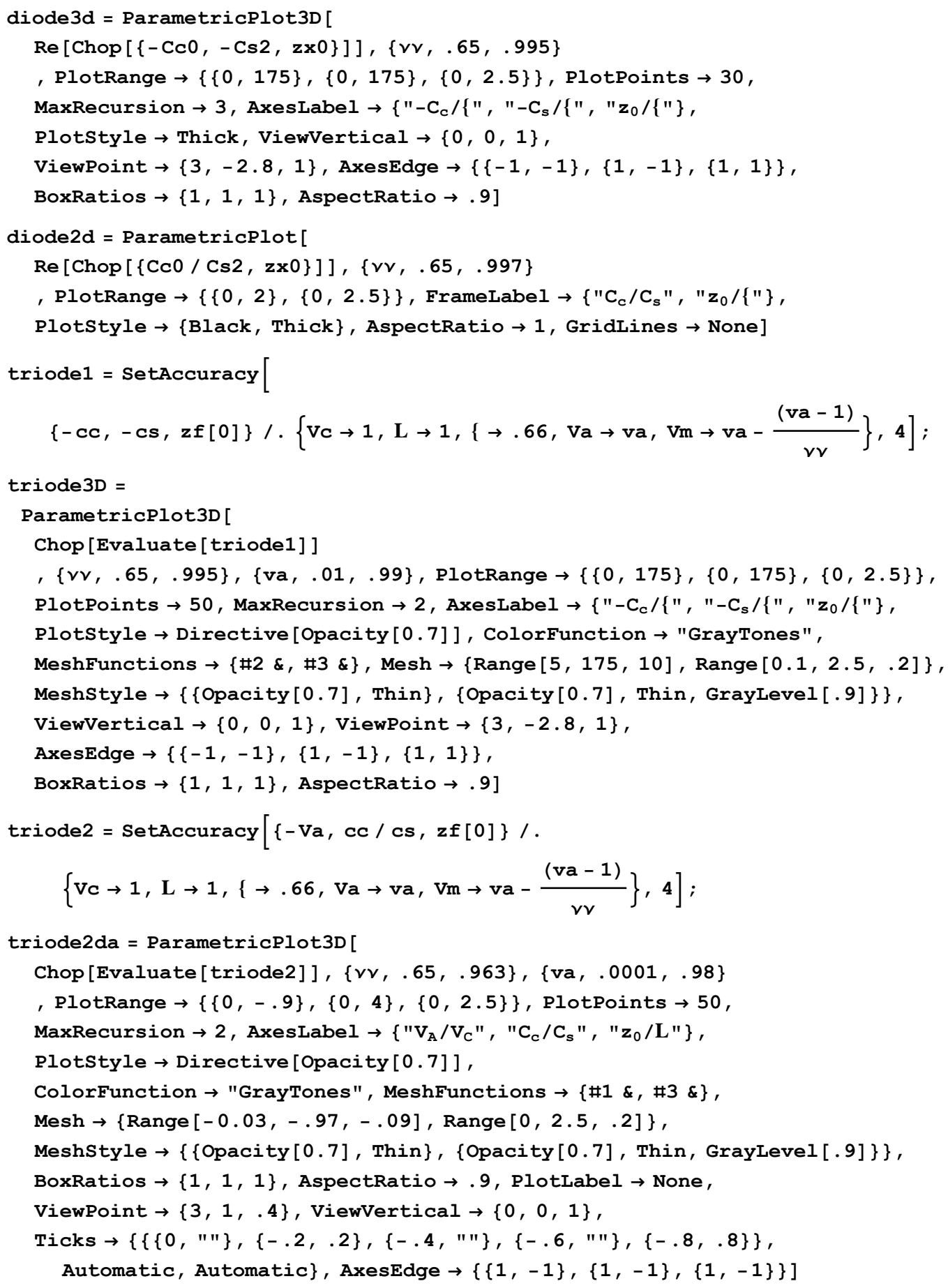




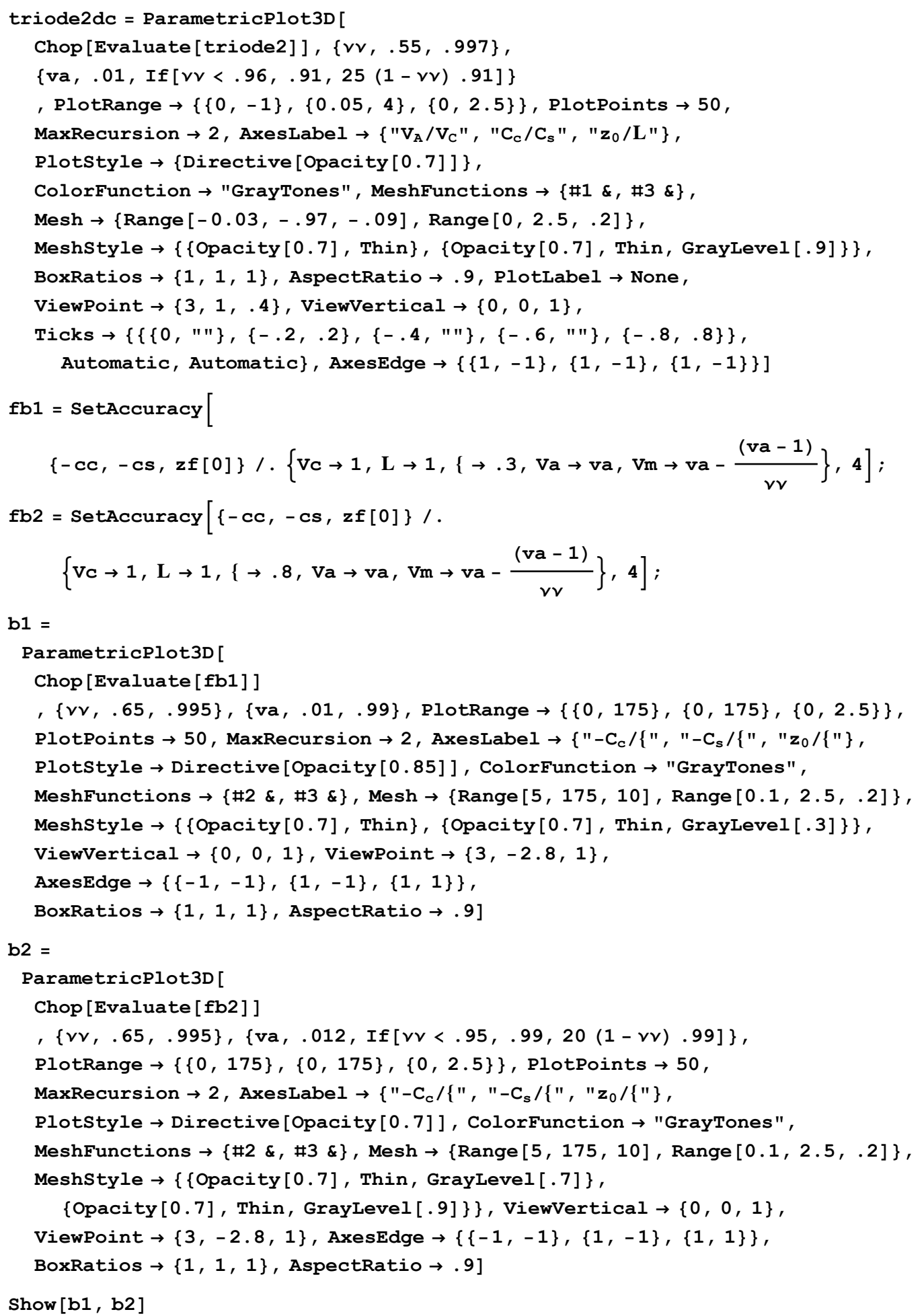




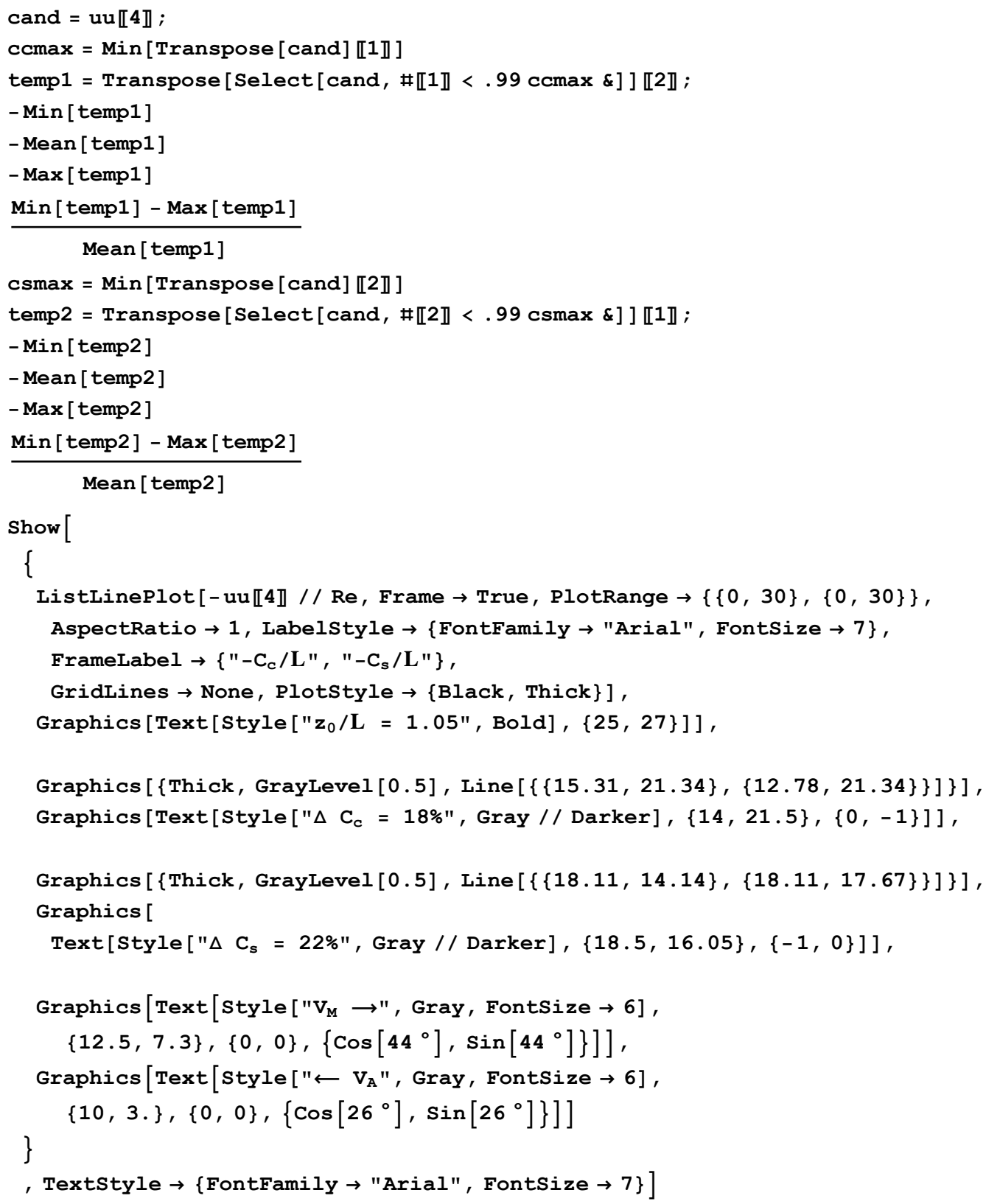




\section{Appendix C Code, Mathematica: mirror theory, updated}

The updated code runs more quickly in Mathematica and can generate the same plots as the code given in the previous appendix. In addition, triode solutions with and without an exit aperture are computed, as well as the effect of replacing the outer hyperbolic field with a constant field. 


\section{Header}

\section{Graphics Options}

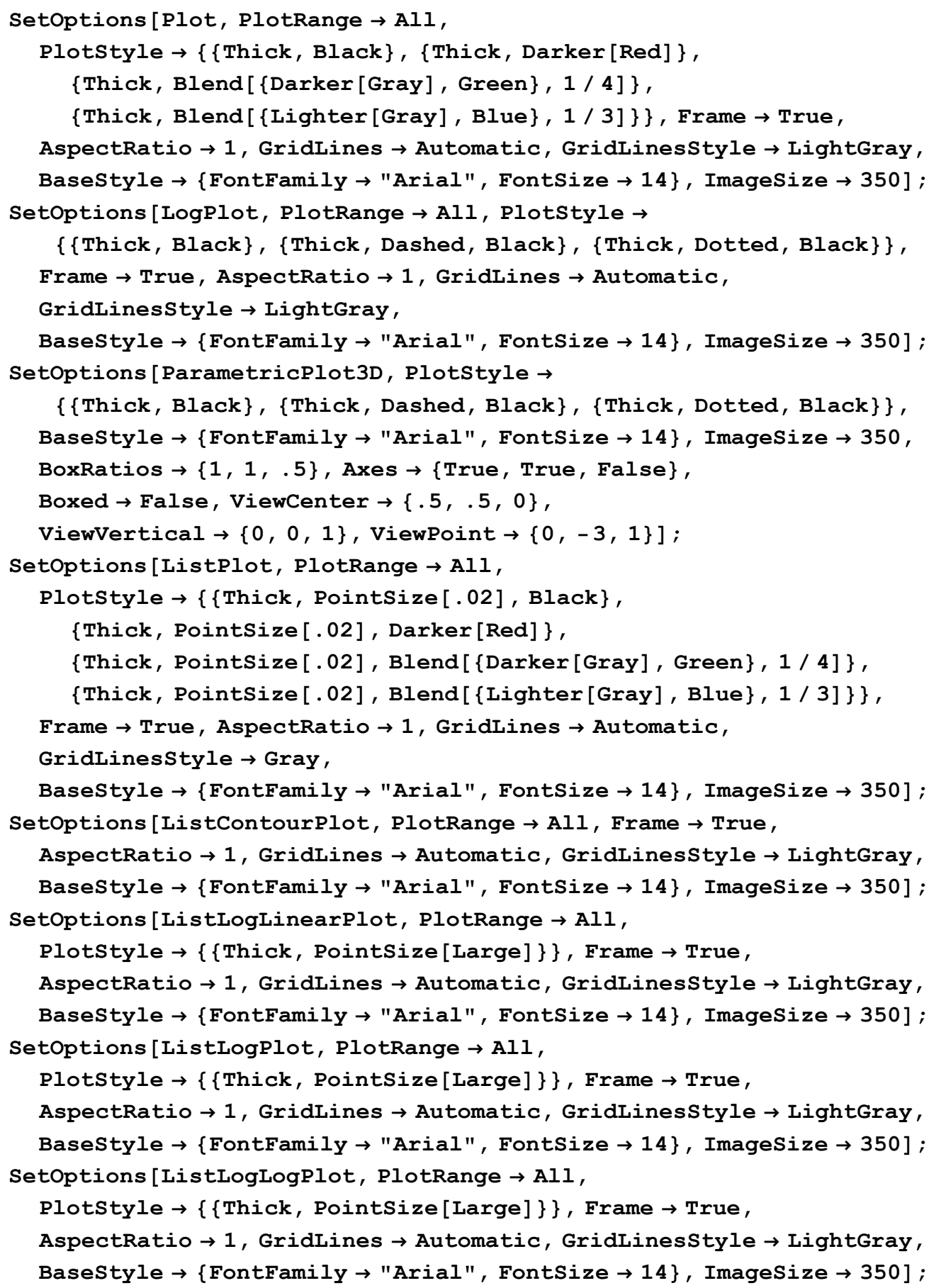




\section{Functions}

Hyperbolic trajectory functions

$\omega=\sqrt{e k / m}, k$ defines shape of hyperbolic potential. Functions of time $t$

$\operatorname{rHyp}\left[\omega_{-}, r 0_{-}, \operatorname{vr} 0_{-}, \theta_{-}\right]:=r 0 \operatorname{Cos}[\theta]+\operatorname{vr} 0 \operatorname{Sin}[\theta] / \omega$;

$\mathrm{zHyp}\left[\omega_{-}, \mathrm{z}_{-}, \mathrm{vz} 0_{-}, \theta_{-}\right]:=\mathrm{z} 0 \operatorname{Cosh}[\sqrt{2} \theta]+\mathrm{vz} 0 \sinh [\sqrt{2} \theta] /(\sqrt{2} \omega)$;

$\operatorname{vrHyp}\left[\omega_{-}, \mathbf{x} 0_{-}, \mathrm{v} 0_{-}, \theta_{-}\right]:=\omega$ Derivative $[0,0,0,1][\mathrm{rHyp}][\omega, \mathbf{x} 0, \mathrm{v} 0, \theta] ;$

vzHyp $\left[\omega_{-}, \mathbf{x} 0_{-}, \mathrm{v} 0_{-}, \theta_{-}\right]:=\omega$ Derivative $[0,0,0,1][\mathbf{z H y p}][\omega, \mathbf{x} 0, \mathrm{v} 0, \theta] ;$

Invert $z(t)$ to get $\theta(z)$

$\psi\left[\omega_{-}, \mathrm{z}_{-}, \mathrm{vz} 0_{-}\right]:=\operatorname{ArcTanh}[-\mathrm{vz} 0 /(\sqrt{2} \omega \mathrm{z} 0)]$

Өof $\left[\omega_{-}, \mathrm{z}_{-}, \mathrm{vz} 0_{-}, z_{-}\right]:=$

$2^{-1 / 2}(\psi[\omega, z 0, \mathrm{vz} 0]+\operatorname{ArcCosh}[(z / z 0) \operatorname{Cosh}[\psi[\omega, z 0, \mathrm{vz} 0]]]) ;$

\section{Davisson-Calbick approximation}

Trajectory through an aperture, from left to right.

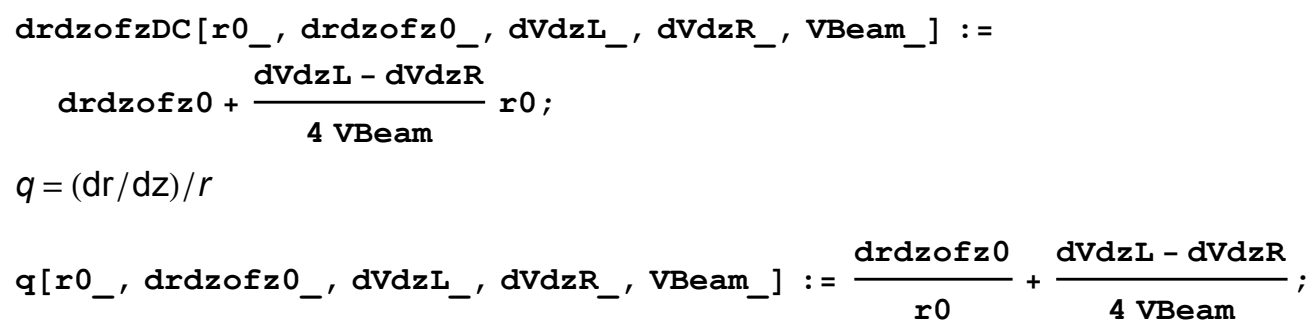

\section{Constant field trajectory functions}

Assumes second electrode is grounded.

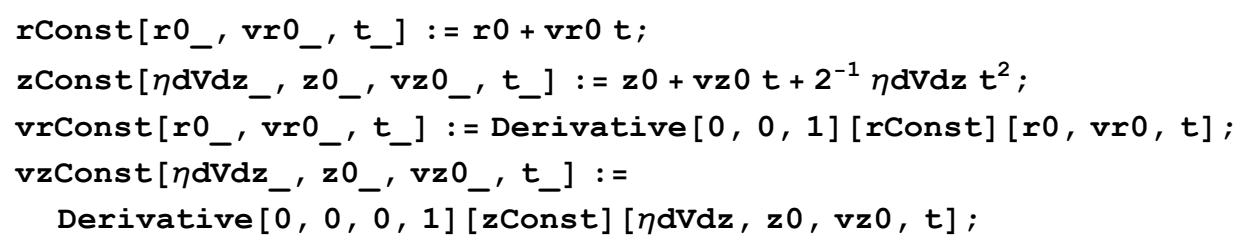




\section{Trajectories}

Variables: $v_{L}=V_{L} / V_{C}, v_{A}=V_{A} / V_{C}, v_{M}^{-1}=V_{M} / V_{C}$.

Constants: $\omega_{1}=\sqrt{e k_{1} / m}, k_{1}=V_{C}\left(v_{A}-v_{M}{ }^{-1}\right) / \ell^{2}$.

Lens half width $=s$.

Turn-around radial extent, limiting variable $r_{C}$

Diode alone

Position and velocity at the aperture

$$
\begin{aligned}
& z C\left[r C_{-}\right]:=\sqrt{\left\{2 \frac{v M-1}{v A V M-1}+r C^{2} / 2\right.} ; \\
& r A=\operatorname{Refine}[r \operatorname{Hyp}[\omega, r \mathrm{C}, 0, \theta \mathrm{A}[r \mathrm{C}]], \omega>0] \\
& \mathbf{z A}=\operatorname{Refine}[\mathbf{z H y p}[\omega, \mathbf{z C}[r \mathrm{C}], 0, \theta \mathbf{A}[\mathrm{rC}]], \omega>0] ; \\
& \operatorname{vrA}=\operatorname{Refine}[\operatorname{vrHyp}[\omega, \mathrm{rC}, 0, \theta \mathrm{A}[\mathrm{rC}]], \omega>0] \text {; } \\
& \mathrm{vzA}=\operatorname{Refine}[\operatorname{vzHyp}[\omega, \mathbf{z C}[\mathrm{rC}], 0, \theta \mathrm{A}[\mathrm{rC}]], \omega>0] \text {; } \\
& \operatorname{drdzA}=\operatorname{Refine}[\operatorname{vrA} / \mathrm{vzA}, \omega>0] \text {; } \\
& \operatorname{drdzAd}=\text { Refine }[\mathrm{drdzofzDC}[\mathrm{rA}, \operatorname{drdzA} \text {, } \\
& \left.2 \mathrm{VC}\left(\nu \mathrm{A}-\nu \mathrm{M}^{-1}\right)\{-2 \mathrm{zA}, 0,-\mathrm{VC}(1-\nu \mathrm{A})],\{\omega>0, V C<0\}\right] \\
& r \mathrm{C} \operatorname{Cos}[\theta \mathrm{A}[\mathrm{rC}]] \\
& -\frac{1}{2 l^{2}(1-v A)} \\
& r C\left(\nu \mathrm{A}-\frac{1}{v \mathrm{M}}\right) \sqrt{\frac{r \mathrm{C}^{2}}{2}+\frac{\rho^{2}(-1+v \mathrm{M})}{-1+v \mathrm{~A} \vee \mathrm{M}}} \operatorname{Cos}[\theta \mathrm{A}[\mathrm{rC}]] \operatorname{Cosh}[\sqrt{2} \theta \mathrm{A}[r \mathrm{C}]]- \\
& \underline{\operatorname{rCSCh}[\sqrt{2} \theta \mathrm{A}[\mathrm{rC}]] \operatorname{Sin}[\theta \mathrm{A}[\mathrm{rC}]]} \\
& \sqrt{2} \sqrt{\frac{r C^{2}}{2}+\frac{\rho^{2}(-1+v M)}{-1+\nu A V M}}
\end{aligned}
$$

Approximations made at the aperture

$$
\begin{aligned}
& \theta A 0\left[r C_{-}\right]:=\theta o f z[\omega, \mathbf{z C}[r C], 0,\{] ; \theta A 0[r C] \\
& r A 1\left[r C_{-}\right]:=r H y p[\omega, r C, 0, \theta A 0[r C]] ; r A 1[r C] \\
& \theta A 1\left[r C_{-}\right]:=\theta o f z\left[\omega, z C[r C], 0, \sqrt{\left\{^{2}+r A 1[r C]^{2} / 2\right.}\right] ; \theta A 1[r C]
\end{aligned}
$$

Image location $z^{\prime}$ and ray angle squared $\alpha^{\prime 2}$.

$\mathbf{z D i o d e}=\mathbf{z A}-(\text { Expand }[\operatorname{drdzAd} / \mathrm{rA}])^{-1}$

$\alpha$ SquaredDiode $=\operatorname{ArcTan}[-\operatorname{drdzAd}]^{2}$ 


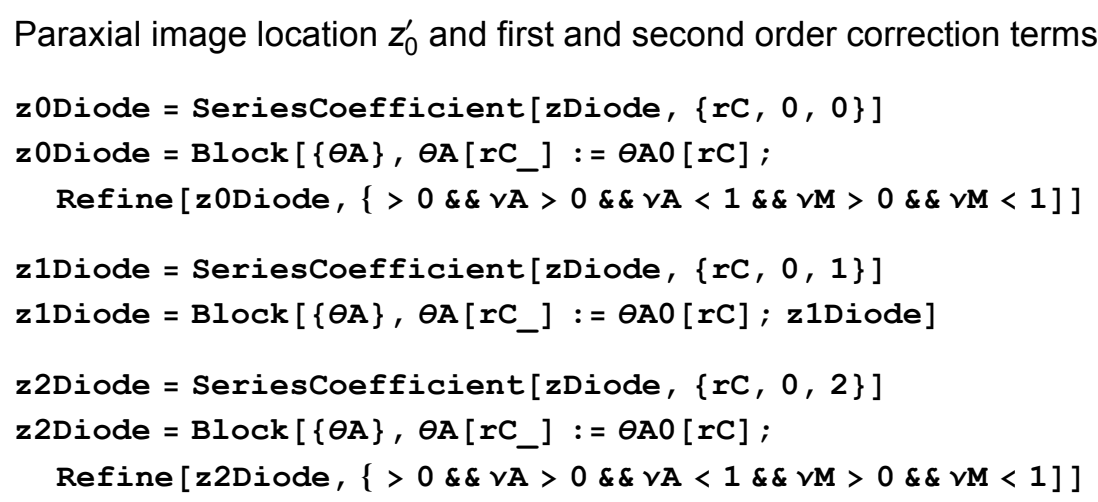

Second order angle expansion $a^{\prime 2}=\left(\alpha^{\prime 2}\right)_{1} r_{C}+\left(\alpha^{\prime 2}\right)_{2} r_{C}^{2}+O\left(r_{C}^{3}\right),\left(\alpha^{\prime 2}\right)_{1}=0$

$\alpha$ Squared1Diode $=$ SeriesCoefficient $[\alpha$ SquaredDiode,$\{r C, 0,1\}]$

$\alpha$ Squared1Diode $=\operatorname{Block}\left[\{\theta \mathrm{A}\}, \theta \mathrm{A}\left[\mathrm{rC}_{-}\right]:=\theta \mathrm{A} 0[\mathrm{rC}] ; \alpha \mathrm{Squared} 1 \mathrm{Diode}\right]$

$\alpha$ Squared2Diode $=$ SeriesCoefficient $[\alpha$ SquaredDiode,$\{r C, 0,2\}]$

$\alpha$ Squared2Diode $=\mathrm{Block}\left[\{\theta \mathrm{A}\}, \theta \mathrm{A}\left[\mathrm{rC} \_\right]:=\theta \mathrm{A} 0[\mathrm{rC}]\right.$;

Refine [aSquared2Diode, $\{>0 \& \& \vee A>0 \& \& \nu A<1 \& \& \vee M>0 \& \& \vee M<1]$ ]

Coefficient of chromatic aberration, $C_{C}=V_{C} \frac{2 \partial z_{0}}{\partial V_{C}}=2\left(v_{M} \partial_{v_{M}} z_{0}-v_{A} \partial_{v_{A}} z_{0}\right)$

CcDiode $=2\left(\nu M \partial_{V M} z\right.$ ODiode $-V A \partial_{V A} z$ ODiode $) ;$

Coefficient of spherical aberration, $z^{\prime}=z_{0}-2^{-1} C_{s} \alpha^{\prime 2} \longrightarrow C_{s}=-2 z_{2}^{\prime} /\left(\alpha^{\prime 2}\right)_{2}$

CsDiode $=-2$ z2Diode $/ \alpha$ Squared2Diode ;

Plots

z0DiodePlotFunction $=\mathbf{z}$ 0Diode $/ .\{\rightarrow 1 / . V A \rightarrow 0 ;$

Plot [z0DiodePlotFunction, $\{V M, .65, .995\}$, PlotRange $\rightarrow\{0,8\}]$

CcDiodePlotFunction $=$ CcDiode $/ .\{\rightarrow 1 / . V A \rightarrow 0$;

Plot [CcDiodePlotFunction, $\{V M, .7, .995\}$, PlotRange $\rightarrow\{0,-160\}$ ]

CsDiodePlotFunction $=$ CsDiode $/ .\{\rightarrow 1 / . \vee A \rightarrow 0$;

Plot [CsDiodePlotFunction, $\{V M, .7, .996\}$, PlotRange $\rightarrow\{0,-160\}$ ]

Plot [CcDiodePlotFunction / CsDiodePlotFunction, $\{v M, .7, .9963\}$, PlotRange $\rightarrow\{0,2\}]$

Apertureless triode, two hyperbolic regions

Position and velocity at the inner aperture 
$z C\left[r C_{-}\right]:=\sqrt{\left\{^{2} \frac{\nu M-1}{V A V M-1}+r C^{2} / 2\right.} ;$

Clear $[r A] ; r A\left[r C_{-}\right]:=\operatorname{Refine}[r H y p[\omega 1, r C, 0, \theta A[r C]], \omega 1>0]$;

Clear $\left.[\mathbf{z A}] ; \mathbf{z A}\left[\mathrm{rC}_{-}\right]:=\operatorname{Refine}\left[\mathrm{zHyp}\left[\omega 1, \mathrm{zC}_{[\mathrm{rC}}\right], 0, \theta \mathrm{A}[\mathrm{rC}]\right], \omega 1>0\right]$;

Clear [vrA] ; vrA [rC_] : = Refine [vrHyp $[\omega 1, r C, 0, \theta A[r C]], \omega 1>0]$;

Clear[vzA]; vzA [rC_] : = Refine [vzHyp $[\omega 1, \mathbf{z C}[r \mathrm{C}], 0, \theta \mathrm{A}[\mathrm{rC}]], \omega 1>0]$;

$\operatorname{drdzA}=\operatorname{Refine}[\mathrm{vrA}[\mathrm{rC}] / \mathrm{vzA}[\mathrm{rC}], \omega 1>0]$;

Aperture deflection

$\mathrm{drdzAd}=\operatorname{drdzofzDC}\left[r A[r C], \operatorname{drdzA}, 2 \mathrm{vC}\left(\nu \mathrm{A}-\mathrm{rM}^{-1}\right)\left\{^{-2} z \mathrm{~A}[\mathrm{rC}]\right.\right.$,

$2(-\mathrm{VC} V \mathrm{~A})\left(\mathrm{L}^{2}-\left\{^{2}\right)^{-1} \mathrm{zA}[\mathrm{rC}],-\mathrm{VC}(1-V \mathrm{~A})\right]$

factor $=(r \mathrm{CCos}[\theta \mathrm{A}[\mathrm{rC}]] \operatorname{Cosh}[\sqrt{2} \theta \mathrm{A}[\mathrm{rC}]] \mathrm{zC}[\mathrm{rC}])$;

drdzAd = factor Refine [Expand [ factor ${ }^{-1}$ drdzAd],$\left.\omega 1>0 \& \& \mathrm{VC}<0\right]$

$\mathrm{vzAd}=\frac{\omega 1}{\mathrm{rC}}\left(\frac{(\omega 1)^{-2} \operatorname{vrA}[r \mathrm{rC}]^{2}+(\omega 1)^{-2} \mathrm{vzA}[\mathrm{rC}]^{2}}{r \mathrm{C}^{-2}+\mathrm{rC}^{-2} \mathrm{drdzAd} \mathrm{d}^{2}}\right)^{1 / 2}$

$\operatorname{vrAd}=\operatorname{drdzAd} \mathrm{vzAd}$

Position and velocity at the outer aperture

$\omega 2=\omega 1\left(\frac{-V A V M}{V A V M-1} \frac{\left\{^{2}\right.}{L^{2}-\left\{^{2}\right.}\right)^{1 / 2} ;$

$r B=\operatorname{Refine}[r \operatorname{ryp}[\omega 2, \operatorname{rA}[r \mathrm{rC}], \operatorname{vrAd}, \theta \mathrm{B}[\mathrm{rC}]], \omega 1>0]$

$\mathrm{zB}=\operatorname{Refine}[\mathrm{zHyp}[\omega 2, \mathrm{zA}[\mathrm{rC}], \mathrm{vzAd}, \theta \mathrm{B}[\mathrm{rC}]], \omega 1>0]$

$\operatorname{vrB}=\operatorname{Refine}[\operatorname{vrHyp}[\omega 2, \operatorname{rA}[r \mathrm{C}], \operatorname{vrAd}, \theta \mathrm{B}[\mathrm{rC}]], \omega 1>0] ;$

$\mathrm{vzB}=\operatorname{Refine}[\mathrm{vzHyp}[\omega 2, \mathrm{zA}[\mathrm{rC}], \mathrm{vzAd}, \theta \mathrm{B}[\mathrm{rC}]], \omega 1>0] ;$

$\operatorname{drdzB}=\operatorname{Refine}[\mathrm{vrB} / \mathrm{vzB}, \omega 1>0]$

Approximations made at the inner aperture

$\theta \mathrm{A} 0\left[\mathrm{rC}_{-}\right]:=\theta \circ \mathrm{fz}[\omega 1, \mathbf{z C}[\mathrm{rC}], 0,\{] ; \theta \mathrm{A} 0[\mathrm{rC}]$

$r A 1\left[r C_{-}\right]:=r \operatorname{ryp}[\omega 1, r \mathrm{C}, 0, \Theta A 0[r C]] ; r A 1[r C]$

$\theta \mathrm{A} 1\left[r \mathrm{r}_{-}\right]:=\theta 0 \mathrm{zz}\left[\omega 1, \mathrm{zC}[\mathrm{rC}], 0, \sqrt{\left\{^{2}+r \mathrm{~A} 1[r \mathrm{C}]^{2} / 2\right.}\right] ; \theta \mathrm{A} 1[\mathrm{rC}]$

Approximations made at the outer aperture

$\theta \mathrm{BO}\left[\mathrm{rC}_{-}\right]:=\theta_{0 \mathrm{fz}}[\omega 2, \mathrm{zA}[\mathrm{rC}], \mathrm{vzA}[\mathrm{rC}], \mathrm{L}] ; \theta \mathrm{B} 0[\mathrm{rC}]$

$r B 1\left[r C_{-}\right]:=r H y p[\omega 2, r A[r C], v z A[r C], \theta B 0[r C]] ; r B 1[r C]$

$\theta \mathrm{B} 1\left[\mathrm{rC} \_\right]:=\theta \circ \mathrm{zz}\left[\omega 2, \mathrm{zA}[\mathrm{rC}], \mathrm{vzA}[\mathrm{rC}], \sqrt{\mathrm{L}^{2}+\mathrm{rB} 1[\mathrm{rC}]^{2} / 2}\right] ; \theta \mathrm{B} 1[\mathrm{rC}]$

Image location $z^{\prime}$ and ray angle squared $\alpha^{\prime 2}$. Explicity using the small angle approximation $\alpha \approx \tan \alpha=-r^{\prime}$. 


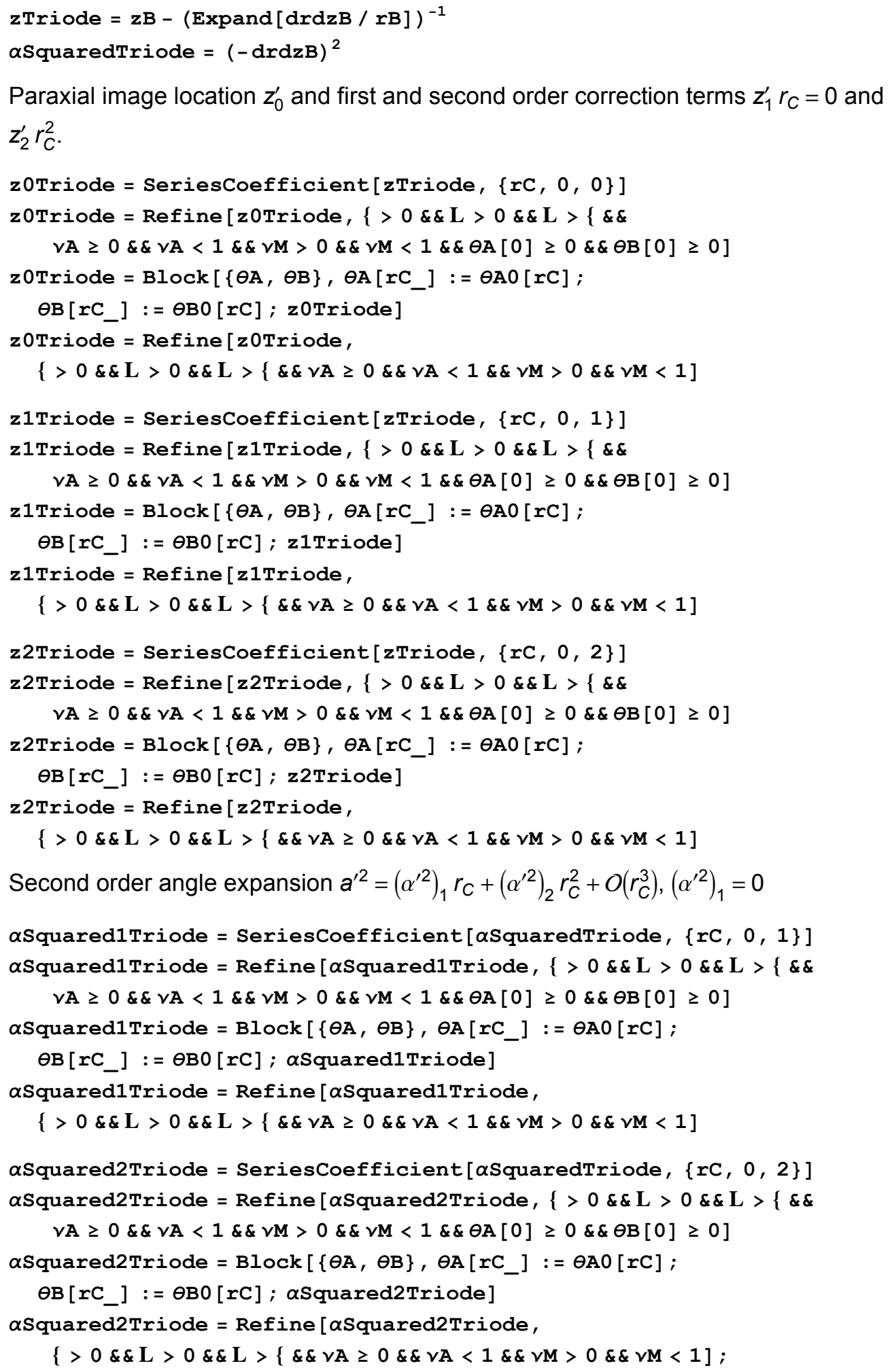


Coefficient of chromatic aberration, $C_{C}=V_{C} \frac{2 \partial z_{0}}{\partial V_{C}}=2\left(v_{M} \partial_{v_{M}} z_{0}-v_{A} \partial_{v_{A}} z_{0}\right)$

CcTriode $=2\left(\nu \mathrm{M} \partial_{\nu \mathrm{M}} \mathrm{z} 0\right.$ Triode $-\nu \mathrm{A} \partial_{\nu \mathrm{A}} \mathrm{z} 0$ Triode $) ;$

Coefficient of spherical aberration, $z^{\prime}=z_{0}-2^{-1} C_{s} \alpha^{\prime 2} \longrightarrow C_{s}=-2 z_{2}^{\prime} /\left(\alpha^{\prime 2}\right)_{2}$

CsTriode $=-2 \mathrm{z} 2 \mathrm{Triode} / \alpha$ Squared2Triode $;$

Plots for $v_{A}=0$ (should give diode behavior)

z0TriodePlotFunction $0=z 0 T r i o d e / .\left\{\rightarrow 1 / . V A \rightarrow 10^{-7} / . \mathbf{L} \rightarrow 2\right.$;

Plot [z0TriodePlotFunction0, $\{v \mathrm{M}, .65, .995\}$, PlotRange $\rightarrow\{0,8\}]$

CcTriodePlotFunction $0=$ CcTriode $/ .\left\{\rightarrow 1 / . \nu \mathrm{A} \rightarrow 10^{-7} / . \mathbf{L} \rightarrow 2\right.$;

Plot [CcTriodePlotFunctiono, $\{V M, .7, .995\}$, PlotRange $\rightarrow\{0,-160\}$ ]

CsTriodePlotFunction $0=$ CsTriode $/ .\left\{\rightarrow 1 / . \vee \mathrm{A} \rightarrow 10^{-7} / . \mathrm{L} \rightarrow 2\right.$;

Plot [CsTriodePlotFunctiono, $\{V M, .7, .996\}$, PlotRange $\rightarrow\{0,-160\}$ ]

Plot [CcTriodePlotFunction0 / CsTriodePlotFunction0,

$\{v M, .7, .9963\}$, PlotRange $\rightarrow\{0,2\}]$

Plots for $v_{A} \neq 0$ and $\ell / \mathcal{L}=0.783 / 1.665$ (design triode specs)

z0TriodePlotFunction $=$ z0Triode $/ .\{\rightarrow 0.783 / . \mathrm{L} \rightarrow 1.665$;

ParametricPlot $[\{\{v M, z 0 T r i o d e P l o t F u n c t i o n\}$,

$\{\nu \mathrm{M}, \mathrm{z} 0 \mathrm{TriodePlotFunction} 0\}\},\{v \mathrm{M}, .65, .995\}$,

$\left\{v A, 10^{-7}, .8\right\}$, PlotRange $\rightarrow\{\{.65,1\},\{0,8\}\}$, AspectRatio $\left.\rightarrow 1\right]$

CcTriodePlotFunction $=$ CcTriode $/ .\{\rightarrow 0.783 / . \mathrm{L} \rightarrow 1.665$;

ParametricPlot $[\{\{v M$, CcTriodePlotFunction $\}$,

$\{\nu M, C c T r i o d e P l o t F u n c t i o n 0\}\},\{V M, .7, .995\},\left\{\nu A, 10^{-7}, .8\right\}$,

PlotRange $\rightarrow\{\{.7,1\},\{0,-160\}\}$, AspectRatio $\rightarrow 1]$

CsTriodePlotFunction $=$ CsTriode $/ .\{\rightarrow 0.783 / . \mathrm{L} \rightarrow 1.665$;

ParametricPlot $[\{\{V M, C s T r i o d e P l o t F u n c t i o n\}$,

$\{\nu \mathrm{M}$, CsTriodePlotFunction 0$\}\},\{\nu \mathrm{M}, .7, .996\},\left\{\vee \mathrm{A}, 10^{-7}, .8\right\}$,

PlotRange $\rightarrow\{\{.7,1\},\{0,-160\}\}$, AspectRatio $\rightarrow 1]$

ParametricPlot $[\{\{v M, C c T r i o d e P l o t F u n c t i o n / C s T r i o d e P l o t F u n c t i o n\}$,

$\{V M$, CcTriodePlotFunction0/CsTriodePlotFunction 0$\}\},\{v M, .7, .997\}$,

$\left\{\nu \mathrm{A}, 10^{-7}, .8\right\}$, PlotRange $\rightarrow\{\{.7,1\},\{0,2\}\}$, AspectRatio $\left.\rightarrow 1\right]$

Plots for $v_{A} \neq 0$ and $\ell / \mathcal{L}=0.783 /(1.665+0.186)$ (front-less triode with lens)

z0TriodePlotFunctionAlt $=\mathbf{z} 0$ Triode $/ .\{\rightarrow 0.783 / . \mathbf{L} \rightarrow(1.665+0.186) ;$

ParametricPlot $[\{\{V M, z 0 T r i o d e P l o t F u n c t i o n A l t\}$,

$\{V M, z 0 T r i o d e P l o t F u n c t i o n 0\}\},\{v M, .65, .995\}$,

$\left\{v \mathrm{~A}, 10^{-7}, .8\right\}$, PlotRange $\rightarrow\{\{.65,1\},\{0,8\}\}$, AspectRatio $\left.\rightarrow 1\right]$ 


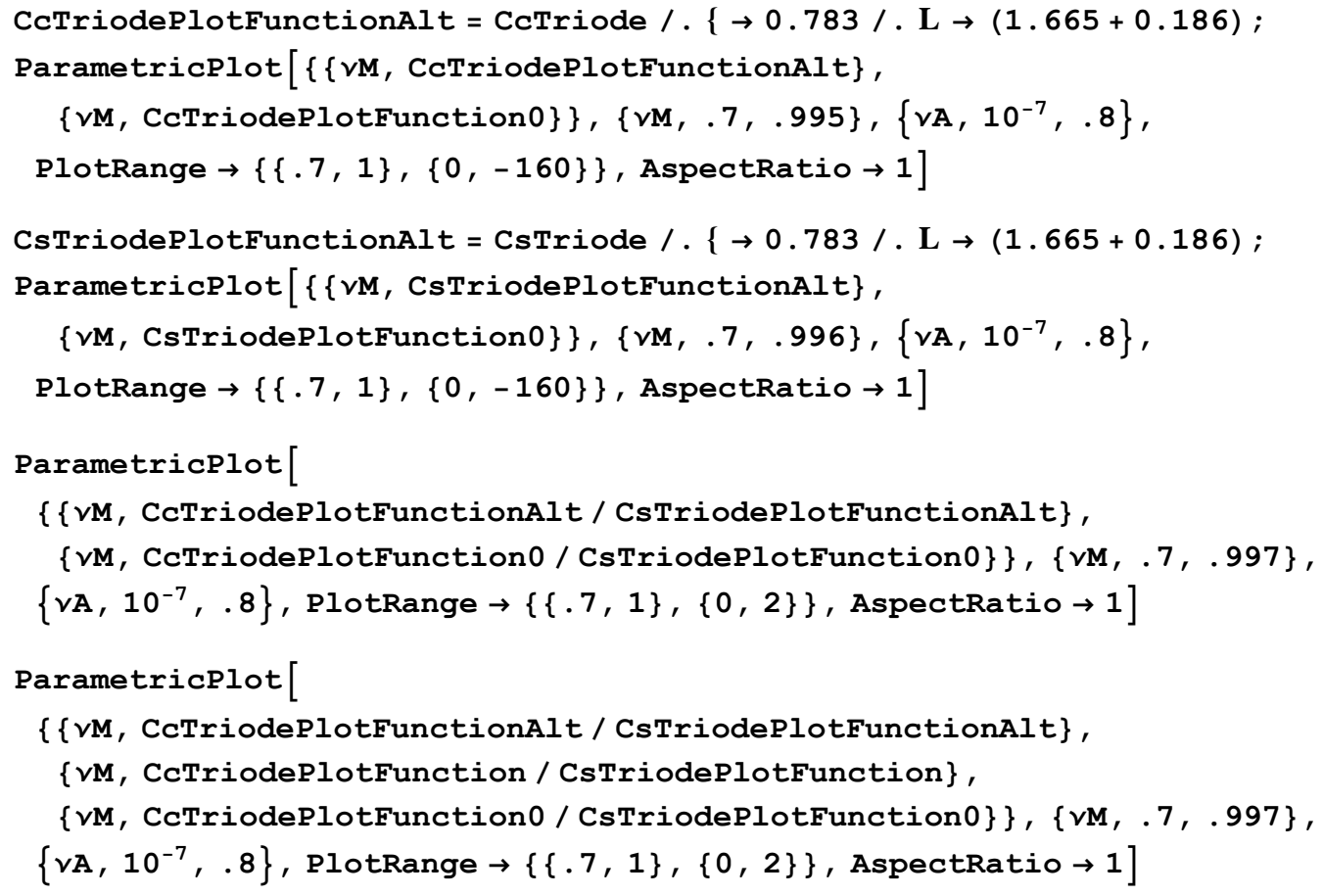

Position and velocity at the inner aperture

$$
\mathrm{zC}\left[\mathrm{rC} \mathrm{C}_{-}\right]:=\sqrt{\left\{^{2} \frac{V \mathrm{M}-1}{V \mathrm{~A} V \mathrm{M}-1}+r \mathrm{C}^{2} / 2\right.} ;
$$

Clear $[r A] ; r A\left[r C \_\right]:=\operatorname{Refine}[r H y p[\omega, r C, 0, \theta A[r C]], \omega>0]$;

Clear $[\mathrm{zA}] ; \mathbf{z A}[\mathrm{rC}]]:=\operatorname{Refine}[\mathbf{z H y p}[\omega, \mathbf{z C}[\mathrm{rC}], 0, \theta \mathrm{A}[\mathrm{rC}]], \omega>0]$;

Clear [vrA]; vrA [rC_] : = Refine $[\operatorname{vrHyp}[\omega, r C, 0, \theta \mathbb{A}[r \mathrm{C}]], \omega>0]$;

Clear [vzA] ; vzA [rC_] : = Refine [vzHyp $[\omega, \mathbf{z C}[r \mathrm{rC}], 0, \theta \mathrm{A}[\mathrm{rC}]], \omega>0]$;

Clear[drdzA]; drdzA [rC_] := Refine $[\operatorname{vrA}[r C] / v z A[r C], \omega>0]$;

Aperture deflection 


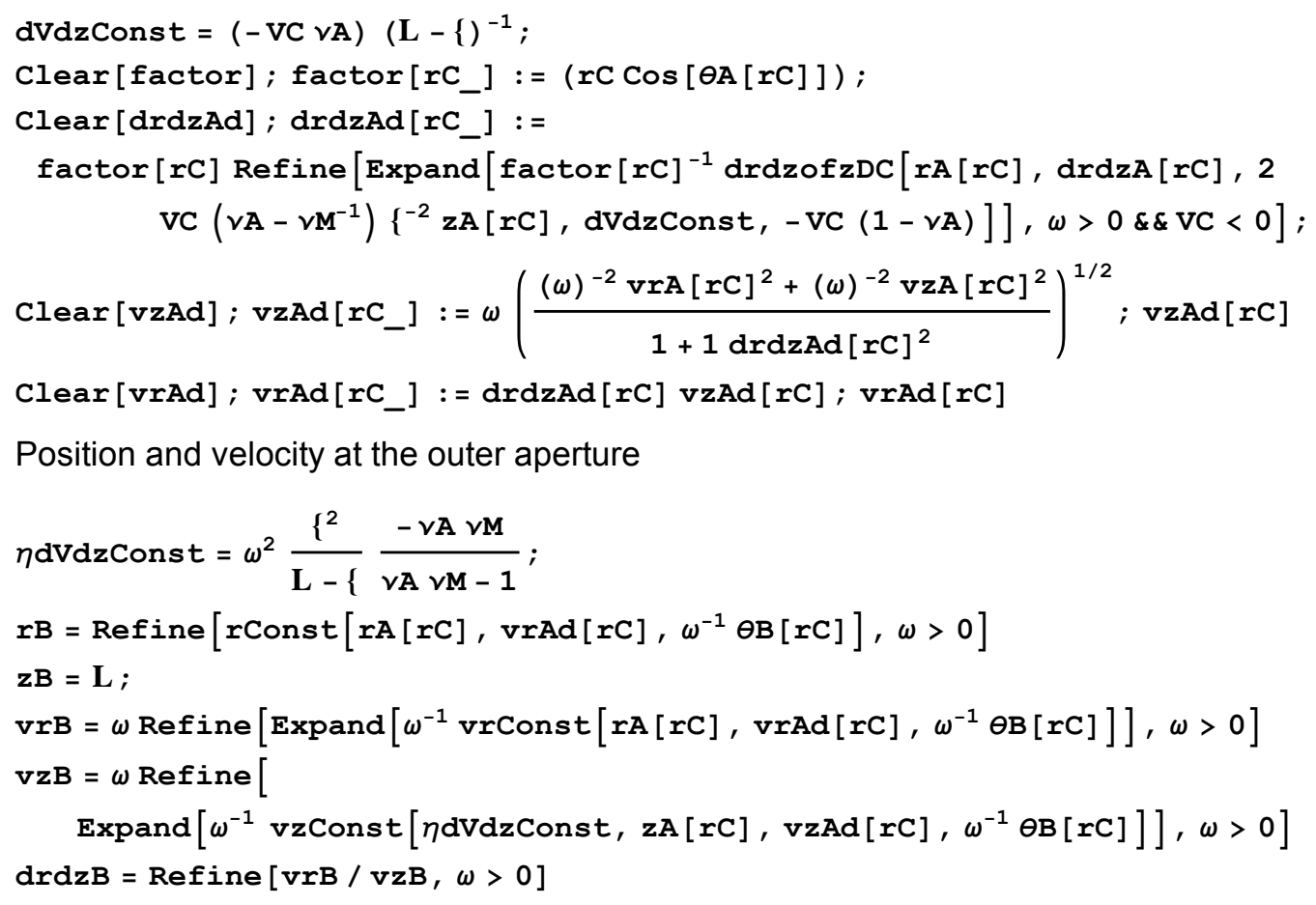

Position and velocity at the outer aperture

Approximations made at the inner aperture

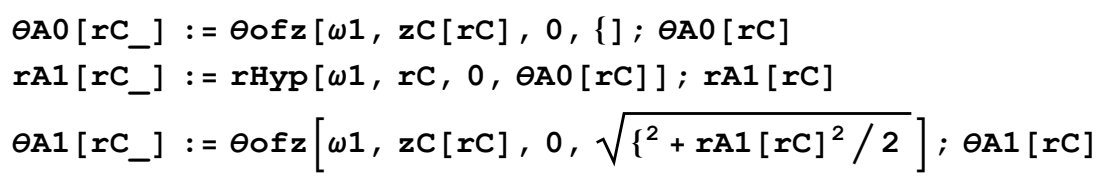

Exact time solution at the outer aperture

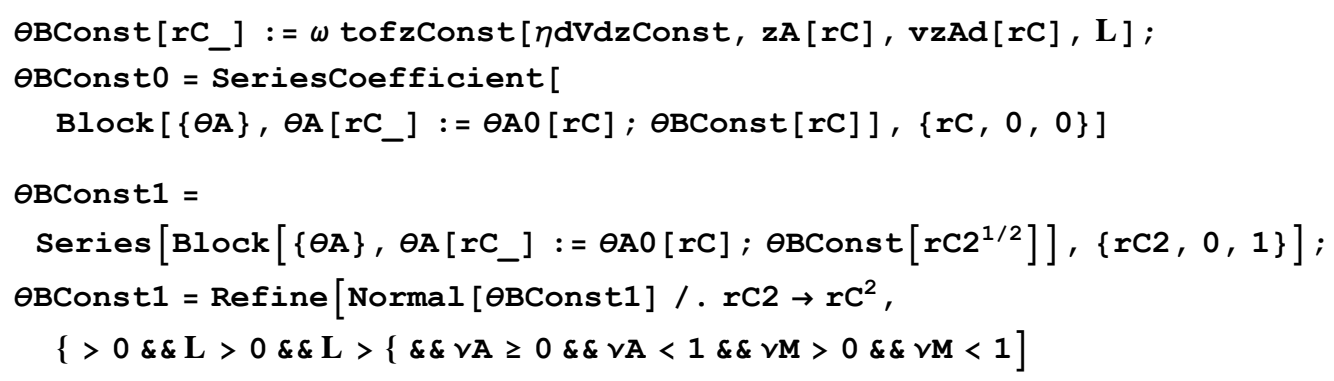

Paraxial image location $z_{0}^{\prime}$ and first and second order correction terms $z_{1}^{\prime} r_{C}=0$ and $z_{2}^{\prime} r_{C}^{2}$. 


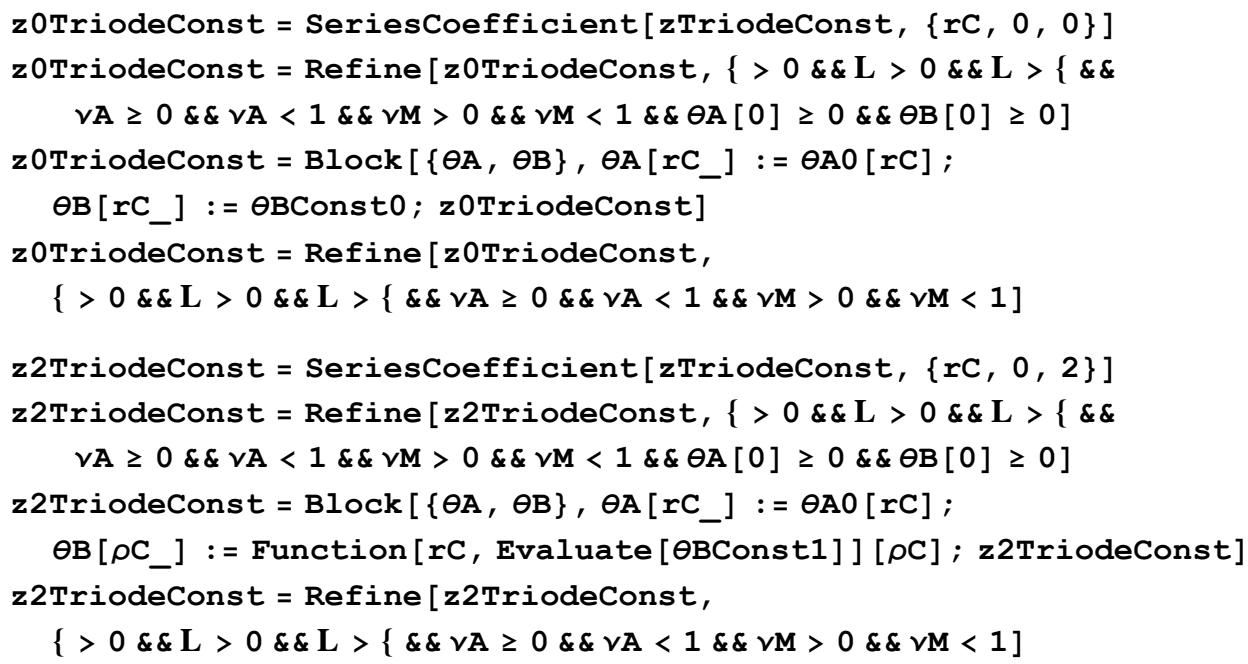

Plots for $v_{A}=0$ (should give diode behavior)

z0TriodeConstPlotFunction0 = z0TriodeConst $/ .\left\{\rightarrow 1 / . v \mathrm{~A} \rightarrow 10^{-7} / . \mathbf{L} \rightarrow 2\right.$; Plot [z0TriodeConstPlotFunctiono, $\{V M, .65, .995\}$, PlotRange $\rightarrow\{0,8\}$ ]

CcTriodeConstPlotFunction0 = CcTriodeConst $/ .\left\{\rightarrow 1 / . \vee \mathrm{A} \rightarrow 10^{-7} / . \mathrm{L} \rightarrow 2\right.$; Plot [CcTriodeConstPlotFunctiono, $\{V M, .7, .995\}$, PlotRange $\rightarrow\{0,-160\}$ ] CsTriodeConstPlotFunction $0=$ CsTriodeConst $/ .\left\{\rightarrow 1 / . V A \rightarrow 10^{-7} / . \mathbf{L} \rightarrow 2\right.$; Plot [CsTriodeConstPlotFunctiono, $\{V M, .7, .996\}$, PlotRange $\rightarrow\{0,-160\}$ ] Plot [CcTriodeConstPlotFunction0 / CsTriodeConstPlotFunction0, $\{V M, .7, .9963\}$, PlotRange $\rightarrow\{0,2\}]$

Plots for $v_{A} \neq 0$ and $\ell / \mathcal{L}=0.783 / 1.665$ (design triode specs) 


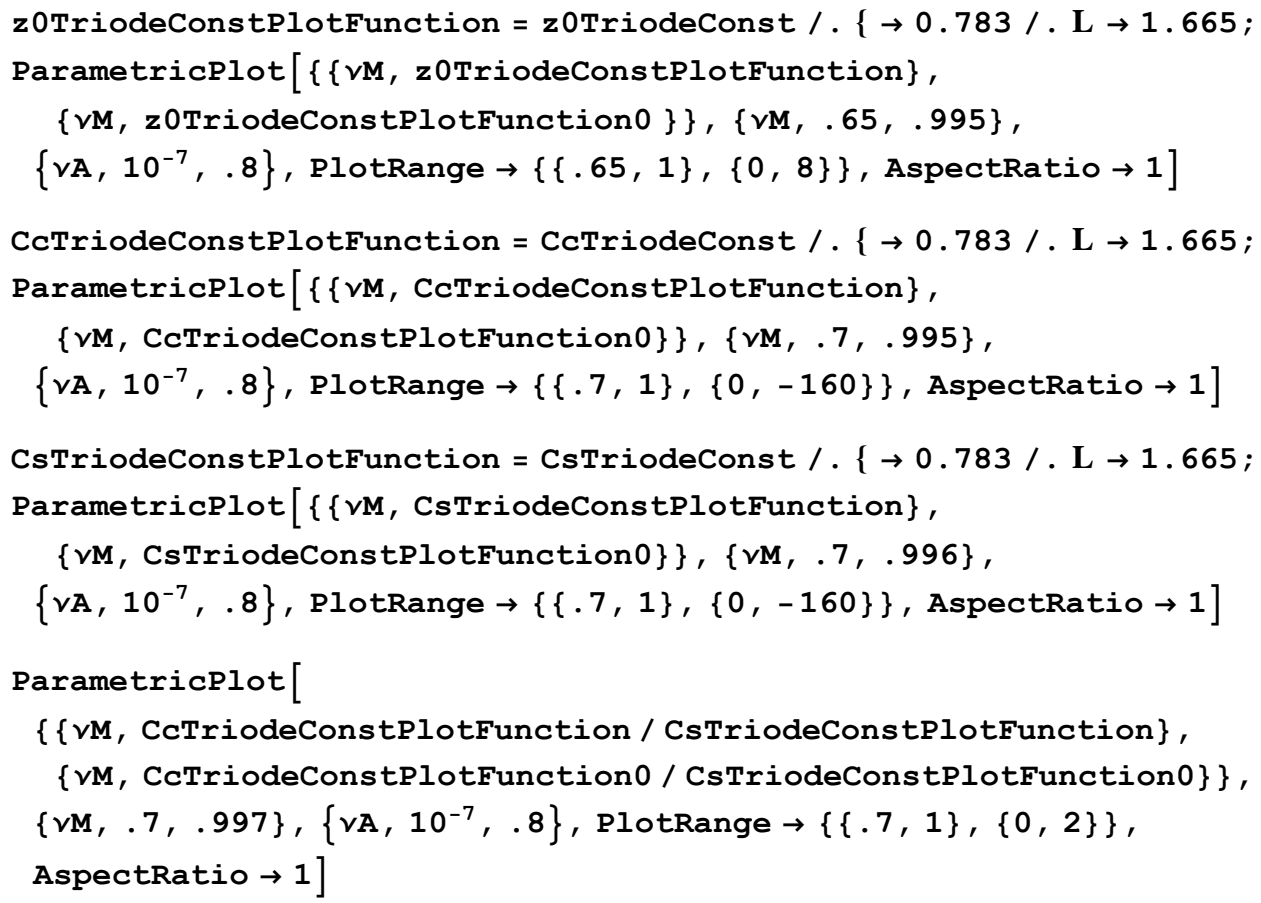

Plots for $v_{A} \neq 0$ and $\ell / \mathcal{L}=0.783 /(1.665+0.186)$ (front-less triode with lens)

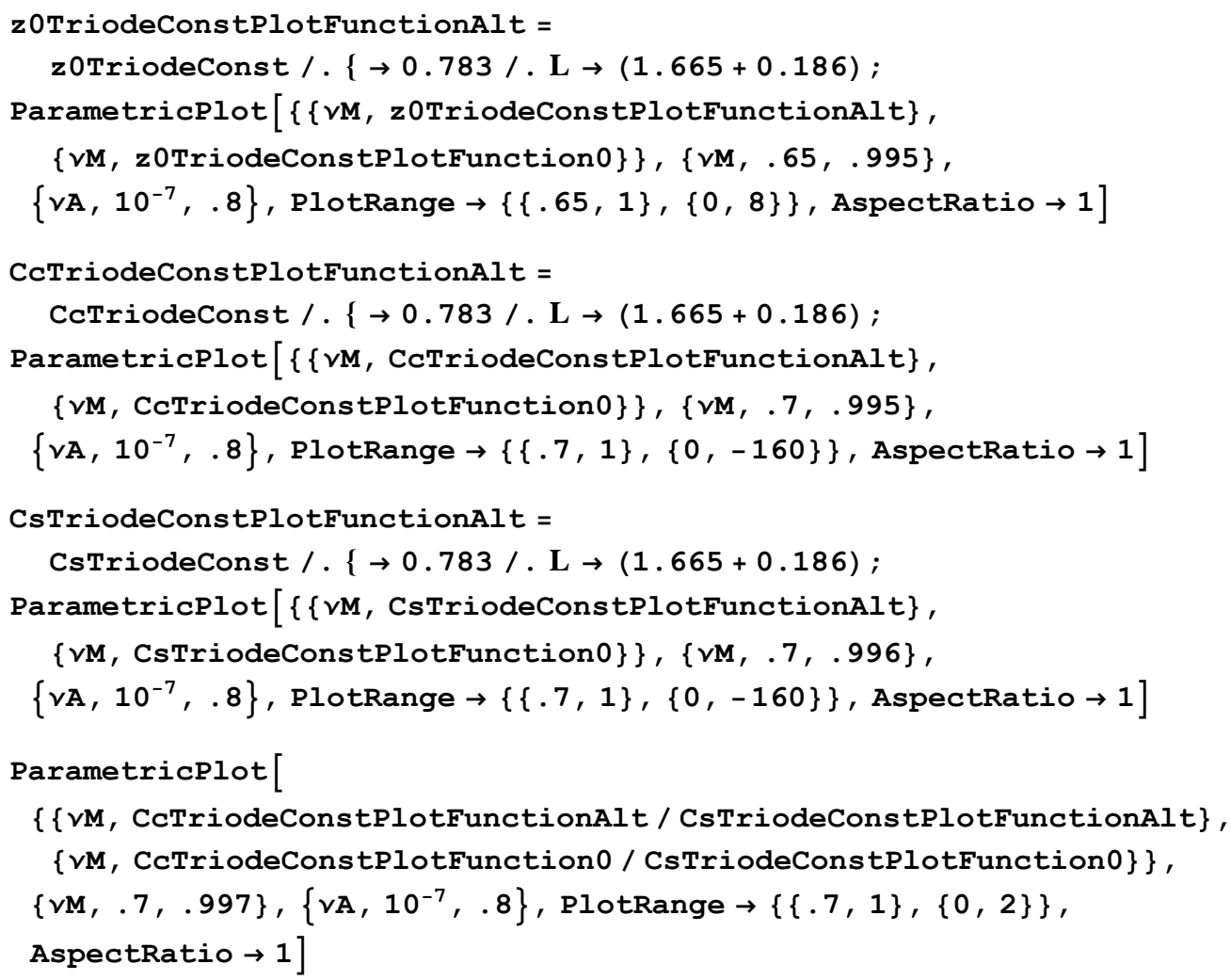




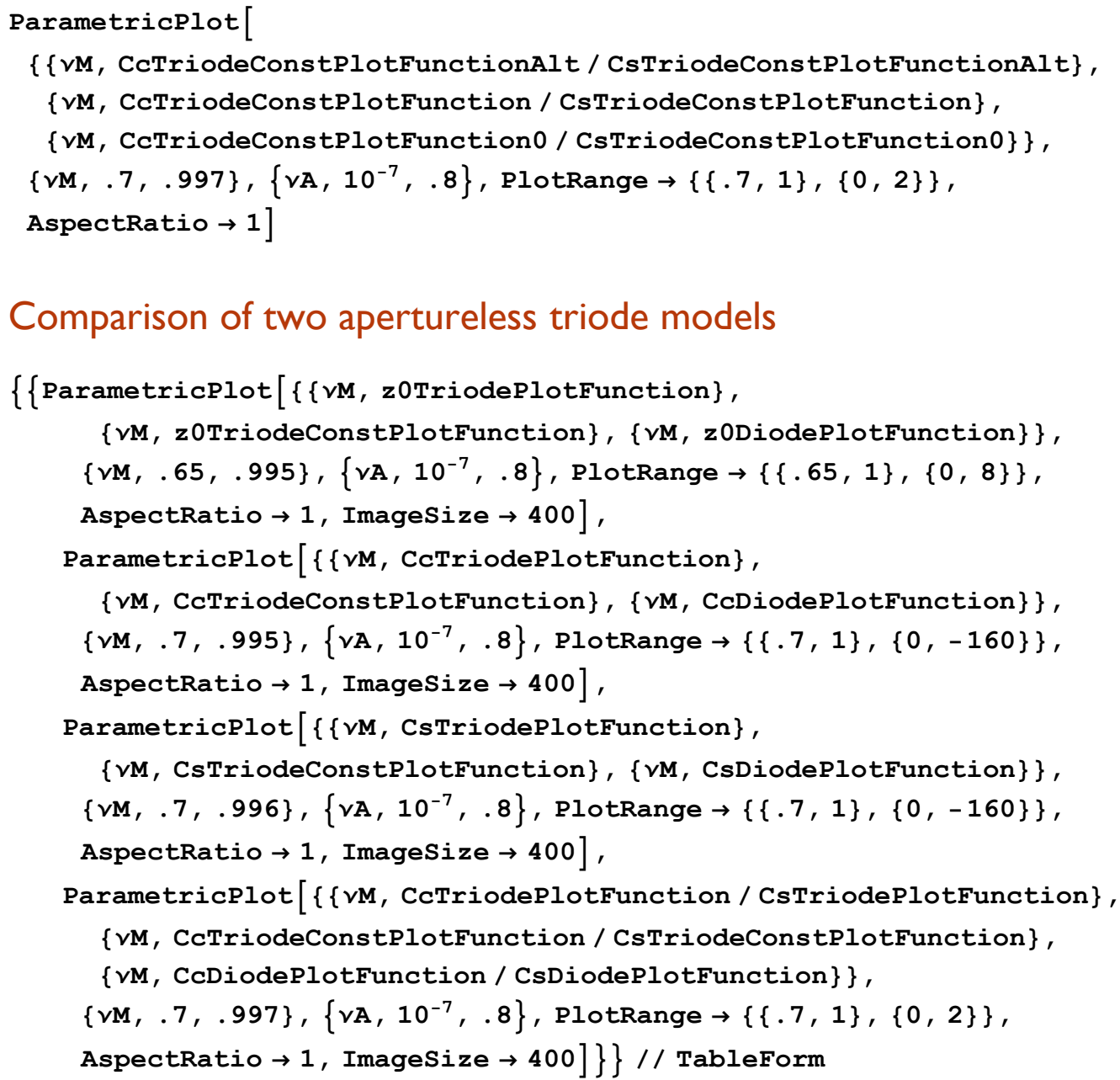




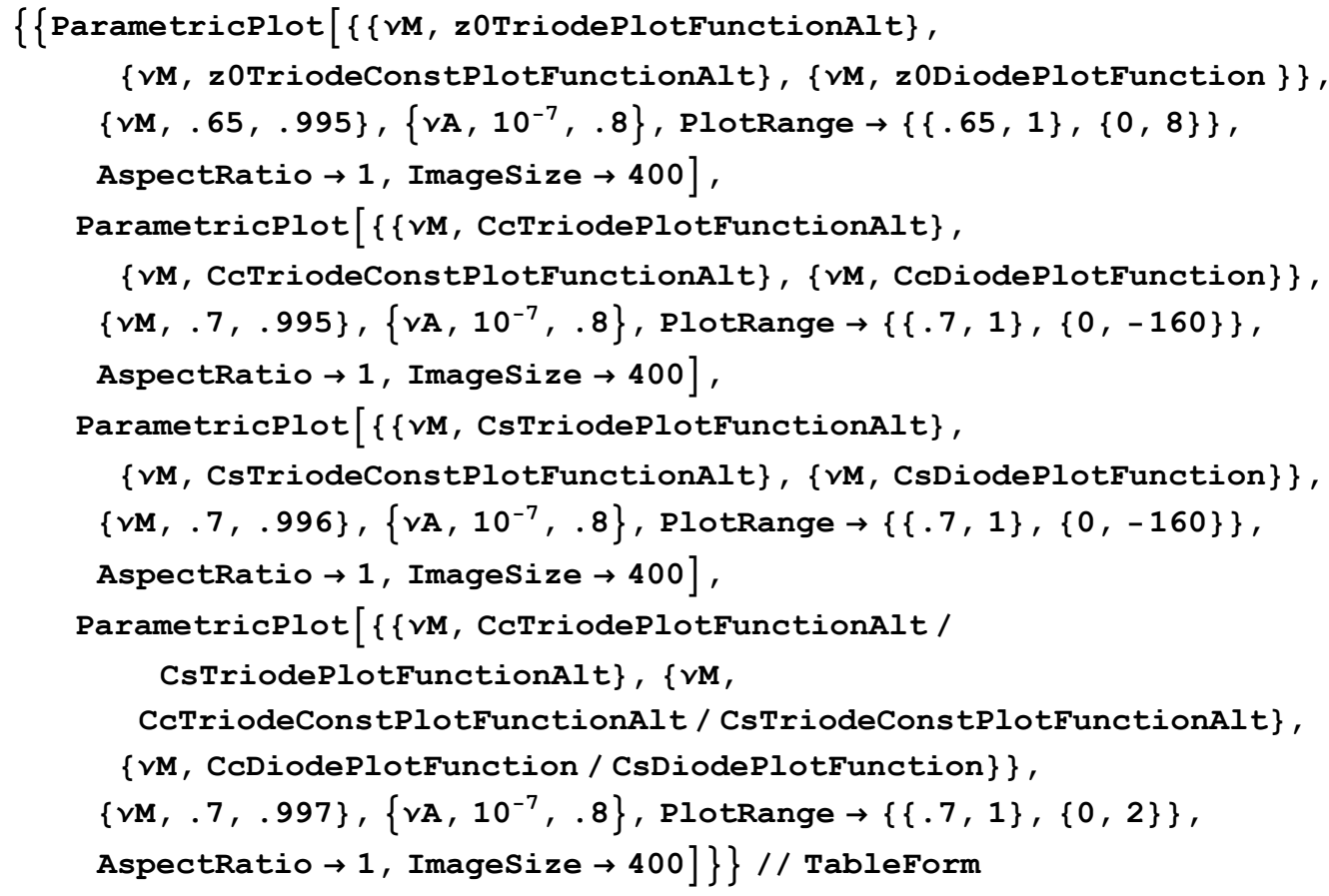

Triode with lens aperture, two hyperbolic regions

Reflection location contour

Clear $[\mathbf{z C}]$;

$\mathrm{zC}\left[r C_{-}\right]:=\left\{\sqrt{\frac{1-\nu \mathrm{M}}{1-\nu \mathrm{A} V \mathrm{M}}+r \mathrm{C}^{2} /\left(2\left\{^{2}\right)\right.} ;\right.$

Position and velocity at the inner aperture

Clear $[r A] ; r A\left[r C \_\right]:=\operatorname{Refine}[r H y p[\omega 1, r C, 0, \theta \mathbb{A}[r C]], \omega 1>0] ; r A[r C]$

Clear $[\mathbf{z A}]$;

$\mathbf{z A}[r \mathrm{rC}]:=\operatorname{Refine}[\mathrm{zHyp}[\omega 1, \mathbf{z C}[\mathrm{rC}], 0, \theta \mathrm{A}[\mathrm{rC}]], \omega 1>0] ; \mathrm{zA}[\mathrm{rC}]$

Clear [vrA] ;

$\operatorname{vrA}\left[r C_{-}\right]:=\operatorname{Refine}[\operatorname{vrHyp}[\omega 1, r \mathrm{C}, 0, \theta \mathrm{A}[r \mathrm{rC}]], \omega 1>0] ; \operatorname{vrA}[r \mathrm{r}]$

Clear [vzA];

vzA [rC_] := Refine [vzHyp $[\omega 1, \mathbf{z C}[r \mathrm{r}], 0, \theta \mathrm{A}[\mathrm{rC}]], \omega 1>0] ; \mathrm{vzA}[\mathrm{rC}]$

Clear [drdzA] ; drdzA [rC_] : = Refine $[v r A[r C] / v z A[r C], \omega 1>0] ; \operatorname{drdzA}[r C]$

Inner aperture deflection 


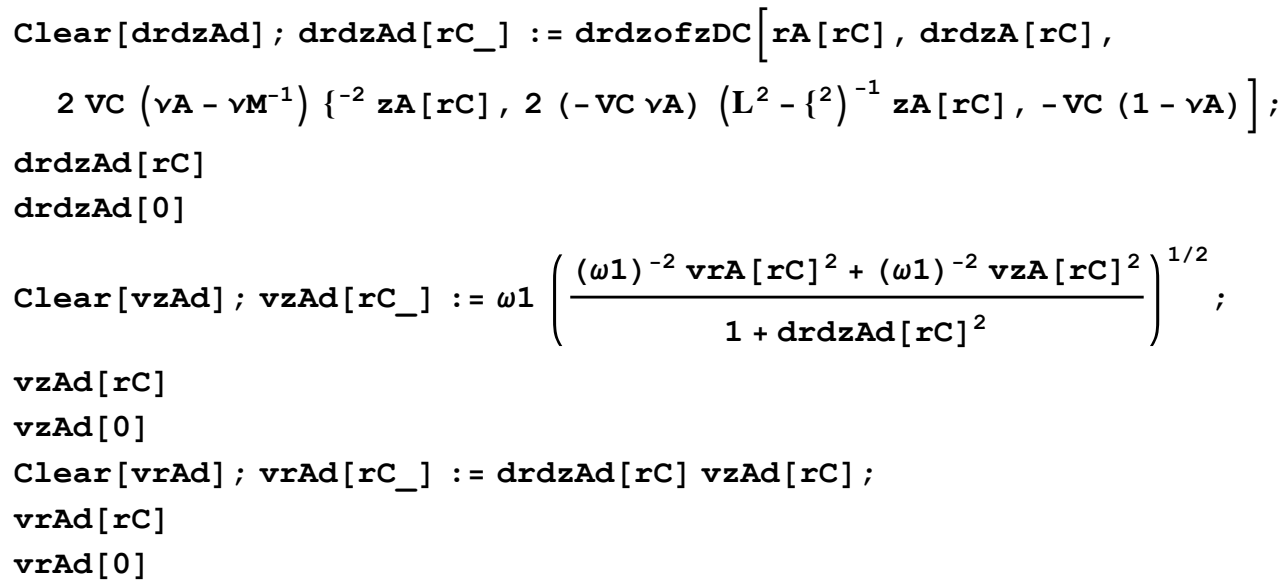

Position and velocity at the outer aperture

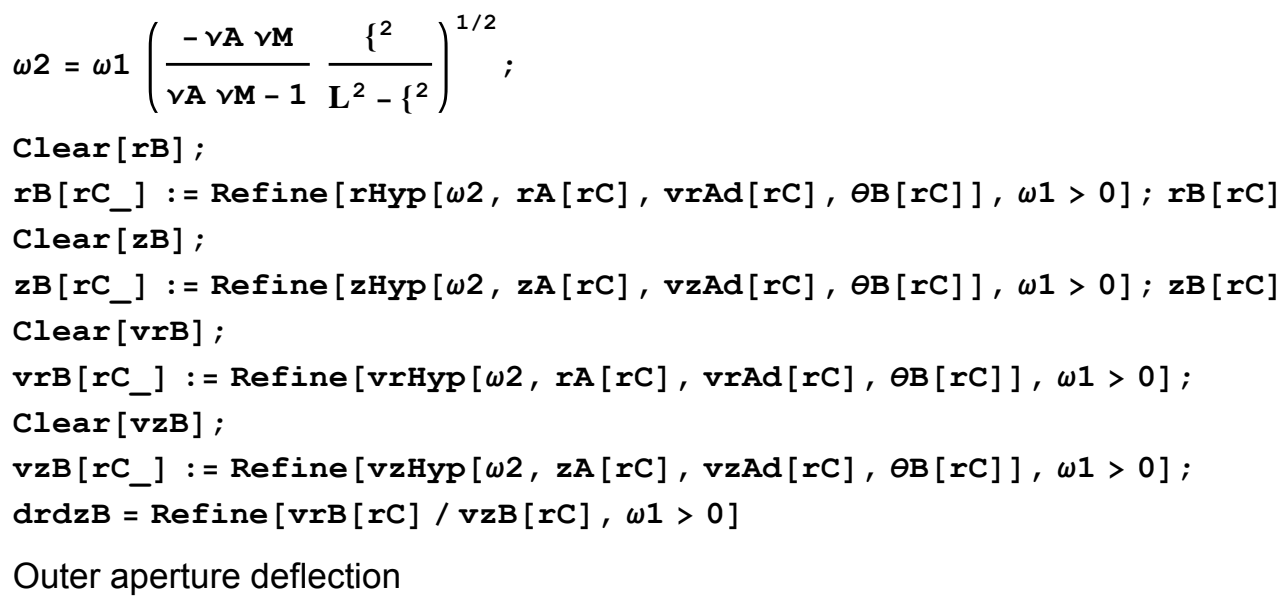

Outer aperture deflection

$\operatorname{drdzBd}=\operatorname{drdzofzDC}[r \mathrm{~B}[r \mathrm{C}], \operatorname{drdzB}$,

$2(-\mathrm{VC} \vee \mathrm{A})\left(\mathrm{L}^{2}-\left\{^{2}\right)^{-1} \mathrm{zB}[\mathrm{rC}], \mathrm{VC} \vee \mathrm{L} / \mathrm{s},-\mathrm{VC}\right] / . \mathrm{VC} \rightarrow 1$

$\mathrm{qB}=\mathrm{q}\left[\mathrm{rB}[\mathrm{rC}], \mathrm{drdzB}, 2(-\mathrm{vC} v \mathrm{~A})\left(\mathrm{L}^{2}-\left\{^{2}\right)^{-1} \mathrm{zB}[r \mathrm{C}], \mathrm{vC} v \mathrm{~L} / \mathrm{s},-\mathrm{vC}\right] / . \mathrm{VC} \rightarrow 1\right.$

Approximations made at the inner aperture

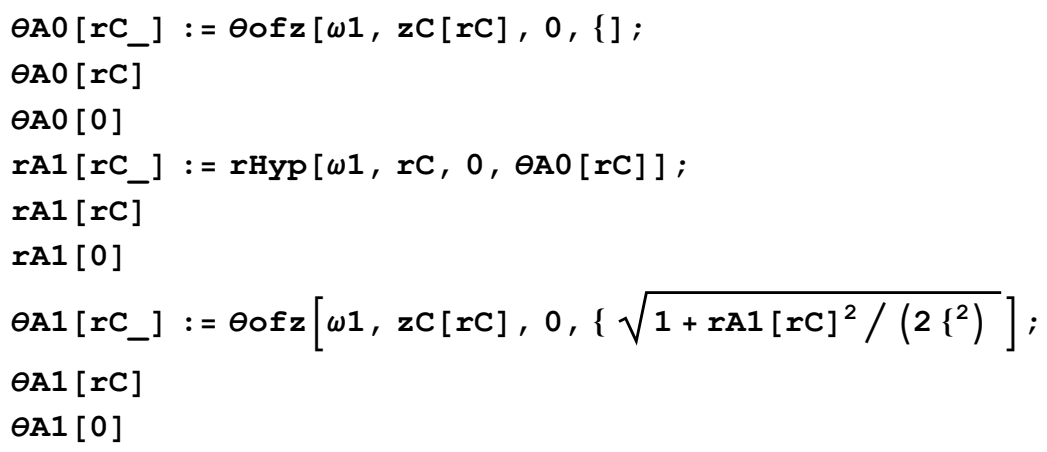


Approximations made at the outer aperture

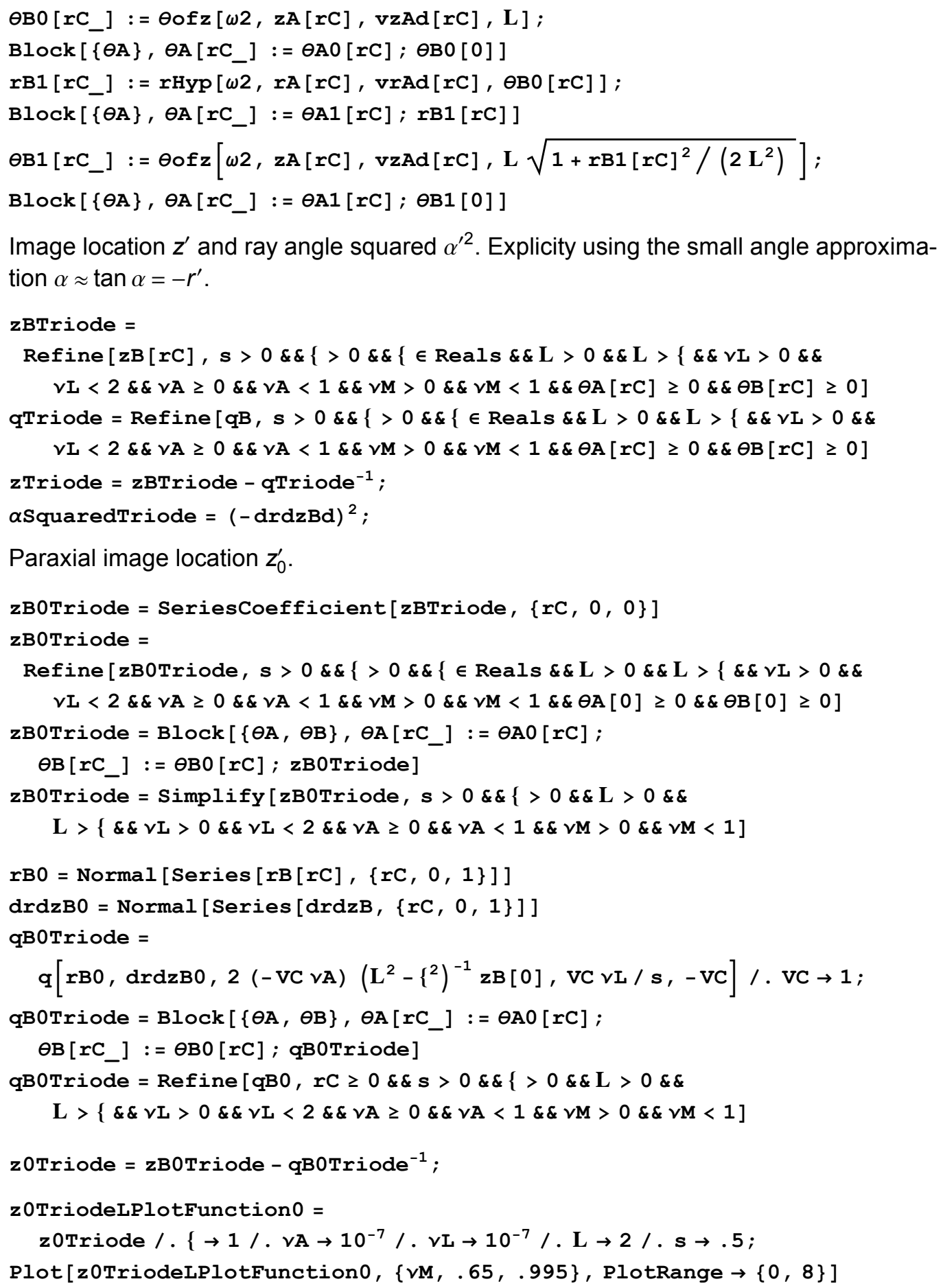




\section{Appendix D Code, simion: swept-back lens}

The cylindrical cross-section of lens geometries can be imported in SIMION from the design specification, e.g., App. Appendix A or more precisely App. ??. SIMION 8.0.6 was then used to determine the potential distribution inside the lens. Alternatively, a scale-independent geometry file (GEM) can generate the lens geometry, though at considerably more effort. This latter approach was used in earlier attempts, and below is the Lua code which generates the GEM file, the SIMION potential array (PA), and then refines the array to determine the electric potential distribution. Scaling and refinement options are found on the last line of the code.

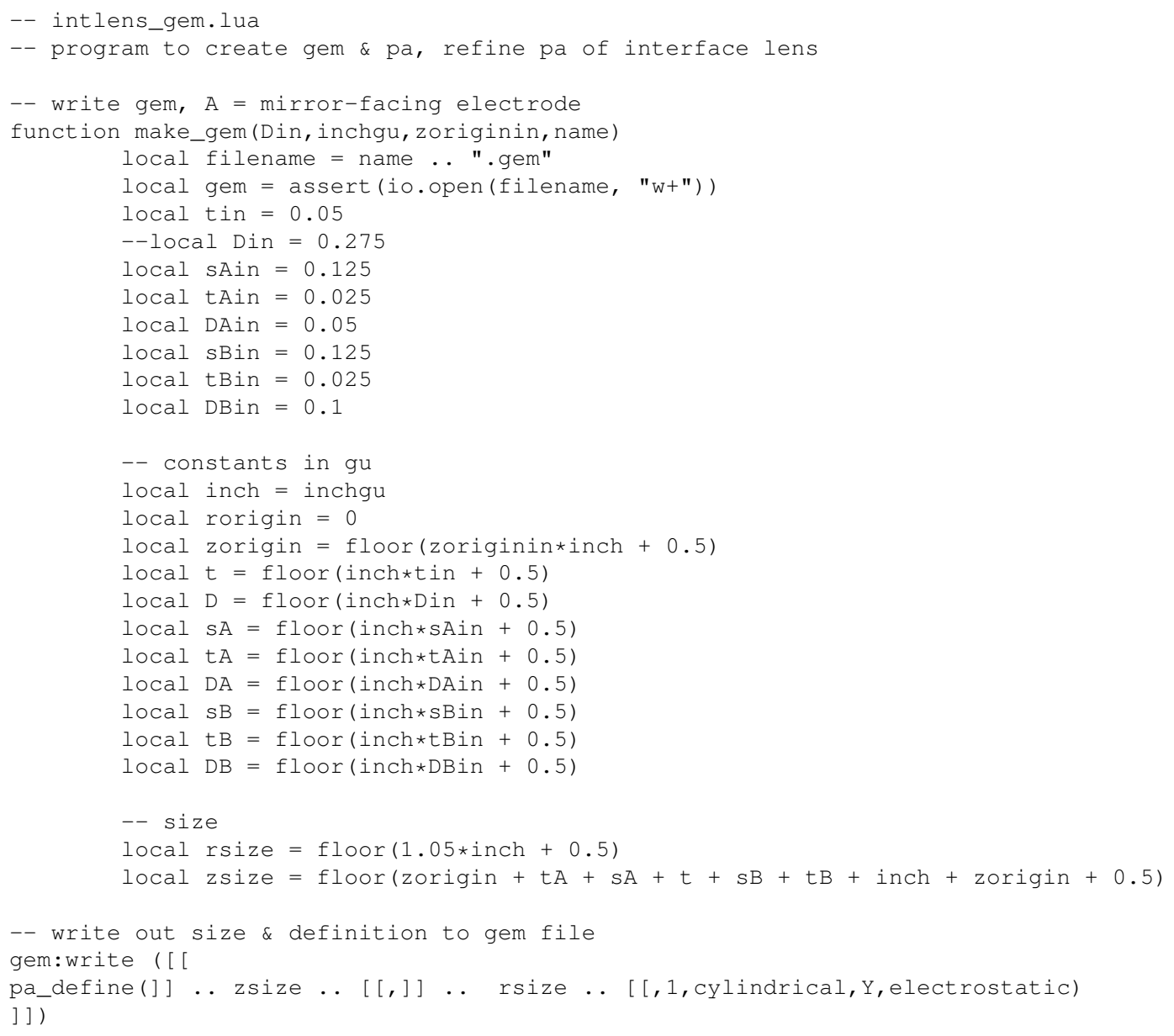




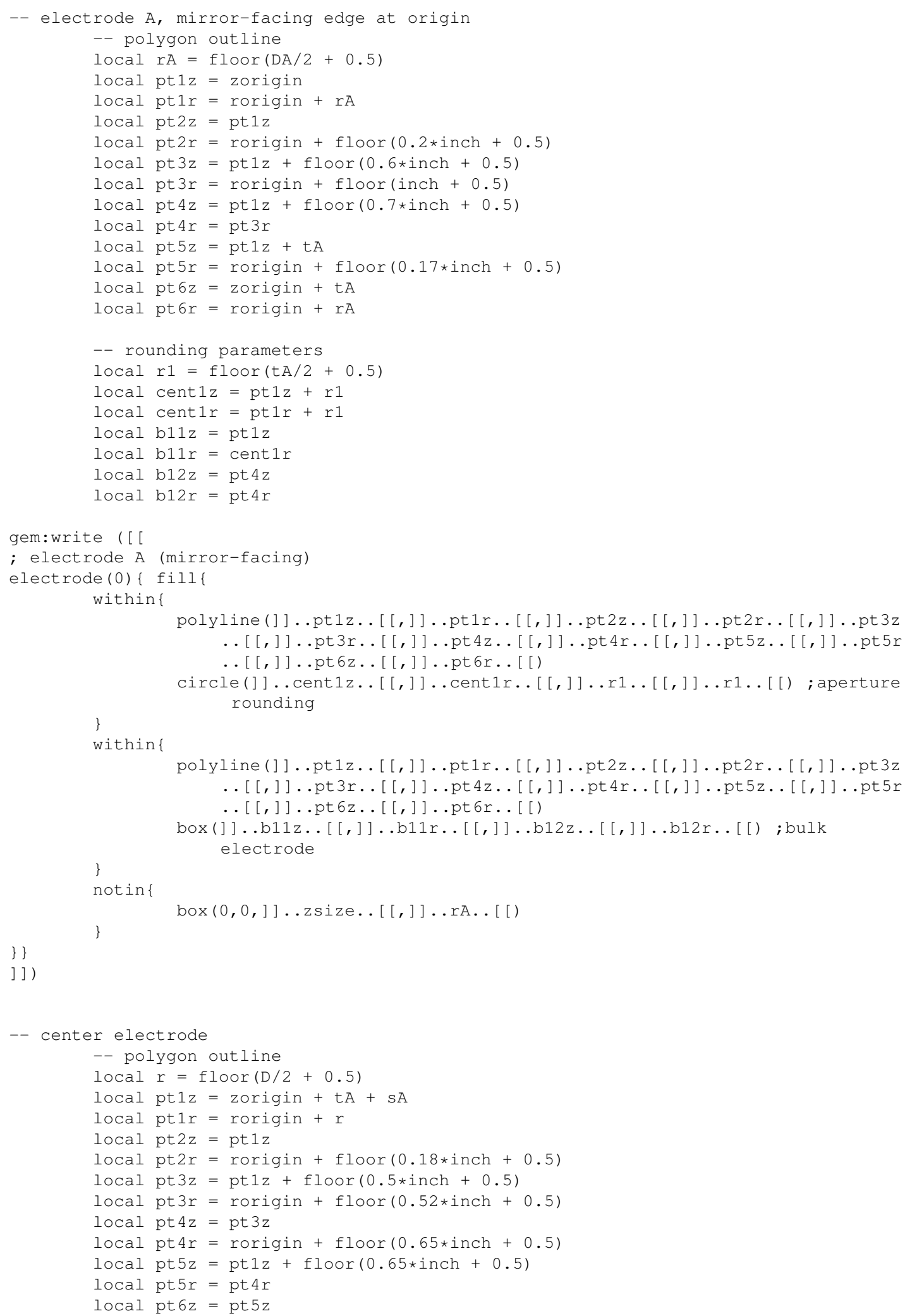




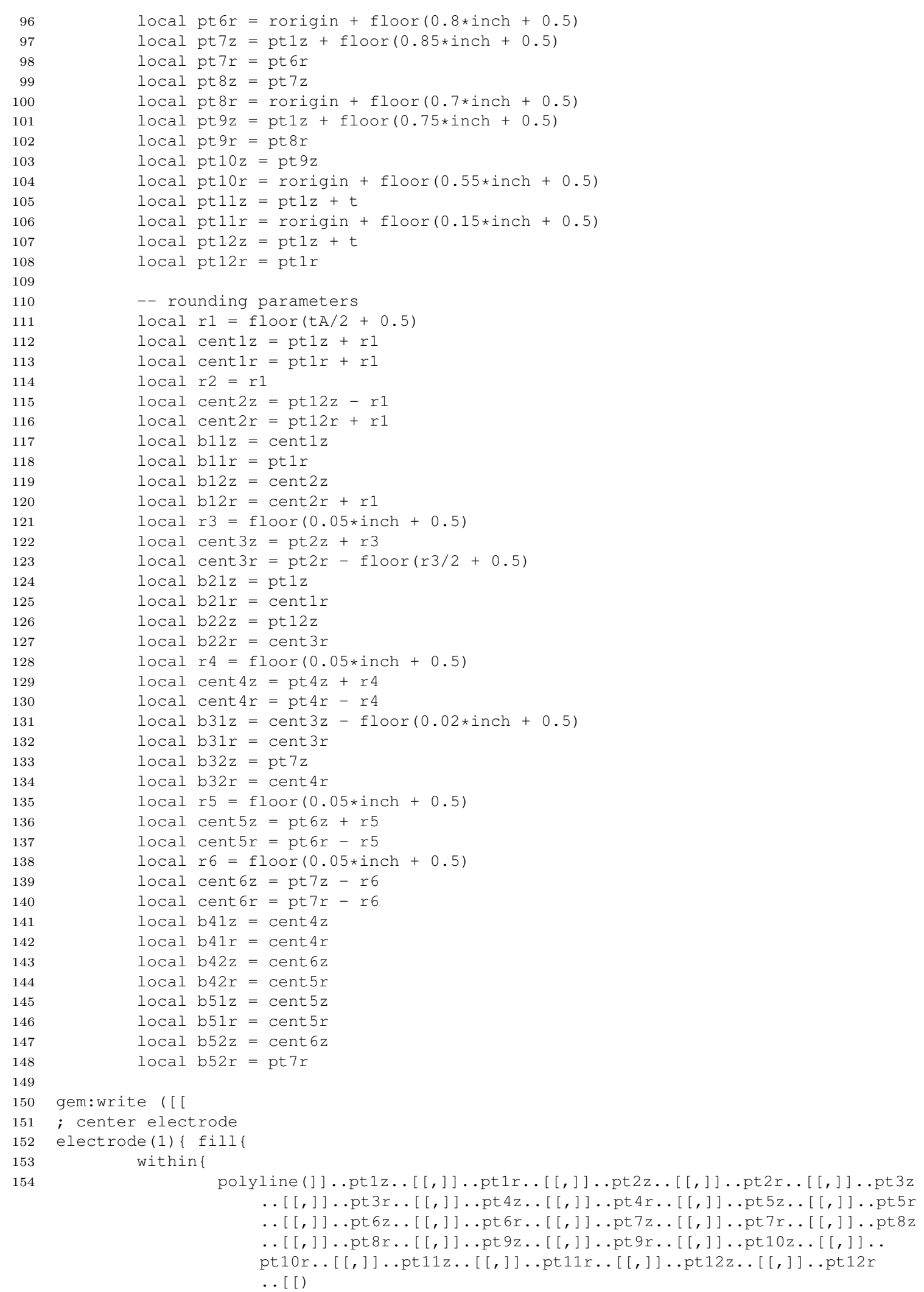




155
156
157
158

160

161

162

163

164

165

166

167

168

169

170

171
172

172
173

174

175

176

177

178

179

180

181

182

183

184

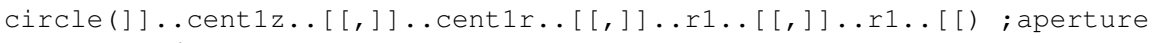
rounding

\}

within\{

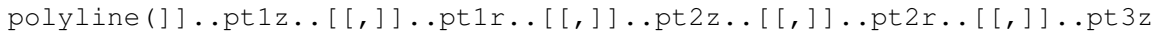
$\ldots[[],] \ldots \operatorname{pt} 3 r \ldots[[],] \ldots \operatorname{pt} 4 z \ldots[[],] \ldots \operatorname{pt} 4 r \ldots[[],] \ldots \operatorname{pt} 5 z \ldots[[],] \ldots \operatorname{pt} 5 r$ $\ldots[[],] \ldots \operatorname{pt} 6 z \ldots[[],] \ldots \operatorname{pt} 6 r \ldots[[],] \ldots \operatorname{pt} 7 z \ldots[[],] \ldots \operatorname{pt} 7 r \ldots[[],] \ldots \operatorname{pt} 8 z$ $\ldots[[],] \ldots \operatorname{pt} 8 \mathrm{p} \ldots[[],] \ldots \operatorname{pt} 9 z \ldots[[],] \ldots \operatorname{pt} 9 r \ldots[[],] \ldots \operatorname{pt} 10 z \ldots[[],] \ldots$

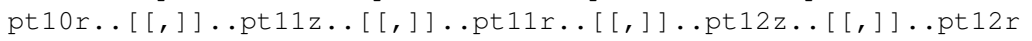
.. [ [ )

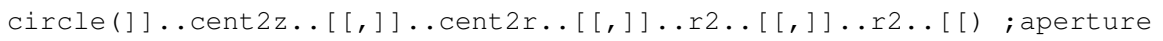
rounding

\section{] ] )}

gem:write ([ [

within\{

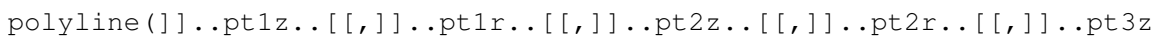
$\ldots[[],] \ldots \operatorname{pt} 3 r \ldots[[],] \ldots \operatorname{pt} 4 z \ldots[[],] \ldots \operatorname{pt} 4 r \ldots[[],] \ldots \operatorname{pt} 5 z \ldots[[],] \ldots \operatorname{pt} 5 r$ $\ldots[[],] \ldots \operatorname{pt} 6 z \ldots[[],] \ldots \operatorname{pt} 6 r \ldots[[],] \ldots \operatorname{pt} 7 z \ldots[[],] \ldots \operatorname{pt} 7 r \ldots[[],] \ldots \operatorname{pt} 8 z$ $\ldots[[],] \ldots \operatorname{pt} 8 r \ldots[[],] \ldots \operatorname{ptgz} \ldots[[],] \ldots \operatorname{pt} 9 r \ldots[[],] \ldots \operatorname{pt} 10 z \ldots[[],] \ldots$

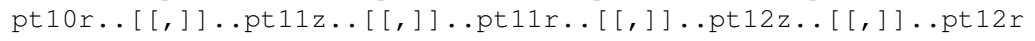
.. [ [ )

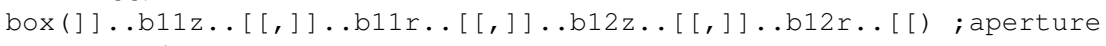
rounding, bulk

\}

; within \{

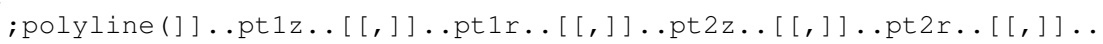

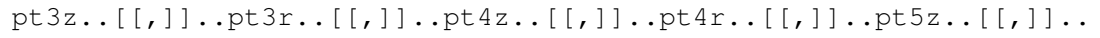
pt5r..[[,] $] \ldots \operatorname{pt} 6 z \ldots[[],] \ldots \operatorname{pt} 6 r \ldots[[],] \ldots \operatorname{pt} 7 z \ldots[[],] \ldots \operatorname{pt} 7 r \ldots[[],] \ldots$

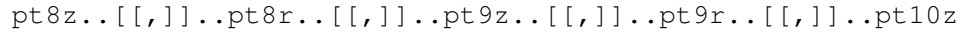
$\ldots[[],] \ldots \operatorname{pt} 10 r \ldots[[],] \ldots \operatorname{pt} 11 z \ldots[[],] \ldots \operatorname{pt} 11 r \ldots[[],] \ldots \operatorname{pt} 12 z \ldots[[],] \ldots$ pt $12 r \ldots[$ [ ]

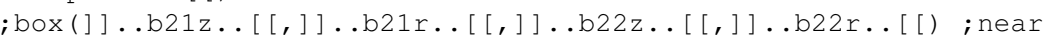
aperture bulk electrode

; \}

] ])

gem:write ( [ [

within \{

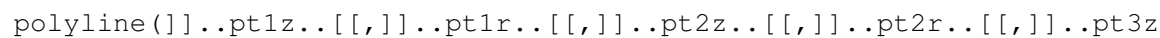
$\ldots[[],] \ldots \operatorname{pt} 3 r \ldots[[],] \ldots \operatorname{pt} 4 z \ldots[[],] \ldots \operatorname{pt} 4 r \ldots[[],] \ldots \operatorname{pt} 5 z \ldots[[],] \ldots \operatorname{pt} 5 r$ $\ldots[[],] \ldots \operatorname{pt} 6 z \ldots[[],] \ldots \operatorname{pt} 6 r \ldots[[],] \ldots \operatorname{pt} 7 z \ldots[[],] \ldots \operatorname{pt} 7 r \ldots[[],] \ldots \operatorname{pt} 8 z$ $\ldots[[],] \ldots \operatorname{pt} 8 \mathrm{p} \ldots[[],] \ldots \operatorname{pt} 9 z \ldots[[],] \ldots \operatorname{ptgr} \ldots[[],] \ldots \operatorname{pt} 10 z \ldots[[],] \ldots$

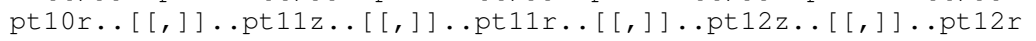
.. [ [ ]

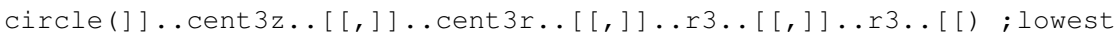
edge rounding

\}

within \{

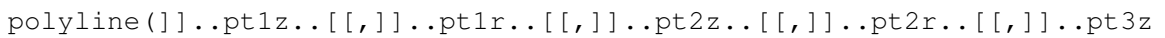
$\ldots[[],] \ldots \operatorname{pt} 3 r \ldots[[],] \ldots \operatorname{pt} 4 z \ldots[[],] \ldots \operatorname{pt} 4 r \ldots[[],] \ldots \operatorname{pt} 5 z \ldots[[],] \ldots \operatorname{pt} 5 r$ $\ldots[[],] \ldots \operatorname{pt} 6 z \ldots[[],] \ldots \operatorname{pt} 6 r \ldots[[],] \ldots \operatorname{pt} 7 z \ldots[[],] \ldots \operatorname{pt} 7 r \ldots[[],] \ldots \operatorname{pt} 8 z$ $\ldots[[],] \ldots \operatorname{pt8r} \ldots[[],] \ldots \operatorname{ptgz\ldots [}[],] \ldots \operatorname{ptgr\ldots [}[],] \ldots \operatorname{pt} 10 z \ldots[[],] \ldots$

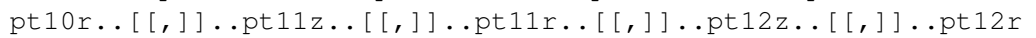

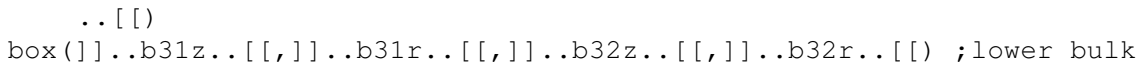
electrode

] ])

gem:write ( [ [

within \{

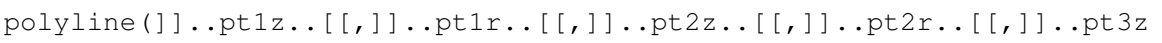
$\ldots[[],] \ldots \operatorname{pt} 3 r \ldots[[],] \ldots \operatorname{pt} 4 z \ldots[[],] \ldots \operatorname{pt} 4 r \ldots[[],] \ldots \operatorname{pt} 5 z \ldots[[],] \ldots \operatorname{pt} 5 r$ $\ldots[[],] \ldots \operatorname{pt} 6 z \ldots[[],] \ldots \operatorname{pt} 6 r \ldots[[],] \ldots \operatorname{pt} 7 z \ldots[[],] \ldots \operatorname{pt} 7 r \ldots[[],] \ldots \operatorname{pt} 8 z$ $\ldots[[],] \ldots \operatorname{pt} 8 r \ldots[[],] \ldots \operatorname{ptgz} \ldots[[],] \ldots \operatorname{pt} 9 r \ldots[[],] \ldots \operatorname{pt} 10 z \ldots[[],] \ldots$ 


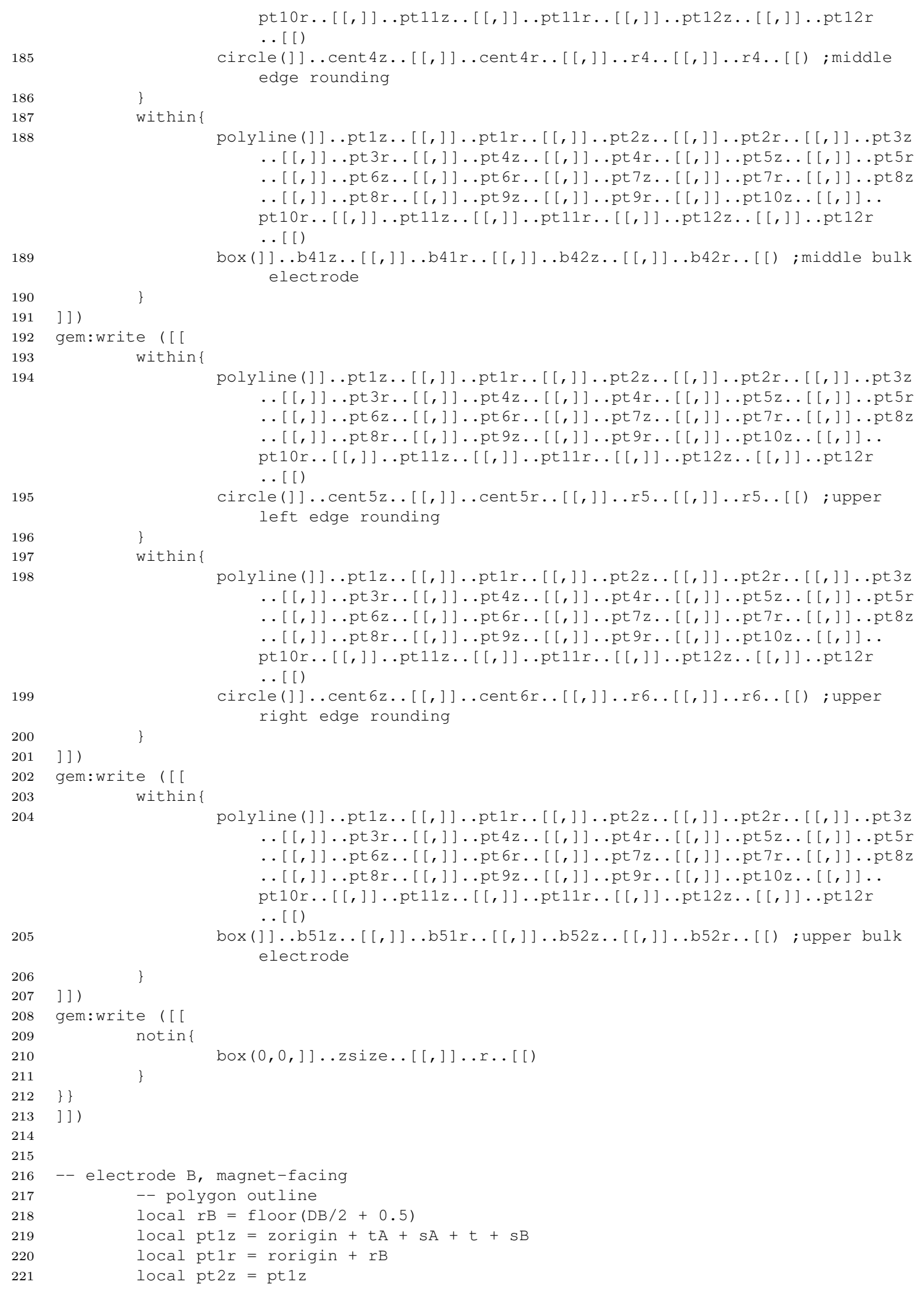




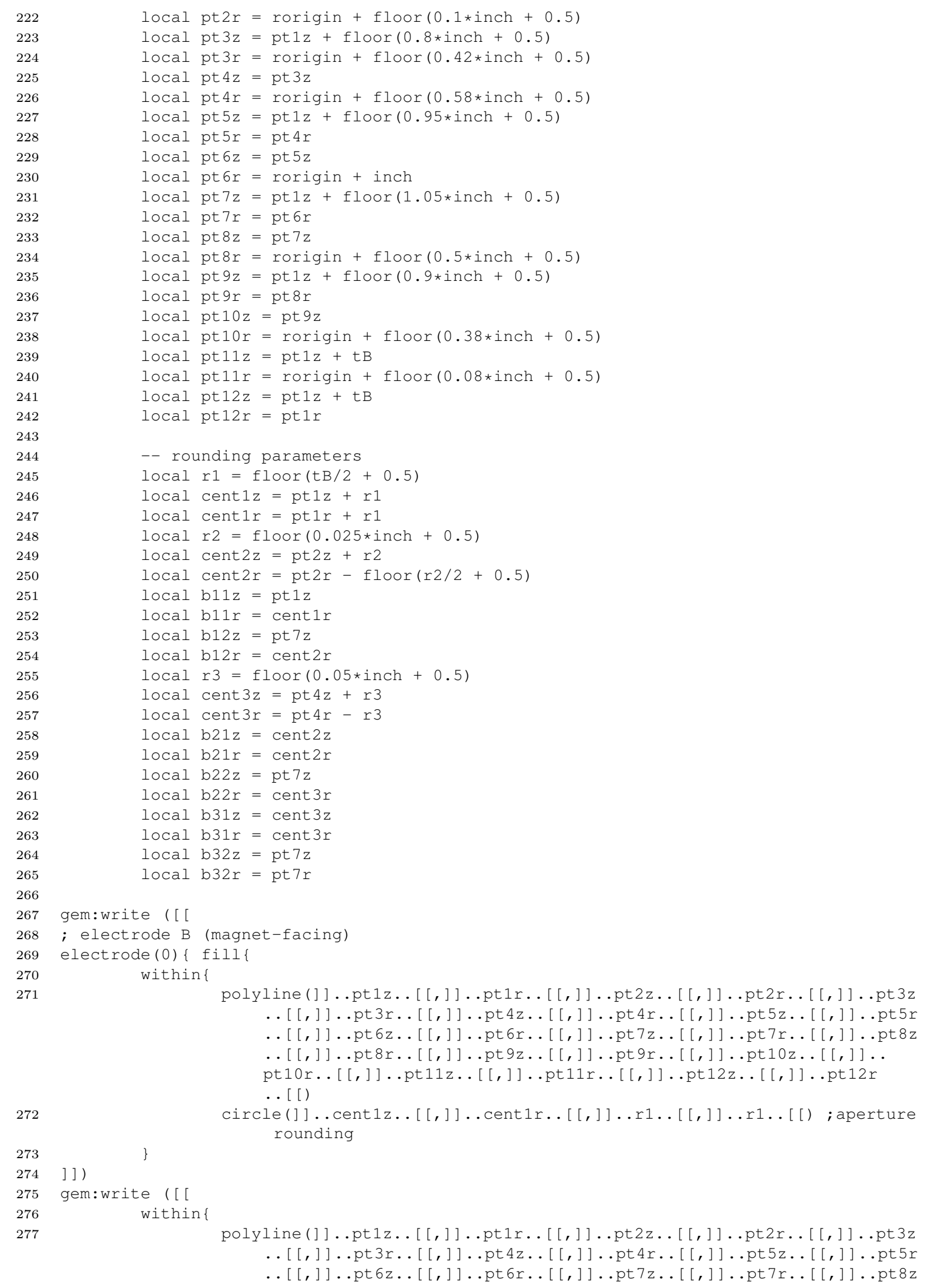




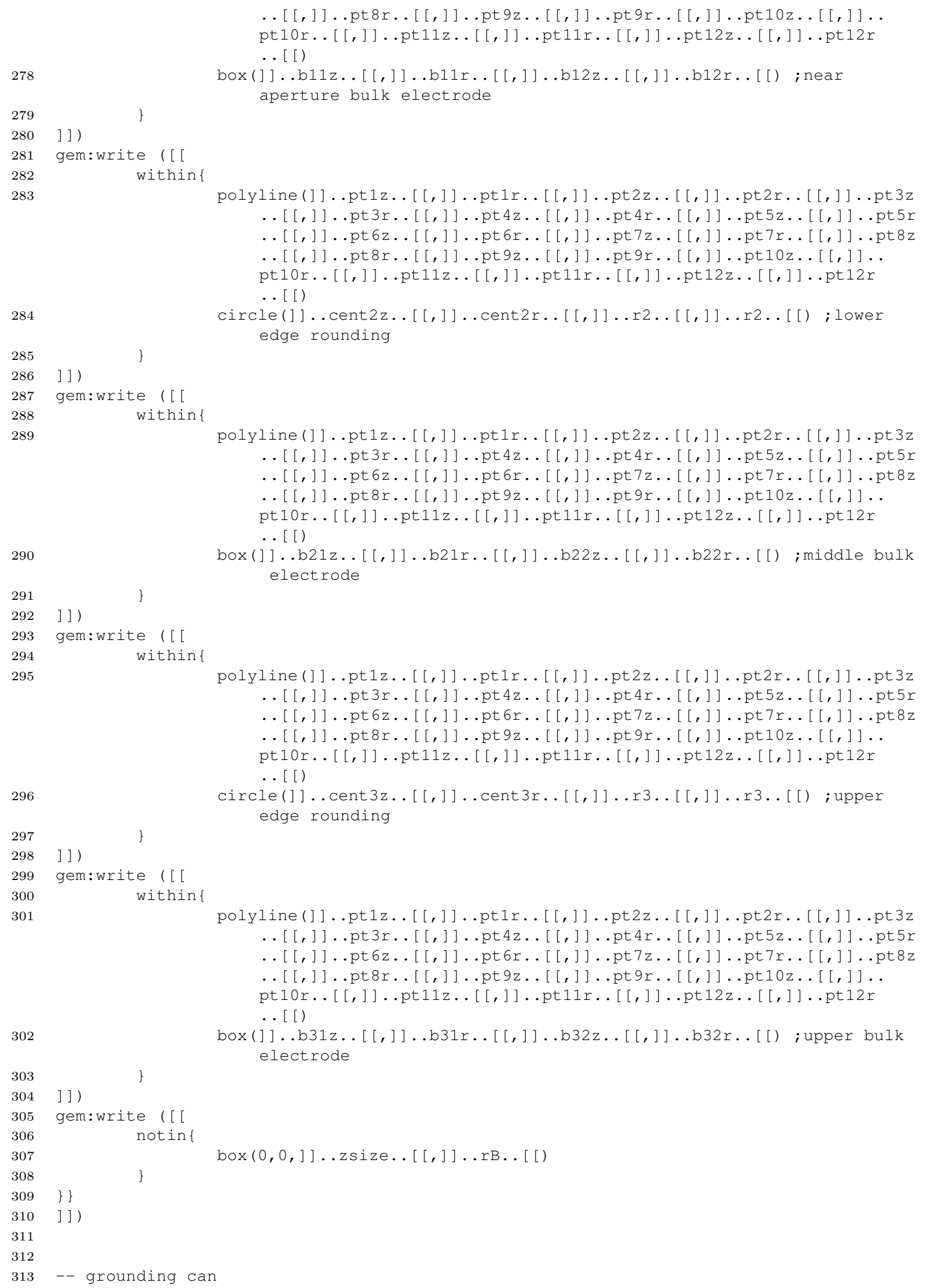




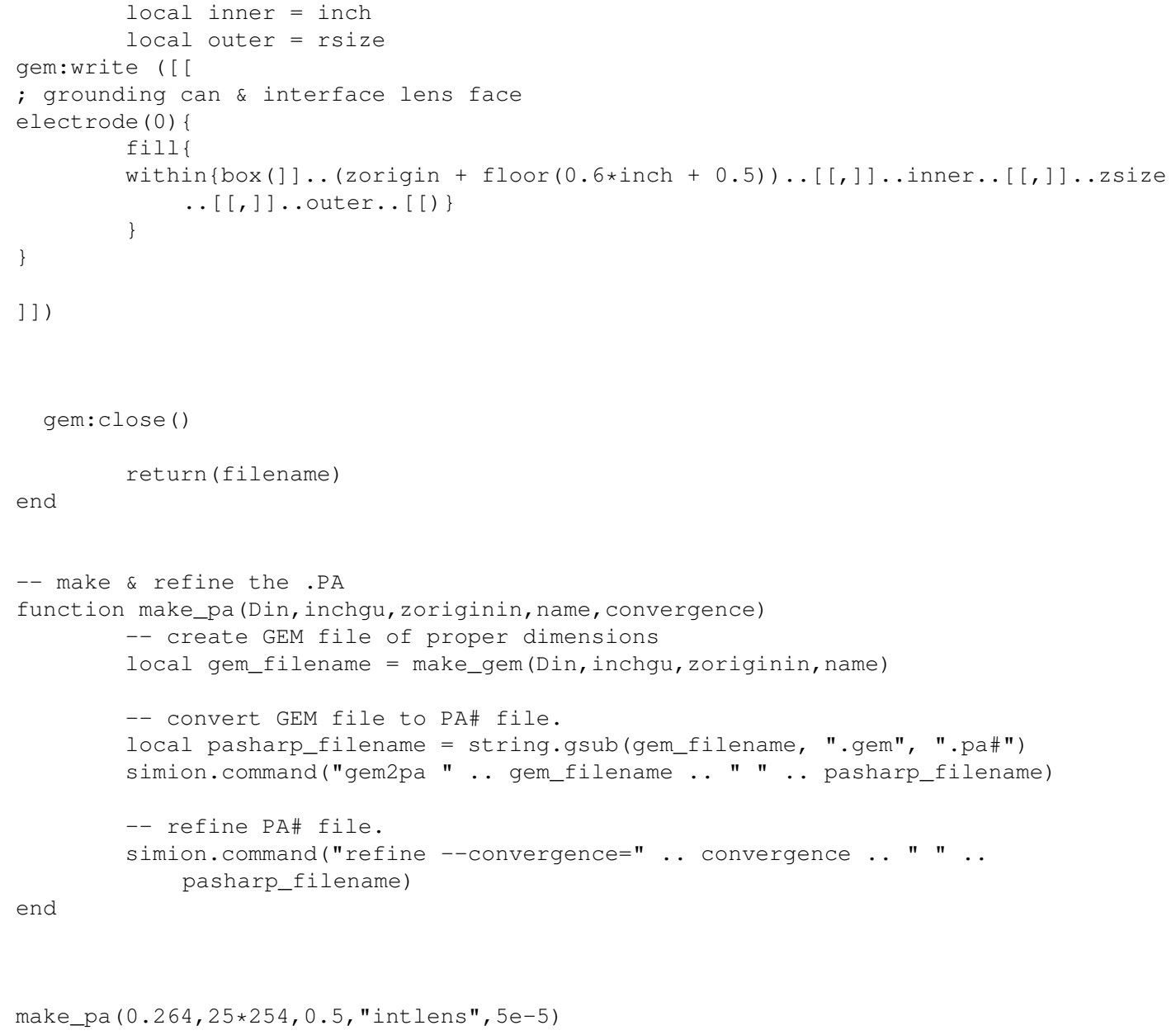

Electron trajectory data is collected in SIMION following a procedure similar to the experimental characterization described in Ref. [60]. Trajectory data was written to file according to a user program written in Lua. The following code is an example of the user program used to analyze trajectories in a swept-back lens. With only a few details specific to the design, it can be readily adapted to other lens geometries.

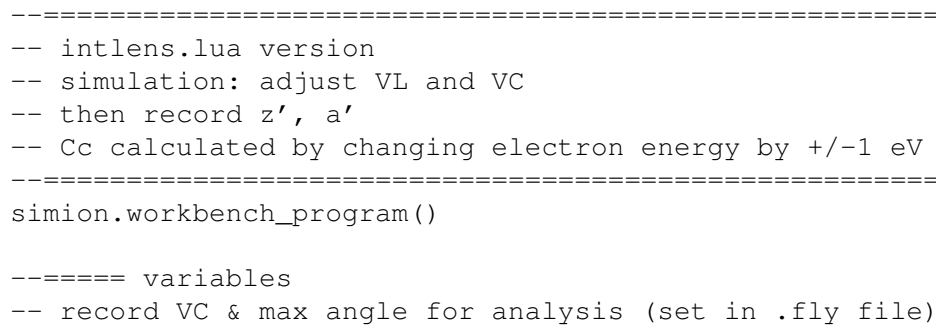




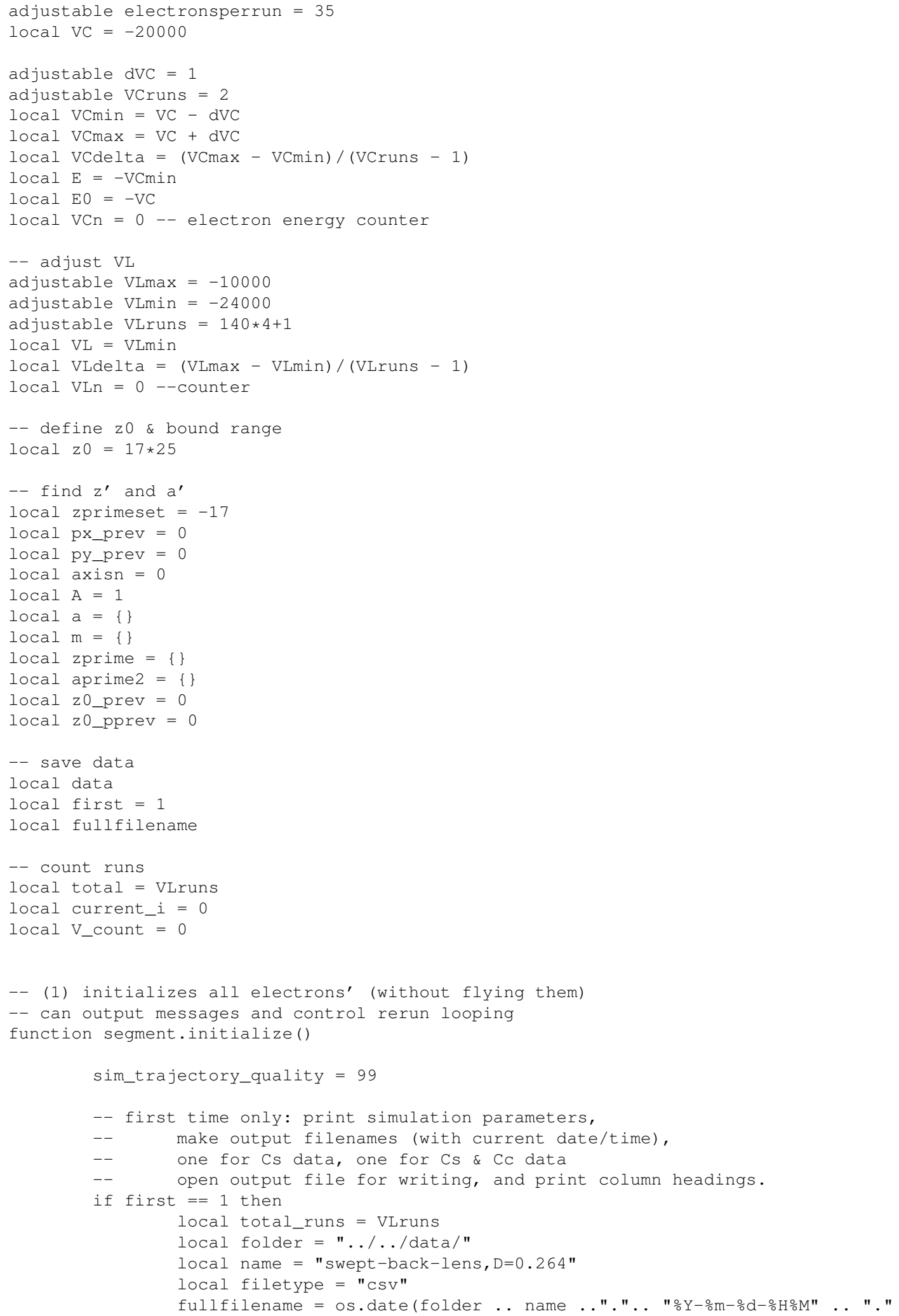




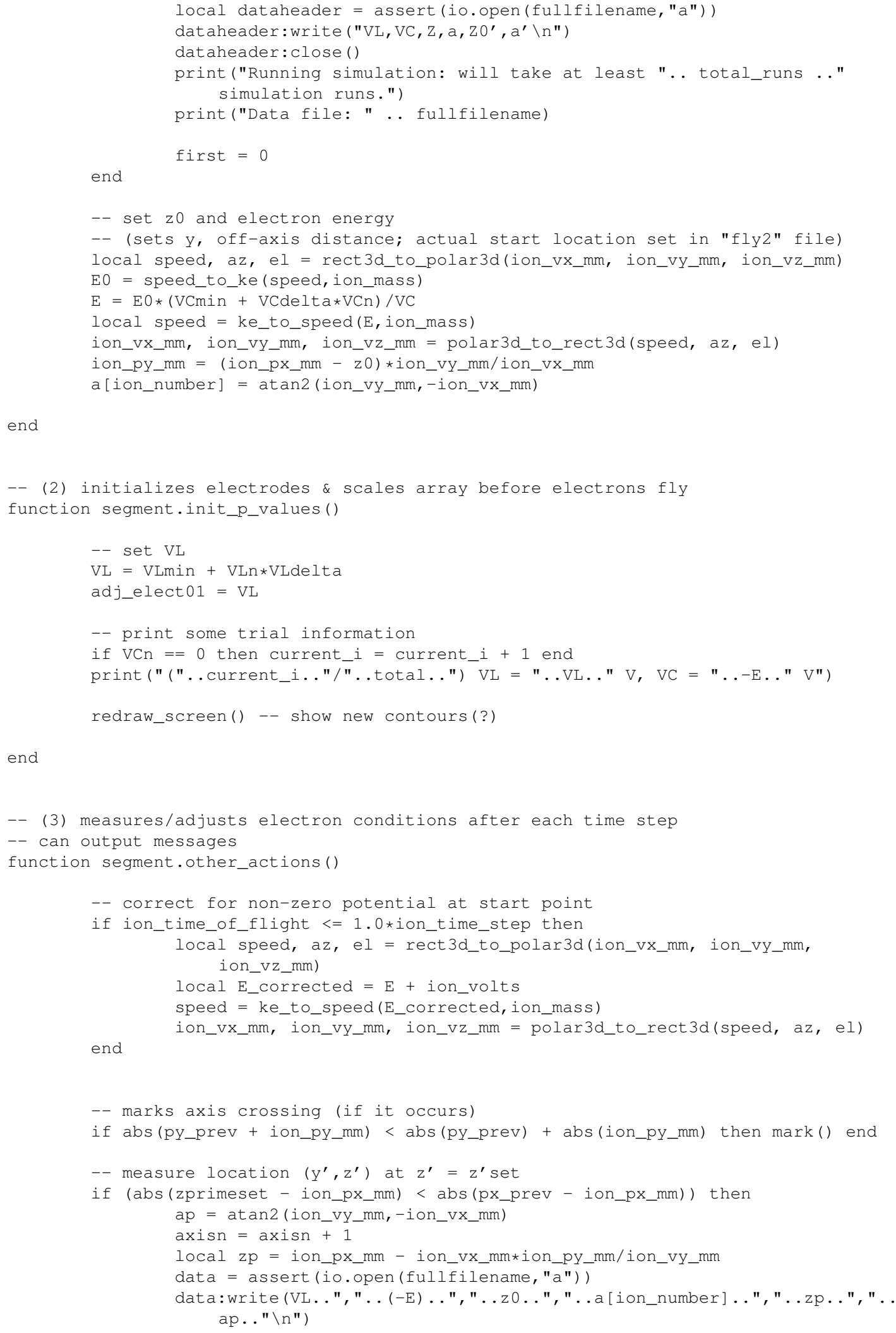




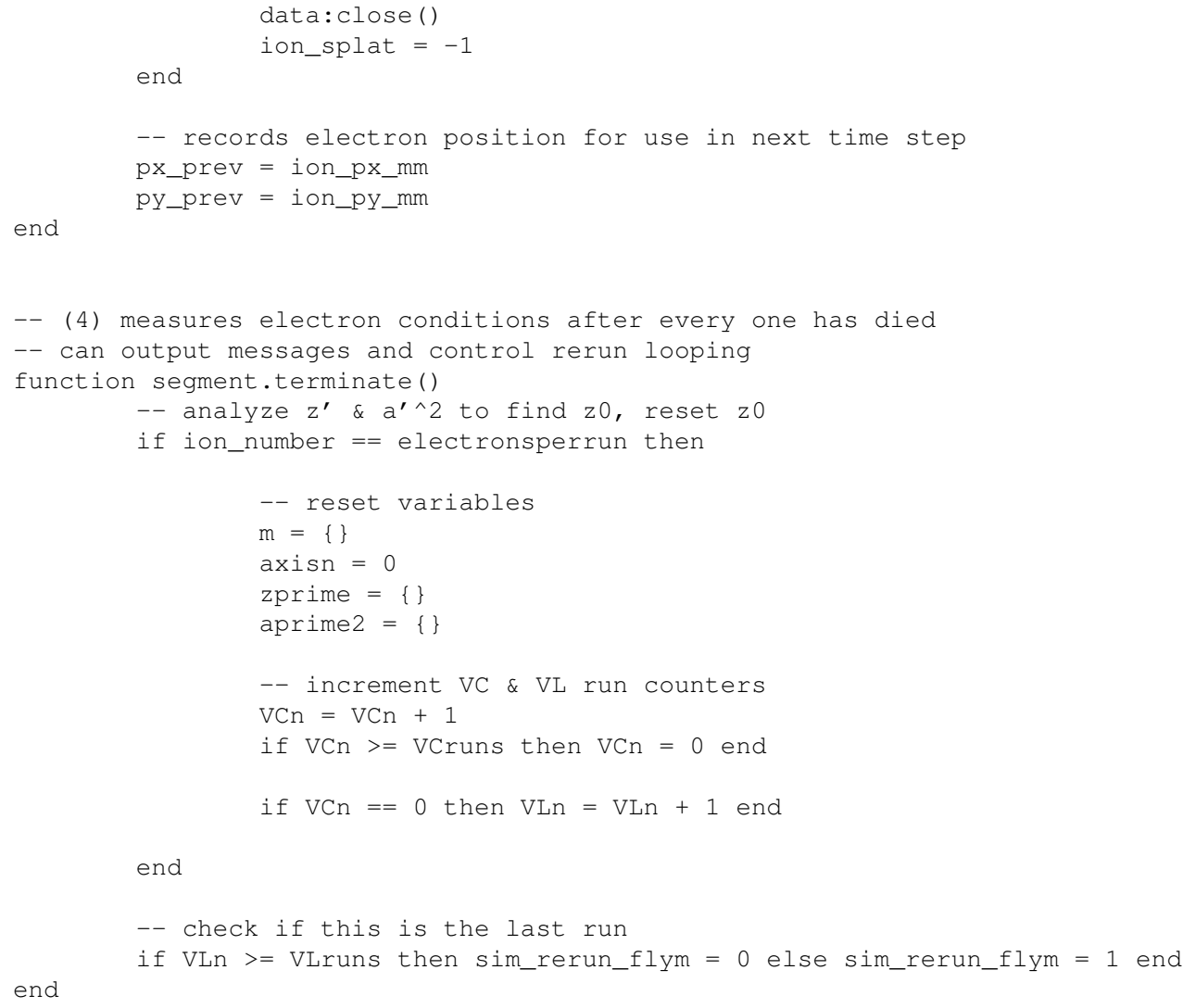




\section{Appendix E Code, Mathematica: swept-back lens}

Electron trajectory data collected from SIMION according to App. Appendix D is then analyzed in Mathematica 9 with the following code to determine the optical properties $f, g, S_{f}, S_{g}, C_{f}$, and $C_{g}$, as defined in Ref. [60]. While this code is applied to the swept-back lens design, it is general enough to be applied to any lens with good SIMION data. 


\section{Header}

\section{Graphics Options}

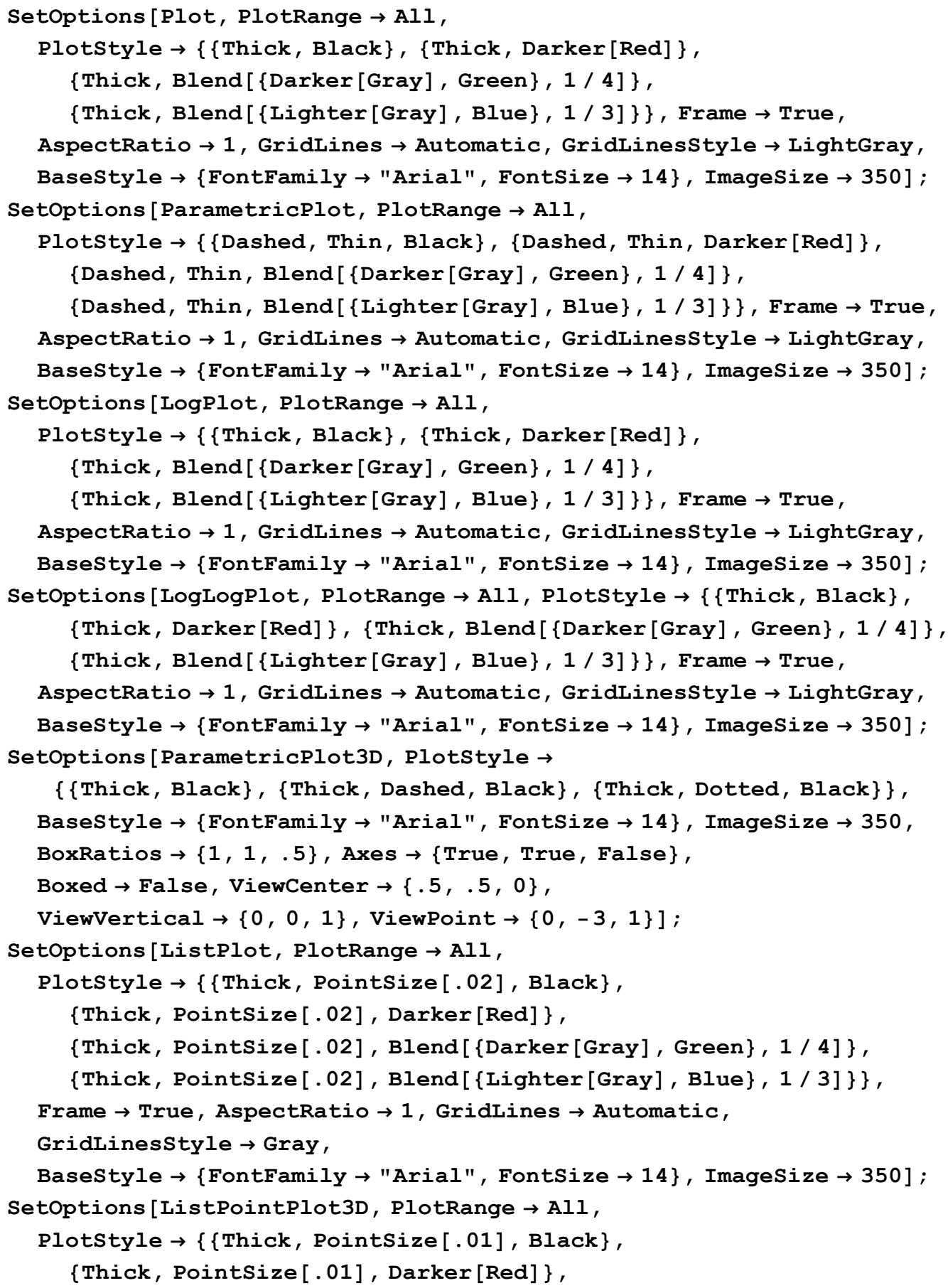


\{Thick, PointSize[.01], Blend[\{Darker[Gray], Green\}, $1 / 4]\}$,

\{Thick, PointSize[.01], Blend[\{Lighter[Gray], Blue\}, 1/3]\}\},

BoxRatios $\rightarrow 1$, Basestyle $\rightarrow$ FontFamily $\rightarrow$ "Arial", FontSize $\rightarrow 14\}$,

ImageSize $\rightarrow$ 350];

Setoptions [ListContourPlot, PlotRange $\rightarrow$ All, Frame $\rightarrow$ True,

AspectRatio $\rightarrow 1$, Gridlines $\rightarrow$ Automatic, GridLinesStyle $\rightarrow$ LightGray,

BaseStyle $\rightarrow$ \{FontFamily $\rightarrow$ "Arial", FontSize $\rightarrow 14$ \}, ImageSize $\rightarrow$ 350];

Setoptions [ListLogLinearPlot, PlotRange $\rightarrow \mathrm{All}$,

PlotStyle $\rightarrow\{\{$ Thick, PointSize [.02], Black $\}$,

\{Thick, PointSize[.02], Darker[Red]\},

\{Thick, PointSize[.02], Blend[\{Darker[Gray], Green\}, $1 / 4]\}$,

\{Thick, PointSize[.02], Blend[\{Lighter[Gray], Blue\}, $1 / 3]\}$,

Frame $\rightarrow$ True, AspectRatio $\rightarrow 1$, Gridlines $\rightarrow$ Automatic,

GridLinesStyle $\rightarrow$ LightGray,

BaseStyle $\rightarrow$ \{FontFamily $\rightarrow$ "Arial", FontSize $\rightarrow 14$ \}, ImageSize $\rightarrow$ 350];

Setoptions [ListlogPlot, PlotRange $\rightarrow$ All,

PlotStyle $\rightarrow\{\{$ Thick, PointSize [.02], Black $\}$,

\{Thick, PointSize[.02], Darker[Red]\},

\{Thick, PointSize [.02], Blend[\{Darker[Gray], Green\}, $1 / 4]\}$,

$\{$ Thick, PointSize[.02], Blend[\{Lighter[Gray], Blue\}, $1 / 3]\}$,

Frame $\rightarrow$ True, AspectRatio $\rightarrow 1$, Gridlines $\rightarrow$ Automatic,

GridLinesstyle $\rightarrow$ LightGray,

BaseStyle $\rightarrow$ \{FontFamily $\rightarrow$ "Arial", FontSize $\rightarrow 14$ \}, ImageSize $\rightarrow$ 350 ];

Setoptions [ListloglogPlot, PlotRange $\rightarrow$ All,

Plotstyle $\rightarrow$ \{ Thick, PointSize [.02], Black,

\{Thick, PointSize[.02], Darker[Red]\},

\{Thick, PointSize[.02], Blend[\{Darker[Gray], Green\}, $1 / 4]\}$,

\{Thick, PointSize[.02], Blend[\{Lighter[Gray], Blue\}, 1/3] \} ,

Frame $\rightarrow$ True, AspectRatio $\rightarrow 1$, Gridlines $\rightarrow$ Automatic,

GridLinesStyle $\rightarrow$ LightGray,

BaseStyle $\rightarrow$ \{FontFamily $\rightarrow$ "Arial", FontSize $\rightarrow$ 14 $\}$, ImageSize $\rightarrow$ 350];

\section{Characterization}

Thick lens equations for focal length and distance from Rempfer 1985

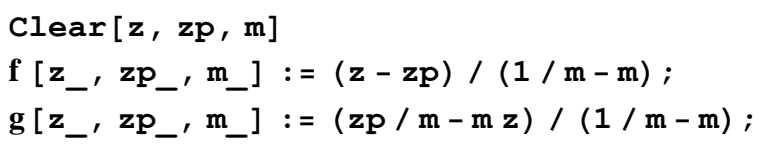

Aberration formulas at the Gaussian plane (not circle of least confusion)

Clear $[\mathrm{m}, \mathrm{f}, \mathrm{Sf}, \mathrm{Sg}, \mathrm{Cf}, \mathrm{Cg}]$

$\operatorname{Csp}\left[\mathrm{m}_{-}, \mathrm{f}_{-}, \mathrm{Sf}_{-}, \mathrm{Sg}_{-}\right]:=-\left(\left(1+\mathrm{m}^{2}\right) \mathrm{Sg}+2 \mathrm{~m} \mathrm{Sf}\right)(1+\mathrm{m})^{2} \mathrm{f}$;

$\mathrm{Ccp}\left[\mathrm{m}_{-}, \mathrm{f}_{-}, \mathrm{Cf}_{-}, \mathrm{Cg}_{-}\right]:=\left(\left(1+\mathrm{m}^{2}\right) \mathrm{Cg}+2 \mathrm{~m} \mathrm{Cf}\right) \mathrm{f}$; 


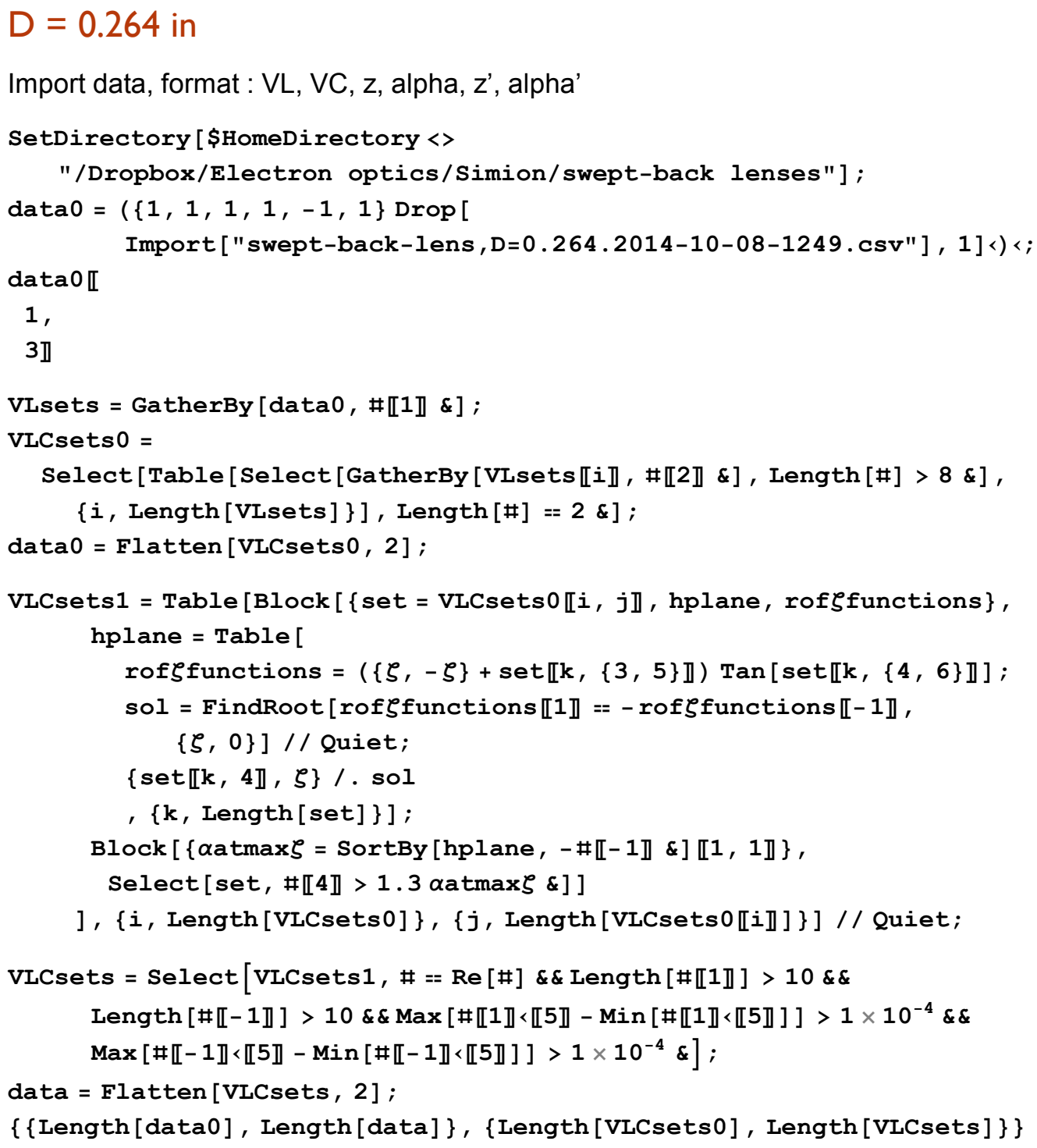




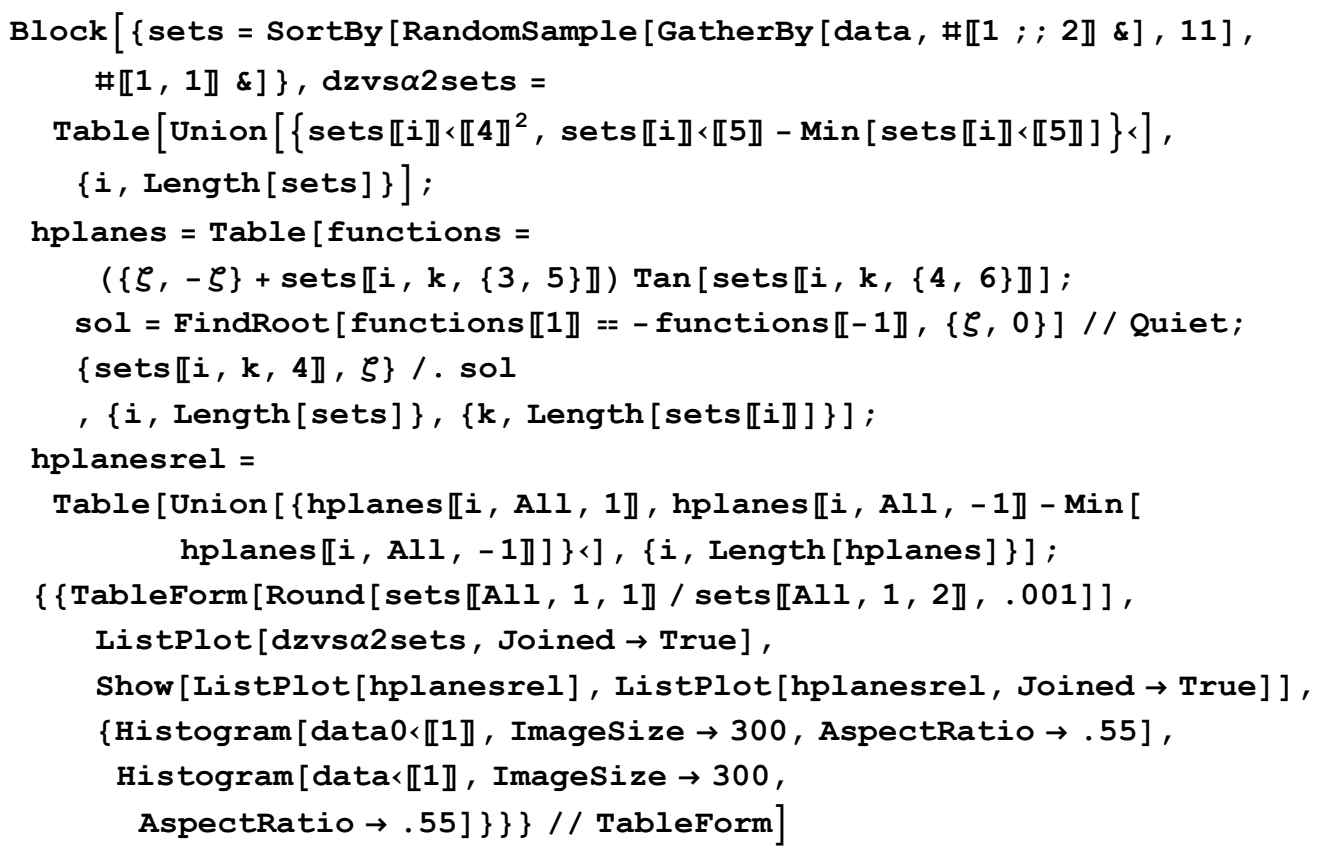




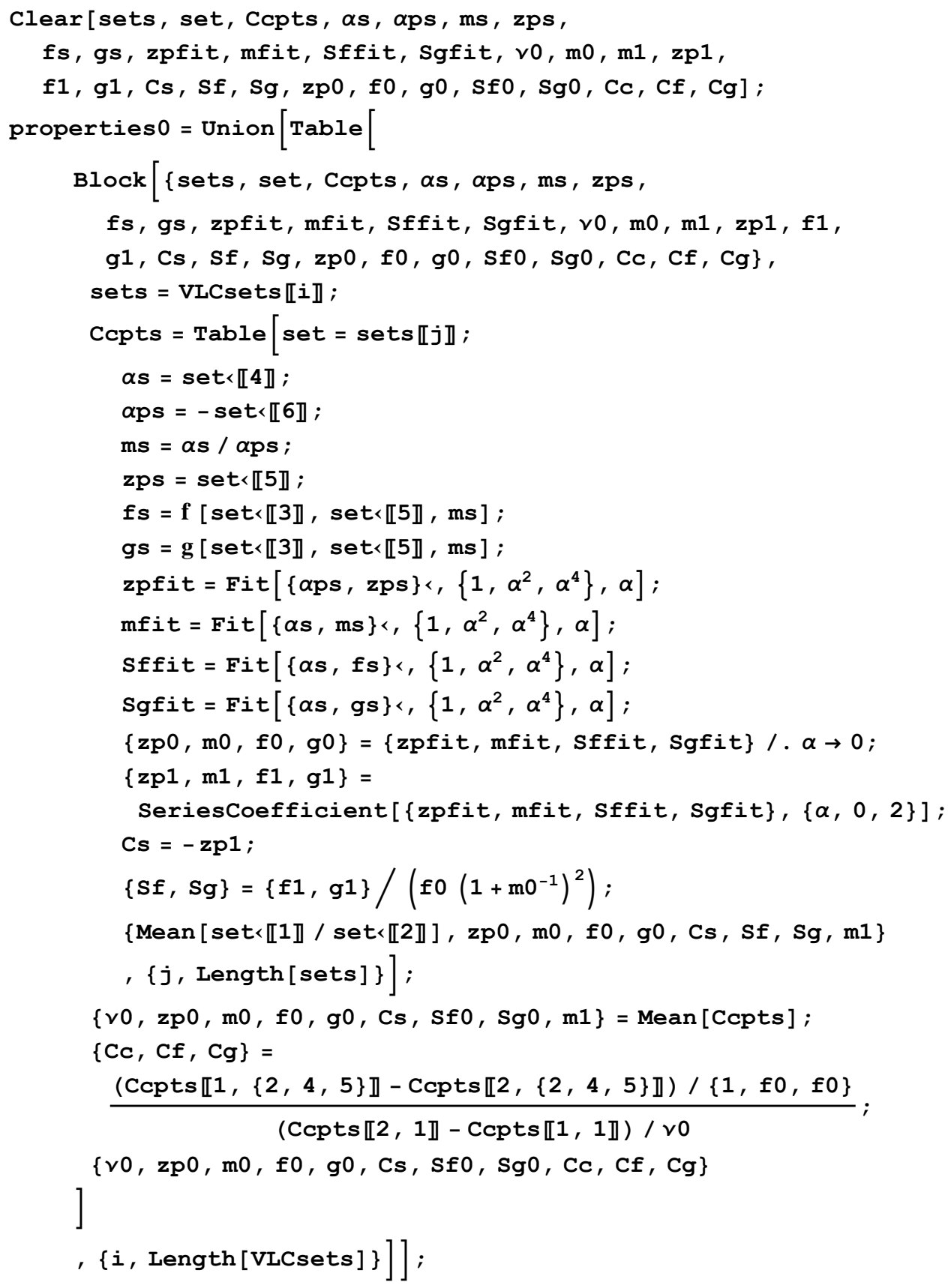




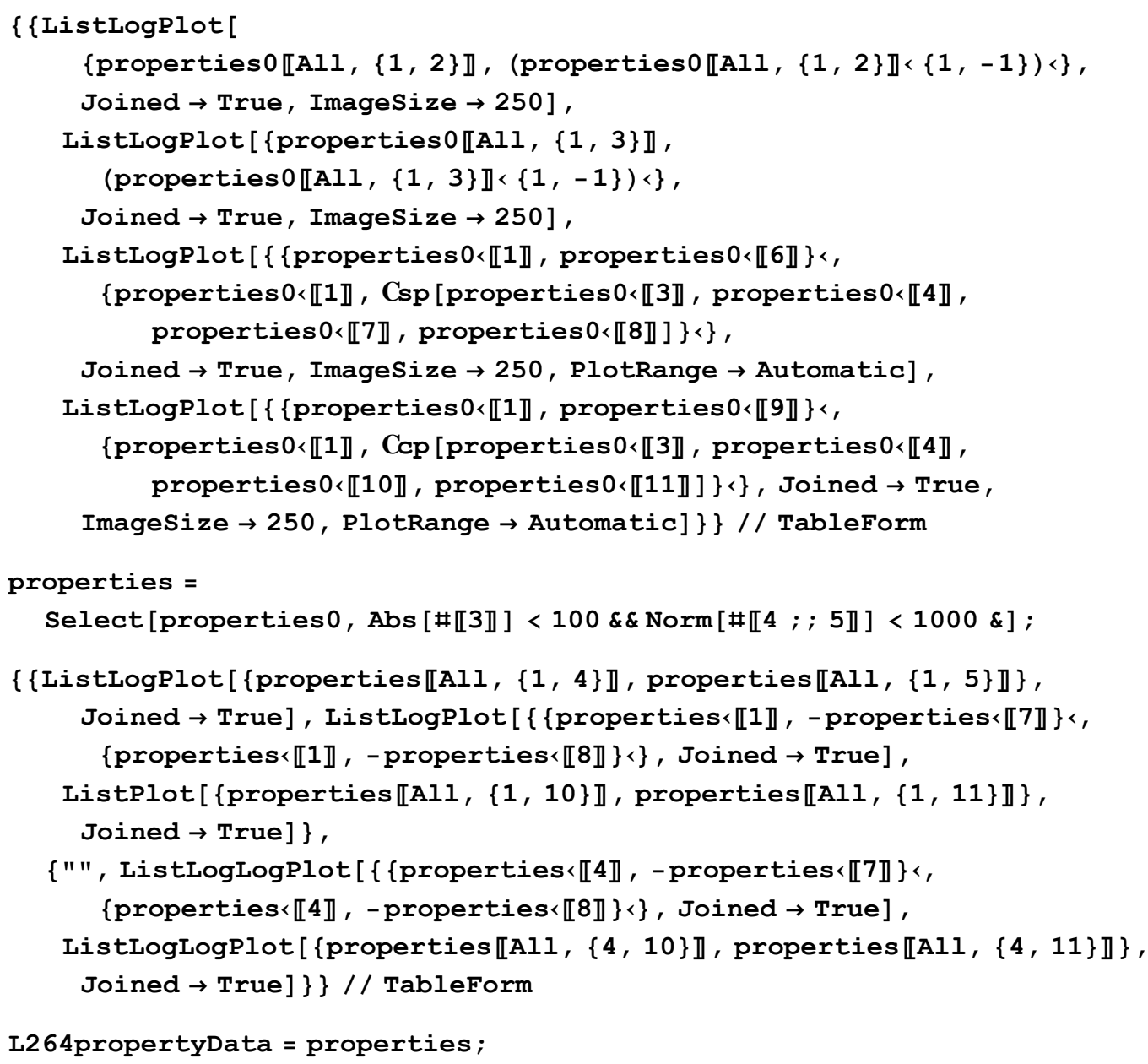

Fit properties to analytic functions

$$
D=0.264 \text { in }
$$

properties = L264propertyData ;

\{ Table [Block [

$\{v s=$ properties $\langle\llbracket 1 \rrbracket$, sum $=.5$ (properties $\langle[i \rrbracket+$ properties $\langle\llbracket i+1 \rrbracket)$, dif $=-.5$ (properties $<\llbracket i \rrbracket-$ properties $<\llbracket i+1 \rrbracket)\}$,

$\{$ ListLogPlot [ $\{v s, A b s[$ sum] $\}<$, AspectRatio $\rightarrow .55]$, ListPlot [Select $[\{v s, \operatorname{dif}\}<, \# \llbracket 1 \rrbracket>.2 \&]$, AspectRatio $\rightarrow .55]\}]$, $\{i, 4,10,3\}]\} / /$ TableForm 


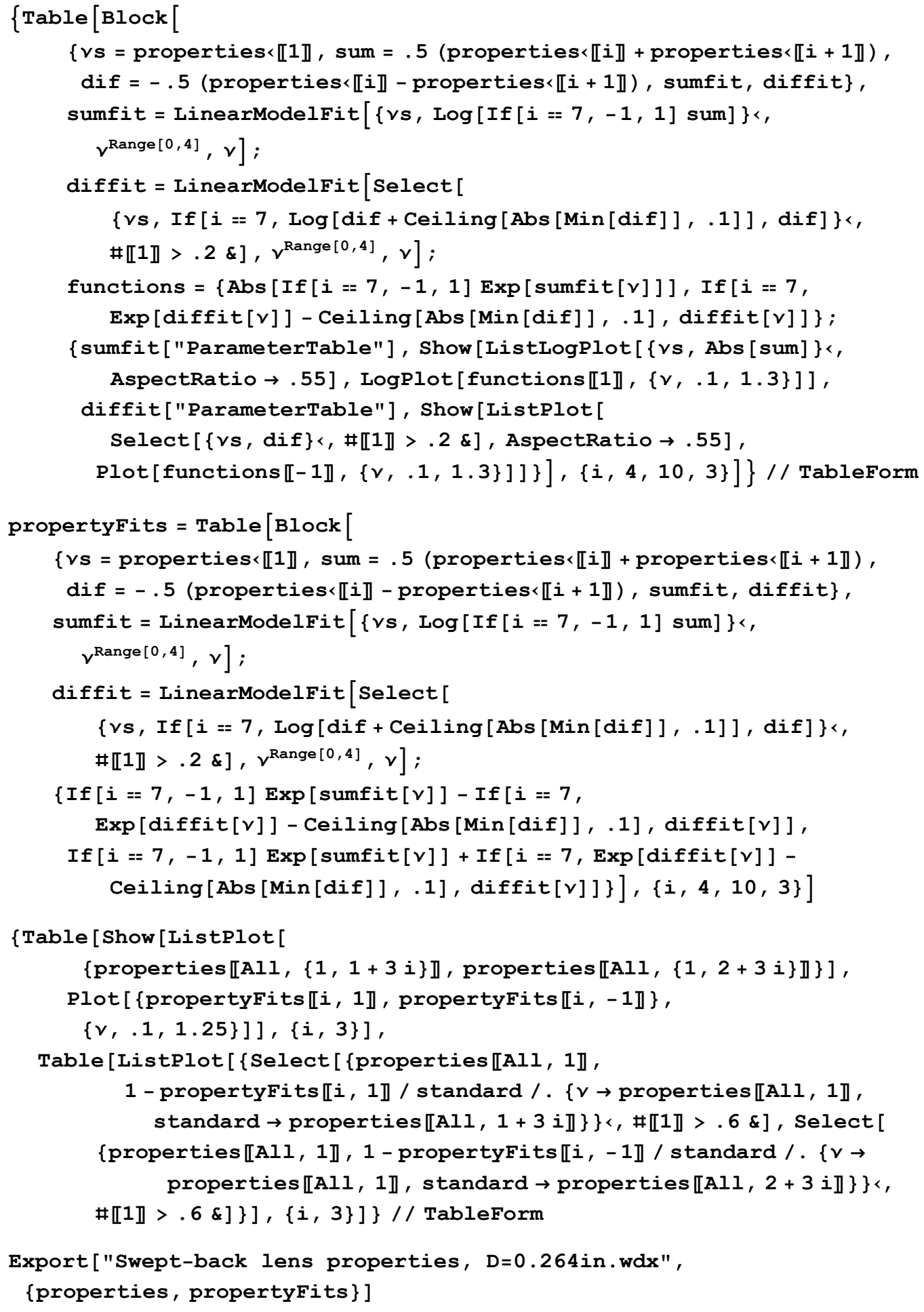




\section{Appendix F Code, Mathematica: objective branch}

After characterizing the swept-back lens and auxiliary lens (of the projection design), they can be combined with the estimated aberration coefficients of the cathode to provide a complete model of the objective branch of our PEEM. The following code computes the focusing curves that maintain constant magnification, with $0.1 \times$ increments given. The spherical and chromatic aberration in transfer space is also computed. 


\section{Header}

\section{Graphics Options}

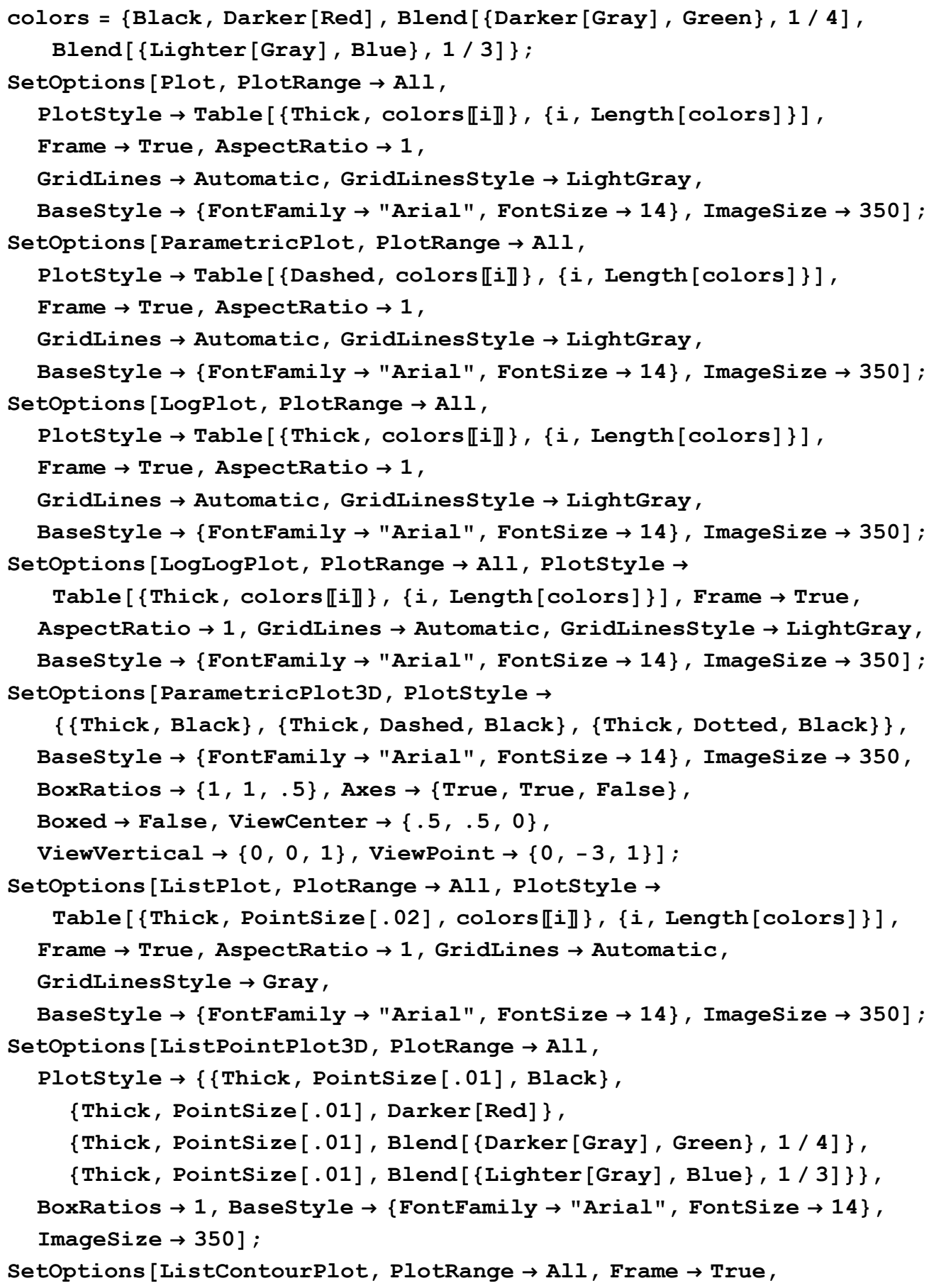


AspectRatio $\rightarrow 1$, GridLines $\rightarrow$ Automatic, GridLinesStyle $\rightarrow$ LightGray, BaseStyle $\rightarrow$ \{FontFamily $\rightarrow$ "Arial", FontSize $\rightarrow 14$ \}, ImageSize $\rightarrow$ 350]; Setoptions [ListlogLinearPlot, PlotRange $\rightarrow$ All, PlotStyle $\rightarrow$ \{ Thick, PointSize [.02], Black,

$\{$ Thick, PointSize[.02], Darker [Red] \},

\{Thick, PointSize[.02], Blend[\{Darker[Gray], Green\}, 1/4]\},

\{Thick, PointSize[.02], Blend[\{Lighter[Gray], Blue\}, 1/3]\}\},

Frame $\rightarrow$ True, AspectRatio $\rightarrow 1$, Gridlines $\rightarrow$ Automatic,

GridLinesstyle $\rightarrow$ LightGray,

BaseStyle $\rightarrow$ \{FontFamily $\rightarrow$ "Arial", FontSize $\rightarrow 14$ \}, ImageSize $\rightarrow$ 350];

Setoptions [ListlogPlot, PlotRange $\rightarrow$ All, Plotstyle $\rightarrow$

Table [ Thick, PointSize[.02], colors [i]\}, \{i, Length[colors] \}],

Frame $\rightarrow$ True, AspectRatio $\rightarrow 1$, Gridlines $\rightarrow$ Automatic,

GridLinesStyle $\rightarrow$ LightGray,

BaseStyle $\rightarrow$ \{FontFamily $\rightarrow$ "Arial", FontSize $\rightarrow 14$ \}, ImageSize $\rightarrow$ 350];

Setoptions [ListlogLogPlot, PlotRange $\rightarrow$ All, PlotStyle $\rightarrow$

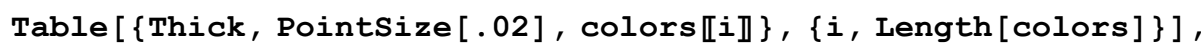

Frame $\rightarrow$ True, AspectRatio $\rightarrow 1$, Gridlines $\rightarrow$ Automatic,

GridLinesstyle $\rightarrow$ LightGray,

BaseStyle $\rightarrow$ \{FontFamily $\rightarrow$ "Arial", FontSize $\rightarrow 14\}$, ImageSize $\rightarrow$ 350 ] ;

\section{Analysis}

\section{Measurements (pixels)}

Measurements taken from PEEM CAD diagram PDF, 90 pixels/in.

Front of cathode

$C f[z]=2550.192$;

Alternate cathode front measurements (diagram unclear).

2548.748

2549.649 ;

2550.192 ;

Front aperture of objective lens

$\{$ Of $[r$, left, front $]$, Of $[z$, left, front $]\}=\{1773.243,2535.472\}$

$\{$ Of $[r$, right, front $]$, of $[z$, right, front $]\}=\{1777.745,2535.472\} ;$

$\{$ Of $[r$, left, back $]$, of $[z$, left, back $]\}=\{1773.243,2533.222\}$;

$\{$ Of $[r$, right, back $], \operatorname{Of}[z$, right, back $]\}=\{1777.745,2533.222\}$;

$\operatorname{Of}[z$, front $]=\operatorname{Mean}[\{$ Of $[z$, left, front $]$, Of $[z$, right, front $]\}]$;

Of $[z, \operatorname{back}]=\operatorname{Mean}[\{\operatorname{Of}[z$, left, back $]$, of $[z$, right, back $]\}]$;

of $[z$, center $]=\operatorname{Mean}[\{$ Of $[z$, front $]$, of $[z$, back $]\}]$

Center aperture of objective lens 
$\{$ Oc $[r$, left, front $]$, Oc $[z$, left, front $]\}=\{1766.497,2521.974\}$;

$\{$ Oc $[r$, right, front $]$, Oc $[z$, right, front $]\}=\{1784.491,2521.974\}$;

$\{$ Oc $[r$, left, back $]$, Oc $[z$, left, back $]\}=\{1766.497,2517.477\}$;

$\{$ Oc $[r$, right, back $]$, Oc $[z$, right, back $]\}=\{1784.491,2517.477\}$;

Oc $[z$, front $]=\operatorname{Mean}[\{$ Oc $[z$, left, front $]$, Oc $[z$, right, front $]\}]$;

Oc $[z, \operatorname{back}]=\operatorname{Mean}[\{$ Oc $[z$, left, back $]$, Oc $[z$, right, back $]\}]$;

Oc $[z$, center $]=\operatorname{Mean}[\{$ Oc $[z$, front $], O c[z, \operatorname{back}]\}]$;

$\mathrm{DO}=\mathrm{Abs}[$ Mean $[\{$ Oc $[r$, left, front $]$, Oc $[r$, left, back $]\}]-$

Mean [\{Oc [r, right, front], Oc [r, right, back]\}] ]

to $=\mathrm{Abs}[\mathrm{Oc}[\mathrm{z}$, front $]-\mathrm{Oc}[\mathrm{z}, \mathrm{back}]]$

Back aperture of objective lens

$\{\mathrm{Ob}[r$, left, front $], \mathrm{Ob}[z$, left, front $]\}=\{1770.994,2506.224\}$;

$\{\mathrm{Ob}[\mathrm{r}$, right, front $], \mathrm{Ob}[\mathrm{z}$, right, front $]\}=\{1779.989,2506.224\}$;

$\{\mathrm{Ob}[r$, left, back $], \mathrm{Ob}[z$, left, back $]\}=\{1770.994,2503.974\}$;

$\{\mathrm{Ob}[r$, right, back $], \mathrm{Ob}[\mathrm{z}$, right, back $]\}=\{1779.989,2503.974\}$;

$\mathrm{Ob}[z$, front $]=\operatorname{Mean}[\{\mathrm{Ob}[z$, left, front $], \mathrm{Ob}[z$, right, front $]\}]$;

Objective lens spacing

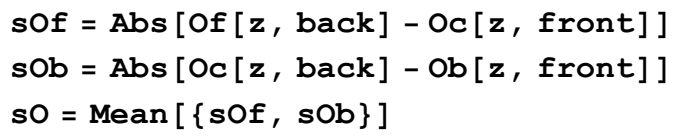

Front aperture of auxilary lens

$\{\mathrm{Xf}[r$, left, front $], \operatorname{Xf}[z$, left, front $]\}=\{1768.742,2094.474\}$;

$\{\mathrm{Xf}[\mathrm{r}$, right, front $], \mathrm{Xf}[\mathrm{z}$, right, front $]\}=\{1782.242,2094.474\} ;$

$\{\mathrm{Xf}[r$, left, back $], \operatorname{Xf}[z$, left, back $]\}=\{1768.742,2089.978\}$;

$\{\mathrm{Xf}[r$, right, back $], \operatorname{Xf}[z$, right, back $]\}=\{1782.242,2089.978\}$;

$\mathrm{Xf}[\mathrm{z}, \operatorname{back}]=\operatorname{Mean}[\{\mathrm{Xf}[\mathrm{z}$, left, back $], \mathrm{Xf}[\mathrm{z}, \operatorname{right}, \mathrm{back}]\}]$;

Center aperture of auxilary lens

$\left\{\mathrm{Xc}[\mathrm{r}\right.$, left, front $], \mathrm{Xc}_{\mathrm{z}} \mathrm{z}$, left, front $\left.]\right\}=\{1768.288,2078.733\}$;

$\left\{X C[r\right.$, right, front $], X_{C}[z$, right, front $\left.]\right\}=\{1782.695,2078.733\}$;

$\{X c[r$, left, back $], X c[z$, left, back $]\}=\{1768.288,2065.231\}$;

$\{\mathrm{Xc}[\mathrm{r}, \mathrm{right}, \mathrm{back}], \mathrm{Xc}[\mathrm{z}, \mathrm{right}, \mathrm{back}]\}=\{1782.695,2065.231\}$;

$\mathrm{Xc}[\mathrm{z}$, front $]=\operatorname{Mean}[\{\mathrm{Xc}[\mathrm{z}$, left, front $], \mathrm{Xc}[\mathrm{z}$, right, front $]\}]$;

$\mathrm{Xc}[z, \operatorname{back}]=\operatorname{Mean}[\{\mathrm{Xc}[z$, left, back $], \mathrm{Xc}[\mathrm{z}$, right, back $]\}]$;

$\mathrm{Xc}[\mathbf{z}$, center $]=\operatorname{Mean}[\{\mathrm{Xc}[\mathbf{z}$, front $], \mathrm{Xc}[\mathbf{z}, \operatorname{back}]\}]$;

$\mathrm{DX}=\mathrm{Abs}[$ Mean $[\{\mathrm{Xc}[\mathrm{r}$, left, front $], \mathrm{Xc}[\mathrm{r}$, left, back $]\}]-$

$\operatorname{Mean}[\{\mathrm{Xc}[r, \mathrm{right}$, front $], \mathrm{Xc}[r, \mathrm{right}, \mathrm{back}]\}]]$

$\mathrm{tX}=\mathrm{Abs}[\mathrm{Xc}[\mathrm{z}$, front $]-\mathrm{Xc}[\mathrm{z}, \mathrm{back}]]$ 
Back aperture of auxilary lens

$\{\mathrm{Xb}[\mathrm{r}$, left, front $], \mathrm{Xb}[\mathrm{z}$, left, front $]\}=\{1772.119,2053.980\}$;

$\{\mathrm{Xb}[r$, right, front $], \mathrm{Xb}[\mathrm{z}$, right, front $]\}=\{1778.869,2053.980\}$;

$\{\mathrm{Xb}[r$, left, back $], \mathrm{Xb}[\mathrm{z}$, left, back $]\}=\{1772.119,2049.479\}$;

$\{\mathrm{Xb}[\mathrm{r}, \mathrm{right}, \mathrm{back}], \mathrm{Xb}[\mathrm{z}$, right, back $]\}=\{1778.869,2049.479\}$;

$\mathrm{Xb}[\mathrm{z}$, front $]=\operatorname{Mean}[\{\mathrm{Xb}[\mathrm{z}$, left, front $], \mathrm{Xb}[\mathrm{z}$, right, front $]\}]$;

Auxilary lens spacing

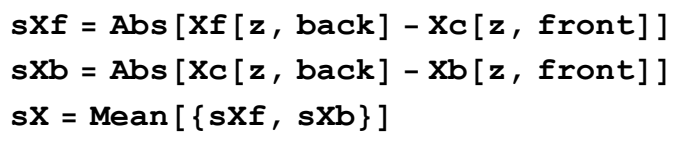

Center of magnet $A$

$\operatorname{MAc}[z]=\operatorname{Mean}[\{1874.793,1869.395\}] ;$

Relevant lengths ( $\mathrm{mm}$ )

$I_{a}=$ cathode length, distance from the sample surface to the front electrode

lamm $=1 \mathrm{a} 25.4 / 90$

Measured $I_{a}$

$1 \mathrm{amm}=3.95 ;$

$D_{O}=$ objective lens center aperture diameter

$\mathrm{DOmm}=\mathrm{DO} 25.4 / 90$

$D_{X}=$ auxiliary lens center aperture diameter

$\mathrm{DXmm}=\mathrm{DX} 25.4 / 90$

$\mathrm{CO}=$ distance from cathode sample plane to center of objective lens

$C f[z]-O C[z$, center $]$

$\mathrm{COmm}=\operatorname{Abs}[\mathrm{Cf}[\mathrm{z}]-\mathrm{Oc}[\mathrm{z}$, center $]] 25.4 / 90$

$\mathrm{OX}=$ distance from center of objective lens to center of auxilary lens

OXmm $=\mathrm{Abs}[\mathrm{Oc}[\mathrm{z}$, center $]-\mathrm{Xc}[\mathrm{z}$, center $]] 25.4 / 90$

$X A=$ distance from center of auxilary lens to center of magnet $A$

$\mathrm{XAmm}=\operatorname{Abs}[\mathrm{Xc}[\mathrm{z}$, center $]-\mathrm{MAc}[\mathrm{z}]] 25.4 / 90$

Objective lens properties from Simlon

Import swept-back lens property fits 


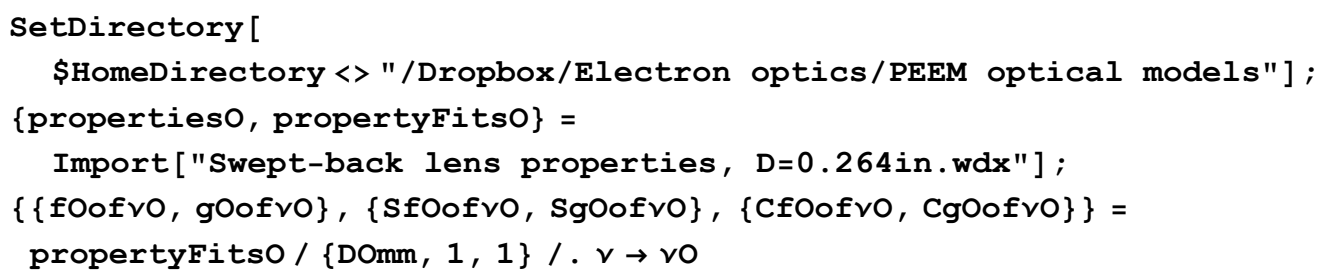

Auxiliary lens properties from Simlon

Import swept-back lens property fits

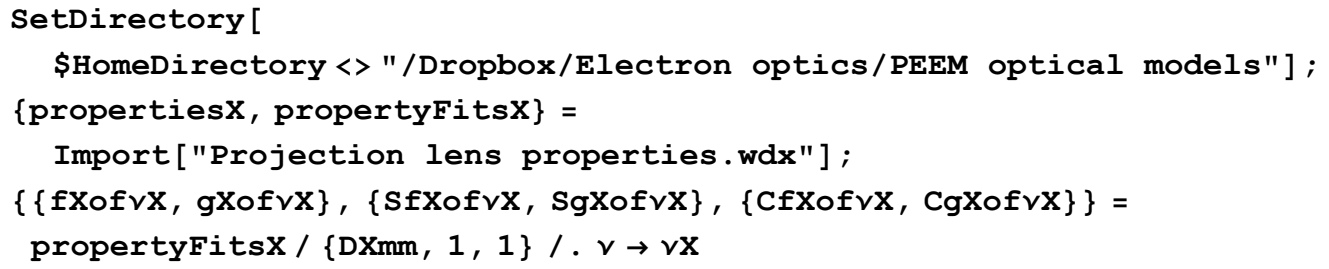

Optics, focusing as function of $z_{C}(\mathrm{~mm})$

Thick lens equations

Clear $[f, g, z, z p, m]$;

$z\left[f_{-}, g_{-}, z_{-}\right]:=g+\frac{f^{2}}{z p-g} ;$

$z p\left[f_{-}, g_{-}, z_{-}\right]:=g+\frac{f^{2}}{z-g}$;

$m\left[f_{-}, g_{-}, z_{-}, z_{-}\right]:=\sqrt{\frac{z p-g}{z-g}} ;$

Accelerating field virtual image location, measured from sample surface.

Magnification of accelerating field and first aperture.

$\mathbf{z C p}=-1 \mathrm{amm}+\delta \mathbf{z C}$

$\mathrm{mC}=2 / 3$;

Objective lens object and image locations.

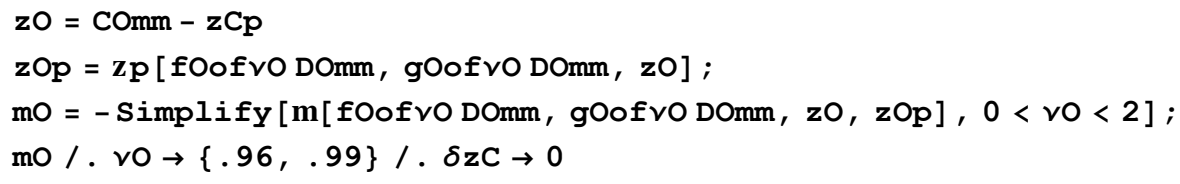




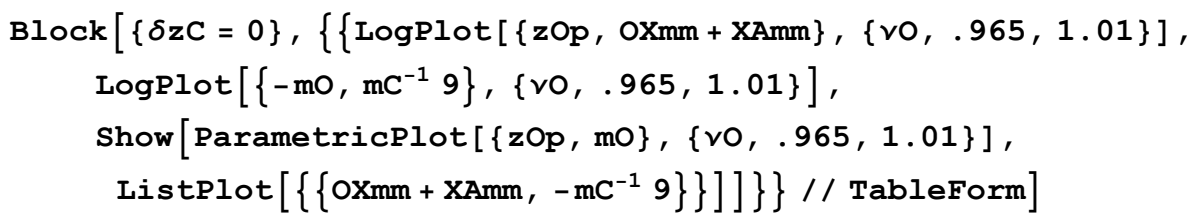

Auxilary lens object and image locations, with image centered in magnet $A$ (where possible).

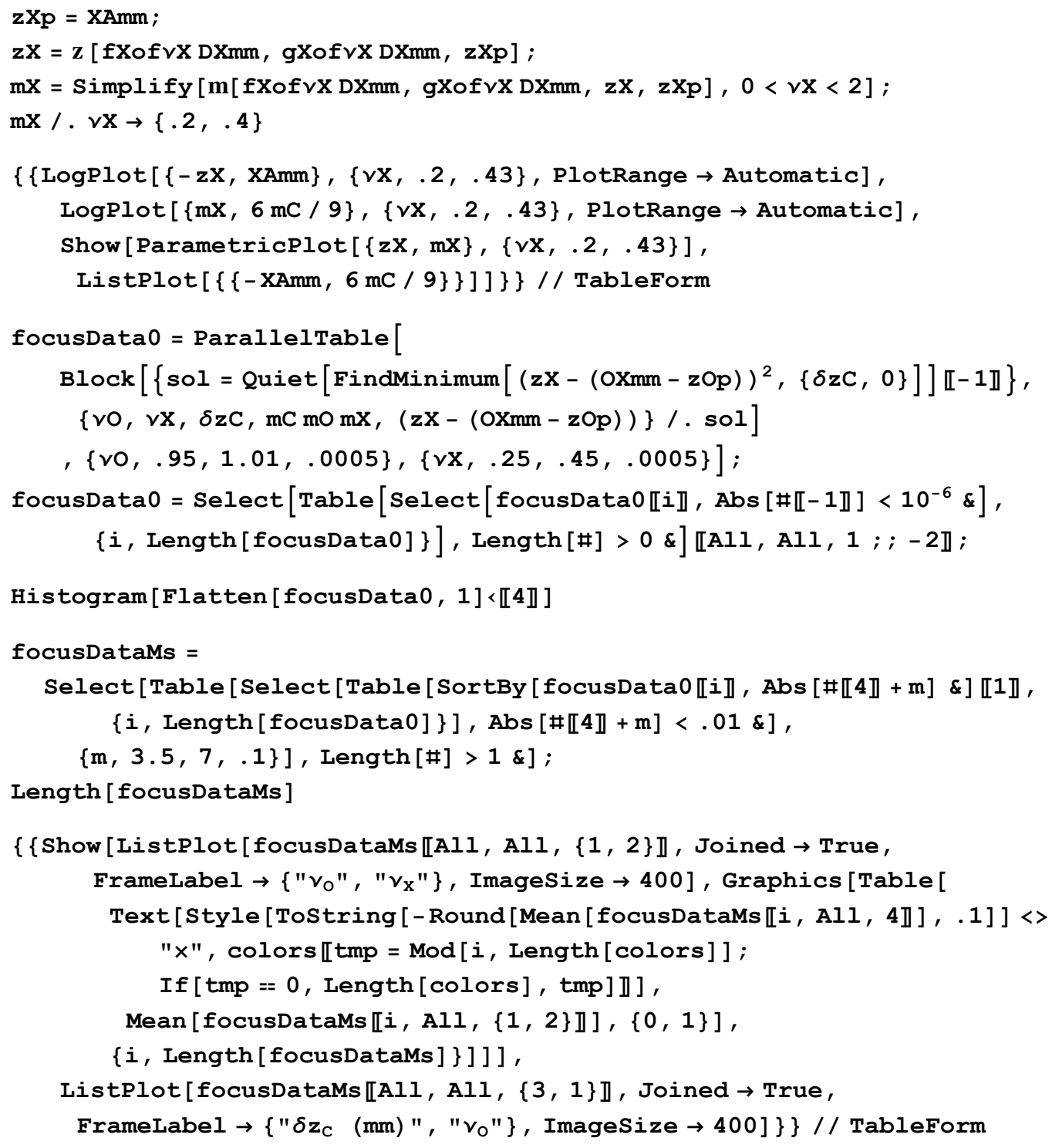


Show [ListPlot [ focusDataMs $\llbracket 1 ; ;-1 ; ; 5, A l l,\{1,2\}]$, Joined $\rightarrow$ True, Framelabel $\rightarrow\left\{" \mathrm{v}_{\mathrm{o}} / \mathrm{V}_{\mathrm{C}} ", " \mathrm{v}_{\mathrm{x}} / \mathrm{V}_{\mathrm{C}} "\right\}$, ImageSize $\rightarrow 300$, GridLines $\rightarrow$ None], Graphics [Table [Text [Style [ ToString [-Round [Mean [focusDataMs [i, All, 4]] , .1] ] > "x", colors $\mathbb{1}$ $\operatorname{tmp}=\operatorname{Mod}[i$, Length [colors] $] ; \operatorname{If}[\operatorname{tmp}==0$, Length [colors], tmp] $]$, Mean [focusDataMs $\llbracket i, A l 1,\{1,2\} \rrbracket],\{0,1\}]$,

$\{i, 1$, Length [focusDataMs ], 5\}] ] ]

\section{Optics, focusing as function of $z_{C}$, aberration $(\mathrm{mm})$}

Cathode object magnificaiton and aberration for Cu illuminated with $4.83 \mathrm{eV}$ light

$\mathrm{CsC}=\left(\frac{1 \mathrm{amm}+\delta \mathrm{zC}}{4}\right) 807$.
$\mathrm{CcC}=\left(\frac{1 \mathrm{amm}+\delta \mathrm{zC}}{4}\right) 844$.

Lens image aberration formulas

Clear $[\mathrm{m}, \mathrm{f}, \mathrm{Sf}, \mathrm{Sg}]$

$\operatorname{Csp}\left[m_{-}, f_{-}, S f_{-}, S g_{-}\right]:=-\left(\left(1+m^{2}\right) S g+2 m S f\right)(1+m)^{2} f$;

$\mathrm{Ccp}\left[\mathrm{m}_{-}, \mathrm{f}_{\text {_ }}, \mathrm{Cf}_{-}, \mathrm{Cg}_{-}\right]:=\left(\left(1+\mathrm{m}^{2}\right) \mathrm{Cg}+2 \mathrm{~m} \mathrm{Cf}\right) \mathrm{f}$;

CsSys $=\mathrm{mC}^{4} \mathrm{mo}^{4} \mathrm{mX}^{4} \mathrm{CsC}+\mathrm{mX}^{4} \mathrm{Csp}[\mathrm{mo}$, fOofvo DOmm, SfOofvo, SgOofvo] +

Csp [mX, fXof $\vee X D O m m, \operatorname{SfX} o f \vee X, \operatorname{SgXof} \vee X]$;

CsSysNoC $=\mathrm{mX}^{4}$ Csp $[\mathrm{mo}$, fOofvo DOmm, SfOofvo, SgOofvo $]+$

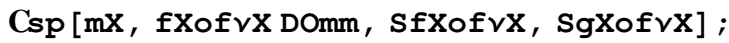

CcSys $=\mathrm{mC}^{2} \mathrm{mO}^{2} \mathrm{mX}^{2} \mathrm{CcC}+\mathrm{mX}^{2} \mathrm{Ccp}[\mathrm{mo}$, fOofvo DOmm, CfOofvo, CgOofvo] +

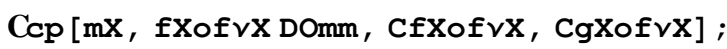

CcSysNoC $=m x^{2}$ Ccp $[\mathrm{mo}$, fOofvo DOmm, CfOofvo, CgOofvo $]+$

$\operatorname{Ccp}[\mathrm{mX}$, fXof $v X \mathrm{DOmm}, \operatorname{CfX} o f v X, \operatorname{CgXof} v X]$;

focusAberDataMs =

Table [Block [ $\{v 0=$ focusDataMs $\llbracket i, j, 1 \rrbracket, v X=$ focusDataMs $\llbracket i, j, 2 \rrbracket$, $\delta \mathrm{zC}=$ focusDataMs $\llbracket i, j, 3 \rrbracket, m=$ focusDataMs $\llbracket i, j, 4 \rrbracket\}$,

Join [focusDataMs $\llbracket i, j \rrbracket,\{$ CsSys, CcSys $\},\left\{\right.$ CsSys $/ \mathrm{m}^{4}$, CcSys $\left./ \mathrm{m}^{2}\right\}$, $\{$ CsSysNoC, CcSysNoC $\},\left\{\right.$ CsSysNoC $/ \mathrm{m}^{4}$, CcSysNoC $\left.\left.\left./ \mathrm{m}^{2}\right\}\right]\right]$

$,\{i$, Length[focusDataMs] $\},\{j$, Length [focusDataMs $\llbracket i \rrbracket]\}]$;

$\left\{\left\{\right.\right.$ Show [ListLogLogPlot [focusAberDataMs $\llbracket A l 1, A l 1,\{5,6\} \rrbracket / 10^{3}$, Joined $\rightarrow$ True, Framelabel $\rightarrow\left\{\right.$ "C $\left._{s}^{\prime}(m) ", " C_{c}^{\prime}(m) "\right\}$, ImageSize $\left.\rightarrow 400\right]$, ParametricPlot $\left[\log \left[\left\{\operatorname{CsC} 6^{4}, \mathrm{CcC} 6^{2}\right\} / 10^{3}\right],\{\delta \mathrm{zC}, 0, .5\}\right.$, Plotstyle $\rightarrow\{\{$ Thick, Red $\}\}]$, Graphics [Table[Text [ Style [ToString [-Round [Mean [focusAberDataMs [i, All, 4]], .1]] 〈> $" x ", \operatorname{colors}[$ tmp $=\operatorname{Mod}[i$, Length [colors] $]$; If $[$ tmp $=0$, Length [colors], tmp] ] ], 


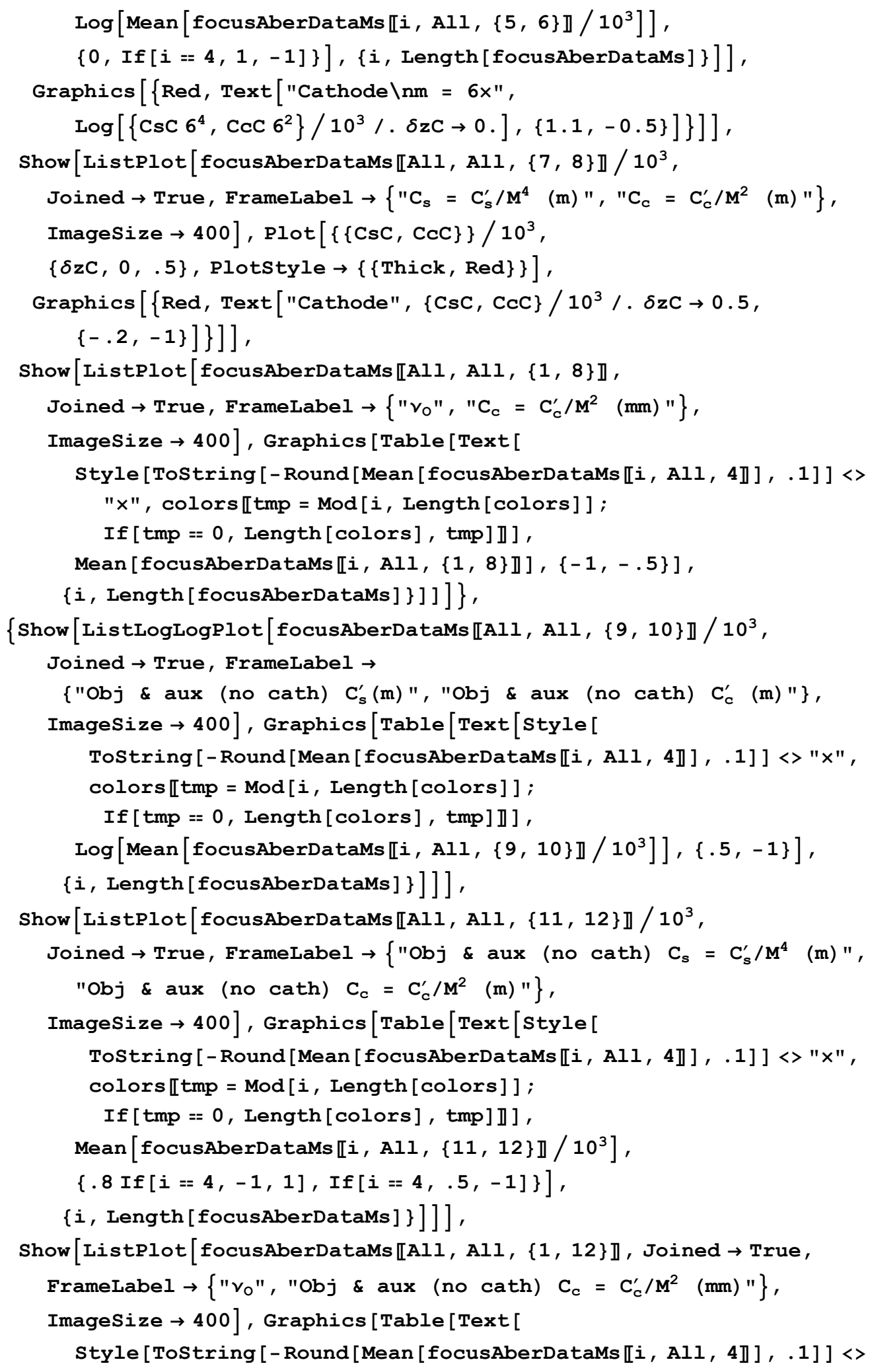




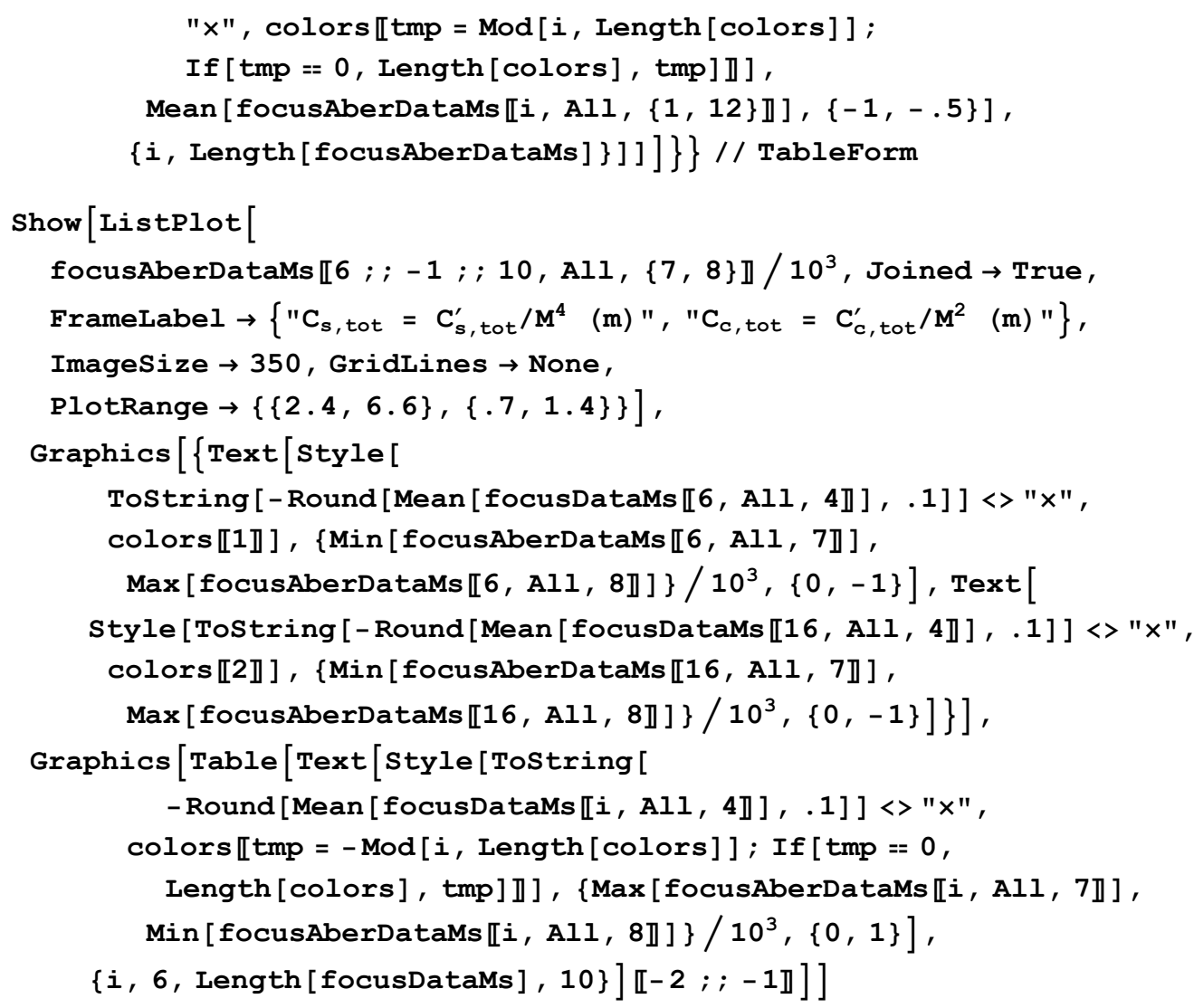




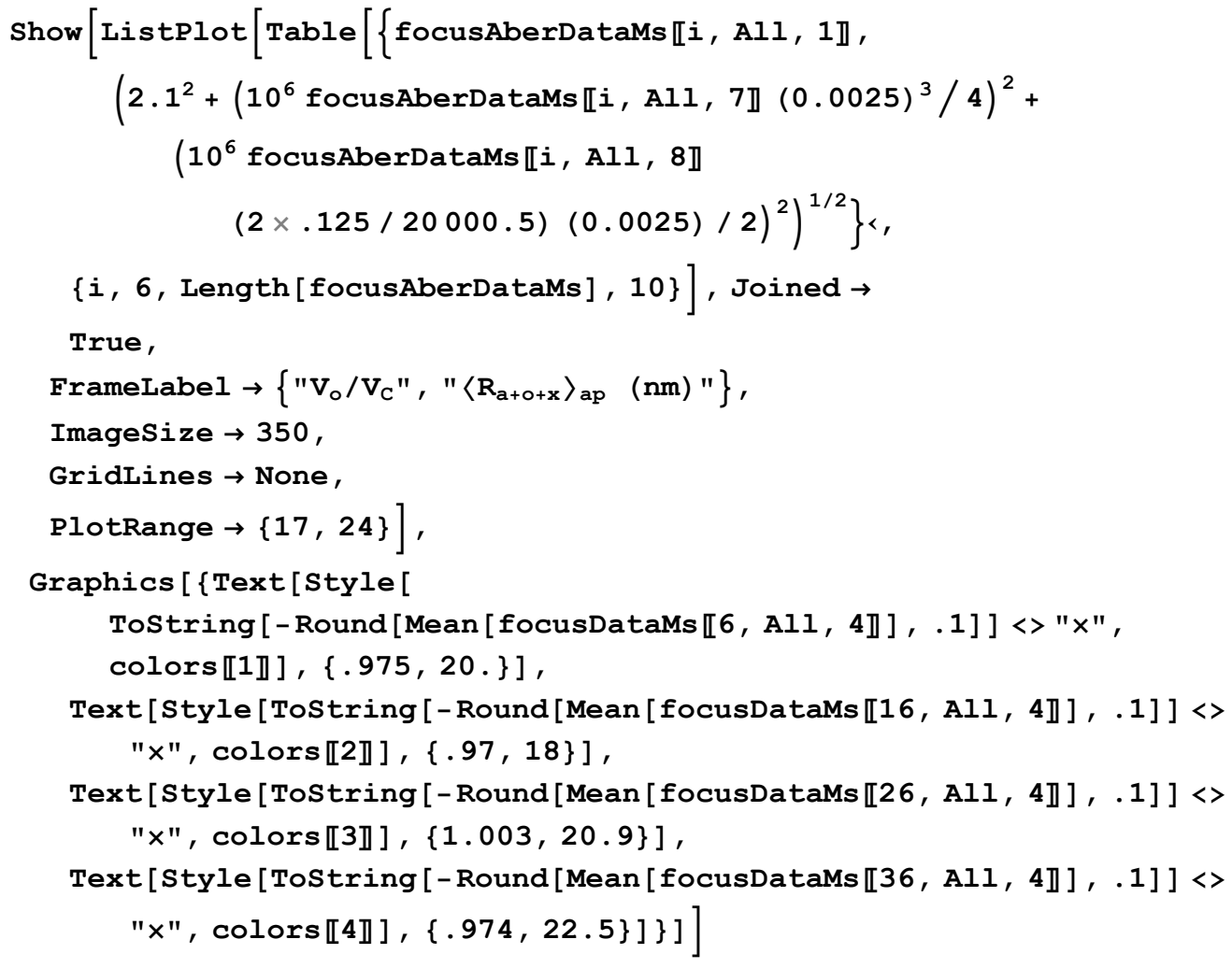

\section{Aberration comparison to diode}

Hyperbolic trajectory functions

$\omega=\sqrt{e k / m}, k$ defines shape of hyperbolic potential. Functions of time $t$

$r$ Hyp $\left[\omega_{-}, r 0_{-}, \operatorname{vr} 0_{-}, \theta_{-}\right]:=r 0 \operatorname{Cos}[\theta]+\operatorname{vr} 0 \operatorname{Sin}[\theta] / \omega$;

$\mathrm{zHyp}\left[\omega_{-}, \mathrm{z}_{-}, \mathrm{vz} 0_{-}, \theta_{-}\right]:=\mathrm{z} 0 \operatorname{Cosh}[\sqrt{2} \theta]+\mathrm{vz} 0 \operatorname{Sinh}[\sqrt{2} \theta] /(\sqrt{2} \omega) ;$

$\operatorname{vrHyp}\left[\omega_{-}, \mathbf{x} 0_{-}, \mathrm{v} 0_{-}, \theta_{-}\right]:=\omega$ Derivative $[0,0,0,1][\mathrm{rHyp}][\omega, \mathbf{x} 0, \mathrm{v} 0, \theta]$; vzHyp $\left[\omega_{-}, \mathbf{x} 0_{-}, v 0_{-}, \theta_{-}\right]:=\omega$ Derivative $[0,0,0,1][\mathbf{z H y p}][\omega, \mathbf{x} 0, \mathrm{v} 0, \theta]$;

Invert $z(t)$ to get $\theta(z)$

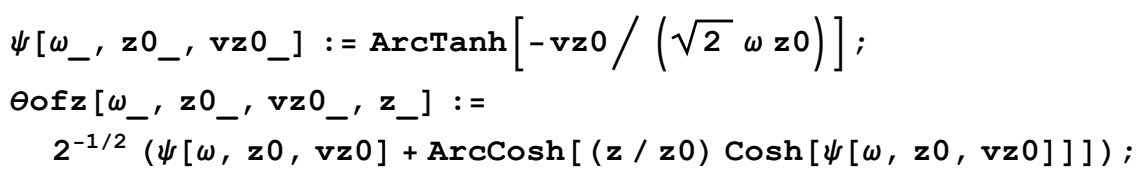

Davisson-Calbick approximation

Trajectory through an aperture, from left to right. 


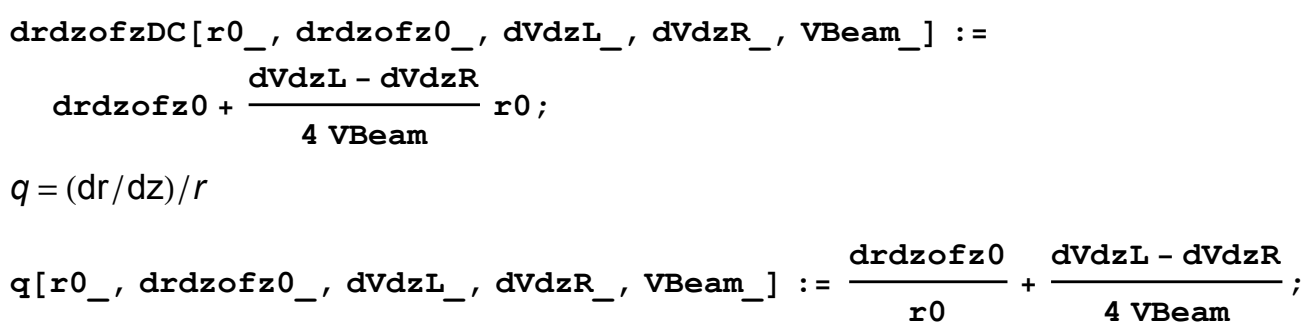

Put it all together

Variables: $v_{L}=V_{L} / V_{C}, v_{A}=V_{A} / V_{C}, v_{M}^{-1}=V_{M} / V_{C}$.

Constants: $\omega_{1}=\sqrt{e k_{1} / m}, k_{1}=V_{C}\left(v_{A}-v_{M}{ }^{-1}\right) / \ell^{2}$.

Lens half width $=s$.

Turn-around radial extent, limiting variable $r_{C}$

Position and velocity at the aperture

$z C\left[r C_{-}\right]:=\sqrt{\left\{2 \frac{\nu M-1}{V A V M-1}+r C^{2} / 2\right.} ;$

$r A=\operatorname{Refine}[\mathrm{rHyp}[\omega, \mathrm{rC}, 0, \theta \mathrm{A}[\mathrm{rC}]], \omega>0]$

$\mathbf{z A}=\operatorname{Refine}[\mathbf{z H y p}[\omega, \mathbf{z C}[r \mathrm{C}], 0, \theta \mathrm{A}[\mathrm{rC}]], \omega>0] ;$

$\operatorname{vrA}=\operatorname{Refine}[\operatorname{vrHyp}[\omega, \mathrm{rC}, 0, \theta \mathrm{A}[\mathrm{rC}]], \omega>0]$;

$\mathrm{vzA}=\operatorname{Refine}[\mathrm{vzHyp}[\omega, \mathbf{z C}[\mathrm{rC}], 0, \theta \mathrm{A}[\mathrm{rC}]], \omega>0] ;$

$\operatorname{drdzA}=\operatorname{Refine}[\mathrm{vrA} / \mathrm{vzA}, \omega>0]$;

$\operatorname{drdzAd}=$ Refine $[\mathrm{drdzofzDC}[\mathrm{rA}, \mathrm{drdzA}$,

$\left.2 \mathrm{VC}\left(\nu \mathrm{A}-v^{-1}\right)\left\{{ }^{-2} \mathrm{zA}, 0,-\mathrm{VC}(1-\nu \mathrm{A})\right],\{\omega>0, v C<0\}\right]$

Approximations made at the aperture

$\theta \mathrm{AO}\left[\mathrm{rC} \_\right]:=\theta 0 f \mathbf{z}[\omega, \mathbf{z C}[\mathrm{rC}], 0,\{] ; \theta \mathrm{A} 0[\mathrm{rC}]$

$r A 1\left[r C_{-}\right]:=r H y p[\omega, r C, 0, \theta A 0[r C]] ; r A 1[r C]$

$\theta \mathrm{A} 1\left[\mathrm{rC} \_\right]:=\theta 0 \mathrm{zz}\left[\omega, \mathrm{zC}[\mathrm{rC}], 0, \sqrt{\left\{^{2}+\mathrm{rA} 1[\mathrm{rC}]^{2} / 2\right.}\right] ; \theta \mathrm{A} 1[\mathrm{rC}]$

Image location $z^{\prime}$ and ray angle squared $\alpha^{\prime 2}$.

$\mathbf{z D i o d e}=\mathbf{z A}-(\text { Expand }[\mathrm{drdzAd} / \mathrm{rA}])^{-1} ;$

$\alpha$ SquaredDiode $=\operatorname{ArcTan}[-\mathrm{drdzAd}]^{2}$;

Paraxial image location $z_{0}^{\prime}$ and first and second order correction terms $z_{1}^{\prime} r_{C}=0$ and $z_{2}^{\prime} r_{C}^{2}$

$z$ 0Diode $=$ SeriesCoefficient $[z$ Diode,$\{r C, 0,0\}] ;$

z0Diode $=\operatorname{Block}\left[\{\theta \mathrm{A}\}, \theta \mathrm{A}\left[\mathrm{rC} \_\right]:=\theta \mathrm{A} 0[\mathrm{rC}] ;\right.$

Refine [z0Diode, $\{>0 \& \& \nu A>0 \& \& \nu \mathbb{A}<1 \& \& \nu M>0 \& \& \nu M<1]$ ];

$\mathbf{z} 1$ Diode $=$ SeriesCoefficient $[\mathbf{z D i o d e},\{\mathrm{rC}, 0,1\}] ;$

z1Diode $=\operatorname{Block}\left[\{\theta \mathrm{A}\}, \theta \mathrm{A}\left[\mathrm{rC}_{-}\right]:=\theta \mathrm{A} 0[\mathrm{rC}] ;\right.$ z1Diode $] ;$ 
z2Diode = SeriesCoefficient $[z$ Diode,$\{r C, 0,2\}]$;

$\mathbf{z} 2 \mathrm{Diode}=\operatorname{Block}\left[\{\theta \mathrm{A}\}, \theta \mathrm{A}\left[\mathrm{rC} \_\right]:=\theta \mathrm{A} 0[\mathrm{rC}]\right.$;

Refine [z2Diode, $\{>0 \& \& \vee A>0 \& \& V A<1 \& \& V M>0 \& \& V M<1]$ ] ;

Second order angle expansion $a^{\prime 2}=\left(\alpha^{\prime 2}\right)_{1} r_{C}+\left(\alpha^{\prime 2}\right)_{2} r_{C}^{2}+O\left(r_{C}^{3}\right),\left(\alpha^{\prime 2}\right)_{1}=0$

$\alpha$ Squared2Diode $=$ SeriesCoefficient $[\alpha$ SquaredDiode,$\{r C, 0,2\}]$;

$\alpha$ Squared2Diode $=\operatorname{Block}\left[\{\theta \mathrm{A}\}, \theta \mathrm{A}\left[\mathrm{rC} \_\right]:=\theta \mathrm{A} 0[\mathrm{rC}] ;\right.$

Refine [ $\alpha$ Squared2Diode, $\{>0 \& \& \nu \mathrm{A}>0 \& \& \nu \mathrm{A}<1 \& \& V \mathrm{M}>0 \& \& V \mathrm{M}<1]$ ] ;

Coefficient of chromatic aberration, $C_{C}=V_{C} \frac{2 \partial z_{0}}{\partial V_{C}}=2\left(v_{M} \partial_{v_{M}} z_{0}-v_{A} \partial_{v_{A}} z_{0}\right)$

CcDiode $=2\left(\nu M \partial_{V M} z\right.$ ODiode $-V A \partial_{V A} z$ ODiode $) ;$

Coefficient of spherical aberration, $z^{\prime}=z_{0}-2^{-1} C_{s} \alpha^{\prime 2} \rightarrow C_{s}=-2 z_{2}^{\prime} /\left(\alpha^{\prime 2}\right)_{2}$

CsDiode = - 2 z2Diode / $\alpha$ Squared2Diode ;

Import interface lens properties

SetDirectory [

\$HomeDirectory <> "/Dropbox/Electron optics/PEEM optical models"];

\{propertiesL, propertyFitsL $\}=$

Import ["Swept-back lens properties, $D=0.242$ in.wdx"] ;

$\{\{f I, g I\},\{S f I, S g I\},\{C f I, C g I\}\}=$ propertyFitsI $/ . v \rightarrow v I$

MImm $=(715.250-558.565) 25.4 / 90$

IBmm $=(972.102-715.250) 25.4 / 90$

Thick lens equations for focal length and distance from Rempfer 1985

Clear $[f, g, z, z p, m]$;

$z\left[f_{-}, g_{-}, z p_{-}\right]:=g+\frac{f^{2}}{z p-g} ;$

$\operatorname{zp}\left[f_{-}, g_{-}, z_{-}\right]:=g+\frac{f^{2}}{z-g} ;$

$m\left[f_{-}, g_{-}, z_{-}, z_{-}\right]:=\sqrt{\frac{z p-g}{z-g}} ;$

mo $\left[f_{-}, g_{-}, z_{-}\right]:=f /(z-g)$;

$\operatorname{mi}\left[\mathrm{f}_{-}, \mathrm{g}_{-}, \mathrm{zp}_{-}\right]:=(\mathrm{zp}-\mathrm{g}) / \mathrm{f}$;

Aberration formulas at the Gaussian plane (not circle of least confusion) 


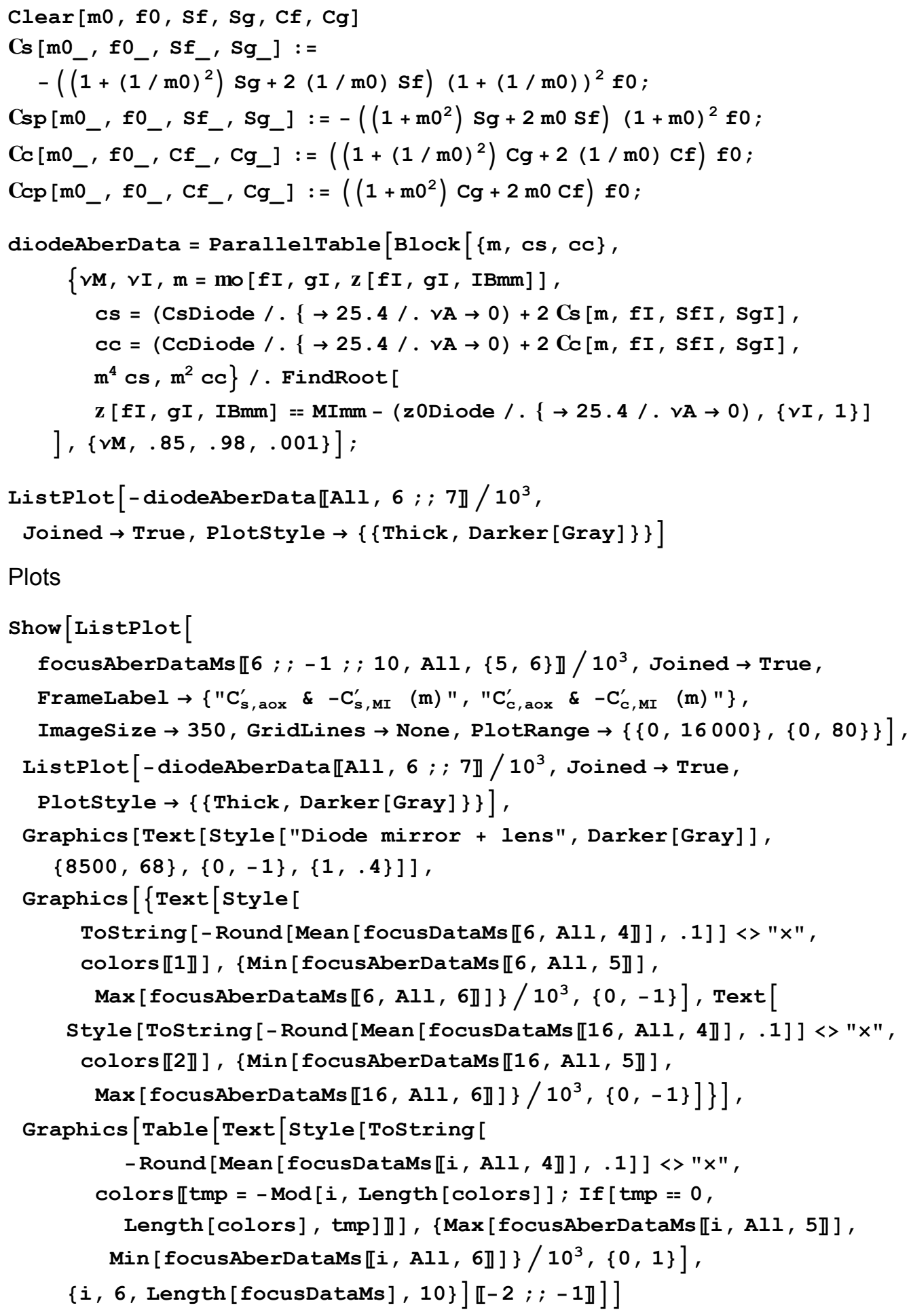




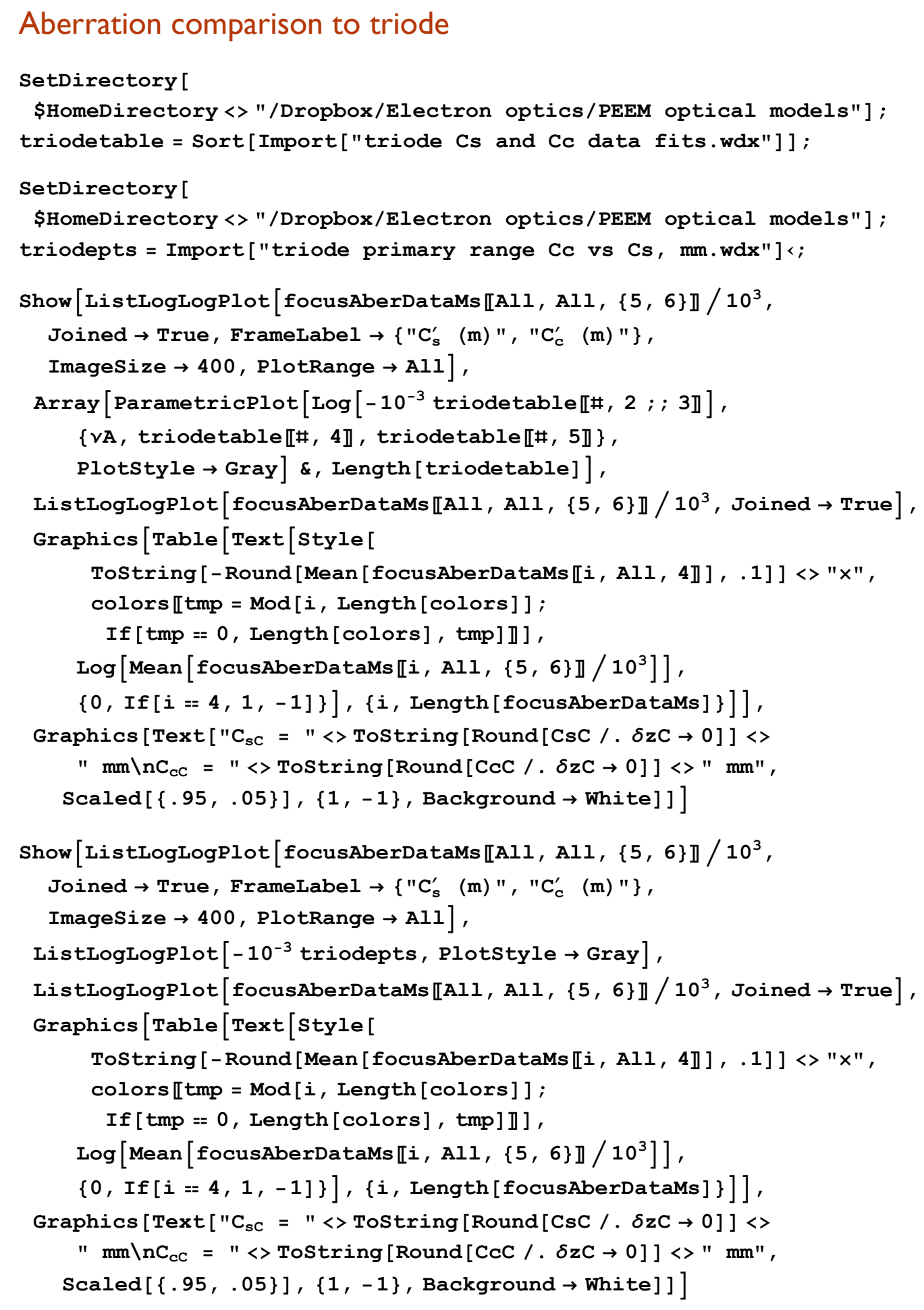




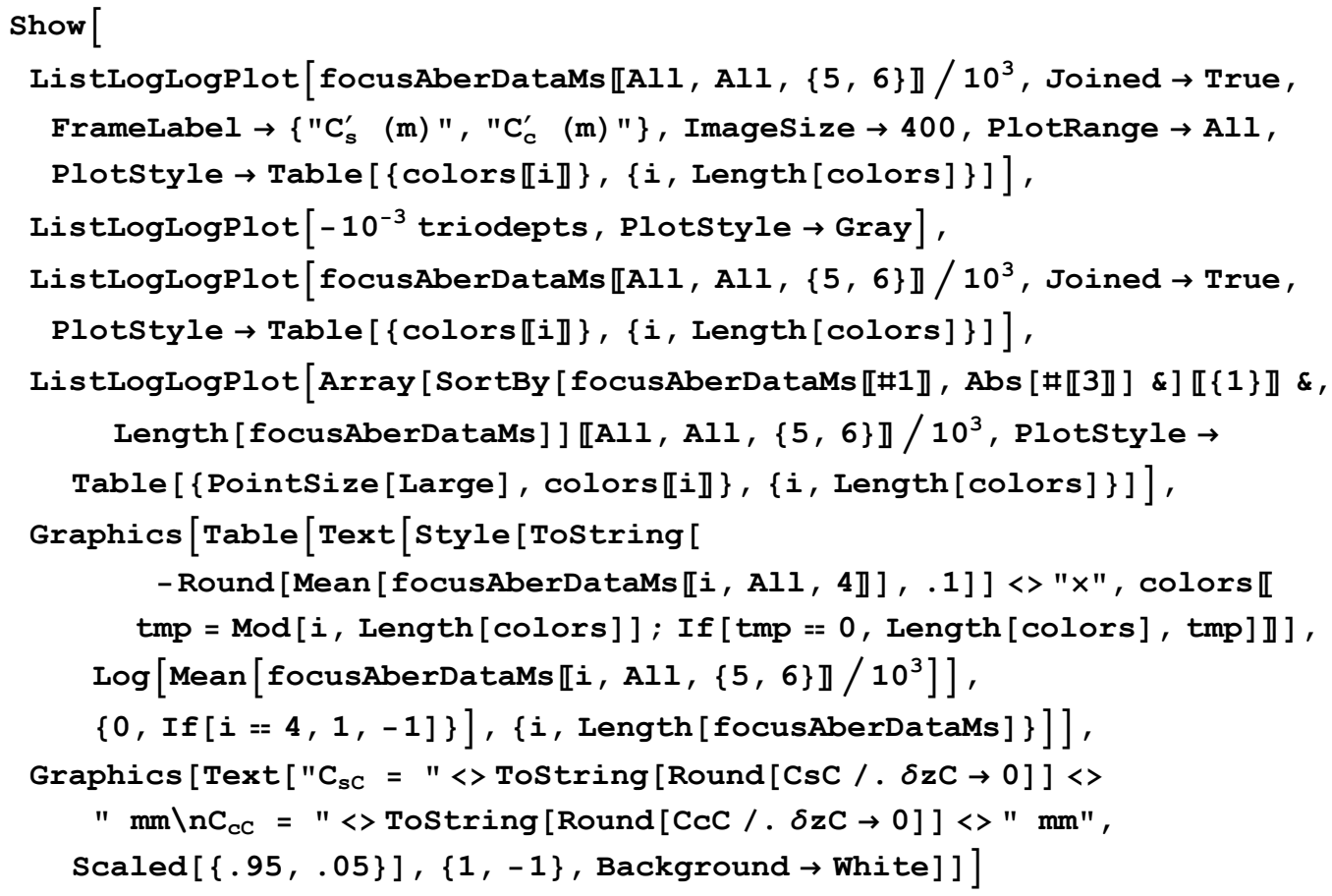

\section{Export tables}

Fit focus data to polynomials of $\nu \bigcirc$

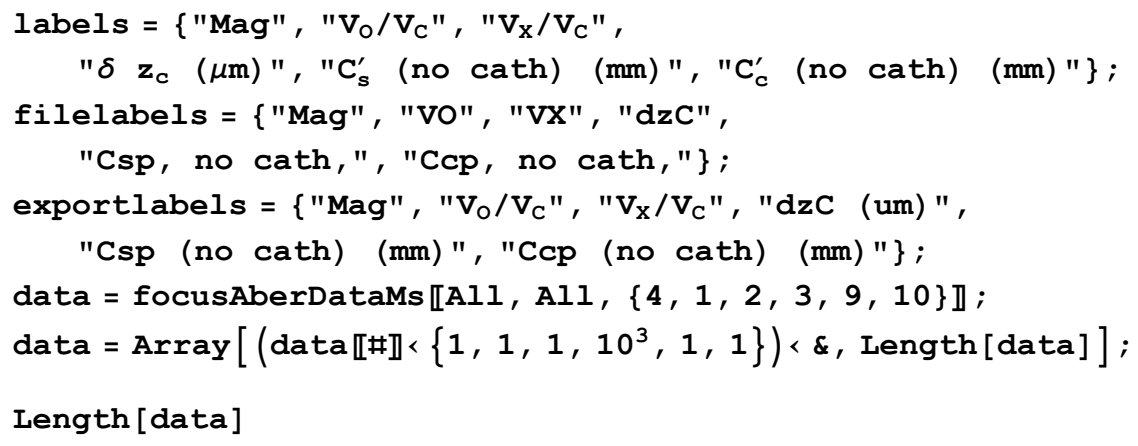




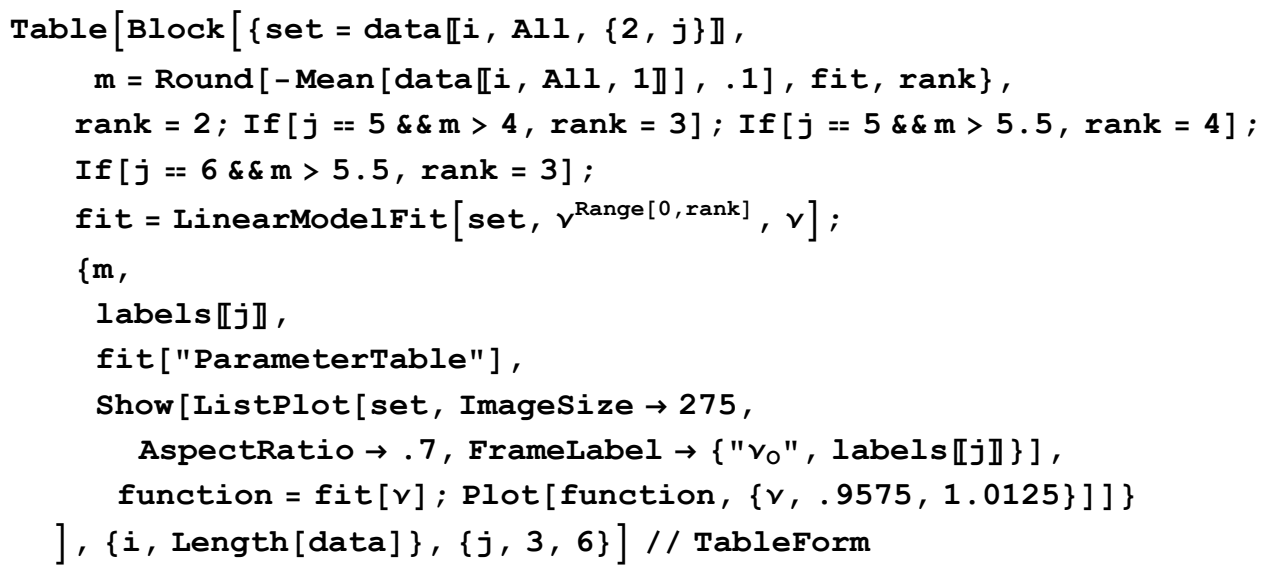

Tables with all magnifications

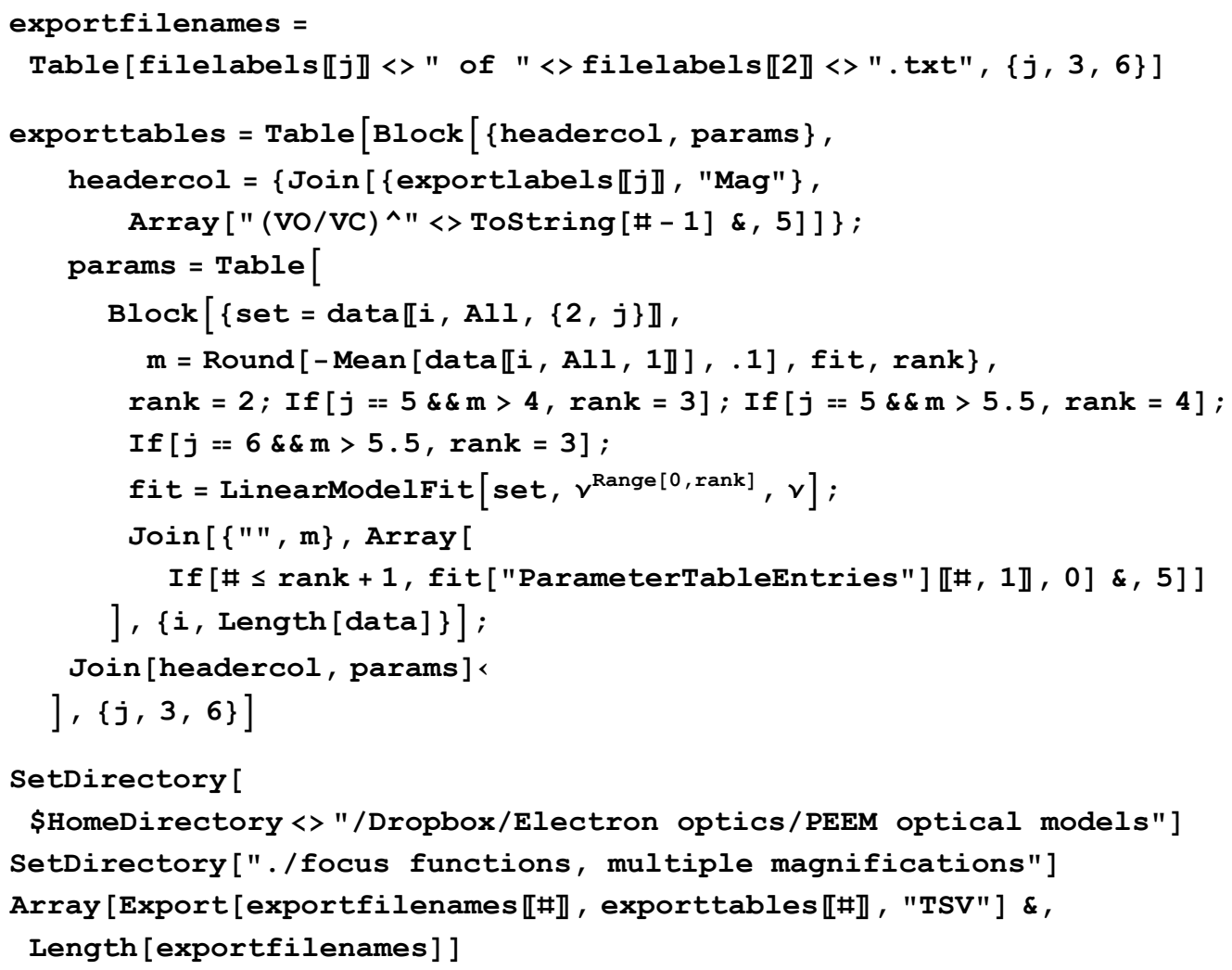

Tables separated by magnification

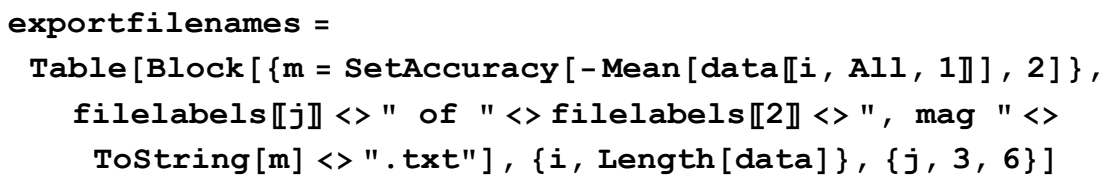




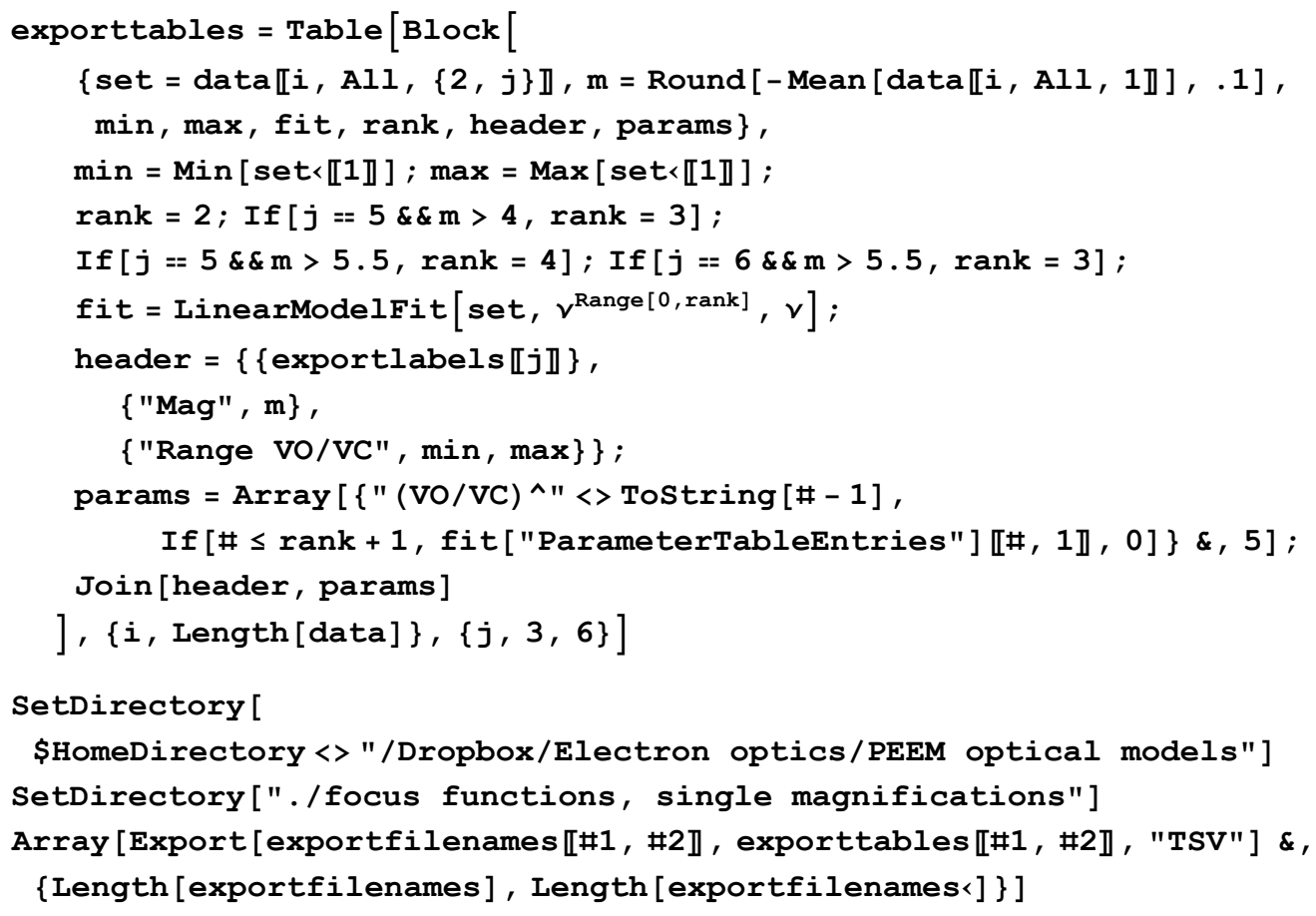

Fit focus data to polynomials of $\delta \mathrm{zC}$

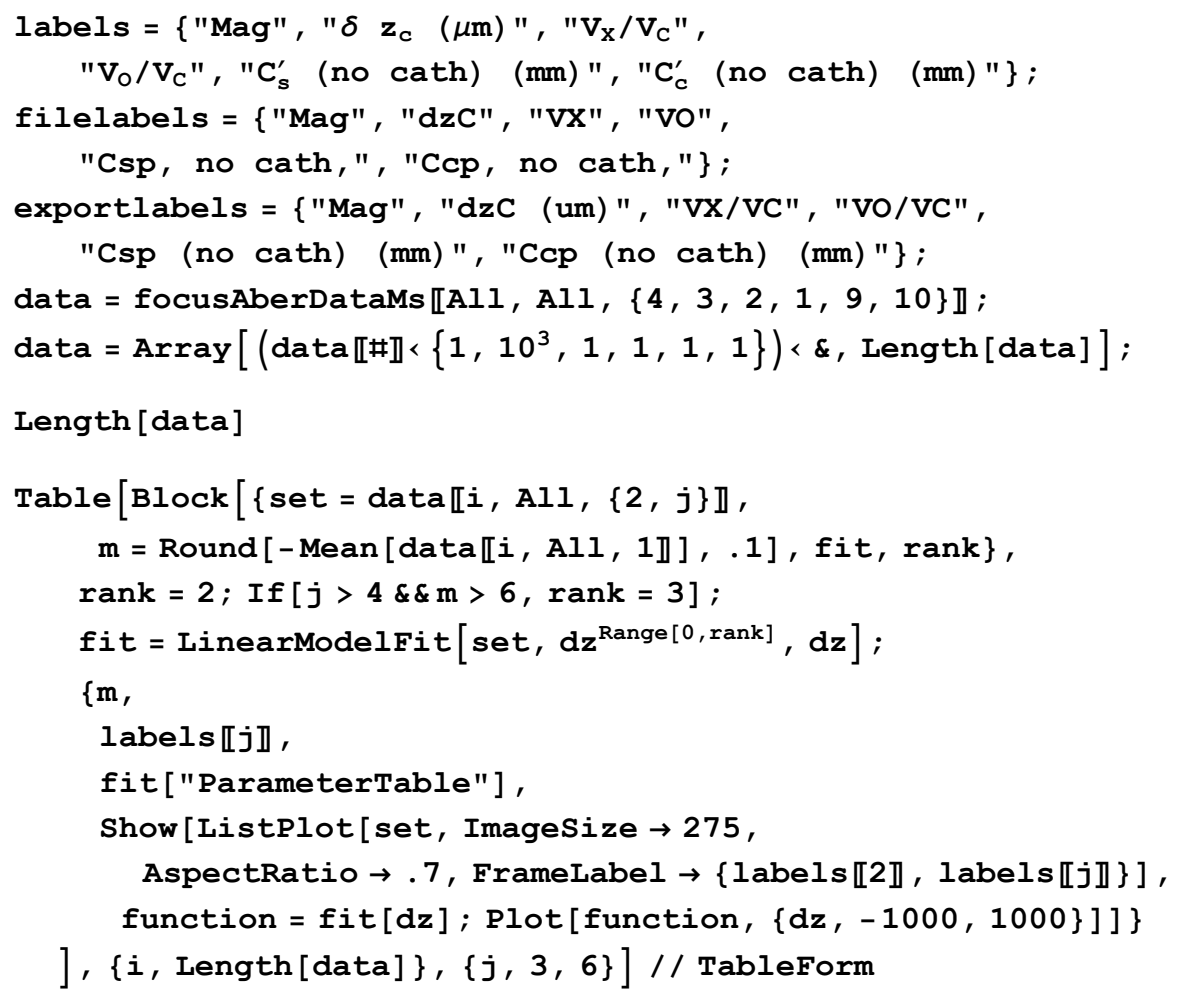


Tables with all magnifications

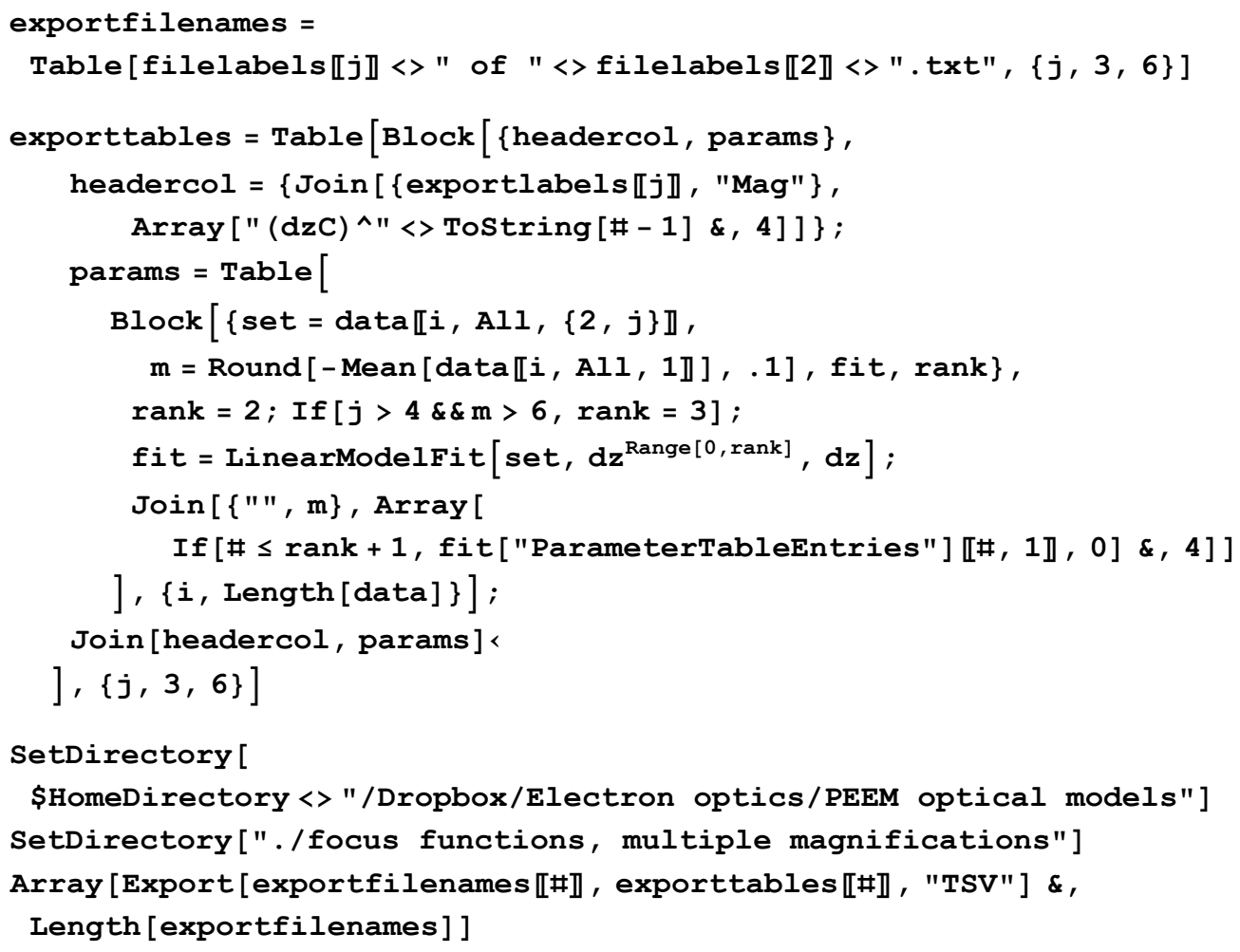

Tables separated by magnification

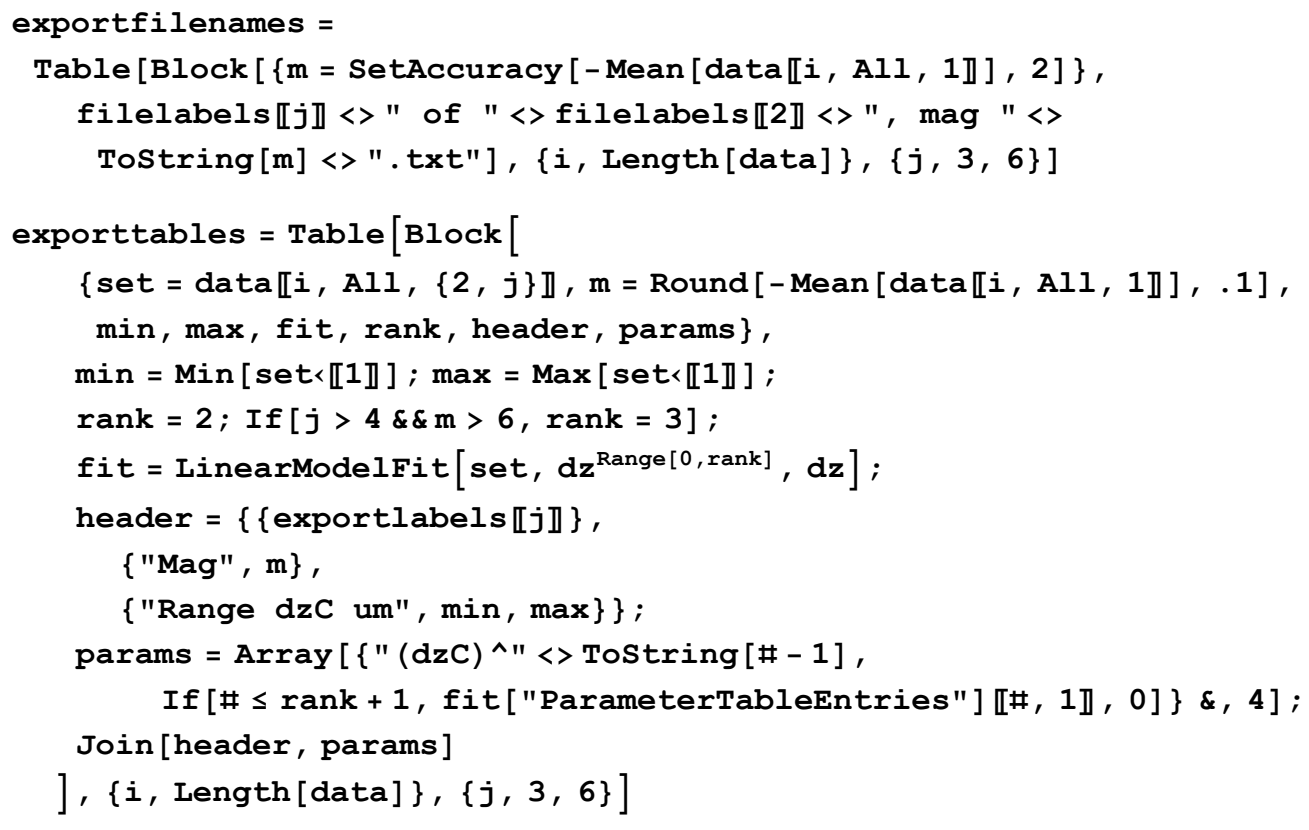


SetDirectory [

\$HomeDirectory <> "/Dropbox/Electron optics/PEEM optical models"]

SetDirectory["./focus functions, single magnifications"]

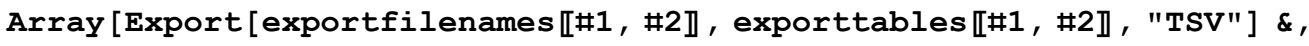
\{Length [exportfilenames], Length [exportfilenames<] \}] 


\section{Appendix G Code, simion: diode hyperbolic mirror}

It was necessary to characterize the diode hyperbolic mirror to compare the strength of the SIMION analysis to the theory in a situation where the theory was known to be at least approximately accurate. In addition, the diode mirror characterization allowed a simple estimation of the aberration in our PEEM. By running the calculation at the same potentials as used in the instrument, the aberration correction of the diode could be measured. Assuming that the diode mirror is operating as designed, this is the also the aberration of our PEEM. It was later determined that the diode mirror may not be correcting aberration as well as hoped - see Fig. 3.5. The interface lens is not included with diode mirror since the separation between the two is sufficiently large that they do not influence each other.

The following code calculates the optical properties of the mirror in SIMION. by first finding the symmetric mode object distance, and then determining the paraxial image distance and spherical aberration coefficient. The incident beam energy is then adjusted, and the symmetric paraxial image distance and spherical aberration coefficient are measured again. The chromatic aberration coefficient is calculated from the difference between the two image distances, and spherical aberration is taken as the mean of the two spherical aberration coefficients. For the immersion triode mirror and lens this method was significantly refined, so it is worth looking at the code there as well. The Mathematica code compiling the data is fairly straightforward, so it is not included here.

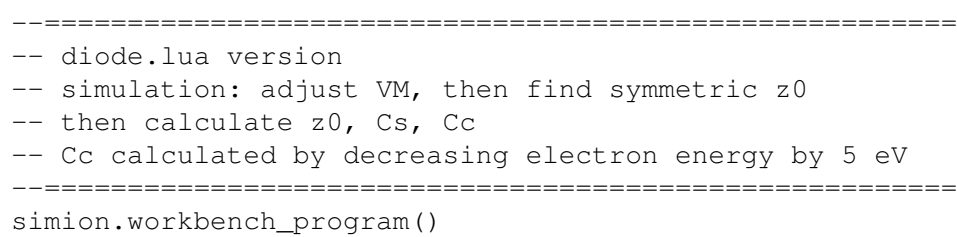




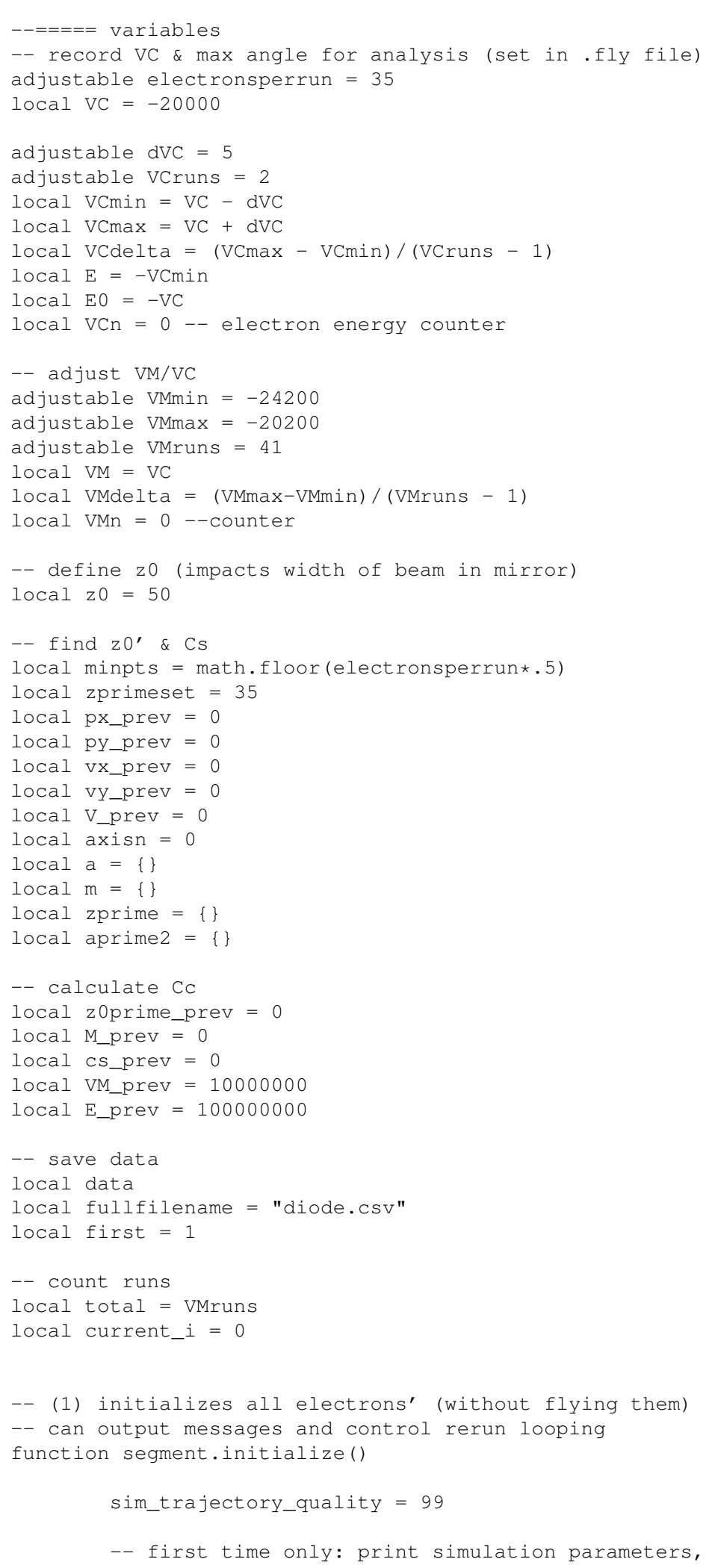




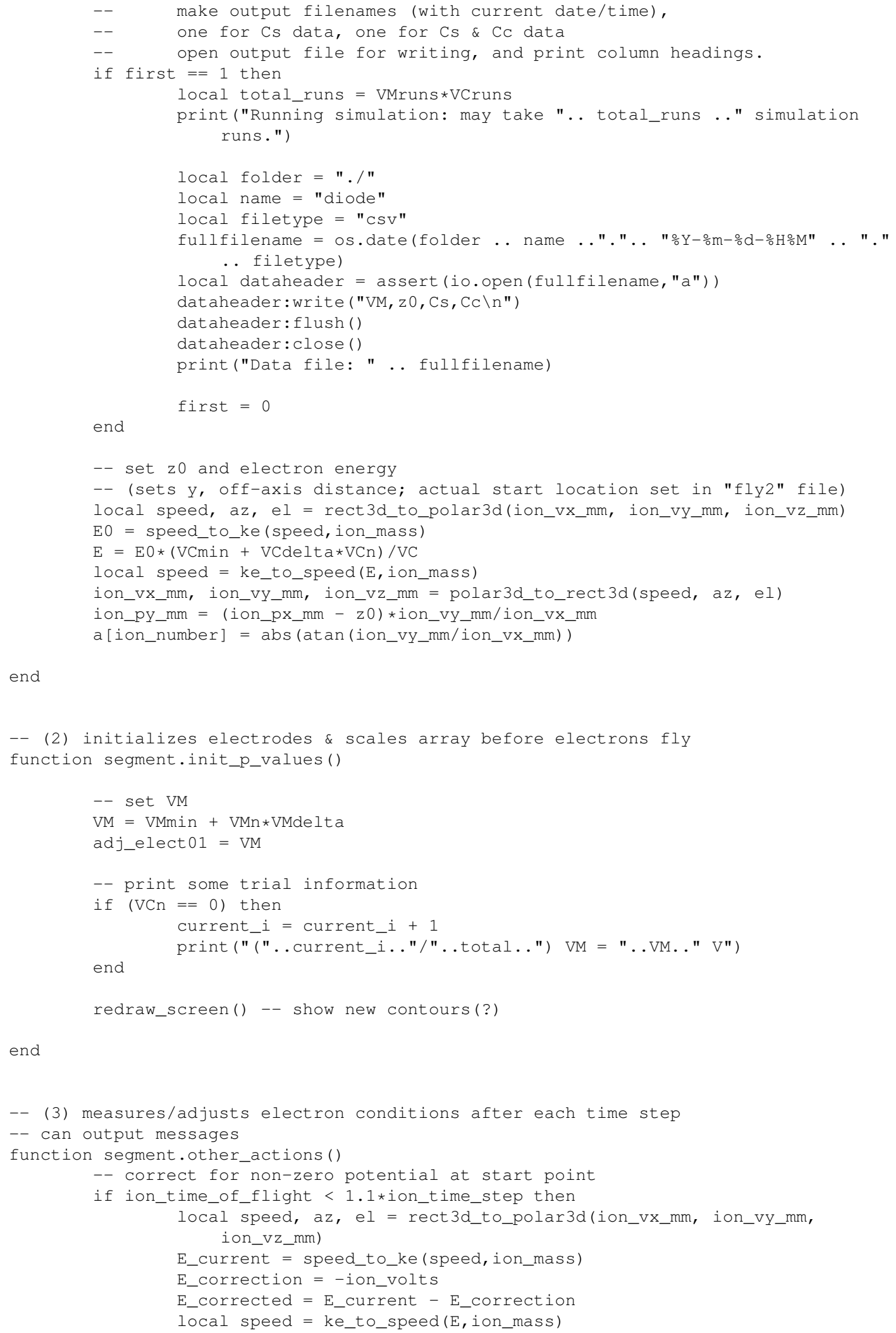




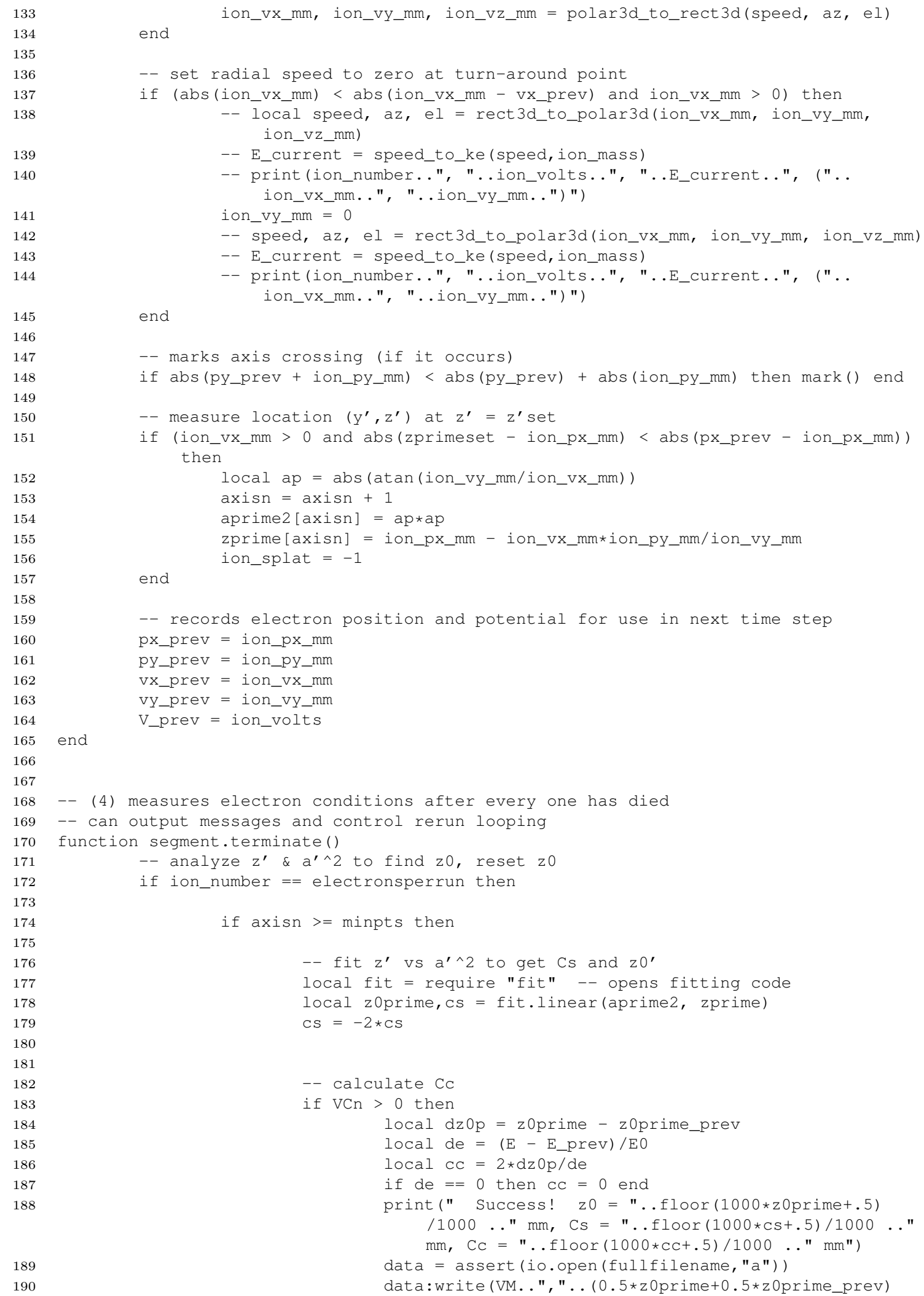




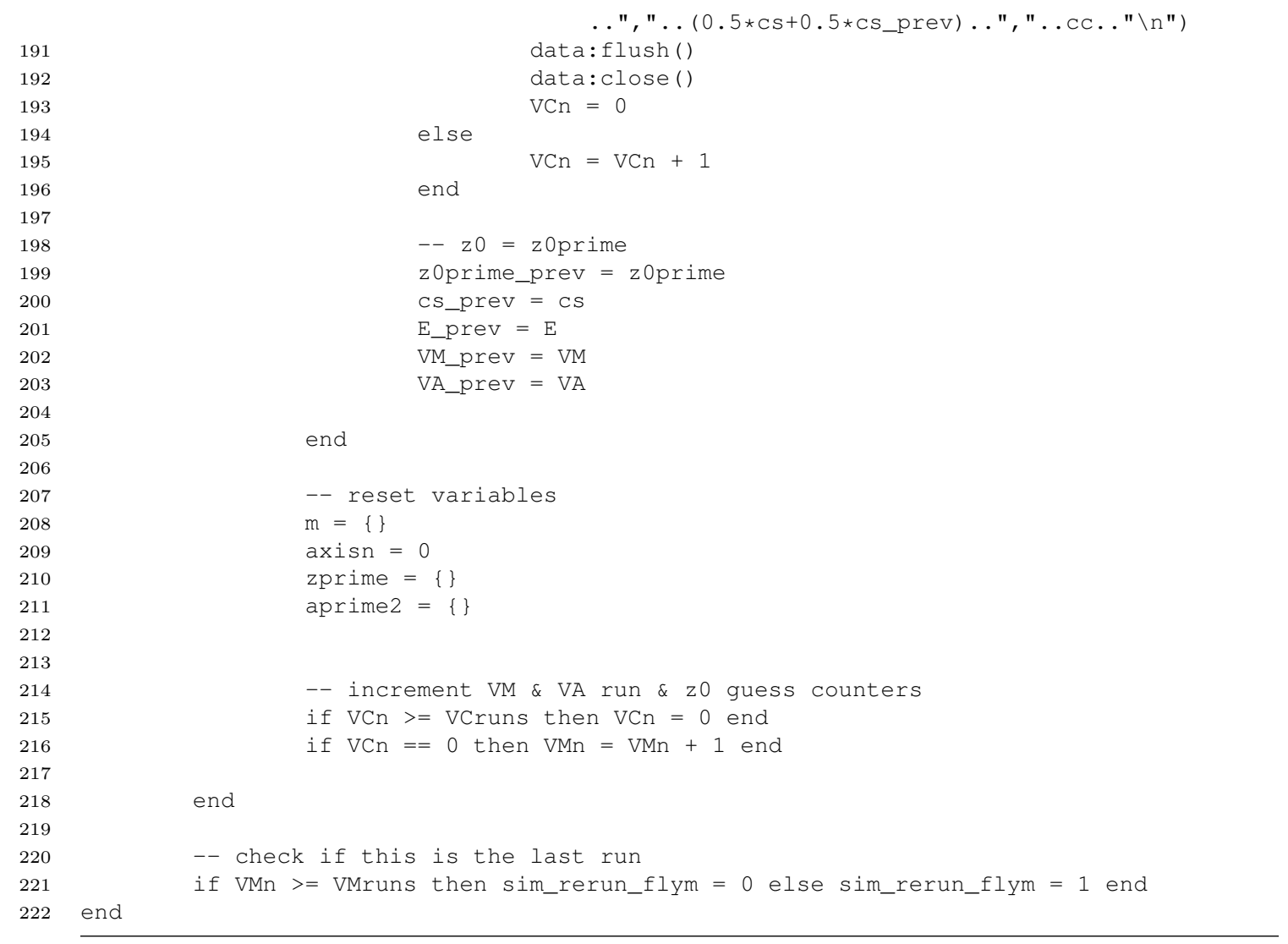




\section{Appendix H Code, simion: triode hyperbolic mirror}

It was necessary to fully characterize the triode hyperbolic mirror and interface lens in SIMION to very high degree in order to provide sufficient control of aberration correction and focus of the mirror branch. The triode mirror was installed without a grounded front electrode, such that there is a significant potential gradient between the center electrode at $V_{A}$ and the outer grounded electrode of the interface lens. As such, it was necessary to model the triode mirror and lens together to capture the complex interaction between them.

The final implementation of this analysis includes several refinements over the initial code. First, the object distance is fixed, and symmetric mode is determined by tuning the lens potential. Over the range in interest, this potential is fairly linear; however, over wider ranges this method could break down. Second, three beam energies are used: the central energy, one below, and one above. Third, the computation only obtains a symmetric mode image distance for the central beam energy. It was found that tuning object position for symmetric mode in the other cases introduces chromatic aberration into the point source object. The desired model is one in which the object has no aberration and the image has all the aberration, instead of some in the image and some in the object, as here. This led to faster analysis, and less uncertainty in the computation. Finally, this code dumps basic trajectory data into the data file, and Mathematica is used to calculate the optical properties.

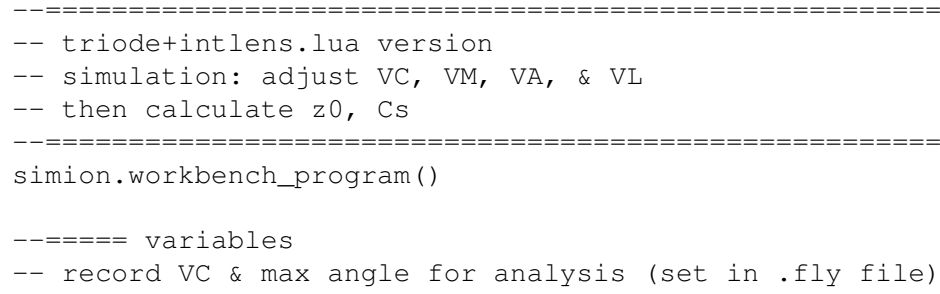




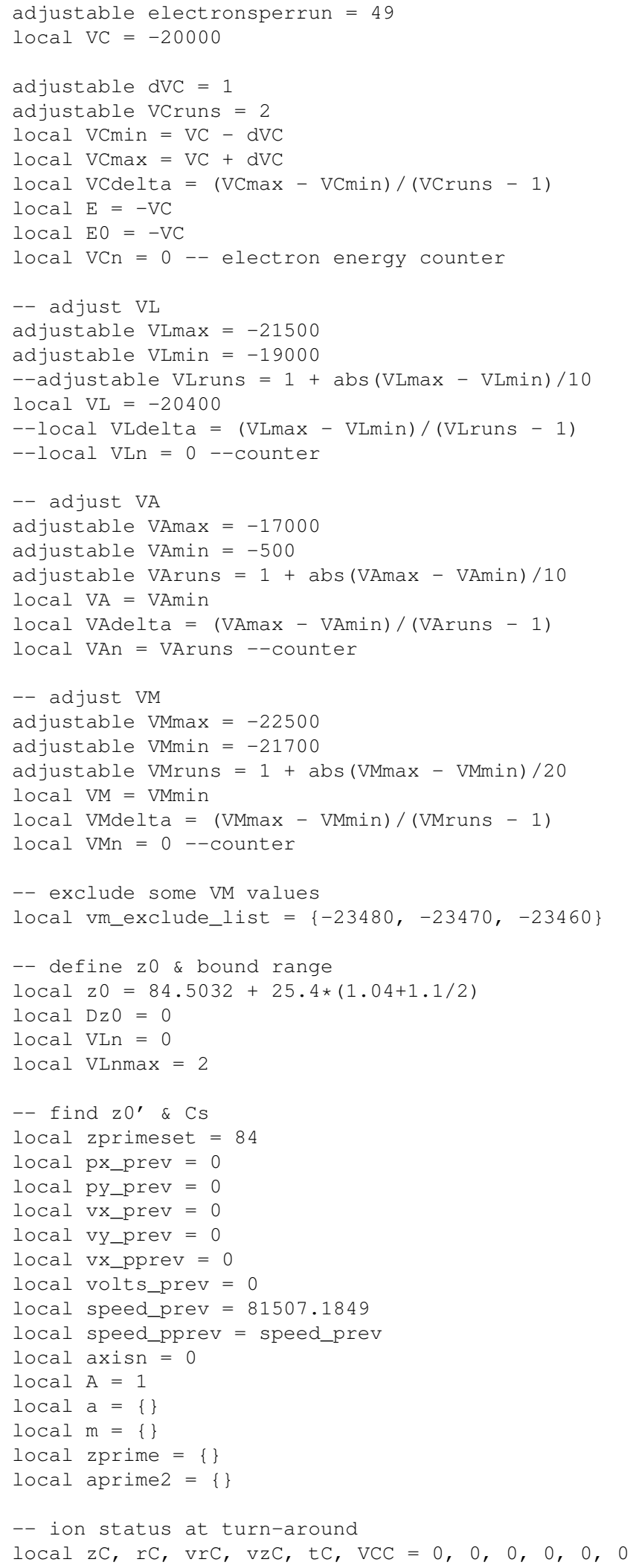




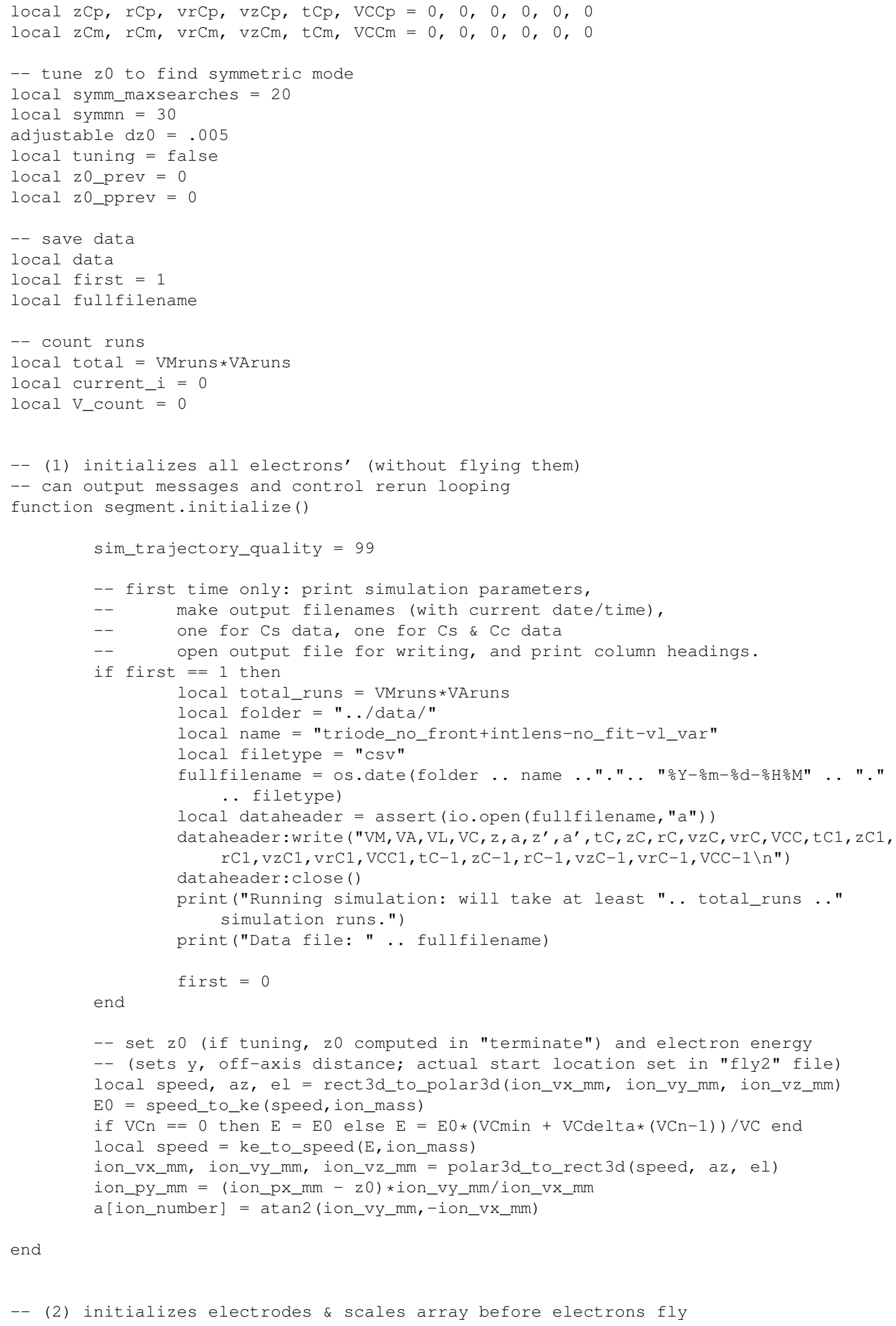




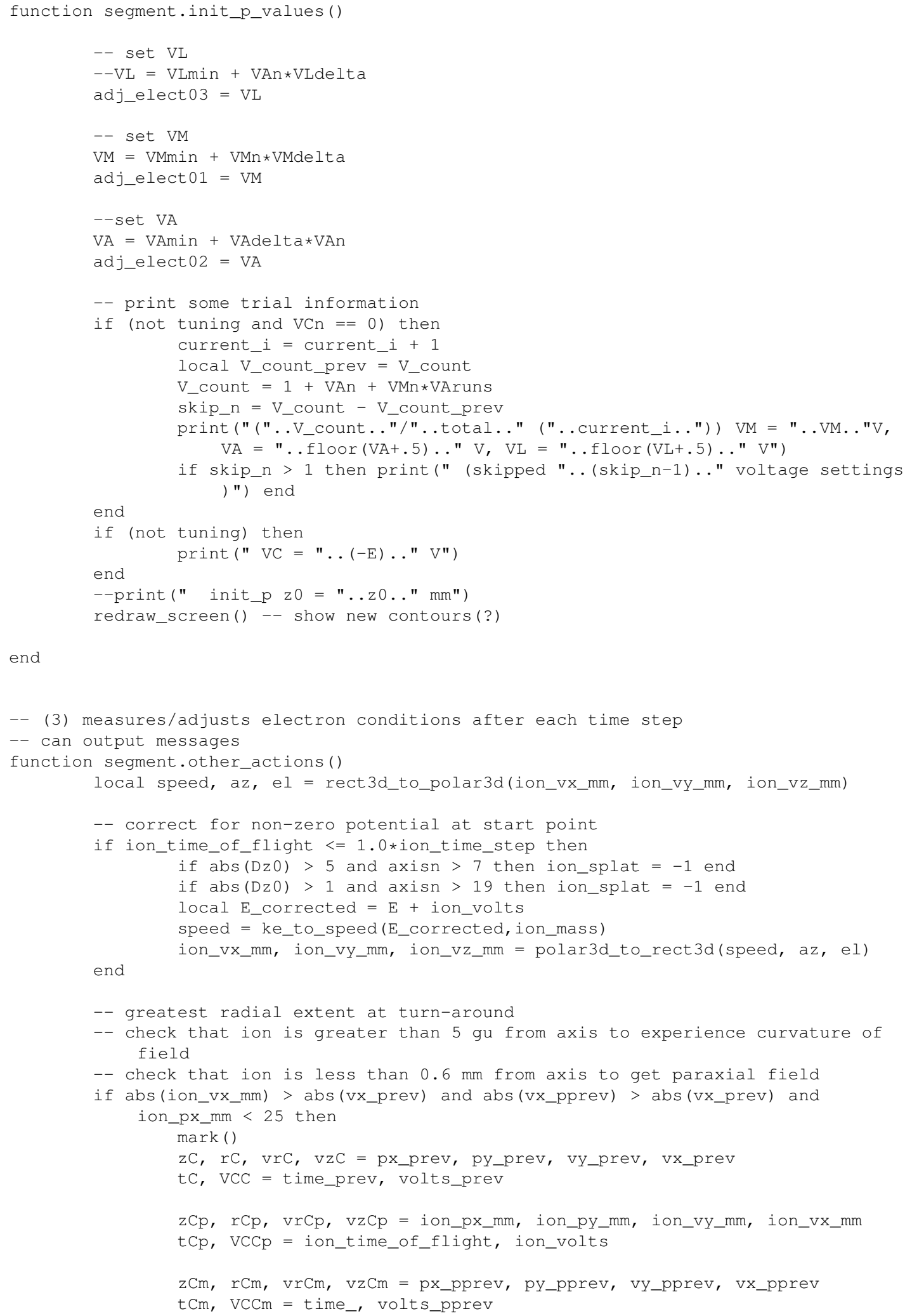




195
196
197
198
199
200
201
202

203
204
205
206
207

243 if (abs (ion_py_gu) < 5 or abs (ion_py_mm) > 0.6) then ion_splat $=-1$ end

end

-- marks axis crossing (if it occurs)

if abs (py_prev + ion_py_mm) < abs (py_prev) + abs (ion_py_mm) then mark() end

-- measure location $\left(y^{\prime}, z^{\prime}\right)$ at $z^{\prime}=z^{\prime}$ set

if (ion_vx_mm > 0 and abs (zprimeset - ion_px_mm) < abs (px_prev - ion_px_mm)) then

$\operatorname{axisn}=\operatorname{axisn}+1$

zprime [axisn] = ion_px_mm - ion_vx_mm*ion_py_mm/ion_vy_mm

local ap = atan2 (ion_vy_mm, ion_vx_mm)

data $=\operatorname{assert}($ io.open (fullfilename, "a"))

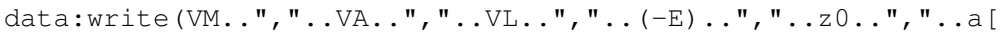
ion_number].."," ..zprime[axisn] . .", . .ap.."," ..zC.."," ..rC.."," . .

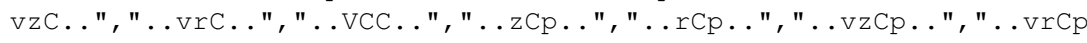

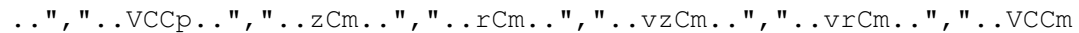
.." $\backslash \mathrm{n} ")$

data:close ()

if $\mathrm{abs}(\mathrm{ap})>1$ then $\mathrm{ap}=\operatorname{atan} 2$ (-ion_vy_mm, ion_vx_mm) end $\mathrm{m}[\mathrm{axisn}]=\mathrm{abs}(\mathrm{ap} / \mathrm{a}[$ ion_number] $)$ aprime2[axisn] = ap*ap

end ion_splat $=-1$

-- kills electrons $1 \mathrm{~mm}$ before hitting mirror electrode

if ion_px_mm < sqrt $(2) *$ ion_py_mm +1 then ion_splat $=-1$ end

-- records electron position for use in next time step

px_pprev $=$ px_prev

py_pprev = py_prev

px_prev = ion_px_mm

py_prev = ion_py_mm

volts_pprev = volts_prev

time_pprev = time_prev

vx_pprev = vx_prev

vy_pprev = vy_prev

time_prev = ion_time_of_flight

vx_prev = ion_vx_mm

vy_prev = ion_vy_mm

speed_pprev = speed_prev

speed_prev $=$ speed

volts_prev = ion_volts

end

-- (4) measures electron conditions after every one has died

-- can output messages and control rerun looping

function segment.terminate()

-- analyze $z^{\prime} \& a^{\prime \wedge} 2$ to find $z 0$, reset $z 0$

if ion_number $==$ electronsperrun then

z0_pprev = z0_prev

$\mathrm{zO}$ _prev $=\mathrm{z} 0$

if $\operatorname{axisn}>=7$ then

local fit $=$ require "fit" -- opens fitting code

-- compute paraxial magnification

local M, slope, aprime4coeff = fit.parabola(aprime2, m)

-- fit $z^{\prime}$ vs $a^{\prime \wedge} 2$ to get $C s$ and $z 0^{\prime}$

local z0prime, cs, aprime4coeff = fit.parabola(aprime2, zprime) 


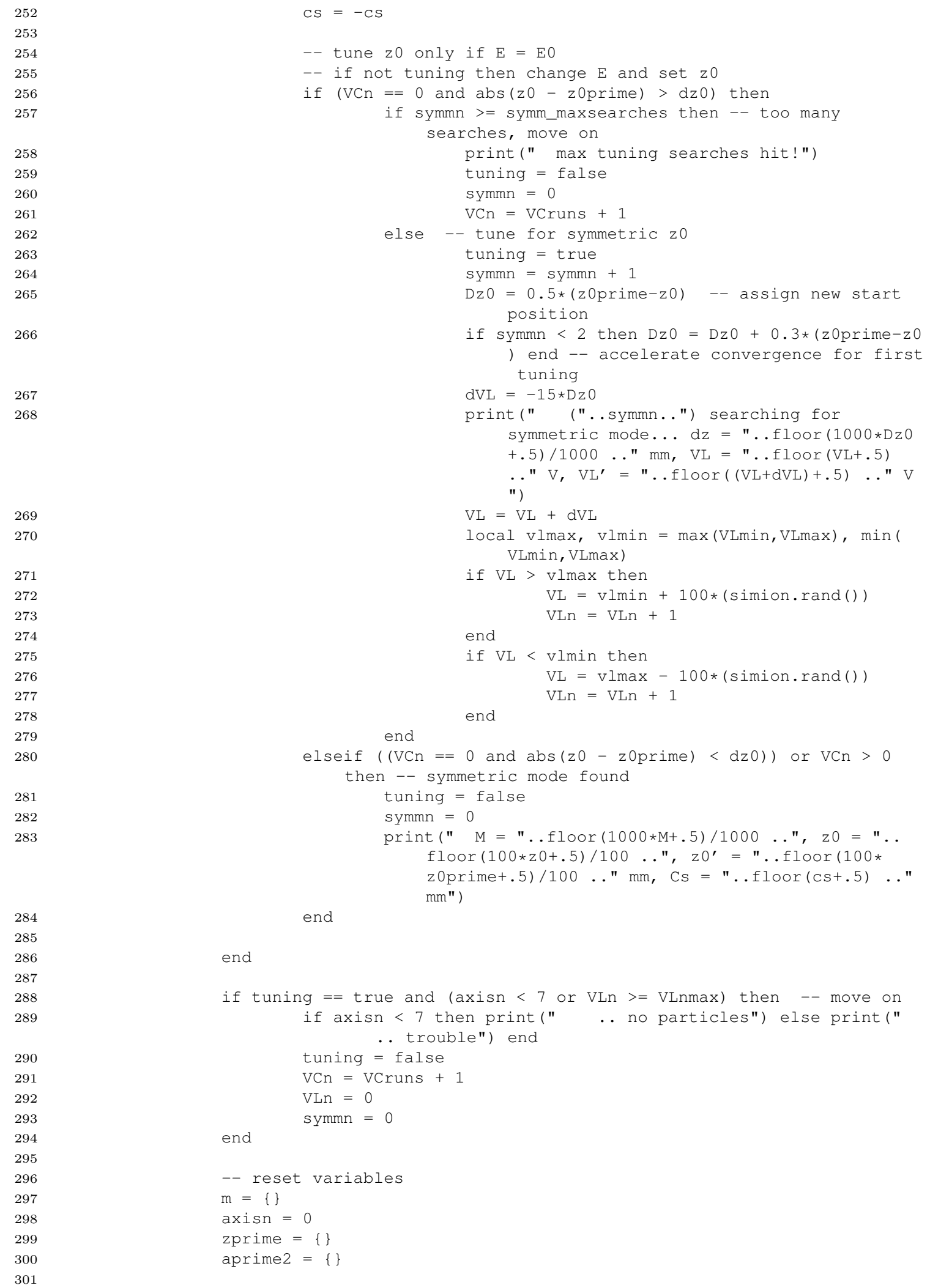




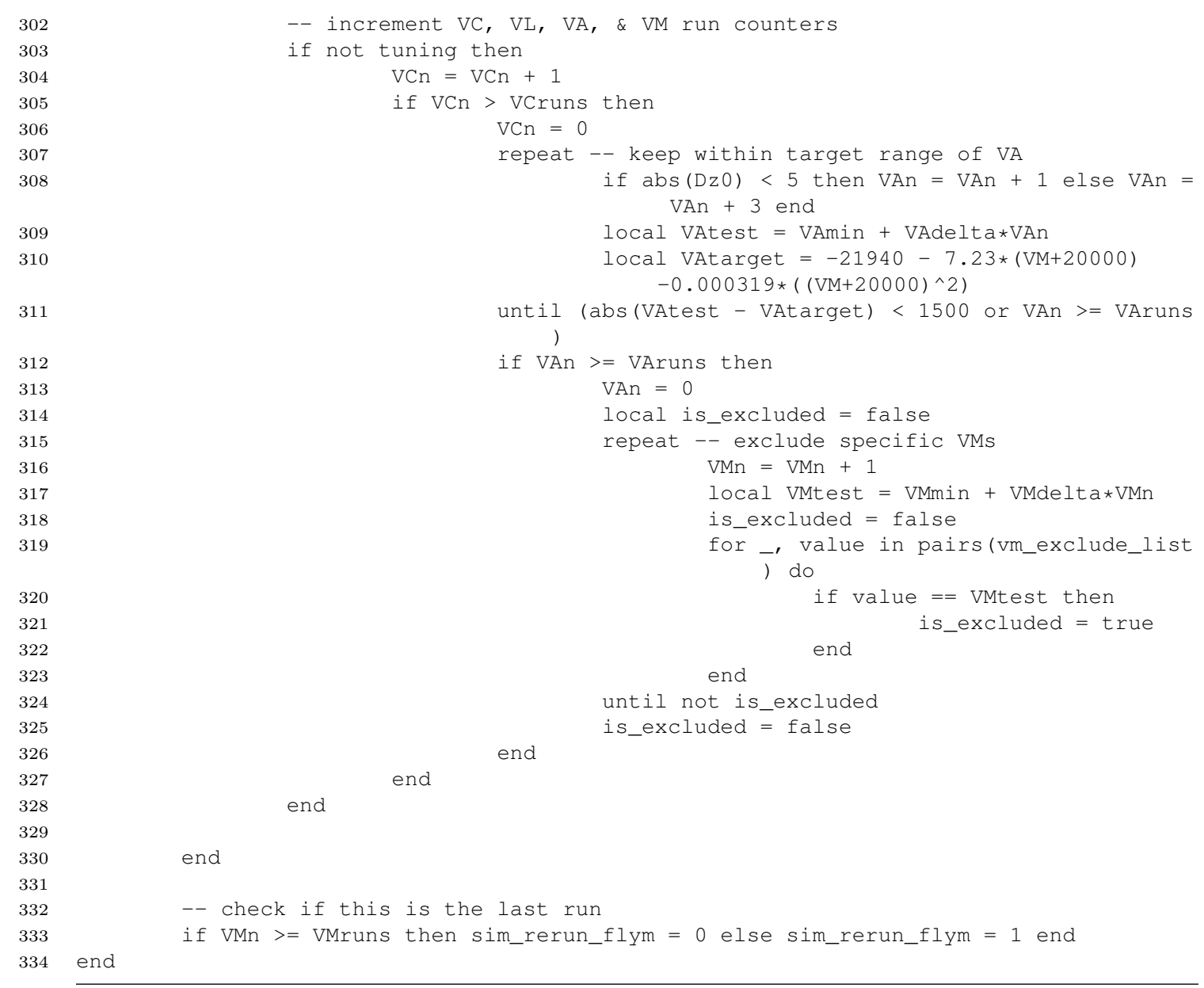




\section{Appendix I Code, Mathematica: triode hyperbolic mirror}

The SIMION trajectory information from executing the previous Lua code is analyzed and sifted through to characterize the mirror branch with Mathematica. Simion uses a stochastic process to determine the symmetric mode paraxial image distance, introducing some random uncertainty to the properties calculated with that data set. In this Mathematica code, data is sorted by constant mirror potential $V_{M}$ and fit to analytic curves which average out this stochastic noise in order to obtain more accurate control parameters for the mirror branch. Finally, a grid of correction values of spherical and chromatic aberration coefficients is generated for computer control. 


\section{Header}

\section{Graphics Options}

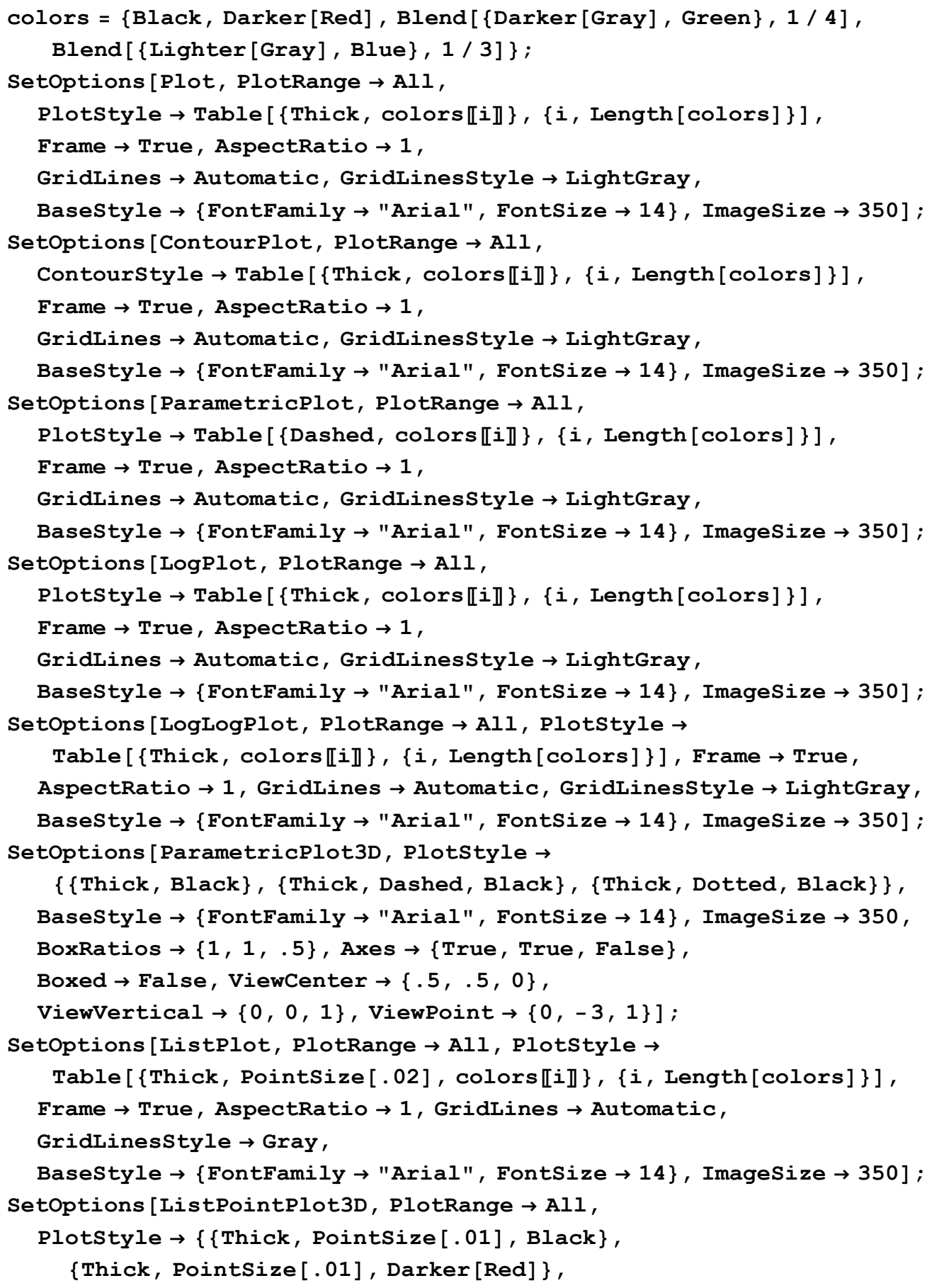


\{Thick, PointSize[.01], Blend[\{Darker[Gray], Green\}, $1 / 4]\}$,

$\{$ Thick, PointSize [.01], Blend[\{Lighter[Gray], Blue\}, 1/3]\}\},

BoxRatios $\rightarrow 1$, Basestyle $\rightarrow$ FontFamily $\rightarrow$ "Arial", FontSize $\rightarrow 14$,

ImageSize $\rightarrow$ 350];

Setoptions [ListPlot3D, PlotRange $\rightarrow$ All, BoxRatios $\rightarrow 1$,

BaseStyle $\rightarrow$ \{FontFamily $\rightarrow$ "Arial", FontSize $\rightarrow 14$ \}, ImageSize $\rightarrow$ 350];

Setoptions [ListContourPlot, PlotRange $\rightarrow$ All, Frame $\rightarrow$ True,

AspectRatio $\rightarrow 1$, GridLines $\rightarrow$ Automatic, Gridlinesstyle $\rightarrow$ LightGray,

BaseStyle $\rightarrow$ \{FontFamily $\rightarrow$ "Arial", FontSize $\rightarrow 14$ \}, ImageSize $\rightarrow$ 350];

Setoptions [ListlogLinearPlot, PlotRange $\rightarrow \mathrm{All}$,

Plotstyle $\rightarrow\{\{$ Thick, PointSize [.02], Black $\}$,

\{Thick, PointSize[.02], Darker[Red]\},

\{Thick, PointSize[.02], Blend[\{Darker[Gray], Green\}, $1 / 4]\}$,

\{Thick, PointSize[.02], Blend[\{Lighter[Gray], Blue\}, 1/3]\}\},

Frame $\rightarrow$ True, AspectRatio $\rightarrow 1$, Gridlines $\rightarrow$ Automatic,

GridLinesStyle $\rightarrow$ LightGray,

BaseStyle $\rightarrow$ \{FontFamily $\rightarrow$ "Arial", FontSize $\rightarrow 14$ \}, ImageSize $\rightarrow$ 350] ;

Setoptions [ListlogPlot, PlotRange $\rightarrow$ All, PlotStyle $\rightarrow$

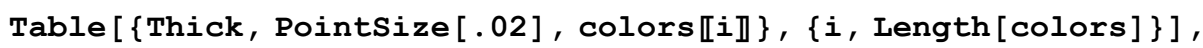

Frame $\rightarrow$ True, AspectRatio $\rightarrow 1$, Gridlines $\rightarrow$ Automatic,

GridLinesStyle $\rightarrow$ LightGray,

BaseStyle $\rightarrow$ \{FontFamily $\rightarrow$ "Arial", FontSize $\rightarrow 14$ \}, ImageSize $\rightarrow$ 350] ;

Setoptions [ListlogLogPlot, PlotRange $\rightarrow$ All, Plotstyle $\rightarrow$

Table [ Thick, PointSize [.02], colors [i]\}, \{i, Length[colors] \}],

Frame $\rightarrow$ True, AspectRatio $\rightarrow 1$, Gridlines $\rightarrow$ Automatic,

GridLinesstyle $\rightarrow$ LightGray,

BaseStyle $\rightarrow$ \{FontFamily $\rightarrow$ "Arial", FontSize $\rightarrow 14$ \}, ImageSize $\rightarrow$ 350] ;

\section{Thick lens equations}

Thick lens equations for focal length and distance from Rempfer 1985

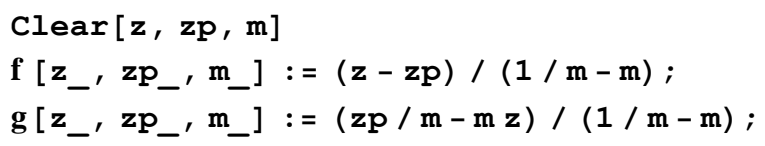




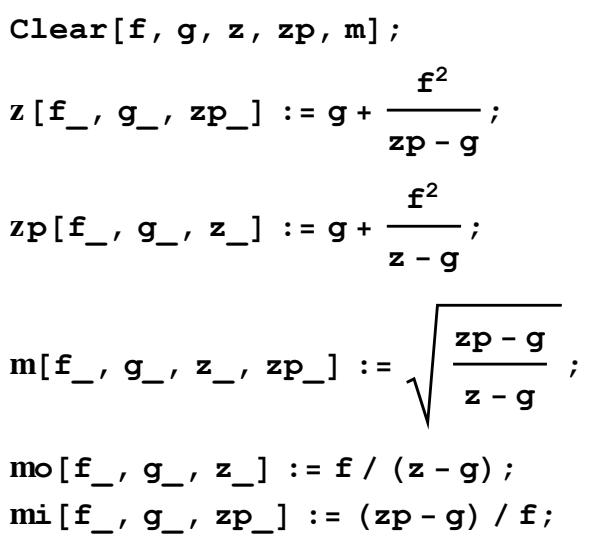

Aberration formulas at the Gaussian plane (not circle of least confusion)

Clear [m0, fo, Sf, Sg, Cf, Cg]

Cs [mo_, fo_, $\left.\mathrm{Sf}_{-}, \mathrm{Sg}_{-}\right]:=$

$-\left(\left(1+(1 / \mathrm{mo})^{2}\right) \mathrm{Sg}+2(1 / \mathrm{mo}) \mathrm{Sf}\right)(1+(1 / \mathrm{m} 0))^{2} \mathrm{fo}$

$\operatorname{Csp}\left[\mathrm{mo}, \mathrm{fO}_{-}, \mathrm{Sf}_{-}, \mathrm{Sg}_{-}\right]:=-\left(\left(1+\mathrm{mo}^{2}\right) \mathrm{Sg}+2 \mathrm{mo} \mathrm{Sf}\right)(1+\mathrm{mo})^{2} \mathrm{fo}$;

$\mathrm{Cc}\left[\mathrm{mo}, \mathrm{fo}, \mathrm{Cf}, \mathrm{Cg}_{-}\right]:=\left(\left(1+(1 / \mathrm{mo})^{2}\right) \mathrm{Cg}+2(1 / \mathrm{mo}) \mathrm{Cf}\right) \mathrm{fo}$;

$\mathrm{Ccp}\left[\mathrm{mo} \mathrm{f}_{-}, \mathrm{fo}_{-}, \mathrm{Cg}_{-}\right]:=\left(\left(1+\mathrm{mo}^{2}\right) \mathrm{Cg}+2 \mathrm{mo} \mathrm{Cf}\right) \mathrm{f0}$;

Relative focal length and magnification variations, $\delta \mathrm{f}=\left(f-f_{0}\right) / f_{0}$ and $\delta \mathrm{g}=\left(g-g_{0}\right) / f_{0}$ and $\delta \mathrm{m}=\left(m-m_{0}\right) / m_{0}$, and object/image distance changes, $\Delta z \& \Delta z^{\prime}$, with spherical and chromatic aberration

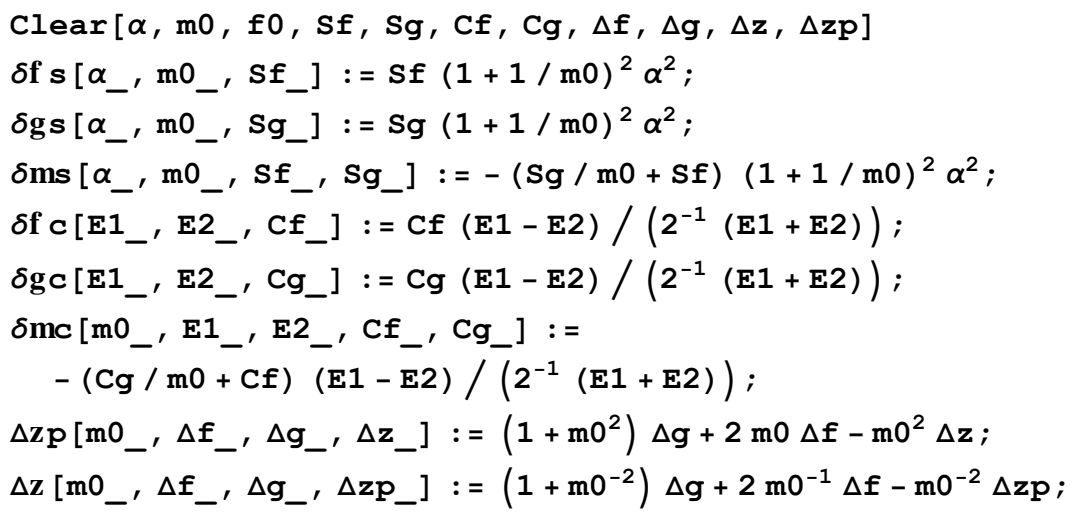

\section{Triode mirror and lens measurement $(\mathrm{mm})$}

$\mathrm{LM}=$ lens center to mirror cone vertex

$\ell=\mathrm{AM}=$ mirror center electrode aperture to mirror cone vertex

$\mathrm{LA}=\mathrm{LM}-\ell=$ lens center to mirror center electrode

$\mathcal{L}=$ distance from lens front aperture to mirror cone vertex

$Z=$ distance from mirror cone vertex to magnet $B$ center 


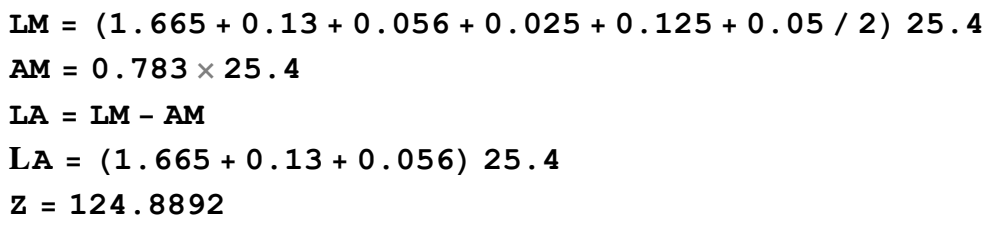

Objective lens properties from previous Simlon analysis

Import swept-back lens property fits

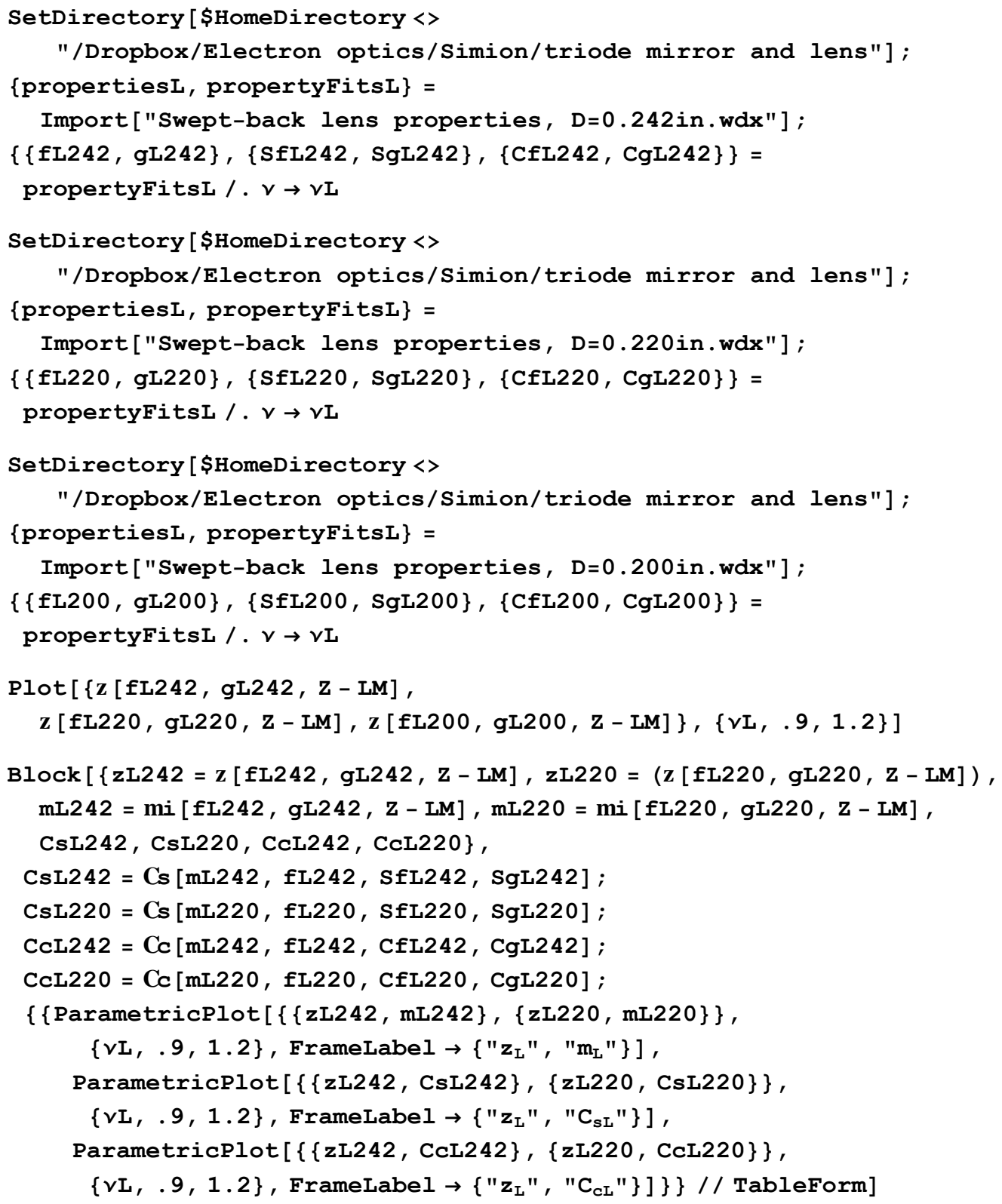




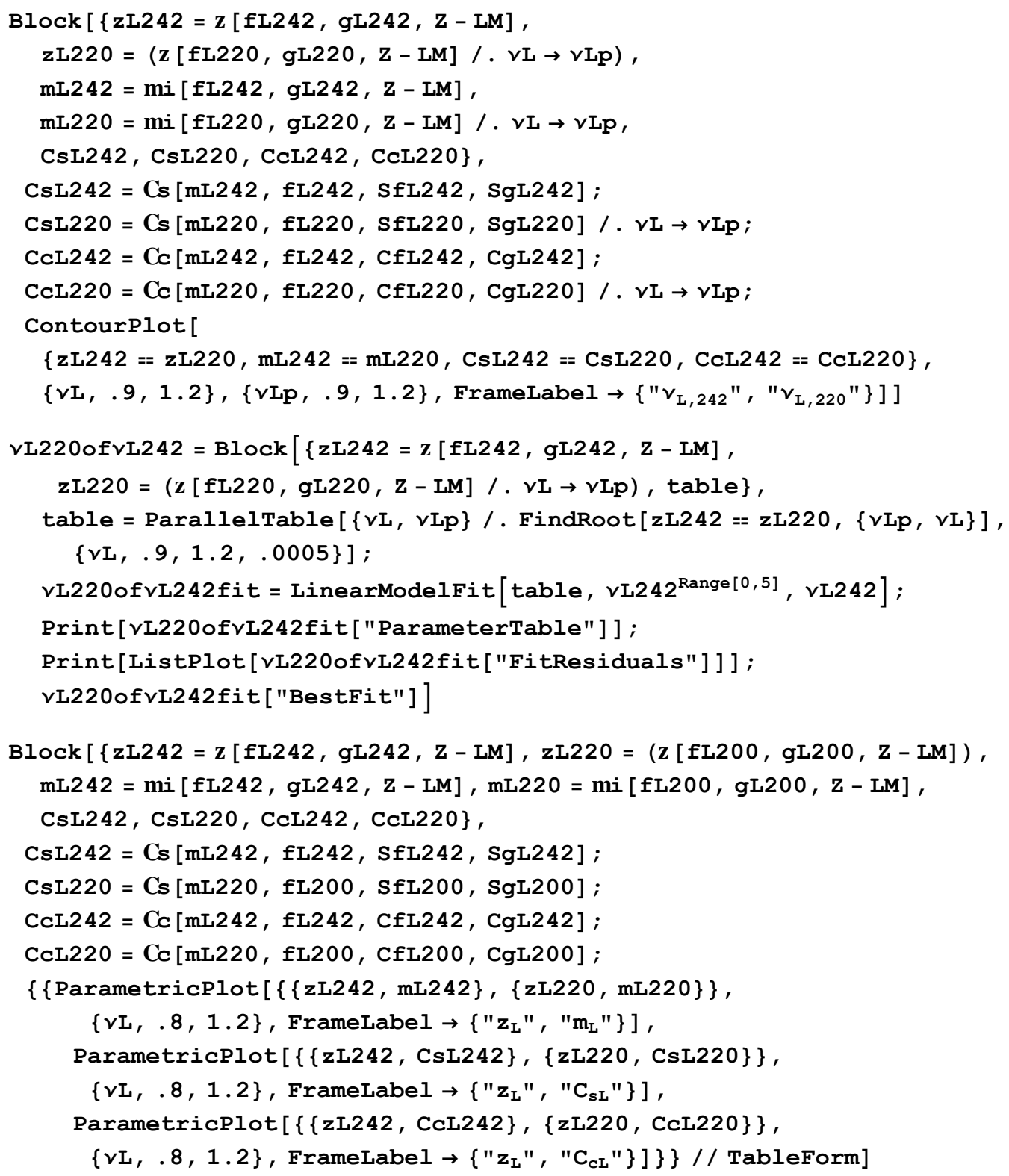




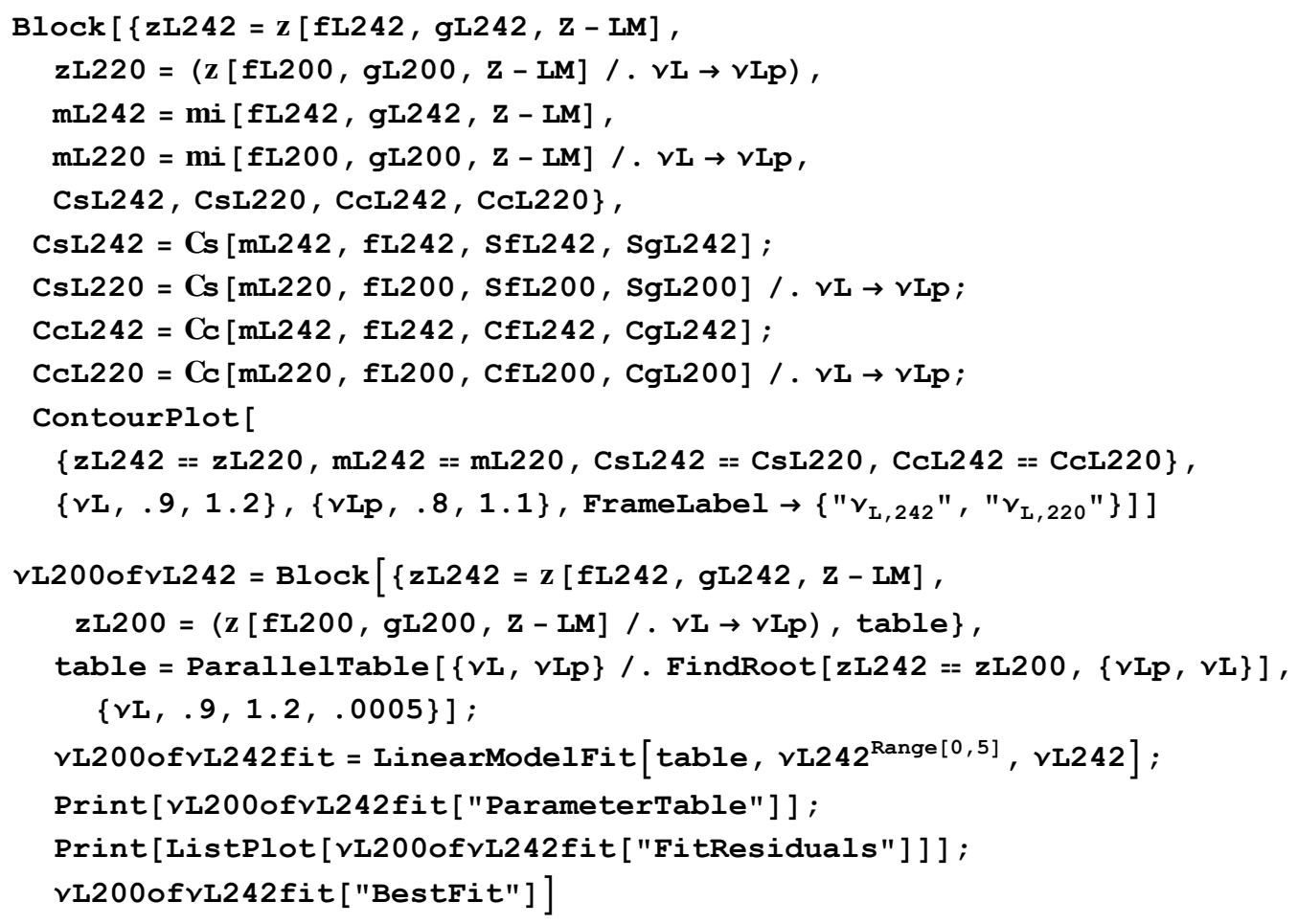

\section{Triode mirror and lens Simlon data}

Import data

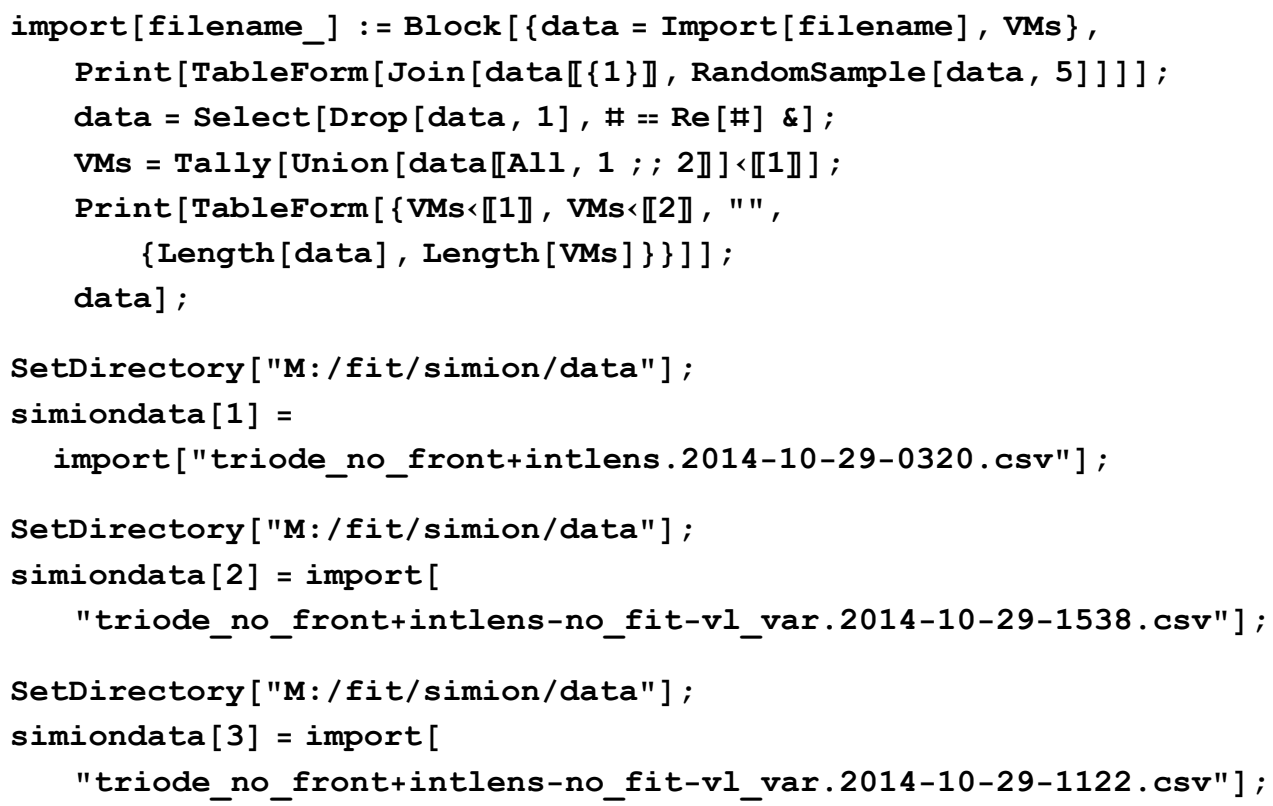




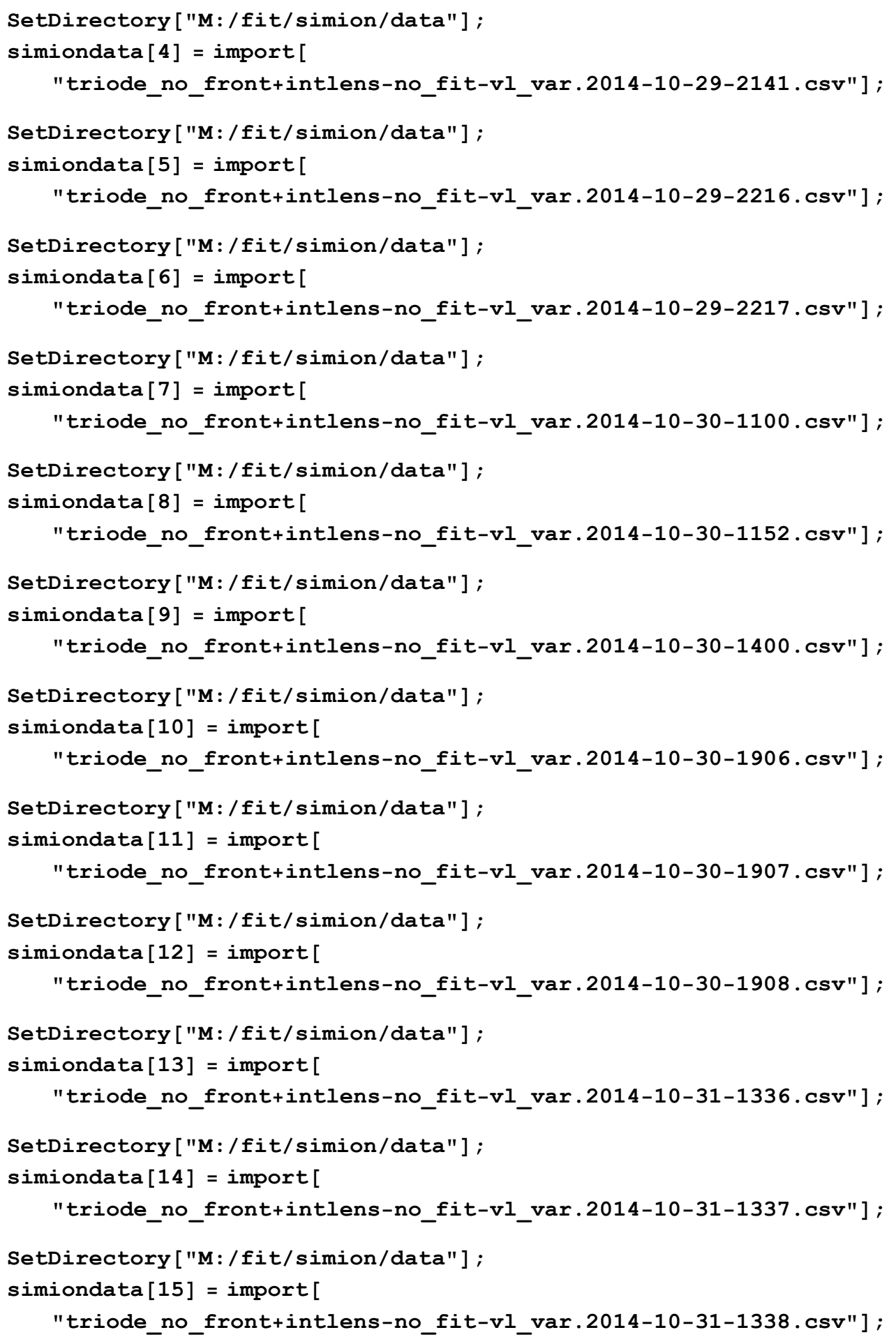

Consolidate data 
data $0=$ Flatten $[$ Delete $[$ Array $[$ simiondata $[\#] \&, 15],\{\{5\},\{6\},\{10\}\}]$,

$1]$; Length [data0]

dz0Tolerance $=0.005$;

$\{$ Cs0guess, Cc0guess $\}=\{-994652,,-23120.5\}$;

ap2 cutoff $=1.4 \times 10^{-7}$;

data1 = Select $\left[\right.$ data $0, \# \llbracket 8 \rrbracket^{2}>\alpha p 2$ cutoff \& $]$;

Length [data1]

Gather symmetric sets

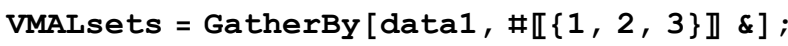

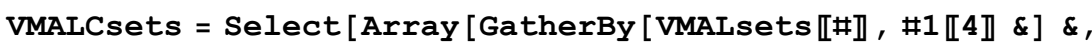
Length [VMALsets] ], Length [\#1] = $3 \&$ ];

VMALCsets = Array [SortBy [VMALCsets $\# 1, \# 2 \rrbracket, \# \llbracket 6 \rrbracket \&] \&$,

\{Length [VMALCsets], 3\}];

data2 = Flatten [VMALCsets, 2];

\{Length [VMALsets], Length [VMALCsets], Length[data2] \}

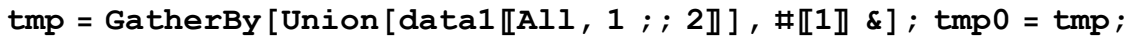

tmp $=\operatorname{Array}[\{$ tmp $\#, 1,1 \rrbracket$, Length $[$ tmp $\llbracket \#]$,

$\operatorname{Max}[t m p \llbracket \#, A l 1,2 \rrbracket]-\operatorname{Min}[\operatorname{tmp} \llbracket \#, A l 1,2 \rrbracket]\} \&$, Length[tmp]] ;

$\{\{$ ListPlot [tmp0], ListPlot [tmp $[A l 1,\{1,2\}]]$,

ListPlot $[$ tmp $\llbracket A 11,\{2,3\} \rrbracket]\}\} / /$ TableForm

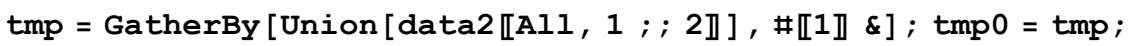

tmp $=\operatorname{Array}[\{\operatorname{tmp} \llbracket \#, 1,1 \rrbracket$, Length $[$ tmp $\llbracket \rrbracket]$,

$\operatorname{Max}[t m p[\#, A l 1,2 \rrbracket]-\operatorname{Min}[t m p \llbracket \#, A l 1,2 \rrbracket]\} \&$, Length[tmp] ] ;

$\{\{$ ListPlot $[$ tmp0], ListPlot $[\operatorname{tmp} \llbracket A l 1,\{1,2\} \rrbracket]$,

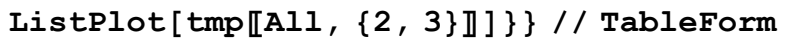

tmp2 $=10^{-3}$ Sort $[$ Select $[$ tmp $\llbracket A 11,\{1,3\} \rrbracket$,

$\# \llbracket 2 \rrbracket<2600 \& \&(\# \llbracket 1 \rrbracket<-23000|| \# \llbracket 1 \rrbracket>-21500|| \# \llbracket 2 \rrbracket>1250) \&]]$;

ListPlot $[$ tmp2, tmp2 $\}$, Framelabel $\rightarrow\left\{" \mathrm{~V}_{\mathrm{M}}(\mathrm{kV}) ", " \mathrm{~V}_{\mathrm{A}}\right.$ Range $\left.(\mathrm{kV}) "\right\}$,

Joined $\rightarrow$ \{True, False $\}$ Filling $\rightarrow$ Bottom $]$

\section{Organize and calculate optical properties of full column}

Organize data \& calculate $Z_{0}^{\prime}, M_{0}, C_{s}^{\prime}, C_{c}^{\prime}$

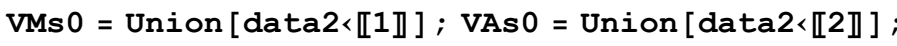

VCs0 = Union $[$ data $2<\llbracket 4 \rrbracket] ;$

$\{$ Nsets $0, \operatorname{NVMs} 0, \operatorname{NVAs} 0, \operatorname{NVCs} 0\}=$

\{Length [VMALCsets], Length [VMs0], Length [VAs 0], Length [VCs0] \} 


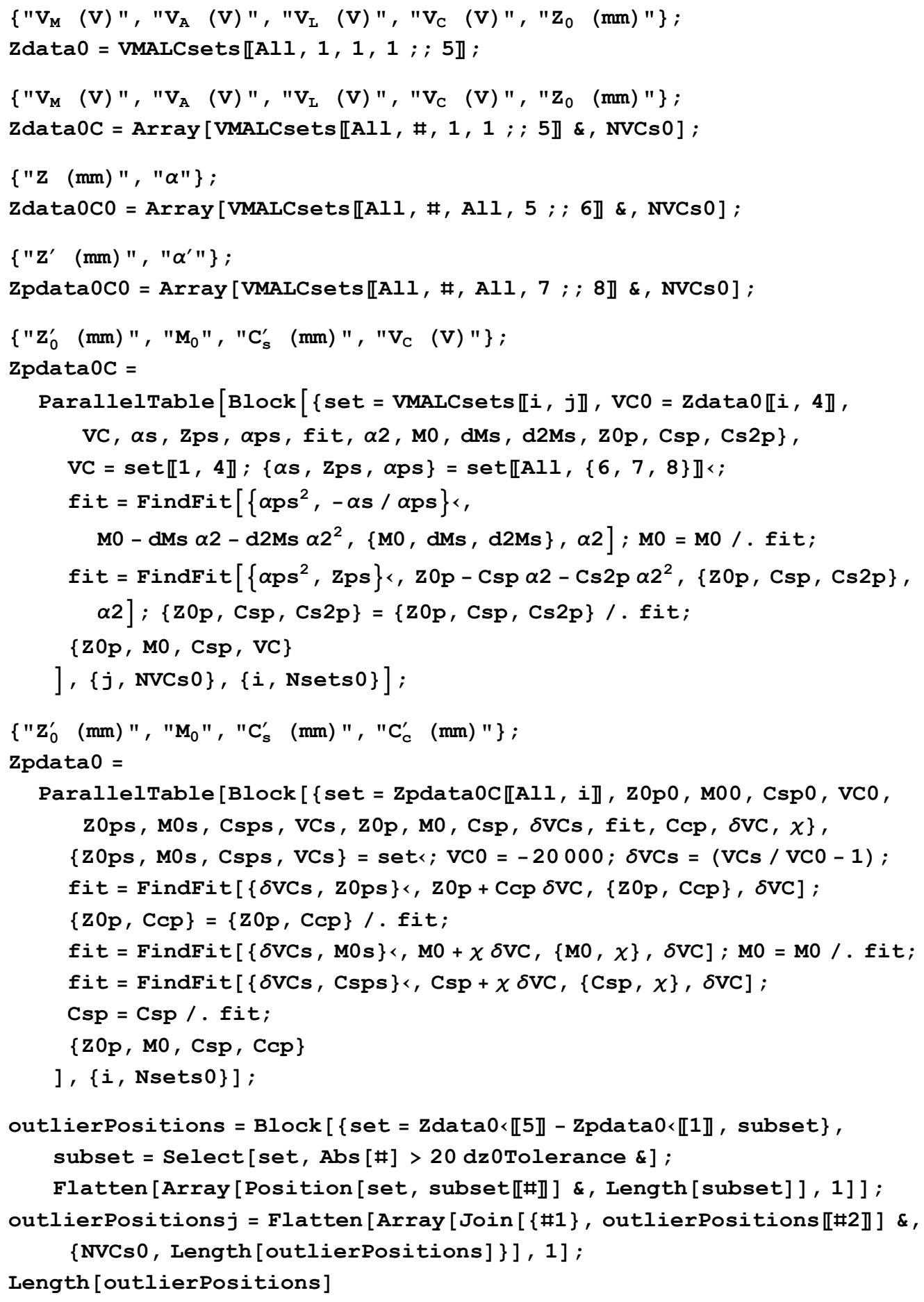




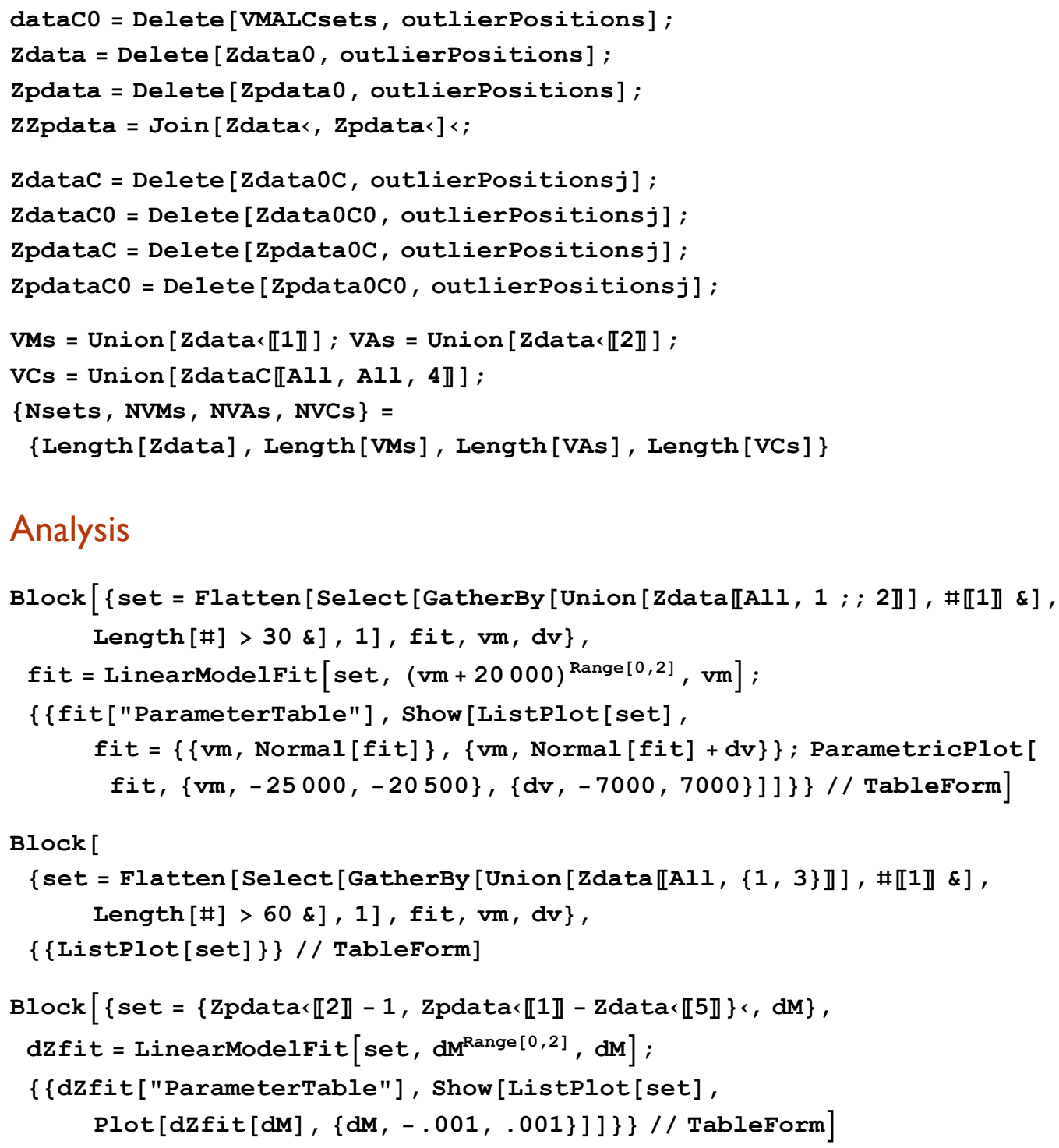




\section{Analysis by constant VM}

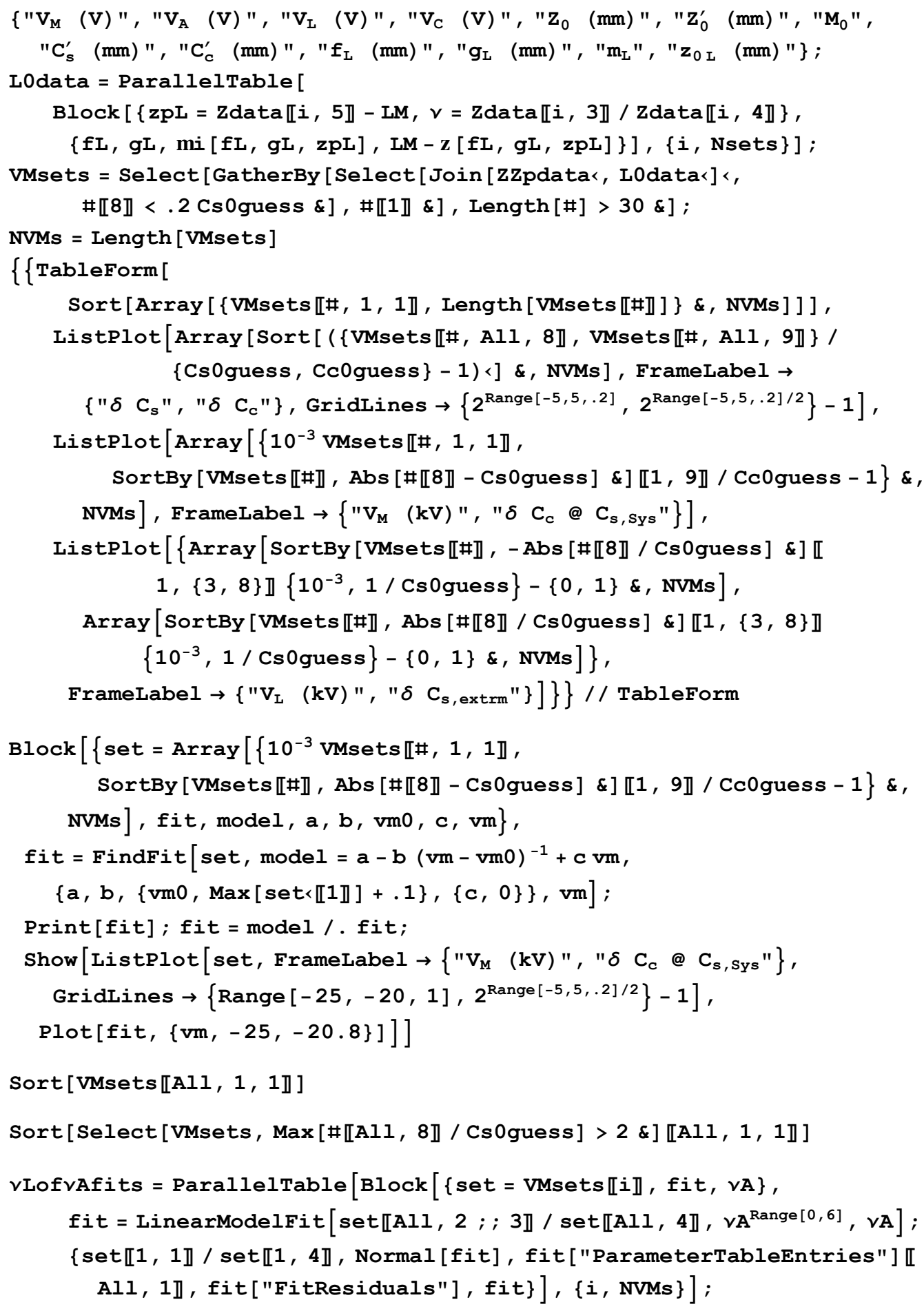


CspofvAfits $=$ ParallelTable $[$ Block $[$ set $=$ VMsets $\llbracket i \rrbracket$, fit, VA $\}$

fit $=$

LinearModelFit $\left[\{\right.$ set $<\llbracket 2 \rrbracket / \operatorname{set}<\llbracket 4 \rrbracket, \operatorname{set}<\llbracket 8 \rrbracket\}<, \nu A^{\text {Range }[0,6], V A]} ;$

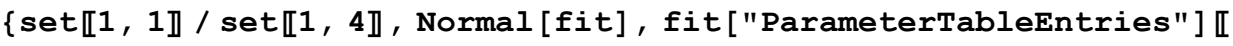

All, 1], fit ["FitResiduals"], fit $\}],\{i$, NVMs $\}]$;

CcpofvAfits $=$ ParallelTable $[$ Block $[$ set $=$ VMsets $\llbracket i \rrbracket$, fit, $v A\}$,

fit $=$

LinearModelFit $\left[\{\right.$ set $<\llbracket 2 \rrbracket / \operatorname{set}<\llbracket 4 \rrbracket$, set $<\llbracket 9 \rrbracket\}<, \nu \mathbb{A}^{\text {Range }[0,6], V A]}$;

$\{\operatorname{set} \llbracket 1,1 \rrbracket / \operatorname{set} \llbracket 1,4 \rrbracket$, Normal [fit] , fit ["ParameterTableEntries"] [

All, 11, fit["FitResiduals"], fit $\}],\{i$, NVMs $\}]$;

$\{$ ListPlot [

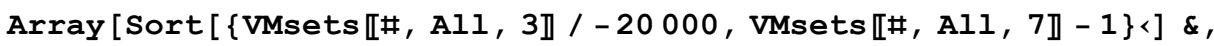

NVMs], Joined $\rightarrow$ True, AspectRatio $\rightarrow .5]$,

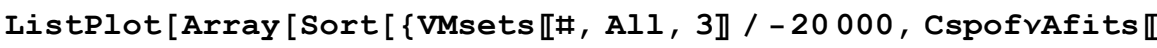

$\#, 4 \rrbracket\}<] \&$, NVMs] , Joined $\rightarrow$ True, AspectRatio $\rightarrow .5]$,

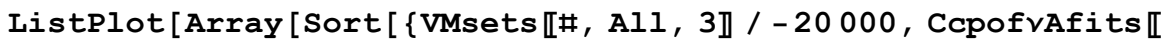

\#, 4】\}<]\&, NVMs], Joined $\rightarrow$ True, AspectRatio $\rightarrow .5]\}$,

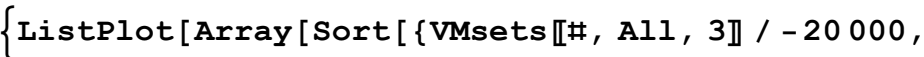

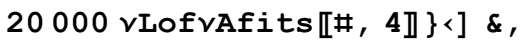

NVMs], Joined $\rightarrow$ True, AspectRatio $\rightarrow .5]$,

ListPlot [Array $[$ Sort $[\{$ VMsets $\llbracket, A l 1,3 \rrbracket /-20000$,

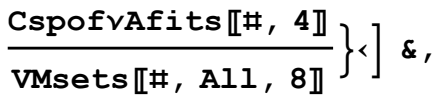

NVMs], Joined $\rightarrow$ True, AspectRatio $\rightarrow .5$ ],

ListPlot [Array $[$ Sort $[\{$ VMsets $\llbracket, A l 1,3 \rrbracket /-20000$,

$\left.\frac{\text { Ccpofvafits } \llbracket \#, 4 \rrbracket}{\text { VMsets } \llbracket \#, A l 1,9 \rrbracket}\right\}<\&$,

NVMs], Joined $\rightarrow$ True, AspectRatio $\rightarrow .5]\}$,

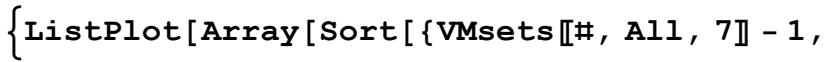

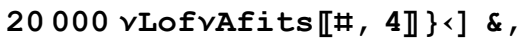

NVMs], Joined $\rightarrow$ True, AspectRatio $\rightarrow .5]$,

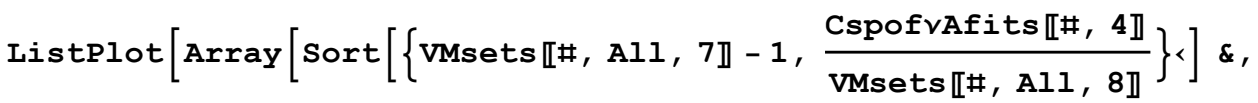

NVMs], Joined $\rightarrow$ True, AspectRatio $\rightarrow .5$ ],

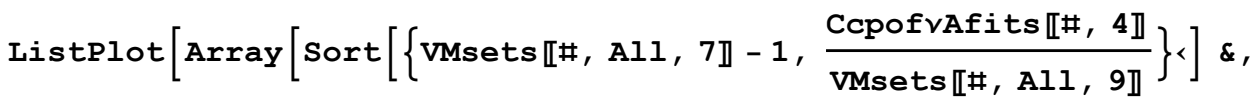

NVMs], Joined $\rightarrow$ True, AspectRatio $\rightarrow .5]\}\} / /$ TableForm 


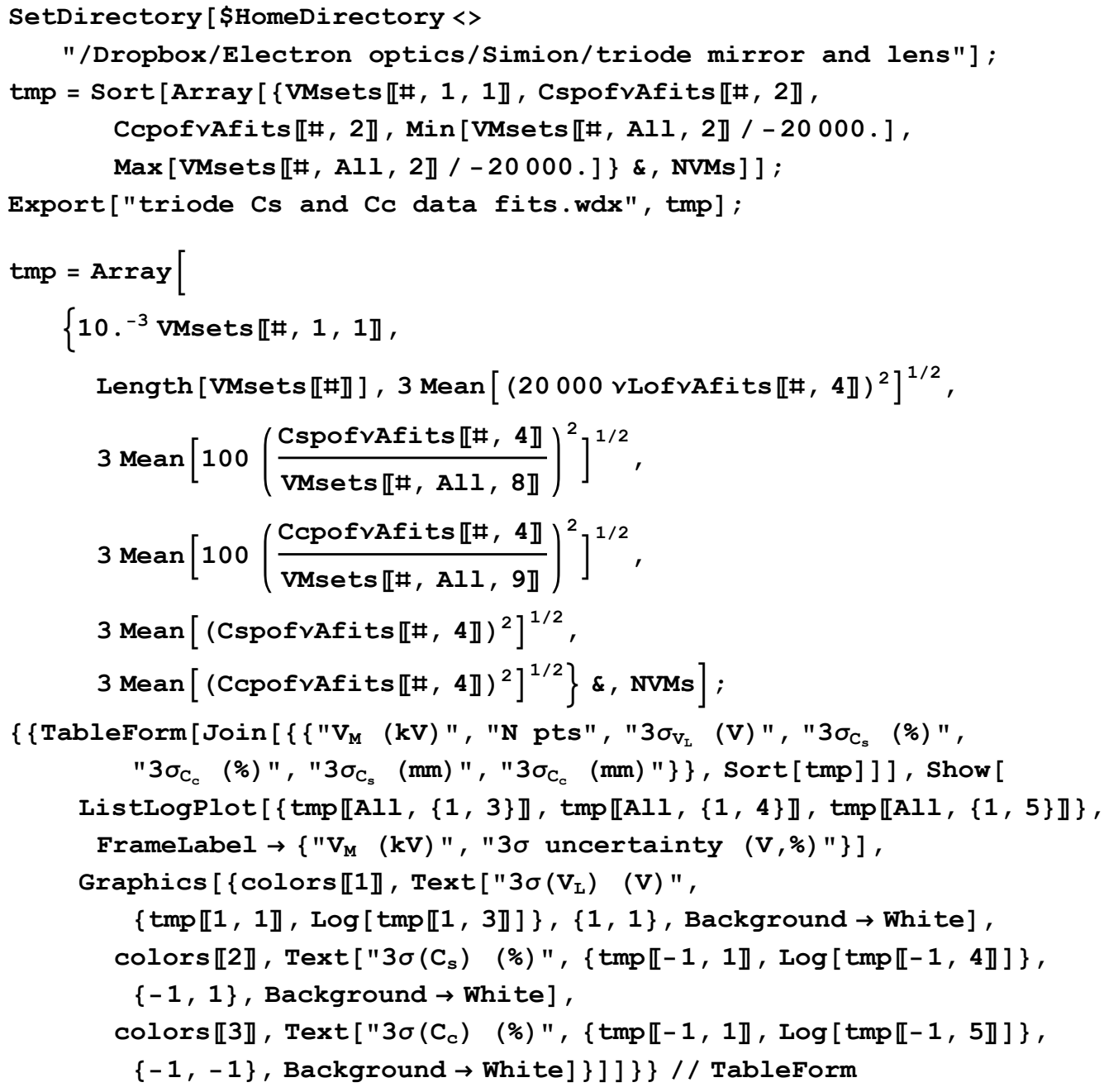


Aberration sensitivity to voltage fluctuations at constant focus

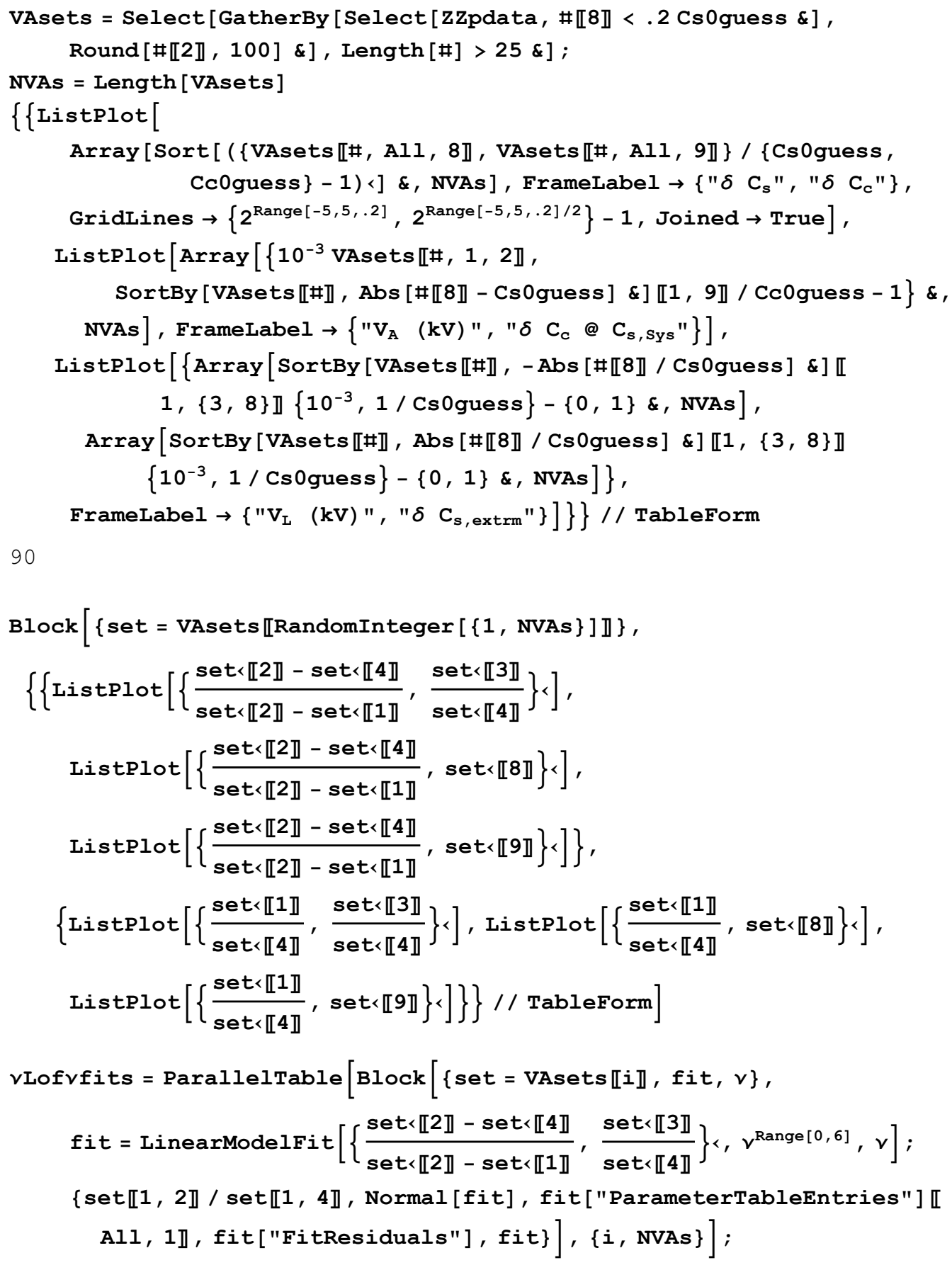




$$
\begin{aligned}
& \text { Cspofvfits }=\text { ParallelTable }[\text { Block }[\{\text { set }=\text { VAsets } \llbracket i \rrbracket, \text { fit }, v\} \text {, } \\
& \text { fit }=\text { LinearModelFit }\left[\left\{\frac{\operatorname{set}<\llbracket 2 \rrbracket-\operatorname{set}<\llbracket 4 \rrbracket}{\operatorname{set}<\llbracket 2 \rrbracket-\operatorname{set}<\llbracket 1 \rrbracket}, \operatorname{set}<\llbracket 8 \rrbracket\right\}<, v^{\text {Range }[0,6], v] ; ~}\right.
\end{aligned}
$$

$\{\operatorname{set} \llbracket 1,2 \rrbracket / \operatorname{set} \llbracket 1,4 \rrbracket$, Normal [fit] , fit ["ParameterTableEntries"] [ All, 1], fit["FitResiduals"], fit $\}],\{i$, NVAs $\}]$;

Ccpofvfits $=$ ParallelTable $[$ Block $[$ set $=$ VAsets $\llbracket i \rrbracket$, fit, $v\}$,

$$
\text { fit }=\text { LinearModelFit }\left[\left\{\frac{\operatorname{set}<\llbracket 2 \rrbracket-\operatorname{set}<\llbracket 4 \rrbracket}{\operatorname{set}<\llbracket 2 \rrbracket-\operatorname{set}<\llbracket 1 \rrbracket}, \operatorname{set}<\llbracket 9 \rrbracket\right\}<, v^{\text {Range }[0,6], \nu] ; ~}\right.
$$

$\{\operatorname{set} \llbracket 1,2 \rrbracket / \operatorname{set} \llbracket 1,4 \rrbracket$, Normal[fit] , fit ["ParameterTableEntries"] [

All, 1】, fit["FitResiduals"], fit $\}],\{i$, NVAs $\}]$;

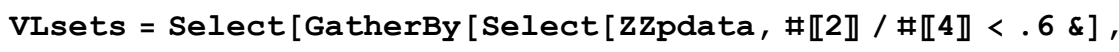

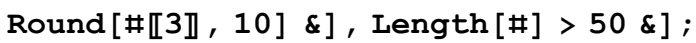

NVLs = Length [VLsets]

ListPlot [Array [

Sort $[(\{$ VLsets $\llbracket \#, A l 1,8 \rrbracket, V L s e t s \llbracket \#, A l 1,9 \rrbracket\} /\{C s 0$ guess, Cc0guess $\}-$

$1)<] \&$, NVLs] , FrameLabel $\rightarrow\left\{" \delta \mathrm{C}_{\mathrm{s}} ", " \delta \mathrm{C}_{\mathrm{c}} "\right\}$,

GridLines $\rightarrow\left\{2^{\text {Range }[-5,5, .2]}, 2^{\text {Range }[-5,5, .2] / 2}\right\}-1$, Joined $\rightarrow$ True $]$

Block $[\{$ set $=$ VLsets $\llbracket$ RandomInteger $[\{1, \operatorname{NVLs}\}] \rrbracket\}$,

$$
\begin{aligned}
& \left\{\left\{\text { ListPlot } \left[\left\{\frac{\operatorname{set}<\llbracket 2 \rrbracket-\operatorname{set}<\llbracket 4 \rrbracket}{\operatorname{set}<\llbracket 2 \rrbracket-\operatorname{set}<\llbracket 1 \rrbracket}, \frac{\operatorname{set}<\llbracket 2 \rrbracket}{\operatorname{set}<\llbracket 4 \rrbracket}\right\}<,\right.\right.\right. \\
& \text { ListPlot }\left[\left\{\frac{\text { set }<\llbracket 2 \rrbracket-\operatorname{set}<\llbracket 4 \rrbracket}{\text { set }<\llbracket 2 \rrbracket-\operatorname{set}<\llbracket 1 \rrbracket}, \operatorname{set}<\llbracket 8 \rrbracket\right\}<\right] \text {, } \\
& \text { ListPlot } \left.\left[\left\{\frac{\operatorname{set}<\llbracket 2 \rrbracket-\operatorname{set}<\llbracket 4 \rrbracket}{\operatorname{set}<\llbracket 2 \rrbracket-\operatorname{set}<\llbracket 1 \rrbracket}, \operatorname{set}<\llbracket 9 \rrbracket\right\}<\right]\right\} \text {, } \\
& \left\{\text { ListPlot } [ \{ \frac { \text { set } < \llbracket 2 \rrbracket } { \operatorname { s e t } < \llbracket 4 \rrbracket } , \frac { \text { set } < \llbracket 1 \rrbracket } { \operatorname { s e t } < \llbracket 4 \rrbracket } \} < ] , \text { ListPlot } \left[\left\{\frac{\text { set }<\llbracket 2 \rrbracket}{\text { set }<\llbracket 4 \rrbracket}, \operatorname{set}<\llbracket 8 \rrbracket\right\}<\right.\right.\text {, } \\
& \text { ListPlot } \left.\left.\left.\left[\left\{\frac{\text { set }<\llbracket 2 \rrbracket}{\operatorname{set}<\llbracket 4 \rrbracket}, \operatorname{set}<\llbracket 9 \rrbracket\right\}<\right]\right\}\right\} / / \text { TableForm }\right]
\end{aligned}
$$

VMof VAconstVLfits $=$ ParallelTable $[$ Block $[\{$ set $=$ VLsets $\llbracket i \rrbracket$, fit, $V A\}$,

fit $=$ LinearModelFit $\left[\left\{\frac{\operatorname{set}<\llbracket 2 \rrbracket}{\operatorname{set}<\llbracket 4 \rrbracket}, \frac{\operatorname{set}<\llbracket 1 \rrbracket}{\operatorname{set}<\llbracket 4 \rrbracket}\right\}<, \nu \mathbb{A}^{\text {Range }[0,6],}, V_{A}\right]$;

$\{\operatorname{set} \llbracket 1,3 \rrbracket / \operatorname{set} \llbracket 1,4 \rrbracket$, Normal [fit] , fit ["ParameterTableEntries"] [

All, 1], fit["FitResiduals"], fit $\}],\{i, N V L s\}]$; 


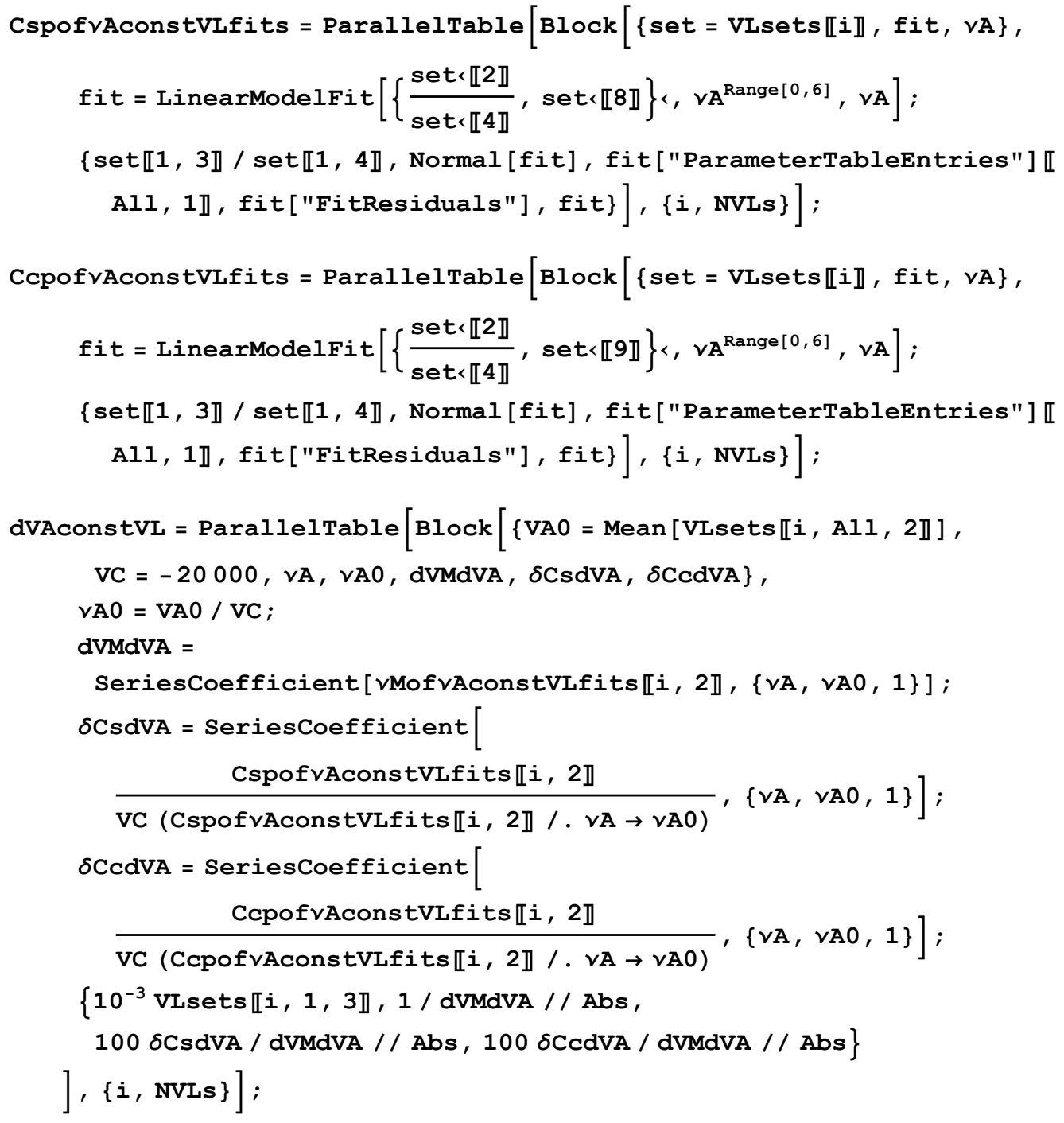




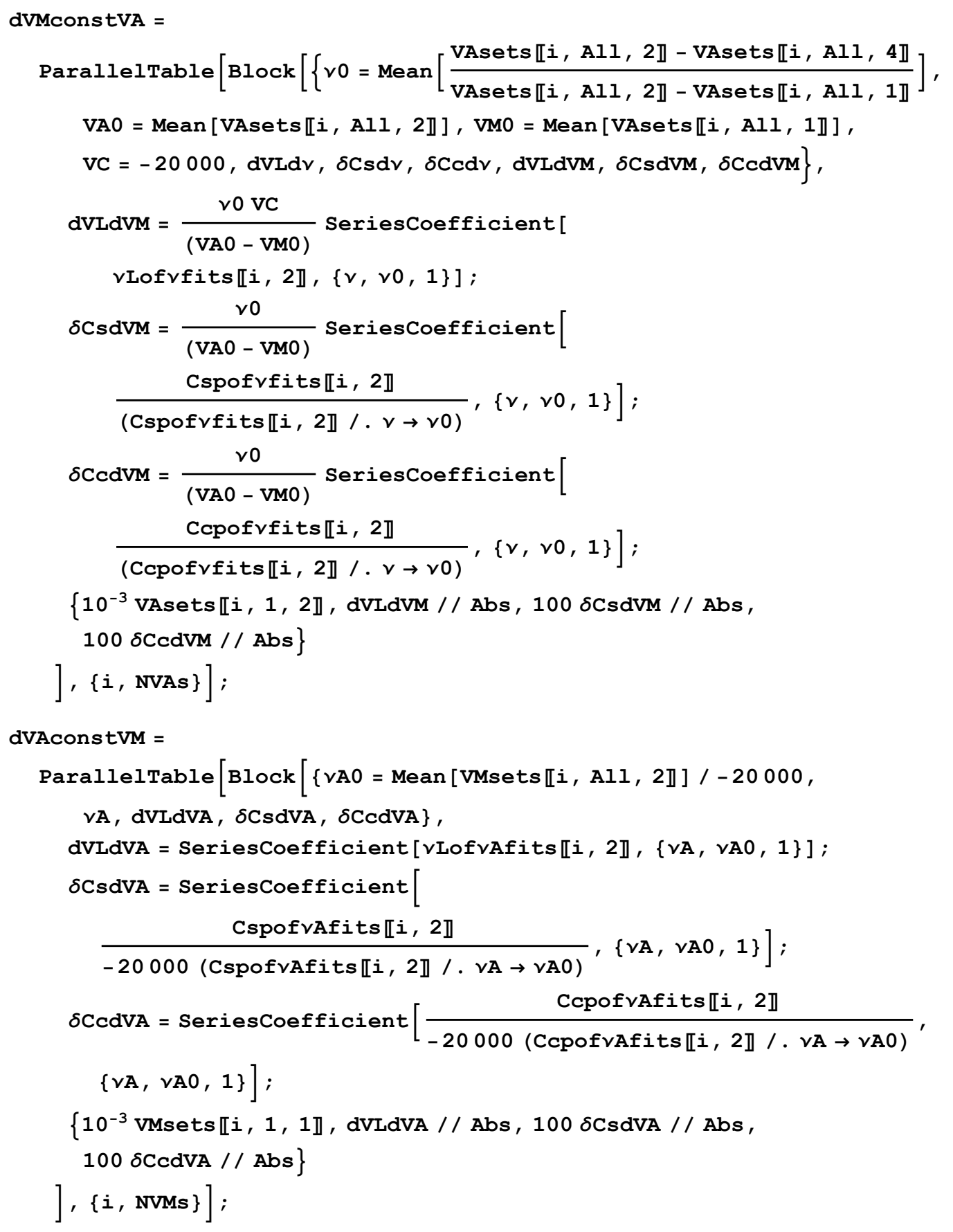




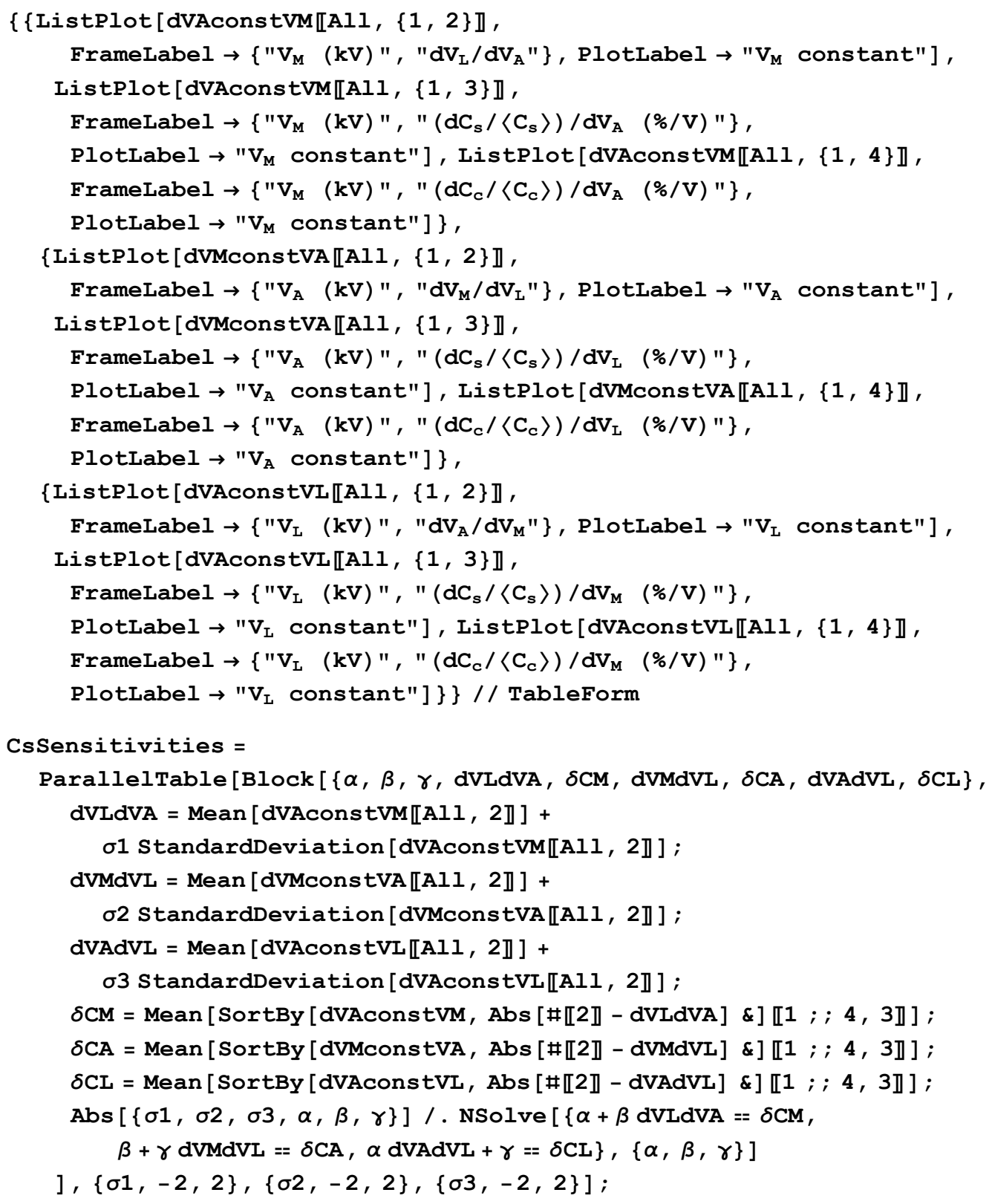




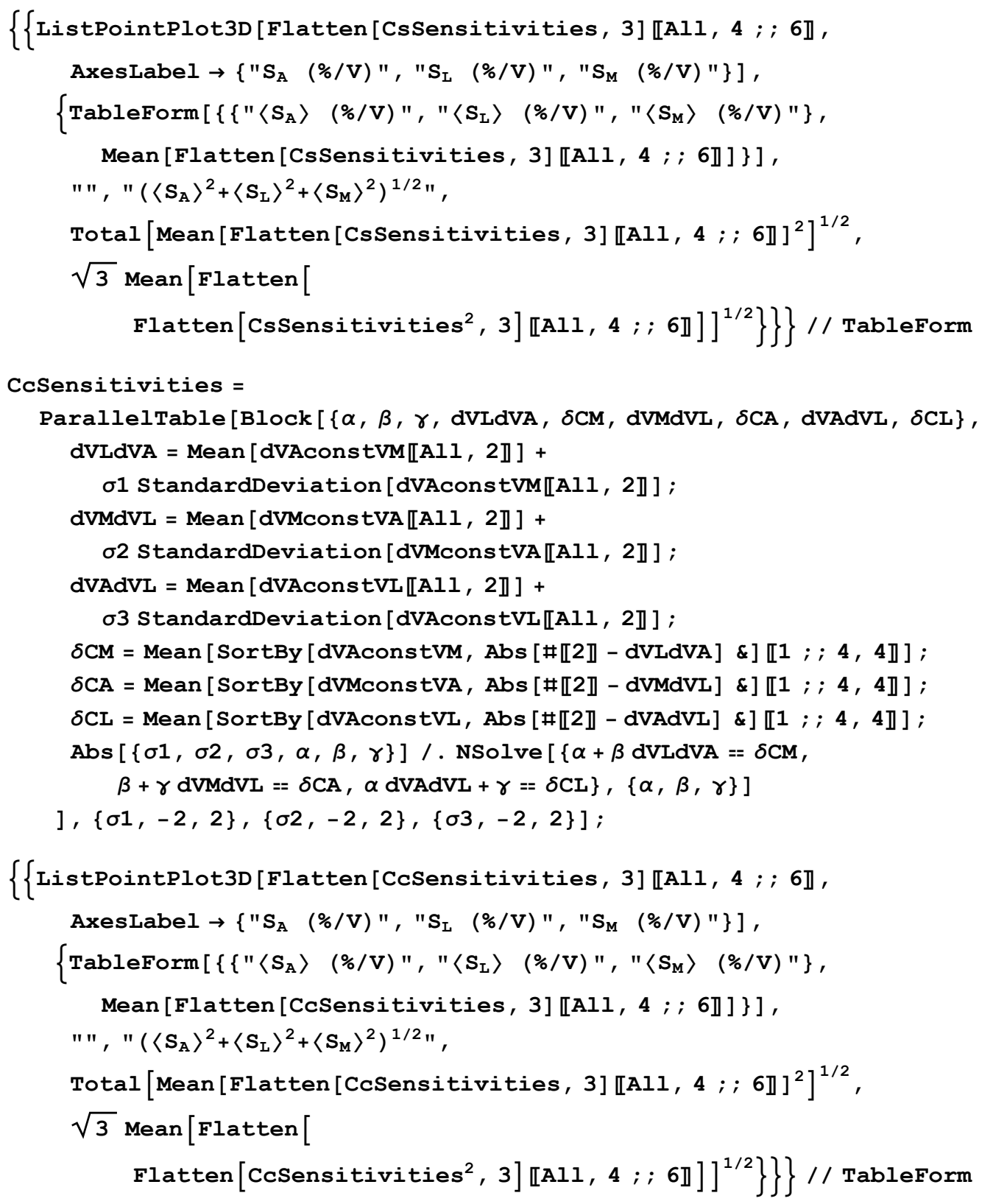

Export tables, apply $V_{L}$ voltage correction for different bore diameter $\{\delta c s i, \delta c c i\}=\{1,1.5\}$; 


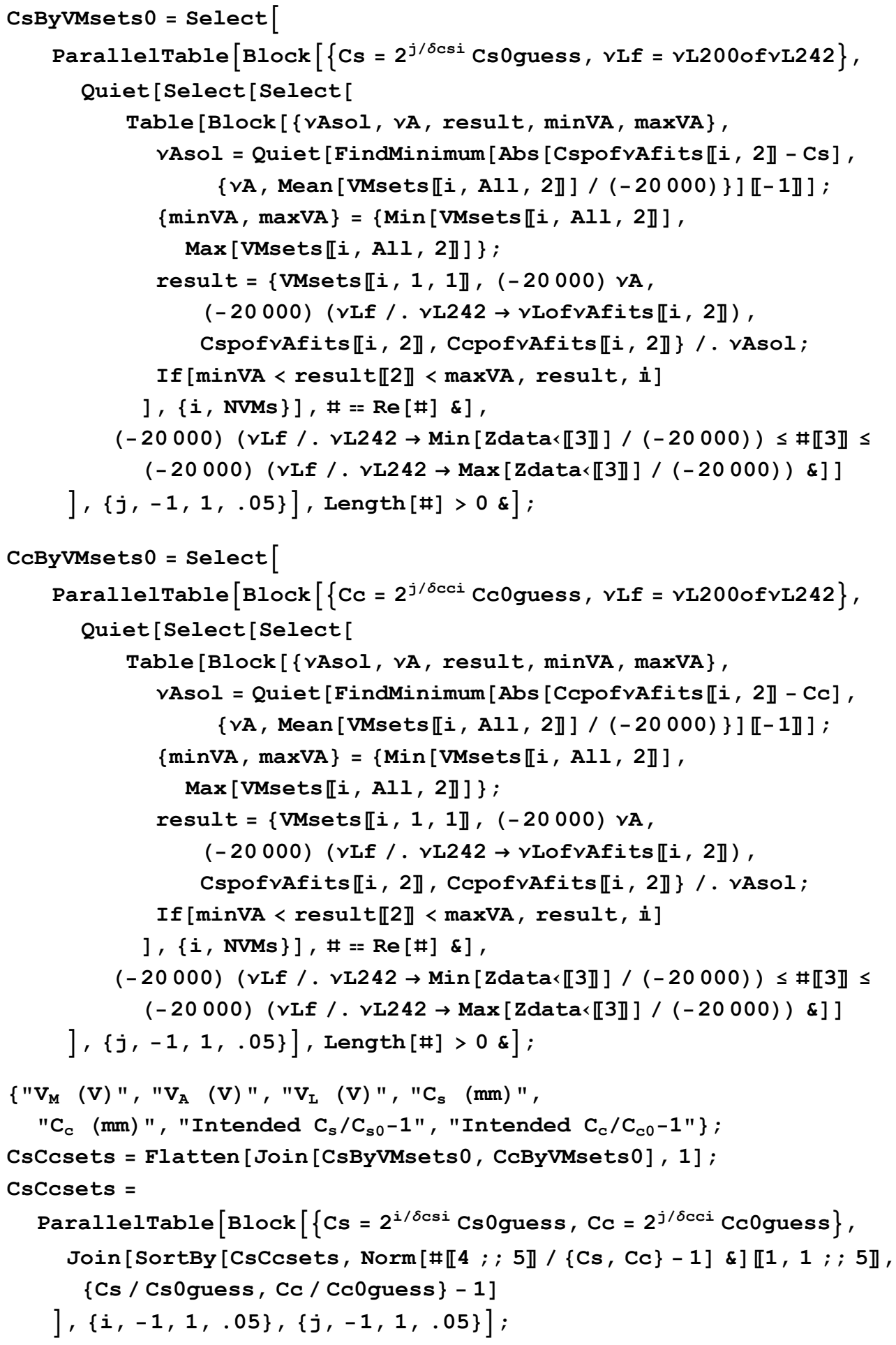




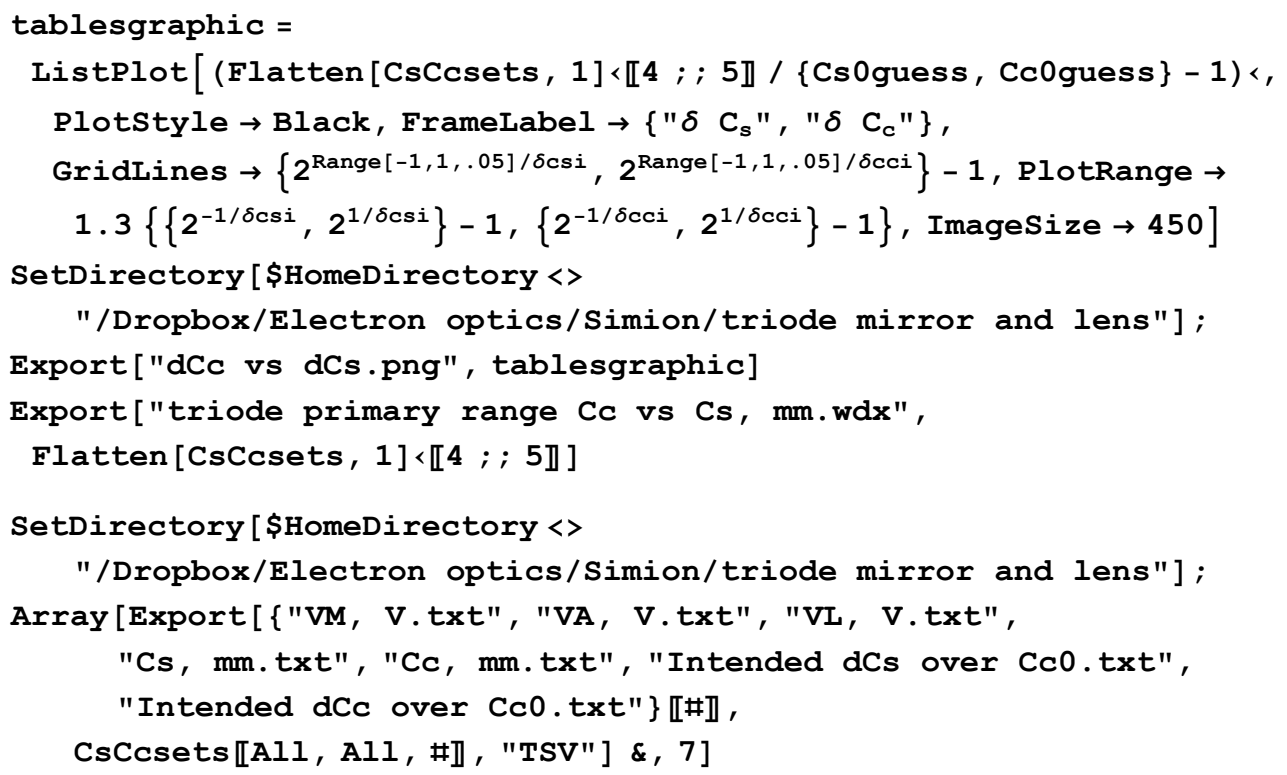




\section{Appendix J PEEM control: triode mirror branch tables}

The results of characterizing the triode mirror branch in SIMION, containing the triode mirror and lens as pictured in Fig. 5.2b. The aberration values here cover the center of the triode corrector range, and vary logarithmically away from the central value. There are approximately $5 \times 5$ values in each quadrant, one-sixteenth of the number of points in Figs. 5.4 and 5.5 but with similar extent. Each pair of aberration correction values is accompanied by the mirror column potentials that create that condition. These are evaluated for an interface lens with bore diameter of $6.15 \mathrm{~mm}$ (0.242 in).

The columns here have units of $\mathrm{mm}\left(C_{s}, C_{c}\right)$ and $\mathrm{V}\left(V_{M}, V_{A}, V_{I}=V_{L}\right)$.

\begin{tabular}{lllll}
$C_{s}(\mathrm{~mm})$ & $C_{c}(\mathrm{~mm})$ & $V_{M}(\mathrm{~V})$ & $V_{A}(\mathrm{~V})$ & $V_{L}(\mathrm{~V})$ \\
\hline-1989304 & -30505.0 & -22000 & -9306.9 & -21134.2 \\
-1989304 & -21267.8 & -23100 & -3462.2 & -21356.5 \\
-1989304 & -36870.1 & -21600 & -11548.1 & -21098.9 \\
-1989304 & -33342.3 & -21800 & -10416.4 & -21110.2 \\
-1989304 & -19207.5 & -23500 & -1396.2 & -21435.3 \\
-1989304 & -23073.3 & -22800 & -5024.2 & -21290.4 \\
-1989304 & -25355.3 & -22510 & -6558.2 & -21233.4 \\
-1989304 & -27856.6 & -22240 & -8003.6 & -21179.6 \\
-1953159 & -18350.8 & -23600 & -857.3 & -21430.9 \\
-1921541 & -18275.4 & -23600 & -844.1 & -21418.9 \\
-1731789 & -18982.3 & -23300 & -2305.3 & -21280.9 \\
-1731789 & -17686.2 & -23600 & -758.4 & -21342.6 \\
-1731789 & -23150.7 & -22610 & -5924.6 & -21137.5 \\
-1731789 & -25427.2 & -22340 & -7370.6 & -21083.3 \\
-1731789 & -27783.6 & -22100 & -8673.3 & -21037.2 \\
-1731789 & -21258.0 & -22900 & -4392.2 & -21198.1 \\
-1731789 & -33440.9 & -21680 & -11013.3 & -20980.8 \\
-1731789 & -36809.9 & -21500 & -12049.6 & -20977.3 \\
-1731789 & -30468.0 & -21880 & -9887.8 & -21002.2 \\
-1672798 & -17409.8 & -23600 & -726.3 & -21314.5 \\
-1609364 & -17121.9 & -23600 & -691.0 & -21283.9 \\
-1507611 & -19042.0 & -23100 & -3233.8 & -21132.6
\end{tabular}




\begin{tabular}{lllll}
$C_{s}(\mathrm{~mm})$ & $C_{c}(\mathrm{~mm})$ & $V_{M}(\mathrm{~V})$ & $V_{A}(\mathrm{~V})$ & $V_{L}(\mathrm{~V})$ \\
\hline-1507610 & -23105.3 & -22460 & -6629.3 & -20998.4 \\
-1507610 & -27739.0 & -21980 & -9250.9 & -20909.0 \\
-1507610 & -17600.3 & -23400 & -1672.0 & -21197.0 \\
-1507610 & -21373.9 & -22710 & -5294.6 & -21053.3 \\
-1507610 & -30559.2 & -21760 & -10481.6 & -20875.7 \\
-1507610 & -25411.3 & -22200 & -8040.4 & -20948.6 \\
-1507610 & -33141.6 & -21600 & -11397.5 & -20865.8 \\
-1507610 & -37139.1 & -21400 & -12571.3 & -20875.3 \\
-1504621 & -16730.8 & -23600 & -633.7 & -21234.9 \\
-1406650 & -16350.1 & -23600 & -577.1 & -21187.3 \\
-1312451 & -37859.1 & -21300 & -13110.2 & -20786.8 \\
-1312451 & -30466.1 & -21670 & -10923.0 & -20766.6 \\
-1312451 & -27836.8 & -21860 & -9842.8 & -20788.5 \\
-1312451 & -15921.3 & -23600 & -517.4 & -21137.9 \\
-1312451 & -21007.8 & -22600 & -5785.3 & -20929.6 \\
-1312451 & -19158.6 & -22900 & -4186.7 & -20994.5 \\
-1312451 & -17668.8 & -23200 & -2606.7 & -21059.1 \\
-1312451 & -23113.2 & -22320 & -7298.7 & -20871.3 \\
-1312451 & -25356.9 & -22080 & -8615.1 & -20824.3 \\
-1312451 & -33348.0 & -21500 & -11909.1 & -20758.5 \\
-1142555 & -33866.8 & -21400 & -12440.1 & -20668.1 \\
-1142555 & -19413.3 & -22710 & -5104.7 & -20862.6 \\
-1142555 & -21051.9 & -22460 & -6453.0 & -20809.1 \\
-1142555 & -30243.2 & -21600 & -11259.2 & -20666.1 \\
-1142555 & -15983.9 & -23400 & -1452.6 & -21009.3 \\
-1142555 & -25470.3 & -21960 & -9207.6 & -20711.6 \\
-1142555 & -17309.0 & -23100 & -3028.4 & -20945.1 \\
-1142555 & -27875.4 & -21760 & -10337.7 & -20681.0 \\
-1142555 & -36199.2 & -21300 & -13048.0 & -20685.5 \\
-1142555 & -23107.2 & -22200 & -7874.3 & -20755.9 \\
-1103635 & -15404.0 & -23500 & -904.2 & -21009.0 \\
-994652 & -32501.1 & -21400 & -12379.2 & -20576.5 \\
-994652 & -27815.1 & -21680 & -10729.9 & -20583.9 \\
-994652 & -17538.0 & -22900 & -3997.3 & -20818.9 \\
-994652 & -23141.5 & -22090 & -8407.6 & -20648.9 \\
-994652 & -16130.7 & -23200 & -2404.3 & -20883.9 \\
-14901.3 & -23500 & -826.1 & -20946.7 \\
-39452 & -19190.8 & -22610 & -5557.7 & -20758.1
\end{tabular}




\begin{tabular}{lllll}
$C_{s}(\mathrm{~mm})$ & $C_{c}(\mathrm{~mm})$ & $V_{M}(\mathrm{~V})$ & $V_{A}(\mathrm{~V})$ & $V_{L}(\mathrm{~V})$ \\
\hline-994652 & -30629.2 & -21500 & -11781.8 & -20572.5 \\
-865895 & -36018.3 & -21200 & -13562.2 & -20539.4 \\
-865895 & -25459.0 & -21780 & -10090.0 & -20514.9 \\
-865895 & -23100.1 & -22000 & -8842.5 & -20554.0 \\
-865895 & -27921.2 & -21600 & -11134.1 & -20496.2 \\
-865895 & -15937.2 & -23100 & -2842.1 & -20785.1 \\
-865895 & -14686.6 & -23400 & -1251.5 & -20847.4 \\
-865895 & -21041.7 & -22240 & -7503.4 & -20601.3 \\
-865895 & -17387.5 & -22800 & -4447.8 & -20720.4 \\
-865895 & -19268.3 & -22480 & -6182.6 & -20650.8 \\
-865895 & -33391.0 & -21300 & -12932.8 & -20505.6 \\
-859008 & -31220.8 & -21400 & -12318.6 & -20487.9 \\
-753806 & -37720.3 & -21100 & -14160.9 & -20511.3 \\
-753805 & -32220.3 & -21300 & -12880.1 & -20426.7 \\
-753805 & -15781.8 & -23000 & -3291.4 & -20693.0 \\
-753805 & -17505.1 & -22660 & -5125.8 & -20618.5 \\
-753805 & -19242.8 & -22380 & -6658.8 & -20558.7 \\
-753805 & -30202.9 & -21400 & -12268.0 & -20415.7 \\
-753805 & -14535.6 & -23300 & -1688.5 & -20754.7 \\
-753805 & -21058.8 & -22140 & -7990.7 & -20508.5 \\
-753805 & -25282.7 & -21720 & -10375.7 & -20433.1 \\
-753805 & -23072.2 & -21920 & -9229.7 & -20465.7 \\
-753805 & -28455.4 & -21500 & -11667.1 & -20414.4 \\
-656859 & -36502.6 & -21100 & -14114.0 & -20434.7 \\
-656226 & -14411.0 & -23200 & -2136.8 & -20668.2 \\
-656226 & -16207.1 & -22800 & -4291.2 & -20583.6 \\
-656226 & -27534.0 & -21500 & -11614.6 & -20344.8 \\
-656225 & -19155.5 & -22300 & -7033.1 & -20474.8 \\
-656225 & -17775.6 & -22510 & -5873.8 & -20520.6 \\
-656225 & -25372.6 & -21650 & -10729.3 & -20358.4 \\
-656225 & -23104.4 & -21840 & -9627.6 & -20384.8 \\
-656225 & -33575.0 & -21200 & -13460.2 & -20380.0 \\
-656225 & -21036.2 & -22060 & -8376.6 & -20425.9 \\
-656225 & -31184.8 & -21300 & -12830.8 & -20354.6 \\
-571278 & -30266.6 & -21300 & -12784.7 & -20288.7 \\
-571278 & -16155.5 & -22710 & -4711.6 & -20505.0 \\
-571277 & -143599.0 & -21100 & -14069.5 & -20363.5 \\
-23111.2 & -21780 & -9920.5 & -20314.7 \\
-17528.0 & -22460 & -6083.6 & -20449.7
\end{tabular}




\begin{tabular}{lllll}
$C_{s}(\mathrm{~mm})$ & $C_{c}(\mathrm{~mm})$ & $V_{M}(\mathrm{~V})$ & $V_{A}(\mathrm{~V})$ & $V_{L}(\mathrm{~V})$ \\
\hline-571277 & -21070.4 & -21980 & -8773.3 & -20350.6 \\
-571277 & -28359.9 & -21400 & -12170.1 & -20281.1 \\
-571277 & -19307.7 & -22200 & -7531.3 & -20395.2 \\
-571277 & -25249.8 & -21600 & -10971.7 & -20289.7 \\
-497406 & -34417.3 & -21100 & -14028.0 & -20298.4 \\
-497326 & -19184.2 & -22140 & -7810.5 & -20325.4 \\
-497326 & -27574.7 & -21400 & -12125.6 & -20222.0 \\
-497326 & -15991.8 & -22650 & -4975.2 & -20436.8 \\
-497326 & -29448.9 & -21300 & -12741.7 & -20228.5 \\
-497326 & -23115.4 & -21720 & -10219.4 & -20248.4 \\
-497326 & -21062.9 & -21920 & -9063.5 & -20283.6 \\
-497326 & -14355.4 & -23000 & -3066.0 & -20511.7 \\
-497326 & -17523.4 & -22380 & -6467.5 & -20377.9 \\
-497205 & -37993.5 & -21000 & -14717.6 & -20392.0 \\
-495151 & -25951.9 & -21500 & -11518.0 & -20220.9
\end{tabular}




\section{Appendix K PEEM control: objective branch tables}

The objective branch is composed of the cathode, objective, and auxiliary lenses. The distance from the cathode to the objective varies slightly with sample, so the objective must be able to change focal distance to bring the virtual specimen into focus. The auxiliary lens ensures that the image has a consistent position in magnetic deflector A, as illustrated in Fig. 3.1 and App. Appendix A. As such, the objective and auxiliary lens must vary continuously with variations in sample height $\delta z_{C}$. The two lenses work together as a zoom pair, giving the freedom to choose image magnification over the range $3.5-7.5 \times$. The quoted magnification includes the demagnification of the first objective aperture. To give continuous focusing at constant magnification, the lens potentials are modeled as polynomials of sample height $\delta z_{C}$, which can vary over several hundred microns. The aberration of the pair can similarly be calculated. The total aberration of the objective branch in transfer space, including the cathode, can be estimated from a sum of the objective-auxiliary aberration and the cathode aberration multiplied by the magnification, as given by Eqs. (3.26) and (3.27). Presented here are the polynomial coefficients of the fits of $V_{O}, V_{X}, C_{s}^{\prime}$, and $C_{c}^{\prime}$ as functions of $\delta z_{C}$ for discrete magnifications. The potentials are given in units of $V_{C}$, the aberration coefficients are given in $\mathrm{mm}$, and the variable $\delta z_{C}$ is given in microns.

\section{Appendix K.1 Objective lens potential}

Objective lens potential $V_{O}$ in units of $V_{C}$ can be expressed as a polynomial of sample height $d z_{C}$ in microns. The polynomial coefficients, noted here, change with magnification. Additional magnifications are possible. 


\begin{tabular}{llll} 
Mag & $\left(d z_{C}\right)^{0}$ & $\left(d z_{C}\right)^{1}$ & $\left(d z_{C}\right)^{2}$ \\
\hline 3.50 & $9.652 \mathrm{E}-01$ & $2.126 \mathrm{E}-05$ & $1.063 \mathrm{E}-09$ \\
4.00 & $9.685 \mathrm{E}-01$ & $2.135 \mathrm{E}-05$ & $1.026 \mathrm{E}-09$ \\
4.50 & $9.710 \mathrm{E}-01$ & $2.140 \mathrm{E}-05$ & $1.012 \mathrm{E}-09$ \\
5.00 & $9.730 \mathrm{E}-01$ & $2.143 \mathrm{E}-05$ & $9.972 \mathrm{E}-10$ \\
5.50 & $9.746 \mathrm{E}-01$ & $2.146 \mathrm{E}-05$ & $9.902 \mathrm{E}-10$ \\
6.00 & $9.759 \mathrm{E}-01$ & $2.149 \mathrm{E}-05$ & $9.824 \mathrm{E}-10$ \\
6.50 & $9.771 \mathrm{E}-01$ & $2.150 \mathrm{E}-05$ & $9.739 \mathrm{E}-10$ \\
7.00 & $9.781 \mathrm{E}-01$ & $2.151 \mathrm{E}-05$ & $9.728 \mathrm{E}-10$
\end{tabular}

Appendix K.2 Auxiliary lens potential

Auxiliary lens potential $V_{X}$ in units of $V_{C}$ can be expressed as a polynomial of sample height $d z_{C}$ in microns. The polynomial coefficients, noted here, change with magnification. Additional magnifications are possible.

\begin{tabular}{llll} 
Mag & $\left(d z_{C}\right)^{0}$ & $\left(d z_{C}\right)^{1}$ & $\left(d z_{C}\right)^{2}$ \\
\hline 3.50 & $4.15 \mathrm{E}-01$ & $7.59 \mathrm{E}-06$ & $-1.61 \mathrm{E}-10$ \\
4.00 & $4.00 \mathrm{E}-01$ & $9.19 \mathrm{E}-06$ & $-1.64 \mathrm{E}-10$ \\
4.50 & $3.84 \mathrm{E}-01$ & $1.11 \mathrm{E}-05$ & $-2.54 \mathrm{E}-10$ \\
5.00 & $3.66 \mathrm{E}-01$ & $1.34 \mathrm{E}-05$ & $-3.72 \mathrm{E}-10$ \\
5.50 & $3.48 \mathrm{E}-01$ & $1.60 \mathrm{E}-05$ & $-5.70 \mathrm{E}-10$ \\
6.00 & $3.27 \mathrm{E}-01$ & $1.91 \mathrm{E}-05$ & $-8.30 \mathrm{E}-10$ \\
6.50 & $3.05 \mathrm{E}-01$ & $2.30 \mathrm{E}-05$ & $-1.21 \mathrm{E}-09$ \\
7.00 & $2.79 \mathrm{E}-01$ & $2.83 \mathrm{E}-05$ & $-1.99 \mathrm{E}-09$
\end{tabular}

Appendix K.3 Spherical aberration

Transfer space spherical aberration $C_{s}^{\prime}$ without the cathode in units of mm expressed as a polynomial of sample height $d z_{C}$ in microns. The polynomial coefficients, noted here, change with magnification. Additional magnifications are possible. 


\begin{tabular}{lllll} 
Mag. & $\left(d z_{C}\right)^{0}$ & $\left(d z_{C}\right)^{1}$ & $\left(d z_{C}\right)^{2}$ & $\left(d z_{C}\right)^{3}$ \\
\hline 3.50 & $3.560 \mathrm{E}+05$ & $-6.139 \mathrm{E}+01$ & $3.537 \mathrm{E}-03$ & $0.000 \mathrm{E}+00$ \\
4.00 & $5.728 \mathrm{E}+05$ & $-1.015 \mathrm{E}+02$ & $5.748 \mathrm{E}-03$ & $0.000 \mathrm{E}+00$ \\
4.50 & $8.875 \mathrm{E}+05$ & $-1.670 \mathrm{E}+02$ & $1.187 \mathrm{E}-02$ & $0.000 \mathrm{E}+00$ \\
5.00 & $1.340 \mathrm{E}+06$ & $-2.775 \mathrm{E}+02$ & $2.589 \mathrm{E}-02$ & $0.000 \mathrm{E}+00$ \\
5.50 & $1.996 \mathrm{E}+06$ & $-4.716 \mathrm{E}+02$ & $6.079 \mathrm{E}-02$ & $0.000 \mathrm{E}+00$ \\
6.00 & $2.989 \mathrm{E}+06$ & $-8.617 \mathrm{E}+02$ & $1.547 \mathrm{E}-01$ & $0.000 \mathrm{E}+00$ \\
6.50 & $4.595 \mathrm{E}+06$ & $-1.719 \mathrm{E}+03$ & $5.808 \mathrm{E}-01$ & $-1.405 \mathrm{E}-04$ \\
7.00 & $7.618 \mathrm{E}+06$ & $-4.177 \mathrm{E}+03$ & $2.160 \mathrm{E}+00$ & $-6.689 \mathrm{E}-04$
\end{tabular}

Appendix K.4 Chromatic aberration

Transfer space chromatic aberration $C_{c}^{\prime}$ without the cathode in units of $\mathrm{mm}$ can be expressed as a polynomial of sample height $d z_{C}$ in microns. The polynomial coefficients, noted here, change with magnification. Additional magnifications are possible.

\begin{tabular}{lllll} 
Mag. & $\left(d z_{C}\right)^{0}$ & $\left(d z_{C}\right)^{1}$ & $\left(d z_{C}\right)^{2}$ & $\left(d z_{C}\right)^{3}$ \\
\hline 3.50 & $1.301 \mathrm{E}+03$ & $-6.558 \mathrm{E}-02$ & $9.133 \mathrm{E}-07$ & $0.000 \mathrm{E}+00$ \\
4.00 & $1.645 \mathrm{E}+03$ & $-8.480 \mathrm{E}-02$ & $1.630 \mathrm{E}-07$ & $0.000 \mathrm{E}+00$ \\
4.50 & $2.038 \mathrm{E}+03$ & $-1.114 \mathrm{E}-01$ & $1.579 \mathrm{E}-06$ & $0.000 \mathrm{E}+00$ \\
5.00 & $2.484 \mathrm{E}+03$ & $-1.473 \mathrm{E}-01$ & $3.719 \mathrm{E}-06$ & $0.000 \mathrm{E}+00$ \\
5.50 & $2.989 \mathrm{E}+03$ & $-1.934 \mathrm{E}-01$ & $8.990 \mathrm{E}-06$ & $0.000 \mathrm{E}+00$ \\
6.00 & $3.567 \mathrm{E}+03$ & $-2.591 \mathrm{E}-01$ & $1.791 \mathrm{E}-05$ & $0.000 \mathrm{E}+00$ \\
6.50 & $4.239 \mathrm{E}+03$ & $-3.573 \mathrm{E}-01$ & $4.125 \mathrm{E}-05$ & $-6.740 \mathrm{E}-09$ \\
7.00 & $5.039 \mathrm{E}+03$ & $-5.267 \mathrm{E}-01$ & $9.457 \mathrm{E}-05$ & $-1.882 \mathrm{E}-08$
\end{tabular}

